INVESTIGATION OF MULTISCALE AND MULTIPHASE FLOW, TRANSPORT AND REACTION IN HEAVY OIL RECOVERY PROCESSES

Final Report

May 6, 1999-January 5, 2003

By

Yannis C. Yortsos

Date Published: March 2003

Work Performed Under Contract No. DE-AC26-99BC15211

University of Southern California Los Angeles, California

National Energy Technology Laboratory National Petroleum Technology Office U.S. DEPARTMENT OF ENERGY Tulsa, Oklahoma 


\section{DISCLAIMER}

This report was prepared as an account of work sponsored by an agency of the United States Government. Neither the United States Government nor any agency thereof, nor any of their employees, makes any warranty, expressed or implied, or assumes any legal liability or responsibility for the accuracy, completeness, or usefulness of any information, apparatus, product, or process disclosed, or represents that its use would not infringe privately owned rights. Reference herein to any specific commercial product, process, or service by trade name, trademark, manufacturer, or otherwise does not necessarily constitute or imply its endorsement, recommendation, or favoring by the United States Government or any agency thereof. The views and opinions of authors expressed herein do not necessarily state or reflect those of the United States Government.

This report has been reproduced directly from the best available copy. 
$\mathrm{DOE} / \mathrm{BC} / 15211-22$

Distribution Category UC-122

\title{
Investigation of Multiscale and Multiphase Flow, Transport and Reaction in Heavy Oil Recovery Processes
}

\author{
March 2003
}

Work Performed Under DE-AC26-99BC15211

\author{
Prepared for \\ U.S. Department of Energy \\ Assistant Secretary for Fossil Energy \\ Jerry Casteel, Project Manager \\ National Energy Technology Laboratory \\ National Petroleum Technology Office \\ One West Third Street, Suite 1400 \\ Tulsa, OK 74103 \\ Prepared by \\ Petroleum Engineering Program \\ Department of Chemical Engineering \\ University of Southern California \\ University Park Campus - EDCO 316, USC \\ Los Angeles, CA 90090-1211
}




\section{TABLE OF CONTENTS}

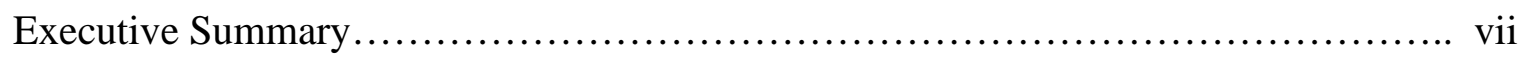

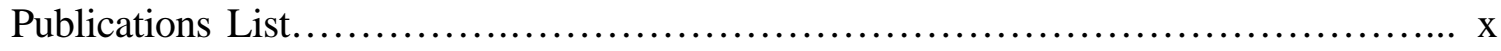

Ph D Theses List....................................................................... xiii

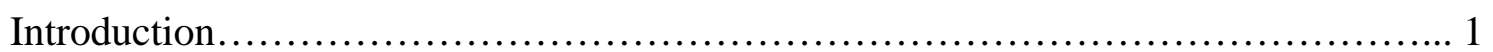

I. Internal Drives in Porous Media............................................. 2

A Model for the Gas Evolution in a Porous Medium Driven by Solute Diffusion... 5

An Effective Continuum Model for the Gas Evolution in Internal Steam Drives.... 67

The Critical Gas Saturation in Porous Media in the Presence of Gravity........... 103

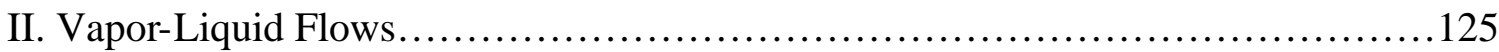

Darcian Dynamics: A New Approach for the Mobilization of Ganglia in Porous

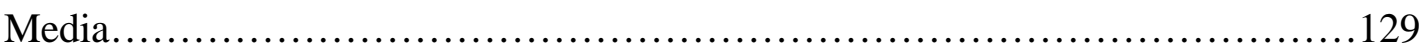

Stability of Vapor-Liquid Counter-Current Flow (Heat Pipes) in the Presence of

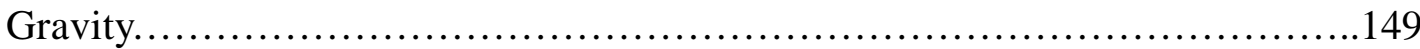

A 2-D Pore-Network Model of the Drying of Single-Component Liquids in Porous

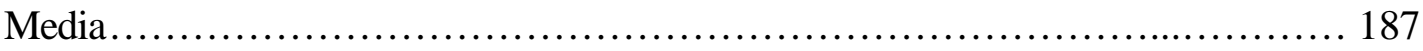

III. Dynamics of In-Situ Combustion at Various Scales ............................ 225

The Dynamics of In-Situ Combustion Fronts in Porous Media.................... 229

A Pore-Network Model of In-Situ Combustion in Porous Media.................. 273

The Effect of Heterogeneity on In-situ Combustion: The Propagation of Combustion

Fronts in Layered Porous Media................................................. 299

IV. Flow and Displacement of Fluids with Yield Stress and Unstable Flows In Porous

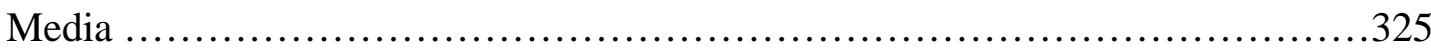

Mobilization and Displacement of Fluids with Yield Stress in Porous Media......329 Asymptotic Regimes in Unstable Miscible Displacements in Random Porous Media 



\begin{abstract}
This is final report for contract DE-AC26-99BC15211. The report describes progress made in the various thrust areas of the project, which include internal drives for oil recovery, vapor-liquid flows, combustion and reaction processes and the flow of fluids with yield stress. The report consists mainly of a compilation of various topical reports, technical papers and research reports published produced during the three-year project, which ended on May 6, 2002 and was no-cost extended to January 5, 2003. Advances in multiple processes and at various scales are described.
\end{abstract}

In the area of internal drives, significant research accomplishments were made in the modeling of gas-phase growth driven by mass transfer, as in solution-gas drive, and by heat transfer, as in internal steam drives. In the area of vapor-liquid flows, we studied various aspects of concurrent and countercurrent flows, including stability analyses of vapor-liquid counterflow, and the development of novel methods for the pore-network modeling of the mobilization of trapped phases and liquid-vapor phase changes. In the area of combustion, we developed new methods for the modeling of these processes at the continuum and pore-network scales. These models allow us to understand a number of important aspects of in-situ combustion, including steady-state front propagation, multiple steady-states, effects of heterogeneity and modes of combustion (forward or reverse). Additional aspects of reactive transport in porous media were also studied. Finally, significant advances were made in the flow and displacement of non-Newtonian fluids with Bingham plastic rheology, which is characteristic of various heavy oil processes. Various accomplishments in generic displacements in porous media and corresponding effects of reservoir heterogeneity are also cited.

A total of 44 publications in refereed journals and/or in scientific conferences resulted from this project. In addition, several papers are under preparation at the time of the writing of this report. The support provided by the contract also allowed the completion of $6 \mathrm{PhD}$ Theses, with two more under way. This research output would have been impossible without this support, for which the PI and the students are grateful. 



\section{EXECUTIVE SUMMARY}

This is the final report of an investigation on the various multi-phase and multiscale transport and reaction processes associated with heavy oil recovery. As we reported in detail in three previous annual reports, the thrust areas in this study include the following: Internal drives, vapor-liquid flows, combustion and reaction processes, fluid displacements, the effect of instabilities and heterogeneities, and the flow of fluids with yield stress. These find respective applications to: foamy oils, the evolution of dissolved gas, internal steam drives, the mechanics of concurrent and countercurrent vapor-liquid flows, associated with thermal methods and steam injection, such as SAGD, the in-situ combustion, the upscaling of displacements in heterogeneous media, the flow of foams, and fluids with yield stress in porous media.

Internal drives, namely displacements driven by an applied supersaturation in dissolved gases or heat content, are common to many processes associated with heavy oil recovery. The result of such processes is the growth of a gas phase, driven by mass or heat transfer, depending on the kind of the applied supersaturation. We have conducted a number of fundamental studies of this multifaceted problem. Two separate processes were studied: The growth of the gas phase from a supersaturated liquid due to pressure decline; and the growth of a gas phase from a superheated liquid, also due to pressure decline. The first is common to solution gas-drive processes, as in the case of foamy oil production; the second to internal steam drives. Common between the two are nucleation and phase change processes. We have examined in great detail the mechanisms of nucleation and phase change and formulated novel approaches for their modeling. Our results show that for the quantitative description and control of these problems, including the very important property of the critical gas saturation, it is necessary to match correctly the nucleation characteristics of the medium. Based on these models, and with the appropriate selection of the nucleation parameters, good agreement with experimental results is found. The advances related to phase change are reported in publications I1-I8 listed below, and in the PhD Thesis of I.N. Tsimpanogiannis, also listed below. In this final report, we present characteristic examples of these works. 
The simultaneous flow of vapor and liquid phases is common to steam injection. Counter-current flows are encountered in Steam-Assisted-Gravity-Drainage (SAGD), and in steam injection in horizontal wells. Concurrent flows are found in typical displacements, in solution gas-drives near wells, and various other contexts. An important aspect to these problems is the flow of the gas as a disconnected phase in the form of trapped bubbles or ganglia, which are mobilized, entrained, flow, coalesce and/or become stranded. To solve this fundamental problem we used a completely new approach, introduced for the first time in this work, based on what we term Darcian Dynamics. It is a computationally fast approach for the evaluation of quantities in concurrent and counter-current flows. The approach allows us to determine the probabilities of mobilization and/or stranding, which are necessary for the modeling of such flows, including relative permabilities. In parallel, we considered the stability of counter-current vapor-liquid flows. We developed a linear stability analysis for a twophase heat pipe zone (vapor-liquid counterflow) in a porous medium, overlying a superheated vapor zone. The competing effects of gravity, condensation and heat transfer on the stability of a planar base state are analyzed in the linear stability limit. The rate of growth of unstable disturbances is expressed in terms of the wave number of the disturbance, and dimensionless numbers, such as the Rayleigh number, a dimensionless heat flux and other parameters. A critical Rayleigh number is identified and shown to be different than in natural convection under single-phase conditions. In addition, we investigated in detail drying processes involved in vapor-liquid systems. The progress reported here describes in a fundamental way the evaporation of a liquid phase in a porous medium using a pore-network approach. The effect of the various parameters on the process, including macroscopic liquid films, was extensively analyzed. Publications II1-II8 and the PhD Thesis by P. Amili detail the work conducted in these two areas. In this report we present characteristic examples illustrating this work.

A well-established method for the recovery of heavy oils is in-situ combustion. Two particular aspects were analyzed in this project: The description of the process at the pore-network scale, and its upscaling at the large scale for field applications. For the first 
time in the literature, a detailed pore-network simulator was developed (Chuan $\mathrm{Lu}, \mathrm{PhD}$ Thesis under completion). This simulator models the process at the small scale. It predicts a number of important phenomena, such as the onset of fingering in reverse combustion, the existence of a percolation threshold in the fuel density, and other microstructure-related properties. A number of publications in this area are forthcoming. In parallel, we conducted a systematic study of combustion at the continuum-large scale, using an approach based on front dynamics, in order to describe the movement of combustion fronts in porous media. This approach is essential for the upscaling of the process at the field scale. We applied this technique to in-situ combustion both in a homogeneous and a heterogeneous reservoir. Importantly, we show that heterogeneity, combined with heat losses, can lead to extinction phenomena, otherwise absent in homogeneous systems. The PhD Thesis of Y.I. Akkutlu details the progress made in this area. More generally, related to this problem is the area of reactive flow in porous media. We have studied, in this context, a number of problems, including acidization processes, and the upscaling of the information from the pore-scale to the scale of the continuum. The $\mathrm{PhD}$ Thesis of $\mathrm{P}$. Kechagia addressed some of these aspects and was partly supported by this contract. The publications related to all these areas are listed below as III1-III11. In this report we present characteristic examples of the work conducted, one involving a pore-network approach, and two others involving the continuum model, all for the in-situ combustion problem.

Heavy oils and heavy-oil associated recovery processes involve non-Newtonian rheology. Of specific importance is that of Bingham plastics (fluids with yield stress), which characterize the flow of foams, heavy oils containing asphaltenes, as well as the rheology during the formation of wormholes in sand production. This topic forms the main aspect of the Thesis of M. Chen. In addition, we have conducted a number of studies on flow and displacements in porous media, particularly on the effect of heterogeneity, the role of fractured reservoirs, the identification of heterogeneity and the effect of viscous instabilities on displacement processes. While many of these are generic to displacements in porous media, they are also applicable to the displacement of heavy oil. The work in this general area is described in publications IV1-IV17 and in the PhD Theses of L. Zhan, 
M. Shariati and M. Chen. In this report we present two characteristic examples of the work conducted.

A total of 44 publications in refereed journals and/or in scientific conferences resulted from this project. A list is attached at the end of the summary. In addition, several papers are under preparation at the time of the writing of this report. Finally, the support provided by this contract allowed the completion of $6 \mathrm{PhD}$ Theses, with two more under way. These are also listed.

\section{PUBLICATIONS LIST}

\section{Internal drives in porous media}

1. Tsimpanogiannis, I.N., and Yortsos, Y.C., The Critical Gas Saturation in a Porous Medium in the Presence of Gravity, J. Colloid Interface Sci., submitted (2003).

2. Tsimpanogiannis, I.N., and Yortsos, Y.C., An Effective Continuum Model for the Gas Evolution in Internal Steam Drives, SPEJ, to appear (2003).

3. Tsimpanogiannis, I.N., and Yortsos, Y.C., Model for the Gas Evolution in a Porous Medium Driven by Solute Diffusion, AIChEJ 48, 2690-2710 (2002).

4. Tsimpanogiannis, I.N., and Yortsos, Y.C., A Continuum Model for the Evolution of Gas During Solution-Gas Drive, SPEJ, accepted (2002).

5. Yortsos, Y.C., and Stubos, A.K, Phase Change in Porous Media, Current Opinions in Colloid and Interface Science 6, 208-216 (2001).

6. Tsimpanogiannis, I.N., and Yortsos, Y.C., An Effective Continuum Model for the Gas Evolution in Internal Steam Drives, paper SPE 75242 presented at the $13^{\text {th }}$ SPE/DOE Symposium on Improved Oil Recovery, Tulsa, OK (April 13-17, 2002).

7. Tsimpanogiannis, I.N., and Yortsos, Y.C., A Continuum Model for the Evolution of Gas During Solution-Gas Drive, paper SPE 71502 presented at the 75th SPE Annual Fall Meeting, New Orleans, LA (Oct. 1-3, 2001).

8. Tsimpanogiannis, I.N., Yortsos, Y.C., and Stubos, A.K., Evaporation of a Stagnant Liquid, Ind. Eng. Chem. Res. 39, 1505-1513 (2000).

\section{Vapor-liquid flows in porous media}

1. Yiotis, A., Boudouvis, A.G., Stubos, A.K., Tsimpanogiannis, I.N., and Yortsos, Y.C., The Effect of Liquid Films on the Isothermal Drying of Porous Media, Phys. Rev. Lett., submitted (2003). 
2. Amili, P., and Yortsos, Y.C., Darcian Dyanmics, Phys. Fluids, to be submitted (2003).

3. Amili, P., and Yortsos, Y. C., Mobilization of Ganglia in Porous Media Using Darcian Dynamics, paper SPE 75191, presented at the $13^{\text {th }}$ SPE/DOE Symposium on Improved Oil Recovery, Tulsa, OK (April 13-17, 2002).

4. Yiotis, A.G., Stubos, A.K., Bountouvis, A., and Yortsos, Y.C., A 2-D Pore-Network Model of the Drying of Single-Component Liquids in Porous Media, Adv. Water Res. 24, 437-458 (2001).

5. Amili, P., and Yortsos, Y. C., Darcian Dynamics, paper presented at the AIChE Fall Meeting, Reno, NV (November 5-8, 2001).

6. Yiotis, A., Stubos, A. K., Bountouvis, A., and Yortsos, Y. C., A Pore-Network Model of Drying in Porous Media, paper presented at the AIChE Fall Meeting, Reno, NV (November 5-8, 2001).

7. Laroche, C., Chen, M., Kamath, J., and Yortsos, Y.C., Determining Relative Permeability Exponents Near the Residual Saturation, paper SPE 71489 presented at the 75th SPE Annual Fall Meeting, New Orleans, LA (Oct. 1-3, 2001).

8. Amili, P. and Yortsos, Y. C., Stability of Heat Pipes in Vapor-Dominated Systems, paper presented at the ASME Fall Meeting, Nashville, TN (November 14-19, 1999).

\section{Combustion and reaction processes in porous media}

1. Akkutlu, Y.I, and Yortsos, Y.C., The Effect of Heterogeneity on In-situ Combustion: ThePropagation of Combustion Fronts in Layered Porous Media, SPEJ, submitted; also highlighted in Jour. Pet. Tech., page 56 (June 2002).

2. Kechagia, P., Tsimpanogiannis, I.N., Yortsos, Y.C. and Lichtner, P., On the Upscaling of Reaction-Transport Processes in Porous Media with Fast Kinetics, Chem. Eng. Sci. 57, 2565-2577 (2002).

3. Akkutlu, Y.I, and Yortsos, Y.C., The Effect of Heterogeneity on In-situ Combustion: ThePropagation of Combustion Fronts in Layered Porous Media, paper SPE 75128, presented at the $13^{\text {th }}$ SPE/DOE Symposium on Improved Oil Recovery, Tulsa, OK (April 13-17, 2002).

4. Akkutlu, I.Y., and Yortsos, Y.C., The Dynamics of Combustion Fronts in Porous Media, Combustion and Flame, submitted (2001).

5. Lu, C., Kechagia, P., Yortsos, Y. C., and Lichtner, P., Non-Local Upscaling of ReactionTransport in Porous Media Using a Hybrid Algorithm, paper presented at the AIChE Fall Meeting, Reno, NV (November 5-8, 2001).

6. Lu, C., and Yortsos, Y.C., A Pore-Network Model of Combustion in Porous Media, paper SPE 69705 presented at the International Thermal Operations and Heavy Oil Symposium (ITOHOS), Margarita Island, Venezuela (March 12-14, 2001). 
7. Kechagia, P., Yortsos, Y.C. and Lichtner, P., A Non-Local KPZ Equation to Model Interface Growth, Phys. Rev. E 64, 016315-1-15 (2001).

8. Lu, C., and Yortsos, Y. C., A Pore-Network Model of Smoldering Combustion, paper presented at the AIChE Fall Meeting, Los Angeles, CA (November 12-17, 2000).

9. Kechagia, P., Yortsos, Y. C. and Lichtner, P., On the Constraint of Local Equilibrium in the Upscaling of Reaction and Transport Problems in Heterogeneous Porous Media, paper presented at the AIChE Fall Meeting, Los Angeles, CA (November 12-17, 2000).

10. Akkutlu, I., and Yortsos, Y.C., The Dynamics of Combustion Fronts in Porous Media, paper SPE 63225 proceedings of the 74th SPE Annual Fall Meeting, Dallas, TX (Oct. 1-4, 2000).

11. Lu, C. and Yortsos, Y.C., The Dynamics of Combustion in Porous Media at the PoreNetwork Scale, paper presented at the 7th European Conference on the Mathematics of Oil Recovery, Baveno, Lago Maggiore, Italy (Sept. 5-8, 2000).

\section{Heterogeneity, instabilities and fluid flow in porous media}

1. Talon, L., Martin, J., Rakotomalala, N., Salin, D. and Yortsos, Y.C., Lattice BGK Simulations of Macrodispersion in Heterogeneous Porous Media, Water Res. Res., to appear (2003).

2. Yang, Z., Yortsos, Y.C., and Salin, D., Asymptotic Regimes of Unstable Miscible Displacements in Random Porous Media, Adv. Water Res., special anniversary issue, 25, 885-898 (2002).

3. Zhan, L. and Yortsos, Y.C., The Shape of a Gravity Finger in a Rectangular Channel in a Homogeneous Porous Medium, Transport in Porous Media 49, 77-97 (2002)..

4. Laroche, C., Chen, M., Yortsos, Y.C., and Kamath, J., Time Scaling of the Rates of Produced Fluids in Laboratory Displacements, SPEJ, submitted (2001).

5. Shariati, M. and Yortsos, Y.C., Stability of Miscible Displacements Across Stratified Porous Media, Phys. Fluids 13, 2245-2257 (2001).

6. Yortsos, Y.C., Xu, B., and Salin, D., Delineation of Microscale Regimes in Fully Developed Drainage and Implications for Continuum Models, Comp. Geosc. 5, 257-278 (2001).

7. Zhan, L. and Yortsos, Y.C., A Direct Method for the Identification of the Permeability Field of an Anisotropic Porous Medium, Water Res. Res. 37, 1929-1938 (2001).

8. Lajeunesse, E., Martin J., Rakotomalala, N., Salin, D., and Yortsos, Y.C., The Threshold of Instability in Miscible Displacements in a Hele-Shaw Cell at High Rates, Phys. Fluids 13, 799 (2001). 
9. Zeng, J., Yortsos, Y. C., and Salin, D., On the Brinkman Correction in Hele-Shaw Flows, paper presented at the APS-DFD meeting, San Diego, CA (Nov. 18-20, 2001).

10. Yortsos, Y.C., The Permeability Variogram from Pressure Transients of Multiple Wells, in "Theory, Modeling, and Field Investigation in Hydrogeology: A Special Volume in Honor of Shlomo P. Neuman's 60th Birthday" (D. Zhang, and C.L. Winter, eds.), Geological Society of America Special Paper 348, 19-23 (2000).

11. Yortsos, Y.C., Physical Considerations in the Upscaling of Immiscible Displacements in a Fractured Medium, in "Dynamics of Fluids in Fractured Rock" (B. Faybisenko, P.A. Witherspoon and S.M. Benson, eds.), Geophysical Monograph Series 122, American Geophysical Union, 235-251 (2000).

12. Zhan, L., and Yortsos, Y.C., Identification of the Permeability Field of a Porous Media from the Injection of a Passive Tracer, Phys. Rev. E 62, 863-879 (2000).

13. Zhan, L. and Yortsos, Y.C., A Direct Method for the Identification of the Permeability Field of an Anisotropic Porous Medium, paper SPE 62976 proceedings of the 74th SPE Annual Fall Meeting, Dallas, TX (Oct. 1-4, 2000).

14. Zhang, Y., Shariati, M. and Yortsos, Y.C., The Spreading of Immiscible Fluids in Porous Media Under the Influence of Gravity, Transport in Porous Media 38, 117-140 (2000).

15. Shariati, M., and Yortsos, Y. C., The Effect of Heterogeneity on the Stability of Miscible Displacements in Porous Media, paper presented at the AGU Fall Meeting, San Francisco, CA (December 16, 1999).

16. Shariati, M., and Yortsos, Y. C., Effect of Heterogeneity on the Stability of Displacement Processes in Porous Media with Non-Monotonic Viscosity, paper presented at the AIChE Fall Meeting, Dallas, TX (November 1-5, 1999).

17. Zhan, L., and Yortsos, Y. C., Identification of the Permeability Heterogeneity of Porous Media from the Displacement of a Passive Tracer, paper presented at the AIChE Fall Meeting, Dallas, TX (November 1-5, 1999).

\section{PHD THESES LIST}

1. Min Chen, Investigations on the Flow and Displacement of Fluids with Yield Stress, University of Southern California, December 2003 (expected).

2. Chuan Lu, Pore-Network Models for Combustion Processes in Porous Media, University of Southern California, May 2003 (expected).

3. Ioannis N. Tsimpanogiannis, Studies on Processes Involving Liquid-to-Gas Phase Change in Porous Media, University of Southern California, May 2002.

4. Yucel Akkutlu, The Dynamics of Combustion in Porous Media, University of Southern California, April 2002. 
5. Pouya Amili, Two Studies in Simultaneous Two-Phase Flow in Porous Media: I. Heat Pipe Stability, II. Darcian Dynamics, University of Southern California, January 2002.

6. Persefoni Kechagia, Non-local Upscaling Models for Reaction and Transport Processes in Porous Media, University of Southern California, May 2001.

7. Lang Zhan, Identification of the Permeability Heterogeneity of Porous Media by the Injection of a Passive Tracer, University of Southern California, June 2000.

8. Maryam Shariati, Effect of Non-Monotonic Mobility at Various Scales in Porous Media, University of Southern California , May 2000. 


\section{ACKNOWLEDGEMENTS}

This research was made possible through the Department of Energy contract DE-AC2699BC15211, the contribution of which is gratefully acknowledged. The PI would like to express his gratitude to the NPTO Project officers in charge of the contract, Dr. Jerry Casteel and the late Mr. Thomas Reid for their help and support. 



\section{INTRODUCTION}

The project undertaken is a thorough investigation of various multi-phase and multiscale transport and reaction processes associated with heavy oil recovery. The thrust areas of the project include the following: Internal drives, vapor-liquid flows, combustion and reaction processes, fluid displacements and the effect of instabilities and heterogeneities and the flow of fluids with yield stress. These find respective applications in foamy oils, the evolution of dissolved gas, internal steam drives, the mechanics of concurrent and countercurrent vapor-liquid flows, associated with thermal methods and steam injection, such as SAGD, in-situ combustion, the upscaling of displacements in heterogeneous media and the flow of foams, Bingham plastics and heavy oils in porous media. Funding of the project was for three years, from May 6, 1999 to May 5, 2002, extended at no cost to January 5, 2003.

In this final report, characteristic samples of the progress made in the various areas outlined above, are described. Work was conducted in all areas, with progress being greater in some areas compared to others. During the period of the work, a total of up to 8 students were supported by the project. A total of 44 publications, 11 technical reports, and $8 \mathrm{PhD}$ Theses (two currently under completion) have resulted from this effort. These were listed in the preceding section and will also be analyzed in more detail in the sections to follow. The report is essentially a compilation of some of these publications.

This report is organized as follows: For each of the four first thrust areas, namely internal drives, vapor-liquid flows, combustion dynamics, and flows with yield stress, instabilities and heterogeneity, we provide a brief summary of the work performed, followed by two reports each. 


\section{INTERNAL DRIVES IN POROUS MEDIA}

In many processes associated with heavy oil recovery, internal drives, namely these driven by applied supersaturation in dissolved gases or heat content, are common. These include, but are not limited to the evolution of gas in foamy oils, internal steam drives, the evaporation of volatile components during gas injection or the injection of steam, and other processes. The main result is the growth of a gas phase, which is driven by mass or heat transfer, depending on the kind of the applied supersaturation. We have conducted various studies of this multifaceted problem. In this report, we present results in two areas, one associated with the nucleation and growth of a gas phase from a supersaturated liquid, and another describing the evolution of a gas phase during an internal steam drive. The studies describe a mathematical model of the dynamics of gas evolution as a function of the rate of application of the supersaturation for solution gas drive and for internal steam drive. Phase equilibria thermodynamics, particulary in pore networks, nucleation of phase change, and growth kinetics, emphasizing the pore-network structure, are highlighted. Then, we extend the theory for the critical gas saturation to problems involving gravity.

The first two studies derive effective continuum models to describe the nucleation and subsequent growth of a gas phase from a supersaturated, slightly compressible binary liquid in a porous medium, driven by solute diffusion or by heat transfer in the respective cases. The evolution of the gas results either from the reduction of the system pressure at a constant rate or the withdrawal of liquid at constant rate. The models addresse two stages before the onset of bulk gas flow, nucleation and gas phase growth. We assume negligible gradients due to gravity or viscous forces, thus the critical gas saturation, which signals the onset of bulk gas flow, is only a function of the nucleation fraction. The effect of gradients was analyzed in a separate work (Tsimpanogiannis and Yortsos, 2003). We show that the important quantities characterizing the process, such as the fraction of pores that host activated sites, the deviation from thermodynamic equilibrium, the maximum supersaturation in the system and the critical gas saturation depend crucially on the nucleation characteristics of the medium. 
We use heterogeneous nucleation models primarily in the form of pre-existing gas, trapped in hydrophobic cavities, but also in terms of a rate-dependent nucleation, to investigate in detail the nucleation behavior. Using scaling analysis and a simpler analytical model we show that the relevant quantities during nucleation can be expressed in terms of a simple combination of dimensionless parameters, which include rate effects, for either type of nucleation model. The theory predicts that the maximum supersaturation in the system is a weakly increasing function of rate, which in the region of typical experimental parameters, can be approximated as a power law with a small exponent. This function depends sensitively on the probability density function of the nucleation cavity sizes. It also predicts that the final nucleation fraction, thus the critical gas saturation, is a power law of the decline rate. The theoretical exponents are shown to be in good agreement with experimental data. The subsequent evolution of the gas phase and the approach to the critical gas saturation is also described.

Then, we address one important additional effect on the critical gas saturation, that of buoyancy. We use 2-D pore-network simulations, based on Invasion Percolation in a Gradient (IPG), and corresponding scaling relations, to obtain the dependence of the critical gas saturation on the gravity Bond number, under conditions of slow growth, namely when mass transfer is sufficiently fast. For a region of intermediate Bond numbers, the critical gas saturation is found to scale as a power-law of the Bond number with an exponent of -0.91 .

Publications resulting from this part of the project are listed below:

1. Tsimpanogiannis, I.N., and Yortsos, Y.C., The Critical Gas Saturation in a Porous Medium in the Presence of Gravity, J. Colloid Interface Sci., submitted (2003).

2. Tsimpanogiannis, I.N., and Yortsos, Y.C., An Effective Continuum Model for the Gas Evolution in Internal Steam Drives, SPEJ, to appear (2003).

3. Tsimpanogiannis, I.N., and Yortsos, Y.C., Model for the Gas Evolution in a Porous Medium Driven by Solute Diffusion, AIChEJ 48, 2690-2710 (2002).

4. Tsimpanogiannis, I.N., and Yortsos, Y.C., A Continuum Model for the Evolution of Gas During Solution-Gas Drive, SPEJ, accepted (2002). 
5. Yortsos, Y.C., and Stubos, A.K, Phase Change in Porous Media, Current Opinions in Colloid and Interface Science 6, 208-216 (2001).

6. Tsimpanogiannis, I.N., and Yortsos, Y.C., An Effective Continuum Model for the Gas Evolution in Internal Steam Drives, paper SPE 75242 presented at the $13^{\text {th }}$ SPE/DOE Symposium on Improved Oil Recovery, Tulsa, OK (April 13-17, 2002).

7. Tsimpanogiannis, I.N., and Yortsos, Y.C., A Continuum Model for the Evolution of Gas During Solution-Gas Drive, paper SPE 71502 presented at the 75th SPE Annual Fall Meeting, New Orleans, LA (Oct. 1-3, 2001).

8. Tsimpanogiannis, I.N., Yortsos, Y.C., and Stubos, A.K., Evaporation of a Stagnant Liquid, Ind. Eng. Chem. Res. 39, 1505-1513 (2000).

The following $\mathrm{PhD}$ Thesis was also completed in this specific area:

1. Ioannis N. Tsimpanogiannis, Studies on Processes Involving Liquid-to-Gas Phase Change in Porous Media, University of Southern California, May 2002. 


\title{
A Model for the Gas Evolution in a Porous Medium Driven by Solute Diffusion
}

\author{
Ioannis N. Tsimpanogiannis and Yannis C. Yortsos
}

\section{INTRODUCTION}

The liquid-to-gas phase change in a porous medium and the subsequent growth of the gas phase is encountered in many applications driven by mass or heat transfer. These span various fields of scientific interest and a range of length scales. Examples include the solution gas-drive process for the recovery of oil from oil reservoirs (Sheng et al., 1999a, 1999b), boiling in porous media (Thome, 1990; Satik and Yortsos, 1996), thermal methods for oil recovery (Prats, 1982), nuclear waste disposal (Doughty and Pruess, 1990), soil remediation (Ho and Udell, 1995) and others. In this paper, we focus on the isothermal gas phase growth from a supersaturated, slightly compressible, binary liquid in a porous medium. This is driven by mass transfer, the extent of which is controlled by the application of either a constant-rate decline of the system pressure or the withdrawal of the liquid at a constant rate.

Consider the removal of an initially supersaturated liquid from a porous medium of a fixed volume (Fig. 1). As the pressure continuously declines, due to liquid expansion, the bubble point of the liquid is eventually reached. Then, nucleation of a gas phase starts, at rates depending on the nucleation properties of the medium. Nucleation is manifested either in the release of pre-existing gas bubbles, trapped in hydrophobic cavities, or in the form of heterogeneously nucleated nuclei. Emphasis will be placed on the former mechanism, although the conventional model will also be used. Because of the competing processes of bubble growth, which depletes the solute from the liquid, thus reducing the supersaturation, and the liquid withdrawal, which reduces the pressure, thus increasing the supersaturation, a supersaturation maximum is attained, following which, nucleation terminates. Identifying the maximum supersaturation and its dependence on process parameters is a key issue. The subsequent gas evolution is controlled by the available supersaturation, the solute mass transfer from the liquid to the gas and the capillary characteristics of the porous medium. The gas phase appears first in the form of small bubbles growing within the confines of single pores (Fig. 2a), but ultimately takes the form of large clusters, spanning a number of pores (Fig. 2b). Competition for mass transfer between the growing bubbles or clusters, capillary 
effects at pore constrictions, viscous and gravity forces, and the possibility of coalescence or the snap-off of gas-liquid interfaces are important factors in determining the gas-phase evolution.

Eventually, gas flows as a bulk phase. The onset of flow is signaled when the gas porevolume fraction, $S_{g}$, becomes equal to the so-called critical gas saturation, $S_{g c}$, a value which depends on the underlying growth and flow mechanisms. If viscous or gravity gradients are negligible, gas flow occurs for the first time when isolated gas clusters connect to form a sample-spanning (percolation) cluster (Yortsos and Parlar, 1989). If they do not, gas flow and production occur through the continuous motion of finite-size gas clusters, subject to various mechanisms of interaction, including coalescence. This simultaneous flow of gas and liquid is quite complex, particularly under strong pressure gradients, for example in the case of high-viscosity oils, where "foamy" oil flow takes place (Smith, 1988; Maini, 1996, 1999). In this paper, we will only consider the stage before the onset of gas flow, however, and in the absence of significant gravity or viscous gradients.

A number of studies have been reported on this problem. A review of the early literature can be found in Li and Yortsos (1995a, 1995b). Experimental work for the case of constant pressure decline rate in consolidated porous media using light oils was reported by Moulu and Longeron (1989), Moulu (1989) and Scherpenisse et al. (1994). Sheng et al. (1999b), Wong et al. (1999) and Urgelli et al. (1999) conducted experiments with heavy oils. Visualization experiments with light oils were reported by Li and Yortsos (1995a), Hawes et al. (1997), Mackay et al. (1998) and Dominguez et al. (2000). Bora et al. (2000) reported experiments with heavy oils. These studies have shown that the critical gas saturation is an increasing function of the liquid withdrawal rate, a finding explained by the increasing number of nucleation centers at larger depletion rates. Scherpenisse et al. (1994) provided useful, but qualitative, scaling arguments showing that maximum supersaturation and critical gas saturation are power-law functions of the depletion rate. A theoretical analysis of bubble growth by solute diffusion in which mass transfer and porous medium capillarity dominate, was provided by Li and Yortsos (1995a, 1995b). The authors conducted visualization experiments in glass micromodels and pore-network simulations to explain patterns and rates of growth of the gas phase at the pore-network scale. Along similar lines, Du and 
Yortsos (1999) provided a pore-network analysis of the critical gas saturation, in the absence of gravity/viscous gradients. They confirmed an earlier hypothesis by Yortsos and Parlar (1989) that in the absence of spatial gradients, the onset of critical gas saturation coincides with the percolation threshold of an invasion percolation process, originating from multiple nucleation centers. They also showed that $S_{g c}$ is a power law of the final nucleation fraction (defined more precisely below), $f_{q f}$, namely

$$
S_{g c}=f_{q f}^{1-D_{f} / E}
$$

Here, $E$ (equal to 2 or 3 ) is the (Euclidean) dimension of the pore network and $D_{f}$ is the mass fractal dimension of the percolation cluster (equal to 1.82 for 2-D Invasion Percolation (IP) with trapping, and 2.53 for 3 -D IP with or without trapping, Feder, 1988). The dependence in (1) was established regardless of the nucleation sequence (instantaneous or sequential) or the particular regime of bubble growth (see Li and Yortsos, 1995a, 1995b).

The presence of gradients will affect the above scaling. Pore-network simulations conducted by McDougal and Sorbie (1999) and Wang and Mohanty (1999) in the related topic of gas condensation, showed that $S_{g c}$ decreases as the hydrostatic pressure gradient increases, a trend also anticipated in Scherpenisse et al. (1994). In a parallel study (Tsimpanogiannis and Yortsos, 2002), we have analyzed the effect of gravity and/or viscous forces on $S_{g c}$, and developed scaling laws for the dependence of $S_{g c}$ on $f_{q f}$ and on two dimensionless parameters, the Bond and capillary numbers, defined respectively as

$$
B=\frac{\Delta \rho g k}{\gamma} \text { and } C a=\frac{q \mu}{\gamma}
$$

Here $\Delta \rho$ denotes the density difference between liquid and gas, $k$ is permeability, $\gamma$ the liquid-gas interfacial tension, $q$ the liquid flow rate and $\mu$ the liquid viscosity. Equation (1) is obtained in the limits $B \ll 1$ and $C a \ll 1$ (more specifically, $B \leq 10^{-5}$ and $C a \leq 10^{-5}$ ), which are the regions of interest of this paper.

In many practical cases, pressure depletion is due to liquid withdrawal at a constant flow rate. Experimental work in consolidated porous media with light oils was reported by Firoozabadi et al. (1992), Firoozabadi and Aronson (1999) and Egermann and Vizika (2000). These studies focused on the critical gas saturation, which was found to be an increasing 
function of the liquid withdrawal rate. As before, this finding was explained by the increasing number of nucleation centers, from which gas clusters grow, at larger depletion rates. Sheng et al. (1999a) and Renard et al. (2000) in two recent reviews focused on aspects of solution gas drive related to the primary recovery of heavy oil.

A modeling attempt to capture the gas phase growth, but not the preceding nucleation period, in an experiment at a constant rate of withdrawal, was made by Firoozabadi and Kashchiev (1997). These authors used an effective continuum model with bubble growth driven by diffusion. The gas phase is modeled as a collection of effective bubbles, mass transfer to which is approximated by simple expressions. Although the paper discusses ratedependent nucleation using classical expressions (see also below), the nucleation issue is in fact bypassed, in that nucleation fraction, the maximum supersaturation, or the effect of depletion rate on the number of bubbles nucleated, are not actually predicted or calculated. Rather, the latter quantities are inferred from the experimental results, and subsequently used as parameters for the gas phase growth following the nucleation period.

Experiments on pressure depletion driven by constant liquid withdrawal rate, particularly with heavy oils, were reported by Pooladi-Darvish and Firoozabadi (1999), Tang and Firoozabadi (1999), Kumar et al. (2000), Andarcia et al. (2001), Arora and Kovscek (2001) and Kamp et al. (2001a, 2001b). In two very recent studies, which appeared at the same time this work was being written, Kamp et al. (2001a) and Arora and Kovscek (2001) presented effective continuum models to interpret the pressure depletion of heavy oils, focusing, in particular, on the foamy oil issue. Because of the high viscous forces in these experiments, these models must also account for two-phase flow, which was done using conventional relative permeability functions. In the present context, these studies are of interest insofar as nucleation is concerned. The latter is incorporated in the form of rate-dependent nucleation in Kamp et al. (2001a), and in the form of activated cavities in Arora and Kovscek (2001). Nucleation parameters were estimated to match experimental data (see more discussion below).

The objective of this paper is to provide a comprehensive model both of the nucleation and the gas-phase growth periods, until the onset of the critical gas saturation. For this purpose, an effective continuum model will be developed. If used to model the later stages of bubble growth, where gas occupies several pores and is influenced by the pore geometry, 
topology and capillarity (e.g. see Li and Yortsos, 1995a, 1995b), effective continuum models have obvious drawbacks. However, they may be adequate for describing nucleation and the early stages of bubble growth. The last two, particularly the nucleation sequence, are the main areas of interest of this paper. We focus on the effect of the nucleation characteristics on the maximum supersaturation, the nucleation fraction and the critical gas saturation, and provide an analysis of the effect of various parameters, such as pressure decline rate, on these quantities. Results for the gas phase growth following the conclusion of nucleation are also presented.

The paper is organized as follows: First, we formulate the problem. A scaling analysis allows to recast the problem in a more useful form, to be used for direct predictions. Then, numerical results are analyzed. It turns out that for their interpretation, a simplified model of the nucleation and growth periods can be developed. We use the simpler model to obtain expressions for the maximum supersaturation as a function of geometric, thermodynamic and process parameters. This allows to obtain useful relations for the dependence of the final nucleation fraction and the critical gas saturation on process parameters. The theoretical predictions are then compared against experimental results.

\section{MATHEMATICAL FORMULATION}

Consider the heterogeneous nucleation and growth of multiple bubbles from a binary liquid, within an effective porous medium. The process is driven by the continuous increase in the supersaturation of the system, $K C_{\infty}(t)-P_{l}(t)$, where we have assumed for simplicity linear thermodynamic equilibria using Henry's law

$$
P_{g}=K C_{\infty}(t)
$$

Here, $K$ is the solubility constant, $C_{\infty}(t)$ the time-varying mass concentration, $P$ stands for pressure, and subscripts $g$ and $l$ denote gas and liquid, respectively. More complex thermodynamics can certainly be incorporated, but the salient features are manifested with the simpler model (3). As noted above, the change in supersaturation can be imposed in two different ways, one in which the pressure declines at a constant rate, and another in which 
the liquid is withdrawn at a constant rate. Because gravitational and/or viscous effects are not included, the pressure is spatially uniform. Instead, emphasis is placed on nucleation and on the effect of the increase of supersaturation on the growth of the gas phase.

\section{a. Nucleation}

As the liquid pressure declines, nucleation sets in. Yortsos and Parlar (1989) reviewed the gas-liquid phase change in porous media and concluded that heterogeneous nucleation is the most plausible mechanism in solution gas drives (see also more recent reviews by Laaksonen et al., 1995, and Jones et al., 1999). In one model, nucleation occurs when a gas bubble, either pre-existing or nucleated inside a cavity at the pore walls, becomes unstable and detaches or otherwise occupies the host pore body (Fig. 3). This type of mechanism is in agreement with visual observations from micromodel experiments (Li and Yortsos, 1995a; El Yousfi et al., 1991, 1997; Bora et al., 2000, and Dominguez et al., 2000). In the cavity model, the condition for the activation of a nucleation site is when the trapping capillary forces are overcome for the first time (Fig. 3). This occurs when the following condition is satisfied between the radius of the nucleation cavity, $r_{c}$, and supersaturation,

$$
\frac{2 \gamma \cos \theta}{r_{c}}=K C_{\infty}(t)-P_{l}(t)
$$

where $\theta$ is the contact angle $(0<\theta<\pi / 2)$. In this model, the onset of nucleation is not kinetically related to the degree of supersaturation, as for example, in conventional approaches (Firoozabadi and Kashchiev, 1997), but rather depends on the size distribution, $\alpha_{c}\left(r_{c}\right)$, of the nucleation cavities.

Consider, now, the activation of nucleation sites. With the decrease in the liquid pressure, the right-hand side of (4) increases, eventually becoming positive. Then, various cavities satisfying (4) become activated and their corresponding host pore bodies occupied by gas. At any time, the current nucleation fraction, $f_{q}$, defined as the number fraction of pores that contain sizes which have been activated, is

$$
f_{q}=\int_{r_{c}}^{\infty} \alpha_{c}(r) d r
$$


where $r_{c}$ is an implicit function of time, through (4). Equation (5) implies a zero nucleation fraction at zero supersaturation $\left(r_{c} \rightarrow \infty\right)$ and a nucleation fraction of one at infinite supersaturation $\left(r_{c} \rightarrow 0\right)$. The cavity size distribution, $\alpha_{c}$, pertains only to the largest cavity in any given pore (as this cavity will be activated first). The actual number of bubbles contained in a given pore may be larger. We will assume that a number of bubbles, $n_{B}$, are contained in each activated pore. Parameter $n_{B}$ will be taken equal to 1 , for the cavity model, as this is suggested in the micromodel experiments, but will be kept arbitrary for rate-dependent heterogeneous nucleation. Equation (5) slightly overestimates the true nucleation fraction, since pores containing sites to be activated later, may already be occupied by gas, due to the growth of gas clusters from neighboring pores. A more appropriate expression in such a case would be

$$
\frac{d f_{q}}{d t}=\left(1-S_{g}\right) \frac{d}{d t}\left[\int_{r_{c}}^{\infty} \alpha_{c}(r) d r\right]
$$

where $S_{g}$ is the gas saturation. However, in most cases, nucleation terminates well before gas bubble growth has occurred to any substantial degree $\left(S_{g} \ll 1\right)$, thus $(5)$ should be an excellent approximation.

It is interesting to illustrate the dependence of $f_{q}$ on the various cavity size distributions. For a Rayleigh distribution

$$
\alpha_{c}(r)=\frac{\pi r}{2 r_{c}^{* 2}} \exp \left(-\frac{\pi r^{2}}{4 r_{c}^{* 2}}\right)
$$

where $r_{c}^{*}$ is a characteristic (here the mean) cavity size, equation (5) reads

$$
f_{q}=\exp \left(-\frac{\pi r_{c}^{2}}{4 r_{c}^{* 2}}\right)=\exp \left[-\frac{\pi \gamma^{2}}{r_{c}^{* 2}\left(K C_{\infty}-P_{l}\right)^{2}}\right]
$$

This exponential relation bears a superficial resemblance to classical nucleation (see below), a result, however, which is purely due to the form of the Rayleigh distribution. Different distributions will result in different functionals. For example, we will also consider stretchedexponential or log-normal expressions of the form

$$
f_{q}=\exp \left(-\frac{r_{c}^{n}}{\sigma r_{c}^{* n}}\right) \quad \text { or } \quad f_{q}=\frac{1}{2} \operatorname{erfc}\left(\frac{\ln \frac{r_{c}}{r_{c}^{*}}}{\sqrt{2} \sigma}\right)
$$


respectively, where $n$ is a positive exponent and $\sigma$ is a measure of the variance. The type of assumed distribution influences the results to be obtained, as will be demonstrated below. In comparing with the experimental results, we postulated a size distribution and then determined its parameters by fitting with the experimental data

As long as the level of supersaturation increases with time, the right-hand-side of equation (8) also increases, implying that additional sites become activated, and the nucleation fraction continuously rises. This is consistent with experimental evidence of sequential nucleation reported by Li and Yortsos (1995a), Hawes et al. (1997), Mackay et al. (1998) and Bora et al. (2000). After the supersaturation reaches a maximum (local or global), equation (8) predicts a decreasing $f_{q}$, which is unphysical. Therefore, in segments of decreasing supersaturation the nucleation fraction is assumed constant. When the supersaturation goes through a global maximum, it signals the end of the nucleation period, in which case the fraction of pores ultimately activated, $f_{q f}$, will be given by equations (8) or (9) at the time of the maximum supersaturation.

The fraction $f_{q f}$ can be directly related to the number of bubbles nucleated per unit pore volume, $N_{f}$, a quantity used in Firoozabadi and Kashchiev (1997) to quantify nucleation. Assuming $n_{B}=1$, we have

$$
N_{f}=\frac{f_{q f} N_{T}}{V_{p}}
$$

where $N_{T}$ is the total number of pores and $V_{p}$ is the total pore volume. By noting that $V_{p}=N_{T} V_{s}$, where $V_{s}$ is a typical volume of a pore (site), we can further write

$$
N_{f}=\frac{f_{q f}}{V_{s}}
$$

This allows us to relate the nucleation fraction to experimental values of $N_{f}$ (see below). We note, in advance, that in typical experiments, $f_{q f}$ is very small, of the order of $10^{-9}-10^{-6}$.

A different approach is to use rate-dependent heterogeneous nucleation. Consider the nucleation rate expression

$$
\frac{d N_{f}}{d t}=K_{h e t} \exp \left[-\frac{16 \pi \gamma^{3} f}{3 k_{B} T\left(K C_{\infty}-P_{l}\right)^{2}}\right]
$$


where $K_{\text {het }}$ is a heterogeneous rate constant, $f$ is a dimensionless number expressing the wettability of the medium vis-a-vis nucleation (ranging between 1 and 0 for perfectly homogeneous and perfectly heterogeneous rate-dependent nucleation, respectively), and $k_{B}$ is Boltzmann's constant. Both $K_{\text {het }}$ and $f$ can be determined from the matching of experimental data. Using the equivalent of (11) we can express (12) in terms of the nucleation fraction $f_{q}$,

$$
\frac{d f_{q}}{d t}=\frac{K_{h e t} V_{s}}{n_{B}} \exp \left[-\frac{16 \pi \gamma^{3} f}{3 k_{B} T\left(K C_{\infty}-P_{l}\right)^{2}}\right]
$$

Compared to (8), equation (13) contains an explicit rate dependence, while the dependence on parameters, such as $\gamma$, is different from the previous, as expected. Both these models will be considered below.

Through the nucleation process, nucleation centers are activated sequentially, giving rise to evolving gas clusters, which grow by mass transfer from the liquid to the gas. Sequential nucleation results into clusters of different ages (the time passed since a particular class of gas clusters has been nucleated/activated). Let $\omega(\tau)$ be the number density of clusters nucleated per total number of pores. Then, $\omega(\tau) d \tau$ is the number of new clusters per total number of pores that become activated in the time interval between $\tau$ and $\tau+d \tau$. Evidently,

$$
\omega(\tau) d \tau=n_{B} d f_{q}
$$

This relation will be used below to simplify the expressions for the gas phase growth.

\section{b. Gas phase growth}

During the growth of the gas phase we can roughly distinguish two periods, one in which the growth is within single pores and another corresponding to gas clusters spanning several pores (Figs. 2a, 2b, respectively). The first period extends throughout and following the nucleation stage, the second is the later stage of growth. In either, growth is driven by diffusive mass transfer of the dissolved gas. During the first period, mass transfer results mostly in the increase of the volume of the gas. During the second, it also leads to an increase in the gas pressure, in case the interface becomes pinned at pore throats (Fig. 2), until the 
time when the smallest capillary threshold at the throats is overcome. Following this, the gas cluster volume expands accordingly. In general, different clusters compete for the available solute in the liquid, the relative mass transfer rates depending on their geometry and relative position. These dynamics were analyzed in Li and Yortsos (1995a, 1995b).

In the absence of competition between adjacent clusters, an isolated cluster $j$ grows at a rate which is proportional to its effective radius, $R_{j}(t, \tau)$, and the driving force $C_{\infty}-C_{i}$, where $C_{\infty}$ is the far-field concentration and $C_{i}$ the equilibrium concentration at the gas-liquid interface. We will proceed, therefore, by assuming that mass transfer is by quasi-steady-state diffusion and that the gas is ideal. Then, we can write the following mass balance for a growing cluster

$$
\left(\frac{M_{w}}{R_{g} T}\right) \frac{d}{d t}\left(P_{l} V_{g}\right) \approx 4 \pi \lambda R_{j} \mathcal{D}\left(C_{\infty}-C_{i}\right)
$$

where $M_{w}$ is the molecular weight of the gas, $R_{g}$ the ideal gas constant, $T$ the temperature, $V_{g}$ the gas cluster volume and $\mathcal{D}$ the diffusion coefficient. The mass transfer term in (15) was obtained assuming quasi-steady-state diffusion to a spherical bubble. This is true even for ramified fractal clusters, as was verified by Satik and Yortsos (1996) for a percolation cluster. Dimensionless parameter $\lambda$ is an $O(1)$ geometric constant to account for possible corrections to the mass transfer model depending on the growth period (see below). In equation (15) we have neglected the capillary pressure, $P_{c}$, which in typical applications is small compared to the liquid pressure. From Henry's law, we also have

$$
C_{i}=\frac{P_{v}}{K}=\frac{P_{l}+P_{c}}{K} \approx \frac{P_{l}}{K}
$$

where the second equality is again an excellent approximation in the typical applications examined here.

The gas volume $V_{g}$ takes a different expression in the two different periods. For growth within a single pore, $V_{g} \approx V_{c}\left(\frac{R_{j}}{r_{c}^{*}}\right)^{3}$, where $V_{c}$ is a characteristic cavity volume (defined here as $\left.\frac{4}{3} \pi r_{c}^{* 3}\right)$. For growth of a cluster spanning several pores, we have $V_{g} \approx A^{*} V_{s}\left(\frac{R_{j}}{r_{s}^{*}}\right)^{D_{f}}$, where $V_{s}$ is the average site volume, $r_{s}^{*}$ is a characteristic pore body size, $D_{f}$ is the mass fractal dimension, equal approximately to 2.5 for a 3 -D cluster, and $A^{*}$ is a dimensionless geometric prefactor. To capture both periods with the same equation we write 


$$
\left(\frac{A V_{c} M_{w}}{R_{g} T}\right) \frac{d}{d t}\left[P_{l}\left(\frac{R_{j}}{r_{c}^{*}}\right)^{D_{f}}\right]=4 \pi \lambda R_{j} \mathcal{D}\left(C_{\infty}-C_{i}\right)
$$

with the understanding that $D_{f}$ varies between 3 and 2.5 , and $A$ between 1 and $\frac{A^{*} V_{s}}{V_{c}}\left(\frac{r_{c}^{*}}{r_{s}^{*}}\right)^{D_{f}}$, during the nucleation period and growth periods, respectively.

The nucleation period and the early part of the growth period are adequately represented by equation (17). However, growth during the later stages of the second period, where gas clusters span several pores, cannot in reality be captured by (17). Competing clusters affect growth rates in a non-trivial manner. The latter would still be proportional to a mean driving force, $C_{\infty}-C_{i}$, where now $C_{\infty}$ is the volume-averaged concentration in the liquid, and $R_{j}$ stands for the average size of a cluster. However, the mass transfer coefficient $\lambda$ may be variable in time and space, while coalescence of clusters will also occur. Accounting for these complexities is a difficult problem, the solution of which requires a pore-network approach (Li and Yortsos 1995a, 1995b).

Under the above assumptions, the gas phase will be described as a collection of clusters of size $R(t, \tau)$, the dynamics of each of which is described by equation (17), with $R_{j}$ replaced by $R$, namely

$$
\left(\frac{A V_{c} M_{w}}{R_{g} T}\right) \frac{\partial}{\partial t}\left[P_{l}\left(\frac{R}{r_{c}^{*}}\right)^{D_{f}}\right]=4 \pi \lambda R \mathcal{D}\left(C_{\infty}-C_{i}\right)
$$

subject to the initial condition $R(\tau, \tau)=r_{c}(\tau)$, where $r_{c}$ satisfies (4). In the formulation of Firoozabadi and Kashchiev (1997), the equivalent of equation (18) was integrated under a number of simplifying assumptions to obtain an explicit dependence of $R$ on time. Such an approximation will not be used here.

Consider, next, the mass balance for the solute in the liquid phase. We have

$$
\frac{d}{d t}\left[V_{p}\left(1-S_{g}\right) C_{\infty}\right]=-4 \pi \lambda \mathcal{D}\left(C_{\infty}-C_{i}\right) N_{T} \int_{0}^{t} R(t, \tau) \omega(\tau) d \tau-C_{\infty} Q(t)
$$

where the integration is over all existing clusters and $Q(t)$, the volumetric flow rate of the liquid out of the porous medium, is in general a function of time. Equivalently, we can rewrite $(19)$ as 


$$
\frac{d}{d t}\left[V_{p}\left(1-S_{g}\right) C_{\infty}\right]=-4 \pi \lambda \mathcal{D}\left(C_{\infty}-C_{i}\right) N_{T} n_{B} \int_{0}^{f_{q}} \hat{R}(t, f) d f-C_{\infty} Q(t)
$$

where we introduced the notation $\hat{R}(t, f(\tau)) \equiv R(t, \tau)$, for the radius of a cluster at time $t$, nucleated when the nucleation fraction was $f(\tau)$. For the case of instantaneous nucleation, e.g. as postulated in Firoozabadi and Kashchiev (1997), $\hat{R}\left(t, f_{q}\right)=R(t) \delta\left(f_{q}-f_{q f}\right)$, where $f_{q f}$ is the final nucleation fraction and $\delta$ is the Dirac delta function. Then, the above integral reduces to $R(t) f_{q f}$. However, $f_{q f}$ is the very quantity we must determine, is not known a priori, and needs to be computed as part of the overall process, as discussed in detail below.

The volumetric flow rate $Q(t) / V_{p}$ is related to the pressure decline rate through the mass balance on the liquid, which reads

$$
\frac{d}{d t}\left[\rho_{l}\left(1-S_{g}\right)\right]=-\rho_{l} \frac{Q(t)}{V_{p}}
$$

where $\rho_{l}$ is the liquid density. For a slightly compressible liquid,

$$
\rho_{l}=\rho_{b} \exp \left[c\left(P_{l}-P_{b}\right)\right]
$$

where the liquid compressibility, $c$, takes values in the range $1.45 \times 10^{-4}-1.45 \times 10^{-3} \mathrm{MPa}^{-1}$. Then,

$$
\frac{Q(t)}{V_{p}}=-\left(1-S_{g}\right) c \frac{d P_{l}}{d t}+\frac{d S_{g}}{d t}
$$

Finally, the gas saturation is related to the radius of the growing clusters and the nucleation fraction through the relation

$$
S_{g}=A v \int_{0}^{t}\left(\frac{R(t, \tau)}{r_{c}^{*}}\right)^{D_{f}} \omega(\tau) d \tau=A v n_{B} \int_{0}^{f_{q}}\left(\frac{\hat{R}\left(t, f_{q}\right)}{r_{c}^{*}}\right)^{D_{f}} d f_{q}
$$

where we introduced the volume ratio $v \equiv \frac{V_{c}}{V_{s}}$. This parameter can be calculated from knowledge of the average cavity and pore sizes. Subject to the relevant initial conditions, the system of equations (18), (20), (23) and (24) can be integrated. Integration proceeds until the time when the critical gas saturation (1) is reached.

\section{c. Dimensionless formulation and scaling}


For the solution of the problem, we recast the equations in dimensionless form. Denote dimensionless quantities by subscript $D$ and scale concentrations by $C_{b}=\frac{P_{b}}{K}$, pressure by $P_{b}$, where subscript $b$ refers to the bubble point, and cluster size by $r_{c}^{*}$. The choice of the characteristic time depends on the process. We will take $t^{*}=\frac{P_{b}}{a}$, where $a$ is the pressure decline rate, for the case of constant pressure decline rate and $t^{*}=\frac{V_{p}}{Q}$, for the case of liquid withdrawal at constant volumetric flow rate $Q$.

For the case of constant pressure decline rate, the dimensionless mass balances for the solute in the gas and liquid phases read

$$
\left(1-t_{D}\right) \frac{\partial \hat{R}_{D}^{D_{f}}}{\partial t_{D}}=\frac{\Pi_{2}}{A \Pi_{1}}\left(C_{D \infty}-P_{D l}\right) \hat{R}_{D}+\hat{R}_{D}^{D_{f}}
$$

and

$$
\left(1-S_{g}\right) \frac{d C_{D \infty}}{d t_{D}}=-\frac{1}{\Pi_{1}}\left(C_{D \infty}-P_{D l}\right) \int_{0}^{f_{q}} \hat{R}_{D}\left(t_{D}, f_{q}\right) d f_{q}-\Pi_{3}\left(1-S_{g}\right) C_{D \infty}
$$

where we used the equilibrium relationship

$$
C_{D i}=P_{D l}\left(t_{D}\right)
$$

and assumed that the process begins $\left(t_{D}=0\right)$ when the pressure is at the bubble point. In the above, we have defined three dimensionless groups,

$$
\Pi_{1}=\frac{V_{p} a}{4 \pi \lambda \mathcal{D} P_{b} N_{T} r_{c}^{*}}=\frac{V_{s} a}{4 \pi \lambda \mathcal{D} P_{b} n_{B} r_{c}^{*}}, \quad \Pi_{2}=\frac{R_{g} T}{v n_{B} M_{w} K} \quad \text { and } \quad \Pi_{3}=c P_{b}
$$

Parameter $\Pi_{1}$ expresses the ratio of the characteristic times for diffusion at the pore scale to that for the decline of pressure. Although a small number in typical applications (see Table 1), it plays a key role in determining the nucleation fraction and the critical gas saturation. Parameter $\Pi_{2}$ is the product of the geometric constant $v n_{B}$ with a thermodynamic constant, expressing the ratio of the equilibrium concentrations in the liquid and the gas phases. These parameters are either known or can be estimated from comparison with experimental data.

For the case of constant liquid withdrawal rate the analogous equations read

$$
P_{D l}\left(t_{D}\right) \frac{\partial \hat{R}_{D}^{D_{f}}}{\partial t_{D}}+\hat{R}_{D}^{D_{f}} \frac{d P_{D l}}{d t_{D}}=\frac{\Pi_{2}}{A \Pi_{4}}\left(C_{D \infty}-P_{D l}\right) \hat{R}_{D}
$$


and

$$
\left(1-S_{g}\right) \frac{d C_{D \infty}}{d t_{D}}=-\frac{1}{\Pi_{4}}\left(C_{D \infty}-P_{D l}\right) \int_{0}^{f_{q}} \hat{R}_{D}\left(t, f_{q}\right) d f_{q}-C_{D \infty}+C_{D \infty} \frac{d S_{g}}{d t_{D}}
$$

where

$$
\Pi_{4}=\frac{Q}{4 \pi \lambda \mathcal{D} N_{T} n_{B} r_{c}^{*}}=\frac{V_{s} Q}{4 \pi \lambda \mathcal{D} V_{p} n_{B} r_{c}^{*}}
$$

Here, parameter $\Pi_{4}$ expresses the ratio of the characteristic time for diffusion to that for the emptying of the pore volume. Typically, this is also a small number (Table 1). As will be shown below, $\Pi_{4} / \Pi_{3}$ plays a role equivalent to $\Pi_{1}$.

Finally, in both cases, we have the following relations: The liquid mass balance becomes

$$
\frac{d P_{D l}}{d t_{D}}=\frac{1}{\Pi_{3}\left(1-S_{g}\right)}\left(\frac{d S_{g}}{d t_{D}}-1\right)
$$

The gas saturation is

$$
S_{g}=A v n_{B} \int_{0}^{f_{q}} \hat{R}\left(t_{D}, f_{q}\right)^{D_{f}} d f_{q}
$$

The cavity size that becomes activated at a given time is

$$
r_{D q}=\frac{\Pi_{c}}{C_{D \infty}\left(t_{D}\right)-P_{D l}\left(t_{D}\right)}
$$

where we introduced the dimensionless cavity capillary pressure threshold, $\Pi_{c}=\frac{2 \gamma \cos \theta}{r_{c}^{* *} P_{b}}$. The latter is an important parameter in the overall dynamics. In terms of the supersaturation

$$
s \equiv C_{D \infty}\left(t_{D}\right)-P_{D l}\left(t_{D}\right)
$$

or, more conveniently, in terms of the rescaled supersaturation

$$
s_{D} \equiv \frac{s}{\Pi_{c}}
$$

equation (34) can be further expressed as $r_{D q}=s_{D}^{-1}$. The nucleation fraction is then given by the various expressions 


$$
f_{q}=\exp \left(-\frac{\pi}{4 s_{D}^{2}}\right), \quad f_{q}=\exp \left(-\frac{1}{\sigma s_{D}^{n}}\right), \quad f_{q}=\frac{1}{2} \operatorname{erfc}\left(-\frac{\ln s_{D}}{\sqrt{2} \sigma}\right)
$$

depending on the size distribution used, or by

$$
\frac{d f_{q}}{d t_{D}}=h_{1} \exp \left(-\frac{h_{2}}{s^{2}}\right)
$$

in the rate-dependent nucleation case. In the latter we introduced the dimensionless parameters

$$
h_{1}=\frac{K_{h e t} t^{*} V_{s}}{n_{B}} \text { and } h_{2}=\frac{16 \pi \gamma^{3} f}{3 P_{o}^{2} k_{B} T}
$$

Parameter $h_{1}$ is inversely proportional to $\Pi_{1}$ or $\Pi_{4}$. If this dependence is extracted, then equation (39) reads as

$$
h_{1}=\frac{\zeta}{\Pi_{1}} \quad \text { or } \quad h_{1}=\frac{\zeta}{\Pi_{4}}, \quad \text { where } \zeta \equiv \frac{K_{h e t} V_{s}^{2}}{4 \pi \lambda \mathcal{D} n_{B}^{2} r_{c}^{*}}
$$

The initial conditions for the simulations were $C_{D \infty}=1, P_{D l}=1$ and $R_{D}(\tau, \tau)=s_{D}^{-1}(\tau)$.

The above system contains one key parameter, $\Pi_{1}$ or $\Pi_{4}$, describing the effect of the rate of increase of the supersaturation. Because it is small, a further rescaling of the nucleation fraction and the cluster size is necessary. After some analysis, it is not difficult to show that for the cavity nucleation model, the following scaling is valid (for example, for the constant pressure decline rate), $f_{q} \sim \Pi_{1}^{\frac{D_{f}}{D_{f}^{-1}}}$ and $f_{q} R^{D_{f}} \sim O(1)$ (where, given that the nucleation fraction varies only during the first period, $D_{f}=3$ ). This scaling contains the main effect of the pressure decline rate on the nucleation fraction. Thus, we may define a rescaled nucleation fraction

$$
\phi_{q}=f_{q} \Pi_{1}^{-\frac{3}{2}} \quad \text { or } \quad \phi_{q}=f_{q}\left(\frac{\Pi_{4}}{\Pi_{3}}\right)^{-\frac{3}{2}}
$$

and rescaled cluster sizes

$$
\rho_{D}=\Pi_{1}^{\frac{1}{2}} \hat{R}_{D} \quad \text { or } \quad \rho_{D}=\left(\frac{\Pi_{4}}{\Pi_{3}}\right)^{\frac{1}{2}} \hat{R}_{D}
$$

in the two different cases. In the new notation, the governing equations become as follows: 
For the case of constant rate of pressure decline,

$$
\left(1-t_{D}\right) \frac{\partial \rho_{D}^{D_{f}}}{\partial t_{D}}=\frac{\Pi_{2}}{A} s \rho_{D}+\rho_{D}^{D_{f}}
$$

and

$$
\left(1-S_{g}\right) \frac{d s}{d t_{D}}=-s \int_{0}^{\phi_{q}} \rho_{D}\left(t_{D}, \phi_{q}\right) d \phi_{q}-\left(s+1-t_{D}\right)\left[\Pi_{3}\left(1-S_{g}\right)\right]+1-S_{g}
$$

while for the constant liquid withdrawal rate,

$$
\Pi_{3} P_{D l}\left(t_{D}\right) \frac{\partial \rho_{D}^{D_{f}}}{\partial t_{D}}+\Pi_{3} \rho_{D}^{D_{f}} \frac{d P_{D l}}{d t_{D}}=\frac{\Pi_{2}}{A} s \rho_{D}
$$

and

$$
\Pi_{3}\left(1-S_{g}\right) \frac{d s}{d t_{D}}=-s \int_{0}^{\phi_{q}} \rho_{D}\left(t_{D}, \phi_{q}\right) d \phi_{q}+\left(\frac{d S_{g}}{d t_{D}}-1\right)\left[\Pi_{3}\left(P_{D l}+s\right)-1\right]
$$

The last two equations are also accompanied by equation (32). The gas saturation expression for either case becomes

$$
S_{g}=A v n_{B} \int_{0}^{\phi_{q}} \rho\left(t_{D}, \phi_{q}\right)^{D_{f}} d \phi_{q}
$$

For the case of rate-dependent nucleation, the rescaled nucleation fraction reads as

$$
\frac{d \phi_{q}}{d t_{D}}=h_{1}^{*} \exp \left(-\frac{h_{2}}{s^{2}}\right)
$$

where $h_{1}^{*}=h_{1} \Pi_{1}^{-\frac{3}{2}}$ or $h_{1}^{*}=h_{1}\left(\frac{\Pi_{4}}{\Pi_{3}}\right)^{-\frac{3}{2}}$ in the respective cases. The solution of the system of the rescaled equations will be sought numerically in the following sections.

\section{NUMERICAL RESULTS}

The system of differential equations was solved numerically using a fourth-order RungeKutta method (Press et al., 1994). A typical calculation requires the time to be marched forward. A difficulty is that the total number of classes of gas clusters is not known a priori, but it is an outcome of the computation during the nucleation process. In theory, this 
number is infinite, and the problem becomes one of solving an infinite system of differential equations. In practice, the number of equations is constrained by the size of the time step. At each time step we examine whether nucleation of a new class of gas clusters is possible, namely whether the supersaturation is increasing. If so, a new class of gas clusters is added. Then, the simultaneous growth of all different classes of clusters is computed. When the supersaturation reaches a maximum, further nucleation stops. Computations during the nucleation process were also facilitated with an asymptotic analysis, to be described in more detail later.

\section{a. Constant Pressure Decline Rate}

In the typical case, parameters which can vary over a significant range are $\Pi_{1}$ and $\Pi_{c}$ (and possibly $\Pi_{2}$ ). An additional variable is the type of the cavity size distribution used in the calculation of the nucleation fraction. The sensitivity to these parameters was examined in the simulations.

The effect of $\Pi_{1}$ and $\Pi_{c}$ on the rescaled nucleation fraction, $\phi_{q}$, the mean rescaled radius, $\rho_{D, m}$, the rescaled supersaturation, $s_{D}$, and the gas saturation, $S_{g}$, is shown in Figs. 4-7. In these calculations, we used a Rayleigh size distribution, $\Pi_{2}$ and $\Pi_{3}$ were kept constant to the values $2.33 \times 10^{6}$ and $8.7 \times 10^{-3}$, respectively, while $\Pi_{1}$ varied over several orders of magnitude (from $10^{-8}$ to $10^{-2}$ ).

The variation of $\phi_{q}$ as a function of the dimensionless time, $t_{D}$, and of the parameters $\Pi_{1}$ and $\Pi_{c}$ is shown in Fig. 4. It is found that $\phi_{q}$ increases very rapidly in a small time interval, and then stabilizes to a final value at the conclusion of nucleation. Such behavior is characteristic of nucleation processes, and has features similar to those reported by El Yousfi et al. (1991, 1997). It is demonstrated here for the first time for the case of nucleation from pre-existing, trapped gas. The rapid variation of $\phi_{q}$ is approximately a stretched exponential of the form

$$
\phi_{q} \sim \exp \left(-\frac{\pi \Pi_{c}^{2}}{4 t_{D}^{2}}\right), \quad \phi_{q} \sim \exp \left(-\frac{\Pi_{c}^{n}}{\sigma t_{D}^{n}}\right), \quad \phi_{q} \sim \operatorname{erfc}\left(\frac{\ln \frac{\Pi_{c}}{\sigma t_{D}}}{\sqrt{2} \sigma}\right)
$$

for the different cases, as during the early nucleation period we have $s \sim t_{D}$ (see below). Equation (49) suggests that, e.g. for the Rayleigh distribution case, a plot of $-\ln \phi_{q}$ vs. 
$t_{D}^{-2}$ is linear with slope $\frac{\pi \Pi_{c}^{2}}{4}$. Because of the resulting very sharp rise, this process can be interpreted as instantaneous nucleation (IN). However, proceeding with such an assumption does not allow for the computation of the final nucleation values. Instead, we must consider the details of the approach to the final values using the progressive nucleation model, used here. Fig. 4a shows that for constant $\Pi_{c}$, the effect of $\Pi_{1}$ on the rescaled nucleation fraction is not very significant at small $\Pi_{1}$, but that it becomes stronger (roughly a power law) as $\Pi_{1}$ takes larger values. The relatively weak dependence on $\Pi_{1}$ verifies the correctness of the scaling (41). At the same time, the stronger dependence at larger $\Pi_{1}$ is significant, and as shown below, is needed in order to explain experimental data. In terms of the actual nucleation fraction, these findings imply that an increase in $\Pi_{1}$ leads to an increase in the final fraction, $f_{q f}$, according to a power law scaling, namely $f_{q f} \sim \Pi_{1}^{\frac{3}{2}}$ at very small $\Pi_{1}$, and $f_{q f} \sim \Pi_{1}$ at larger $\Pi_{1}$. The effect of $\Pi_{c}$ is also significant. As $\Pi_{c}$ increases, the final nucleation fraction $\phi_{q f}$ (hence $f_{q f}$ ) decreases (Fig. $4 \mathrm{~b}$ ). The increase of $f_{q f}$ with an increase in $\Pi_{1}$ and a decrease in $\Pi_{c}$ is expected. Larger values of $\Pi_{1}$ result from a faster decline rate, a greater departure from equilibrium, the establishment of a greater supersaturation, hence the activation of more nucleation sites. Likewise, smaller $\Pi_{c}$ imply that nucleation is facilitated at increasingly smaller supersaturations, as larger size cavities can be activated. An approximate analysis shown below will provide an explanation of the behavior observed.

Fig. 5 shows the corresponding effects on the mean rescaled size $\rho_{D m}$. There are two regions, one corresponding to the nucleation period, and another to growth after nucleation. The two periods can be roughly approximated as power-law regimes (as a function of time) with slopes approximately equal to 1 and 0.63 , respectively. The effect of $\Pi_{1}$ is relatively insignificant at small $\Pi_{1}$, confirming the validity of the scaling (41). The effect of $\Pi_{c}$ is more significant. Smaller values of $\Pi_{c}$ lead to an increase in the nucleation fraction, and a corresponding decrease in the size of the gas clusters at the conclusion of nucleation.

Fig. 6a shows plots of the rescaled supersaturation $s_{D}$ as a function of time for different $\Pi_{1}$ and $\Pi_{c}$. At the beginning of the process and during nucleation, the supersaturation increases with time almost linearly, suggesting that $C_{D \infty}$ does not vary significantly in that period. As nucleation and growth take place, the rate of supersaturation increase slows down and, at some point, $s_{D}$ reaches a maximum value, $s_{D m}$. It is at that point where 
nucleation terminates. Following this point, the supersaturation decreases monotonically. The value $s_{D m}$ is plotted in Fig. $6 \mathrm{~b}$ as a function of $\Pi_{1}$ and $\Pi_{c}$. Note that $s_{D m}$ is in general of the order of $10^{-1}$. The dependence on the parameters is weak at small $\Pi_{1}$ and large $\Pi_{c}$, but becomes stronger at larger $\Pi_{1}$ and smaller $\Pi_{c}$. This behavior is consistent with that of the nucleation fraction discussed above. From a compilation of experimental results, Scherpenisse et al. (1994) suggested that $s_{D m}$ behaves roughly as a power law of the pressure decline rate with exponent $1 / 4$. Our analysis indicates that such a power law is not universally valid, although it may apply in a certain range of $\Pi_{1}$. It is interesting that the sensitivity of $s_{D m}$ to $\Pi_{c}$ and $\Pi_{1}$ (and in particular to the latter) is not as large as one might have intuitively anticipated. Nonetheless, its effect on the nucleation fraction can be significant, due to the exponential dependence, as can be seen for example in the following expression (for a Rayleigh distribution)

$$
\phi_{q}=\exp \left[-\frac{\pi}{4 s_{D}^{2}}-\frac{D_{f}}{\left(D_{f}-1\right)} \ln \Pi_{1}\right]
$$

Because of the exponential dependence on $s_{D}^{-2}$ and because $s_{D m}$ is of the order of $10^{-1}$, even small changes in $s_{D}$ have a very large effect on the nucleation fraction. This large sensitivity enhances the weak sensitivity of $s_{D m}$ on $\Pi_{1}$ and $\beta$ and leads overall to a non-trivial effect.

The evolution of the gas saturation is shown in Fig. 7. It follows that of $f_{q}$, during the nucleation period, and that of $\rho_{D m}$, during the period of growth. The latter gives a power-law segment of slope 0.63 . The effect of $\Pi_{c}$ is indirect, in that smaller values of $\Pi_{c}$ promote larger values of $S_{g}$ due to an increase in both $f_{q f}$ and $\rho_{D}$. The difference between equilibrium and actual curves depends on the value of $\Pi_{1}$, increasing as the latter increases, but remaining constant following the end of nucleation. Fig. 8 shows the effects of $\Pi_{1}$ and $\Pi_{c}$ on the critical gas saturation $S_{g c}$. In our work, the latter pertains to the formation of a sample-spanning cluster, in the absence of viscous or gravity effects. Thus, Fig. 8 actually reflects the variation of $f_{q f}$. Fig. 8 shows that $S_{g c}$ can be considered a power-law both of $\Pi_{1}$ and of $\Pi_{c}$ with exponents that vary between 0.16 and 0.25 with respect to $\Pi_{1}$ and between -0.33 and -0.22 , with respect to $\Pi_{c}$, respectively. The trends are consistent with the experimental evidence (Scherpenisse et al., 1994; Bora et al., 2000). In Fig. 8 we allowed $S_{g c}$ to take values that may be larger than what is required for the validity of our model. 
This was done only for the shake of parametric sensitivity.

\section{b. Constant Rate of Liquid Withdrawal}

Except for the evolution of pressure with time, similar results are obtained for the case of constant rate of liquid withdrawal. The effect of the parameters is also very similar to the constant pressure decline rate, subject to the change $\Pi_{1} \rightarrow \Pi_{4} / \Pi_{3}$ and to the rescaling of time by $\Pi_{3}$. Thus, we anticipate a scaling of the form: $f_{q f} \sim \Pi_{4}^{\frac{3}{2}}$ at small $\Pi_{4}$, and $f_{q f} \sim \Pi_{4}$ at larger $\Pi_{4}$; and of the form: $f_{q f} \sim \Pi_{c}^{-2}$ at large $\Pi_{c}$, and $f_{q f} \sim \Pi_{c}^{-1.3}$ at smaller $\Pi_{4}$. The critical gas saturation has the analogous scaling: $S_{g c} \sim \Pi_{4}^{0.25}$ at small $\Pi_{4}$, and $S_{g c} \sim \Pi_{4}^{0.16}$ at larger $\Pi_{4}$; and $S_{g c} \sim \Pi_{c}^{-0.22}$ at large $\Pi_{c}$, and $S_{g c} \sim \Pi_{c}^{-0.33}$ at smaller $\Pi_{4}$. The discussion and interpretation of the findings is similar to the case of constant pressure decline rate and will not elaborated further. Additional figures and discussion are presented in Tsimpanogiannis (2002).

What is different in the case of constant rate of liquid withdrawal, is the evolution of pressure with time (Fig. 9). In the calculations, shown in Fig. 9 we used a Rayleigh size distribution, $\Pi_{2}$ and $\Pi_{3}$ were kept constant to the values $6.84 \times 10^{5}$ and $1.5 \times 10^{-2}$, respectively, while $\Pi_{4}$ varied over several orders of magnitude (from $10^{-12}$ to $10^{-6}$ ). During the nucleation period, the pressure declines almost linearly with time, $P_{D l} \approx 1-\frac{t_{D}}{\Pi_{3}}$, following equation $(32)$. This decrease slows down as nucleation sets in, and when the maximum supersaturation is approached, the pressure reaches a local minimum. Following this minimum, the pressure increases, reaches a maximum and subsequently decreases, roughly paralleling the equilibrium curve. The pressure minimum decreases as $\Pi_{4}$ increases (Fig. 9), the dependence being roughly the same as that of $s_{D m}$, namely weak at small $\Pi_{4}$ and stronger at larger $\Pi_{4}$ (where the $1 / 4$ power law may be applicable).

The non-equilibrium behavior reflects the competition between mass transfer and solute availability and can be explained as follows. The ideal gas law requires $P_{g} V_{g}=n R_{g} T$. The rate of change, $d n / d t$, of the moles in the gas phase is dictated by the mass transfer rate. At the end of the nucleation period, near $s_{D m}$, this rate is the highest. Now, if the rate by which

the gas volume expands, $d V_{g} / d t$ (which is almost equal to $Q$ ), is not sufficiently large, the increase in volume due to mass transfer cannot be compensated, thus the pressure, $P_{g}$, must 
increase. An increasing pressure leads to a successively decreasing supersaturation (since $C_{i}$ increases), thus to a continuous decrease of the mass transfer rate. Eventually, this decrease becomes sufficiently large for the volume expansion rate to balance mass transfer. Then, the pressure goes through a maximum, following which it begins to decline.

In the above, we used the cavity-based nucleation model. We must stress that qualitatively similar results were obtained for the model based on rate-dependent nucleation. These will not be shown. In a subsequent section, the numerical solutions obtained will be compared against available experimental results. However, before doing so it is beneficial to provide an interpretation of the main findings, using a simpler model.

\section{INTERPRETATION USING A SIMPLER MODEL}

To interpret the results obtained we consider a simpler model that captures the essential features of the problem. Consider, first, the nucleation period.

\section{A. Nucleation}

\section{a. Constant Pressure Decline Rate}

To approximately describe the nucleation period, we simplify as follows the equations for the gas phase growth and the supersaturation

$$
\frac{\partial \rho_{D}^{3}}{\partial t_{D}} \approx \Pi_{2} s \rho_{D}
$$

and

$$
\frac{d s}{d t_{D}} \approx 1-s \int_{0}^{\phi_{q}(s)} \rho_{D} d \phi_{q}
$$

respectively. These are subject to the initial conditions

$$
s(0)=0 \quad \text { and } \quad \rho_{D}(\tau, \tau)=\frac{\Pi_{1}^{\frac{1}{2}}}{s_{D}(\tau)}
$$

At early times and for small $\Pi_{1}$, the approximate solution of (51)-(53) is 


$$
s \approx t_{D} \text { and } \rho_{D} \approx\left[\frac{\Pi_{1} \Pi_{c}^{2}}{s^{2}(\tau)}+\frac{\Pi_{2}\left[s^{2}-s(\tau)^{2}\right]}{3}\right]^{\frac{1}{2}}
$$

The dimensionless supersaturation is equal to the dimensionless time and the mean cluster size becomes eventually a power-law of time with exponent 1 . These results are consistent with the numerical results during the nucleation period (Figs. 5 and 6 ).

We will use (52) to approximate the approach to the maximum supersaturation. The latter is reached when $\frac{d s}{d t_{D}}=0$, namely when

$$
s \int_{0}^{\phi_{q}} \rho_{D} d \phi_{q} \approx 1
$$

From (54) we approximately read, $\rho_{D} \approx\left(\frac{\Pi_{2}}{3}\right)^{\frac{1}{2}} s\left(t_{D}\right)$. Then, using the definition of $\phi_{q}$ leads to an algebraic equation for the rescaled maximum supersaturation, $s_{D m}$. For example, for the case of Rayleigh distribution we have the equation

$$
\frac{\pi}{4 s_{D m}^{2}}-2 \ln s_{D m} \approx \ln \Lambda-\frac{1}{2} \ln 3-\frac{3}{2} \ln \Delta
$$

where we introduced the combination of variables

$$
\Delta \equiv \Pi_{1} \Pi_{c}^{-\frac{4}{3}} \Pi_{2}^{-\frac{1}{3}}
$$

and for the case of constant pressure decline rate, $\Lambda \equiv 1$. Likewise for the case of a stretched exponential, and of a log-normal distribution, we have

$$
\begin{gathered}
\sigma^{-1} s_{D m}^{-n}-2 \ln s_{D m} \approx \ln \Lambda-\frac{1}{2} \ln 3-\frac{3}{2} \ln \Delta \\
s_{D m}^{2} \operatorname{erfc}\left(\frac{\ln \frac{1}{s_{D m}}}{\sqrt{2} \sigma}\right) \approx 2 \sqrt{3} \Delta^{\frac{3}{2}} \Lambda^{-1}
\end{gathered}
$$

where, again, for the constant pressure decline rate, $\Lambda=1$. Equations (56)-(59) represent key findings of this paper. First, they suggest that the dependence of the maximum supersaturation on the various parameters, other than the thermodynamic ones, enters only through $\Delta$. The solution of (56) for the Rayleigh distribution is plotted in Fig. 10, as a function of $\Delta$. We see that $s_{D m}$ varies weakly, in the range $0.1-1$, as $\Delta$ varies over several 
orders of magnitude (between $10^{-10}$ and $10^{3}$ ). For small $\Delta$, the maximum supersaturation is practically constant. As $\Delta$ takes larger values, $s_{D m}$ increases weakly and eventually more strongly, as $\Delta$ approaches the order of one (compare also with Fig. 6). Also shown in the logarithmic coordinates of Fig. 10 is a line with slope $1 / 4$, corresponding to the $1 / 4$ power law postulated by Scherpenisse et al. (1994) to describe several experimental data. Although the power law does not capture the overall behavior, it can approximate the results in a certain window of $\Delta$. Plotted in the same figure are also the results of the numerical solution of the full problem for a number of different parameter values. The agreement between the numerical results and the simple analytical model is remarkable and demonstrates the validity of the simple equation (56). For the stretched exponential nucleation model, the variation is much stronger in the logarithmic plot. The solution of (56), (58) and (59) corresponding to different distributions was investigated in detail in Tsimpanogiannis (2002). As the tail of the cavity size distribution becomes longer (which occurs for smaller values of $n>0$ and/or for larger $\sigma$ ) the dependence of $s_{D m}$ on $\Delta$ becomes stronger. In addition, the region where a power-law scaling with exponent $\approx 1 / 4$ tentatively fits the results, increases and also corresponds to a range with smaller values of $\Delta$.

Equations (56)-(59) can be used to approximate the final nucleation fraction, $\phi_{q f}$, and the time (or pressure) at the end of nucleation. For all cases we have

$$
\phi_{q f} \approx s_{D m}^{-2} \Pi_{c}^{-2}\left(\frac{\Pi_{2}}{3}\right)^{-\frac{1}{2}} \Lambda^{-1}
$$

thus, the final nucleation fraction reads

$$
f_{q f} \approx s_{D m}^{-2} \Pi_{1}^{\frac{3}{2}} \Pi_{c}^{-2}\left(\frac{\Pi_{2}}{3}\right)^{-\frac{1}{2}} \Lambda^{-1}
$$

This equation represents another important result of this paper and leeds to the following conclusions:

(a) In the region where $s_{D m}$ varies weakly with $\Delta$ (at small $\Delta$ ) the final nucleation fraction varies as a power law of $\Pi_{1}$, with slope equal to $3 / 2$. This is consistent with the anticipated increase in the nucleation fraction as the rate of pressure decline increases. The equation suggests a power-law dependence on the capillary properties of the cavity. One should interpret this carefully, however, since information on the cavity properties is included in all 
three parameters $\Pi_{1}, \Pi_{2}$ and $\Pi_{c}$ (through $r_{c}^{*}$ and $v$ ). For example, if we were to consider only the dependence on $r_{c}^{*}$, we would find the power-law scaling $f_{q f} \sim r_{c}^{* 2}$, indicating a smaller nucleation fraction as the cavity size decreases. This is as expected.

(b) In the region where $s_{D m}$ may be approximated by a power-law dependence on $\Delta$, e.g. as $s_{D m} \sim \Delta^{m}$, we have the scaling

$$
f_{q f} \sim \Delta^{\frac{3}{2}-2 m}
$$

Such a dependence on $\Delta$ leads to a decrease in the exponent in the power-law scaling of $f_{q f}$ on $\Pi_{1}$. For example, if we take $m \approx 1 / 4$ (as suggested by Scherpenisse et al., 1994), we read

$$
f_{q f} \sim \Pi_{1} \quad \text { and } \quad f_{q f} \sim r_{c}^{* \frac{4}{3}}
$$

A linear dependence of the rate on $\Pi_{1}$ was postulated in Scherpenisse et al. (1994) and McDougal and Sorbie (1999), to fit available experimental data.

(c) The time, hence the pressure, $P_{m}$, when nucleation ends can be approximated using (54). We find

$$
\frac{P_{b}-P_{m}}{P_{b}} \approx \Pi_{c} s_{D m}
$$

thus, the supersaturation at the end of nucleation is directly related to $s_{D m}$. It follows that in the region where $s_{D m}$ is insensitive to $\Delta$, the pressure supersaturation varies only linearly with $\Pi_{c}$. A rate dependence, observed experimentally in some cases, enters only insofar as $s_{D m}$ varies with $\Delta$. Assuming again a power-law variation with $m \approx 1 / 4$, the maximum pressure supersaturation varies as follows

$$
\frac{P_{b}-P_{m}}{P_{b}} \sim \Pi_{1}^{\frac{1}{4}} \quad \text { and } \quad \frac{P_{b}-P_{m}}{P_{b}} \sim r_{c}^{-\frac{2}{3}}
$$

The 1/4 power-law dependence was found to fit well experimental data (see below). It is interesting that the maximum supersaturation relative to the bulk bubble point is only weakly dependent on the rate of pressure decline, for example varying by only a factor of 2 when the pressure decline rate varies by two orders of magnitude, in the range considered. 


\section{b. Constant Rate of Liquid Withdrawal}

A similar analysis applies for the case of constant liquid withdrawal. After various simplifications, the equations for the gas phase growth and the supersaturation read

$$
\Pi_{3} \frac{d \rho_{D}^{3}}{d t_{D}} \approx \Pi_{2} s \rho_{D}
$$

and

$$
\Pi_{3} \frac{d s}{d t_{D}} \approx 1-\left(1+\frac{\kappa}{\Pi_{3}}-\kappa\right) s \int_{0}^{\phi_{q}} \rho_{D} d \phi_{q}
$$

respectively, where we have introduced the thermodynamic parameter $\kappa \equiv \frac{R_{g} T}{M_{w} K}$. These are subject to the initial conditions

$$
s(0)=0 \quad \text { and } \quad \rho_{D}(\tau, \tau)=\frac{\left(\frac{\Pi_{4}}{\Pi_{3}}\right)^{\frac{1}{2}}}{s_{D}(\tau)}
$$

At early times and for small $\frac{\Pi_{4}}{\Pi_{3}}$, the solution is

$$
s \approx \frac{t_{D}}{\Pi_{3}} \quad \text { and } \quad \rho_{D} \approx\left[\frac{\Pi_{4}}{\Pi_{3}} \frac{\Pi_{c}^{2}}{s^{2}(\tau)}+\frac{\Pi_{2}\left[s^{2}-s(\tau)^{2}\right]}{3}\right]^{\frac{1}{2}}
$$

The early-time behavior is identical to the constant pressure decline rate, if $t_{D}$ is replaced by $t_{D} / \Pi_{3}$ and $\Pi_{1}$ with $\frac{\Pi_{4}}{\Pi_{3}}$. We note again, that the linear scaling of the cluster size with time is consistent with the full numerical solution.

Proceeding as previously we find that the maximum rescaled supersaturation, $s_{D m}$, is now given by

$$
s \rho_{D} \phi_{q} \approx \frac{\Pi_{3}}{\kappa}
$$

where $\rho_{D}$ satisfies Eq. (69). Thus, the solution of (70) is the same as that obtained for the constant pressure decline rate problem, except that one must replace $\Pi_{1}$ with $\frac{\Pi_{4}}{\Pi_{3}}$, and take $\Lambda \equiv \kappa / \Pi_{3}$. For exactly the same reasons, the final nucleation fraction can be directly obtained from (61). The previous analysis for the constant pressure decline rate applies directly to the constant rate of liquid withdrawal, subject to the aforementioned substitution. 
A comparison between the solution of the full problem (for the cases of the Rayleigh distribution and a stretched exponential with $n=0.5$ and $\sigma=1.0$ ) and of the approximate equation (70) is also shown in Fig. 10. We note an excellent agreement. The scalings obtained are also consistent with the solution of the full equations. Additional comparisons with stretched exponential cavity distribution, which have lower values of $n$ and $\sigma$, will be presented below.

As noted above, a difference for the problem involving a constant rate of liquid withdrawal, is that the pressure reaches a local minimum. To identify it, we will proceed as follows. Integrating equation (32), we obtain

$$
\Pi_{3} P_{D l} \approx A v \phi_{q} \rho_{D}^{3}-t_{D}+\Pi_{3}
$$

where we made the same approximation for the integral, as in (67). Finding the minimum in pressure requires equating the derivative of (71) to zero. Using (69) for $\rho_{D}$, it is not difficult to show that the following equation is satisfied by $s_{D n}$ at that point,

$$
\phi_{q}\left(3 s_{D n}^{2}+\frac{\pi}{2}\right) \approx \frac{\Pi_{3}}{A v}\left(\frac{3}{\Pi_{2}}\right)^{\frac{3}{2}}\left(\frac{1}{\Pi_{c}}\right)^{2}
$$

where we used a Rayleigh distribution. For the stretched exponential case we have,

$$
\phi_{q}\left(3 s_{D n}^{2}+\frac{n s_{D n}^{2-n}}{\sigma}\right) \approx \frac{\Pi_{3}}{A v}\left(\frac{3}{\Pi_{2}}\right)^{\frac{3}{2}}\left(\frac{1}{\Pi_{c}}\right)^{2}
$$

Based on these equations, one can show that the pressure reaches its local minimum before the supersaturation reaches its maximum, suggesting that nucleation continues slightly after the minimum in pressure, albeit for a very brief period of time. We can solve the above to determine the pressure minimum. For the Rayleigh distribution, we approximately find

$$
\frac{P_{b}-P_{n}}{P_{b}} \approx \Pi_{c} s_{D n}\left(\frac{2 s_{D n}^{2}+\frac{\pi}{2}}{3 s_{D n}^{2}+\frac{\pi}{2}}\right)
$$

and for the stretched exponential,

$$
\frac{P_{b}-P_{n}}{P_{b}} \approx \Pi_{c} s_{D n}\left(\frac{2 \sigma s_{D n}^{n}+n}{3 \sigma s_{D n}^{n}+n}\right)
$$


Given that $s_{D n}$ is generally of the order of 0.1 , equations (74) and (75) are very similar to those for the maximum supersaturation in the constant-pressure decline rate case (equation (64)). Furthermore, because of the closeness of $s_{D n}$ to $s_{D m}$, we may use the sensitivity analysis we conducted before to assess the dependence of $\frac{P_{b}-P_{n}}{P_{b}}$ to the various parameters. Thus, in the region where $s_{D m}$ is insensitive to $\Delta$, the supersaturation $\frac{P_{b}-P_{n}}{P_{b}}$ varies linearly with $\Pi_{c}$. When $s_{D m}$ is more sensitive, with an assumed power-law variation with an exponent $1 / 4$, the supersaturation at the minimum pressure varies roughly as

$$
\frac{P_{b}-P_{m}}{P_{b}} \sim\left(\frac{\Pi_{4}}{\Pi_{3}}\right)^{\frac{1}{4}} \Pi_{c}^{\frac{2}{3}} \Pi_{2}^{-\frac{1}{12}}
$$

Such a dependence can be used to guide the matching of the experimental data, as discussed below.

\section{c. The Rate-Dependent Nucleation Model}

We close this section by applying a similar analysis, but now for the rate-dependent nucleation model. We recall the rescaled expression

$$
\frac{d \phi_{q}}{d t_{D}}=h_{1}^{*} \exp \left(-\frac{h_{2}}{s^{2}}\right)
$$

where $h_{1}^{*}=h_{1} \Pi_{1}^{-\frac{3}{2}}$. We proceed as before to evaluate the time when the maximum supersaturation is reached. For this, we first use the relation $s \approx t_{D}$, to obtain

$$
\frac{d \phi_{q}}{d s} \approx h_{1}^{*} \exp \left(-\frac{h_{2}}{s^{2}}\right)
$$

the solution of which is readily found

$$
\phi_{q}=h_{1}^{*}\left[\operatorname{sexp}\left(-\frac{h_{2}}{s^{2}}\right)-\sqrt{\pi h_{2}} \operatorname{erfc}\left(\frac{\sqrt{h_{2}}}{s}\right)\right]
$$

The maximum supersaturation occurs when the right-hand-side of (52) vanishes, which in this model approximately occurs when the following equation is satisfied

$$
s_{D m}^{* 3} \exp \left(-\frac{1}{s_{D m}^{* 2}}\right)-s_{D m}^{* 2} \sqrt{\pi} \operatorname{erfc}\left(\frac{1}{s_{D m}^{*}}\right)=\Delta_{h}^{\frac{3}{2}} \Lambda^{-1}
$$


Here, we defined the reduced supersaturation $s_{D}^{*}=s / \sqrt{h_{2}}$ and the dimensionless parameter

$$
\Delta_{h}=\Pi_{1} h_{1}^{-\frac{2}{3}} h_{2}^{-1} \Pi_{2}^{-\frac{1}{3}} \quad\left(=\Pi_{1}^{\frac{5}{3}} \zeta^{-\frac{2}{3}} h_{2}^{-1} \Pi_{2}^{-\frac{1}{3}} \text { or }=\frac{\Pi_{4}}{\Pi_{3}} \zeta^{-\frac{2}{3}} h_{2}^{-1} \Pi_{2}^{-\frac{1}{3}}\right)
$$

For relatively small $s_{D m}^{*}$, the solution of the above reads

$$
\frac{1}{s_{D m}^{* 2}}-5 \ln s_{D m}^{*} \approx \ln \Lambda-\frac{1}{2} \ln 3-\ln 2-\frac{3}{2} \ln \Delta_{h}
$$

A plot of the solution of $(80)$ is shown in Fig. 11. We note features very similar to the cavity nucleation model, namely a region of weak sensitivity at small $\Delta_{h}$ and of stronger sensitivity at higher $\Delta_{h}$. The rescaled supersaturation for the rate-dependent nucleation model is slightly higher in the region of small $\Delta_{h}$, but its rate of increase at higher $\Delta_{h}$ is weaker than for the cavity model. Given the dependence of $\Delta_{h}$ on rate, the dependence of the maximum supersaturation could, at first, be considered stronger. For example, for $s_{D m}^{*}$ to vary as a power-law of the rate with exponent $m$, it suffices for it to follow a power law with respect to $\Delta_{h}$ with exponent $3 \mathrm{~m} / 5$. Interestingly, however, this higher sensitivity is counterbalanced by the lower sensitivity to $\Delta_{h}$ at higher values of $\Delta_{h}$, compared to the cavity model. For example, if we were to demand $m=1 / 4$, then we should consider a range of $\Delta_{h}$ in Fig. 11 where the exponent is of the order of $3 / 20$. As shown in the Figure, this roughly corresponds to the same range as that of $\Delta$, for the cavity model (Fig. 10).

The nucleation fraction at the time of the maximum supersaturation can be estimated as before. We find

$$
f_{q f}=s_{D m}^{*-2} h_{2}^{-1}\left(\frac{\Pi_{2}}{3}\right)^{-\frac{1}{2}} \Pi_{1}^{\frac{3}{2}} \Lambda^{-1}
$$

As expected, the nucleation rate increases with a decreasing $h_{2}$, namely with smaller values

of the interfacial tension $\gamma$ and the nucleation parameter $f$. The combination $h_{1}^{\frac{1}{2}} h_{2}^{\frac{3}{4}}$ plays here the role of $\Pi_{c}$. For the same reasons as before, the pressure at the end of nucleation, which is also approximately the minimum pressure, is given by

$$
\frac{P_{b}-P_{m}}{P_{b}} \approx \sqrt{h_{2}} s_{D m}^{*}
$$


In matching experimental data using this model, we would need to infer two parameters, the rate constant $K_{\text {het }}$ and the heterogeneous parameter $f$. This is discussed below.

Working likewise for the case of constant liquid withdrawal rate we find that the previous equations are valid if we replace $\Pi_{1}$ with $\frac{\Pi_{4}}{\Pi_{3}}$, and take $\Lambda \equiv \kappa / \Pi_{3}$.

\section{B. Gas cluster growth}

The modeling of the growth regime, where nucleation has terminated, can also be simplified if we consider only one class of clusters. For the case of constant pressure decline rate we approximate

$$
\frac{d C_{D \infty}}{d t_{D}} \approx-\left(C_{D \infty}-1+t_{D}\right) z-C_{D \infty} \Pi_{3}
$$

and

$$
\left(1-t_{D}\right) \frac{d z^{D_{f}}}{d t_{D}}=k_{1}^{-1}\left(C_{D \infty}-1+t_{D}\right) z+z^{D_{f}}
$$

where, we introduced the variable

$$
z \equiv \phi_{q f} \rho_{D}
$$

and the parameter

$$
k_{1}=\frac{\phi_{q f}^{1-D_{f}}}{\Pi_{2}}
$$

The final value of the rescaled nucleation fraction, $\phi_{q f}$, as well as the initial values for $C_{D \infty}$

and $\rho_{D}$ needed for the calculations, are obtained from the previous analysis. Likewise, for the case of constant liquid withdrawal rate, we have similar equations

$$
\frac{d C_{D \infty}}{d t_{D}} \approx-\frac{1}{\Pi_{3}}\left(C_{D \infty}-P_{D l}\right) z-C_{D \infty}+C_{D \infty} \kappa k_{2} \frac{d z^{D_{f}}}{d t_{D}}
$$

and

$$
\Pi_{3} P_{D l} \frac{d z^{D_{f}}}{d t_{D}}+z^{D_{f}}\left(\kappa k_{2} \frac{d z^{D_{f}}}{d t_{D}}-1\right)=k_{2}^{-1}\left(C_{D \infty}-P_{D l}\right) z
$$


along with

$$
\frac{d P_{D l}}{d t_{D}} \approx \frac{1}{\Pi_{3}}\left(\kappa k_{2} \frac{d z^{D_{f}}}{d t_{D}}-1\right)
$$

and where we defined

$$
k_{2}=\frac{\phi_{q f}^{1-D_{f}}}{\Pi_{2}}
$$

To use the simplified growth model, we take initial values for $C_{D \infty}, \rho_{D}, P_{D l}$, and $\phi_{q f}$ corresponding to the time the local minimum pressure is reached. Note that $P_{D l}$ might be known experimentally, while one can take $C_{D \infty} \approx 1$ without introducing significant error.

Comparison of the full solution with the approximate model is shown in Fig. 12. We note a good agreement. In particular, the approximate model captures well the pressure increase, following the minimum, its subsequent leveling and the gradual decline paralleling the equilibrium curve. The system in consideration has parameters corresponding to the Berea sandstone experiments of Firoozabadi et al. (1992) with $Q=1.44 \mathrm{~cm}^{3} / \mathrm{day}$. This system will be discussed in further detail in the next section.

\section{COMPARISON WITH EXPERIMENTS}

\section{a. Constant Pressure Decline Rate}

The above models were subsequently checked against published experimental results. We attempted to match the following quantities and their dependence on parameters, particularly on rate: the final nucleation fraction, the maximum supersaturation, $s_{D m}$, the critical gas saturation, $S_{g c}$, and the evolution of pressure or saturation as a function of time.

Experimental data for the maximum supersaturation, for the case of constant pressure decline rate, were reported by Moulu and Longeron (1989) and Scherpenisse et al. (1994). In these experiments, the maximum estimated value for the capillary number was approximatelly $10^{-8}$ which is well within the range of the validity of the model. In addition, the macroscopic capillary number, $C a_{m}=C a \frac{L}{\sqrt{k}}$, where $L$ is the length of the core, was of the order of $10^{-3}$ indicating negligible viscous pressure gradients. Matching the results requires 
the use of a window in the $s_{D m}$ vs. $\Delta$ relationship, where a $1 / 4$ power-law is approximately observed. The corresponding windows were identified in Fig. 11 both for the rate-dependent nucleation model and the cavity model. The combination $\Delta$ contains a number of geometric variables, which are not known a priori. The cavity size distribution is also unknown. We used best estimates for $V_{s}$ and a range of values for the cavity size characteristics, to indicate the range of $\Delta$ where the various experimental results fall for various size distributions considered. Theory and experiments for the mixture C1/C5 in the Berea sandstone experiments by Scherpeniesse et al. (1994) match well, assuming a stretched exponential distribution with values $n=0.215$ and $\sigma=0.045$. In these experiments we used for $r_{s}^{*}$ and $r_{c}^{*}$ the suggested values by the authors. For the experiments involving the mixture $\mathrm{C} 1 / \mathrm{C} 3 / \mathrm{C} 10$ in a limestone core, reported by Moulu and Longeron (1989), the corresponding best-fit values were $n=0.152$ and $\sigma=0.049$, respectively. These indicate a significantly stretched (long-tailed) cavity-size distribution, with small characteristic sites (see also Table 2 for additional data regarding the experiments).

The predictions of the rate-dependent nucleation model were also tested. Here, the parameters to be estimated include $f$ and the heterogeneous nucleation rate $K_{\text {het }}$, since

$$
\Delta_{h} \sim K_{h e t}^{-\frac{2}{3}} f^{-1}
$$

Matching of the experiments of Scherpenisse et al. (1994) required the following parameter values: $f=2 . \times 10^{-4}$ and $K_{\text {het }}=0.02119\left(\mathrm{~cm}^{3} \mathrm{~s}\right)^{-1}$ (Fig. 11). Correspondingly, for the experiments by Moulu and Longeron (1989) the following best-fit parameter values were found: $f=3.6 \times 10^{-6}$ and $K_{h e t}=6.45 \times 10^{-6}\left(\mathrm{~cm}^{3} \mathrm{~s}\right)^{-1}$. Both these sets of values are extreme for the heterogeneousmodel considered.

Estimates for the final nucleation fraction for the experiments by Scherpenisse et al. (1994) are shown in Fig. 13. The final nucleation fraction in the experiments was estimated as suggested by Scherpenisse et al. (1994). Then, $f_{q f}$ was calculated using equation (11). As anticipated, $f_{q f}$ is quite small, of the order of $10^{-10}-10^{-7}$. The power-law scaling with exponent 1, predicted by the theory in this range, is well supported by the data. Finally, a comparison of the evolution of the gas saturation, $S_{g}$, as a function of the dimensionless pressure $P_{D l}=P / P_{b}$, using the full solution for the Berea sandstone experiments of Scherpenisse 
et al. (1994) is presented in Fig. 14. Good agreement is observed for the early part of the curve, namely before the gas saturation starts to approach an asymptotic value. The latter stage corresponds to flow of gas out of the sample, which the present theory does not take into account. The dependence of $S_{g c}$ on $f_{q f}$ was tested against the data of Scherpenisse et al. (1994). Shown in Fig. 15 are predictions for both the critical gas saturation as a function of $\Delta$ from the simpler model and from the full solution. Although there is a slight tendency for the theory to underestimate the data, we note a quite good agreement. In particular, the power-law scaling with exponent 0.16 , predicted by the theory, is well supported by the data.

One of the most interesting effects is that of the pressure decline rate. Quantifying its effect is significant, as one can then control the extent of nucleation, the value of the critical gas saturation, and the time of the onset of bulk gas flow, which for practical purposes signifies the end of the liquid production. An important result of this work has been the derivation of simple algebraic equations (for example (56)-(59), (80)) that relate the critical supersaturation to a combination of dimensionless parameters, which involve the rate, given the particular nucleation characteristics of the system. The model developed shows that the effect on the nucleation fraction, hence on the critical gas saturation, is a power law, with an exponent which is equal $3 / 2$ at low rates, decreases to 1 at higher rates, and ultimately becomes zero at very high rates. The critical supersaturation was predicted to be independent of the rate at small rates and to depend as a weak power law (with an exponent equal to $1 / 4$ or larger) at higher rates. It can be also readily shown that for very large depletion rates, the maximum supersaturation is insensitive to the particular size distribution, scaling as a power law with exponent $3 / 4$. In that limit, the final nucleation fraction is also independent of the rate of depletion.

It interesting that for the experimentally reported rate effect to be matched requires a considerably stretched (long-tailed) cavity size distribution and small cavity sizes. Equivalently, if the heterogeneous, rate-dependent nucleation model is used, matching the experiments requires very small values of the wetability parameter and of the nucleation rate constant. A long-tailed cavity size distribution leads to a larger nucleation fraction, provided that the decline rate is not extremely large. Then, more cavity sizes are nucleated at the early stages 
of the process, as (large) sizes are available for activation even under very small supersaturations. It is possible that this is due to the nucleation of large cavities, exposed at the openings of the core for which a very small supersaturation is needed. Because of the existence of nucleated bubbles early during the process, the maximum supersaturation obtained for a long-tailed distribution is smaller than for a narrow distribution (such as a Rayleigh), when the rates are not too large. Because as the rate of depletion becomes very large, the supersaturation becomes eventually the same for all distributions, there is a large window in the rate dependence, where the behavior is like the experimentally reported $1 / 4$ power law. A similar explanation holds for the case of the rate-dependent heterogeneous nucleation model, where a very small value of the wetability parameter favors the nucleation of bubbles even at small supersaturation.

\section{b. Constant Rate of Liquid Withdrawal}

The model was also compared against experimental results for the case of constant rate of liquid withdrawal. We matched the local minimum pressure and the related the maximum supersaturation, $P_{n}$ and $s_{D n}$ respectively, the critical gas saturation, $S_{g c}$, and the evolution of pressure as a function of time.

Consider, first, matching the minimum pressure and its rate-dependence. Using the simpler model, this can be done by fitting parameters $r_{c}^{*}, r_{s}^{*}$ (if not available) and the cavity size distribution. We first performed this matching for the mixture $\mathrm{C} 1 / \mathrm{C} 10$ in the Berea sandstone experiments of Firoozabadi et al. (1992). In these experiments, the estimated values for $C a$ and $C a_{m}$ were $10^{-9}$ and $10^{-3}$, respectively, well within the assumptions of our theory.

Best-fit values $r_{s}^{*}=1.0 \times 10^{-2} \mathrm{~cm}, r_{c}^{*}=2.0 \times 10^{-3} \mathrm{~cm}, n=0.1014$ and $\sigma=0.0339$ (for a stretched exponential cavity distribution) were found for a good match. The calculated minimum pressures, $P_{n}$, using the full solution and the above parameters are very close to the experimental, as shown in Table 3. Comparison of the evolution of the system pressure as a function of the dimensionless time, $t_{n}=t_{D}$, using the full solution, and the Berea experiments of Firoozabadi et al. (1992) is presented in Fig. 16. A good agreement is also observed. 
A comparison with the chalk experiments of Firoozabadi et al. (1992), for two different reported volumetric flow rates, was also undertaken. Again, $C a \simeq 10^{-8}$ and $C a_{m} \simeq 10^{-2}$ are within the assumptions of our model. Here, the effect of rate is rather weak, and a Rayleigh distribution was found to be adequate. Based on $r_{s}^{*}=1.0 \times 10^{-4} \mathrm{~cm}$, a value of $r_{c}^{*}=1.98 \times 10^{-6} \mathrm{~cm}$ is needed to match the minimum pressure supersaturation. Figure 17 shows a comparison of the evolution of the liquid pressure as a function of the dimensionless time, $t_{n}=t_{D}$, using the full solution. Again good agreement is observed for the pressure evolution, while an excellent match is achieved for the minimum pressures (see Table 3 ). In both Figs. 16 and 17, the predictions from the simpler model closely follow the full solution.

Finally, the theory was tested against the sand-pack experiments of Kumar et al. (2000). In contrast to the previous, the estimated values for $C a$ and $C a_{m}$ are considerably higher (equal to about $10^{-4}$ and 10 , respectively). This sugests that our model may not adequately capture the physical processes particularly at later times Using the best-fit values of $r_{s}^{*}=$ $1.0 \times 10^{-2} \mathrm{~cm}$ and $r_{c}^{*}=1.0 \times 10^{-3} \mathrm{~cm}$, and a stretched exponential cavity distribution, with parameters $n=0.3466$ and $\sigma=0.0134$, provides for good matching. With these parameters, the ability of the full problem to predict the minimum pressure is good, as can be seen in Table 4. The evolution of the pressure as a function of dimensionless time, $t_{n}$, and for different flow rates is shown in Fig. 18, based on the simpler growth model. With the exception of the high rate curve, which is not perfectly matched, agreement is good, considering the scatter of the experimental data.

A feature that is not well matched in either of these experiments, however, are the values of $S_{g c}$. The predicted values, using the full solution, are shown in Tables $3-4$ respectively. The theory systematically over-predicts the experimental data. This disagreement may be due to the different definition of $S_{g c}$. In this paper, the critical gas saturation is defined as the gas saturation, when a sample spanning cluster is formed, in the absence of gravity or viscous gradients in the system (which will result in increasing $S_{g c}$ ). In the experiments, however, bubbles can be mobilized due to the presence of gradients before the onset of a sample-spanning cluster. The disagreement is more profound for the cases of the Berea sandstone or the sand-pack, than it is for the chalk. It is also very pronounced in the Kumar et al. (2000) experiments, as explained above. This is consistent, since chalk is a tighter 
porous medium, the capillary and Bond numbers are smaller and the earlier mobilization of the gas bubbles is less likely.

For completeness, we also estimated the wetability parameter, $f$, and the heterogeneous nucleation rate, $K_{h e t}$, assuming a rate-dependent nucleation model. We found the following: for the Berea sandstone experiments (Firoozabadi et al., 1992), $f=3.305 \times 10^{-5}$ and $K_{\text {het }}=$ $7.219 \times 10^{-7}\left(\mathrm{~cm}^{3} \mathrm{~s}\right)^{-1}$; for the chalk experiments (Firoozabadi et al., 1992), $f=1.240 \times 10^{-5}$ and $K_{\text {het }}=2.299 \times 10^{5}\left(\mathrm{~cm}^{3} \mathrm{~s}\right)^{-1}$; and for the sand-pack experiments (Kumar et al., 2000), $f=1.790 \times 10^{-5}$ and $K_{\text {het }}=1.683 \times 10^{-5}\left(\mathrm{~cm}^{3} \mathrm{~s}\right)^{-1}$.

As in the case of constant pressure decline rate and with the exception of the experiments in chalk, matching of the experimental results with the theory required the use of either stretched, long-tailed cavity size distributions, or very small wetability parameters in the rate-dependent nucleation model. Such distributions offer the ability to nucleate bubbles even at small supersaturations, and can provide the reported experimental dependence on rate.

\section{CONCLUSIONS}

In this paper we developed an effective continuum model to describe the nucleation and subsequent growth of a gas phase from a supersaturated, slightly compressible binary liquid in a porous medium, driven by solute diffusion. The evolution of the gas results either from the reduction of the system pressure at a constant rate, or from the withdrawal of the liquid at constant rate. The model addresses two stages before the onset of bulk gas flow, nucleation and gas phase growth. We assume negligible gradients due to gravity or viscous forces, thus the critical gas saturation, which signals the onset of bulk gas flow, is only a function of the nucleation fraction.

We showed that the important quantities characterizing the process, such as the fraction of pores that host activated sites, the deviation from thermodynamic equilibrium, the maximum supersaturation in the system and the critical gas saturation depend crucially on the nucleation characteristics of the medium. We used a heterogeneous nucleation models

primarily in the form of pre-existing gas, trapped in hydrophobic cavities, but also in terms 
of a rate-dependent nucleation, to investigate in detail the nucleation behavior. Using scaling analysis and a simpler analytical model we showed that the relevant quantities during nucleation can be expressed in terms of a simple combination of dimensionless parameters, which include rate effects, for either type of nucleation model.

The theory predicts that the maximum supersaturation in the system is a weakly increasing function of rate, which in the region of typical experimental parameters, can be approximated as a power law with a small exponent. This function depends sensitively on the probability density function of the nucleation cavity sizes. It also predicts that the final nucleation fraction, thus the critical gas saturation, is a power law of the decline rate (or the withdrawal rate). The theoretical exponents were shown to fit the experimental data provided that a stretched exponential distribution for the cavity size distribution is used. 


\section{REFERENCES}

Andarcia, L., Kamp, A.M., and P. Vaca, "Heavy Oil Solution Gas Drive in the Venezuelan Orinoco Belt: Laboratory Experiments and Field Simulation" paper SPE 69715, presented at the SPE International Thermal Operations and Heavy Oil Symposium, Margarita Island, Venezuela (12-14 March 2001).

Arora, P., and A.R. Kovscek, "Mechanistic Modeling of Solution-Gas Drive in Viscous Oils," paper SPE 6971\%, presented at the SPE International Thermal Operations and Heavy Oil Symposium, Margarita Island, Venezuela (12-14 March 2001).

Bora, R., Maini, B.B., and A. Chakma, "Flow Visualization Studies of Solution Gas Drive Process in Heavy Oil Reservoirs Using a Glass Micromodel," SPE Res. Eval. Eng., 3, $224(2000)$.

Dominguez, A., Bories, S., and M. Prat, "Gas Cluster Growth by Solute Diffusion in Porous Media. Experiments and Automaton Simulation on Pore Network," Int. J. Multiphase Flow, 26, 1951 (2000).

Doughty, C. and K. Pruess, "A Similarity Solution for Two-Phase Fluid and Heat Flow Near High-Level Nuclear Waste Packages Emplaced in Porous Media," Int. J. Heat Mass Transfer, 33, 1205 (1990).

Du, C., and Y.C. Yortsos, "A Numerical Study of the Critical Gas Saturation in a Porous Medium," Transport in Porous Media, 35, 205 (1999).

Egermann, P., and O. Vizika, "Critical Gas Saturation and Relative Permeability During Depressurization in the Far Field and the Near-Wellbore Region," paper 63149, presented at the SPE Annual Technical Conference and Exhibition, Dallas, TX, 1-4 October 2000.

El Yousfi, A., Zarcone, C., Bories, S. and R. Lenormand, "Mècanismes de Formation d'une Phase Gazeuse par détente d'un liquide en Milieu Poreux," C.R. Acad. Sci. Paris, Série II, 313, 1093 (1991).

El Yousfi, A., Zarcone, C., Bories, S. and R. Lenormand, "Physical Mechanisms for Bubble Growth During Solution Gas Drive," paper SPE 38921 presented at the SPE Annual Technical Conference and Exhibition, San Antonio, TX (5-8 October 1997).

Feder, J., Fractals, Plenum, New York (1988). 
Firoozabadi, A., and A. Aronson, "Visualization and Measurement of Gas Evolution and Flow of Heavy and Light Oil in Porous Media," SPE Res. Eval. Eng., 2, 550 (1999).

Firoozabadi, A., and D. Kashchiev, "Pressure and Volume Evolution During Gas Phase Formation in Solution Gas Drive Processes," SPE Journal, 1, 219 (1997).

Firoozabadi, A, Ottesen, B, and M. Mikkelsen, "Measurements of Supersaturation and Critical Gas Saturation," SPE Form. Eval., 337 (December 1992).

Jones, S.F., Evans, G.M., and K.P. Galvin, "Bubble Nucleation from Gas Cavities - A Review," Adv. Colloid Interface Sci., 80, 27 (1999).

Hawes, R.I., Dawe, R.A., and R.N. Evans, “The Release of Solution Gas from Waterflood Residual Oil," SPE Journal, 2, 379 (1997).

Ho, C.K., and K.S. Udell, "Mass Transfer Limited Drying of Porous Media Containing an Immobile Binary Liquid Mixture," Int. J. Heat Mass Transfer, 38, 339 (1995).

Kamp, A.M., Joseph, D.D., and R. Bai, “A New Modeling Approach for Heavy Oil Flow in Porous Media," paper SPE 69720, presented at the SPE International Thermal Operations and Heavy Oil Symposium, Margarita Island, Venezuela (12-14 March 2001a).

Kamp, A.M., Heny, C., Andarcia, L., Lago, M. and A. Rodriguez, "Experimental Investigation of Foamy Oil Solution Gas Drive," paper SPE 69725, presented at the SPE International Thermal Operations and Heavy Oil Symposium, Margarita Island, Venezuela (12-14 March 2001b).

Kumar, R., Pooladi-Darvish, M., and T. Okazawa, "An Investigation Into Enhanced Recovery Under Solution Gas Drive in Heavy Oil Reservoirs," paper SPE 59336, presented at the SPE/DOE Improved Oil Recovery Symposium, Tulsa, OK (3-5 April 2000).

Laaksonen, A., Talanquer, V., and D.W. Oxtoby, "Nucleation: Measurements, Theory, and Applications," Annu. Rev. Phys. Chem., 46, 489 (1995).

Li, X., and Y.C. Yortsos, "Visualization and Simulation of Bubble Growth in Pore Networks," AIChE J., 41, 214 (1995a).

Li, X., and Y.C. Yortsos, "Theory of Multiple Bubble Growth in Porous Media by Solute Diffusion," Chem. Eng. Sci., 50, 1247 (1995b).

Mackay, E..J., Henderson, G.D., Tehrani, D.H., and A. Danesh, "The Importance of the Interfacial Tension on Fluid Distribution During Depressurization," SPE Res. Eva. Eng., 
1, 408 (1998).

McDougall, S.R., and K.S. Sorbie, "Estimation of Critical Gas Saturation During Pressure Depletion in Virgin and Water-flooded Reservoirs," Petroleum Geoscience, 5, 229 (1999).

Maini, B., "Foamy Oil Flow in Primary Production of Heavy Oil Under Solution Gas Drive," paper SPE 56541, presented at the SPE Annual Technical Conference and Exhibition, Houston, TX (3-6 October 1999).

Maini, B., "Foamy Oil Flow in Heavy Oil Production," J. Canadian Petroleum Technology, 35(6), 21 (1996).

Moulu, J.C., "Solution-Gas Drive: Experiments and Simulation," J. Pet. Sci. Eng., 2, $379(1989)$.

Moulu, J.C., and D.L. Longeron, "Solution-Gas Drive: Experiments and Simulation," Proc., Fifth European Symposium on Improved Oil Recovery, Budapest, Hungary (April 1989).

Pooladi-Darvish, M., and A. Firoozabadi, "Solution-gas Drive in Heavy Oil Reservoirs," J. Canadian Petroleum Technology, 38(4), 54 (1999).

Prats, M., Thermal Recovery, SPE Monograph, Dallas, TX, 1982.

Press, W.H., Teukolsky, W.T., Vettering, S.A., and B.P. Flannery, Numerical Recipes, 2nd ed. Cambridge University Press, (1992).

Renard, G., Nauroy, J.-F., Deruyter, Ch., Moulu, J.-C., Sarda, J.-P., and J.-F. Le Romancer, "Production Froide des Huiles Visqueuses," Oil 8 Gas Science and Technology Rev. IFP, 55, 35 (2000).

Scherpenisse, W., Wit, K., Zweers, A.E., Shoei, G., and A. van Wolfswinkel, "Predicting Gas Saturation Buildup during Depressurization of a North Sea Oil Reservoir," paper SPE 2884D, presented at the European Petroleum Conference, London, UK (25-27 October 1994).

Satik, C., and Y.C. Yortsos, "A Pore Network Study of Bubble Growth in Porous Media Driven by Heat Transfer," ASME J. of Heat Transfer, 118, 155 (1996).

Sheng, J.J., Maini, B.B., Hayes, R.E., and W.S. Tortike, "Critical Review of Foamy Oil Flow," Transport in Porous Media, 35, 157 (1999a).

Sheng, J.J., Hayes, R.E., Maini, B.B., and W.S. Tortike, "Modeling Foamy Oil Flow in 
Porous Media," Transport in Porous Media, 35, 227 (1999b).

Smith, G.E., "Fluid Flow and Sand Production in Heavy-Oil Reservoirs Under SolutionGas Drive," SPE Prod. Eng., 3, 169 (1988).

Tang, G.-Q., and A. Firoozabadi, "Gas and Liquid-Phase Relative Permeabilities for Cold Production from Heavy Oil," paper SPE 56540 presented at the SPE Annual Technical Conference and Exhibition, Houston, TX (3-6 October 1999).

Thome, J.R., Enhanced Boiling Heat Transfer, Hemisphere Publishing Co., New York (1990)

Tsimpanogiannis, I.N., PhD Dissertation, Univ. of Southern California, In preparation (2002).

Tsimpanogiannis, I.N., and Y.C. Yortsos, "A Numerical Study of the Critical Gas Saturation in a Porous Medium in the Presence of Viscous or Gravity Gradients," In preparation (2002).

Urgelli, D., Durandeau, M., Foucault, H., and J.-F. Besnier, "Investigation of Foamy Oil Effect from Laboratory experiments," paper SPE 54083 presented at the SPE International Thermal Operations and Heavy Oil Symposium, Bakersfield, CA, 17-19 March 1999.

Wang, X., and K.K. Mohanty, "Critical Condensate Saturation in Porous Media," J. Colloid Interface Sci., 214, 416 (1999).

Wong, R.C.K., Guo, F., Weaver, J.S., and W.E. Barr, "Heavy Oil Flow Under SolutionGas Drive: Pressure Depletion Tests," J. Canadian Petroleum Technology, 38, 31 (1999).

Yortsos, Y.C., and M. Parlar, "Phase Change in Binary Systems in Porous Media: Application to Solution Gas Drive," paper SPE 19697, presented at the SPE Annual Technical Conference and Exhibition, San Antonio, TX (8-11 October 1989). 


\begin{tabular}{|c|c|c|}
\hline Parameter & $C P D R$ & $C L W R$ \\
\hline $\mathrm{MW}(g / m o l)$ & 18.6 & 16.0 \\
\hline $\mathrm{D}\left(\mathrm{cm}^{2} / \mathrm{s}\right)$ & $2.40 \times 10^{-5}$ & $1.35 \times 10^{-5}$ \\
\hline$\gamma(m N / m)$ & 8.2 & 13.0 \\
\hline $\mathrm{T}(K)$ & 314.6 & 314.6 \\
\hline$P_{b}(M P a)$ & 6.0 & 7.384 \\
\hline $\mathrm{K}\left(M P a m^{3} / k g\right)$ & $8.604 \times 10^{-3}$ & $1.537 \times 10^{-1}$ \\
\hline$c\left(M P a^{-1}\right)$ & $1.45 \times 10^{-3}$ & $2.17 \times 10^{-3}$ \\
\hline$r_{s}(\mathrm{~cm})$ & $9.0 \times 10^{-4}$ & $1.0 \times 10^{-2}$ \\
\hline$r_{c}(\mathrm{~cm})$ & $8.0 \times 10^{-6}$ & $2.0 \times 10^{-3}$ \\
\hline$A^{*}$ & 1. & 1. \\
\hline$n_{B}$ & 1. & 1. \\
\hline$\lambda$ & 1. & 1. \\
\hline$a(P a / s)$ & 26.82 & - \\
\hline $\mathrm{Q}\left(\mathrm{cm}^{3} / \mathrm{s}\right)$ & - & $1.67 \times 10^{-5}$ \\
\hline$V_{p}\left(\mathrm{~cm}^{3}\right)$ & - & 132.24 \\
\hline$\Pi_{1}$ & $5.859 \times 10^{-6}$ & - \\
\hline$\Pi_{2}$ & $2.327 \times 10^{6}$ & $1.330 \times 10^{3}$ \\
\hline$\Pi_{3}$ & $8.700 \times 10^{-3}$ & $1.603 \times 10^{-2}$ \\
\hline$\Pi_{4}$ & - & $1.559 \times 10^{-6}$ \\
\hline$\Pi_{c}$ & $3.417 \times 10^{-2}$ & $1.760 \times 10^{-4}$ \\
\hline
\end{tabular}

Table 1: Characteristic values of the various parameters. CPDR: Constant Pressure Decline Rate; CLWR: Constant Liquid Withdrawal Rate 


\begin{tabular}{|c|c|c|c|c|c|c|c|}
\hline 1 & 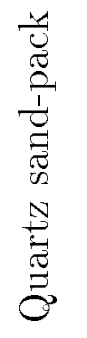 & 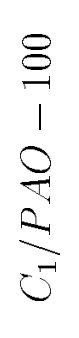 & 8 & $\Xi$ & $\begin{array}{l}\dot{0} \\
\stackrel{0}{\circ}\end{array}$ & 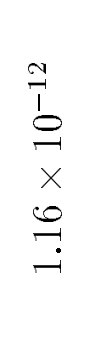 & 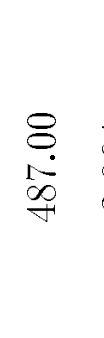 \\
\hline 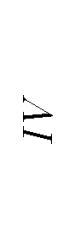 & 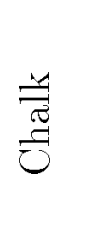 & $\frac{\dot{U}}{U}$ & $\underset{20}{\stackrel{20}{+}}$ & 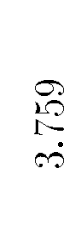 & 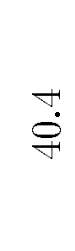 & 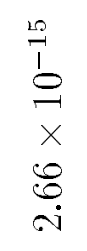 & 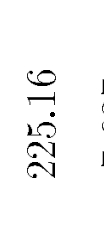 \\
\hline$\Xi$ & 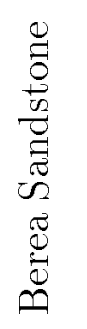 & $\frac{O}{U}$ & $\underset{\stackrel{\vec{\sigma}}{\dot{\sigma}}}{\vec{\sigma}}$ & $\begin{array}{l}0 \\
\vec{\sigma} \\
\sigma\end{array}$ & : & 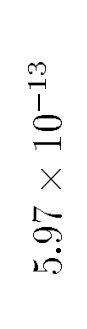 & 范 \\
\hline$\approx$ & 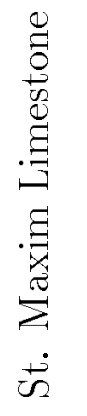 & $\frac{U^{\infty}}{U^{m}}$ & 20 & 10 & $\stackrel{\leftrightarrow:}{\stackrel{8}{8}}$ & 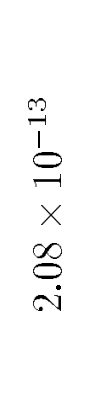 & $\ddot{\Xi}$ \\
\hline- & 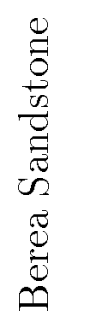 & $\underbrace{20}$ & $\stackrel{20}{2}$ & $\begin{array}{l}20 \\
2\end{array}$ & 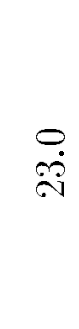 & 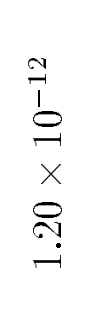 & $\stackrel{\Xi}{\approx}$ \\
\hline 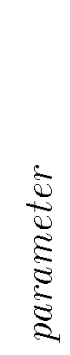 & 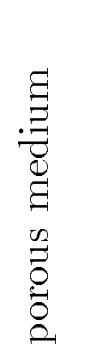 & 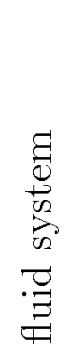 & 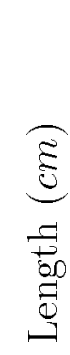 & 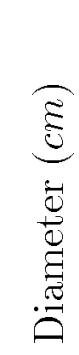 & $\begin{array}{l}0 \\
0 \\
0 \\
0 \\
0 \\
0 \\
0 \\
0 \\
0\end{array}$ & 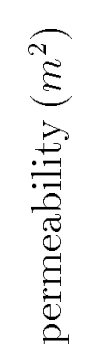 & 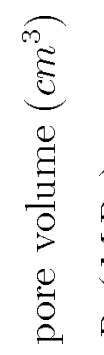 \\
\hline
\end{tabular}

ఠ్లై

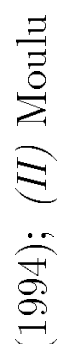

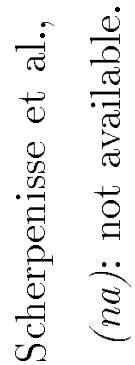

₹ $\underset{8}{\mathscr{8}}$

范

ஏ

$\stackrel{\square}{0}$

离

吾光

$\Xi$

造

5

क क

$=$

号:

$\frac{\pi}{2} \quad \frac{\pi}{\pi}$

घ

$\stackrel{5}{\Xi}$

$\frac{0}{0} E$

ह

స

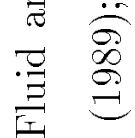

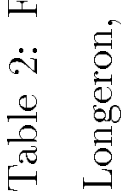




\begin{tabular}{|c|c|c|}
\hline$\Xi$ & 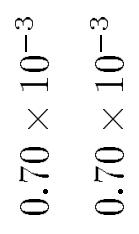 & 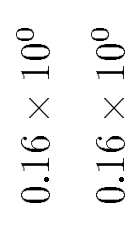 \\
\hline$\Xi$ & 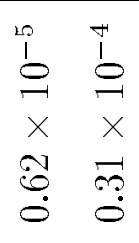 & 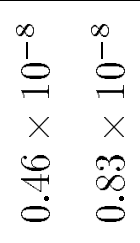 \\
\hline$\stackrel{\infty}{9}$ & 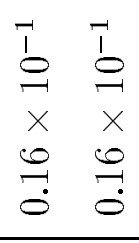 & 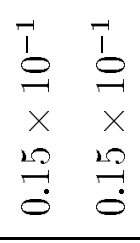 \\
\hline$\Xi$ & $\begin{array}{ll}\overrightarrow{0} & \overrightarrow{0} \\
\stackrel{0}{0} & x \\
\times & \times \\
\overrightarrow{20} & \overrightarrow{20} \\
\infty & \infty\end{array}$ & 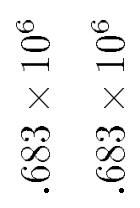 \\
\hline$\triangleleft$ & 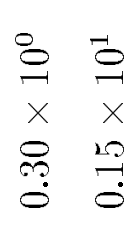 & 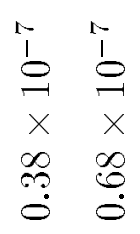 \\
\hline 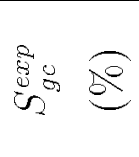 & 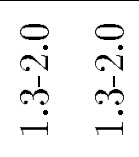 & 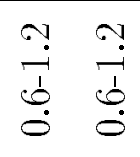 \\
\hline$\tilde{g}$ & 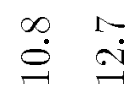 & $\stackrel{0}{\stackrel{9}{\rightarrow}} \stackrel{9}{-}$ \\
\hline ¿ & 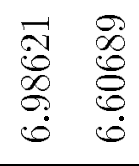 & 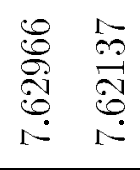 \\
\hline 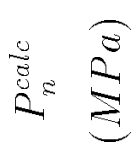 & 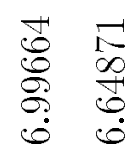 & 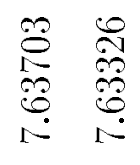 \\
\hline 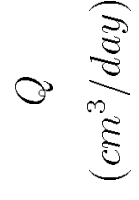 & 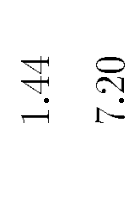 & 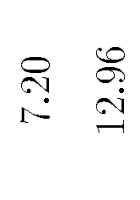 \\
\hline 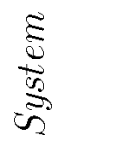 & 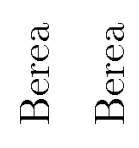 & 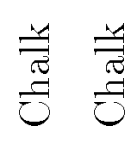 \\
\hline
\end{tabular}

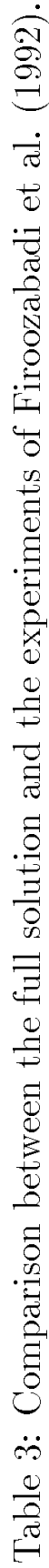




\begin{tabular}{|c|c|c|c|}
\hline$ت$ & 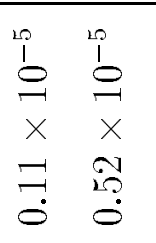 & 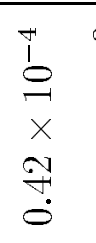 & 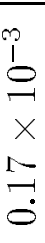 \\
\hline$\triangleleft$ & $\begin{array}{cc}8 & 8 \\
0 & 0 \\
\times & \times \\
0 & \times \\
0 & 0 \\
0 & 0\end{array}$ & $\begin{array}{l}\overrightarrow{0} \\
\stackrel{0}{1} \\
\times \\
\dot{0} \\
\dot{0} \\
\dot{0}\end{array}$ & 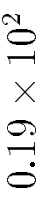 \\
\hline s & $\stackrel{\vec{\leftrightarrow}}{\leftrightarrow}$ & $\stackrel{\sim}{+}$ & $\stackrel{8}{\circ}$ \\
\hline$\vec{z}$ & $\stackrel{\leftrightarrow}{g}$ & 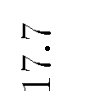 & $\begin{array}{l}0 \\
\stackrel{i}{N}\end{array}$ \\
\hline 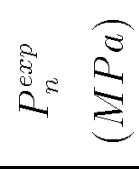 & 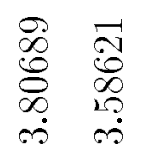 & 范 & 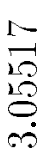 \\
\hline$\stackrel{\Xi}{\tilde{z}}=\stackrel{\Xi}{\Xi}$ & 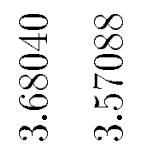 & 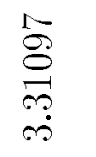 & 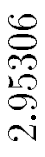 \\
\hline 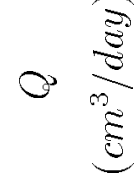 & \begin{tabular}{cc}
$\mathscr{N}$ & $\infty$ \\
$\stackrel{:}{-}$ & $\infty$ \\
\hdashline & $\infty$
\end{tabular} & $\begin{array}{l}\mathbb{8} \\
\text { i } \\
i\end{array}$ & 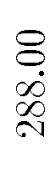 \\
\hline
\end{tabular}

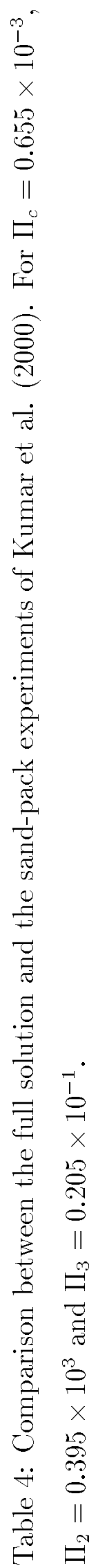




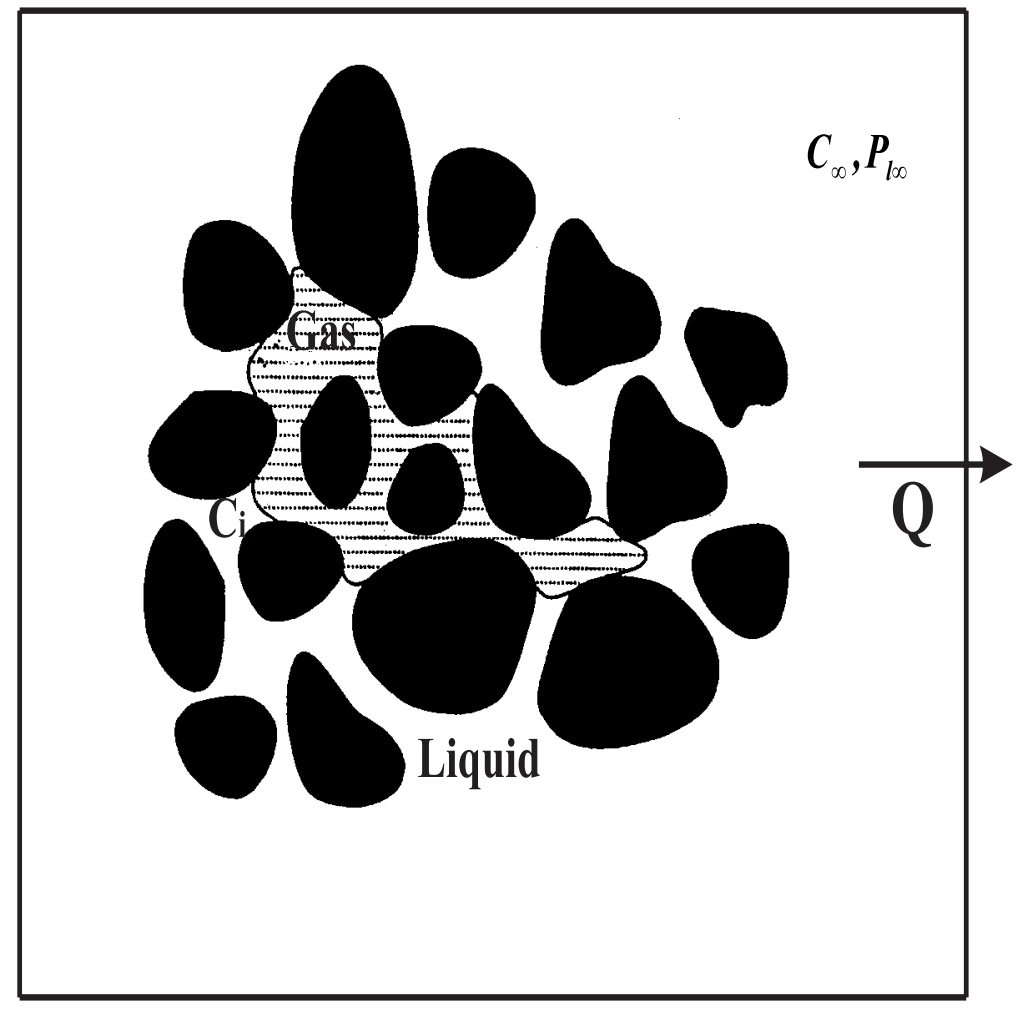

Figure 1: Schematic of a gas cluster growth in a porous medium, driven by the decline of pressure at constant rate. 
( a )

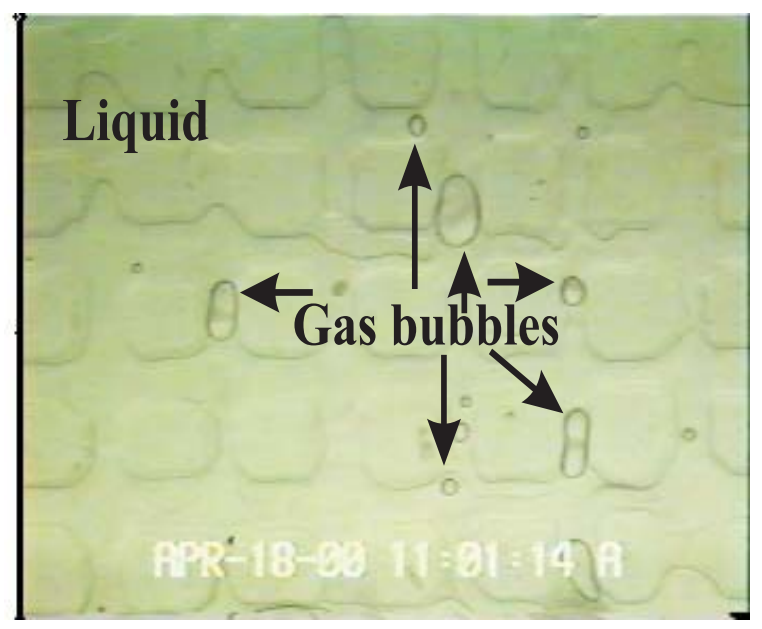

( b )

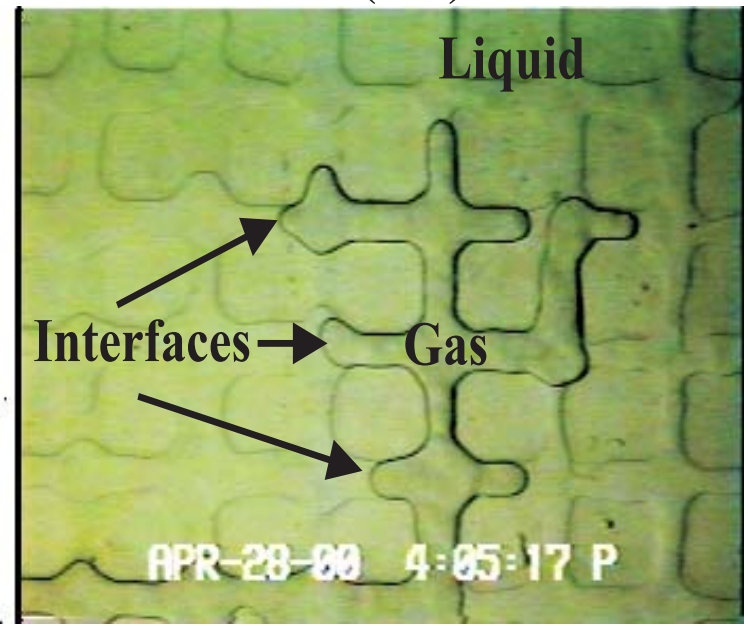

Figure 2: Micromodel snapshots indicating: (a) Gas bubbles confined within single pore throats/bodies; (b) a gas bubble spanning several pore bodies. 


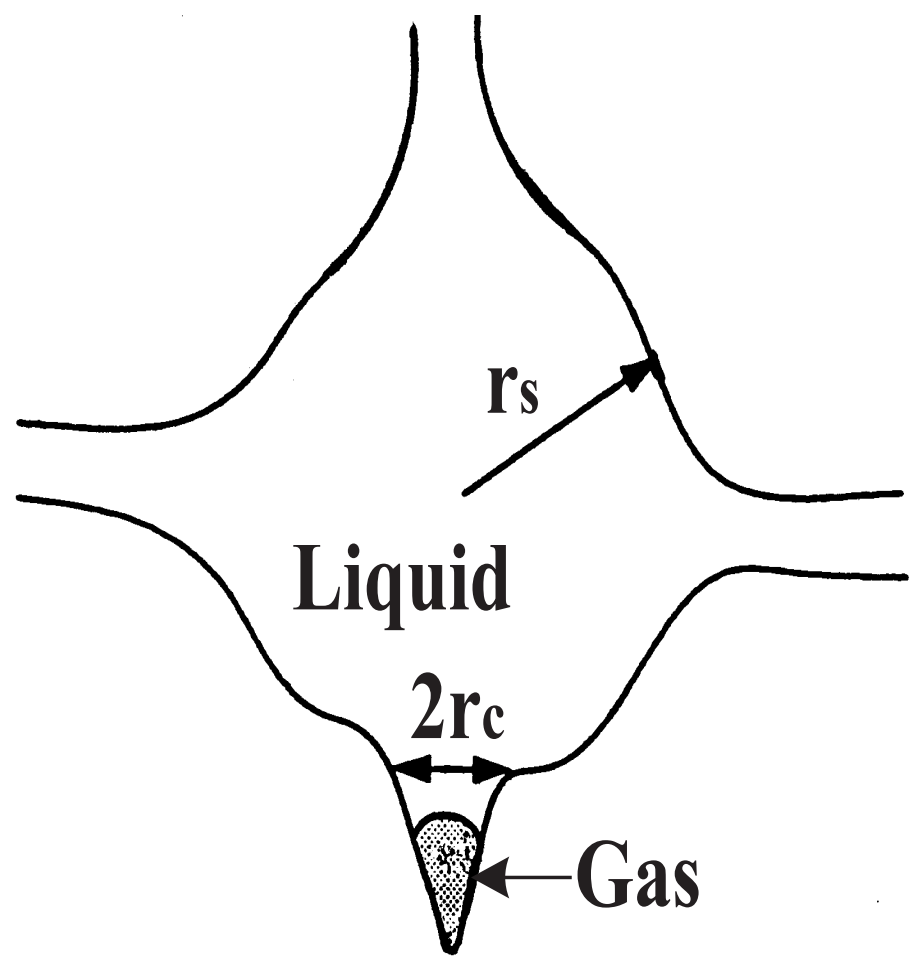

Figure 3: Schematic of a nucleation cavity in a host pore body. 
(a)

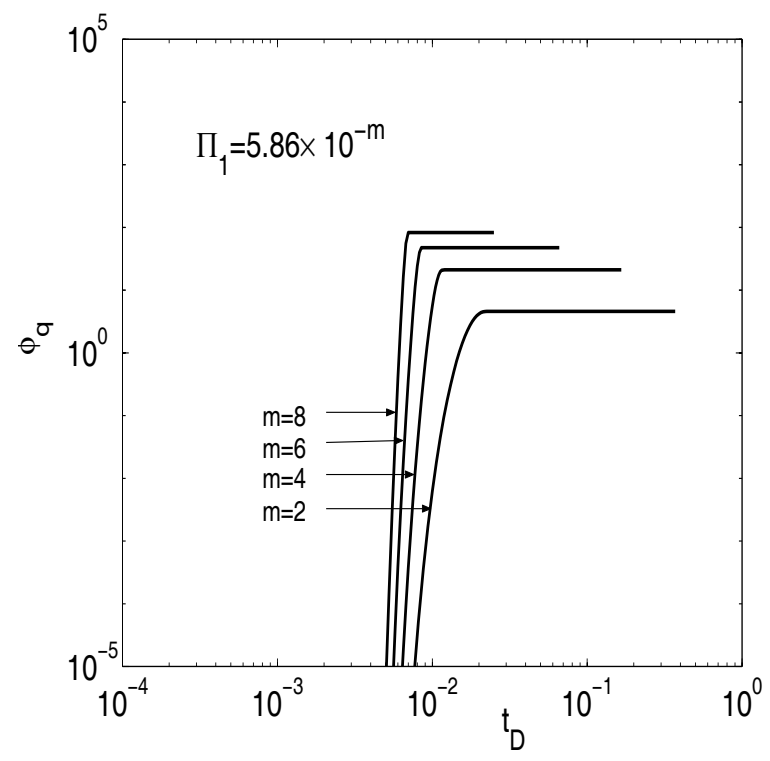

(b)

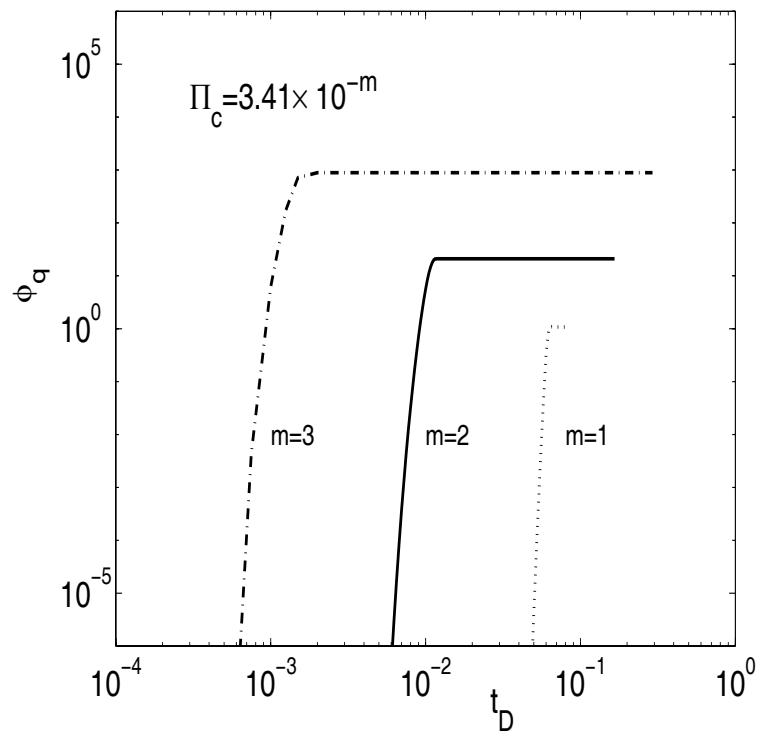

Figure 4: The variation of the rescaled nucleation fraction, $\phi_{q}$, as a function of the dimensionless time, $t_{D}$. (a) Effect of $\Pi_{1}=5.86 \times 10^{-m}$, for $\Pi_{c}=3.41 \times 10^{-2}, \Pi_{2}=2.33 \times 10^{6}$ and $\Pi_{3}=8.7 \times 10^{-3}$. (b) Effect of $\Pi_{c}=0.34 \times 10^{-m}$, for $\Pi_{1}=5.86 \times 10^{-4}$ and $\Pi_{3}=8.7 \times 10^{-3}$. 


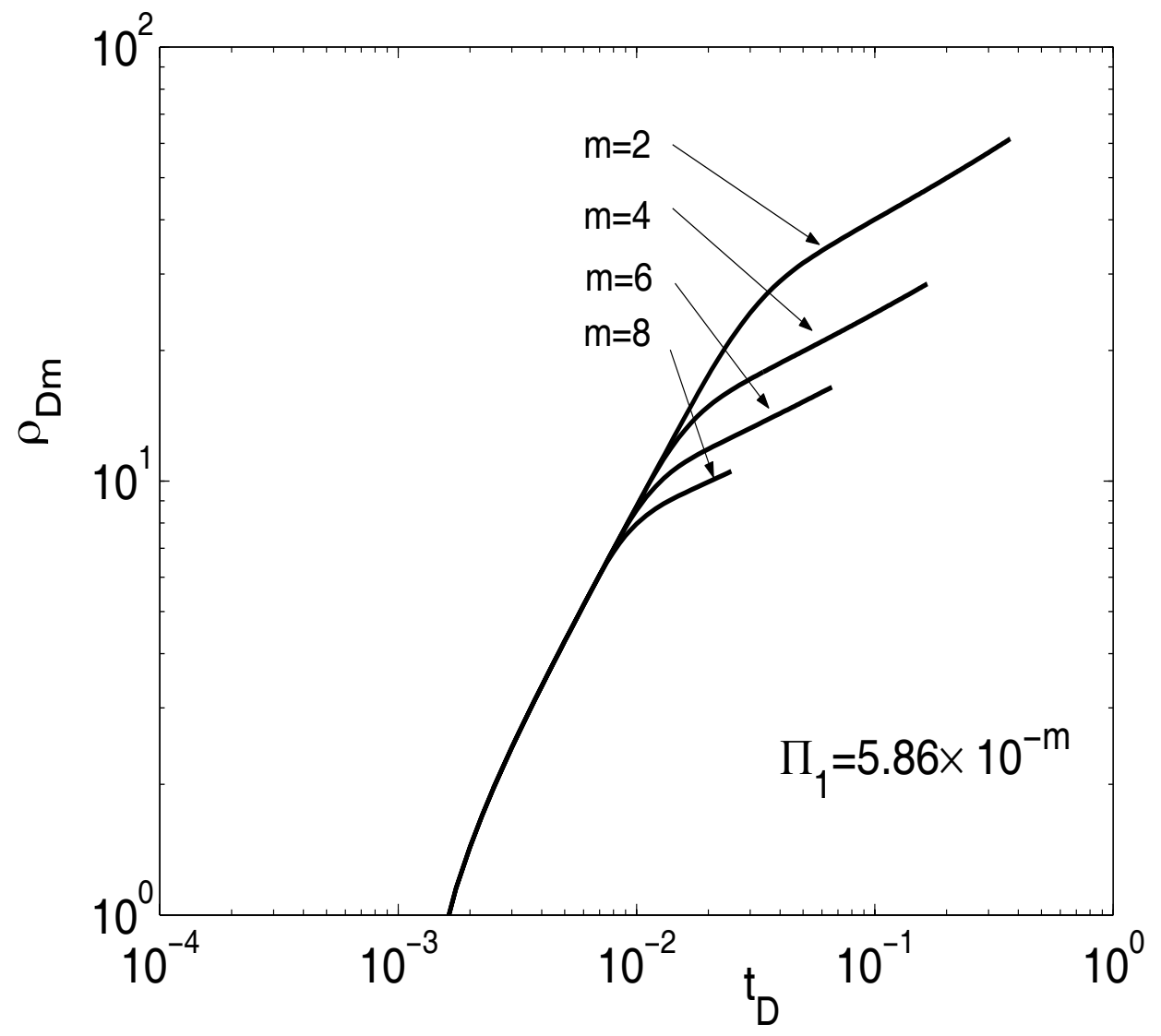

Figure 5: The variation of the mean rescaled dimensionless radius, $\rho_{D m}$, as a function of the dimensionless time, $t_{D}$. Effect of $\Pi_{1}=5.86 \times 10^{-m}$, for $\Pi_{c}=3.41 \times 10^{-2}, \Pi_{2}=2.33 \times 10^{6}$ and $\Pi_{3}=8.7 \times 10^{-3}$. 
(a)

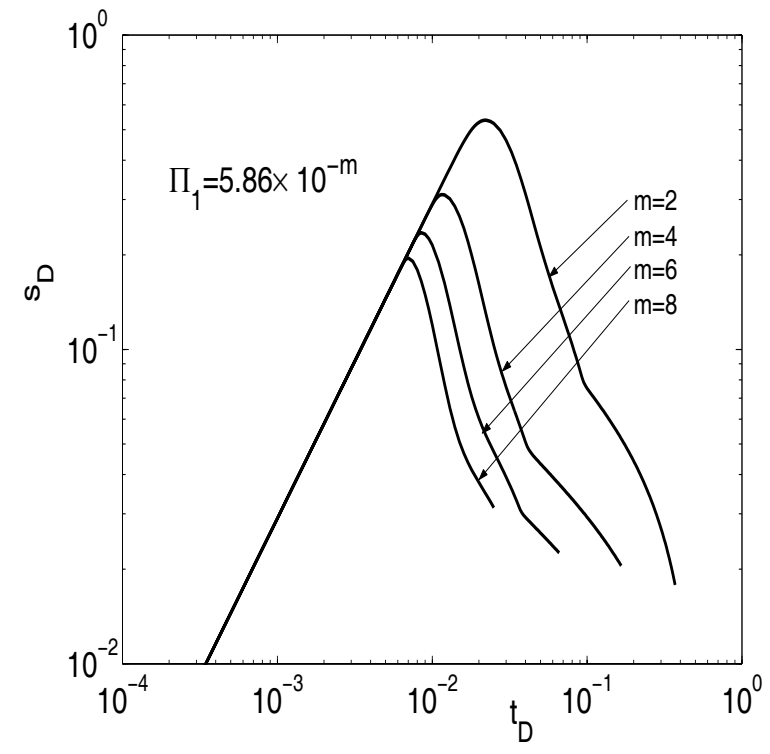

(b)

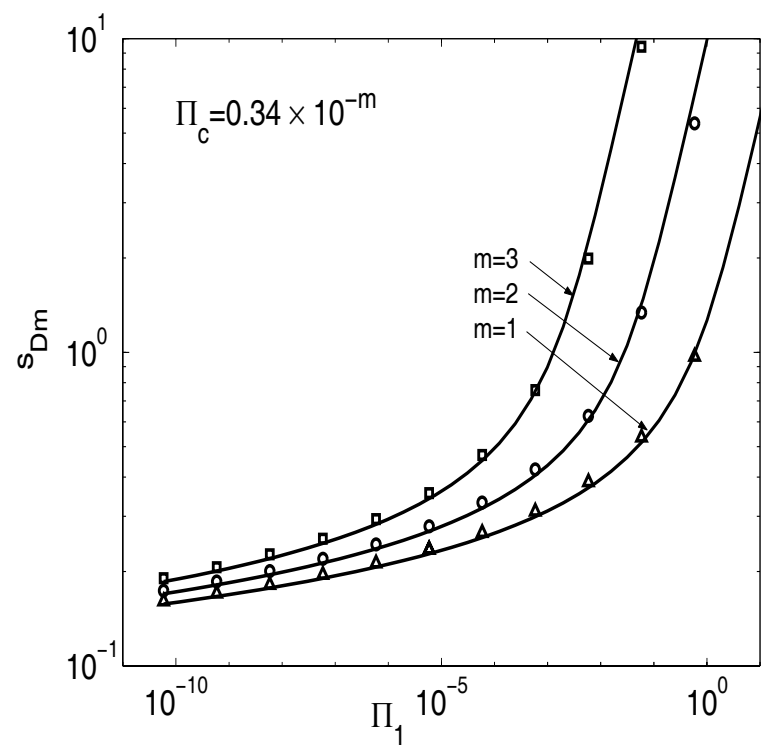

Figure 6: Numerical results for: (a) The variation of the rescaled supersaturation, $s_{D}$, as a function of the dimensionless time, $t_{D}$. Effect of $\Pi_{1}=5.86 \times 10^{-m}$, for $\Pi_{c}=3.41 \times 10^{-2}$, $\Pi_{2}=2.33 \times 10^{6}$ and $\Pi_{3}=8.7 \times 10^{-3}$. (b) The effect of the dimensionless parameter $\Pi_{1}$ on the maximum rescaled supersaturation, $s_{D m}$, for $\Pi_{c}=0.34 \times 10^{-m}$. Points correspond to the full numerical solution, solid lines correspond to the simpler model. 


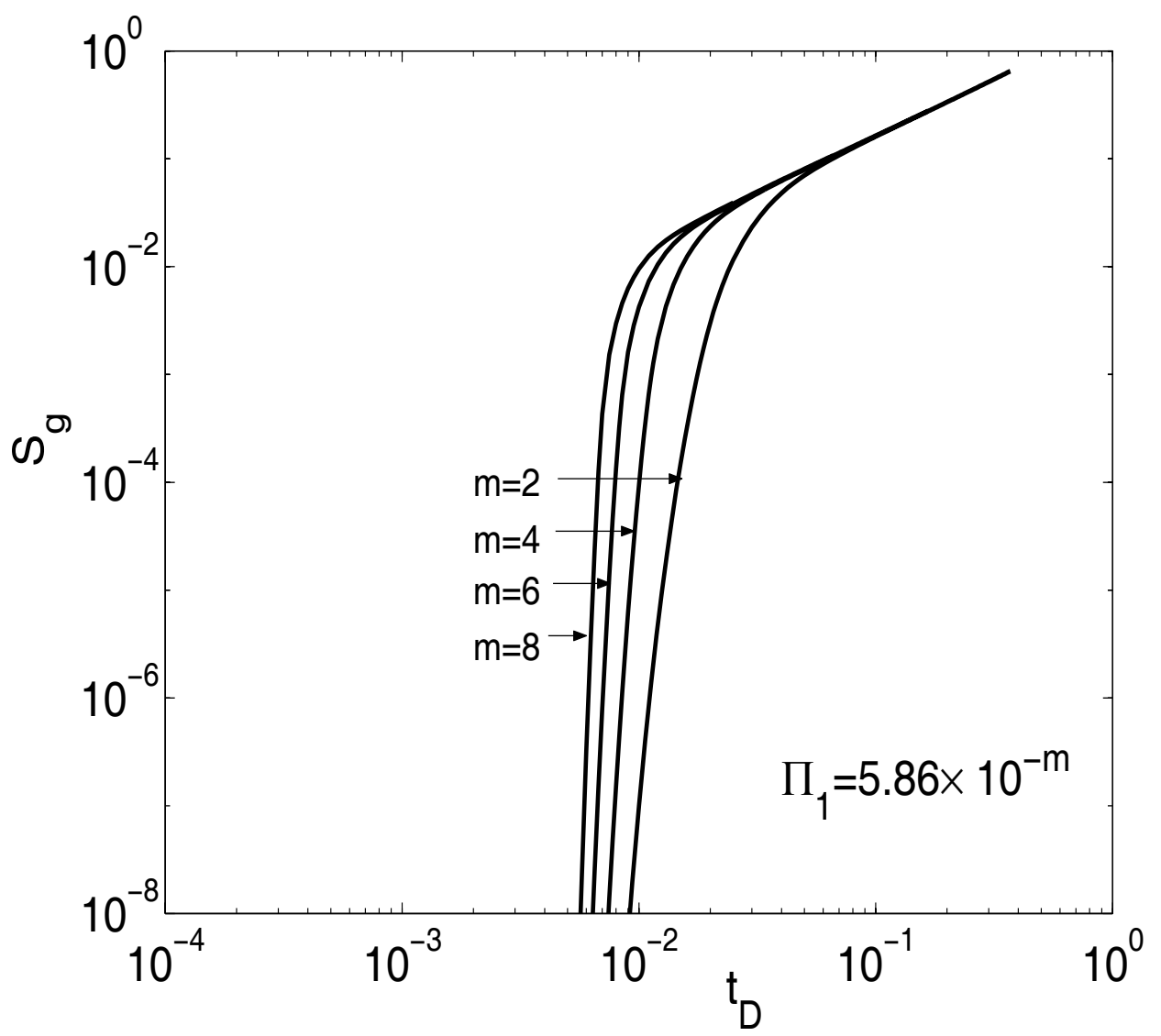

Figure 7: The variation of the gas saturation, $S_{g}$, as a function of the dimensionless time, $t_{D}$. Effect of $\Pi_{1}=5.86 \times 10^{-m}$, for $\Pi_{c}=3.41 \times 10^{-2}, \Pi_{2}=2.33 \times 10^{6}$ and $\Pi_{3}=8.7 \times 10^{-3}$. 


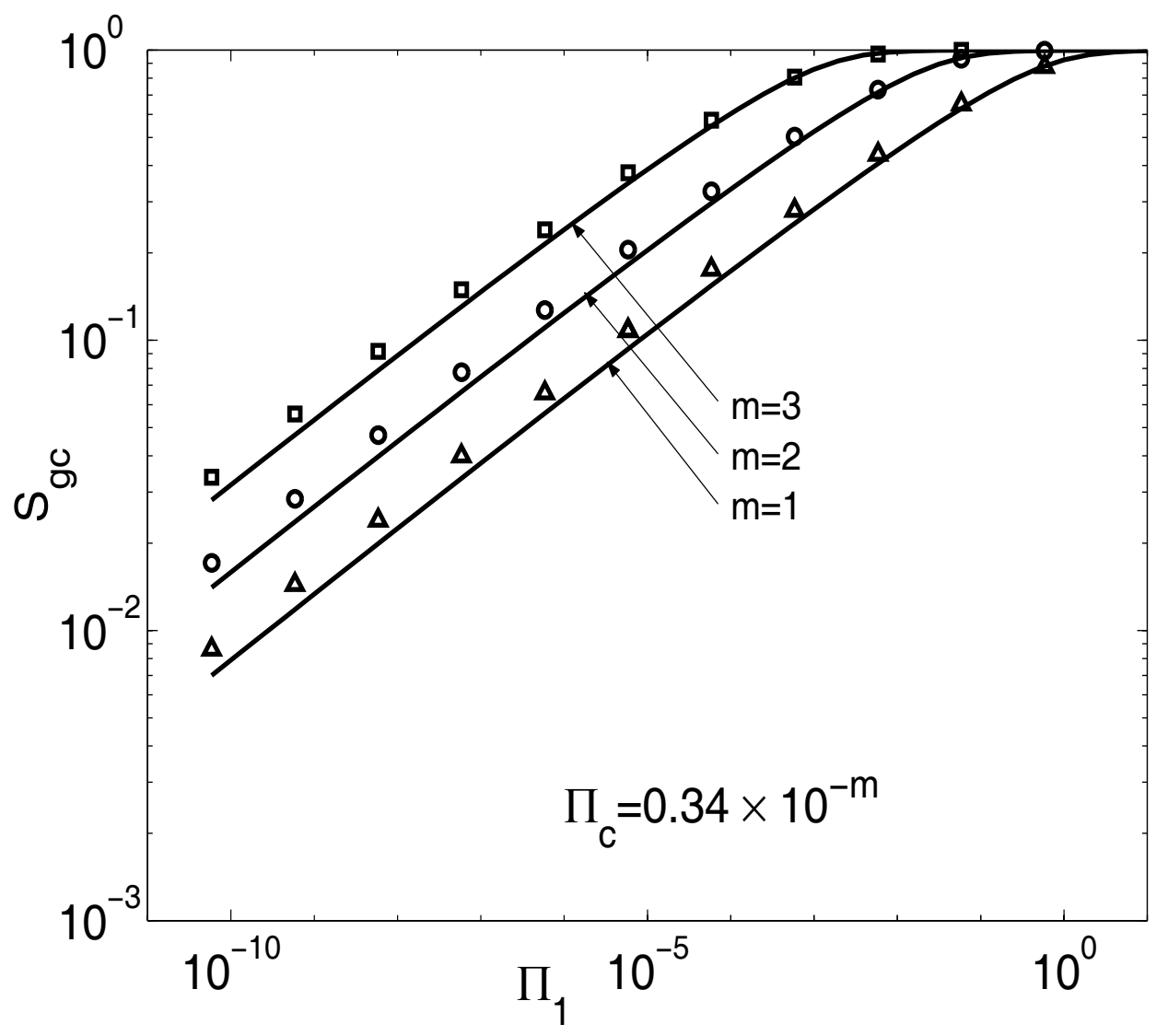

Figure 8: The effect of the dimensionless parameter $\Pi_{1}$ on the critical gas saturation, $S_{g c}$, for $\Pi_{c}=0.34 \times 10^{-m}$. Points denote the full numerical solution, solid lines correspond to the simpler model. 


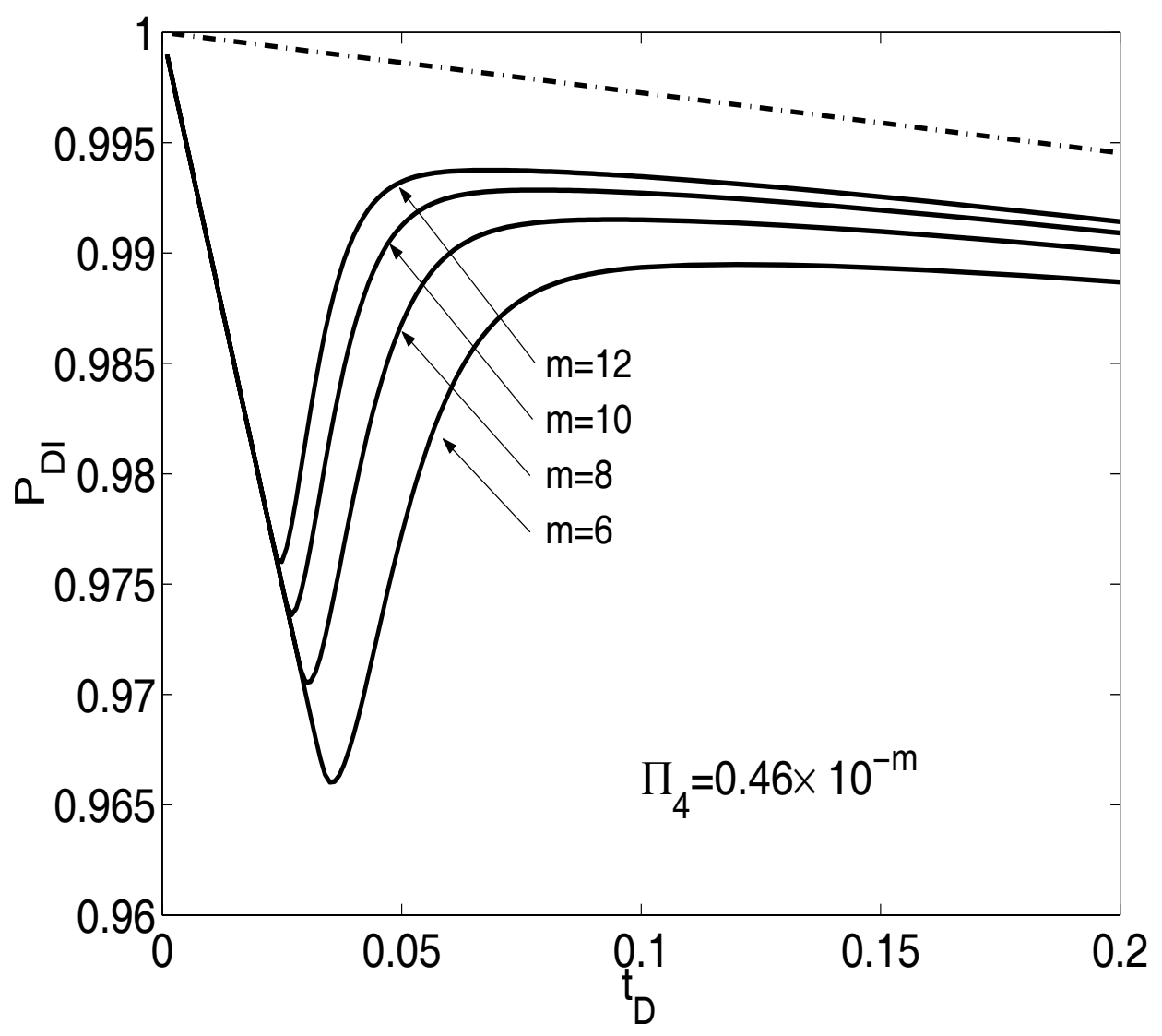

Figure 9: The variation of the dimensionless pressure, $P_{D l}$, as a function of the dimensionless time, $t_{D}$, for the case of constant liquid withdrawal rate. Effect of $\Pi_{4}=0.4631 \times 10^{-m}$, for $\Pi_{c}=1.67 \times 10^{-1}, \Pi_{2}=6.84 \times 10^{5}$ and $\Pi_{3}=1.52 \times 10^{-2}$. Shown in dashed-dotted line is the thermodynamic equilibrium curve. 


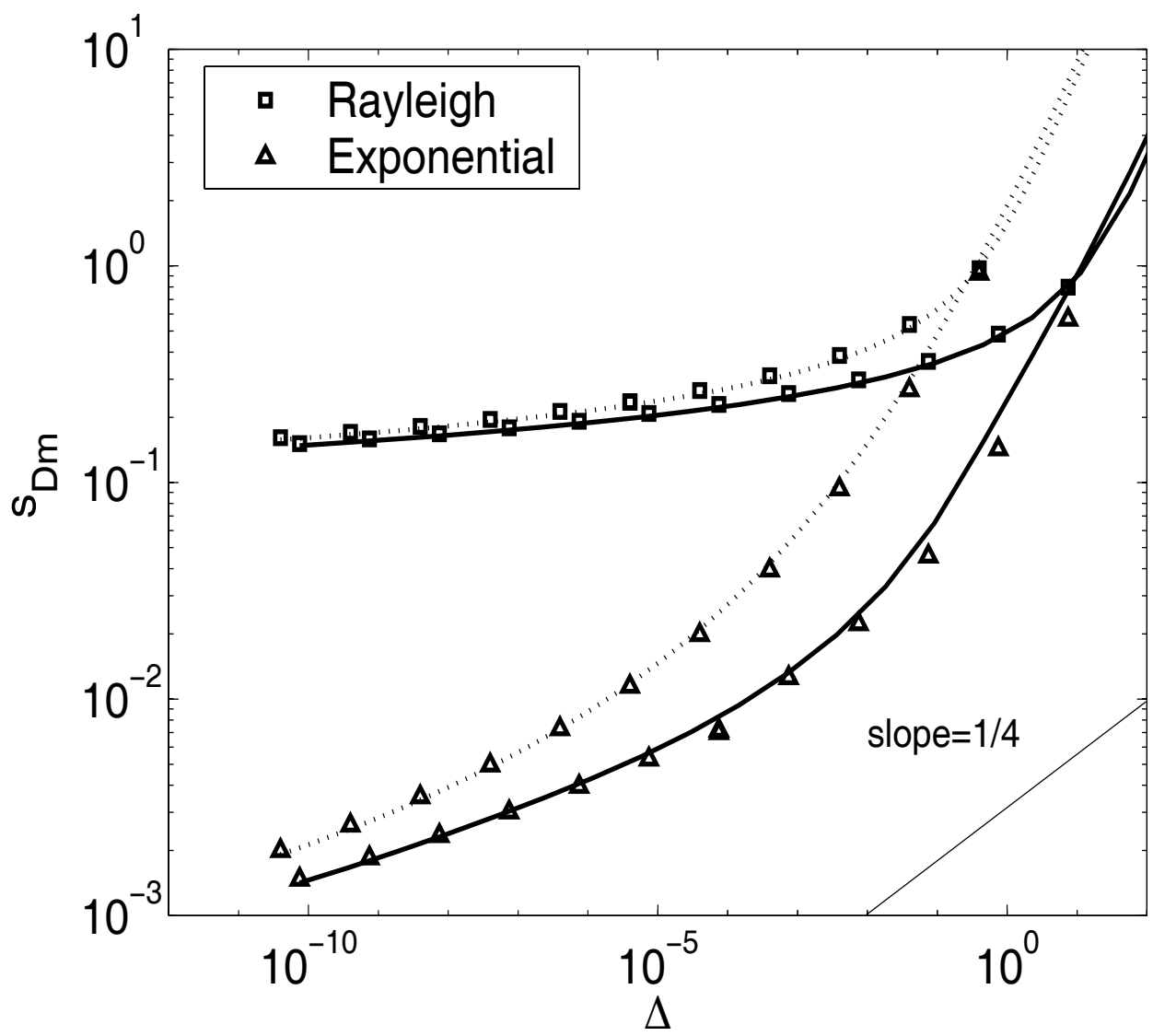

Figure 10: The maximum rescaled supersaturation, $s_{D m}$, as a function of $\Delta$ for the cases of constant pressure decline rate (dotted lines) and constant liquid withdrawal rate (solid lines). Comparison between the simpler model (dotted or solid lines) and the full numerical solution (denoted by triangles for the stretched exponential cavity size distribution with $n=0.5$ and $\sigma=1.0$, and by squares for the Rayleigh cavity size distribution). 


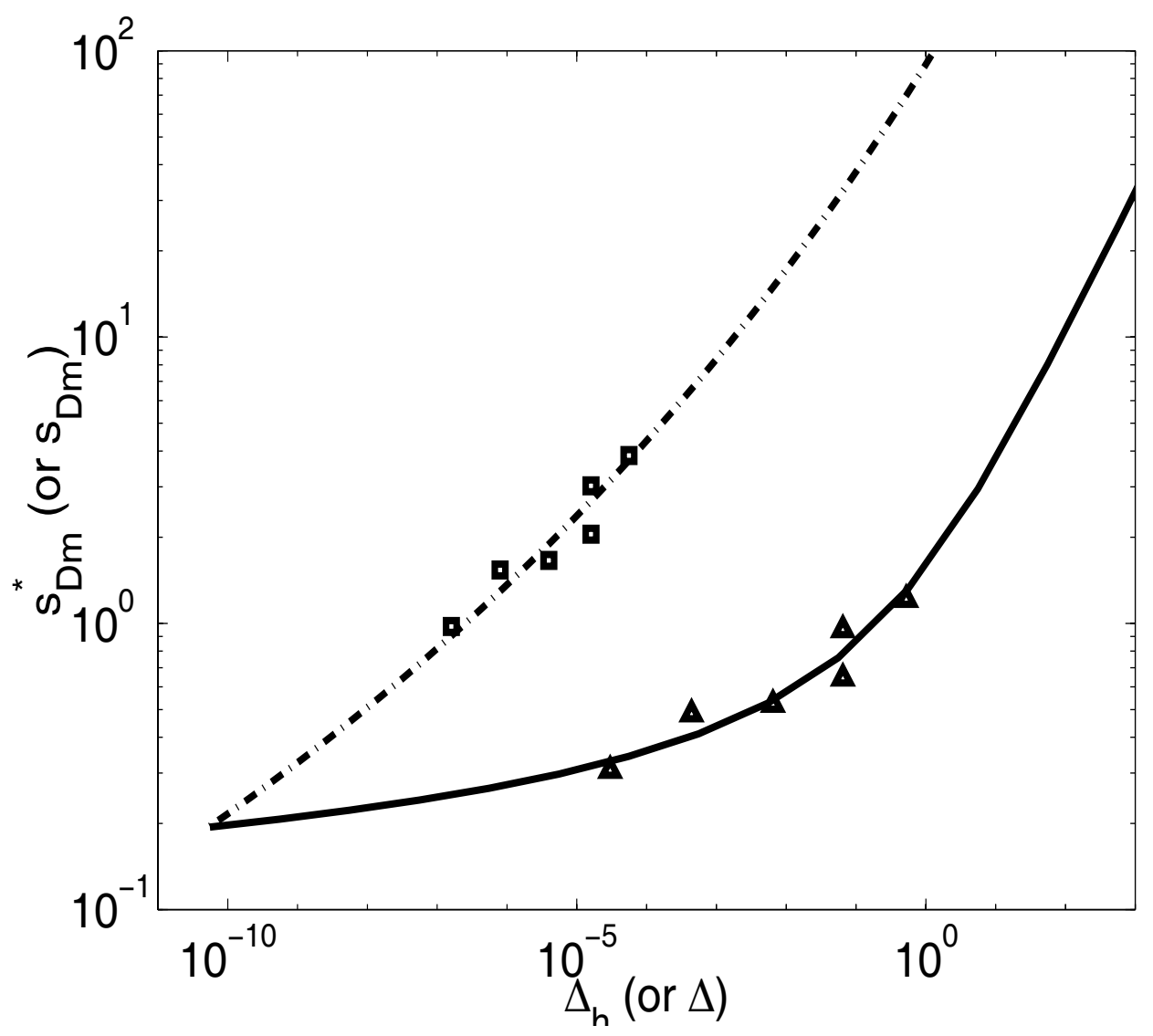

Figure 11: The maximum rescaled supersaturation, $s_{D m}^{*}$, as a function of $\Delta_{h}$ for the simpler model (solid line). Triangles denote $s_{D m}^{*}$ values calculated using experimental data from Scherpenisse et al. (1994). Also plotted are the predictions based on the cavity model (dashed lines for the simpler model, squares denoting $s_{D m}$ values for the same experimnets). 

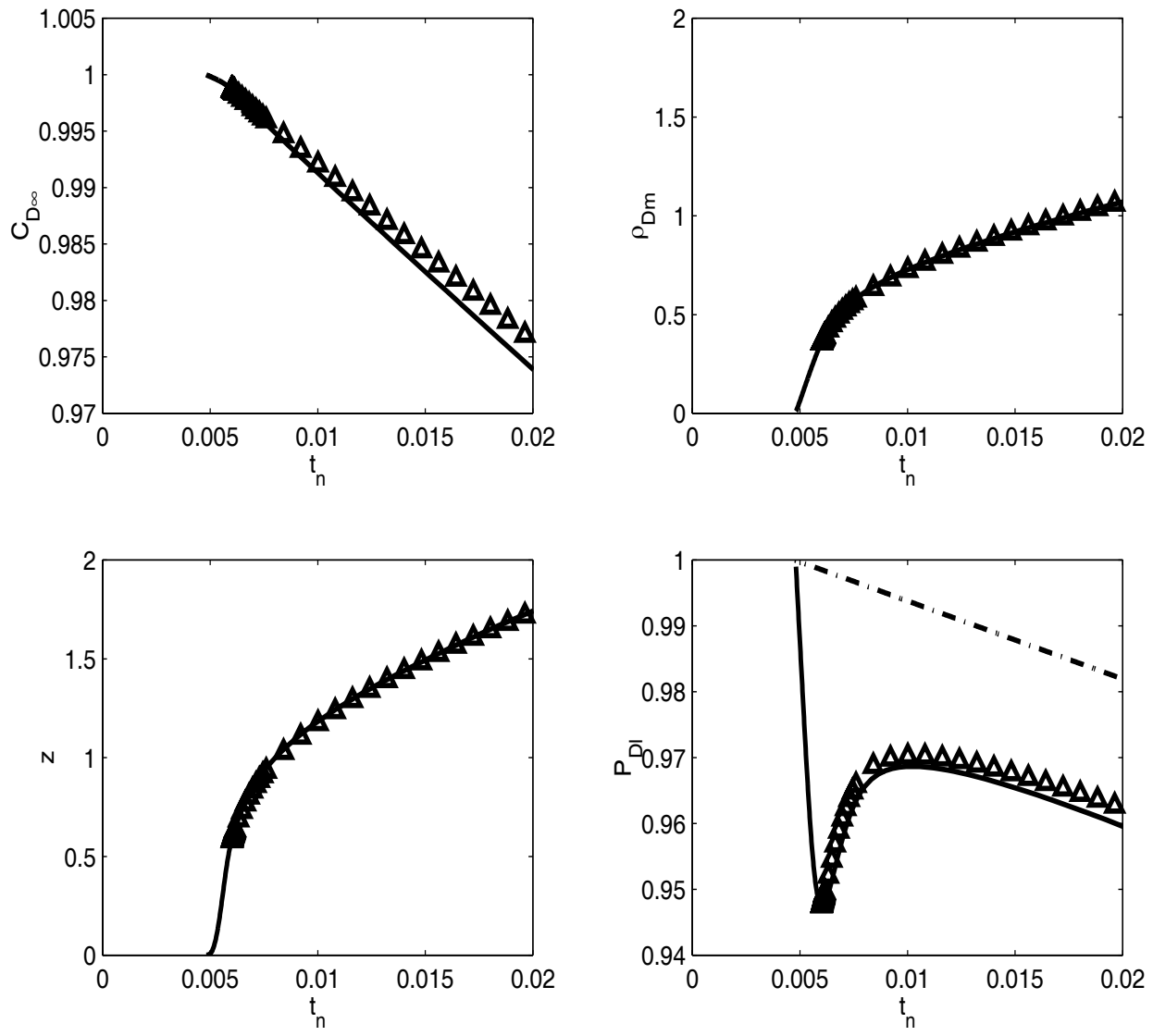

Figure 12: Comparison of the full numerical results (denoted by solid lines) with the approximate model (denoted by triangles) for the case of constant liquid withdrawal rate. Shown in dashed-dotted line in the last panel is the thermodynamic equilibrium curve. 


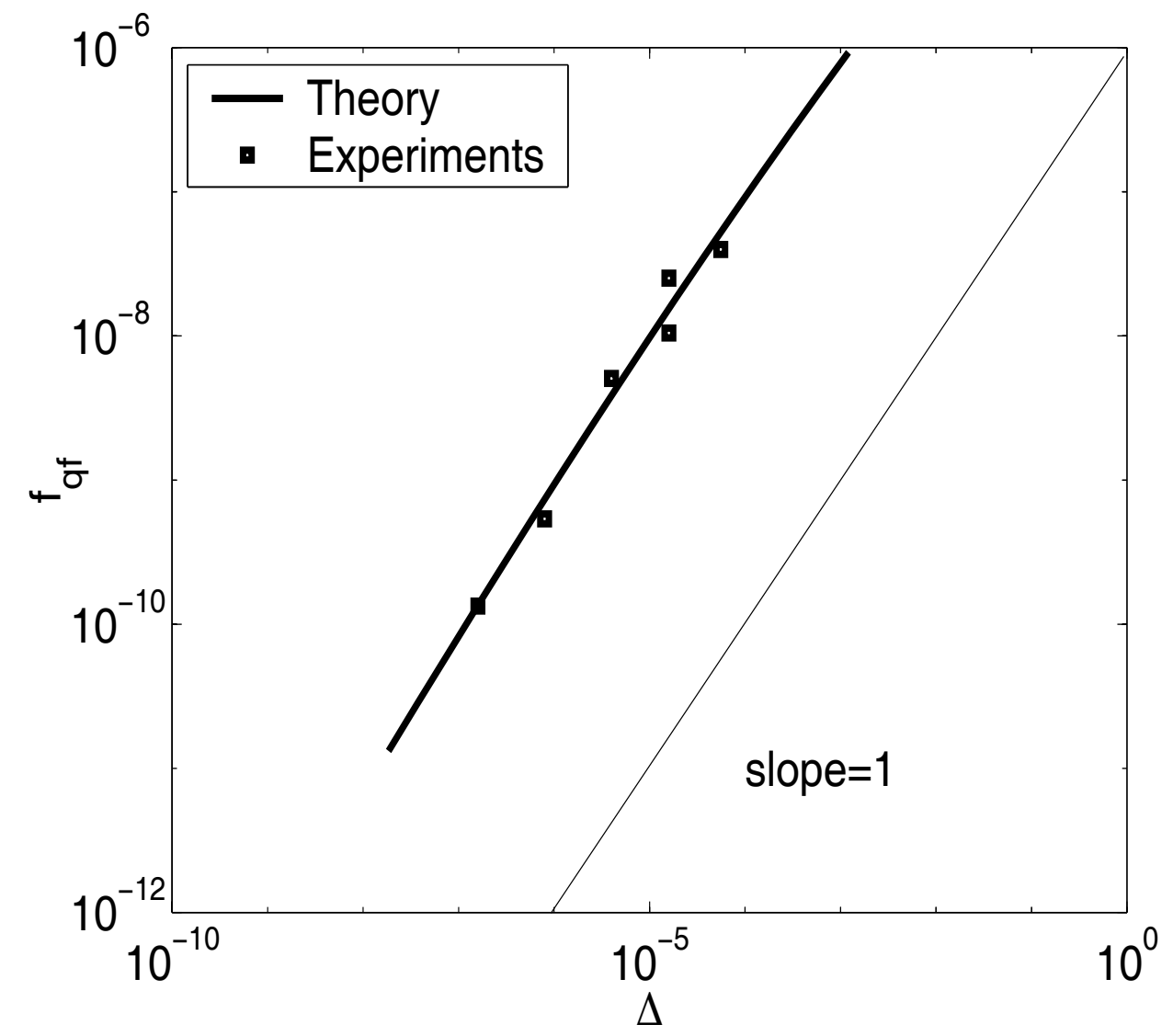

Figure 13: The final nucleation fraction, $f_{q f}$, as a function of $\Delta$, for a stretched exponential ( $n=0.215$ and $\sigma=0.045$ ) cavity size distribution. The solid line corresponds to the simpler model, squares denote values calculated using experimental data from Scherpenisse et al. (1994). 


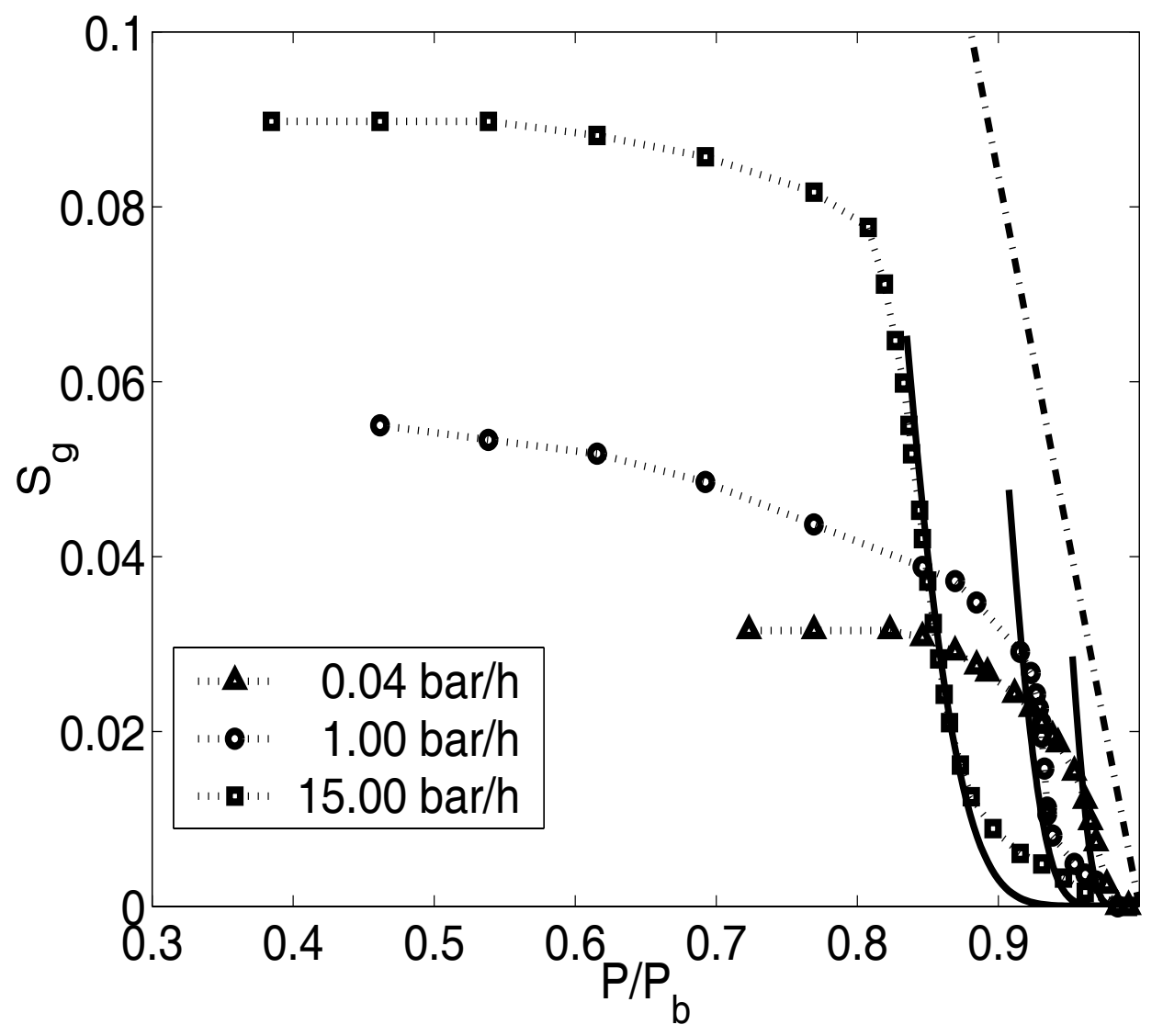

Figure 14: The evolution of the gas saturation as a function of the dimensionless pressure for three depletion rates for the Berea sandstone experiments of Scherpenisse et al. (1994). Points denote experimental values, solid lines correspond to the full numerical solution, the dashed-dotted line corresponds to the thermodynamic equilibrium curve. 


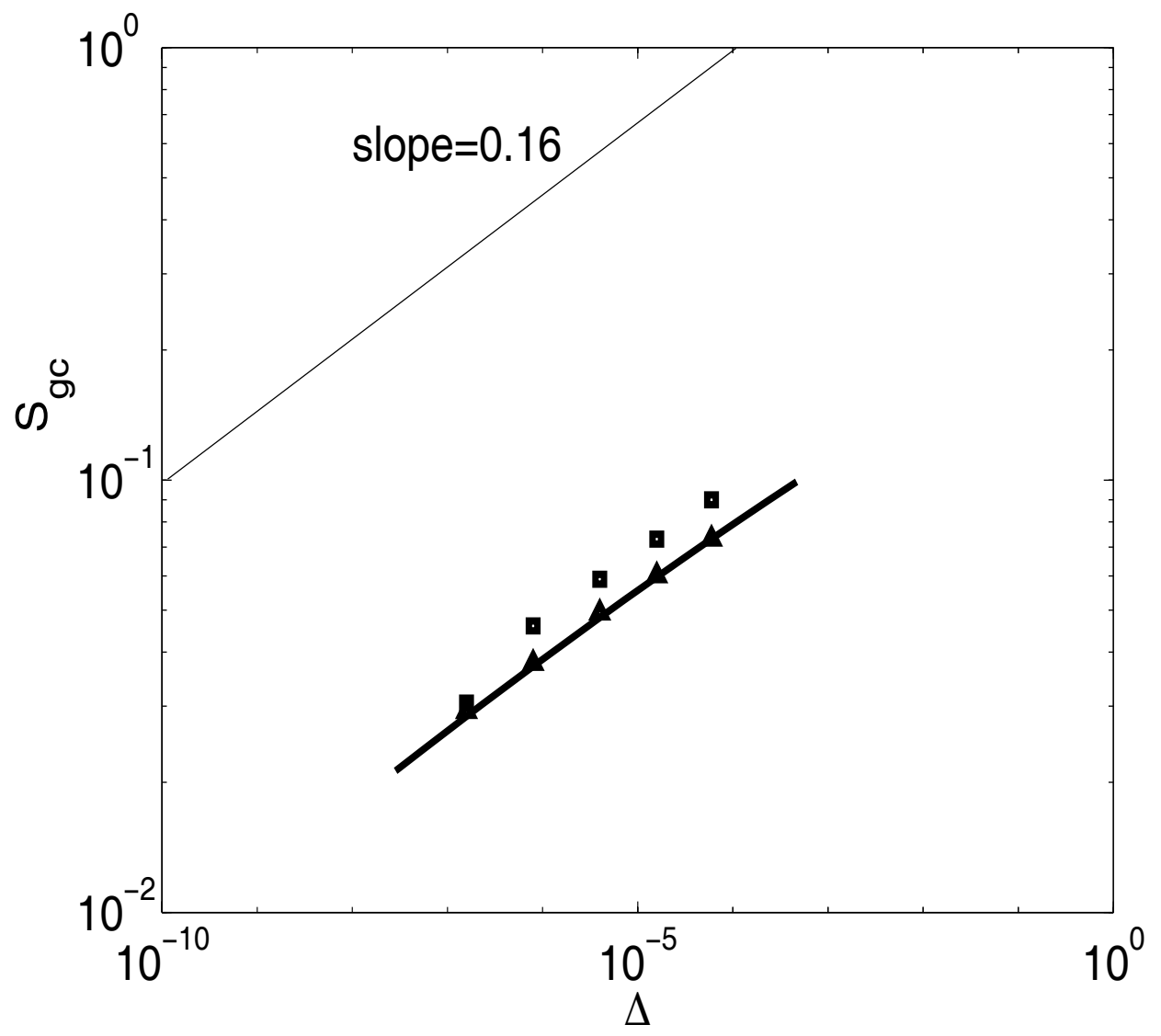

Figure 15: The critical gas saturation, $S_{g c}$, as a function of $\Delta$, for a stretched exponential ( $n=0.215$ and $\sigma=0.045$ ) cavity size distributions. The solid line corresponds to the simpler model, triangles denote the full solution, squares denote experimental data (constant pressure decline rate) from Scherpenisse et al. (1994). 


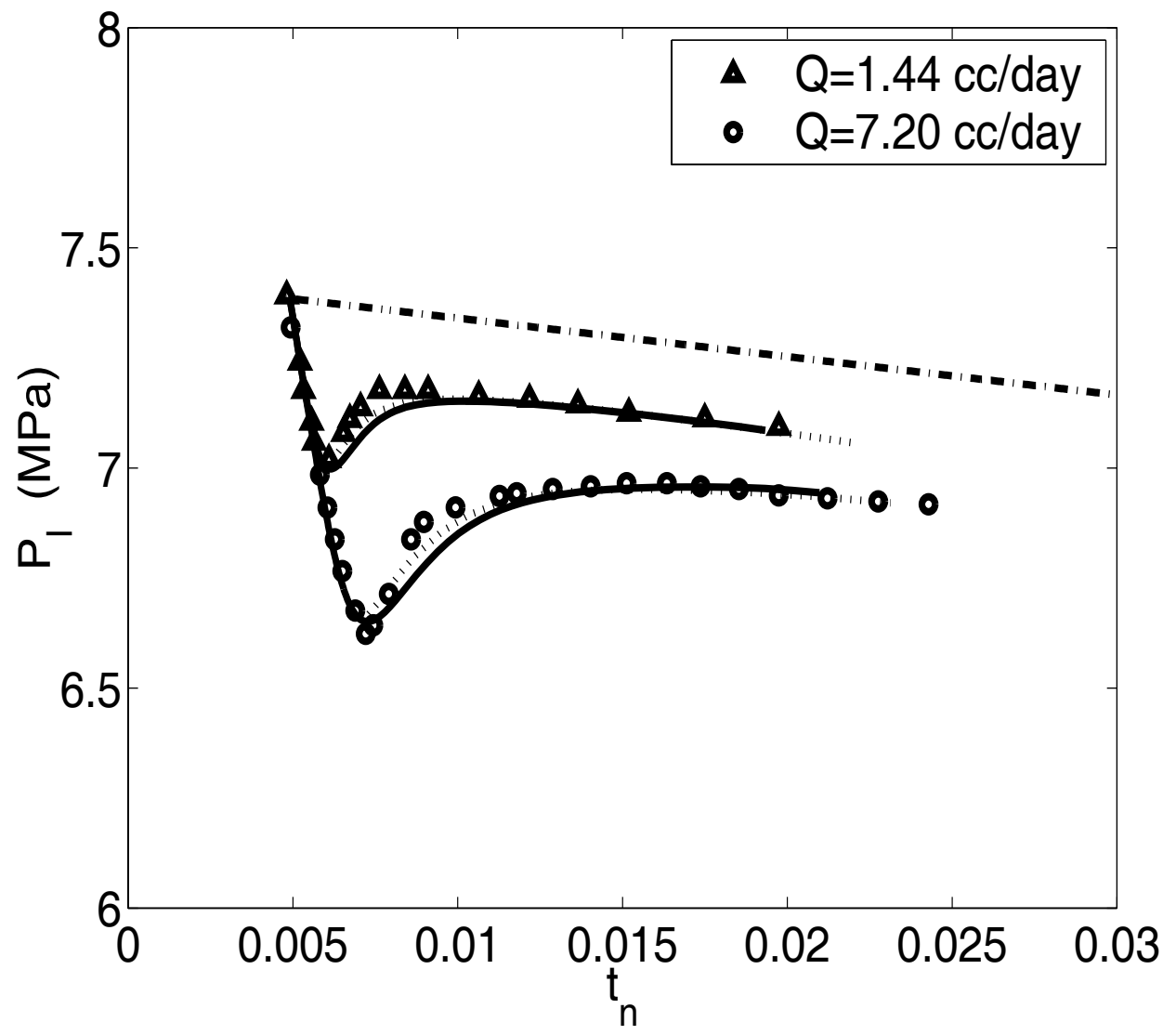

Figure 16: Evolution of pressure as a function of time for the Berea stone experiments of Firoozabadi et al. (1992) for two different withdrawal rates. Solid lines denote the full solution, dotted lines denote the simpler growth model, the dashed-dotted line denotes the thermodynamic equilibrium curve, symbols denote the experimental results. 


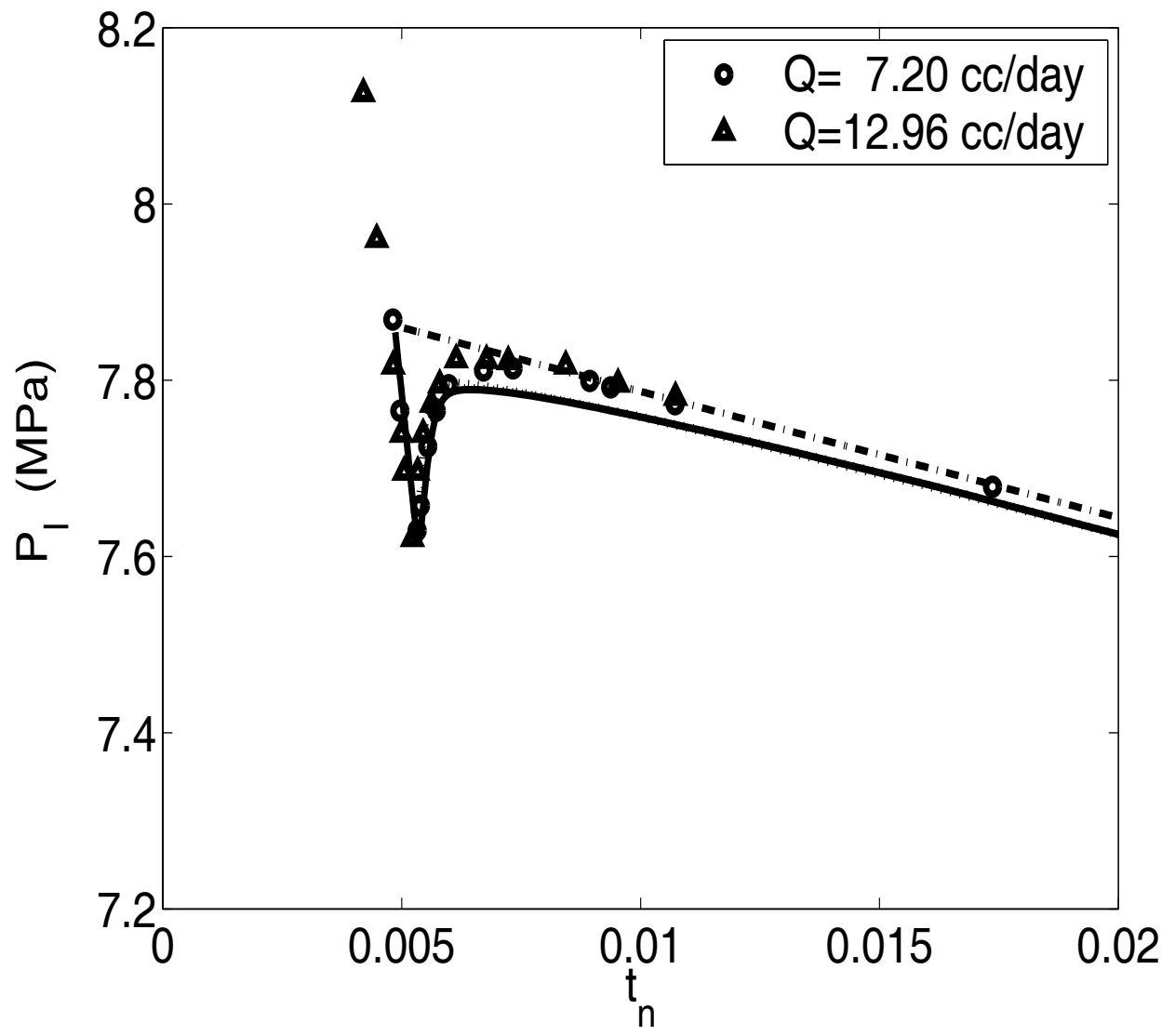

Figure 17: Evolution of pressure as a function of time for the Chalk experiments of Firoozabadi et al. (1992) for two different withdrawal rates. Solid lines (both coincide) denote the full solution, dotted lines denote the simpler growth model, the dashed-dotted line denotes the thermodynamic equilibrium curve, symbols denote the experimental results. 


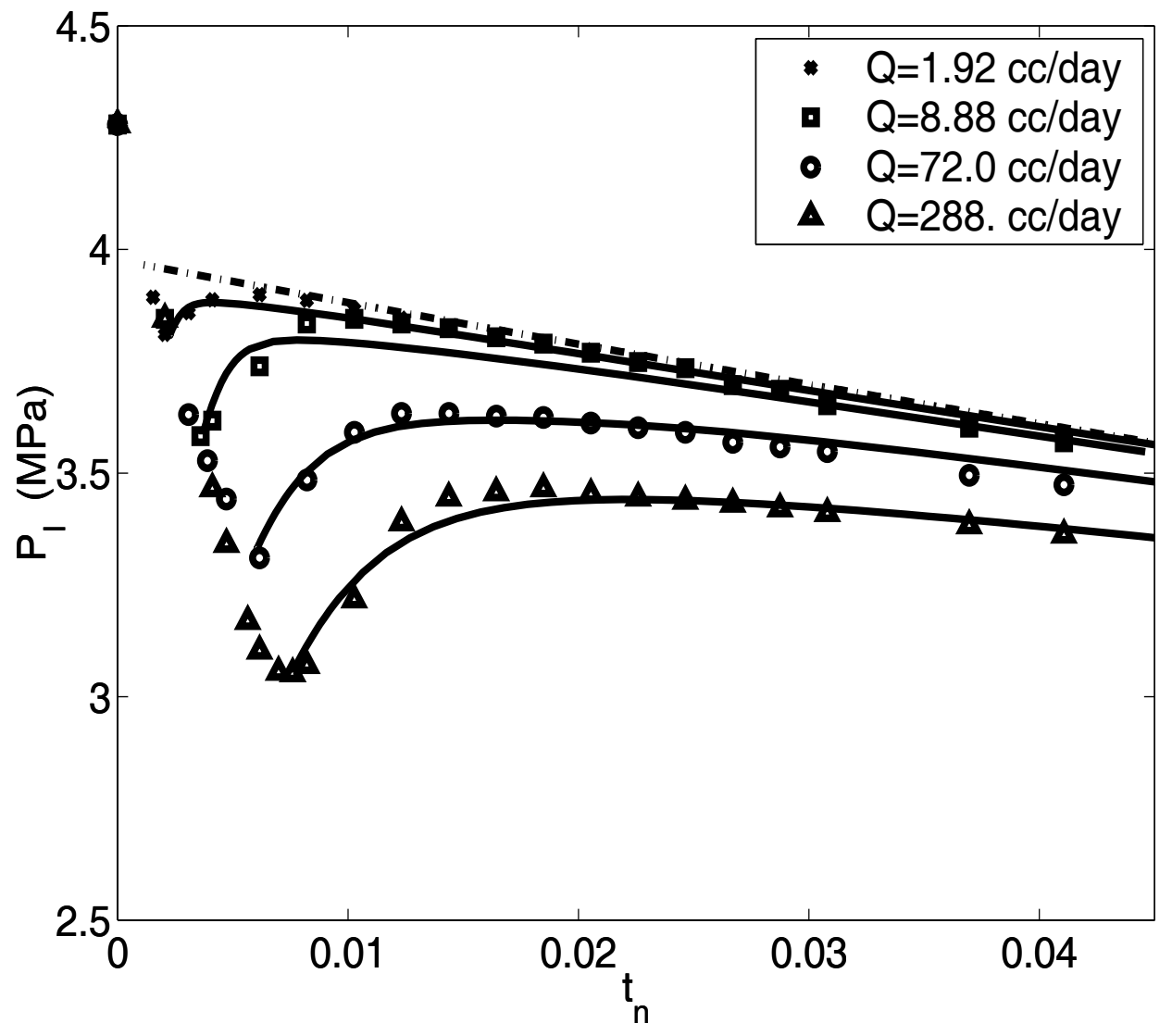

Figure 18: Evolution of pressure as a function of time for the sand-pack experiments of Kumar et al. (2000) for four different withdrawal rates. Solid lines denote the simpler growth model, the dashed-dotted line denotes the thermodynamic equilibrium curve, symbols denote the experimental results. 


\title{
An Effective Continuum Model for the Gas Evolution in Internal Steam Drives
}

\author{
Ioannis N. Tsimpanogiannis, and Yannis C. Yortsos
}

\section{Introduction}

The liquid-to-gas phase change in a porous medium and the subsequent growth of the gas phase is encountered in a plethora of applications driven by mass or heat transfer. Typical examples include solution gas-drive for the recovery of oil from oil reservoirs, boiling in porous media, thermal methods for oil recovery, nuclear waste disposal, soil remediation and others. In this paper, we examine the gas phase growth from a supersaturated, slightly compressible, liquid in a porous medium, driven by heat transfer and controlled by the application of a constant-rate decline of the system pressure. A characteristic example of such a process occurs during cyclic steaming for the recovery of oil from low permeability reservoirs through hydraulic or natural fractures (Dehghani et al. ${ }^{1}$ ). During injection and soaking, steam condenses in the fracture and hot water imbibes into the matrix. During production, the pressure of the system constantly declines, and when it falls sufficiently below the vapor pressure, it results in the appearance of steam in the matrix (in-situ boiling). The in-situ production and subsequent growth of the steam phase inside the matrix are of interest because they result in expelling additional oil.

Dehghani et al. ${ }^{1}$ conducted a series of core experiments in order to study the effect of insitu steam drive on fluid displacement in porous media. Subsequently, Dehghani and Kamath ${ }^{2}$ conducted experiments in a vuggy carbonate core using a recombined oil to study the contribution of the various recovery mechanisms (thermal expansion, thermally enhanced solution gas drive, dry distillation, and in-situ steam drive) in steam injection, followed by pressure reduction.

While of interest both from theoretical and applied viewpoints, a more fundamental understanding of the basic aspects has not been obtained, to our knowledge. It is the objective of this paper to bridge this gap, by providing a model of the nucleation and the gas-phase growth periods. Internal steam drive has many similarities with the process of solution gas-drive. They both describe the evolution of a gas phase due to the increase of the supersaturation of the system, through a relatively slow pressure decline. Nucleation and subsequent phase growth play a key role in both processes. In a recent publication (Tsimpanogiannis and Yortsos ${ }^{3}$ ) we developed a comprehensive effective continuum model to model solution gas-drive under 
various conditions. In this paper, we extend that approach to the specific problem of internal steam drive.

As discussed in Tsimpanogiannis and Yortsos ${ }^{3}$, our effective continuum model is best suited for the early part of the process, where nucleation and the early stages of bubble growth are dominant. The latter two, particularly the nucleation sequence, are the main areas of interest of this paper as well. We focus on the effect of the nucleation characteristics on the maximum supersaturation, the nucleation fraction and the critical gas saturation, and provide an analysis of the effect of various parameters, such as pressure decline rate, on these quantities. Results for the gas phase growth, following the conclusion of nucleation are also presented for completeness.

Solution gas-drive involves a binary system and is controlled by mass transfer. On the other hand, internal steam drive is fundamentally a single-component system, controlled by heat transfer. Of course, in the more general case, oil will be present in the pore space in addition to water. The presence of oil can affect the nucleation process, depending on which phase is wetting the porous medium and can prevent a component having access to cavities on the pore walls where nucleation occurs. It can also affect the growth process by restricting the motion and the coalescence of the gas bubbles, thus delaying the reaching of the critical gas saturation. The effects of the combined presence of oil and water on the solution gas drive process were examined extensively, using visualization experiments in glass micromodels by Hawes et al. ${ }^{4}$, Mackay et al. ${ }^{5}$, and Bora et al. ${ }^{6}$, and with core experiments by Kortekaas and van Poelgeest ${ }^{7}$. Dehghani et al. ${ }^{1}$ and Dehghani and Kamath ${ }^{2}$ examined the effects of the combined presence of oil and gas on internal steam drive processes. Dehgani et al. ${ }^{1}$ also discussed in detail the effect of hot-water flash tests on oil recovery, for various initial oil saturations. However, our emphasis will be on single-component systems.

The paper is organized as follows: First, we formulate the problem closely following Tsimpanogiannis and Yortsos ${ }^{3}$. A scaling analysis of the resulting equation allows to recast the problem in a more useful form, to be used for direct predictions. The numerical results are analyzed. It turns out that for their interpretation, a simplified model of the nucleation and growth periods can be developed. We use this simpler model to obtain expressions for the maximum supersaturation as a function of geometric, thermodynamic and process parameters. This allows us to obtain useful relations for the dependence of the final nucleation fraction (and the critical gas saturation) on process parameters. The theoretical predictions are then compared against experimental results. 


\section{Mathematical Formulation}

Consider an effective porous medium occupied by a single-component liquid. At the beginning of the process, the system is subcooled at the initial temperature, $T_{o}$, with pressure, $P_{o}$, such that $P_{o}>P^{s a t}\left(T_{o}\right)$ where $P^{s a t}(T)$ denotes the equilibrium vapor pressure at temperature $T$. In the practical application discussed by Dehghani and Kamath ${ }^{2}$ this state is achieved by steam injection, followed by steam condensation. Following this initial state, the pressure of the system is slowly decreased. Nucleation and subsequent bubble growth are driven by the continuous increase in the supersaturation, $P^{s a t}\left(T_{\infty}\right)-P_{l}(t)$, where $T_{\infty}$ is the far-field temperature and subscript $l$ denotes liquid. To describe phase equilibria, we will assume a simple Clausius-Clapeyron equation

$$
\frac{d \ln \left[P^{s a t}\left(T_{\infty}\right)\right]}{d T_{\infty}}=\frac{L_{v}}{R_{g} T_{\infty}^{2}},
$$

where $R_{g}$ is the ideal gas constant and $L_{v}$ the molar latent heat of vaporization. Eq. 1 does not include Kelvin vapor pressure lowering effects. However, these can be readily incorporated by replacing $L_{v}$ in Eq. 1 by $L_{v}+P_{c} v_{m}$, where $P_{c}$ is the capillary pressure and $v_{m}$ the molar liquid volume. More complex thermodynamics can certainly be incorporated (Reid et al. ${ }^{8}$ ), but the salient features are manifested with the simpler model of Eq. 1. Conversely, at a specified liquid pressure, $P_{l}$, a degree of superheat is present in the system, given by the difference

$$
\Delta T=T_{\infty}-T^{\text {sat }}\left(P_{l}\right)
$$

where $T^{s a t}\left(P_{l}\right)$ denotes the equilibrium temperature corresponding to $P_{l}$. Here, the change in supersaturation (or superheat) is driven by a constant rate of pressure decline. As mentioned, we will proceed with the assumption that the rate of decline is sufficiently slow, so that inertia effects, as well as effects of spatial gradients (gravitational and/or viscous), are negligible. This requires sufficiently small Rayleigh, Bond, capillary and Peclet numbers. Emphasis will be placed on nucleation and on the effect of the increase of supersaturation on the growth of the gas phase. 
We note that this model can in principle be also applied to describe the onset of boiling in porous media, driven by the application of a constant heat flux. This application is left for a future study, however.

Nucleation. As the liquid pressure declines, nucleation sets in. Yortsos and Parlar ${ }^{9}$ reviewed the gas-liquid phase change in porous media and concluded that heterogeneous nucleation is the most plausible mechanism under sufficiently slow rates of supersaturation. In one model, nucleation occurs when a gas bubble, either pre-existing or nucleated inside a cavity at the pore walls (see Fig. 1 for a schematic), becomes unstable and detaches or otherwise occupies the host pore body. This type of mechanism is in agreement with visual observations from micromodel experiments in solution gas drive (Li and Yortsos ${ }^{10}$, El Yousfi et al. ${ }^{11,12}$, Bora et al. ${ }^{6}$, and Dominguez et al. ${ }^{13}$ ) and will also be assumed here. In the cavity model, the activation of a nucleation site occurs when the trapping capillary forces are overcome for the first time. Then, the following condition is satisfied between the radius of the nucleation cavity, $r_{c}$, and the (local) supersaturation,

$$
P_{c} \equiv \frac{2 \gamma \cos \theta^{*}}{r_{c}}=P^{s a t}\left(T_{\infty}\right)-P_{l}(t)
$$

where $\theta^{*}$ is the contact angle $\left(0<\theta^{*}<\pi / 2\right)$. Thus, in the present model, the onset of nucleation is not kinetically related to the degree of supersaturation, as for example, in more conventional approaches for solution gas-drive (Firoozabadi and Kaschiev ${ }^{14}$ ), but rather depends on the size distribution, $\alpha_{c}\left(r_{c}\right)$, of the nucleation cavities.

Consider, now, the activation of nucleation sites. With the decrease in the liquid pressure, the right-hand side of Eq. 3 increases, eventually becoming positive. Then, various cavities satisfying Eq. 3 become activated and their corresponding host pore bodies are occupied by gas. At any time, the current nucleation fraction, $f_{q}$, defined as the number fraction of pores that contain sizes which have been activated, is

$$
f_{q}=\int_{r_{c}}^{\infty} \alpha_{c}(r) d r
$$

where $r_{c}$ is an implicit function of time, through Eq. 3. Eq. 4 implies a zero nucleation fraction at zero supersaturation and a nucleation fraction of one at infinite supersaturation. As elaborated in 
Tsimpanogiannis and Yortsos $^{3}$, the cavity size distribution, pertains only to the largest cavity in any given pore (as this cavity will be activated first). Eq. 4 slightly overestimates the true nucleation fraction, since pores containing potential sites to be activated later, may already be occupied by gas, due to the growth of gas clusters from neighboring pores. However, in most cases, nucleation terminates well before gas bubble growth has occurred to any substantial degree $\left(S_{g}<<1\right)$, thus Eq. 4 should be an excellent approximation.

It is apparent that $f_{q}$ will have a different dependence on parameters, depending on the assumed cavity size distribution. In the present paper, we will consider distribution of the Rayleigh type, namely

$$
f_{q}=\exp \left(-\frac{\pi r_{c}^{2}}{4 r_{c}^{* 2}}\right)=\exp \left[-\frac{\pi \gamma^{2}}{r_{c}^{* 2}\left[P^{s a t}(T \infty)-P_{l}\right]^{2}}\right]
$$

where $r_{c}{ }^{*}$ is a characteristic (here the mean) cavity size, as well as a stretched-exponential

$$
f_{q}=\exp \left(-\frac{r_{c}^{n}}{\sigma r_{c}^{* n}}\right)
$$

where $n$ is a positive exponent and $\sigma$ is a measure of the variance. The type of distribution influences significantly the results to be obtained, as will be demonstrated below.

As long as the level of supersaturation increases with time, the right-hand-side of Eq. 5 also increases, implying that additional sites become activated, and the nucleation fraction continuously rises. This is consistent with experimental evidence of sequential nucleation, e.g. as reported by Satik and Yortsos ${ }^{15}$. After the supersaturation reaches a maximum (local or global), Eq. 5 predicts a decreasing $f_{q}$. This is unphysical. Therefore, in segments of decreasing supersaturation the nucleation fraction will be taken constant. When the supersaturation goes through a global maximum, it signals the end of the nucleation period, in which case the fraction of pores ultimately activated, $f_{q f}$, will be given by Eqs. 5 or 6 at the time of the maximum supersaturation. We note that in typical solution gas-drive experiments, $f_{q f}$ is very small, of the order of $10^{-9}-10^{-6}$. 
Through this process, nucleation centers are activated sequentially, giving rise to evolving gas clusters, which grow by heat transfer from the liquid to the gas. Sequential nucleation results into clusters of different ages (the time passed since a particular class of gas clusters has been nucleated/activated). Let $\omega(\tau)$ be the number density of clusters nucleated per total number of pores. Then, $\omega(\tau) d \tau$ is the number of new clusters per total number of pores that become activated in the time interval between $\tau$ and $\tau+d \tau$. Evidently,

$$
\omega(\tau) d \tau=d f_{q}
$$

This relation will be used below to simplify the expressions for the gas phase growth.

Gas phase growth. During the growth of the gas phase we can roughly distinguish two periods, one in which the growth is within single pores and another corresponding to gas clusters spanning several pores (Tsimpanogiannis and Yortsos ${ }^{3}$ ). The first period extends throughout and following the nucleation stage, the second is the later stage of growth. In either growth is driven by heat transfer. Fig. 2 shows snapshots of a growing gas cluster in a glass micromodel, in the context of solution gas-drive. In general, different clusters compete with each other through a combination of pore geometrical and topological effects for the available heat in the liquid. The heat transfer rates depend on the cluster geometry and their relative position, and an accurate description is needed. In such cases, a pore-network model should instead be used. Pore-network models of bubble growth in single-component systems driven by heat transfer were developed by Satik and Yortsos ${ }^{15}$. In principle, these contain all the necessary physics for a rigorous modeling of the process, particularly when significant spatial gradients develop. Such an effort can be pursued in parallel with the continuum model.

In the absence of competition between adjacent clusters, and under the assumption that heat transfer is conduction-controlled (namely that the Peclet number is sufficiently small), an isolated cluster $j$ grows at a rate which is proportional to its effective radius, $R_{j}(t, \tau)$, and to the driving force, $T_{\infty}-T^{\text {sat }}\left(P_{l}\right)$, where $T_{\infty}$ is the far-field temperature. This is true even for ramified fractal clusters, as was verified by Satik and Yortsos ${ }^{15}$. Assuming that the gas is ideal, we can write the following mass balance for a growing cluster 


$$
\left(\frac{M_{w}}{R_{g} T_{g}}\right) \frac{d}{d t}\left[\left(P_{l}+P_{c}\right) V_{g}\right] \approx 4 \pi R_{j} \frac{k_{e f f}}{\tilde{L}_{v}}\left(T_{\infty}-T^{s a t}\left(P_{l}\right)\right),
$$

where $M_{w}$ is the molecular weight of the gas, $T_{g}$ the temperature in the gas phase, $V_{g}$ the gas cluster volume, $k_{\text {eff }}$ an effective conductivity and $\tilde{L}_{v}$ the mass latent heat of vaporization $\left(L_{v}=\tilde{L}_{v} M_{w}\right)$. In Eq. 8 we have also included the capillary pressure, $P_{c}$, which in the application of interest can be significant. To simplify, we will linearize the phase equilibria around $P_{o}$, namely

$$
T^{\text {sat }}\left(P_{l}\right) \approx T^{\text {sat }}\left(P_{o}\right)+\frac{d T^{\text {sat }}}{d P}\left(P_{l}-P_{o}\right),
$$

and take without significant loss $T_{g} \approx T_{o}$.

The gas volume $V_{g}$ takes a different expression in the two different periods (Tsimpanogiannis and Yortsos ${ }^{3}$ ). For growth within a single pore, $V_{g} \approx V_{c}\left(R_{j} / r_{c}^{*}\right)^{3}$, where $V_{c}$ is a characteristic cavity volume [defined here as $(4 / 3) \pi r_{c}^{* 3}$ ]. For growth of a cluster spanning several pores, we have $V_{g} \approx A^{*} V_{s}\left(R_{j} / r_{s}^{*}\right)^{D_{f}}$, where $V_{s}$ is the average site volume, $r_{s}^{*}$ is a characteristic pore body size, $D_{f}$ is the mass fractal dimension, equal approximately to 2.5 for a 3-D cluster $\left(\right.$ Feder $\left.^{16}\right)$, and $A^{*}$ is a dimensionless geometric pre-factor. To capture both periods with the same equation we will write

$$
\left(\frac{A V_{c} M_{w}}{R_{g} T_{o}}\right) \frac{d}{d t}\left[\left(P_{l}+P_{c}\right)\left(\frac{R_{j}}{r_{c}^{*}}\right)^{D_{f}}\right]=4 \pi R_{j} \frac{k_{e f f}}{\tilde{L}_{v}}\left(T_{\infty}-T^{s a t}\right)
$$

with the understanding that $D_{f}$ varies between 3 and 2.5, and $A$ between 1 and $A=\frac{A^{*} V_{s}}{V_{c}}\left(\frac{r_{c}^{*}}{r_{s}^{*}}\right)^{D_{f}}$, during the nucleation and growth periods, respectively.

Under the above assumptions, the gas phase can be described as a collection of clusters of size $R(t, \tau)$, the dynamics of each of which is described by Eq. 10, with $R_{j}$ replaced by $R$, namely 


$$
\left(\frac{A V_{c} M_{w}}{R_{g} T_{o}}\right) \frac{\partial}{\partial t}\left[\left(P_{l}+P_{c}\right)\left(\frac{R}{r_{c}^{*}}\right)^{D_{f}}\right]=4 \pi R_{j} \frac{k_{e f f}}{\tilde{L}_{v}}\left(T_{\infty}-T^{s a t}\right)
$$

subject to the initial condition $R(t, \tau)=r_{c}(\tau)$, where $r_{c}$ satisfies Eq. 3. Consider, next, the heat balance for the entire system. We have

$$
V_{p}\left[\phi\left(1-S_{g}\right) \rho_{l} C_{p l}+(1-\phi) \rho_{r} C_{p r}\right] \frac{d T_{\infty}}{d t_{d}}=-4 \pi \phi k_{e f f}\left(T_{\infty}-T^{\text {sat }}\right) N_{T} \int_{0}^{t} R(t, \tau) \omega(\tau) d \tau+h A_{\text {surf }}\left(T_{o}-T_{\infty}\right)
$$

where the integration is over all existing clusters, $C_{p}$ denotes heat capacity per unit mass, $\phi$ is porosity, $h$ is the heat transfer coefficient to the surroundings, assumed at temperature $T_{o}$, and $A_{\text {surf }}$ is the corresponding surface area through which such heat is exchanged.

The gas saturation is related to the radius of the growing clusters and the nucleation fraction through the relation

$$
S_{g}=A v \int_{0}^{f_{q}}\left(\frac{\hat{R}\left(t, f_{q}\right)}{r_{c}^{*}}\right)^{D_{f}} d f_{q}
$$

where we introduced the volume ratio $v \equiv V_{c} / V_{s}$, and the notation $\hat{R}(t, f(\tau)) \equiv R(t, \tau)$, for the radius of a cluster at time $t$, nucleated when the nucleation fraction was $f(\tau)$. Note also the liquid mass balance which reads as

$$
\frac{Q(t)}{V_{p}}=-\left(1-S_{g}\right) c \frac{d P_{l}}{d t}+\frac{d S_{g}}{d t}
$$

where $c$ takes values in the range of $1.45 \times 10^{-4}-1.45 \times 10^{-3} \mathrm{MPa}^{-1}$. However, in the present problem it is not needed. Subject to the relevant initial conditions, the system of Eqs. 11, 12, 13, and 14 can be integrated. Integration proceeds until the time when the critical gas saturation is reached. In the present approach, we assume that the critical gas saturation, $S_{g c}$, can be predicted given the nucleation fraction and the capillary and Bond numbers (Du and Yortsos ${ }^{17}$, Tsimpanogiannis and 
Yortsos $^{3}$, Tsimpanogiannis ${ }^{18}$ ). Therefore, for the purposes of estimating $S_{g c}$, it only suffices to model well the events during the nucleation period.

Dimensionless formulation and scaling. For the solution of the problem, we recast the equations in dimensionless form. Denote dimensionless quantities by subscript $D$ and scale temperature by $T_{o}$, pressure by $P_{o}$, cluster size by $r_{c}{ }^{*}$, and time by $t^{*}=P_{o} / a$, where $a$ is the constant pressure decline rate. The dimensionless mass balance for the gas phase is given by

$$
\left(1-t_{D}+\Pi_{c}\right) \frac{\partial\left(\hat{R}_{D}\right)^{D_{f}}}{\partial t_{D}}=\frac{\Pi_{2}}{A \Pi_{1}}\left(T_{D \infty}-T_{D}^{s a t}\right) \hat{R}_{D}+\left(\hat{R}_{D}\right)^{D_{f}}
$$

while the dimensionless heat balance for the system reads

$$
\left(1-S_{g}\right)\left(\Pi_{\rho}+\frac{1-\phi}{\phi}\right) \frac{d T_{D \infty}}{d t_{D}}=-\frac{1}{\Pi_{1}}\left(T_{D \infty}-T_{D}^{s a t}\right) \int_{0}^{f_{q}} \hat{R}_{D}\left(t_{D}, f_{q}\right) d f_{q}+\Pi_{H}\left(1-T_{D \infty}\right)
$$

In the above, we have defined the dimensionless groups

$$
\begin{aligned}
& \Pi_{1}=\frac{V_{s} \rho_{r} C_{p r} a}{4 \pi P_{o} k_{e f f} r_{c}^{*}}=\frac{V_{p} \rho_{r} C_{p r} a}{4 \pi P_{o} N_{T} k_{e f f} r_{c}^{*}}, \\
& \Pi_{2}=\frac{R_{g} T_{o}^{2}}{v M_{w} \tilde{L}_{v}} \frac{\rho_{r} C_{p r}}{P_{o}}, \quad \Pi_{H}=\frac{h A_{s u r f} P_{o}}{V_{p} \rho_{r} C_{p r} a \phi}, \\
& \Pi_{\rho}=\frac{\rho_{l} C_{p l}}{\rho_{r} C_{p r}} \text { and } \Pi_{c}=\frac{2 \gamma \cos \theta^{*}}{r_{c}^{*} P_{o}}
\end{aligned}
$$

Parameter $\Pi_{1}$ expresses the ratio of the characteristic times for heat diffusion at the pore scale to that for the decline of pressure. Although a small number in typical applications, it plays a key role in determining the nucleation fraction and the critical gas saturation.

In addition, we have the following relations: The gas saturation is 


$$
S_{g}=A v \int_{0}^{f_{q}} \hat{R}\left(t_{D}, f_{q}\right)^{D_{f}} d f_{q}
$$

Using the linearized phase equilibria, the dimensionless superheat is

$$
\theta \equiv T_{D \infty}-T_{D}^{s a t}=T_{D \infty}-\left(1-\psi t_{D}\right)
$$

where $\psi \equiv \frac{R_{g} T_{o}}{L_{v}}\left(\right.$ or $\psi \equiv \frac{R_{g} T_{o}}{L_{v}+P_{c} v_{m}}$, when Kelvin effects are important). The cavity size that becomes activated at a given time and temperature can be expressed in terms of the supersaturation

$$
s \equiv P_{D}^{s a t}\left(T_{D \infty}, t_{D}\right)-P_{D l}\left(t_{D}\right)=t_{D}-\frac{1-T_{D \infty}}{\psi},
$$

or, more conveniently, in terms of the rescaled supersaturation

$$
s_{D} \equiv \frac{s}{\Pi_{c}}
$$

Then, the nucleation fraction is

$$
f_{q}=\exp \left(-\frac{\pi}{4 s_{D}^{2}}\right), f_{q}=\exp \left(-\frac{1}{\sigma s_{D}^{n}}\right)
$$

depending on the size distribution used. In the solution of the problem, we assumed that the process begins $\left(t_{D}=0\right)$ when the pressure is at the bubble point corresponding to $T_{o}$. Initial conditions for the simulations were $T_{D \infty}=1, P_{D l}=1$ and $R_{D}(\tau, \tau)=s_{D}^{-1}(\tau)$.

We note that the system contains one key parameter, $\Pi_{1}$, describing the effect of the rate of increase of the supersaturation. Because $\Pi_{1}$ is small, a further rescaling of the nucleation fraction and the cluster size is necessary. After some analysis (Tsimpanogiannis and Yortsos ${ }^{3}$ ), it is not difficult to show that for the cavity nucleation model, the following scaling is valid, 
$f_{q} \sim \Pi_{1}^{\frac{D_{f}}{D_{f}-1}}$ and $f_{q} R^{D_{f}} \sim O(1)$ (where, given that the nucleation fraction varies only during the first period, $\left.D_{f}=3\right)$.

This scaling contains the main effect of the pressure decline rate on the nucleation fraction. Thus, we define a rescaled nucleation fraction and rescaled cluster sizes

$$
\phi_{q}=f_{q} \Pi_{1}^{-3 / 2} \text { and } \rho_{D}=\Pi_{1}^{1 / 2} \hat{R}_{D}
$$

In this notation, the governing equations finally become

$$
\left(1-t_{D}+\Pi_{c}\right) \frac{\partial \rho_{D}^{D_{f}}}{\partial t_{D}}=\frac{\Pi_{2}}{A} \theta \rho_{D}+\rho_{D}^{D_{f}}
$$

and

$$
\left(1-S_{g}\right)\left(\Pi_{\rho}+\frac{1-\phi}{\phi}\right) \frac{d T_{D \infty}}{d t_{D}}=-\theta \int_{0}^{\phi_{q}} \rho_{D}\left(t_{D}, \phi_{q}\right) d \phi_{q}+\Pi_{H}\left(1-T_{D \infty}\right)
$$

while

$$
S_{g}=A v \int_{0}^{\phi_{q}} \rho\left(t_{D}, \phi_{q}\right)^{D_{f}} d \phi_{q}
$$

The numerical solution of the system of the rescaled equations is described below. Before proceeding, we will summarize for the reader's convenience, the model constraints: We consider a single-component, supersaturated, slightly compressible liquid phase, occupying an effective porous medium with an external application of constant heat flux. Gas clusters nucleate when pre-existing, trapped gas in cavities of different sizes, overcome the capillary pressure of the mouth of the cavities. Nucleation is sequential, starting with larger cavities first. The gas cluster growth is proportional to the effective radius of the cluster and the driving force, $T_{\infty}-T^{\text {sat }}\left(P_{l}\right)$. The change in the system supersaturation is provided by the reduction of the pressure. The pressure decline rates are sufficiently slow so that inertia and spatial gradient effects on bubble growth are negligible. The model is applicable for the initial stages of nucleation and growth, during which no significant competition between the various gas clusters occurs. We assume that the heat transfer is conduction controlled and that simple thermodynamics (Clausius-Clapeyron equation) provide a sufficient description of the problem. The critical gas saturation is known from numerical studies and pertains to the formation of a sample-spanning cluster. 


\section{Numerical Results}

The system of differential equations was solved numerically using a fourth-order RungeKutta method (Press et al. ${ }^{19}$ ). At each time step we examine whether nucleation of a new class of gas clusters is possible, namely whether the supersaturation is increasing. If so, a new class of gas clusters is added. Then, the simultaneous growth of all different classes of clusters is computed. When the supersaturation reaches a maximum, further nucleation stops. In the typical case, parameters which can vary over a significant range are $\Pi_{1}$ and $\Pi_{c}$ (and possibly $\Pi_{2}$ ). An additional important variable is the type of the cavity size distribution used in the calculation of the nucleation fraction. The sensitivity to these parameters was examined in the simulations.

The effects of $\Pi_{1}$ and $\Pi_{c}$ on the rescaled nucleation fraction, $\phi_{q}$, the mean rescaled radius, $\rho_{D m}$, and the rescaled supersaturation, $s_{D}$, are shown in Figs. 3-7. In these calculations, we used a stretched-exponential ( $n=1.0$ and $\sigma=1.0)$ cavity size distribution, $\Pi_{2}$ was kept constant to the value $0.9697 \times 10^{8}$, we assumed an adiabatic system $\left(\Pi_{H}=0\right)$, while $\Pi_{1}$ varied over several orders of magnitude (from $10^{-14}$ to $10^{-5}$ ).

The variation of $\phi_{q}$ as a function of the dimensionless time, $t_{D}$, and of the parameters $\Pi_{1}$ and $\Pi_{c}$ is shown in Figs. 3-4. The nucleation fraction increases rapidly in a small time interval, and then stabilizes to a final value at the conclusion of nucleation. Such behavior is characteristic of nucleation processes, and has features similar to those reported by Tsimpanogiannis and Yortsos $^{3}$ for solution gas-drive. There is only a slight effect of $\Pi_{1}$, which basically demonstrates the correctness of the scaling, Eq. 23. The effect of $\Pi_{c}$ is significant. As $\Pi_{c}$ increases, the final nucleation fraction $\phi_{q f}$ (hence $f_{q f}$ ) decreases, while the onset of nucleation is delayed (Fig. 4). The increase of $f_{q f}$ with an increase in $\Pi_{1}$ and a decrease in $\Pi_{c}$ is expected. Larger values of $\Pi_{1}$ result from a faster decline rate, a greater departure from equilibrium, the establishment of a greater supersaturation in the system, hence the activation of more nucleation sites. Likewise, smaller $\Pi_{c}$ imply that nucleation is facilitated at increasingly smaller supersaturations, as larger size cavities can be activated more easily.

Fig. 5 shows the corresponding effects on the mean rescaled size $\rho_{D m}$. There are two different regions, one corresponding to the nucleation period, and another to growth after 
nucleation. The first period can be approximated as a linear function of time. The effect of $\Pi_{1}$ is relatively insignificant at small $\Pi_{1}$, again confirming the validity of the scaling, Eq. 23 . The effect of $\Pi_{c}$ (not shown) is more significant. Smaller values of $\Pi_{c}$ lead to an increase in the nucleation fraction, and a corresponding decrease in the size of the gas clusters at the conclusion of nucleation.

Fig. 6 shows a plot of the rescaled supersaturation $s_{D}$ as a function of time for different $\Pi_{1}$. The nucleation period corresponds to the straight-line segment in Fig. 6. The supersaturation increases with time almost linearly, suggesting that $T_{D \infty}$ does not vary significantly in that period. Eventually, the rate of supersaturation increase slows down and, at some point, $s_{D}$ reaches a maximum, $s_{D m}$, at which point nucleation terminates. Following this point, the supersaturation decreases monotonically. The maximum value $s_{D m}$ is plotted in Fig. 7 as a function of $\Pi_{1}$ for two different values of $\Pi_{c}$. Note that $s_{D m}$ is in general of the order of $10^{-3}-10^{-1}$. The dependence on the parameters becomes stronger at larger $\Pi_{1}$ and smaller $\Pi_{c}$.

The evolution of the gas saturation follows that of $f_{q}$, during the nucleation period, and that of $\rho_{D m}$, during the period of growth. The effect of $\Pi_{c}$ is indirect, in that smaller values of $\Pi_{c}$ promote larger values of $S_{g}$ due to an increase in both $f_{q f}$ and $\rho_{D}$. All these trends are similar to the case of solution gas drive, as explained in Tsimpanogiannis and Yortsos ${ }^{3}$. We refer the reader to this publication for various effects, including the effect of $\Pi_{1}$ and $\Pi_{c}$ on the critical gas saturation $S_{g c}$. Because the latter pertains to the formation of a sample-spanning cluster, in the absence of viscous or gravity effects, $S_{g c}$ actually reflects the variation of $f_{q f}$. Thus, $S_{g c}$ can be considered a power-law both of $\Pi_{1}$ and of $\Pi_{c}$ with exponents that vary between 0.16 and 0.25 with respect to $\Pi_{1}$ and between -0.33 and -0.22 , with respect to $\Pi_{c}$, respectively (see Tsimpanogiannis and Yortsos $^{3}$ ).

An important effect, absent from solution gas-drive is the heat transfer to the surroundings. The effect of $\Pi_{H}$ on the rescaled nucleation fraction, $\phi_{q}$ and the gas saturation, $S_{g}$, is shown in Figs. 8-9. In these calculations, we used a stretched exponential $(n=0.2233$ and $\sigma=0.1364)$ size distribution. As $\Pi_{H}$ increases, the level of superheat and thus the level of the supersaturation in the system increase. This leads to an earlier onset of nucleation, as well as a higher degree of nucleation. However, the effect of $\Pi_{H}$ on the rescaled final nucleation fraction, $\phi_{q f}$, and on the maximum superheat is not significant. A change of $\Pi_{H}$ by three orders of 
magnitude, results in a change of $\phi_{q f}$ by a factor of less than 2 (Fig. 8). On the other hand, the gas saturation increases faster as the heat transfer coefficient increases (Fig. 9). This is due to the maintaining of a higher level of superheat, therefore a larger driving force for gas volume growth. Interestingly, as the heat transfer coefficient decreases the gas saturation growth slows down at larger values of the gas saturation. A noticeable difference, at higher values of $\Pi_{H}$, is that the superheat is not completely depleted before the gas saturation becomes equal to one, as happens with the lower values of $\Pi_{H}$.

The numerical solutions obtained will be compared against available experimental results. However, before doing so it is beneficial to provide an interpretation of the numerical findings, using a simpler model.

\section{Interpretation Using a Simpler Model}

To interpret the results obtained we will consider a simpler model that captures the essential features of the problem, just like in Tsimpanogiannis and Yortsos ${ }^{3}$. Consider, first, the nucleation period.

Nucleation. We will use the following simpler set of equations

$$
\left(1+\Pi_{c}\right) \frac{\partial \rho_{D}^{3}}{\partial t_{D}} \approx \Pi_{2} \theta \rho_{D}
$$

and

$$
\left(\Pi_{\rho}+\frac{1-\phi}{\phi}\right) \frac{d \theta}{d t_{D}} \approx \psi\left(\Pi_{\rho}+\frac{1-\phi}{\phi}\right)-\theta \int_{0}^{\phi_{q}(s)} \rho_{D} d \phi_{q}+\Pi_{H}\left(\psi t_{D}-\theta\right)
$$

These are subject to the initial conditions

$$
\theta(0)=0 \text { and } \rho_{D}(\tau, \tau)=\frac{\Pi_{1}^{1 / 2}}{s_{D}(\tau)}
$$

At early times and for small $\Pi_{1}$, the solution is approximately 


$$
\theta \approx \psi t_{D} \text { and } \rho_{D} \approx\left[\frac{\Pi_{2}}{3\left(1+\Pi_{c}\right)} \frac{\theta^{2}}{\psi}\right]^{1 / 2}
$$

Note that the heat transfer term does not affect the early behavior (compare also with Figs. 8-9). The dimensionless superheat is linearly proportional to the dimensionless time and the mean cluster size becomes eventually proportional to time. Both are consistent with the full numerical results during the nucleation period (Figs. 5-7).

We can use Eq. 28 to approximate the approach to the maximum superheat. The latter is reached when $\frac{d \theta}{d t_{D}}=0$, namely when

$$
\theta \int_{0}^{\phi_{q}} \rho_{D} d \phi_{q} \approx\left(\Pi_{\rho}+\frac{1-\phi}{\phi}\right) \psi
$$

Following a similar approach as in Tsimpanogiannis and Yortsos $^{3}$ we can combine Eqs. 30 and 31 with the definition of $\phi_{q}$ to obtain an approximate algebraic equation for the rescaled maximum supersaturation, $s_{D m}$. For example, for the case of Rayleigh distribution we find

$$
\frac{\pi}{4 s_{D m}^{2}}-2 \ln s_{D m} \approx \frac{1}{2} \ln \psi-\ln \left(\Pi_{\rho}+\frac{1-\phi}{\phi}\right)-\frac{1}{2} \ln 3-\frac{3}{2} \ln \Delta
$$

where we introduced the combination of variables

$$
\Delta \equiv \Pi_{1} \Pi_{c}^{-4 / 3}\left(\frac{\Pi_{2}}{1+\Pi_{c}}\right)^{-1 / 3}
$$

Likewise, for the case of a stretched exponential, we get

$$
\sigma^{-1} s_{D m}^{-n}-2 \ln s_{D m} \approx \frac{1}{2} \ln \psi-\ln \left(\Pi_{\rho}+\frac{1-\phi}{\phi}\right)-\frac{1}{2} \ln 3-\frac{3}{2} \ln \Delta
$$

These equations show the dependence of the maximum supersaturation (hence the maximum superheat, since $\left.\theta=\psi \Pi_{c} s_{D}\right)$ on the various parameters. The solutions of Eq. 32 for the Rayleigh distribution and of Eq. 34 for two different cases of a stretched exponential are plotted in Fig. 10, as a function of $\Delta$. For the Rayleigh distribution, $s_{D m}$ varies weakly, in the range 0.1-1, as $\Delta$ varies over several orders of magnitude (between $10^{-14}$ and $10^{-5}$ ). For small $\Delta$, the maximum supersaturation is practically constant. As $\Delta$ takes larger values, $s_{D m}$ first increases weakly and 
then eventually much more strongly, as $\Delta$ approaches the order of one (compare also with Fig. 7). On the other hand, for the stretched exponential, the variation is much stronger in the logarithmic plot, and almost approximates a straight line. Stronger dependence on $\Delta$ is observed for the case when the tail of the cavity size distribution becomes longer (smaller values for $n$ ). Plotted in the same figure are also the results of the numerical solution of the full problem for a number of different parameter values. The agreement between the numerical results and the simple analytical model is very good and demonstrates the validity of the simple model.

Eqs. 32-34 can be used to approximate the final nucleation fraction. For all cases we have

$$
f_{q f} \approx s_{D m}^{-2} \Lambda\left(\Pi_{1}^{3 / 2}\right) \Pi_{c}^{-2}\left[\frac{\Pi_{2}}{3\left(1+\Pi_{c}\right)}\right]^{-1 / 2},
$$

where

$$
\Lambda=\left(\Pi_{\rho}+\frac{1-\phi}{\phi}\right) \psi^{-1 / 2}
$$

The behavior of the maximum supersaturation as a function of the parameter $\Delta$ is very similar to that in solution gas drive (Tsimpanogiannis and Yortsos ${ }^{3}$ ). In particular:

(a) In the region where $s_{D m}$ varies weakly with $\Delta$ (at very small $\Delta$ ) the final nucleation fraction varies as a power law of $\Pi_{1}$, with exponent equal to $3 / 2$.

(b) In the region where $s_{D m}$ may be approximated by a power-law dependence on $\Delta$, e.g. as $s_{D m} \sim \Delta^{m}$, we have the scaling

$$
f_{q f} \sim \Lambda \Delta^{\frac{3}{2}-2 m}
$$

Such a dependence on $\Delta$ leads to a decrease in the exponent in the power-law scaling of $f_{q f}$ on $\Pi_{1}$. For example, if we take $m \approx 1 / 4$ (a value examined in more detail in Tsimpanogiannis and Yortsos ${ }^{3}$ ), we read

$$
f_{q f} \sim \Pi_{1} \text { and } f_{q f} \sim r_{c}^{*}
$$


Gas cluster growth. The modeling of the growth regime, where nucleation has terminated, can be simplified if we consider only one class of clusters. Then, we can also simplify the heat and mass balances as follows

$$
\frac{d \theta}{d t_{D}} \approx \frac{-\theta z+\Pi_{H}\left(\psi t_{D}-\theta\right)}{\left(\Pi_{\rho}+\frac{1-\phi}{\phi}\right)\left(1-v \Pi_{2} k_{1} z^{D_{f}}\right)}+\psi
$$

and

$$
\frac{d z^{D_{f}}}{d t_{D}}=\frac{k_{1}^{-1} \theta z+z^{D_{f}}}{1-t_{D}+\Pi_{c}}
$$

Here, we introduced the variable

$$
z \equiv \phi_{q f} \rho_{D}
$$

and the parameter

$$
k_{1}=\frac{\phi_{q f}^{1-D_{f}}}{\Pi_{2}}
$$

The final value of the rescaled nucleation fraction, $\phi_{q f}$, as well as initial values for $T_{D \infty}$ and $\rho_{D}$, needed for the above calculation, are obtained from the previous step.

\section{Comparison With Experiments}

The theoretical model was next compared to the experimental results of Dehghani et al. ${ }^{1}$. In these experiments, the pressure at the open end of a Colton sandstone core, saturated with water and embedded in a constant temperature bath, was slowly reduced at the rate of 0.7448 bar/h $(10.8 \mathrm{psi} / \mathrm{h})$. The other end of the core was kept closed to flow. Properties of the core of interest to this paper were taken as follows: $r_{s}=3.0 \times 10^{-5} \mathrm{~cm}, r_{c}=3.0 \times 10^{-7} \mathrm{~cm}$. Additional, physical parameters and values of the dimensionless parameters used in the calculations are shown in Table 1.

The gas saturation as a function of time for the single-component experiment and for various axial positions along the core are shown in Fig. 11. We note that the evolution of the gas 
saturation is slower as the distance from the entrance of the core increases. This may reflect either a reduced rate of pressure decline, or a decrease in the heat transfer coefficient as the distance from the open-end increases. For a better comparison of the data, we attempted to collapse all data into a single curve. By replotting the data using as time origin the time the boiling point in the bulk is reached (which here is $t_{o}=84$ minutes), and by rescaling time by a factor $b(L)$, where $L$ is the distance from the open end, we were able to collapse satisfactorily all data in a single curve, as shown in Fig. 12. The match was obtained using a stretchedexponential cavity size distribution with $n=0.35$ and $\sigma=1.0$. The less satisfactory collapse of the data at the early times could be the result of less accurate CT-scan resolution in the low porosity sandstone used in the experiments (Dehghani et al. ${ }^{1}$ ). The variation of the factor $b(L)$ which allows this collapse is shown in Fig. 13. It is a linear function of the dimensionless distance, with the best-fit line $b(L)=1.0106+5.1513\left(L / L_{o}\right)$, where $L_{o}$ is the core length.

\section{Conclusions}

In this paper we developed an effective continuum model to describe the nucleation and subsequent growth of a gas phase from a supersaturated liquid in a porous medium, driven by heat transfer. The evolution of the gas results from the reduction of the system pressure at a constant rate. The model addresses two stages before the onset of bulk gas flow, nucleation and gas phase growth.

We used heterogeneous nucleation models in the form of pre-existing gas, trapped in hydrophobic cavities to investigate the nucleation behavior. Using scaling analysis and a simpler analytical model we showed that the relevant quantities during nucleation can be expressed in terms of a simple combination of dimensionless parameters, which include rate effects. The subsequent evolution of the gas phase was also described using numerical and analytical models.

The theory predicts that the maximum supersaturation in the system is a weakly increasing function of the decline rate. This function depends sensitively on the probability density function of the nucleation cavity sizes. It also predicts that the final nucleation fraction, thus the critical gas saturation, is a power law of the decline rate. The theory was then compared with available experimental data of internal steam drives, such as the blowdown experiments in carbonate rocks (Dehghani et al. ${ }^{1}$ ) and a good match was obtained by appropriate fitting of the nucleation characteristics of the medium. 


\section{Nomenclature}

$a=$ pressure decline rate, $\mathrm{bar} / \mathrm{h}$

$A=$ dimensionless geometric factor

$A^{*}=$ dimensionless geometric factor

$A_{\text {surf }}=$ surface area through which heat is exchanged, $\mathrm{m}^{2}$

$b$ = time rescaling factor, $\min$

$c$ = liquid compressibility, $\mathrm{Pa}^{-1}$

$C_{p l}=$ liquid heat capacity per unit mass, $\mathrm{J} /(\mathrm{Kg} \mathrm{K})$

$C_{p r}=$ rock heat capacity per unit mass, $\mathrm{J} /(\mathrm{Kg} \mathrm{K})$

$D_{f}=$ mass fractal dimension

$f_{q}=$ current nucleation fraction

$f_{q f}=$ final nucleation fraction

$h=$ heat transfer coefficient, $\mathrm{W} /\left(\mathrm{m}^{2} \mathrm{~K}\right)$

$k=$ core absolute permeability, $\mu \mathrm{m}^{2}$

$k_{1}=$ dimensionless parameter (Eq. 44)

$k_{\text {eff }}=$ effective conductivity, $\mathrm{W} /(\mathrm{m} \mathrm{K})$

$L=$ distance from the open end of the core, $\mathrm{cm}$

$L_{o}=$ core length, $\mathrm{cm}$

$L_{v}=$ molar latent heat of vaporization, $\mathrm{J} / \mathrm{mol}$

$\tilde{L_{v}}=$ mass latent heat of vaporization, $\mathrm{J} / \mathrm{Kg}$

$\mathrm{m}=$ dimensionless exponent in Eq. 37

$M_{w}=$ molecular weight, $\mathrm{g} / \mathrm{mol}$

$n$ = positive exponent in cavity distribution

$N_{T}=$ total number of pores

$P_{c}=$ capillary pressure, bar

$P_{l}=$ liquid pressure, bar

$P_{o}=$ initial system pressure, bar

$P^{\text {sat }}=$ equilibrium vapor pressure, bar

$Q=$ volumetric flow rate, $\mathrm{m}^{3} / \mathrm{h}$

$r_{c}=$ radius of a cavity, $\mathrm{cm}$

$r_{c}{ }^{*}=$ characteristic (mean) cavity size, $\mathrm{cm}$ 


$$
\begin{aligned}
& r_{s}=\text { radius of a site, } \mathrm{cm} \\
& R_{g}=\text { ideal gas constant, } 8.314 \mathrm{~J} /(\mathrm{mol} \mathrm{K}) \\
& R_{j}=\text { effective radius of cluster } \mathrm{j}, \mathrm{cm} \\
& \hat{R}=\text { effective radius, } \mathrm{cm} \\
& s \text { = dimensionless supersaturation } \\
& s_{D}=\text { rescaled dimensionless supersaturation } \\
& s_{D m}=\text { maximum rescaled dimensionless supersaturation } \\
& S_{g}=\text { gas saturation } \\
& S_{g c}=\text { critical gas saturation } \\
& t \text { = time, } \mathrm{s} \\
& t_{D}=\text { dimensionless time } \\
& t^{*}=\text { characteristic time, } \mathrm{s} \\
& T=\text { temperature, } \mathrm{K} \\
& T_{D}^{\text {sat }}=\text { dimensionless equilibrium (saturation) temperature } \\
& T_{D \infty}=\text { dimensionless far-field system temperature } \\
& T_{g}=\text { gas phase temperature, } \mathrm{K} \\
& T_{o}=\text { initial system temperature, } \mathrm{K} \\
& T^{\text {sat }}=\text { equilibrium (saturation) temperature, } \mathrm{K} \\
& T_{\infty}=\text { far-field system temperature, } \mathrm{K} \\
& V_{c}=\text { cavity volume, } \mathrm{m}^{3} \\
& V_{g}=\text { gas volume, } \mathrm{m}^{3} \\
& V_{p}=\text { total pore volume, } \mathrm{m}^{3} \\
& V_{s}=\text { site volume, } \mathrm{m}^{3} \\
& z=\text { dimensionless variable (Eq. 41) }
\end{aligned}
$$

$\alpha_{c}\left(r_{c}\right)=$ cavity size distribution

$\gamma=$ interfacial tension, $\mathrm{mN} / \mathrm{m}$

$\Delta=$ dimensionless combination of variables (Eq. 33)

$\theta=$ dimensionless superheat

$\theta^{*}=$ contact angle, degree 
$\Lambda=$ dimensionless combination of variables (Eq. 36)

$\Pi_{1}, \Pi_{2}, \Pi_{\rho}, \Pi_{H}=$ dimensionless numbers

$\Pi_{c}=$ dimensionless capillary pressure

$\rho_{D}=$ rescaled dimensionless radius of a gas cluster

$\rho_{D m}=$ mean rescaled dimensionless radius of a gas cluster

$\rho_{l}=$ liquid density, $\mathrm{Kg} / \mathrm{m}^{3}$

$\rho_{r}=$ rock density, $\mathrm{Kg} / \mathrm{m}^{3}$

$\sigma=$ measure of the variance of the cavity distribution

$\tau=$ dimensionless time

$v=$ volume ratio, $\mathrm{m}^{3} / \mathrm{m}^{3}$

$v_{m}=$ molar liquid volume, $\mathrm{m}^{3} / \mathrm{mol}$

$\phi=$ porosity

$\phi_{q}=$ rescaled nucleation fraction

$\phi_{q f}=$ final rescaled nucleation fraction

$\psi=$ dimensionless parameter $\left(\psi \equiv \frac{R_{g} T_{o}}{L_{v}}\right)$

$\omega(\tau)=$ number density of clusters nucleated per total number of pores

\section{References}

1. Dehghani, K., Kumar, M., deZabala, E.F., Meyer, R.F. and Duran, H.: "An Experimental and Numerical Study of In-Situ Steam-drive During Cyclic Steaming," SPERE (May 1997) 144.

2. Dehghani, K. and Kamath, J.: "High Temperature Blow-Down Experiments in a Vuggy Carbonate Core," paper SPE 56542 presented at the 1999 SPE Annual Technical Conference and Exhibition, Houston, Texas, 3-6 October.

3. Tsimpanogiannis, I.N. and Yortsos, Y.C: "Model for the Gas Evolution in a Porous Medium Driven by Solute Diffusion," AIChE J. (2002) 48, 2690.

4. Hawes, R.I., Dawe, R.A. and Evans, R.N.: "The Release of Solution Gas from Waterflood Residual Oil," SPEJ (1997) 2, 379.

5. Mackay, E.J., Henderson, G.D., Tehrani, D.H. and Danesh, A.: "The Importance of Interfacial Tension on Fluid Distribution During Depressurization," SPEREE (1998) 1, 408. 
6. Bora, R., Maini, B.B. and Chakma, A.: "Flow Visualization Studies of Solution Gas Drive Process in Heavy Oil Reservoirs Using a Glass Micromodel," SPEREE (2000) 3, 224.

7. Kortekaas, T.F.M. and van Poelgeest, F.: "Liberation of Solution Gas During Pressure Depletion of Virgin and Watered-Out Oil Reservoirs," SPERE (1991) 6, 329.

8. Reid, R.C., Prausnitz, J.M. and Poling, B.E.: The Properties of Gases and Liquids, 4th ed. McGraw-Hill, New York, (1986).

9. Yortsos, Y.C. and Parlar, M.: "Phase Change in Binary Systems in Porous Media: Application to Solution Gas Drive," paper SPE 19697 presented at the 1989 SPE Annual Technical Conference and Exhibition, San Antonio, Texas, 8-11 October.

10. Li, X. and Yortsos, Y.C.: "Visualization and Simulation of Bubble Growth in Pore Networks," AIChE J. (1995) 41, 214.

11. El Yousfi, A., Zarcone, C., Bories, S. and Lenormand, R.:"Mecanismes de Formation d'une Phase Gazeuse par detente d' un liquide en Milieu Poreux," C.R. Acad. Sci. Paris, Serie II (1991) 313, 1093.

12. El Yousfi, A., Zarcone, C., Bories, S. and Lenormand, R.: "Physical Mechanisms for Bubble Growth During Solution Gas Drive," paper SPE 38921 presented at the 1997 SPE Annual Technical Conference and Exhibition, San Antonio, Texas, 5-8 October.

13. Dominguez, A., Bories, S. and Prat, M.: "Gas Cluster Growth by Solute Diffusion in Porous Media. Experiments and Automaton Simulation on Pore Network," Int. J. Multiphase Flow (2000) 26, 1951.

14. Firoozabadi, A. and Kashchiev, D.: "Pressure and Volume Evolution During Gas Phase Formation in Solution Gas Drive Processes," SPEJ (1997) 1, 219.

15. Satik, C. and Yortsos, Y.C: "A Pore Network Study of Bubble Growth in Porous Media Driven by Heat Transfer," ASME J. of Heat Transfer (1996) 118, 155.

16. Feder, J.: Fractals, Plenum, New York, (1988).

17. Du, C. and Yortsos, Y.C.: "A Numerical Study of the Critical Gas Saturation in a Porous Medium," Transport in Porous Media (1999) 35, 205.

18. Tsimpanogiannis, I.N.: "Studies on Processes Involving Liquid-to-Gas Phase Change in Porous Media," Ph.D. Dissertation, University of Southern California, Los Angeles, California (2002).

19. Press, W.H., Teukolsky, W.T., Vettering, S.A. and Flannery, B.P.: Numerical Recipes, $2^{\text {nd }}$ Ed. Cambridge University Press, (1992). 


\begin{tabular}{|c|c|}
\hline \multicolumn{2}{|c|}{ TABLE 1- PARAMETER VALUES } \\
\hline $\begin{array}{l}\text { Paramete } \\
\mathbf{r}\end{array}$ & Value \\
\hline$\alpha \quad(b a r / h)$ & 0.7448 \\
\hline$C_{p l}(J /(K g K))$ & 5954.7 \\
\hline$C_{p r}(J /(K g K))$ & 850.0 \\
\hline$k \quad\left(\mu m^{2}\right)$ & $4.145 \times 10^{-4}$ \\
\hline$k_{e f f}(W /(m K))$ & 1.0 \\
\hline$L_{o} \quad(\mathrm{~cm})$ & 11.354 \\
\hline$L_{v}(\mathrm{~J} / \mathrm{mol})$ & 37294.8 \\
\hline$M_{w}(g / m o l)$ & 18.016 \\
\hline$P_{o} \quad$ (bar) & 9.276 \\
\hline$r_{c} \quad(\mathrm{~cm})$ & $3.0 \times 10^{-7}$ \\
\hline$r_{s} \quad(\mathrm{~cm})$ & $3.0 \times 10^{-5}$ \\
\hline$T_{o}(K)$ & 449.4 \\
\hline$\gamma \quad(m N / m)$ & 55.0 \\
\hline$\Pi_{1}$ & $1.333 \times 10^{-10}$ \\
\hline$\Pi_{2}$ & $9.697 \times 10^{7}$ \\
\hline$\Pi_{c}$ & $3.901 \times 10^{1}$ \\
\hline$\Pi_{H}$ & $2.697 \times 10^{2}$ \\
\hline$\Pi_{\rho}$ & $2.981 \times 10^{0}$ \\
\hline$\rho_{r} \quad\left(K g /\left(m^{3}\right)\right)$ & 2350.0 \\
\hline$v$ & $1.0 \times 10^{-6}$ \\
\hline$\phi$ & 0.111 \\
\hline$\psi$ & $1.002 \times 10^{-1}$ \\
\hline
\end{tabular}




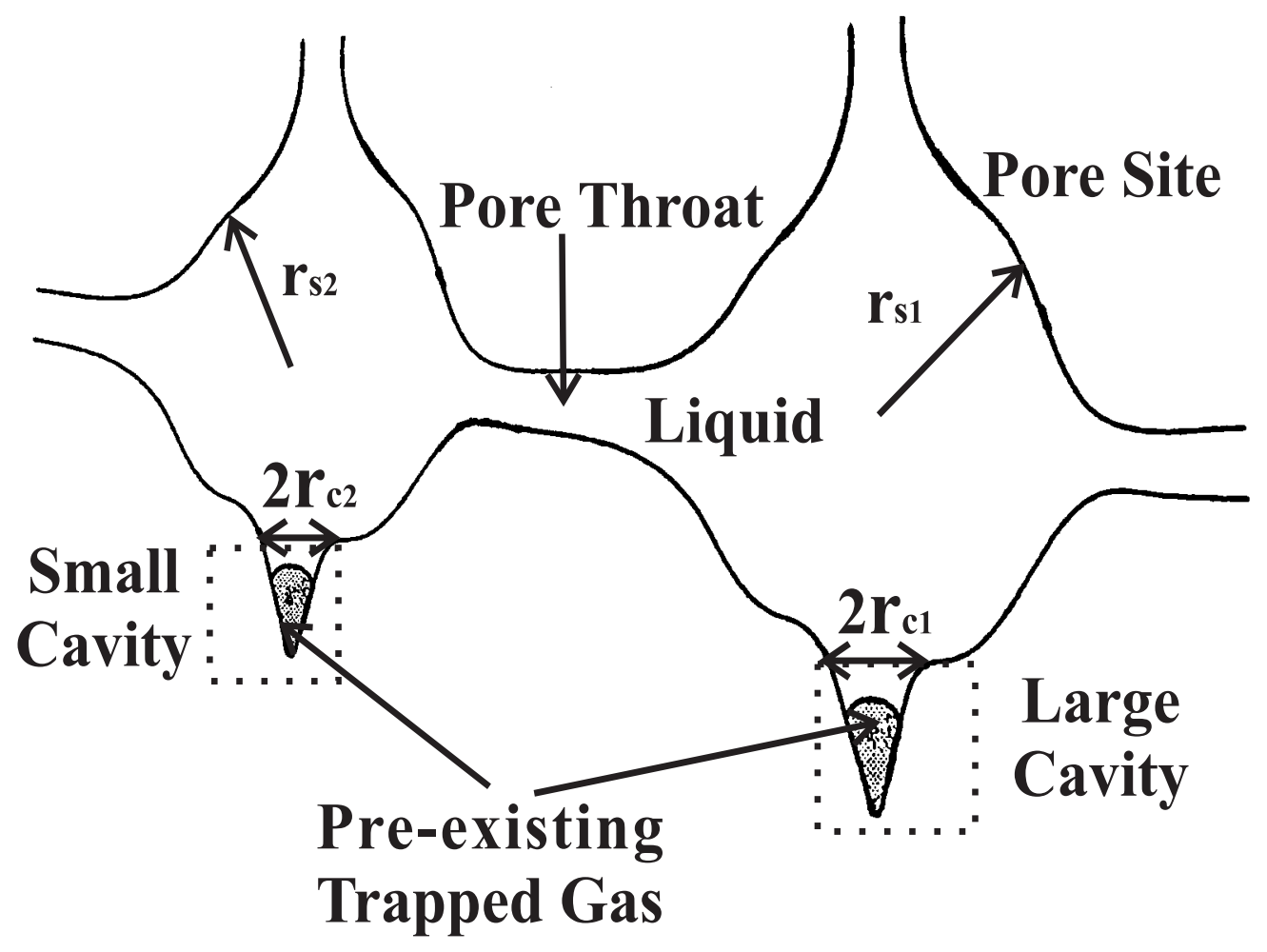

Figure 1: Schematic of nucleation cavities in host pore bodies of different sizes. 

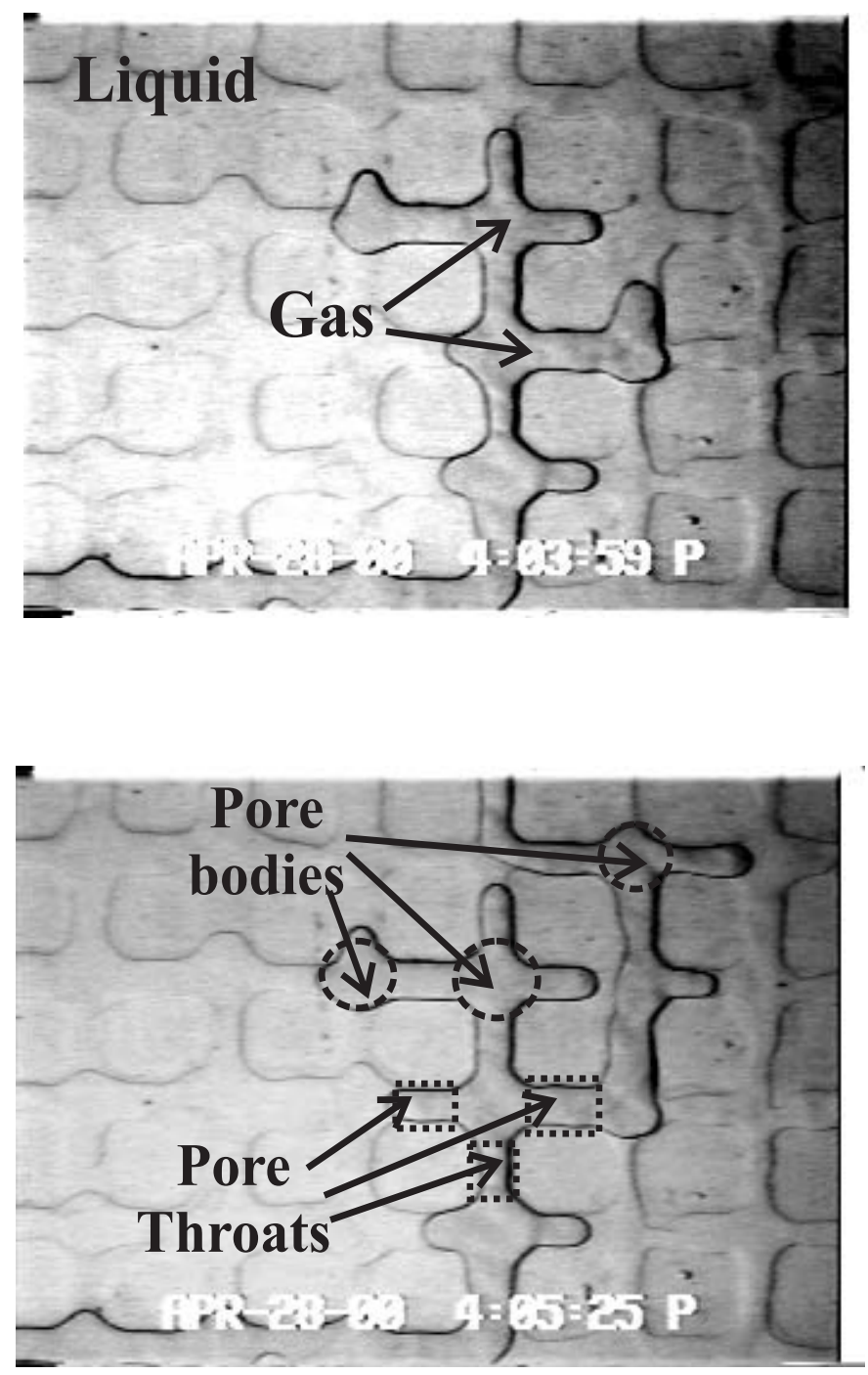

Figure 2: Glass micromodel snapshots indicating the growth of a gas bubble that spans several pore bodies. The sysstem examined is $\mathrm{C}_{10} / \mathrm{CO}_{2}$ and the time interval between the snapshots is $86 \mathrm{~s}$. 


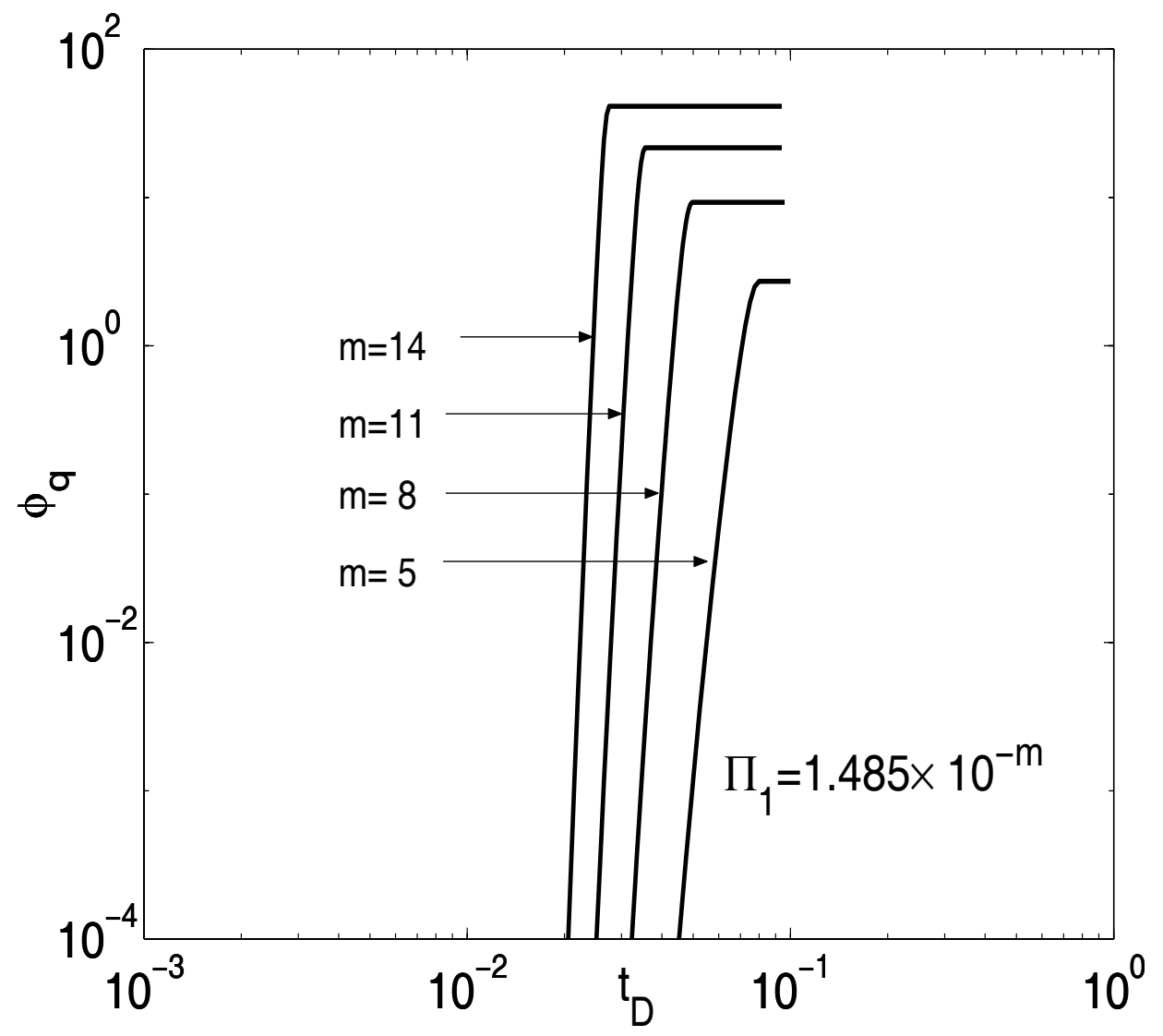

Figure 3: Variation of the rescaled nucleation fraction, $\phi_{q}$, as a function of the dimensionless time, $t_{D}$. Effect of $\Pi_{1}=1.485 \times 10^{-m}$, for $\Pi_{2}=9.6972 \times 10^{7}, \Pi_{\rho}=2.981$ and $\Pi_{H}=0$. 


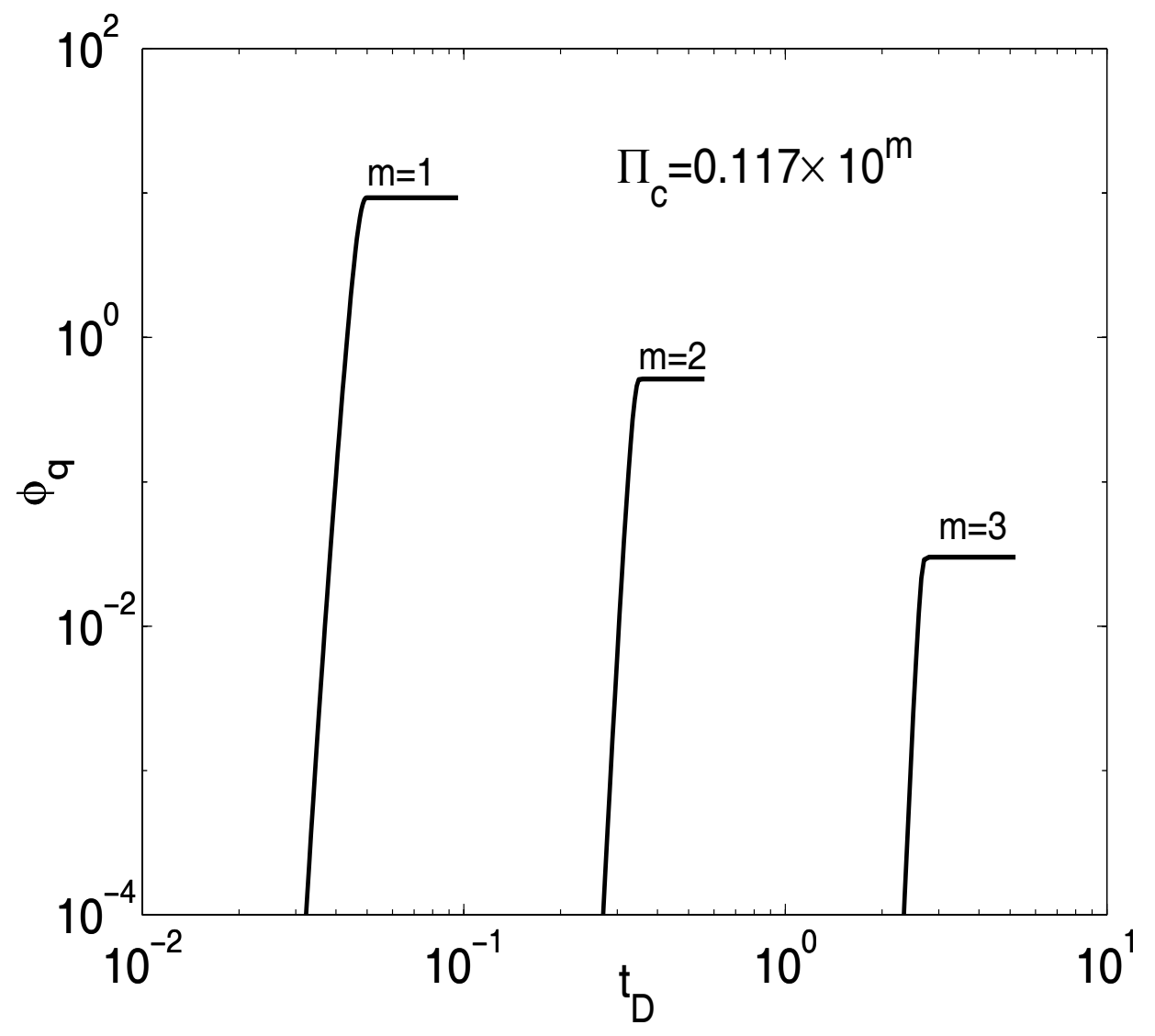

Figure 4: Variation of the rescaled nucleation fraction, $\phi_{q}$, as a function of the dimensionless time, $t_{D}$. Effect of $\Pi_{c}=0.117 \times 10^{m}$, for $\Pi_{1}=1.485 \times 10^{-8}, \Pi_{2}=9.6972 \times 10^{7}, \Pi_{\rho}=2.981$ and $\Pi_{H}=0$. 


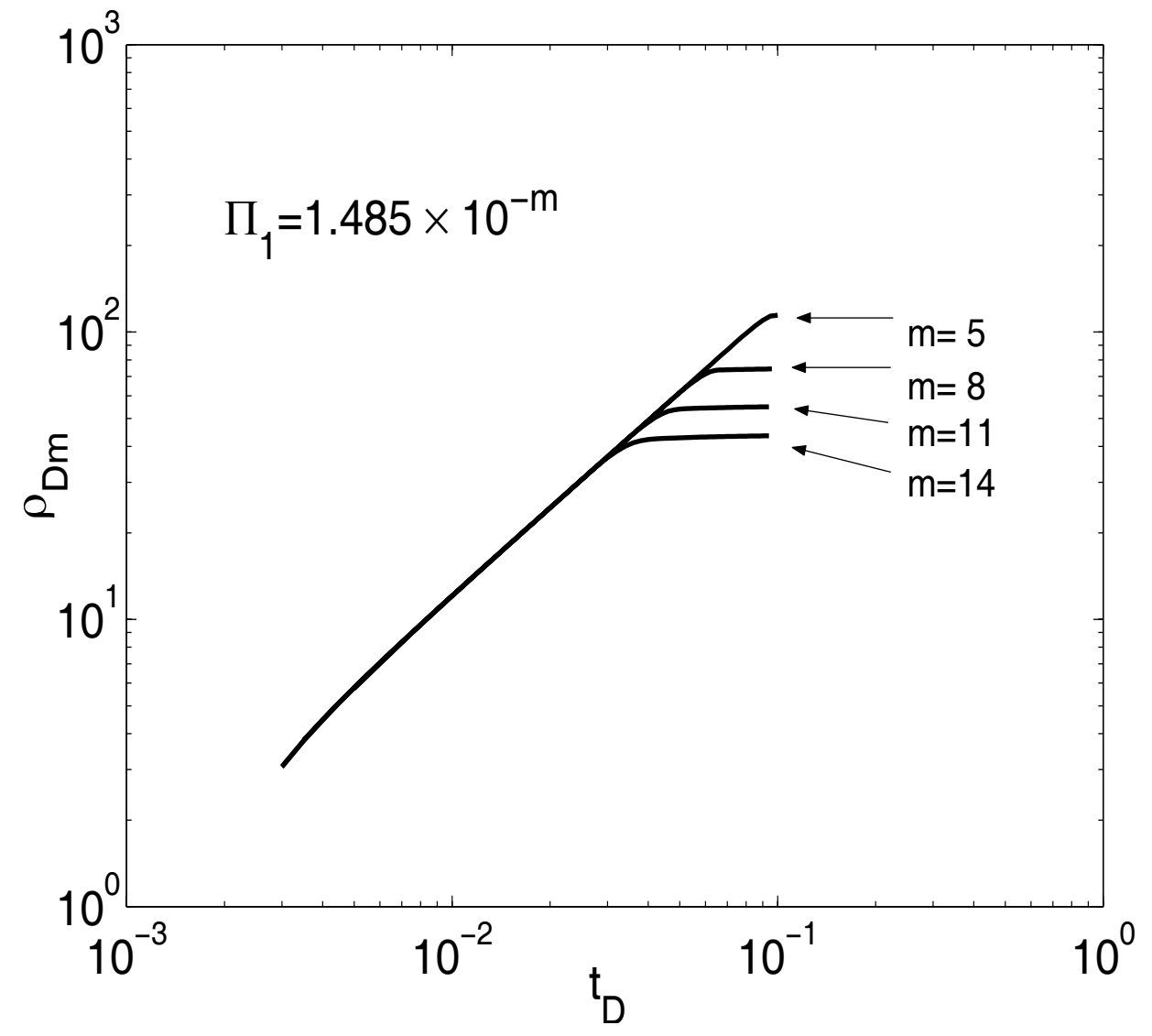

Figure 5: Variation of the mean rescaled dimensionless radius, $\rho_{D m}$, as a function of dimensionless time, $t_{D}$. Effect of $\Pi_{1}=1.485 \times 10^{-m}$, for $\Pi_{2}=9.6972 \times 10^{7}, \Pi_{\rho}=2.981$, $\Pi_{c}=0.117 \times 10^{1}$ and $\Pi_{H}=0$. 


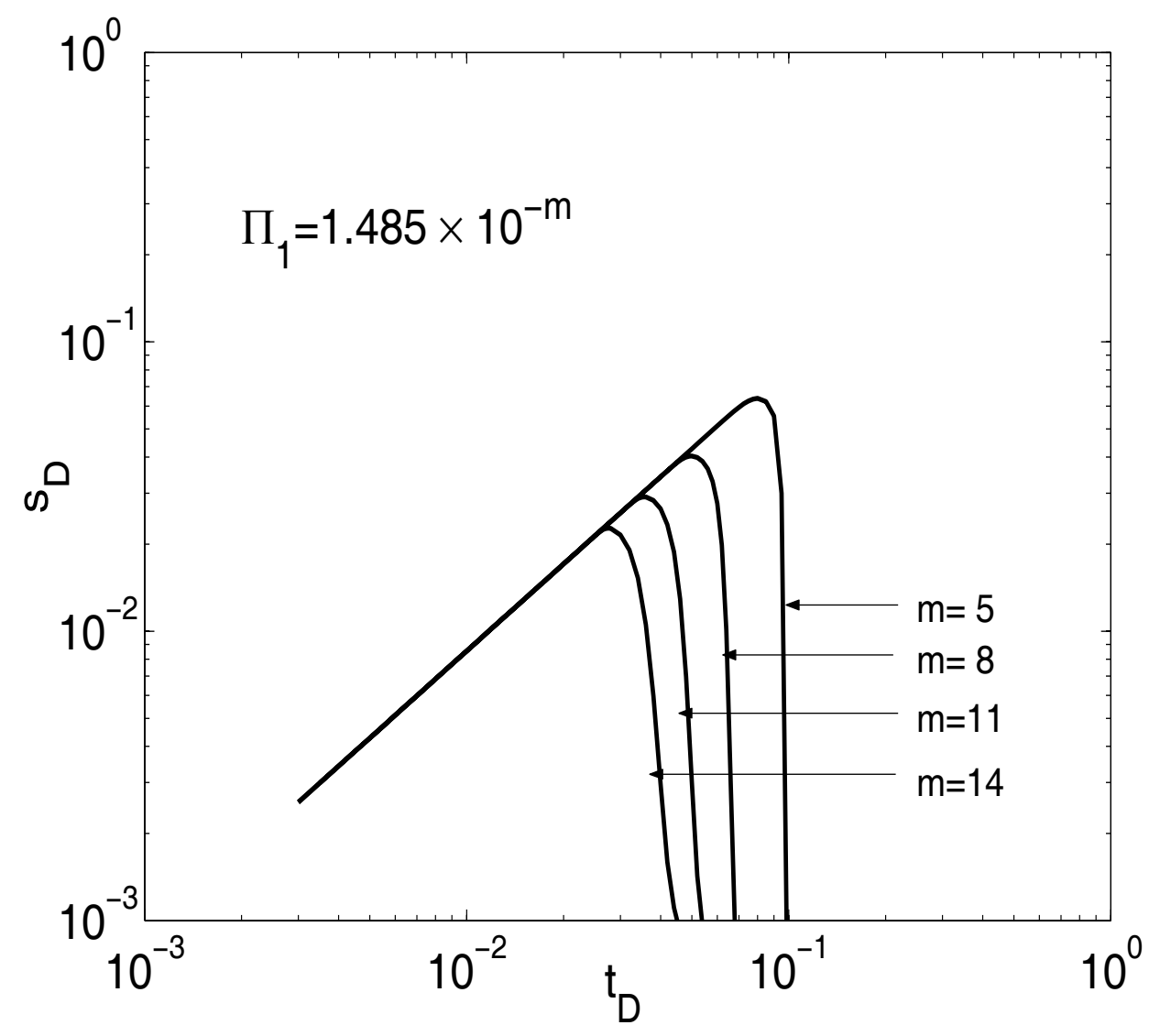

Figure 6: Variation of the rescaled supersaturation, $s_{D}$, as a function of dimensionless time, $t_{D}$. Effect of $\Pi_{1}=1.485 \times 10^{-m}$, for $\Pi_{2}=9.6972 \times 10^{7}, \Pi_{\rho}=2.981$ and $\Pi_{H}=0$. 


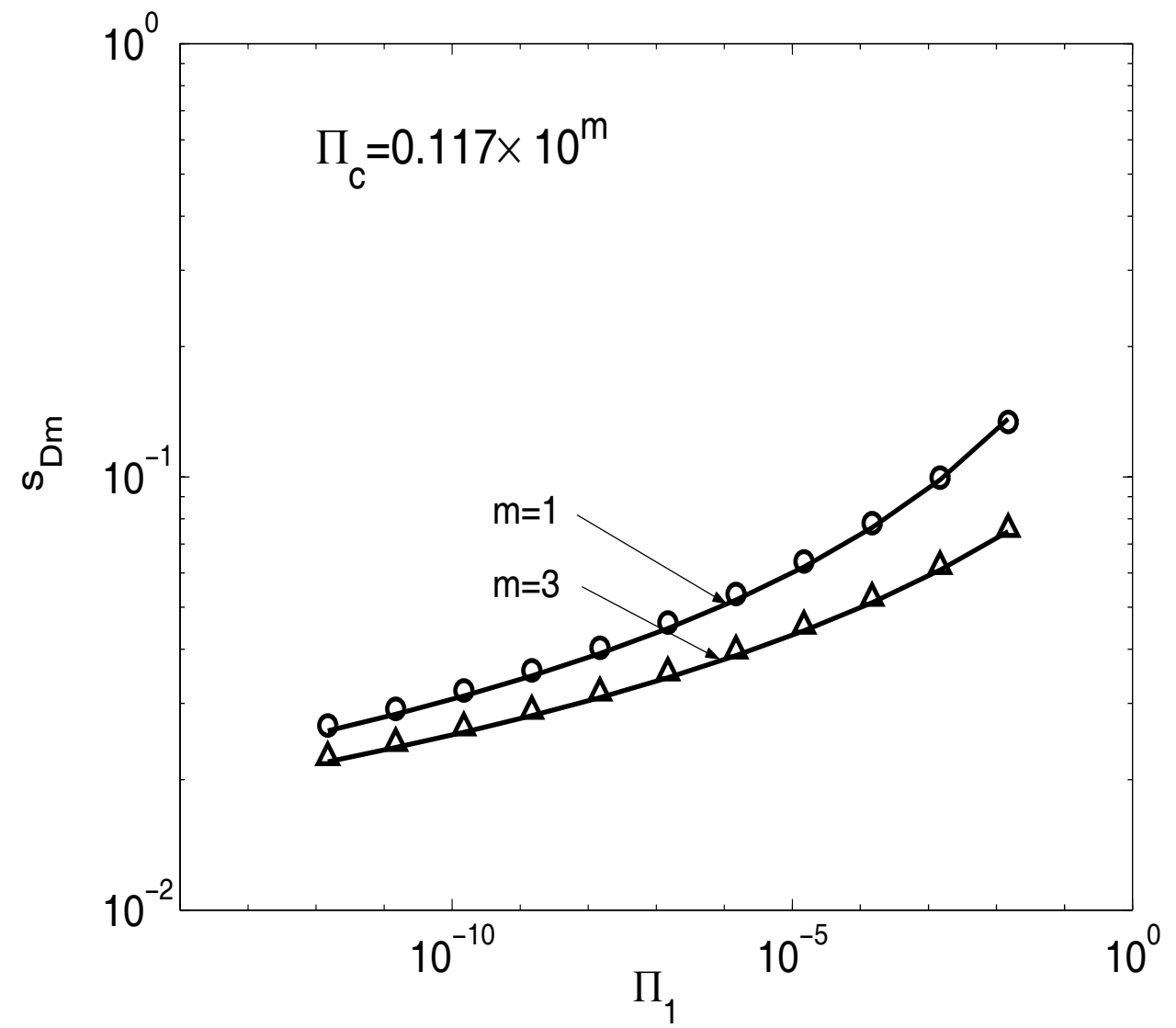

Figure 7: Effect of the parameter $\Pi_{1}$ on the maximum rescaled supersaturation, $s_{D m}$, for $\Pi_{c}=0.117 \times 10^{m}$. Points correspond to the full numerical solution, solid lines correspond to the simpler problem. 


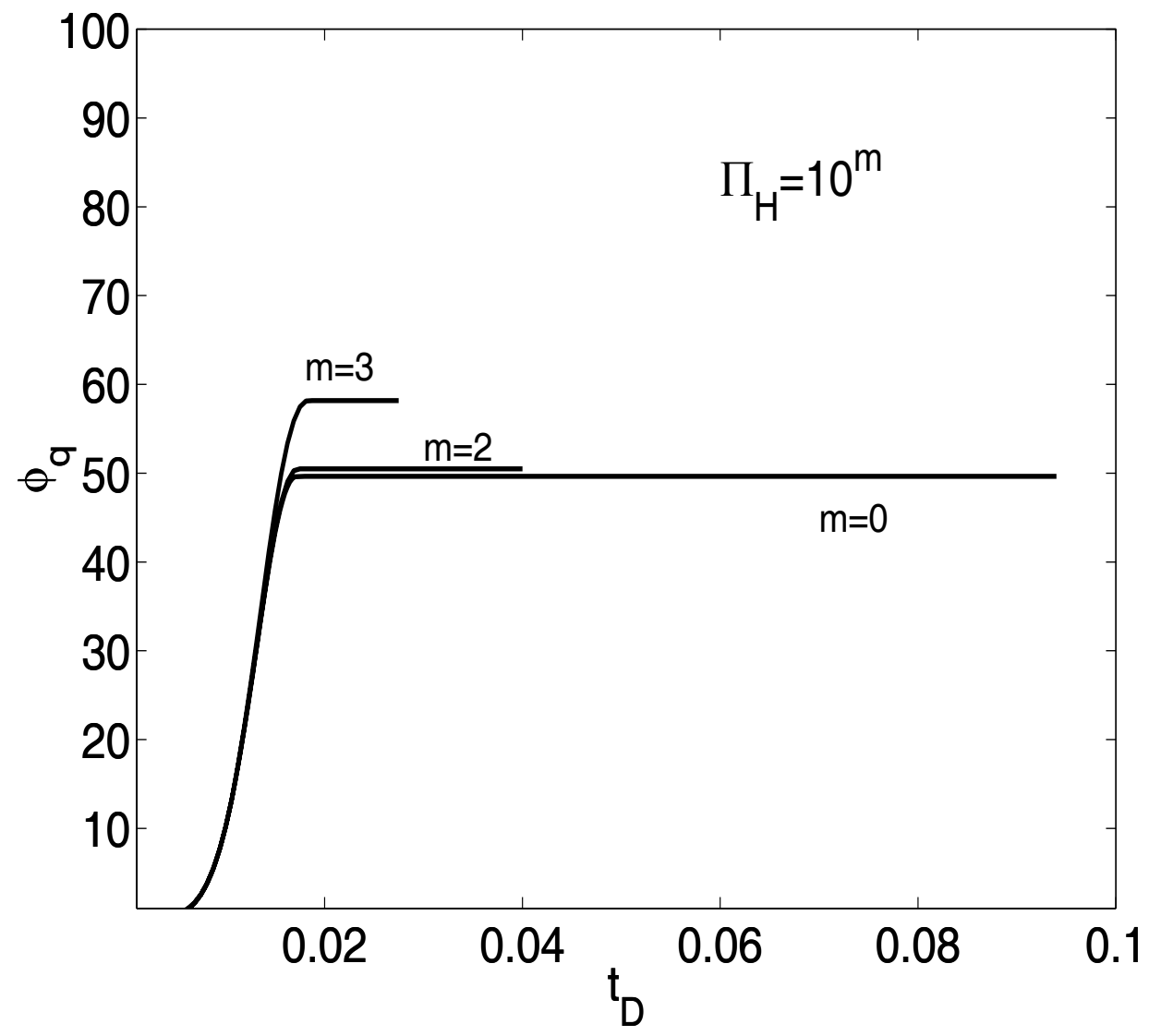

Figure 8: Variation of the rescaled nucleation fraction, $\phi_{q}$, as a function of dimensionless time, $t_{D}$. Effect of $\Pi_{H}=10^{m}$, for $\Pi_{1}=1.485 \times 10^{-8}, \Pi_{2}=9.6972 \times 10^{7}, \Pi_{c}=0.117 \times 10^{1}$ and $\Pi_{\rho}=2.981$. 


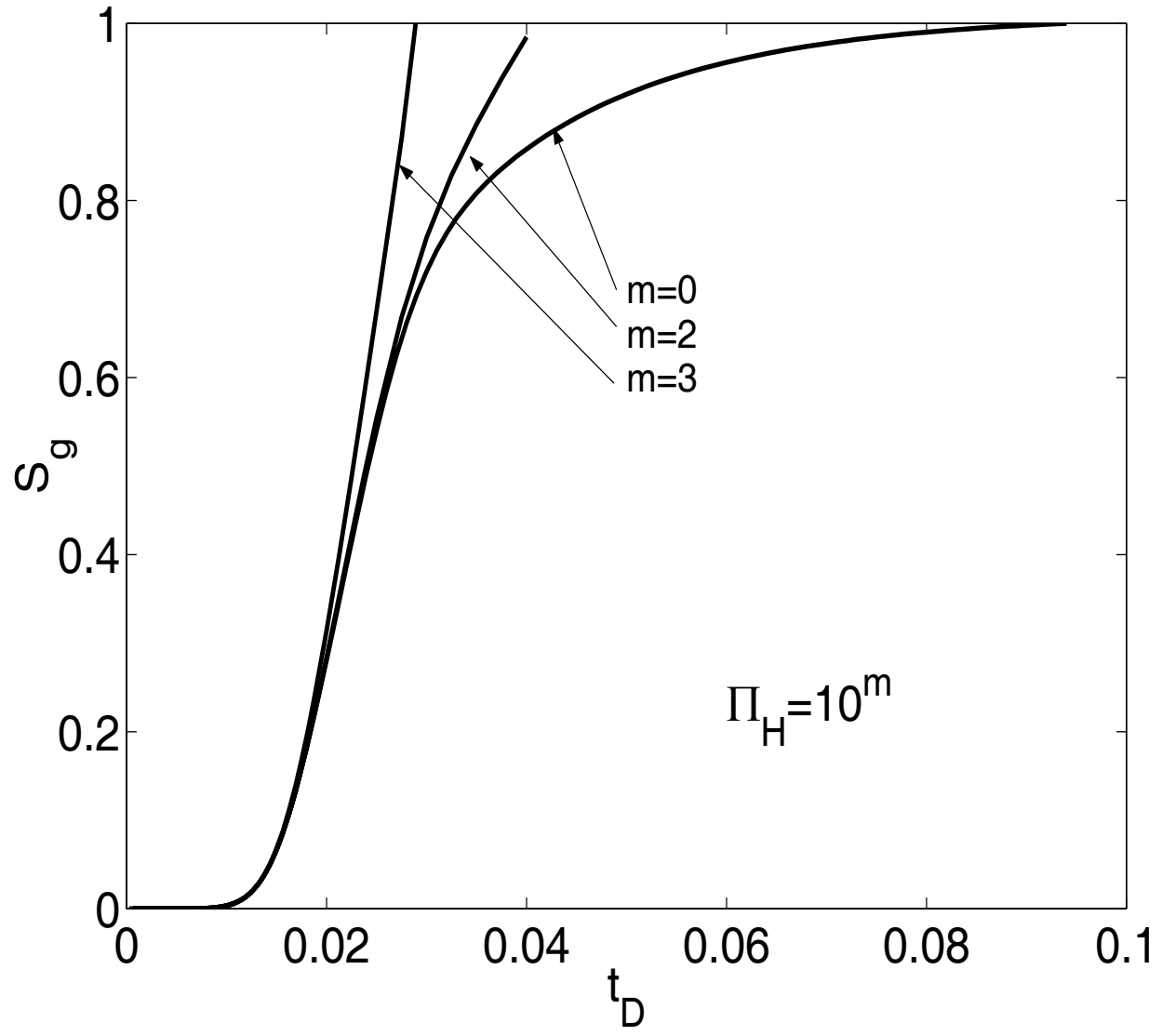

Figure 9: Variation of the gas saturation, $S_{g}$, as a function of dimensionless time, $t_{D}$. Effect of $\Pi_{H}=10^{m}$, for $\Pi_{1}=1.485 \times 10^{-8}, \Pi_{2}=9.6972 \times 10^{7}, \Pi_{c}=0.117 \times 10^{1}$ and $\Pi_{\rho}=2.981$. 


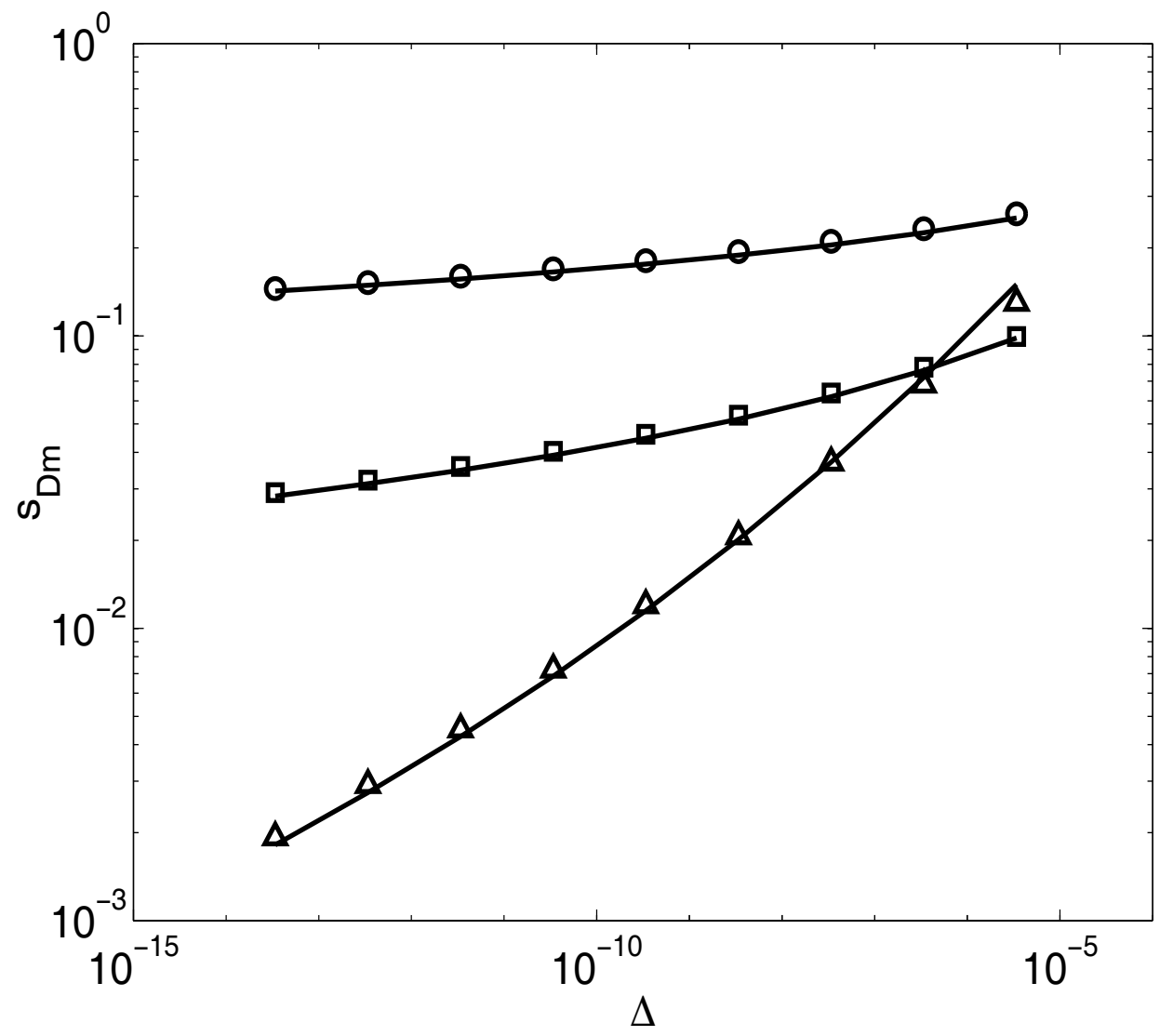

Figure 10: Maximum rescaled supersaturation, $s_{D m}$, as a function of $\Delta$ for various cavity size distributions. Solid lines correspond to the simpler problem, points correspond to the full numerical solution [denoted by circles for the Rayleigh cavity size distribution, by triangles for a stretched exponential $(n=0.2233$ and $\sigma=0.1364)$ and by squares for a stretched exponential $(n=1.0$ and $\sigma=1.0)]$. 


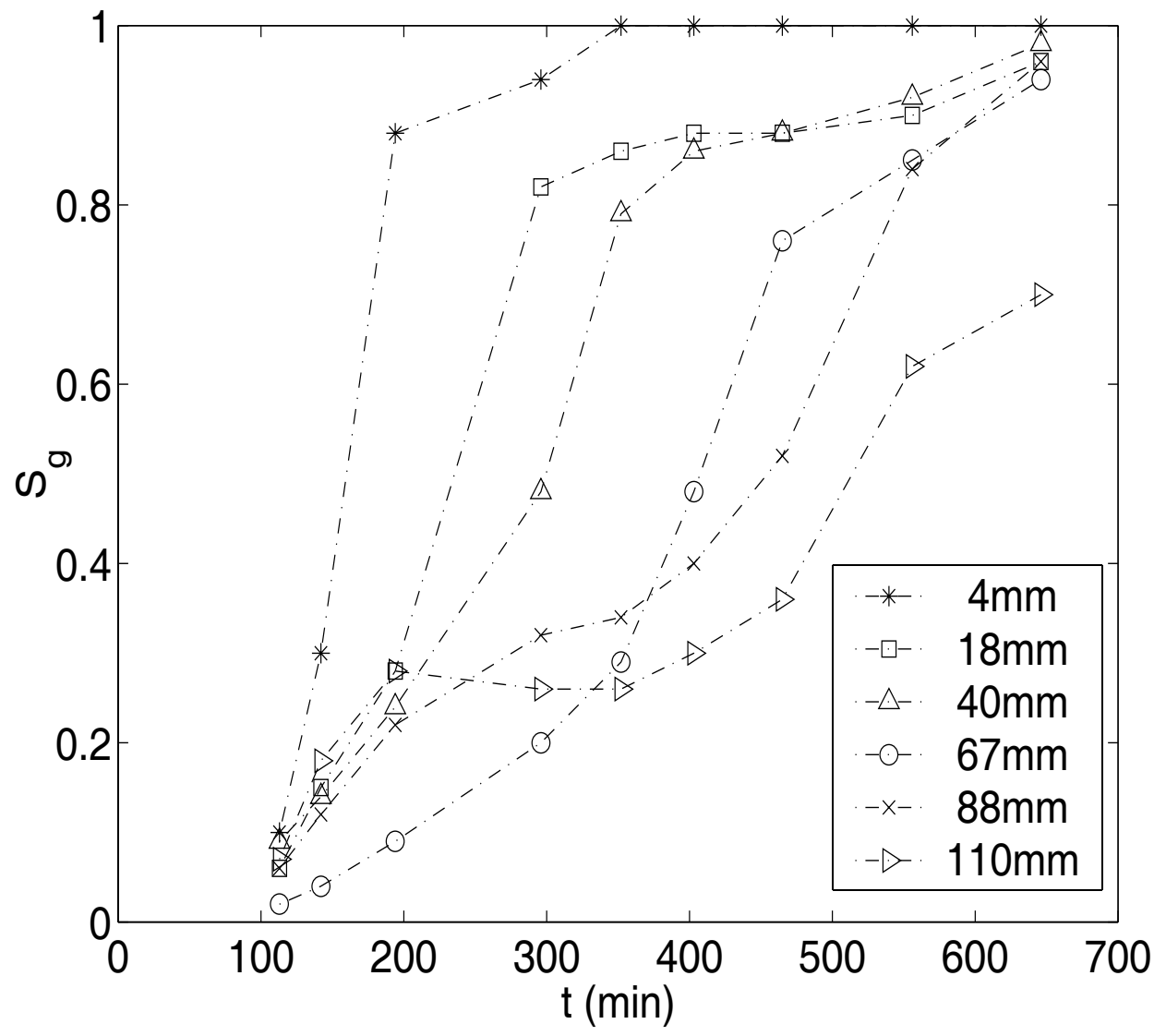

Figure 11: Gas saturation profiles for single-phase flash experiment as a function of time and for various axial positions along the core, L. Experimental data from Dehghani et al., (1997). 


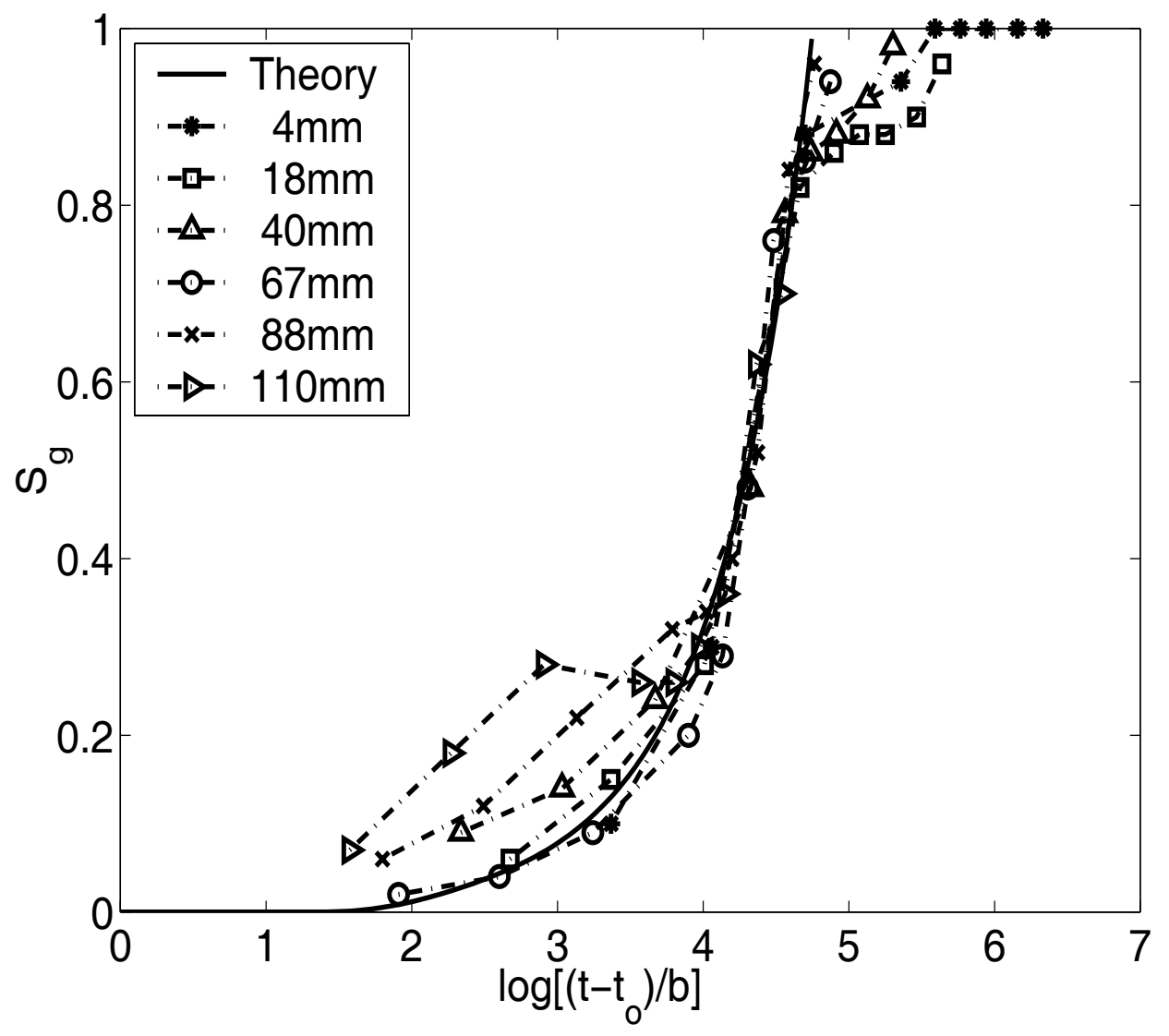

Figure 12: The evolution of the gas saturation as a function of a rescaled time for the experiments of Dehghani et al., (1997). Points denote experimental values and the solid line corresponds to the full numerical solution. 


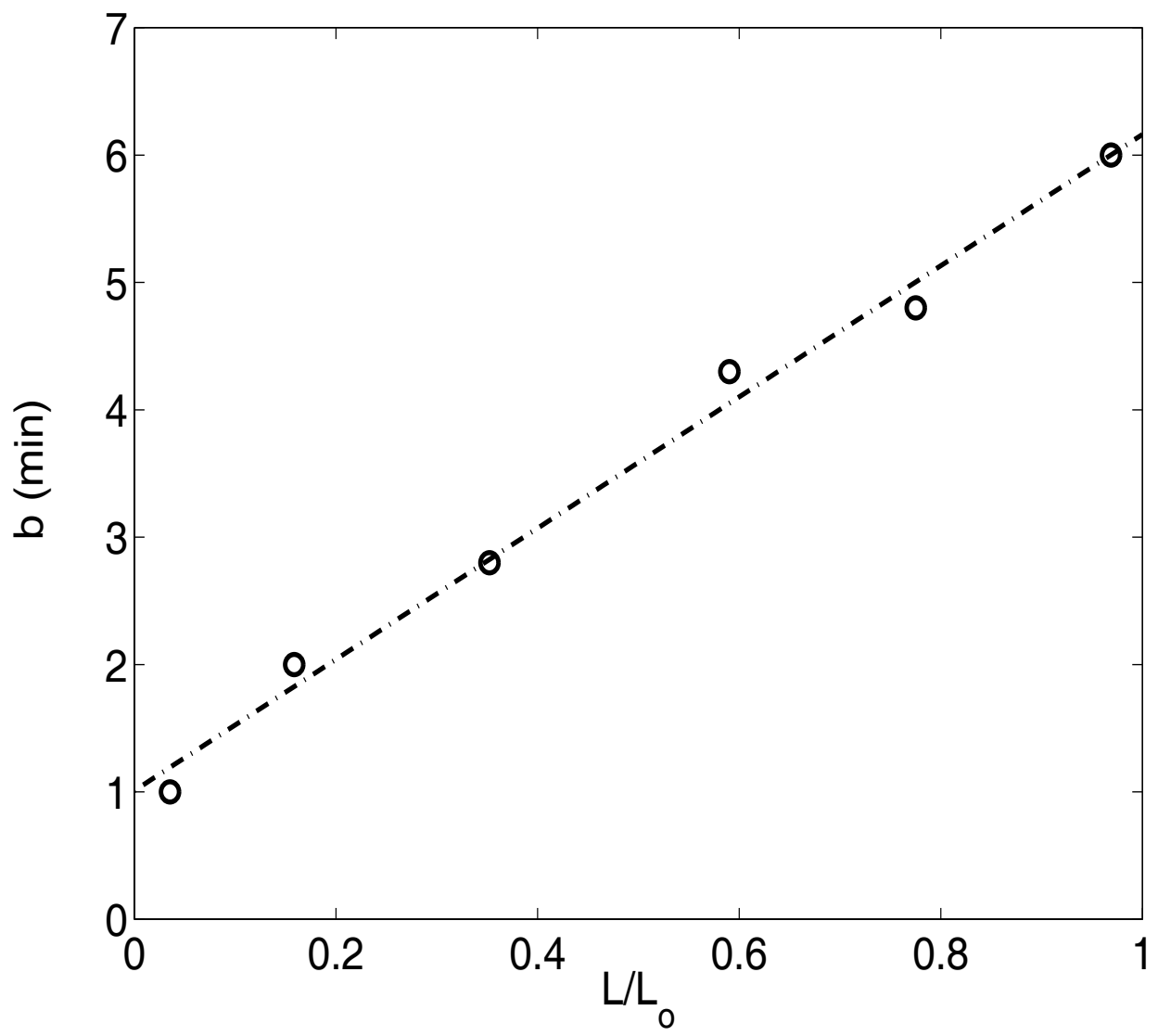

Figure 13: Variation of the rescaling factor, $b$, as a function of the dimensionless axial position along the core, $L / L_{\circ}$. 


\title{
The Critical Gas Saturation in a Porous Medium in the Presence of Gravity
}

\author{
Ioannis N. Tsimpanogiannis and Yannis C. Yortsos
}

\section{INTRODUCTION}

The liquid-to-gas phase change in a porous medium can be described by two distinct, consecutive mechanisms. Gas formation, which is the result of nucleation, and the subsequent gas phase growth, which is the result of mass transfer. Yortsos and Parlar [1] and, subsequently, Li and Yortsos [2] identified these two separate mechanisms and studied them in the context of solution gas-drive. This is a process by which an initially supersaturated liquid releases its dissolved gas by a continuous or abrupt pressure decline. The studies mentioned provided a comprehensive review of nucleation and gas-phase growth.

In the context of solution gas-drive, an important quantity of interest is the critical gas saturation, $S_{g c}$. This denotes the gas saturation (pore-volume fraction) at which the onset of bulk gas flow occurs (Du and Yortsos [3]). Below this value, mostly liquid flows out of the porous medium outlet, the gas remaining pinned in pores by capillarity. At higher gas saturation values, gas is preferentially produced, affecting the liquid-gas flow rate in the produced stream. Although $S_{g c}$ is a parameter of significant importance, ambiguity still exists regarding to its definition, and reported experimental values vary widely. The difficulty was pointed out by Sheng et al. [4], where values as low as $0.5 \%$ and as high as $40 \%$ were reported.

The critical gas saturation can be estimated from production data (Platt and Lewis [5], De Swaan [6]). However, this estimation is not useful for an a priori prediction, and before production data become available. One frequently practiced approach to calculating $S_{g c}$ is based on extrapolating the gas relative permeability curve corresponding to an external gas drive (Chowdiah [7]). The latter is a process in which the gas is externally injected, not generated in-situ as in solution gas-drive. Kamath and Boyer [8], however, have shown that $S_{g c}$ in a depletion experiment, which drives nucleation and gas phase growth, can differ significantly from that determined from an external displacement.

An alternative, self-consistent approach to define $S_{g c}$, and to also obtain robust predictions, is based on visualizations in transparent micromodels and corresponding pore-network simulations. In this context, Yortsos and Parlar[1] and Li and Yortsos [2] defined $S_{g c}$, in the 
absence of gravity or viscous gradients, as the volume fraction when a sample-spanning gas cluster forms for the first time. Clearly, this is related to the nucleation fraction and the percolation properties of the medium. For example, in the case of a single nucleation cluster, sample-spanning would occur when the percolation threshold of the porous medium (or porenetwork) is reached. At that point, the gas cluster coincides with the incipient percolation cluster (and hence occupies a vanishingly small volume fraction, (Feder [9], see also below)).

As long as capillarity dominates the process, and gravity or viscous forces are negligible, a gas cluster remains pinned at the pore throats, until the capillary pressure threshold at the cluster perimeter is exceeded, following which the cluster grows by obeying invasion percolation rules. The gas-liquid interface advances "one-pore-at-a-time", the pore throat to be invaded determined by the least capillary resistance. In the presence of multiple clusters, this rule is obeyed globally among all clusters ("global percolation"), if the pressure decline rate is very slow. Otherwise, it is only locally obeyed in every cluster ("local percolation"), the relative rate by which a cluster grows depending on the mass transfer rates it receives (see Li and Yortsos [10]).

Based on the assumption that viscous or gravity gradients are negligible, Du and Yortsos [3] used pore-network simulations to study the dependence of the critical gas saturation on the geometric parameters of the porous medium. Nucleation centers were distributed at random, simultaneously or sequentially, based on the idea that in solution gas-drive, nucleation from pre-existing cavities is the most plausible mechanism (see also recent review on nucleation by Jones et al. [11]). The growth of such clusters was followed using a variety of percolation mechanisms. The purpose of the study was to test two hypotheses of Yortsos and Parlar [1]: that $S_{g c}$ coincides with the threshold of percolation processes originating from multiple nucleation centers; and that it follows a power-law scaling with the nucleation fraction, $f_{q}=\frac{N_{a}}{L^{E}}$, as follows

$$
S_{g c} \approx f_{q}^{1-D / E}
$$

Here, $N_{a}$ denotes the number of sites that host active nucleation cavities, $L$ is the (dimensionless) lattice size, $E$ is the Euclidean dimension (equal to 2 or 3 ), and $D$ is the mass fractal dimension of the percolation cluster (equal to 1.89 for 2-D ordinary percolation (OP), to 1.82 
for 2-D invasion percolation (IP) with trapping, and to 2.53 for 3 -D invasion percolation (IP) with or without trapping (Feder [9])). Both conjectures were found to be well satisfied. In particular, the dependence (1) was established regardless of the nucleation sequence (instantaneous or sequential) or the particular regime of bubble growth (global or local percolation, with or without dominant mass transfer characteristics). Based on these findings, Tsimpanogiannis and Yortsos [12] subsequently developed an effective continuum model in the absence of gradients, which utilizes (1) in order to obtain the dependence of $S_{g c}$ on other important characteristics, such as the pressure decline rate.

Related studies of the general problem have also included the following: Li and Yortsos $[2,10]$ conducted glass micromodel visualization experiments, coupled with pore-network simulations, to develop insight on the growth of multiple bubbles evolving from a variety of nucleation centers. Their simulations included a number of relevant processes, such as nucleation, phase equilibria at interfaces, mass transfer, and capillary and viscous forces. Hawes et al. [13] developed a pore-network model to reproduce experimental observations considering the depressurization of water-flooded reservoirs. A simplified 3-D, multi-bubble growth model was developed, which simulated the development and mobilization of the gas phase. The effects of grid size, depressurization rate, residual oil saturation, and interfacial tension on the critical gas saturation were studied, in the absence of mass transfer control. McDougall and Sorbie [14] developed a 3-D, three-phase numerical pore-network simulator which included aspects such as nucleation, diffusion, bubble growth, migration and fragmentation, resulting from buoyancy forces. They also considered the variation of the gas/oil interfacial tension with pressure and proposed various correlations for $S_{g c}$ as a function of the gravity Bond number and other parameters.

Despite the above-cited studies, the problem where gravity or viscous forces are important has not been thoroughly analyzed, however. In such cases, additional phenomena, such as cluster migration, coalescence, and the onset of mobilization, take place prior to the onset of the sample-spanning cluster. It is the purpose of this paper to focus on these issues, specifically on the effect of gravity forces. Viscous effects require the computation of the flow field around a number of trapped or migrating ganglia and it is a more complex problem. It is examined in a separate study by Pouya and Yortsos [15], using a Darcian Dynamics 
approach, in which effects of crowding, the orientation of the ganglia, and the rates of mobilization and coalescence are extensively discussed.

When gravity (or viscous) forces overcome capillary thresholds, migration and mobilization of a gas cluster is possible. In such a case, the definition of $S_{g c}$ needs to be modified and be described as the saturation at which mobilization occurs for the first time. Under these conditions, $S_{g c}$ is expected to depend on the gravity Bond number, $B$, or on the capillary number, $C a$, in the respective cases, where

$$
B=\frac{\Delta \rho g k}{\gamma} \text { or } \quad C a=\frac{q \mu}{\gamma}
$$

Here $\Delta \rho=\rho_{w}-\rho_{n w}$ denotes the density difference between the liquid (w) and gas (nw), $k$ is permeability, $\gamma$ the liquid-gas interfacial tension, $q$ the flowing liquid velocity and $\mu$ is the liquid viscosity. Emphasis in this work will be on the effect of $B$.

The paper is organized as follows: First, we introduce a brief description of the physical problem. Then, we describe the aspects of the pore-network simulation, which is based on Invasion Percolation in a Gradient (IPG) (Wilkinson and Willemsen [16], Sapoval et al. [17], Hulin et al. [18], Gouyet et al. [19], Birovljev et al. [20], Chaouche et al. [21]). Subsequently, we analyze the numerical results, develop relations that govern the dependence of the various parameters of interest and provide the relevant discussion.

\section{DESCRIPTION OF THE PHYSICAL PROBLEM}

Consider the liquid expansion of an initially supersaturated liquid from a porous medium of a fixed volume due to pressure decline. As the pressure continuously decreases, the bubble-point of the liquid is eventually reached (Tsimpanogiannis and Yortsos [12]). Then, nucleation of the gas phase starts. Nucleation can be the result of the activation of a cavity containing pre-existing gas bubbles, or the result of heterogeneous nucleation. The activation of a cavity is defined here as the condition at which a pre-existing gas bubble, trapped in a cavity, overcomes the capillary threshold of the mouth of the cavity and migrates to the hosting site. The bubble initially grows, while still confined within a single pore. As time progresses, the bubble invades adjacent pores, and its growth ultimately takes the form of a (ramified) gas cluster occupying a number of pores. As noted, these patterns are consistent 
with visual observations from micromodel experiments ( $\mathrm{Li}$ and Yortsos [2], El Yousfi et al. [22], Bora et al. [23]). The evolution of the gas phase is affected, among other parameters, by capillary, viscous and gravity forces, as well as by the migration, fragmentation and coalescence events between the different gas clusters. Eventually, gas flows out of the system as a bulk phase.

Porous media are now routinely represented as lattices (networks) of pores. To simulate our problem, a pore-network representation will also be assumed in the form of a regular square lattice of size $N \times N$. Initially, all the lattice sites are filled with the defending phase (wetting liquid). We distribute nucleation sites in the form of cavities within sites. Each site contains one cavity of a random size, unrelated to the site size. During the nucleation process we "activate" cavities of size larger than a predetermined threshold value. Alternatively, one can specify the nucleation fraction, $f_{q}$, and occupy a corresponding number of sites by gas (non-wetting invading phase) at random. In the simulations to be reported, no bias due to gravity was included in the nucleation site distribution.

As pointed out, gas-phase growth from multiple centers and in the absence of gradients was simulated by Du and Yortsos [3] using Invasion Percolation. In the presence of gravity (buoyancy) gradients, however, we must consider, instead, IPG. Here, in addition to nucleation, the following processes must be taken into consideration: growth of a gas cluster, cluster coalescence, and the onset of migration of a gas cluster. If a cluster is not mobilized, it grows by occupying the perimeter site with the least effective threshold

$$
\pi_{j}=\tau_{j}-B z_{j}
$$

where $z_{j}$ is the elevation of site $j$. The invasion thresholds $\tau$ were randomly assigned to sites from a uniform distribution on the unit interval. In all cases, periodic boundary conditions at the two lateral sizes are used. Because $B>0$ the gas phase will tend to grow by invading sites with larger $z$. This results in favoring sites further advanced in the direction parallel to the gravity vector, and will eventually produce invasion patterns consistent with Invasion Percolation in a Destabilizing Gradient (IPDG) (Xu et al. [26]). Trapping rules (Wilkinson and Willemsen [16], Dias and Wilkinson [27]) were also included. During a growth step, coalescence of clusters is possible. At each stage of the simulation a record of 
the number of clusters in the system is kept, using a Hoshen-Kopelman algorithm (Hoshen and Kopelman [24], Hoshen et al. [25]). Implicit to the above is that mass transfer is not the rate-determining step and that each cluster grows at a rate independent of mass transfer.

Before a growth step, every individual cluster is examined to check if it satisfies a migration condition. For this calculation, each site $i$ was also assigned a withdrawal threshold, $w_{i}$. While threshold $\tau_{j}$ represents the capillary resistance that must be overcome for site $j$ to be invaded, $w_{i}$ represents the threshold for the non-wetting fluid to withdraw from site $i$ (Wagner et al. [28]). Considering a single withdrawal threshold represents an oversimplification of the real process. One can incorporate dditional mechanisms (Wagner et al. [28], Lenormand et al. [29], see also related experimental work and simulations by Wagner et al. [30], Birovljev et al. [31], Vedvik et al. [32] and Meakin et al. [33]).

For a migration step to occur, the non-wetting fluid must invade site $j$ and at the same time withdraw from site $i$. Figure 1 depicts a schematic of this process. Without loss of generality we assume that the volume of all sites is the same, thus volume is conserved during migration. Cluster migration (hence mobilization) requires that there is a pair of sites $i$ and $j$ at the cluster perimeter, for which the inequality

$$
\Pi_{j i} \equiv \tau_{j}+w_{i}-B l_{j i} \leq 0
$$

is satisfied the first time. Here, $l_{j i}=z_{j}-z_{i}>0$ is the difference in elevation $z$ of the site pair considered. For each gas cluster, all possible combinations of sites (satisfying $z_{j}>z_{i}$ ) are examined. If $\Pi_{i j}<0$, mobilization occurs and the corresponding gas saturation at that point is recorded as $S_{g c}$. We also consider that the system has reached the critical gas saturation when the non-wetting cluster reaches the top of the lattice. However, to avoid artificial early-time arrivals of clusters at the top of the lattice, due to finite size effects, a zone near the top of the lattice (of size sufficiently larger than the mean nucleation center distance) was excluded from the nucleation process.

\section{RESULTS}

Figure 2 shows typical patterns of the growth of the gas phase, for the case where mobilization occurs before any significant coalescence takes place $\left(B=5.0 \times 10^{-3}\right)$. The case 
involving a lower Bond number $\left(B=10^{-4}\right)$, where the critical gas saturation coincides with the formation of a "sample-spanning" cluster, is depicted in Figure 3. Both figures were obtained using a $100 \times 100$ lattice with a number of $N_{a}=10$ clusters activated. In general, we find that the mobilization of a cluster requires a value of the Bond number in the order of $10^{-3}$ or larger (see also below), for non-negligible nucleation fractions. This is also consistent with the range for the critical capillary number for the mobilization of stranded ganglia, found in Amili and Yortsos [15]. For smaller values, gas clusters grow, coalesce, but are not mobilized until they form a sample-spanning cluster.

To illustrate the importance of coalescence, Figure 4 depicts the normalized number of clusters, $N_{c} / N_{a}$ (current number of clusters present in the lattice divided by the initial number of clusters activated), as a function of $S_{g}$ for the same conditions, but in the absence of gradients $(B=0)$. Three different nucleation fractions, corresponding to $N_{a}=4,10,40$, are considered. The point when a "sample-spanning" cluster is formed is shown. Coalescence occurs systematically and extensively. Initially, the fraction of clusters decreases linearly with the total volume of the clusters. As the percolation threshold is approached, however, the rate of coalescence slows down. As anticipated, the smaller the nucleation fraction, $f_{q} \equiv \frac{N_{a}}{L^{E}}$, the smaller the effect of coalescence.

In the following, we will report the variation with the gravity Bond number, $B$, of the following quantities, at the onset of the critical gas saturation: the average mass (number of sites occupied), $M_{c}$, of a cluster at incipient mobilization, the average projection length, $l_{c}$, of the same cluster along the direction of gravity, the critical gas saturation, $S_{g c}$, and the normalized number of clusters, $N_{f} / N_{a}$. The results shown are averages over a large number of different realizations. Up to 1000 realizations were averaged for cases with $B>10^{-2}$, up to 500 realizations for cases with $10^{-3} \leq B \leq 10^{-2}$, and up to 250 realizations for $B<10^{-3}$. Due to the considerable amount of time required for these computations, the figures presented were obtained using $40 \times 40$ lattices, hence they are subject to some finite-size effects.

Figure 5 shows the average mass of the gas cluster, $M_{c}$, at incipient mobilization, as a function of the Bond number and for four different nucleation fractions, corresponding to $N_{a}=1,2,5,10$. For values below approximately $10^{-4}$, the mass is almost constant, for a given nucleation fraction, increasing with the nucleation fraction as predicted by (1) (and 
where one should also keep in mind the finite-size effects). For larger values of $B$, the value of $M_{c}$ decreases as a power-law of $B$, which to a good approximation has an exponent of about -0.91 . Ultimately, $M_{c}$ asymptotes to the value of 1 , which is the high- $B$ limit. The corresponding projected length, $l_{c}$, of the incipient mobilization cluster is shown in Figure 6 , for the same nucleation fractions. The behavior is similar to that for $M_{c}$ : There are two plateaus at low and high $B$, followed by a power-law behavior in the in-between interval. The approximate exponent of the latter is -0.44 . As indicated previously, for the lower Bond numbers the critical gas saturation is obtained after considerable coalescence. The approach of the average mass and the average projection length of the mobilized cluster towards an asymptotic value, at low Bond numbers, is simply the result of finite size effects, since the cluster eventually spans the lattice before getting mobilized. Using a larger lattice size will result in shifting the Bond number, where the asymptotic value is obtained, to lower values. The simulations at lower $B$ in larger lattices require significantly higher computational cost, however.

Figure 7 shows the normalized number of clusters, $N_{f} / N_{a}$ at the onset of $S_{g c}$, as a function of the Bond number and for the same nucleation fractions as in the previous two figures. It is clear that this ratio depends on both the nucleation fraction and the Bond number. At high values of $B$, the ratio of clusters approaches 1 , as mobilization occurs almost immediately following nucleation, and before any coalescence has occured. As the Bond number decreases, coalescence becomes important. At sufficiently low $B$, again corresponding to a range of about $10^{-4}$, the curves approach the limiting value $1 / N_{a}$, where only the "sample spanning" cluster is present when the critical gas saturation is reached. The critical gas saturation is plotted in Figure 8 as a function of the Bond number and for the different cases considered earlier. For a fixed lattice size, the plot has the same three regimes described above:

(i) A low- $B$ plateau, where $S_{g c}$ is only dependent on the nucleation fraction. This regime is the one reported by Du and Yortsos [3], and corresponds to (1). Note, that in 2-D the exponent of (1) is very weak, equal to 0.09 .

(ii) A high- $B$ plateau, where

$$
S_{g c}=f_{q}
$$

In this regime, once a site is activated, it becomes mobilized right away, before it has any 
chance to grow further.

(iii) A power-law, in-between regime, with an apparent exponent of about -0.91 . This regime corresponds also to the range where no significant coalescence occurs. Once coalescence becomes important, the slope changes continuously until the value corresponding to the lower asymptotic regime is obtained.

\section{DISCUSSION}

To analyze the results obtained, we will make use of concepts from Invasion Percolation in a Gradient (IPG). As Invasion Percolation (Wilkinson and Willemsen [16]) has contributed significantly to our understanding of patterns during the slow immiscible displacement of a wetting fluid by a non-wetting fluid in a porous medium and in the absence of gradients. Such a process is controlled by capillary forces only. When gravity or viscous forces are important, percolation patterns do not develop over the entire region of displacement, however. Rather, the resulting patterns are described by IPG.

When the gravity gradient is destabilizing (e.g. in the upward displacement of a liquid phase by gas in a gravity field), Meakin et al. [35] showed that the resulting pattern is a single "finger", made up of a collection of fractal blobs, that retain the structure of an invasion percolation cluster at short length scales. The typical blob has a more or less isotropic structure, since its growth is controlled only by capillary forces. Each of the blobs has a characteristic size equal to the correlation length of IPG, $\xi$, given by (Meakin et al. [35], Wilkinson $[37,38])$

$$
\xi \sim B^{-\frac{\nu}{\nu+1}}
$$

Here, $\nu$ is the correlation length exponent of percolation $(\nu=4 / 3$ for $2-\mathrm{D}$ invasion percolation with trapping and $\nu \simeq 0.88$ for 3 -D invasion percolation with or without trapping). It follows that the exponent in (6) is equal to 0.57 for $2-\mathrm{D}$ and 0.47 for 3 -D. The corresponding mass of the blobs (number of occupied sites), $s$, is then given by

$$
s \sim \xi^{D}
$$

where $D$ is the mass fractal dimension in percolation. 
To find the mass of the cluster when mobilization occurs for the first time, we need first to determine the projected length $l_{c}$, and subsequently proceed by dividing the cluster into $l_{c} / \xi$ segments (blobs) of size $\xi$ and mass $s$. Consider the determination of $l_{c}$. When the length of the cluster, $l$, is such that capillary forces dominate over buoyancy $\left(l \ll l_{c}\right)$, the cluster will remain pinned by capillarity. However, at some point and as the cluster grows, it will reach a critical size, $l_{c}$, at which buoyancy overcomes capillarity. From equation [4] this happens when $\Pi_{i j} \leq 0$ for the first time. The corresponding length between sites is then

$$
l_{i j, c}=\frac{\tau_{j, c}+w_{i, c}}{B}
$$

At that point mobilization of the cluster occurs. At first glance, one might assume that at the onset of mobilization, the ensemble-averaged value of the critical length, $l_{c}$, is inversely proportional to $B$, at least in the in-between regime. However, our pore-network simulations show that this is not true. Instead, we obtain the different scaling

$$
l_{c} \sim B^{-0.44}
$$

as shown in the simulations of Figure 6. The reason for this is due to the gradient percolation rules used for the clusters during the growth stage. Because of the bias of gravity, newly invaded sites do not necessarily correspond to the smallest available threshold, $\tau$. Rather, thresholds of larger values are possible.

Using (9), we can determine the mass of the cluster, $M_{c}$, when mobilization occurs for the first time. Then,

$$
M_{c} \sim \frac{s l_{c}}{\xi} \sim l_{c} \xi^{D-1} \sim B^{-z}
$$

where

$$
z=0.44+(D-1) \frac{\nu}{\nu+1}
$$

Substitution of the $2-\mathrm{D}$ values for percolation gives $z=0.91$ for $2-\mathrm{D}$. We find that this prediction is satisfied quite well in the plot of Figure 5.

The above analysis was for a single cluster that does not interact with any others. This is the case at relatively small nucleation fractions and relatively large $B$, when coalescence 
of clusters does not occur. In such cases, the total mass, $M_{\text {total }}$, is simply the sum over all initial clusters, $N_{a}$

$$
M_{\text {total }}=\sum_{i=1}^{N_{a}} M_{i} \approx N_{a} M_{c}
$$

Then, the total gas saturation (the number of gas-occupied sites divided by the number of total lattice sites), $S_{g}$, is

$$
S_{g}=\frac{S_{\text {total }}}{L^{E}}=\frac{N_{a}}{L^{E}} M_{c}=f_{q} M_{c}
$$

Thus, in the absence of any coalescence (in the in-between region), we expect the scaling

$$
S_{g c} \sim f_{q} B^{-z}
$$

Figure 8 shows that (14) is indeed satisfied in the appropriate regime, specifically when the nucleation fraction is low, or the Bond number high, so that gas clusters are mobilized before they start interacting with each other. As higher values of the nucleation fraction or for lower values of the Bond number, coalescence of clusters occurs and has to be taken into account in the calculation of the total mass of the gas phase and the gas saturation.

When coalescence occurs the number of clusters in the system at any time is less than the originally activated, $N_{a}$. The total mass will be given, now, by

$$
M_{\text {total }}=\sum_{i=1}^{N_{c}} S_{i} \approx N_{c} M_{c}
$$

In particular, at the onset of $S_{g c}$, we will have

$$
S_{g c} \approx \frac{N_{f}}{N_{a}} f_{q} M_{c} \sim f_{f}\left(B, f_{q}\right) \cdot B^{-z}
$$

where we defined $f_{f}\left(B, f_{q}\right)=\frac{N_{f}}{N_{a}} f_{q}$. It is shown in Figure 7 , that the fraction $\frac{N_{f}}{N_{a}}$ is indeed a function of both $B$ and $f_{q}$. In this regime, the power-law dependence of $S_{g c}$ is modified by the continuous coalescence. This is confirmed in the results shown in Figure 8.

\section{CONCLUSIONS}

In this paper, we used pore-network simulations and concepts from Invasion Percolation in a Gradient to study the effect of gravity on the critical gas saturation in a porous medium. 
The latter denotes the volume fraction of the gas phase at the onset of bulk gas flow, during the depressurization of a supersaturated liquid in a porous medium. In the absence of viscous or gravity gradients, $S_{g c}$ is controlled by nucleation, capillary forces and the rate of decline of the supersaturation. Here we addressed the additional effect of buoyancy. We used 2-D porenetwork simulations and developed corresponding scaling relations, to obtain the dependence of $S_{g c}$ on the gravity Bond number, $B$, under conditions of slow growth, namely when mass transfer is sufficiently fast. In particular, we focused on the average mass, and the average projection length, of the clusters at incipient mobilization, and/or when the critical gas saturation of the system is reached. In general, the results show that for all these properties there exist three regimes, in the case of finite-size lattices: two plateaus at high and low values of the Bond number, corresponding, respectively, to instant mobilization after nucleation, and the complete absence of mobilization, and an in-between regime of intermediate Bond numbers, where power-law scalings are observed. In particular, the critical gas saturation scales $S_{g c} \sim B^{-0.91}$, in 2-D lattices. The value of this exponent derives from the result that in the same regime, the projected length of an cluster at incipient mobilization scales with $B$ as $l_{c} \sim B^{-0.44}$, rather than as $l_{c} \sim B^{-1}$.

\section{References}

[1] Yortsos, Y.C., and Parlar, M.,Paper SPE 19697 (1989).

[2] Li, X., and Yortsos, Y.C., AICHE J. 41, 214 (1995).

[3] Du, C., and Yortsos, Y.C., Trans. Porous Media 35, 205 (1999).

[4] Sheng, J.J., Maini, B.B., Hayes, R.E., and Tortike, W.S., Trans. Porous Media 35, $157(1999)$.

[5] Platt, C.R., and Lewis, W.M., J. Pet. Tech. 22, 1507 (1969).

[6] De Swaan, A., J. Pet. Tech. 33, 907 (1981).

[7] Chowdiah, P., Paper SPE 16945 (1987). 
[8] Kamath, J., and Boyer, R.E., SPE Form. Eva. 10, 247 (1995).

[9] Feder, J., "Fractals." Plenum, New York, 1988.

[10] Li, X., and Yortsos, Y.C., Chem. Eng. Sci. 50, 1247 (1995).

[11] Jones, S.F., Evans, G.M., and Galvin, K.P., Adv. Colloid Interface Sci. 80, 27 (1999).

[12] Tsimpanogiannis, I.N., and Yortsos, Y.C., AICHE J. 48, 2690 (2002).

[13] Hawes, R.I., Dawe, R.A., and Evans, R.N., Trans. Inst. Chem. Eng. 74, 197 (1996).

[14] Mc Dougall, S.R., and Sorbie, K.S., Petroleum Geoscience 5, 229 (1999).

[15] Pouya, A., and Yortsos, Y.C., Phys. Fluids, submitted.

[16] Wilkinson, D., and Willemsen, J.F., J. Phys. A 16, 3365 (1983).

[17] Sapoval, M., Rosso, M., and Gouyet, J.F., J. Phys. II (France) 46, L149 (1985).

[18] Hulin, J.P., Clement, E., and Baudet, C., Phys. Rev. Lett. 61, 333 (1988).

[19] Gouyet, J.F., Rosso, M. and Sapoval, B., Phys. Rev. B 37, $1832(1988)$.

[20] Birovljev, A., Furuberg, L., Feder, J., Jossang, T., Maloy, K.J., and Aharany, A., Phys. Rev. Lett. 67, 584 (1991).

[21] Chaouche, M., Rakotomalala, N., Salin, D., Xu, B., and Yortsos, Y.C., Phys. Rev. E 49, 4133 (1994).

[22] El Yousfi, A., Zarcone, C., Bories, S., and Lenormand, R., C.R. Acad. Sci. Paris, Série II 313, 1093 (1991).

[23] Bora, R., Maini, B.B., and Chakma, A., SPE Res. Eval. Eng. 3, 224 (2000).

[24] Hoshen, J., and Kopelman, R., Phys. Rev. B 14, 3438 (1976).

[25] Hoshen, J., Berry, N.W., and Minser, K.S., Phys. Rev. E 56, 1455 (1997). 
[26] Xu, B., Yortsos, Y.C., and Salin, D., Phys. Rev. E 57739 (1998).

[27] Dias, M.M., and Wilkinson, D., J. Phys. A: Math. Gen. 19, 3131 (1986).

[28] Wagner, G., Birovljev, A., Meakin, P., Feder, J., and Jossang, T., Phys. Rev. E 55, 7015 (1997).

[29] Lenormand, R., Zarcone, C., and Sarr, A., J. Fluid Mech. 135, 337 (1983).

[30] Wagner, G., Birovljev, A., Meakin, P., Feder, J., and Jossang, T., Europhys. Lett. 31, $139(1995)$.

[31] Birovljev, A., Wagner, G., Meakin, P., Feder, J., and Jossang, T., Phys. Rev. E 51, $5911(1995)$.

[32] Vedvik, A., Wagner, G., Oxaal, U., Feder, J., Meakin, P., and Jossang, T., Phys. Rev. Lett. 80, 3065 (1998).

[33] Meakin, P., Wagner, G., Vedvik, A., Amundsen, H., Feder, J., and Jossang, T., Marine Petroleum Geology 17, 777 (2000).

[34] Voss, R.F., J. Phys. A: Math. Gen. 17, L373 (1984).

[35] Meakin, P., Feder, J., Frette, V., and Jossang, T., Phys. Rev. A 46, 3357 (1992).

[36] Meakin, P., Wagner, G., Feder, J., and Jossang, T., Physica A 200, 241 (1993).

[37] Wilkinson, D., Phys. Rev. A 30, 520 (1984).

[38] Wilkinson, D., Phys. Rev. A 34, 1380 (1986). 


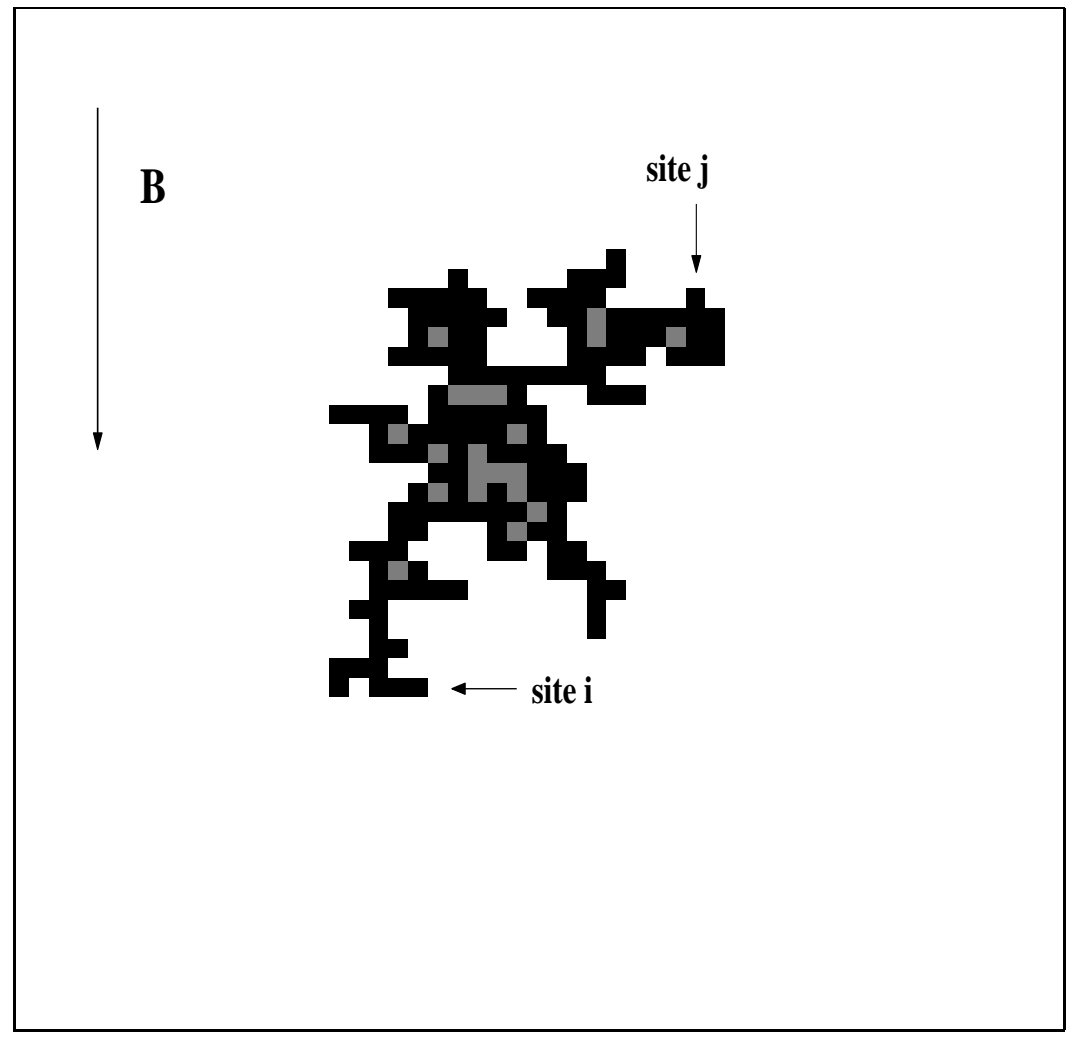

Figure 1: Schematic of a single cluster growing in a $100 \times 100$ lattice with $B=10^{-3}$. (Depicted, here, is the central area of size $50 \times 50$.) Gas-occupied sites are shown in black, liquid-occupied sites are shown in white, trapped-liquid sites are shown in gray. 
(a)

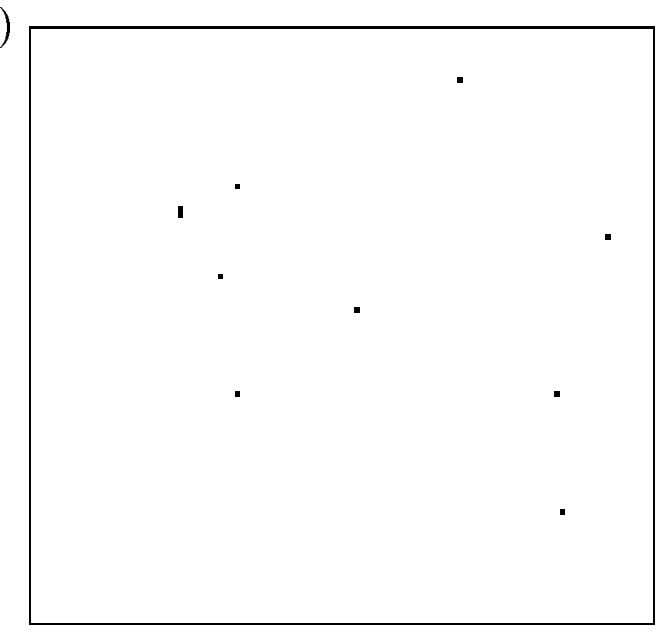

(c)

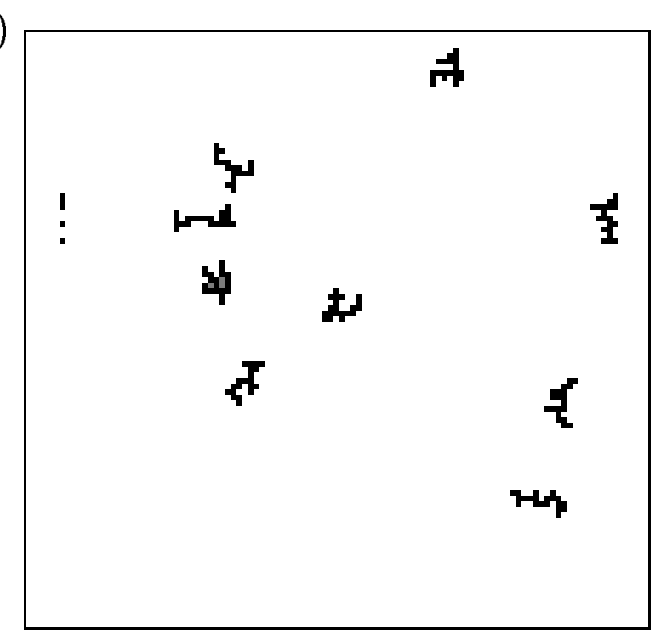

(b)

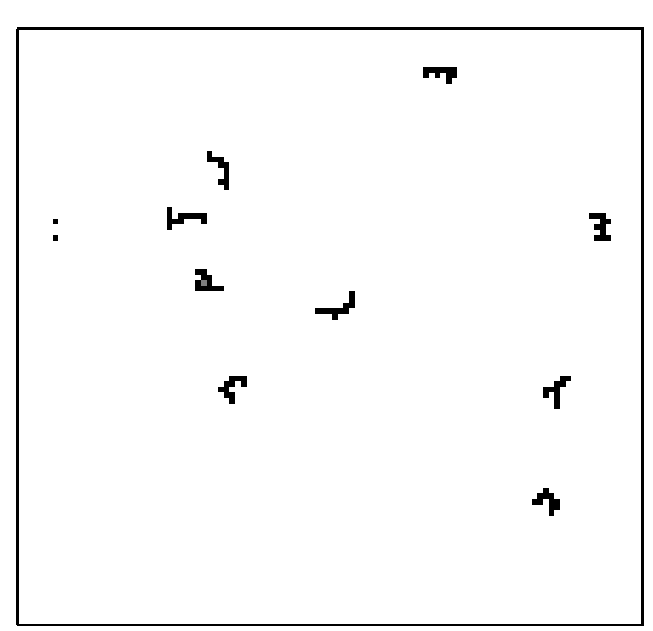

(d)

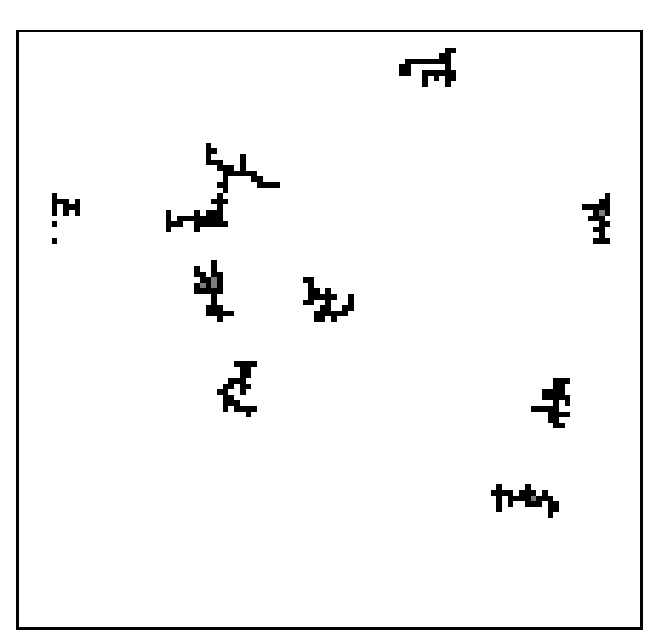

Figure 2: A sequence of four gas-growth patterns in a $100 \times 100$ lattice with $B=5.0 \times 10^{-3}$ and $N_{a}=10$. The number of clusters present, $N_{c}$, are respectively: (a) $10 ;$ (b) $9 ;$ (c) $9 ;$ (d) 9. Periodic boundary conditions are used at the lateral boundaries, and the color notation of Figure 1 applies. The last panel corresponds to the onset of $S_{g c}$. 
(a)

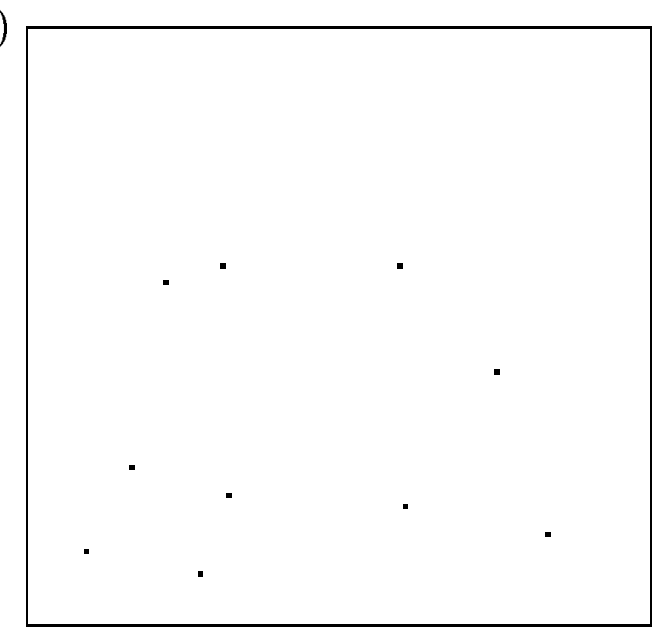

(c)

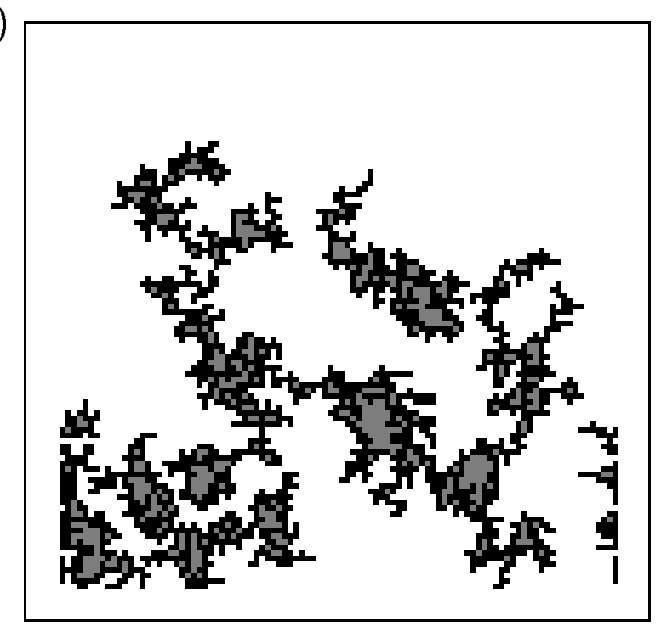

(b)

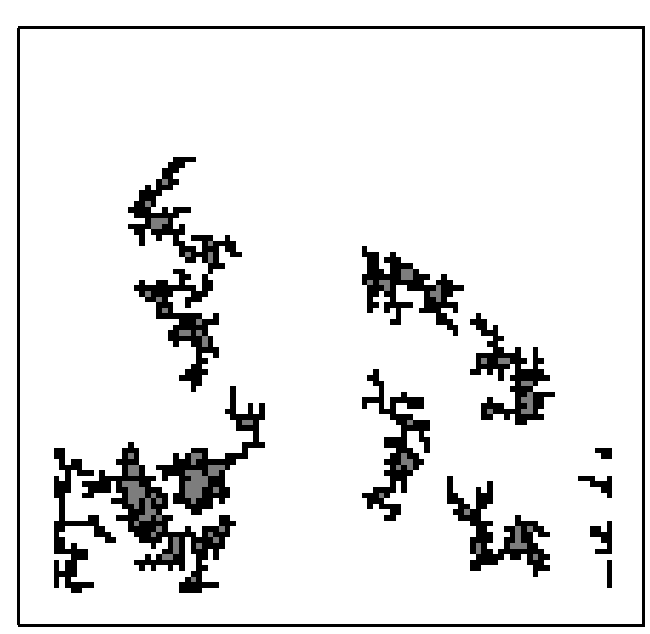

(d)

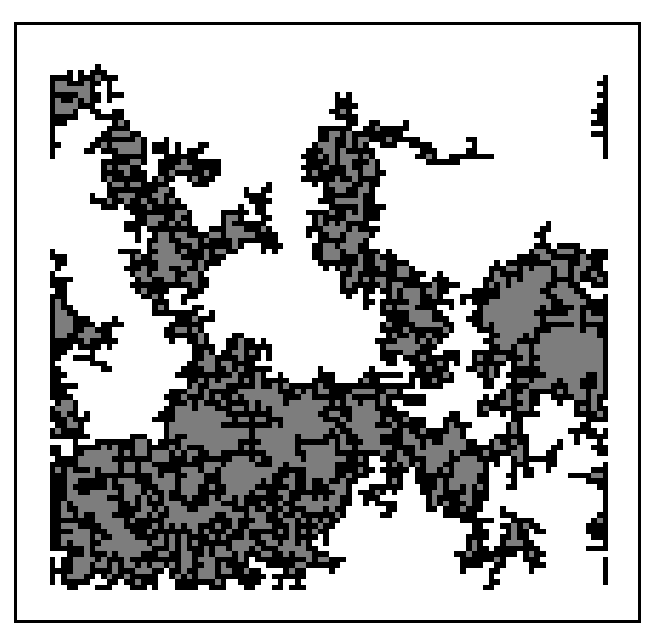

Figure 3: A sequence of four gas-growth patterns in a $100 \times 100$ lattice with $B=10^{-4}$ and $N_{a}=10$. The number of clusters present, $N_{c}$, are respectively: (a) $10 ;$ (b) $9 ;$ (c) 6 ; (d) 3 . Here, coalescence occurs before the onset of $S_{g c}$. Periodic boundary conditions are used at the lateral boundaries, and the color notation of Figure 1 applies. The last panel corresponds to the onset of $S_{g c}$. 


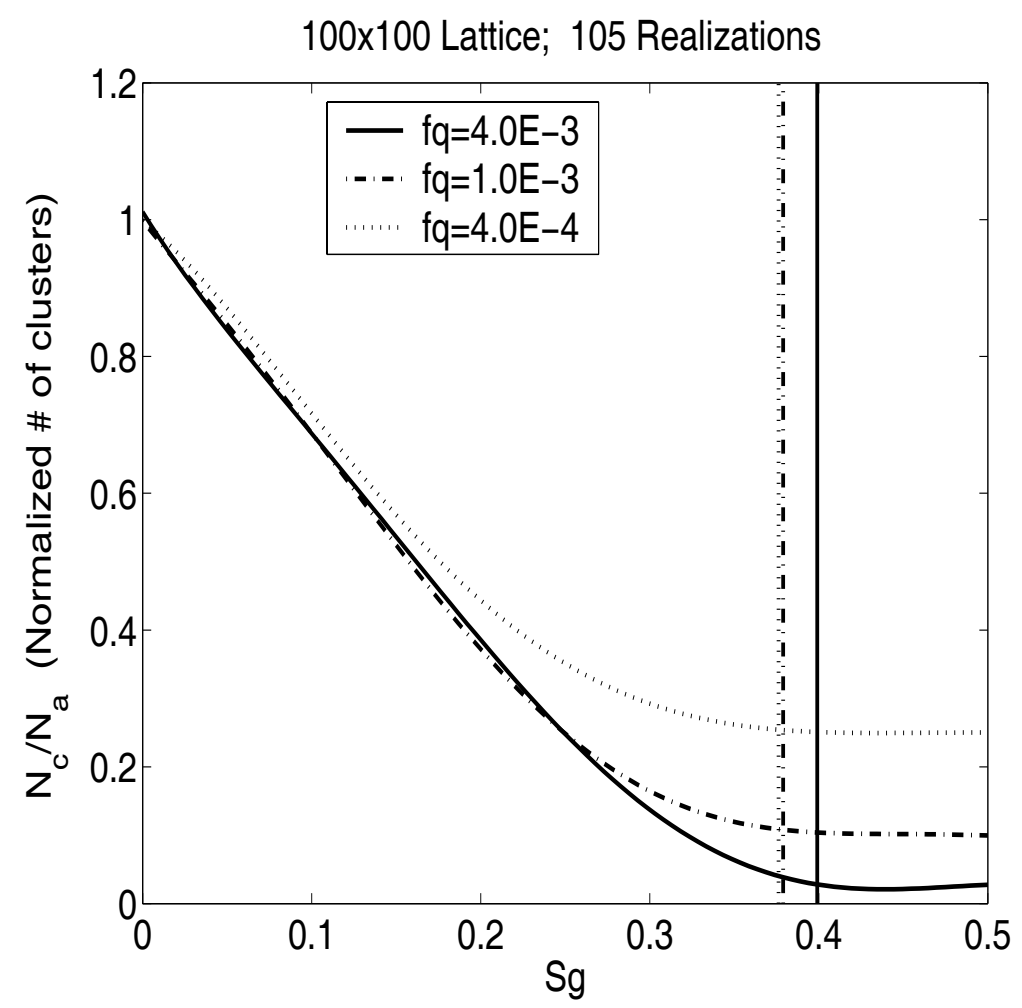

Figure 4: The normalized number of clusters, $N_{c} / N_{a}$, as a function of $S_{g}$ in the absence of gravity, $B=0$, and for three different nucleation fractions, corresponding to $N_{a}=4,10,40$, respectively. The vertical lines denote the point where the "sample-spanning" cluster is formed. 


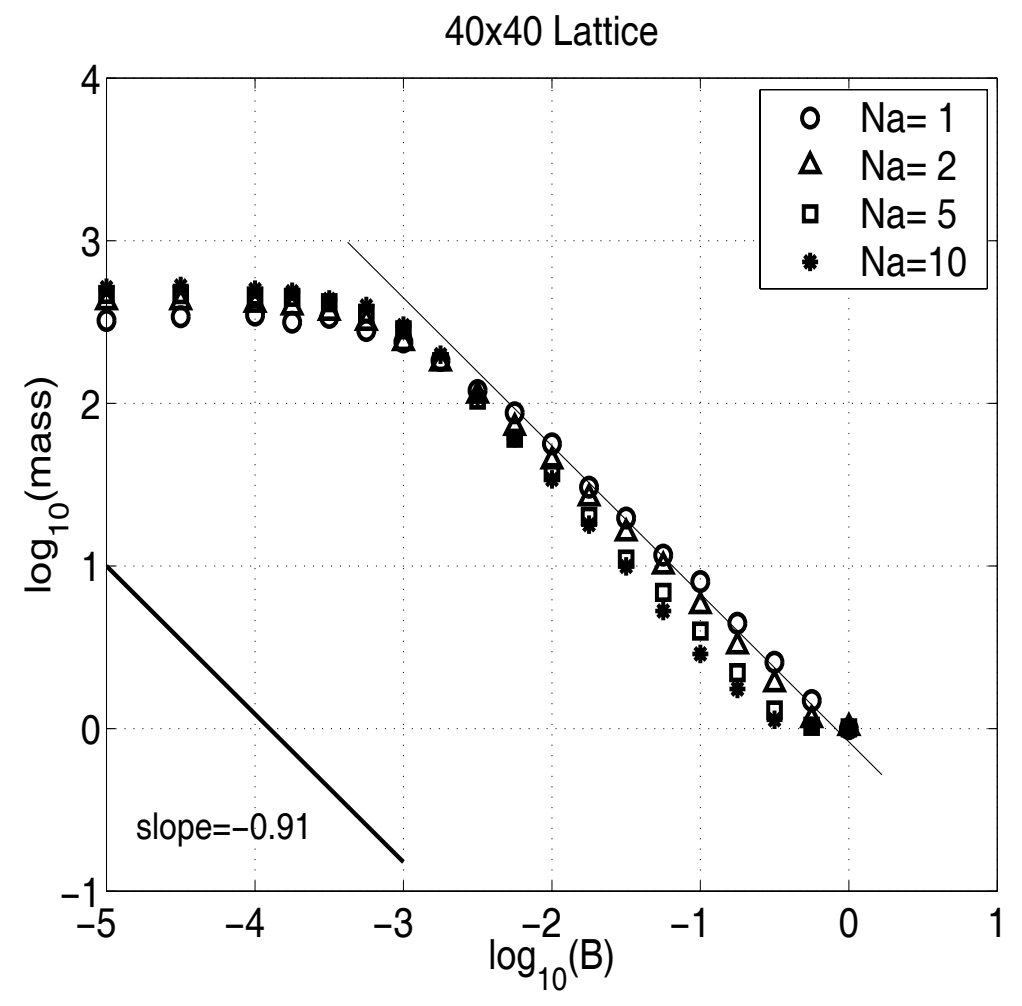

Figure 5: The average mass of the clusters, $M_{c}$, at incipient mobilization, as a function of the Bond number, $B$, for a $40 \times 40$ periodic lattice and for four different nucleation fractions, $f_{q}=\frac{N_{a}}{L^{E}}$. The solid lines denote the theoretical slope in the absence of coalescence. 


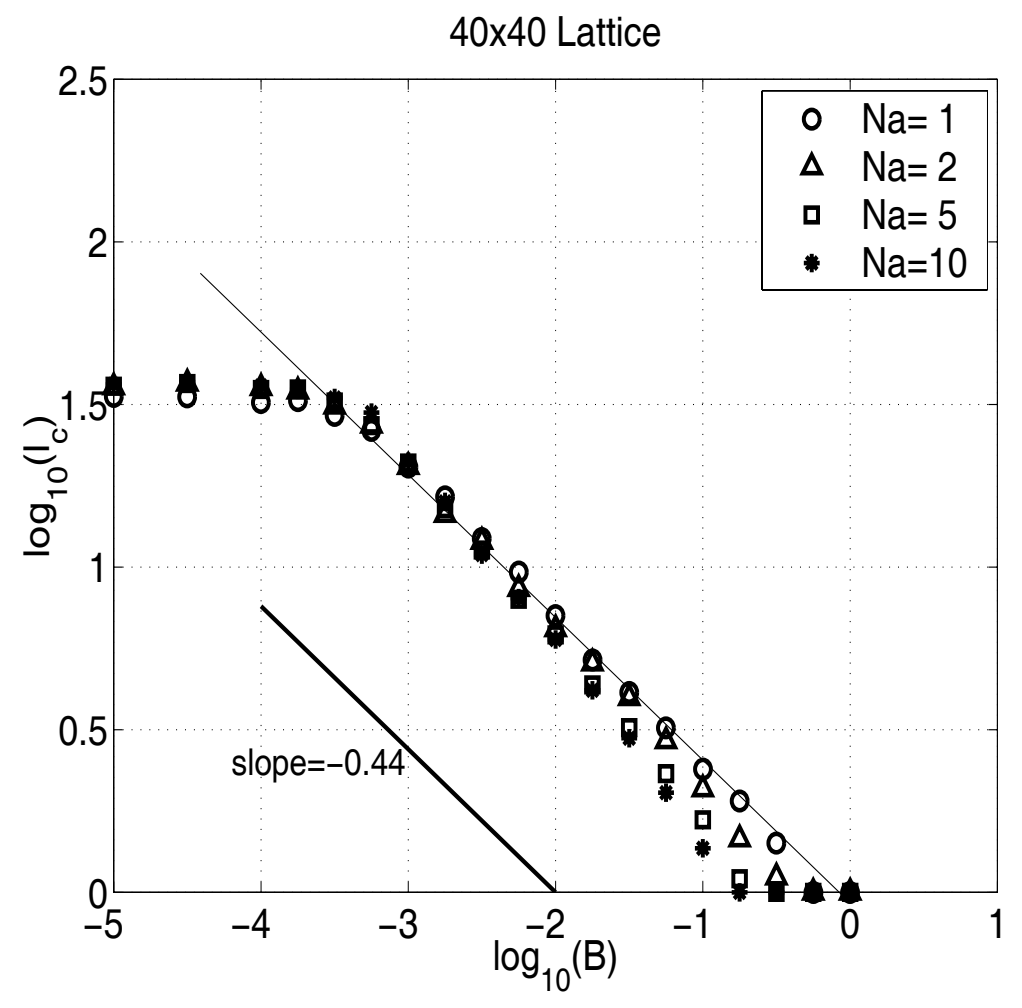

Figure 6: The average projected length, $l_{c}$, of the cluster at incipient mobilization, as a function of the Bond number, $B$, for a $40 \times 40$ periodic lattice and for four different nucleation fractions, $f_{q}=\frac{N_{a}}{L^{E}}$. The solid lines denote the theoretical slope in the absence of coalescence. 


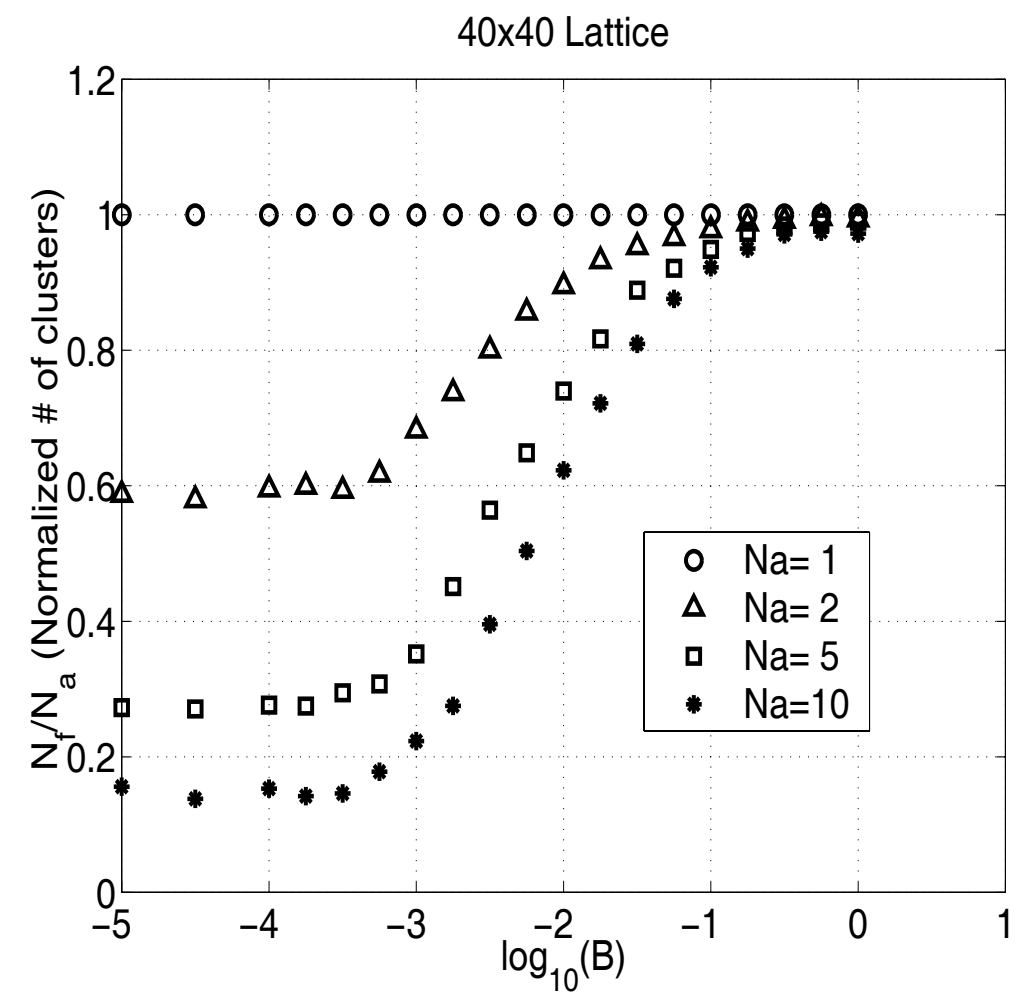

Figure 7: The normalized number of clusters, $N_{f} / N_{a}$, at the onset of the critical gas saturation, as a function of the Bond number, $B$, for a $40 \times 40$ periodic lattice and for four different nucleation fractions, $f_{q}=\frac{N_{a}}{L^{E}}$. 


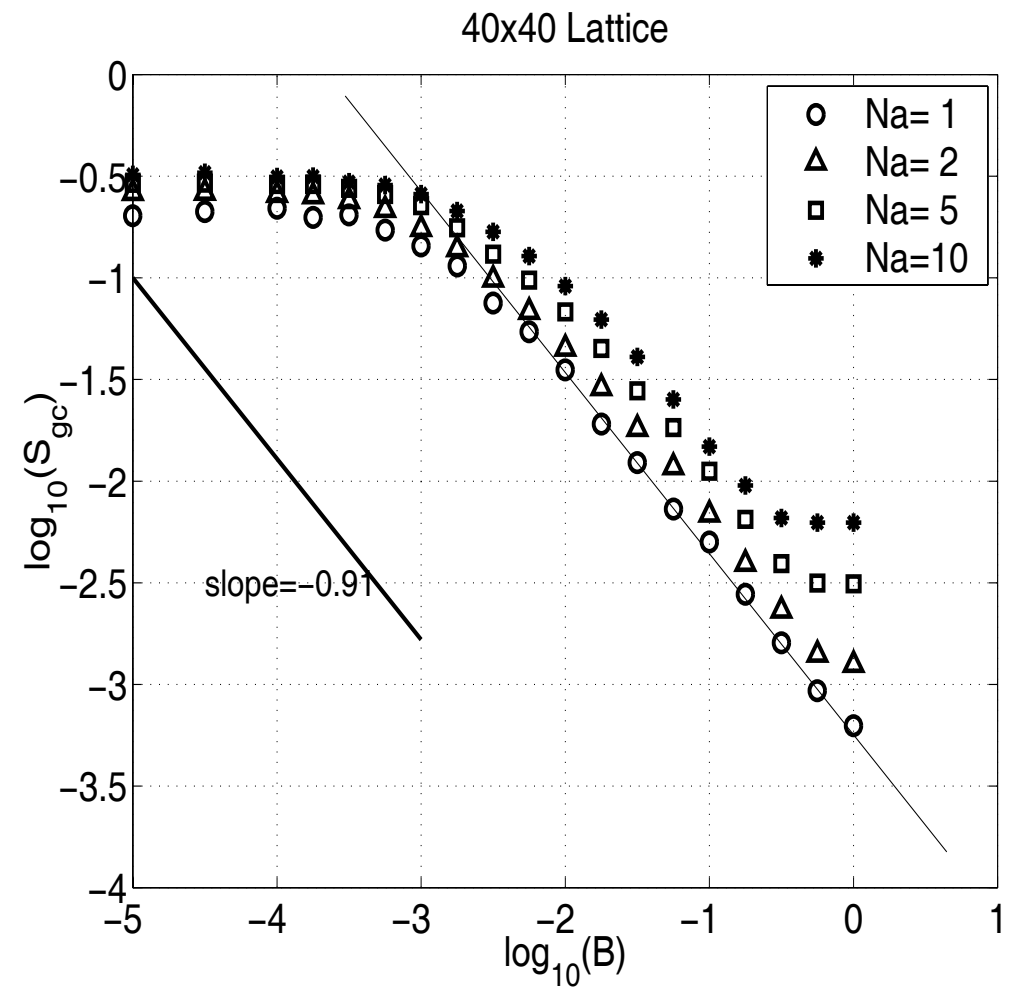

Figure 8: The critical gas saturation, $S_{g c}$, as a function of the Bond number, $B$, for a $40 \times 40$ periodic lattice and for four different nucleation fractions, $f_{q}=\frac{N_{a}}{L^{E}}$. The solid lines denotes the theoretical slope in the absence of coalescence. 


\section{VAPOR-LIQUID FLOWS IN POROUS MEDIA}

The simultaneous flow of vapor and liquid phases is common to steam injection. Counter-current flows are encountered in Steam- Assisted-Gravity-Drainage (SAGD), and in steam injection in horizontal wells. They also appear in the context of heat pipes in a variety of processes (from geothermal to high-level nuclear waste disposal). Concurrent flows are found in typical displacements, in solution gas-drives near wells, and various other contexts. The interaction between heat transfer, heat flux, buoyancy and fluid flow affects the occupancy of phases and the flow characteristics, such as the relative permeabilities. In this section we report on three studies, one dealing with the dynamics of two-phase flows in heterogeneous media where capillarity induces a trapped phase, a second in which we analyze the stability of vapor-liquid counterflows in the presence of gravity and a third one, in which we describe the receding of a liquid phase in a porous medium due to phase change in the context of drying.

A number of problems in porous media involve the simultaneous flow of two immiscible fluids, a liquid and a gas. As a result of the capillarity of the porous medium, however, the flow occurs in an intermittent way, in which one phase may get trapped, immobilized, or conversely mobilized due to the flow of the other phase. This process of ganglia motion has been described in detailed past works by various authors using elaborate porenetwork simulations. In this report, we apply a different approach, in which all the hydrodynamic information of the flowing fluid and the effect of the various ganglia enter at the various ganglia interfaces using a boundary integral method. Methods of this type have been extensively used in potential theory, but have not been applied in the context of flow and mobilization of immiscible fluids in porous media. Their advantage is that the hydrodynamic problem is mapped at the ganglia interfaces and allows a fast and convenient way to describe the forces on the disconnected phase, the possible mobilization, stranding, coalescence, crowding effects, etc. We present the method development by deriving the integral equation to be solved on the fluid interfaces. We then use a numerical method for its solution and examine a variety of problems including: the condition for the mobilization of a stranded ganglion as a function of the capillary 
number and the ganglion shape; its subsequent motion in the random capillary environment and the rate of stranding or coalescence; effects of gravity; and the interaction between neighboring ganglia. The latter does not always enter as a crowding effect, as intuitively expected. The method finds applications to problems where the flowing phase is disconnected, as for example in foamy oil flow or the countercurrent flow of steam-water in the presence of gravity.

In a related study, we considered the linear stability of a two-phase heat pipe zone (vaporliquid counterflow) in a porous medium, overlying a superheated vapor zone. The competing effects of gravity, condensation and heat transfer on the stability of a planar base state are analyzed in the linear stability limit. The rate of growth of unstable disturbances is expressed in terms of the wave number of the disturbance, and dimensionless numbers, such as the Rayleigh number, a dimensionless heat flux and other parameters. A critical Rayleigh number is identified and shown to be different than in natural convection under single-phase conditions. The results find applications to enhanced oil recovery using steam injection, as well as to the conditions of the proposed Yucca Mountain nuclear waste repository. This study complements work of the stability of boiling by previous researchers.

Drying processes in porous media are typically approached using macroscopic continuum models involving phenomenological coefficients. Insight on these coefficients can be obtained by a more fundamental study at the pore- and pore-network levels. In the second report, we present a model based on a pore-network representation of porous media that accounts for various processes at the pore-scale in the context of drying. These include mass transfer by advection and diffusion in the gas phase, viscous flow in liquid and gas phases and capillary effects at the gas-liquid menisci in the pore throats. We consider isothermal drying in a rectilinear horizontal geometry, with no-flow conditions in all but one boundary, at which a purge gas is injected at a constant rate. The problem is mainly characterized by two dimensionless parameters, a diffusion-based capillary number, $\mathrm{Ca}$, and a Peclet number, $\mathrm{Pe}$, in addition to the various geometrical parameters of the pore network. Results on the evolution of the liquid saturation, the trapped liquid islands and 
the drying rate are obtained as a function of time and the dimensionless parameters. The importance of trapped liquid islands on screening mass transfer to the continuous liquid cluster is emphasized. Effects of capillarity and mass transfer on saturation profiles and drying rates are discussed. The results are then used to discuss upscaling to continuum models.

Publications resulting from this part of the project are listed below:

1. Yiotis, A., Boudouvis, A.G., Stubos, A.K., Tsimpanogiannis, I.N., and Yortsos, Y.C., The Effect of Liquid Films on the Isothermal Drying of Porous Media, Phys. Rev. Lett., submitted (2003).

2. Amili, P., and Yortsos, Y.C., Darcian Dyanmics, Phys. Fluids, to be submitted (2003).

3. Amili, P., and Yortsos, Y. C., Mobilization of Ganglia in Porous Media Using Darcian Dynamics, paper SPE 75191, presented at the $13^{\text {th }}$ SPE/DOE Symposium on Improved Oil Recovery, Tulsa, OK (April 13-17, 2002).

4. Yiotis, A.G., Stubos, A.K., Bountouvis, A., and Yortsos, Y.C., A 2-D Pore-Network Model of the Drying of Single-Component Liquids in Porous Media, Adv. Water Res. 24, 437-458 (2001).

5. Amili, P., and Yortsos, Y. C., Darcian Dynamics, paper presented at the AIChE Fall Meeting, Reno, NV (November 5-8, 2001).

6. Yiotis, A., Stubos, A. K., Bountouvis, A., and Yortsos, Y. C., A Pore-Network Model of Drying in Porous Media, paper presented at the AIChE Fall Meeting, Reno, NV (November 5-8, 2001).

7. Laroche, C., Chen, M., Kamath, J., and Yortsos, Y.C., Determining Relative Permeability Exponents Near the Residual Saturation, paper SPE 71489 presented at the 75th SPE Annual Fall Meeting, New Orleans, LA (Oct. 1-3, 2001).

8. Amili, P. and Yortsos, Y. C., Stability of Heat Pipes in Vapor-Dominated Systems, paper presented at the ASME Fall Meeting, Nashville, TN (November 14-19, 1999).

The following $\mathrm{PhD}$ Thesis was also completed in this specific area:

1. Pouya Amili, Two Studies in Simultaneous Two-Phase Flow in Porous Media: I. Heat Pipe Stability, II. Darcian Dynamics, University of Southern California, January 2002. 



\title{
Darcian Dynamics:
}

\section{A New Approach for the Mobilization of Ganglia in Porous Media}

\author{
Pouya Amili and Yannis C. Yortsos
}

\section{INTRODUCTION}

Two-phase flow in porous media has been the subject of many studies due to its importance in applications such as oil recovery, geothermal reservoirs, nuclear waste repositories and so on. The original motivation for this study was the modeling of cocurrent and counter-current vapor-liquid flows in porous media ${ }^{1}$. In many of these applications, due to capillary forces, one of the phases (and particularly the gas phase) becomes disconnected. The resulting bubbles (generically referred to here as ganglia) can be mobilized, if the flow rate of the other phase is sufficiently large, otherwise they become stranded. The existence of trapped ganglia perturbs the flow, which in turn affects trapping and mobilization conditions of other ganglia.

Modeling of trapping, mobilization, and or coalescence of disconnected ganglia in porous media has been investigated using two models, percolation theory and porenetwork simulations. Percolation models ${ }^{2,3,4,5}$ are computationally fast, however they only apply to very small flow rates where the process is quasi-static. In fact, they theoretically correspond to a zero capillary number. On the other hand, pore-network simulations do not have this disadvantage and apply to general dynamic flow conditions $^{6,7,8}$. However, they can be computationally intensive.

In this work, we provide an alternative approach, which utilizes the fact that at the small scale, fluid flow is in fact described by Poiseuille's equation, which then leads to a Darcy law macroscale description. We use homogeneous flow conditions to describe the flow of the flowing phase, and account for the pore microstructure only in so far as capillary forces are concerned. In this approach, therefore, the governing equation is the Laplace equation, for the solution of which we can use the many advances made in potential theory. Specifically, the linear character of the equation allows us to apply a boundary integral method in which all the relevant flow information is mapped onto the 
boundaries of the two phases. (We should note that pore-network models essentially solve the same equation, except that they can also account for heterogeneity in the pore conductances. This cannot be captured by the present method.)

An analogous situation arises in the bulk flow of a suspension of particles, droplets or bubbles, where viscous forces predominate. A significant advance for the understanding of the flow properties of such systems is the method of Stokesian Dynamics, developed by Brady and Bossis ${ }^{9}$. Stokesian Dynamics is based on the linearity of the problem at small Reynolds numbers, where Stokes equations apply, and relies on a boundary integral method to compute the hydrodynamic forces between hydrodynamically interacting particles, as transmitted through the flowing fluid. In fluids in the bulk, these forces are driven by viscous shear, which compete with thermal and interparticle forces. In fluid flow through porous media, on the other hand, the relevant forces are pressure forces, expressed through Darcy's law, and capillary forces. Their competition determines the mobilization, coalescence, break-up and stranding of the various ganglia. The method we propose in this study, and which we will term Darcian Dynamics, is the analogue of Stokesian Dynamics to flow in porous media.

The use of a boundary integral technique enables us to investigate with relatively small computational requirements the interaction in a porous medium between flowing and dispersed phases, held trapped by capillary forces. With this approach, the pressure distribution on the interface of the various ganglia is directly obtained as a function of parameters, such as ganglion size and shape, their position and relative distance, and their population. Thus, the net effect of the viscous forces on each ganglion can be calculated and compared to the capillary forces to determine the possibility of mobilization and the subsequent movement. Effects of gravity can be included in a straightforward manner. Thus, Darcian Dynamics provides a fast way to model the flow behavior and its dependence on the various geometric and flow parameters.

The paper is organized as follows: First, we present the theoretical foundation and the development of the Darcian Dynamics approach. Then, a numerical technique is introduced to solve the resulting boundary integral equation. Numerical results are presented that test the validity of the method and subsequently address the conditions for 
ganglia mobilization, probabilities of break-up and stranding, the effect of gravity and the effects of ganglia interaction.

\section{DARCIAN DYNAMICS}

\section{Boundary integral formulation}

We consider the flow of a fluid in a homogeneous porous medium in the presence of another phase, which is disconnected in the form of ganglia. We assume that the flowing fluid is an incompressible viscous liquid, while the disconnected phase is an inviscid fluid (e.g. vapor or gas). Under the assumptions noted above, the governing equation for the flowing fluid is described by the Laplace equation

$$
\nabla^{2} p=0
$$

As pointed out above, pore-network simulators also solve a discrete form of the above equation. Hence, with the exception of the fact that in pore-network simulation the flow conductance can be heterogeneous, the fundamental premises of the two approaches are identical in this respect.

In this report, we consider the solution of Eqn. (1) in a two-dimensional geometry, in which the injected fluid flows with a far-field velocity $\mathbf{u}_{\mathrm{o}}$ aligned, with no loss of generality, along the direction of the $x$-axis. The existence of the ganglia of the other phase perturbs the flow, thus, we will take the pressure and flow velocity as the superposition of a base flow and a perturbation

$$
\begin{aligned}
& p_{t}=p_{d}+p_{o} \\
& \mathbf{u}_{t}=\mathbf{u}_{d}+\mathbf{u}_{o}
\end{aligned}
$$

where $p_{d}=-\mathbf{u}_{o} x \mu / k$ and subscript $d$ denotes the perturbation due to the presence of the ganglia. At the far field the perturbed flow rate and pressure vanish 
$p_{d} \rightarrow 0, \mathbf{u}_{d} \rightarrow 0 \quad$ and $\quad \mathbf{u}_{t} \rightarrow \mathbf{u}_{o}$,

while at the ganglia interface, the normal flow velocity is zero

$\mathbf{u}_{t} \cdot \mathbf{n}=0$

where $\mathbf{n}$ is the outer normal to the ganglia interface (see Figure 1). In appropriate dimensionless notation, the above can be rewritten as follows

$\nabla^{2} p_{d}=0$

$\begin{cases}\frac{\partial p_{d}}{\partial n}=\cos \theta & \text { on the interface } \\ \mathbf{u}_{t}=1 & \text { at far }- \text { field }\end{cases}$

and where $\theta$ is the angle between the normal $\mathbf{n}$ and the $\mathrm{x}$-direction.

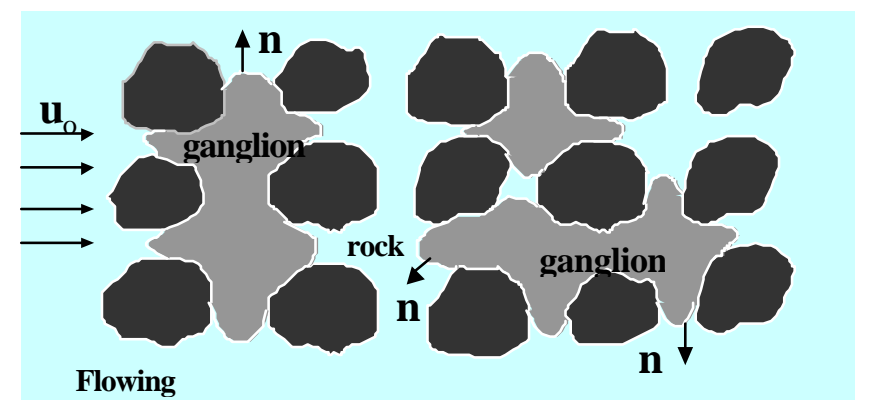

Figure 1. Schematic of the problem considered.

To solve (3) we will use an integral equation approach following advances from electrostatic potential theory. In that terminology, the free-space 2-D potential at location $\rho$ due to a point sink (source) at $\rho$ ' is 
$\phi(\rho)=\frac{1}{2 \pi} \ln |\rho-\rho|$

For sources or sinks distributed on any contour $C$ (here the ganglia interface) with density $Q_{l}$, the potential at point $\rho$ is then given by superposition

$p_{d}(\rho)=\int_{C} \frac{Q_{l}\left(\rho^{\prime}\right)}{2 \pi} \ln \left|\rho-\rho^{\prime}\right| d l_{\rho^{\prime}}$

Differentiating the above equation by carefully accounting for the removable singularities ${ }^{1}$ and using the boundary condition (4) results in

$\lim _{\rho \rightarrow C^{+}} \frac{\partial p_{d}}{\partial n_{\rho}}=\frac{1}{2} Q_{l}(C)+\int_{C_{0}} Q_{l}\left(\rho^{\prime}\right) \frac{\cos \left(\rho-\rho^{\prime}, \mathbf{n}\right)}{2 \pi\left|\rho-\rho^{\prime}\right|} d l_{\rho}=\cos \theta$

This is a Fredholm integral equation of the second kind for $Q_{l}$. For its numerical solution we apply the method of moments ${ }^{1}$ which reduces the equation into a linear matrix equation. From the calculated $Q_{l}$, the pressure follows readily using Eqn. (6).

The Green's function in Eqn. (5) is based on an infinite system. For different geometries, the appropriate Green's functions must be used instead. For example, for flow confined between two impermeable boundaries parallel to the $x$-axis, the corresponding Green's function is ${ }^{1}$

$$
\begin{aligned}
& \phi\left(x, y ; x_{0}, y_{0}\right)=\frac{1}{4 \pi} \ln \frac{1}{2}\left[\cosh \frac{\pi}{d}\left(x-x_{0}\right)-\cos \frac{\pi}{d}\left(y-y_{0}\right)\right]+ \\
& \frac{1}{4 \pi} \ln \frac{1}{2}\left[\cosh \frac{\pi}{d}\left(x-x_{0}\right)-\cos \frac{\pi}{d}\left(y+y_{0}-d\right)\right]
\end{aligned}
$$


where $d$ is the distance between two boundaries. This equation provides the potential at $(x, y)$ caused by a point source at $\left(x_{0}, y_{0}\right)$. If the system is periodic in the flow direction, we have ${ }^{1}$

$\phi\left(x, y ; x_{0}, y_{0}\right)=\frac{1}{4 \pi} \ln \frac{1}{2}\left[\cosh \frac{2 \pi}{a}\left(y-y_{0}\right)-\cos \frac{2 \pi}{a}\left(x-x_{0}\right)\right]$

where $a$ is the period length. This geometry is useful when the number of ganglia is large and a periodic distribution along the flow direction may be assumed. Results will be provided for a number of cases in the various geometries, in the next section of this report.

\section{Mobilization Conditions}

To simulate the flow behavior in a system containing a dispersed and a disconnected phase, conditions for ganglia mobilization must be formulated. Specifically, we need to know if the pressure difference across a ganglion is sufficient to overcome the capillary forces, which hold it trapped. The pore microstructure gives rise to capillary thresholds, which are in general randomly distributed, following the distribution of the pore throats. Thus, we can assign dimensionless capillary pressure thresholds to all ganglia segments, which in the present notation will be expressed in terms of the capillary number of the problem. We find that the capillary threshold at any segment is ${ }^{1}$

$p_{c D}=\frac{\beta}{\operatorname{Car} r_{D}}$

where parameter $\beta=-\frac{16 \operatorname{Ln}\left(p_{c}\right)}{226 F}$ is related to the percolation threshold of the lattice, $p_{c}$, and the formation factor, $F$, and we defined the capillary number $C a=u_{o} \mu / \gamma$, where $\gamma$ is the interfacial tension. The dimensionless radius $r_{D}$ is in general a random variable, which in the following illustrations was distributed using the Rayleigh distribution 
$\alpha(r)=r \exp \left(-\frac{r^{2}}{2}\right)$

The movement of a ganglion is decided by the difference between the viscous pressure and the capillary pressure threshold. At every step of the simulation, we first determine the segments of the ganglia subject to mobilization. For this, we form the pressure difference between pairs of ganglia segments $i$ and $j$, belonging to the same ganglion, and subtract the capillary pressure threshold of the segment with the smallest pressure,

$\Delta p_{i, j}=p_{j}-p_{i}-p_{c D i}$

For a given ganglion, the maximum of $\Delta p_{i, j}$ over all $i$ and $j$ is found. If it is positive, segments $i$ and $j$ are advanced one pore, such that segment $i$ moves outward and segment $j$ inward. Otherwise the ganglion remains stationary. The procedure is repeated at every step, leading to new ganglia configuration, with different shapes, geometry and orientation, from which the fuid pressure can be comouted again. We note that this approach is essentially a quasi-static one, in that only one set of interfaces is advanced at any step. Given that time is not explicit in these calculations, however, this limitation is not significant.

The above were obtained in the absence of gravity. Buoyancy forces can play an equally important role to viscous, however. In the presence of gravity acting along the $\mathrm{x}$ direction, the determination of the pressure field is as above, except that the base pressure must also include the hydrostatic contribution. The effect of gravity actually enters in the mobilization condition (12), which must now include the gravity contribution $l_{i, j} N g_{x}$, where we introduced the gravity number

$$
N g_{x}=\frac{\Delta \rho k g_{x}}{u_{o} \mu}
$$


and $l_{i, j}$ is the projection of the distance between the two segments along the x-axis. The gravity number is also equal to the ratio between the Bond number, $B o=\Delta \rho g k / \gamma$, and the capillary number. Gravity effects will be analyzed below.

\section{NUMERICAL RESULTS}

Using the method of moments, the integral equation was solved for a variety of situations of interest. In this section we first demonstrate the validity of the method by comparison with an analytical solution. Then, we consider the conditions for the mobilization of a single ganglion as a function of its size, confinement, flown rate, capillary environment and gravity. We analyze the sensitivity of the critical capillary number, above which a ganglion is mobilized to various parameters. We also investigate the effect of ganglia interaction.

\section{Validity of the Method}

To test the validity of the method, we compared the numerical predictions with some analytical results. In 2-D, analytical solutions exist for potential flow around a cylinder in an unbounded domain. Then, the dimensionless pressure at the interface is given by the simple equation

$p=-\cos \theta$

$\left(\right.$ see Lamb $\left.{ }^{10}\right)$. Figure 2 shows a comparison between 


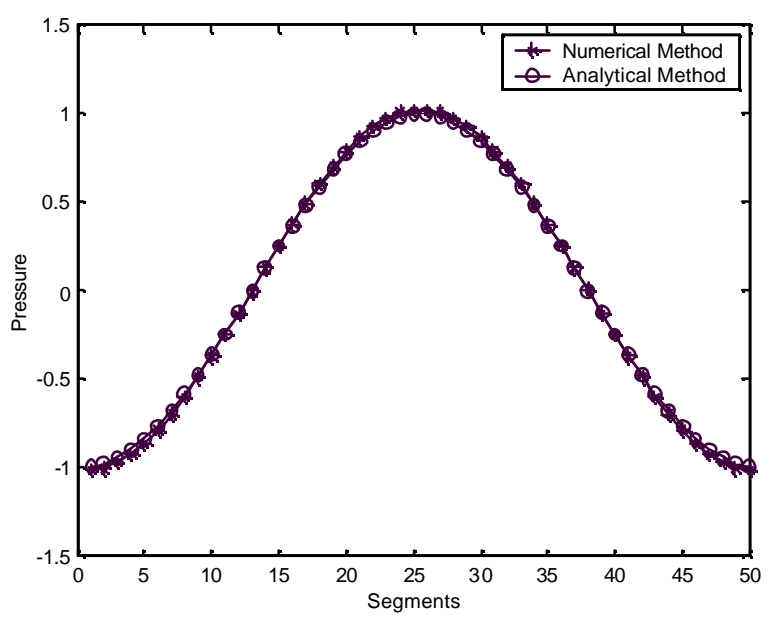

Figure 2. Comparison between analytical and numerical solutions for flow around a circular ganglion.

analytical and numerical results, using Darcian Dynamics, in which the circle was discretized in 50 segments. In Figure 2 the ordinate indicates the location of a segment, numbered clockwise from the downstream end. An excellent agreement is observed. Similar agreement was found for the case of an elliptical shape ganglion, for which an analytical solution is also possible ${ }^{1}$. Having confirmed the validity of the method, we then proceeded to investigate the sensitivity of the flow to various parameters.

\section{Single Ganglion}

First, we studied the mobilization and subsequent movement of a single ganglion. The first parameter studied was the ganglion size.

\section{Ganglion size}

The mobilization of a ganglion depends principally on its size. Increasing the size, increases the pressure gradient across the ganglion, resulting in a higher probability of mobilization. Figure 3 shows the variation of the critical capillary number as a function of size for a square-shaped ganglion of size nxn in a homogeneous capillary environment with the same capillary thresholds in each segment. As expected, there exists a critical capillary number for ganglia mobilization, which is of the order of $10^{-3}$. This value is consistent with what is typically assumed regarding the importance of 
viscous forces in porous media. The critical capillary number decreases as the ganglion size increases, and becomes asymptotically inversely proportional to the linear ganglion size (and in Figure 3 inversely proportional to the square root of the ordinate, except for the periodic geometry case, which limits the ganglion size). The effect of confinement is also apparent. When the flow is confined, the critical capillary number decreases, due to the increased viscous pressure. This is more clearly illustrated in Figure 4. It is shown that the spacing of the confinement has a significant effect as long as it is smaller than a value, which is about 5 times the ganglion size. For larger spacings, the effect is not significant and the critical capillary number approaches its value in an infinite system. We add that the above behavior is qualitatively similar for ganglia of different shapes, although the shape and orientation of a ganglion does affect the numerical value of the critical capillary number.

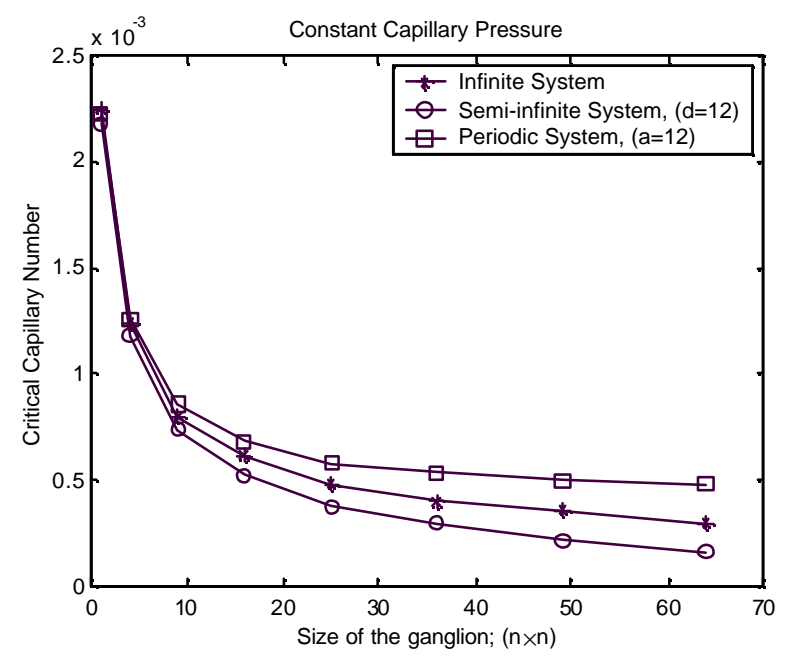

Figure 3. The dependence of the critical capillary number for mobilization on ganglion size for three different geometries: infinite, semi infinite and periodic. 


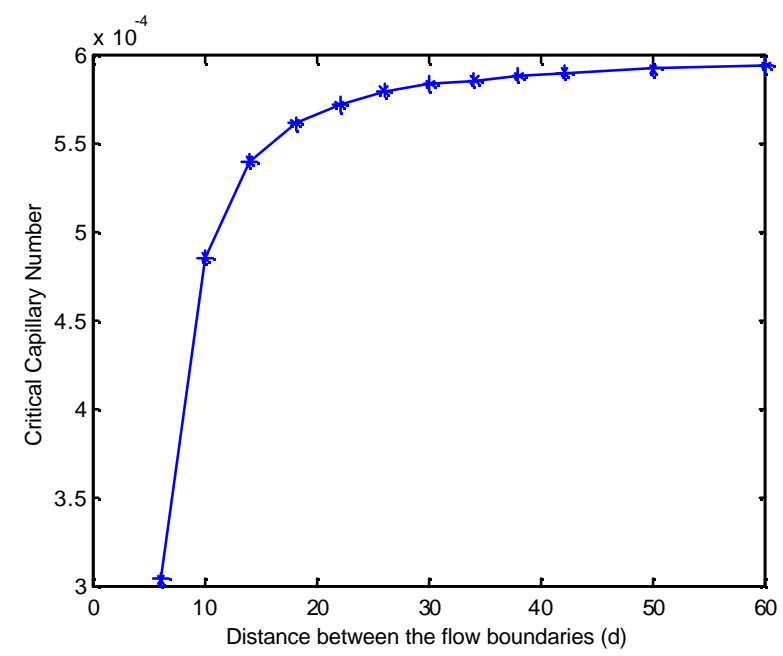

Figure 4. Critical capillary number for the mobilization of a square-shaped ganglion of size $4 \times 4$ confined between two parallel plates, plotted versus the distance between the plates.

\section{Effect of flow rate}

Following the onset of mobilization, ganglia undergo shape changes, break-up, coalescence and/or stranding. The applied flow rate (capillary number), the capillary threshold distribution and the original ganglion shape are important variables for these events. Figure 5 illustrates successive snapshots of the movement of a ganglion in a random capillary environment for a capillary number equal to $8 \times 10^{-4}$, which is larger than the critical for this size. It is apparent that the mobilization and subsequent motion is accompanied by deformation, break-up and stranding of daughter ganglia of smaller size. The resulting ganglia interaction is quite complex and dynamic. Typically, ganglia movement occurs in a random capillary threshold environment, due to the randomness in the pore structure. In a random domain, the critical capillary number is also a random variable. Equivalently, we may define a probability of mobilization, given a value of the applied capillary number. Likewise, we can determine probabilities for break-up, stranding of all ganglia generated, etc. as a function of the capillary number. Figure 6 shows the curves obtained by simulating the motion of a ganglion of size 45 , for several realizations of the random capillary thresholds. The key features of the curves obtained are as follows: (i) mobilization, break-up and stranding are stochastic processes; (ii) the variation of the probabilities for these events vary in a rather narrow interval of the capillary number, of the order of $10^{-4}-10^{-3}$, the width of which also depends on the 
variance of the threshold distribution; (iii) the probability of break-up becomes one for sufficiently large capillary numbers; (iv) the probability of stranding becomes negligible if the flow rate is sufficiently large. It is important to mention here that although a ganglion can go through several break-ups during its movement, the data presented correspond to the first break-up only. It is also important to mention that a value of unity for the probability of stranding in this case means the stranding of all daughter ganglia. Similarly, a value of zero means that at least one daughter ganglion is still mobile before leaving the domain of the simulation.

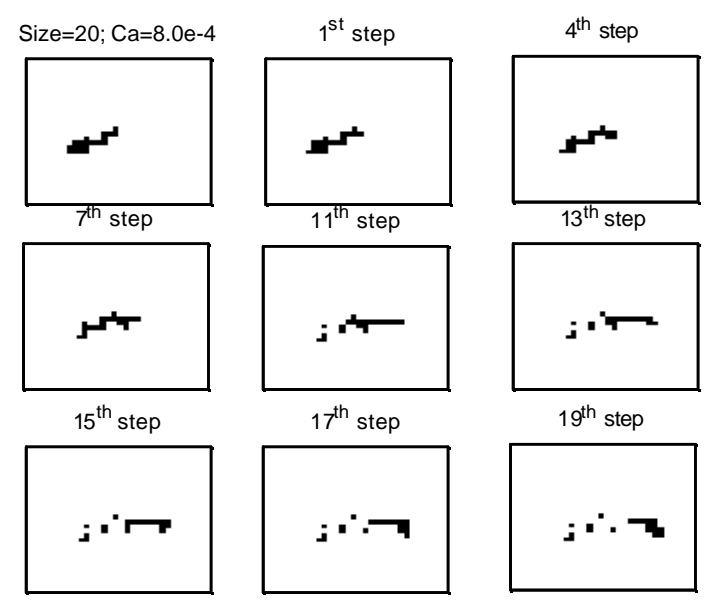

Figure 5. Snapshots of the movement of a ganglion of size 20 in a random capillary threshold environment for $\mathrm{Ca}=8.0 \mathrm{e}-4$. 


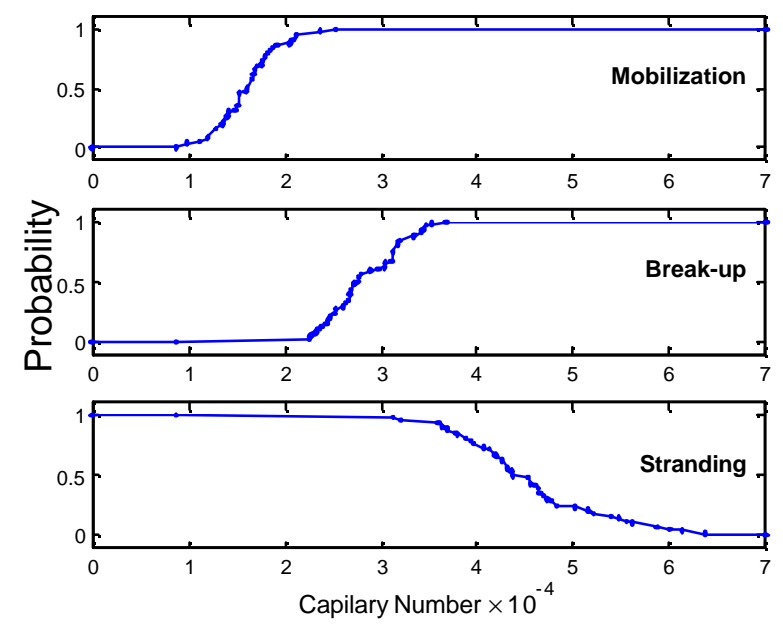

Figure 6. Probabilities of mobilization, break-up, and stranding after the mobilization of a single ganglion of size 45 in a heterogeneous medium, plotted as a function of the capillary number.

\section{Effect of gravity}

When buoyancy forces are considered, both the magnitude and the direction of the gravity vector are important. If gravity aids viscous forces, mobilization is facilitated, and the critical capillary number decreases with an increase in the Bond number. In such cases, the critical capillary number is a decreasing linear function of the Bond number, as can be readily shown. Figure 7 shows the corresponding relationship, obtained from simulations in a homogeneous capillary threshold environment, with a ganglion of size 45. There exists a critical Bond number of the order of about $3 \times 10^{-4}$, above which the ganglion is mobilized by gravity forces alone. In the opposite case, when gravity opposes mobilization (for example, when flow and gravity act in the same direction), the situation is a bit more complex. Now, the critical capillary number increases with the Bond number. Furthermore, when the velocity is small, for sufficiently large Bond numbers, the ganglion is mobilized in the direction opposite to the flow. In other words, there exists now a critical Bond number, which depends in a straight-line relationship on the capillary number. In-between the two lines is the regime where the ganglion remains stationary. The two lines intersect at a critical point, beyond which a single line separates two regimes, one in which mobilization is in the direction of flow (higher $\mathrm{Ca}$ ) and another in which it is in the opposite direction (lower $\mathrm{Ca}$ ). Subsequent to mobilization, 
the ganglion undergoes a sequence of events similar to the previous, except that when gravity effects are significant, there is a preference for the ganglion to elongate along the gravity direction ${ }^{1}$.

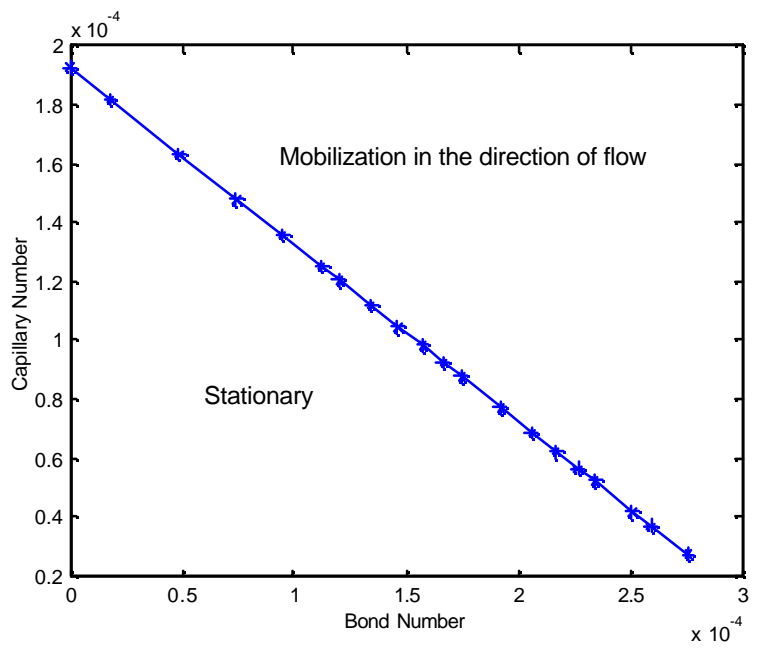

Figure 7. Regimes of mobilization in a diagram involving the capillary and the Bond numbers, for the case when gravity aids the mobilization.

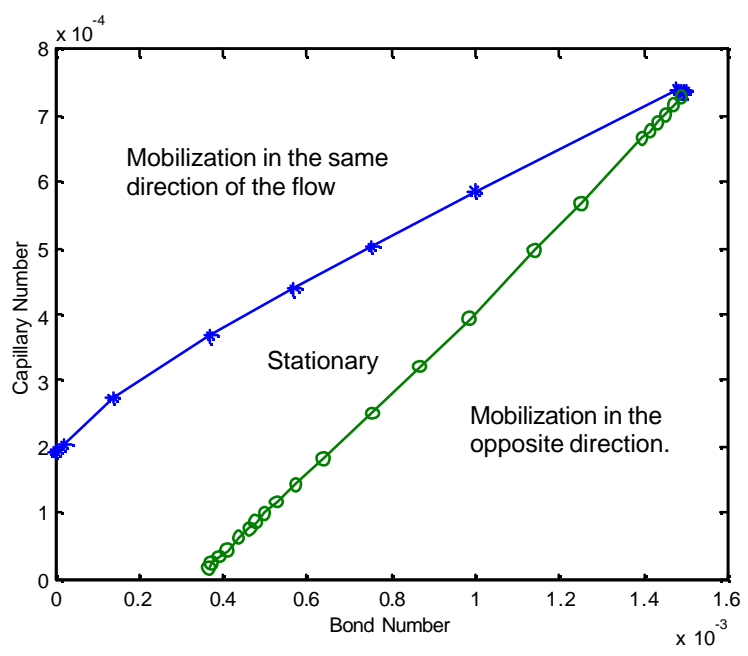

Figure 8. Regimes of mobilization in a diagram involving the capillary and the Bond numbers, for the case when gravity aids the mobilization. 


\section{Ganglia interaction}

The mobilization and subsequent evolution of a ganglion depends also on the presence of neighboring ganglia. A "crowding" effect has been postulated by previous authors ${ }^{11}$, in which the increase of the population density of ganglia increases the viscous forces on the system, thus making it easier to mobilize ganglia, even at relatively small capillary numbers, where viscous effects are not expected to be significant. To investigate such phenomena, we considered the effect of distance and orientation of one ganglion on the mobilization of another identical ganglion. For simplicity, we assumed two identical circular ganglia confined between two parallel plates, as shown in the insets of Figures 9 and 10. First, we examined the case in which the ganglia are stacked in a direction perpendicular to the boundaries (Figure 9).

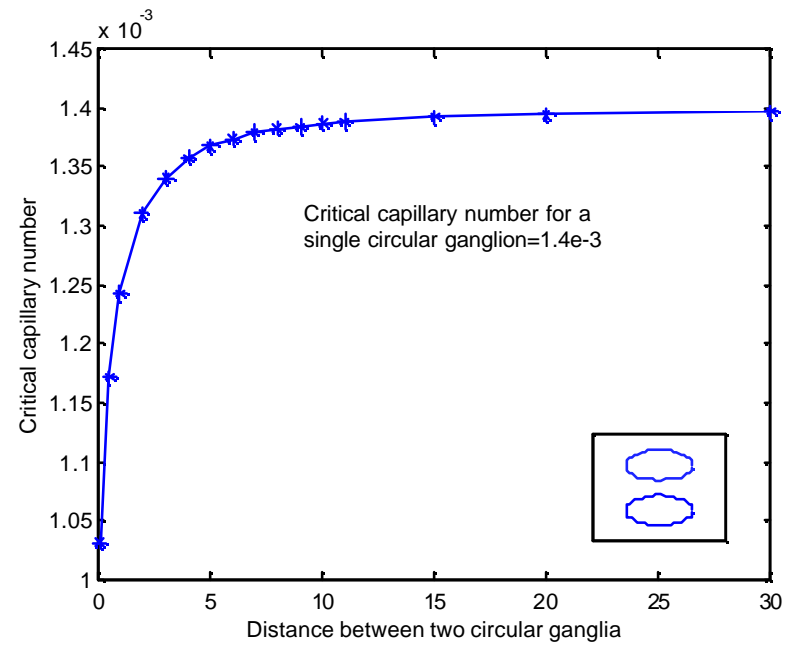

Figure 9. Critical capillary number for ganglia mobilization plotted versus the distance between the two ganglia confined between two parallel plates and stacked perpendicular to the plates (see inset). Homogeneous capillary environment.

Figure 9 shows the critical capillary number for the mobilization of the ganglia (both are mobilized simultaneously due to the symmetrical location) as a function of the distance between them. In this configuration, it is apparent that the presence of the neighboring 
ganglion increases the viscous forces in the system, thus making the mobilization of the other ganglion (and itself) easier. Indeed, there is approximately a $40 \%$ reduction in the value of the critical capillary number as the two ganglia approach one another. A crowding effect is indeed present, leading to enhanced viscous forces.

When the configuration was changed, however, such that the two ganglia were aligned along the flow direction, parallel to the plates, the effect was in fact opposite to the previous (Figure 10). In this case, increasing the distance between the ganglia resulted in a decrease of the critical capillary number, which asymptotically approached the critical capillary number for a single ganglion as the distance becomes sufficiently large. It appears that as the ganglia approach each other, such configurations lead to a better streamlined object, which offers smaller viscous resistance, hence an increase in the critical capillary number. This unexpected effect is opposite to the conventional crowding effect. The effect was confirmed using a different numerical technique, as well. One concludes that not only the density, but also the relative position and orientation of the ganglia are important factors in ganglia mobilization.

An illustration of the non-trivial aspects of these effects is shown in Figure 11, where the effect of the orientation of the two ganglia on the critical number for the mobilization of the upstream ganglion is portrayed. As in Figure 10, the critical capillary number of a single isolated circular ganglion in this geometry is $1.4 \times 10^{-3}$. The second ganglion is then rotated counter-clockwise around the reference one, while maintaining the same distance. Clearly, the configuration in which the ganglia are stacked perpendicular to the flow is the one with the strongest crowding effect, while the alignment along the flow direction in fact favors the trapping of the ganglion. Additional work in this direction, including the subsequent evolution of the mobilized ganglion is discussed in Amili ${ }^{1}$ and is also actively pursued at this time. 


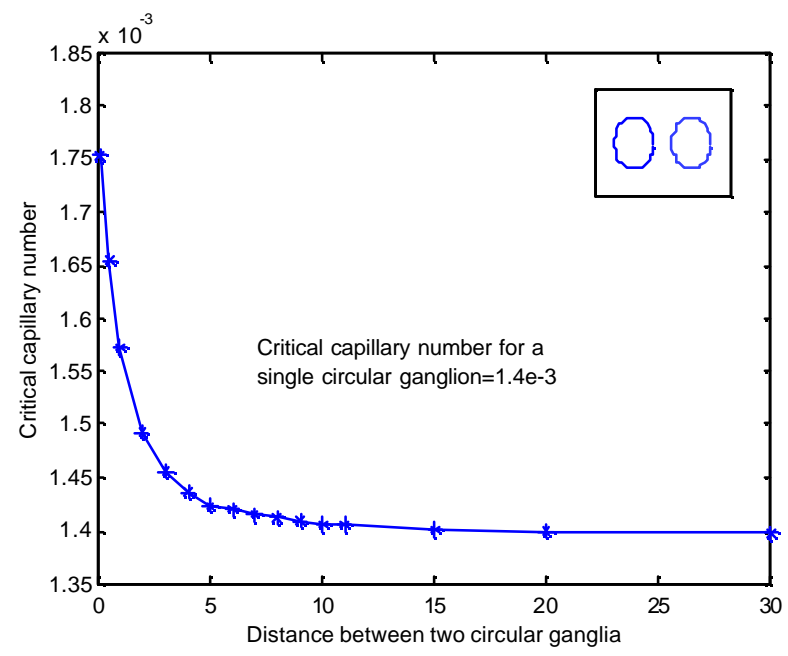

Figure 10. Critical capillary number for ganglia mobilization plotted versus the distance between the two ganglia confined between two parallel plates and stacked perpendicular to the plates (see inset). Homogeneous capillary environment.

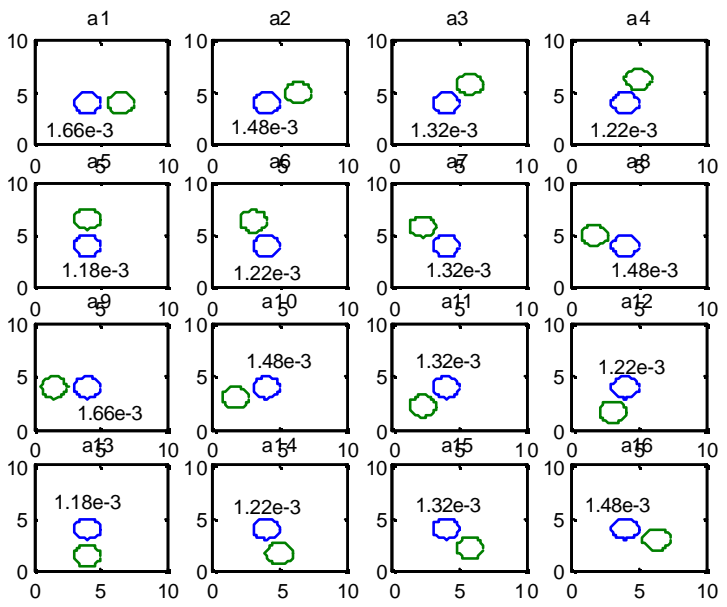

Figure 11. The critical capillary number for the mobilization of the upstream ganglion for many different positions of the two ganglia. (Note that the lower two panels are mirror images of the top two.) Homogeneous capillary environment. 


\section{CONCLUSIONS}

In this report we developed a new method, which we termed Darcian Dynamics, in analogy with Stokesian Dynamics for flow of bulk fluids, to study and simulate twophase (liquid-gas) flow in porous media, when the gas phase is disconnected in the form

of ganglia. The method assumes homogeneous flow conditions for the flowing liquid phase, but heterogeneous capillary thresholds. Using techniques from potential theory, we describe the hydrodynamic interaction in terms of the solution of an integral equation, valid over the ganglia interfaces. We use this method to explore the conditions for the onset of ganglia mobilization and their subsequent motion. Break-up, coalescence and stranding are simulated. The interaction between ganglia and the flowing phase is influenced by many parameters, several of which have been studied in this work. In particular, we examined effects of size, orientation, gravity, and ganglia interaction. The latter does not always enter as a crowding effect, as intuitively expected. The technique is currently being further developed in order to address a variety of interesting problems.

\section{REFERENCES}

1. Amili, P.: “Two Studies in Simultaneous Two-Phase Flow in Porous Media: I. Heat Pipe Stability, II. Darcian Dynamics" Ph.D. dissertation, University of Southern California, Los Angeles, California (2002).

2. Melrose, J. C. and Brander, C. F.: "Role of Capillary Forces in Determining Microscopic Displacement Efficiency for Oil Recovery by water Flooding," Can. J. Pet. Tech. 13, 54-62 (1974).

3. Scriven, L. E. Larson, R. G. and Davis, H. T.: "Percolation Theory of Residual Phases in Porous Media," Nature 268, 409-413 (1977).

4. Lerman ,K. Chandler, R. Koplik, J. and Willemsen, J. F.: "Capillary Displacement and Percolation in Porous Media,” J. Fluid Mech. 119, 249-267 (1982).

5. Wilkinson, D. and Willemsen, J.: "Invasion Percolation: A New Form of Percolation Theory," J. Phys. A 16, 3365-3376 (1983). 
6. Dias, M. M. and Payatakes, A. C.: "Network Models for Two-Phase Flow in Porous Media. Part 1. Immiscible Microdisplacement of Non-Wetting Fluids," J. Fluid Mech. 164, 305-336 (1986).

7. Dias, M. M. and Payatakes, A. C.: "Network Models for Two-Phase Flow in Porous Media. Part 2. Motion of Oil Ganglia," J. Fluid Mech. 164, 337-358 (1986).

8. Constantinides, G. N. and Payatakes, A. C.: "Network Simulation of Steady-State Two-Phase Flow in Consolidated Porous Media," AIChE Journal 42, 369-382 (1996).

9. Brady, J. F. and Bossis, G.: "Stokesian Dynamics," Ann. Rev. Fluid Mech. 20, 111-157 (1988).

10. Sir H. Lamb. Hydrodynamics. Dover, New York (1945).

11. Valavanides, M.S. Constantinides, G.N. Payatakes, A.C.: "Mechanistic Model of Steady-State Two-Phase Flow in Porous Media Based on Ganglion Dynamics," Transp. Porous Media 30, 267-299 (1998). 



\title{
Stability of Vapor-Liquid Counter-Current Flow (Heat Pipes) in the Presence of Gravity
}

\author{
Pouya Amili and Yannis C. Yortsos
}

\section{INTRODUCTION}

Heat pipes are steady-state, vapor-liquid (typically, steam-water), countercurrent flow regimes in porous media driven by the application of heat flux and buoyancy (White et al., 1971). The heat flux, typically applied at the bottom, results in the evaporation of the liquid phase, which descends due to buoyancy, and in the return upward flow of vapor, which ascends also due to density differences. This steady-state motion gives rise to enhanced heat transfer by convection. Heat pipes in horizontal systems are also possible, driven by capillarity instead of gravity. A number of geothermal reservoirs are characterized by heat pipe action, including the Geysers (White et al., 1971), the Larderello, Matsukawa and Kawah Kamjang fields (McGuinness, 1995).

Heat pipes in homogeneous porous media are characterized by two key features: the temperature is approximately constant, and equal to the vapor saturation temperature at the prevailing pressure, and the liquid saturation is approximately uniform spatially (Udell, 1985). In theory, the spatial extent of a heat pipe can be infinitely large, under the conditions that the porous medium is homogeneous and that the temperature decrease due to the pressure drop is not significant (Satik et al., 1991, Stubos et al., 1993, Pestov, 1998). In practice, viscous, capillary, heat transfer and heterogeneity effects limit the spatial extent. For example, in heterogeneous systems, changes in permeability induce saturation changes, driven by both capillary and gravity effects (Stubos et al., 1993), even in 1-D systems. For a constant heat flux directed against the gravity vector, there are two possible steady-states, in the absence of heat conduction or capillary effects. These are determined by the solution of the following equation (Bau and Torrance, 1982, Udell, 1985, Stubos et al., 1993) (see also schematic of Fig. 1). 


$$
\omega \equiv \frac{q v_{\mathrm{v}}}{k g h_{f g}\left(\rho_{l}-\rho_{\mathrm{v}}\right)}=\frac{1}{\frac{v_{l}}{v_{v}} \frac{1}{k_{r l}}+\frac{1}{k_{r \mathrm{v}}}}
$$

Equation (1) expresses the balance of zero net mass flow at steady-state, and the heat transfer of the applied heat flux by the upwards moving vapor. Here, $\omega$ is a dimensionless parameter expressing the magnitude of the applied heat flux, $q$ is the applied heat flux, $v$ is the kinematic viscosity, $k$ is the permeability, $g$ the acceleration of gravity, $h_{f g}$ the latent heat of vaporization and $k_{r}$ the relative permeability, with subscripts $v$ and $l$ denoting vapor and liquid, respectively.

The Right-Hand-Side of equation (1) vanishes at the two end-points (residual saturations) of the saturation $s$ (Fig. 1), since the relative permeabilities vanish there. The solution of (1), obtained by the intersection of the horizontal line corresponding to a constant heat flux, $\omega$, with the saturation curve, has two solutions, at points A and B in Fig. 1, provided that the heat flux is smaller than a critical value, $\omega_{\max }$ (and which is equal to about 0.8 in Fig. 1). In the illustration of Fig. 1, we considered straight-line relative permeabilities with zero residual saturations (the place where the relative permeabilities vanish). The two steady states are designated as a vapor-dominated (VD) or a liquid-dominated (LD) heat pipe, depending on whether the liquid saturation is small (point A) or large (point B), respectively. Above the critical value, a heat pipe per se, in the sense of a constant saturation region, does not form. Instead, a two-phase zone of considerably smaller extent forms, governed by the competition of capillary and gravity forces, where the saturation varies in the range $(1,0)$ (see for example, Stubos et al., 1993).

The heat pipe regime is in turn, connected to single-phase flow regimes above or below it. In applications where the liquid-dominated branch exists, a (subcooled) liquid layer overlies the heat pipe region. The point of transition between the heat pipe and the liquid depends on a variety of factors, including the heterogeneity of the medium (Stubos et al., 1993, Mc Guiness, 1996a, 1996b, Pestov, 1998). Typically, this application is encountered in boiling at low rates in porous media, where a liquid layer above the twophase region is maintained, for example by keeping its temperature below boiling 
(Ramesh and Torrance, 1990, 1993). Conversely, a vapor-dominated heat pipe develops when a superheated vapor lies below the two-phase region. This situation,

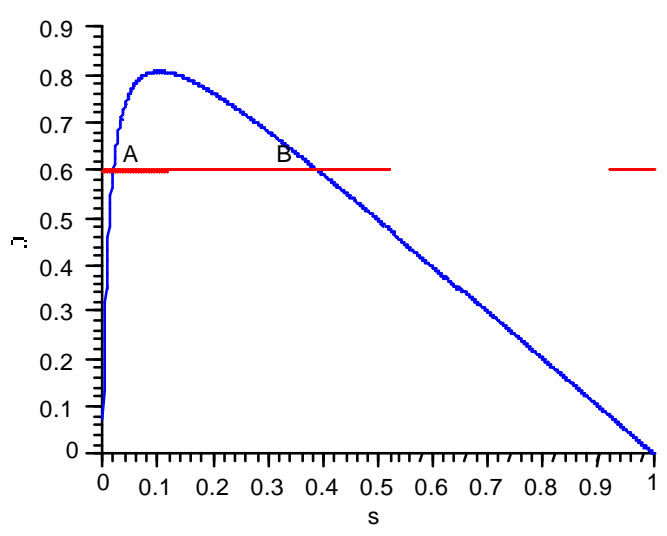

Figure 1. The saturation of the base state. Note the existence of two solutions (if $\omega<$ $\omega_{\max }$ ), at points A (vapor-dominated) and B (liquid-dominated). If $\omega>\omega_{\max }$, a steadystate heat pipe does not exist.

often referred to as "dryout", requires that superheated conditions exist below the twophase regime. In either case, the transition between single-phase and two-phase flow regimes is also controlled by capillary forces (Stubos et al., 1993). While transitions between single-phase and two-phase flow regimes are possible, a transition between the two different heat pipe regimes, namely from liquid-dominated to vapor-dominated or vice-versa, is not possible (Mc Guiness et al., 1993, Stubos et al., 1993). Field measurements and observations have confirmed the existence of VD and LD heat pipes in geothermal reservoirs. For example, the upper parts of the Geysers geothermal field in California (Thomas et al., 1981), the Larderello field in Italy (Pruess et al., 1987) and the Wairakei field in New Zealand (Allis and Hunt, 1986) are modeled as VD fields while LD zones are found in lower parts of the Larderello (Pruess et al., 1987).

Regardless of the particular application, the existence of a heat pipe regime either below an overlying liquid or above an underlying vapor raises questions of gravitational instability. Consider, for example, the case of a liquid-dominated heat pipe. Given that the heat pipe is of a lower (although not by much) density than the overlying liquid, the possibility of a Rayleigh-Taylor gravitational instability is clear (Drazin and Reid, 1981). 
The onset of natural convection in the overlying liquid layer, due to its variable temperature, becomes then an important factor. Recall that in porous media under singlephase flow conditions, the onset of natural convection requires that the single-phase Rayleigh number is greater than a specific value,

$$
R a=\frac{k H g \beta \Delta T}{\alpha \nu}>R a_{\min }=4 \pi^{2}
$$

(Lapwood, 1948, Gebhart et al., 1988). Here, $H$ is the thickness of the single-phase region, across which a temperature difference, $\Delta T$, is applied, $\beta$ is the thermal expansion coefficient of the liquid and $\alpha$ is the effective thermal diffusivity. Stabilizing factors include conduction, the phase change at the liquid-heat pipe interface, and capillary effects.

The stability of liquid-dominated heat pipes was explored by Ramesh and Torrance $(1990,1993)$ in the context of boiling in porous media. A window of unstable wave numbers was found to exist for Rayleigh numbers larger than a critical, $R a_{\min }$. Interestingly, the minimum critical value reported was about half of that for the onset of natural convection in single-phase flow, suggesting that the underlying two-phase region is destabilizing the flow. As in the single-phase case, stability at large wavelengths is associated with viscous flow, while that at smaller wavelengths is due to conduction. Pestov (1998) examined the stability of the two-phase region overlying a vapordominated heat pipe, which she found to be stable. However the stability of the combined vapor-dominated heat pipes has not been explored at this time. An earlier study of the stability of a liquid overlying a vapor zone (thus excluding the two-phase zone) was conducted by Straus and Schubert (1980). It was concluded that such systems are stable, provided that the permeability of the porous media is sufficiently low, the phase change at the interface being responsible for the stability. That conclusion was reached without considering the buoyancy in the single-phase zone, however.

Bau and Torrance (1982) conducted boiling experiments in a vertical circular cylinder heated from below and cooled from above. They observed the formation of a 
two-phase zone underlying a liquid zone. In the absence of convection, before the onset of boiling, the temperature profile in the single-phase region was found to be linear, as expected when conduction dominates. After boiling, an almost isothermal region was formed at the saturation temperature, based on which the vertical countercurrent flow of liquid and vapor was concluded. After exceeding a critical Rayleigh number, convection instabilities developed in both phases.

Vapor-dominated heat pipes find applications in similar contexts as liquiddominated heat pipes. An interesting visualization was provided recently by Kneafsy and Pruess (1999), who studied the mechanisms of heat pipes in a fracture, where superheated conditions were maintained below the two-phase region. Although that study focused mainly on the mechanics of liquid flow, many issues related to flow instability and the possibility that the downwards-percolating liquid may "penetrate" the superheated region, were raised. At present, the stability features of this configuration are not known. However, some of these features should be similar to the liquid-dominated case. For example, we should expect the onset of a natural convection mechanism for the vapor underlying the two-phase region, as well as a gravitional instability due to the two-phase region above being heavier (although only by a small amount) than the underlying vapor. The effect of the phase-transition at the interface is unclear, however, just as it has been unclear for the liquid-dominated heat pipes studied by Ramesh and Torrance (1990). In the context of other problems involving phase change in porous media, for example in steam injection processes for the recovery of heavy oil, condensation of steam at an advancing steam front is less destabilizing, than in non-condensing flows, due to the associated volume reduction. Conversely, the vaporization of liquid is more destabilizing, due to the associated volume expansion. Such effects, if present here, also need to be addressed.

In this paper, we study the linear stability of vapor-dominated (VD) heat pipes by following a linear stability approach, as also done for the LD case by Ramesh and Torrance $(1990,1993)$. In addition to the base-state configuration, other differences exist between our approach and that of Ramesh and Torrance. We consider an infinitely long two-phase zone (heat pipe), as there are no compelling reasons to restrict the two-phase region to a given length. The same difference applies also between our work and 
Pestov's (1998). The stability analysis is also conducted using analytical methods, which allow for an asymptotic treatment of the problem. The paper is organized as follows: First, we present a dimensionless formulation of the base state and discuss the properties of the vapor-dominated solution. Then, the linearized stability analysis is presented based on normal modes. Results and implications are discussed in a subsequent section. Finally, the results are compared with the stability predictions in the case of liquid-dominated systems, namely when the vapor-liquid counterflow underlies a liquid zone.

\section{MATHEMATICAL FORMULATION}

Consider the porous medium configuration shown in the schematic of Fig. 2. Due to the application of a heat flux, at the bottom of a region containing a single-phase liquid, a dryout region of thickness $H$, consisting of a superheated vapor of almost constant pressure, $P_{v}$, will ultimately develop at steady-state. Due to heat conduction, this region will be of a finite extent, and will underlie a two-phase (vapor-liquid) region of (almost) infinite extent. The boundary between the two regions is a planar interface, with saturation temperature corresponding to the saturation pressure, $P_{v}$, to be denoted as $T^{*}$ sat. Before the onset of convective instabilities the two-phase region (heat pipe) corresponds to the 1-D vapor-dominated branch of the solution of equation (1). We assume that the heat flux is sufficiently small so that equation (1) has a solution (namely $\omega<\omega_{\max }$ ). At base-state conditions, the heat transport is solely by conduction in the vapor zone and by convection in the two-phase zone (vertical counterflow). The temperature profile in the vapor zone is linear due to conductive heat transfer and is considered to be constant at the saturation temperature within the two-phase zone. If convective instability develops, heat transfer in both regions becomes convective. To describe the onset of instability we will first describe the mathematical formulation, then the base state and finally we will proceed with its stability analysis. 


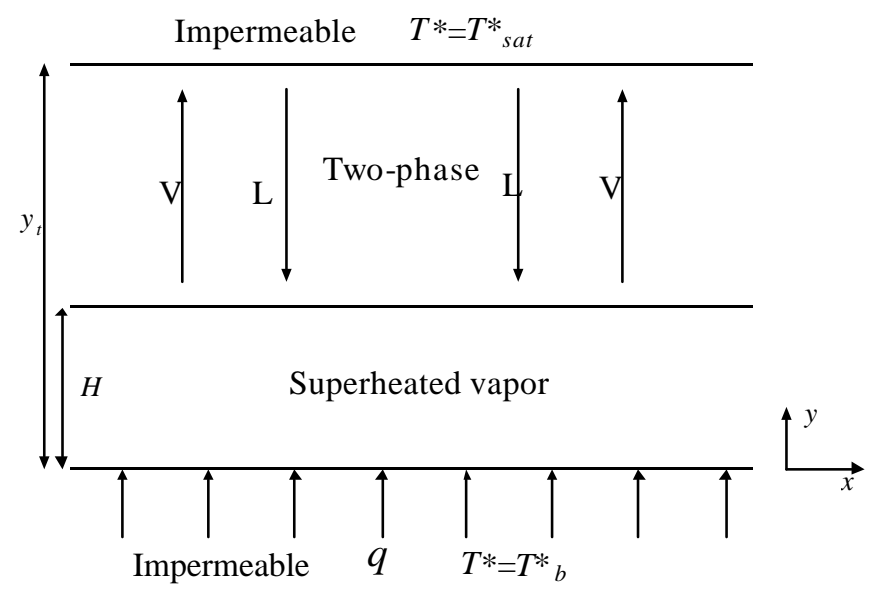

Figure 2. Schematic of a vapor-dominated heat pipe overlying a superheated layer. In our analysis, we will assume that conduction in the two-phase zone (heat pipe) is negligible, thus its temperature is practically constant and its extent practically infinite $\left(y_{t}>>H\right)$.

\section{Governing Equations}

In addition to the standard continuum assumptions, the following assumptions will be made:

1. The porous medium is uniform, isotropic, fully saturated with fluid, and has constant properties.

2. A conventional multi-phase Darcy description applies.

3. Capillary effects are neglected (see, however, Stubos et al., 1993).

4. The compressibility of the vapor is negligible, except in the buoyancy term, which constitutes a Boussinesq type approximation (see also below, however).

5. Heat conduction in the two-phase region is negligible, which is thus isothermal at the saturation temperature, and of practically infinite extent.

6. The relative permeabilities are linear functions of the liquid saturation.

The vapor zone is occupied by a single-phase (superheated steam) whose temperature is bounded between $T_{b}^{*}$ at the bottom and the saturation temperature at the top. In the following, asterisks will denote dimensional quantities, and subscripts $v, l$, and $r$ will 
represent vapor, liquid, and rock, respectively. Based on these assumptions, the following mass, momentum and energy balances can be formulated. In the following, the vertical coordinate $y$ points upwards.

\section{Vapor Region}

$$
\begin{gathered}
\frac{\partial\left(\phi \rho_{v}^{*}\right)}{\partial t^{*}}+\nabla^{*} \cdot\left(\mathrm{v}_{\mathrm{v}}^{*} \rho_{\mathrm{v}}^{*}\right)=0 \\
\mathrm{v}_{\mathrm{v}}^{*}=-\frac{\mathrm{k}}{\mu_{\mathrm{v}}}\left(\nabla^{*} \mathrm{p}^{*}-\rho_{\mathrm{v}}^{*} \mathrm{~g}\right) \\
\phi \rho_{\mathrm{v}} \frac{\partial \mathrm{h}_{\mathrm{v}}}{\partial t^{*}}+(1-\phi) \rho_{r} \frac{\partial \mathrm{h}_{\mathrm{r}}}{\partial t^{*}}+\rho_{\mathrm{v}} \mathrm{v}_{\mathrm{v}}^{*} \cdot \nabla^{*} \mathrm{~h}_{\mathrm{v}}=\mathrm{k}_{\mathrm{e}} \nabla^{* 2} \mathrm{~T}^{*}
\end{gathered}
$$

where $\phi$ is porosity, $\rho$ denotes density and $\boldsymbol{v}$ is velocity and $k_{e}$ is the effective thermal conductivity of the porous medium. Since $\phi$ and $\rho_{v}{ }^{*}$ are assumed constant, this further gives:

$$
\nabla^{*} \cdot\left(\mathrm{v}_{\mathrm{v}}^{*}\right)=0
$$

The enthalpy of the superheated vapor $h_{v}$ is given by:

$$
h_{v}=h_{v s}+\Delta h_{s}=h_{v s}+C_{p v}\left(T^{*}-T_{s a t}^{*}\right)
$$

Two-Phase Region

$$
\begin{aligned}
& \frac{\partial}{\partial t^{*}}\left(\phi \rho_{\mathrm{v}}^{*} \mathrm{~s}_{\mathrm{v}}+\phi \rho_{1}^{*} \mathrm{~s}_{1}\right)+\nabla^{*}\left(\mathrm{v}_{\mathrm{v}}^{*} \rho_{\mathrm{v}}^{*}+\mathrm{v}_{1}^{*} \rho_{1}^{*}\right)=0 \\
& \mathrm{v}_{\mathrm{v}}^{*}=-\frac{\mathrm{k} \mathrm{k}_{\mathrm{rv}}}{\mu_{\mathrm{v}}}\left(\nabla^{*} \mathrm{p}^{*}-\rho_{\mathrm{v}}^{*} \mathrm{~g}\right)
\end{aligned}
$$




$$
\begin{gathered}
\mathrm{v}_{1}^{*}=-\frac{\mathrm{kk}_{\mathrm{rl}}}{\mu_{1}}\left(\nabla^{*} \mathrm{p}^{*}-\rho_{1}^{*} \mathrm{~g}\right) \\
\frac{\partial}{\partial t^{*}}\left(\phi\left(\rho_{\mathrm{v}}^{*} \mathrm{~h}_{\mathrm{v}} \mathrm{s}_{\mathrm{v}}+\rho_{1}^{*} \mathrm{~h}_{1} \mathrm{~s}_{1}\right)+(1-\phi) \rho_{\mathrm{r}} \mathrm{h}_{\mathrm{r}}\right)+\nabla^{*}\left(\rho_{1} \mathrm{v}_{\mathrm{l}}^{*} \mathrm{~h}_{\mathrm{l}}+\rho_{\mathrm{v}} \mathrm{v}_{\mathrm{v}}^{*} \mathrm{~h}_{\mathrm{v}}\right)=\mathrm{k}_{\mathrm{e}} \nabla^{* 2} \mathrm{~T}^{*}
\end{gathered}
$$

where $s$ denotes the liquid saturation, namely the volumetric faction of the pore volume occupied by the phase, and $k_{r l}$ and $k_{r v}$ are taken to be linear functions of the liquid-phase saturation

$$
k_{r l}=s \text { and } k_{r}=1-s
$$

The two regions are further coupled with the following boundary and interface conditions.

\section{Boundary and Interface Conditions}

The bottom boundary (at $y=0$ ) is impermeable

$$
\mathrm{v}_{\mathrm{v}} \cdot \mathrm{n}=0
$$

where $\boldsymbol{n}$ is the unit normal vector, and at constant temperature

$$
T^{*}=T_{b}^{*}
$$

At the interface between the vapor zone and the two-phase zone $(y=\delta)$ the temperature is at its saturation value

$$
T^{*}=T_{s a t}^{*}
$$

while continuity of mass, energy and momentum leads to the following

$$
\left(\rho_{\mathrm{v}} \mathrm{v}_{\mathrm{v}}^{*}+\rho_{1} \mathrm{v}_{1}^{*}\right)^{+} \cdot n-\left(\rho_{\mathrm{v}} \mathrm{v}_{\mathrm{v}}^{*}+\rho_{1} \mathrm{v}_{1}^{*}\right)^{-} \cdot n=\left[\left(\phi \rho_{\mathrm{v}} s_{\mathrm{v}}+\phi \rho_{1} s_{1}\right)^{+}-\left(\phi \rho_{\mathrm{v}} s_{\mathrm{v}}+\phi \rho_{1} s_{1}\right)^{-}\right] \cdot \mathrm{v}_{\mathrm{i}}^{*} \cdot n
$$




$$
\begin{gathered}
\left(\rho_{1} \mathrm{v}_{1}^{*} h_{1}+\rho_{\mathrm{v}} \mathrm{v}_{\mathrm{v}}^{*} h_{\mathrm{v}}-k_{e} \nabla^{*} T^{*}\right)^{+} \cdot n-\left(\rho_{1} \mathrm{v}_{1}^{*} h_{1}+\rho_{\mathrm{v}} \mathrm{v}_{\mathrm{v}}^{*} h_{\mathrm{v}}-k_{e} \nabla^{*} T^{*}\right)^{-} \cdot n= \\
{\left[\phi\left(\rho_{\mathrm{v}} h_{\mathrm{v}} s_{\mathrm{v}}+\rho_{1} h_{1} s_{1}\right)^{+}-\phi\left(\rho_{\mathrm{v}} h_{\mathrm{v}} s_{\mathrm{v}}+\rho_{1} h_{1} s_{1}\right)^{-}\right] \mathrm{v}_{\mathrm{i}}^{*} \cdot n} \\
p^{*+}=p^{*-}
\end{gathered}
$$

where $v_{i}{ }^{*}$ is the velocity of interface, superscripts + and - indicate vapor and liquid, and $n$ is the unit normal vector. Finally, the top of the heat pipe region (at $y=y_{t}$ ? 8 ) is impermeable

$$
\left.\overline{\left(\rho_{\mathrm{v}}\right.} \mathrm{v}^{*}{ }_{\mathrm{v}}+\mathrm{v}^{*}{ }_{1}\right) \cdot n=0
$$

where we defined the density ratio $\overline{\rho_{\mathrm{v}}} \equiv \frac{\rho_{\mathrm{v}}}{\rho_{1}}$.

To proceed further, we will recast the equations in dimensionless form, by using the characteristic variables $H^{2} / \alpha_{v}, H, v_{\mathrm{v}} \alpha_{\mathrm{v}} / \mathrm{k} \rho_{\mathrm{vr}}$ for time, length and pressure and using the dimensionless temperature $\quad T=\frac{T^{*}-T_{s a t}^{*}}{T_{b}^{*}-T_{s a t}^{*}}$, where e introduced the vapor thermal diffusivity $a_{v}=\frac{k_{e}}{\rho_{v} C_{p v}}$. We then, obtain the dimensionless governing equations:

\section{Vapor Region}

$$
\begin{gathered}
\nabla \cdot\left(\mathrm{v}_{\mathrm{v}}\right)=0 \\
\mathrm{v}_{\mathrm{v}}=-\nabla p-R a T j
\end{gathered}
$$

where $\boldsymbol{j}$ is the upwards pointing unit vector,

\section{Two-phase Region}

$$
\beta_{2} \frac{\partial s}{\partial t}+\nabla \cdot\left(\overline{\rho_{\mathrm{v}}} \mathrm{v}_{\mathrm{v}}+\mathrm{v}_{1}\right)=0
$$




$$
\begin{gathered}
\mathrm{v}_{1}=-\frac{k_{\mathrm{rl}}}{\overline{\mu_{\mathrm{v}}}}\left(\nabla p-R a_{2 \phi} \frac{1}{\overline{\rho_{\mathrm{v}}}} j\right) \\
\mathrm{v}_{\mathrm{v}}=-k_{\mathrm{rv}} \nabla p
\end{gathered}
$$

and

$$
-\phi \frac{\partial s}{\partial t}+\nabla\left(\mathrm{v}_{\mathrm{v}}\right)=0
$$

where

$$
\beta_{2}=\phi\left(1-\overline{\rho_{\mathrm{v}}}\right)
$$

\section{Interface conditions}

$$
\begin{gathered}
{\left[\left(\mathrm{v}_{1}^{+}+\overline{\rho_{\mathrm{v}}} \mathrm{v}_{\mathrm{v}}^{+}\right)-\overline{\rho_{\mathrm{v}}} \mathrm{v}_{\mathrm{v}}^{-}-\left[\phi s\left(1-\overline{\rho_{\mathrm{v}}}\right)\right] \mathrm{v}_{\mathrm{i}}\right] \cdot n=0} \\
{\left[\lambda\left(\mathrm{v}_{\mathrm{v}}^{+}-\mathrm{v}_{\mathrm{v}}^{-}\right)+(\phi s \lambda) \mathrm{v}_{\mathrm{i}}+\nabla T^{-}\right] \cdot n=0} \\
p^{+}=p^{-}
\end{gathered}
$$

In the above, we defined the single-phase Rayleigh number $R a=\frac{k H g \beta_{\mathrm{g}}\left(T_{b}^{*}-T_{s a t}^{*}\right)}{\alpha_{\mathrm{v}} \mathrm{v}_{\mathrm{v}}}$, the two-phase Rayleigh number, $R a_{2 \phi}=\frac{k H g\left(1-\overline{\rho_{\mathrm{v}}}\right)}{\alpha_{\mathrm{v}} v_{\mathrm{v}}}$ the ratio of heat capacities of vapor and rock, $\beta_{1}=\frac{\phi \rho_{v} C p_{v}+(1-\phi) \rho_{r} C p_{r}}{\rho_{v} C p_{v}}$, the ratio of liquid and vapor viscosities, $\overline{\mu_{\mathrm{v}}}=\frac{\mu_{1}}{\mu_{\mathrm{v}}}$, and the dimensionless latent heat $\lambda=\frac{L_{\mathrm{v}}}{C_{p v}\left(T_{b}^{*}-T_{s a t}^{*}\right)}$. 


\section{STABILITY ANALYSIS}

To carry out a linear stability analysis, all dependent variables are perturbed in the transverse direction, $x$, and the rates of growth of these disturbances is sought in terms of normal modes. Thus, we take disturbances of the form

$$
\begin{aligned}
& T=T_{0}+\varepsilon T_{1} \\
& p=p_{0}+\varepsilon p_{1} \\
& s=s_{0}+\varepsilon s_{1} \\
& \delta=1+\varepsilon \delta_{1}
\end{aligned}
$$

where $\varepsilon$ is a small parameter, and where the base state (subscript 0 ) satisfies

$$
\begin{gathered}
\frac{d p_{0}}{d y}=R a T_{0} \\
T_{0}=1-y
\end{gathered}
$$

in the vapor region and

$$
\begin{gathered}
\frac{d p_{0}}{d y}=-\frac{\omega M}{k_{\mathrm{rv}}} \\
\mathrm{v}_{10}=M \omega \\
T_{0}=0 \\
s=0
\end{gathered}
$$

in the two-phase region. In the above, we have neglected capillary effects. The perturbations are taken in terms of normal modes 


$$
\begin{aligned}
& T_{1}=\theta(y) \exp (i \kappa \mathrm{x}+\sigma \mathrm{t}) \\
& p_{1}=\pi(y) \exp (i \kappa \mathrm{x}+\sigma \mathrm{t}) \\
& s_{1}=\Sigma(y) \exp (i \kappa \mathrm{x}+\sigma \mathrm{t}) \\
& \delta_{1}=\Delta \exp (i \kappa \mathrm{x}+\sigma \mathrm{t})
\end{aligned}
$$

where $\kappa$ is the wavenumber and $\sigma$ is the rate of growth of the disturbance. These expansions are then substituted in the governing equations and the boundary conditions, and the system is linearized. The final results for the eigenvalue problem are shown below.

\section{Vapor:}

$$
\begin{aligned}
& \frac{\partial \mathrm{u}_{\mathrm{v} 1}}{\partial \mathrm{x}}+\frac{\partial \mathrm{w}_{\mathrm{v} 1}}{\partial \mathrm{y}}=0 \\
& \mathrm{u}_{\mathrm{v} 1}=-\frac{\partial p_{1}^{-}}{\partial x} \\
& \mathrm{w}_{\mathrm{v} 1}=-\frac{\partial p_{1}^{-}}{\partial y}+R a T_{1} \\
& \beta_{1} \frac{\partial T_{1}}{\partial t}-\mathrm{w}_{\mathrm{v} 1}=\frac{\partial^{2} T_{1}}{\partial x^{2}}+\frac{\partial^{2} T_{1}}{\partial y^{2}}
\end{aligned}
$$

Two-phase:

$$
\begin{aligned}
& \frac{\partial \mathrm{u}_{\mathrm{vl}}}{\partial \mathrm{x}}+\frac{\partial \mathrm{w}_{\mathrm{vl}}}{\partial \mathrm{y}}=\phi \frac{\partial s_{1}}{\partial t} \\
& \frac{\partial \mathrm{u}_{l 1}}{\partial \mathrm{x}}+\frac{\partial \mathrm{w}_{l 1}}{\partial \mathrm{y}}=-\phi \frac{\partial s_{1}}{\partial t} \\
& \mathrm{u}_{l 1}=-\frac{k_{\mathrm{r} 10}}{\mu_{\mathrm{v}}} \frac{\partial p_{1}^{+}}{\partial x}
\end{aligned}
$$




$$
\begin{aligned}
& \mathrm{w}_{l 1}=-\frac{1}{\mu_{\mathrm{v}}}\left[k_{\mathrm{r} 10} \frac{d p_{1}^{+}}{d y}+\frac{d k_{\mathrm{rl}}}{\mathrm{ds}}\left(\frac{d p_{0}^{+}}{d y}-\frac{R a_{2 \phi}}{\rho_{\mathrm{v}}}\right) s_{1}\right] \\
& \mathrm{u}_{\mathrm{v} 1}=-k_{r \mathrm{v} 0} \frac{d p_{1}^{+}}{d x} \\
& \mathrm{w}_{\mathrm{v} 1}=-k_{r \mathrm{v} 0} \frac{d p_{1}^{+}}{d y}-\frac{d k_{r \mathrm{v}}}{\mathrm{ds}} \frac{d p_{0}^{+}}{d y} s_{1}
\end{aligned}
$$

Now by substituting Eqns. (37) into (38) and (39), we obtain the final form of the equations as follows:

Vapor Region (denoted by superscript - where appropriate)

$$
\begin{gathered}
\kappa^{2} \pi^{-}+R a \frac{d \theta}{d y}-\frac{d^{2} \pi^{-}}{d y^{2}}=0 \\
\left(\beta_{1} \sigma-R a+\kappa^{2}\right) \theta+\frac{d \pi^{-}}{d y}-\frac{d^{2} \theta}{d y^{2}}=0
\end{gathered}
$$

Two-phase (denoted by superscript + where appropriate)

$$
\begin{aligned}
& \kappa^{2} \pi^{+}+\frac{\phi \sigma \overline{\mu_{\mathrm{v}}}}{k_{r l 0}} \Sigma-\frac{1}{k_{r l 0}}\left(\frac{d p_{0}^{+}}{d y}+\frac{R a_{2 \phi}}{\overline{\rho_{\mathrm{v}}}}\right) \frac{d \Sigma}{d y}-\frac{d^{2} \pi^{+}}{d y^{2}}=0 \\
& \kappa^{2} \pi^{+}-\frac{\phi \sigma}{k_{r v 0}} \Sigma+\frac{1}{k_{r v 0}} \frac{d p_{0}^{+}}{d y} \frac{d \Sigma}{d y}-\frac{d^{2} \pi^{+}}{d y^{2}}=0
\end{aligned}
$$

The differential equations in these regions are to be solved subject to boundary conditions of constant temperature and zero vapor flux at $\mathrm{y}=0$, no-flux conditions for vapor and liquid at $\mathrm{y}=\infty$, and continuity of mass, energy, temperature and pressure at the interface $(\mathrm{y}=\delta)$. These are: 
At $y=0$ :

$$
\left\{\begin{array}{l}
\theta=0 \\
\frac{d \pi^{-}}{d y}=0
\end{array}\right.
$$

At $y=y_{t}$ :

$$
\left\{\begin{array}{l}
-k_{r \mathrm{v} 0} \frac{d \pi^{+}}{d y}+\frac{d p_{0}^{+}}{d y} \Sigma=0 \\
-\frac{k_{r l 0}}{\overline{\mu_{\mathrm{v}}}} \frac{d \pi^{+}}{d y}-\frac{1}{\overline{\mu_{\mathrm{v}}}}\left(\frac{d p_{0}^{+}}{d y}+\frac{R a_{2 \phi}}{\overline{\rho_{\mathrm{v}}}}\right) \Sigma=0
\end{array}\right.
$$

At $\mathrm{y}=1$ :

$$
\begin{aligned}
& \theta=\Delta \\
& \pi^{-}-\pi^{+}+\frac{\Delta}{\lambda k_{r v 0}}=0 \\
& \left(\overline{\rho_{\mathrm{v}} R a}\right) \theta+\left(\gamma_{1} \sigma\right) \Delta+\left[\frac{1}{\overline{\mu_{\mathrm{v}}}}\left(\frac{d p_{0}^{+}}{d y}+\frac{R a_{2 \phi}}{\overline{\rho_{\mathrm{v}}}}\right)-\frac{d p_{0}^{+}}{d y} \overline{\rho_{\mathrm{v}}}\right] \Sigma+\left(\frac{k_{r l 0}}{\overline{\mu_{\mathrm{v}}}}+\overline{\rho_{\mathrm{v}}} k_{r \mathrm{v} 0}\right) \frac{d \pi^{+}}{d y}-\overline{\rho_{\mathrm{v}}} \frac{d \pi^{-}}{d y}=0 \\
& (\lambda R a) \theta-\left(\gamma_{2} \sigma\right) \Delta-\left(\lambda \frac{d p_{0}^{+}}{d y}\right) \Sigma-\frac{d \theta}{d y}-\lambda \frac{d \pi-}{d y}+\lambda k_{r \mathrm{v} 0} \frac{d \pi^{+}}{d y}=0
\end{aligned}
$$

where $\gamma_{1}$ and $\gamma_{2}$ are defined as:

$$
\left\{\begin{array}{l}
\gamma_{1}=\phi\left(1-\overline{\rho_{\mathrm{v}}}\right) s_{0} \\
\gamma_{2}=\phi \lambda s_{0}
\end{array}\right.
$$

For the stability condition, the rate of growth, $\sigma$, has to be determined. Positive rates of growth $(\sigma>0)$ indicate that disturbances will be amplified at an exponential rate with respect to time, leading the system into instability. On the other hand, a non-positive rate 
of growth $(\sigma \leq 0)$ indicates that the system will be stable and disturbances will decay or at most stay constant.

By incorporating the boundary conditions we obtain a fourth-order homogeneous linear system, the determinant of which must vanish for a non-trivial solution to exist. We note that because we have neglected the compressibility of the vapor, the saturation disturbance in our problem turns out to be zero (in contrast to Pestov, 1998). The vanishing of the determinant provides the solution for the rate of growth $\sigma$ (or $\sigma^{*}$ ) as a function of the wavenumber $\kappa$ and the various dimensionless parameters, among which key roles are played by the Rayleigh number and $\omega$. The solution of these equations can be found analytically and numerically and both forms are presented here. The analytical solution assists us to better understand and investigate the asymptotic behavior of the system at small wavenumbers. It also serves to verify the results obtained from the more flexible numerical approach. Details of the procedure follow.

\section{Analytical Solution}

The solution of the eigenvalue problem is the vanishing of the determinant of the following matrices, depending on the value of $\kappa^{2}+\beta_{1} \sigma$.

For $\kappa^{2}+\beta_{1} \sigma \geq R a$ :

$$
\mathrm{F}_{\mathrm{r}}=\left[\begin{array}{cccc}
\tanh \left(r_{1}\right) & \tanh \left(r_{2}\right) & 1 & 0 \\
n_{1} & n_{2} & \frac{-1}{\lambda k_{r v}} & -1 \\
r_{1} n_{1} \tanh \left(r_{1}\right) & r_{2} n_{2} \tanh \left(r_{2}\right) & R a+\beta_{1} \sigma \zeta & c_{1} \kappa \\
r_{1}\left(\frac{1}{\lambda}+n_{1} \tanh \left(r_{1}\right)\right) & r_{2}\left(\frac{1}{\lambda}+n_{2} \tanh \left(r_{2}\right)\right) & R a-\beta_{1} \sigma \zeta & k_{r \mathrm{v}} \kappa
\end{array}\right]
$$

and for $\kappa^{2}+\beta_{1} \sigma<R a$ : 


$$
\mathrm{F}_{i}=\left[\begin{array}{cccc}
\tanh \left(r_{1}\right) & \sin \left(r_{3}\right) & 1 & 0 \\
n_{1} & n_{3} & \frac{-1}{\lambda k_{r \mathrm{v}}} & -1 \\
r_{1} n_{1} \tanh \left(r_{1}\right) & r_{3} n_{4} & R a+\beta_{1} \sigma \varsigma & c_{1} \kappa \\
r_{1}\left(\frac{1}{\lambda}+r_{1} n_{1} \tanh \left(r_{1}\right)\right) & r_{3} n_{4}+\frac{r_{3} \cos \left(r_{3}\right)}{\lambda} & R a-\beta_{1} \sigma \zeta & k_{r \mathrm{v}} \kappa
\end{array}\right]
$$

where the various parameters are defined as follows

$$
\begin{aligned}
& r_{1}=\sqrt{\frac{2 \kappa^{2}+\beta_{1} \sigma+\sqrt{\left(\beta_{1} \sigma\right)^{2}+4 \kappa^{2} R a}}{2}} \\
& r_{2}=\sqrt{\frac{2 \kappa^{2}+\beta_{1} \sigma-\sqrt{\left(\beta_{1} \sigma\right)^{2}+4 \kappa^{2} R a}}{2}} \\
& r_{3}=\sqrt{\frac{-2 \kappa^{2}-\beta_{1} \sigma+\sqrt{\left(\beta_{1} \sigma\right)^{2}+4 \kappa^{2} R a}}{2}} \\
& n_{1}=r_{1}-\frac{\beta_{1} \sigma-R a+\kappa^{2}}{r_{1}} \\
& n_{2}=r_{2}-\frac{\beta_{1} \sigma-R a+\kappa^{2}}{r_{2}} \\
& \left.n_{3}=\frac{\phi\left(1-\frac{\rho_{\mathrm{v}}}{\rho_{0}}\right) s_{0}}{\rho_{\mathrm{v}} \beta_{1}}\right) \sin \left(r_{3}\right) \\
& \left.n_{4}=-\frac{\beta_{1} \sigma-R a+\kappa^{2}}{r_{3}}\right) \cos \left(r_{3}\right) \\
& \left.r_{3}+\frac{\beta_{1} \sigma-R a+\kappa^{2}}{r_{3}}\right)
\end{aligned}
$$

It is important to note that because we have assumed incompressible vapor and isothermal two-phase zone, the saturation disturbance in the problem turns out to be identically equal to zero. Vanishing of the determinant provides the solution for the rate of growth, $\sigma$, as a function of the wave number, $\kappa$, and the various dimensionless 
parameters, among which key roles are played by the Rayleigh number and the dimensionless heat flux.

The solution of the above equations was found numerically. The results were also confirmed by the full numerical solution of the problem as discussed below. However, analytical solutions were possible by considering the asymptotic behavior of the equations in the small wavenumber limit. Then, it is not difficult to show that in the small $\kappa$ limit we have the asymptotic behavior

$$
\sqrt{-\sigma^{*}} \tan \left(\sqrt{-\sigma^{*}}\right)=\kappa
$$

This equation admits infinitely many solutions (as also confirmed numerically) the largest of which approaches $\left(-\pi^{2} / 4\right)$, as $\kappa$ approaches zero. Hence, the system is stable at long wavelengths, but perhaps unstable at intermediate values.

\section{Numerical Solution}

The system of differential equations (40)-(45) can also be written as a system of first-order ordinary differential equations

$$
\begin{gathered}
\frac{d X_{\mathrm{v}}}{d y}=M_{\mathrm{v}} X_{\mathrm{v}} \\
\frac{d X_{2 p}}{d y}=M_{2 p} X_{2 p}
\end{gathered}
$$

where: $\quad X_{\mathrm{v}}=\left[\begin{array}{c}\frac{d \pi^{-}}{d y} \\ \frac{d \theta}{d y} \\ \pi^{-} \\ \theta\end{array}\right]$ and $X_{2 p}=\left[\begin{array}{c}\frac{d \pi^{+}}{d y} \\ \pi^{+} \\ \Sigma\end{array}\right] . \mathrm{M}_{\mathrm{v}}$ is a 4 by 4 matrix defined as: 


$$
M_{\mathrm{v}}=\left[\begin{array}{cccc}
0 & R a & \kappa^{2} & 0 \\
1 & 0 & 0 & \beta_{1} \sigma-R a+\kappa^{2} \\
1 & 0 & 0 & 0 \\
0 & 1 & 0 & 0
\end{array}\right]
$$

while matrix $\mathrm{M}_{2 \mathrm{p}}$ is defined as:

$$
M_{2 p}=\left[\begin{array}{ccc}
0 & \kappa^{2} & A_{6} \\
1 & 0 & 0 \\
0 & 0 & A_{5}
\end{array}\right]
$$

where the various coefficients are

$$
\begin{aligned}
& A_{1}=\frac{\overline{\mu_{\mathrm{v}}} \phi \sigma}{K_{r l}} \\
& A_{2}=\frac{1}{k_{r l}}\left(\frac{d p_{0}^{+}}{d y}+\frac{R a_{2 \phi}}{\overline{\rho_{\mathrm{v}}}}\right) \\
& A_{3}=\frac{\phi \sigma}{k_{r \mathrm{v}}} \\
& A_{4}=\frac{1}{k_{r v}} \frac{d p_{0}^{+}}{d y} \\
& A_{5}=\frac{A_{1}+A_{3}}{A_{2}+A_{4}} \\
& A_{6}=A_{1}-A_{2} * A_{5}
\end{aligned}
$$

In matrix form, the boundary conditions are:

$$
\text { At } \mathrm{y}=0 \text { : } \quad \mathrm{BC}_{1} \times \mathrm{X}_{\mathrm{v}}=0
$$

where, matrix $\mathrm{BC}_{1}$ is 


$$
\begin{aligned}
\mathrm{BC}_{1}=\left[\begin{array}{cccc}
1 & 0 & 0 & 0 \\
0 & 0 & 0 & 1
\end{array}\right] \\
\text { At } \mathrm{y}=1: \quad \quad \mathrm{BC}_{2} \times\left[\begin{array}{c}
X_{\mathrm{v}} \\
X_{2 p}
\end{array}\right]=0
\end{aligned}
$$

where, matrix $\mathrm{BC}_{2}$ is

$$
\mathrm{BC}_{2}=\left[\begin{array}{ccccccc}
0 & 0 & 1 & \frac{1}{\lambda k_{r v}} & 0 & -1 & 0 \\
-\overline{\rho_{\mathrm{v}}} & 0 & 0 & \overline{\rho_{\mathrm{v}}} R a+\gamma_{1} \sigma & \frac{k_{r l 0}}{\overline{\mu_{\mathrm{v}}}}+\overline{\rho_{\mathrm{v}}} k_{r v 0} & 0 & \frac{1}{\overline{\mu_{\mathrm{v}}}}\left(\frac{d p_{0}^{+}}{d y}+\frac{R a_{2 \phi}}{\overline{\rho_{\mathrm{v}}}}\right)-\frac{d p_{0}^{+}}{d y} \overline{\rho_{\mathrm{v}}} \\
-\lambda & -1 & 0 & \lambda R a-\gamma_{2} \sigma & \lambda k_{r v 0} & 0 & -\lambda \frac{d p_{0}^{+}}{d y}
\end{array}\right]
$$

At $\mathrm{y}=\mathrm{y}_{\mathrm{t}}: \quad \quad \mathrm{BC}_{3} \times \mathrm{X}_{2 \mathrm{p}}=0$

where, matrix $\mathrm{BC}_{3}$ is

$$
\mathrm{BC}_{3}=\left[\begin{array}{ccc}
-k_{r v 0} & 0 & \frac{d p_{0}^{+}}{d y} \\
\frac{k_{r l 0}}{\overline{\mu_{\mathrm{v}}}} & 0 & \frac{1}{\overline{\mu_{\mathrm{v}}}}\left(\frac{d p_{0}^{+}}{d y}+\frac{R a_{2 \phi}}{\overline{\rho_{\mathrm{v}}}}\right)
\end{array}\right]
$$

The shooting method described in Davey (1973) was used to solve Equations (48) through (57). The characteristic equation for stability is obtained as the determinant of the following $14 x 14$ matrix:

$$
\mathrm{F}_{n}=\operatorname{det}\left[\begin{array}{cccc}
B C_{1} & 0 & 0 & 0 \\
B_{\mathrm{v}} & -I & 0 & 0 \\
0 & B C_{2} & 0 & \\
0 & 0 & B_{2 p} & -I \\
0 & 0 & 0 & B C_{3}
\end{array}\right]=\mathrm{F}_{\mathrm{n}}(R a, \omega, \sigma, \mathrm{K})=0
$$




\section{RESULTS}

Figure 3 shows a typical graph of $\sigma^{*}=\sigma \beta_{1}$ versus $\kappa$ at a constant heat flux and Rayleigh number greater than the critical. Typically, we find that for small and large wave numbers the system is stable and a window of unstable wave numbers exists for Rayleigh numbers larger than the critical value. Stability at large wavelengths (small $\kappa$ 's) is associated with viscous flow, while that at smaller wavelengths is due to conduction. Typically, we find that the long waves are stable (see inset of Figs. 3 and 4 below). An asymptotic analysis shows that in the limit $\kappa \rightarrow 0$, we have

$$
\sqrt{-\sigma^{*}} \tan \left(\sqrt{-\sigma^{*}}\right)=\kappa
$$

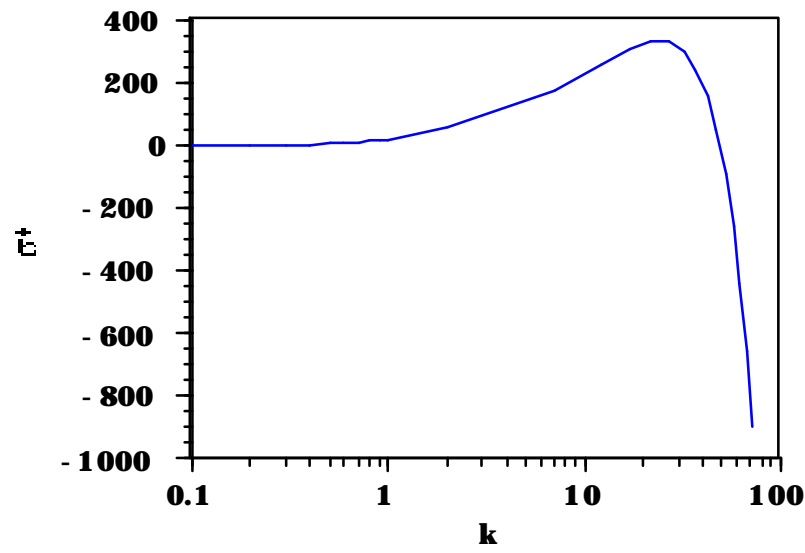

(a)

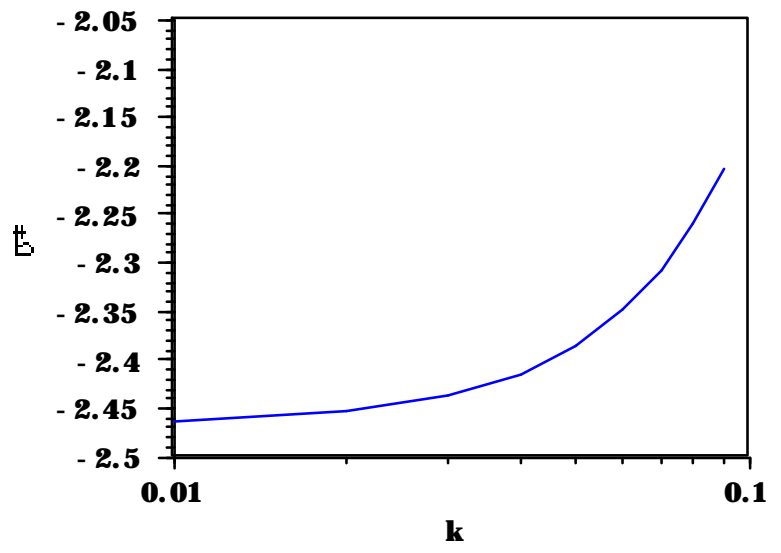

(b)

Figure 3. (a) The $\sigma^{*}-\kappa$ relation for $\mathrm{Ra}>\mathrm{Ra}_{\text {crit }}$ (b) Inset of the plot at small $\kappa$.

where $\kappa \ll 1$. This equation admits infinitely many solutions (which was confirmed numerically as well) the algebraically largest of which is $\left(-\pi^{2} / 4\right)$, as $\kappa$ approaches zero. This is indeed confirmed in Fig. $3 \mathrm{~b}$ and Fig. 4 (see below). The behavior is similar to the boiling problem of Ramesh and Torrance (1993) with an infinite two-phase region. Intermediate wavenumbers can be unstable, depending on whether or not the Rayleigh number is larger than a critical number, as discussed below. Sufficiently small wavelengths are stable, as in Ramesh and Torrance (1990).

Numerically accurate results were obtained by imposing the condition of vanishing of the fourth-order determinant. There are basically two parameters, that can 
vary independently, $\omega$, which contains the dimensionless heat flux, and the two-phase Rayleigh number M. Note that the conventional Rayleigh number is related to these parameters via

$$
R a=\omega M^{2} N
$$

where we introduced the additional dimensionless parameter $N=\left(h_{f g} \rho_{v}\right) /\left[C_{p v}\left(\rho_{1}-\rho_{v}\right)\right.$ $\left.\mathrm{t}_{\mathrm{sat}}\right]$.

Figure 4 shows the rate of growth vs. the wavenumber curve for fixed $M$ and variable $\omega$. It is to be noted that $\omega$ cannot exceed a maximum value, for a heat pipe region to exist. Figure 4 shows that as $\omega$ decreases, the configuration is less unstable, reflecting the facts that the density of the heat pipe region diminishes at smaller $\omega$ and that the overall Rayleigh number is also smaller.

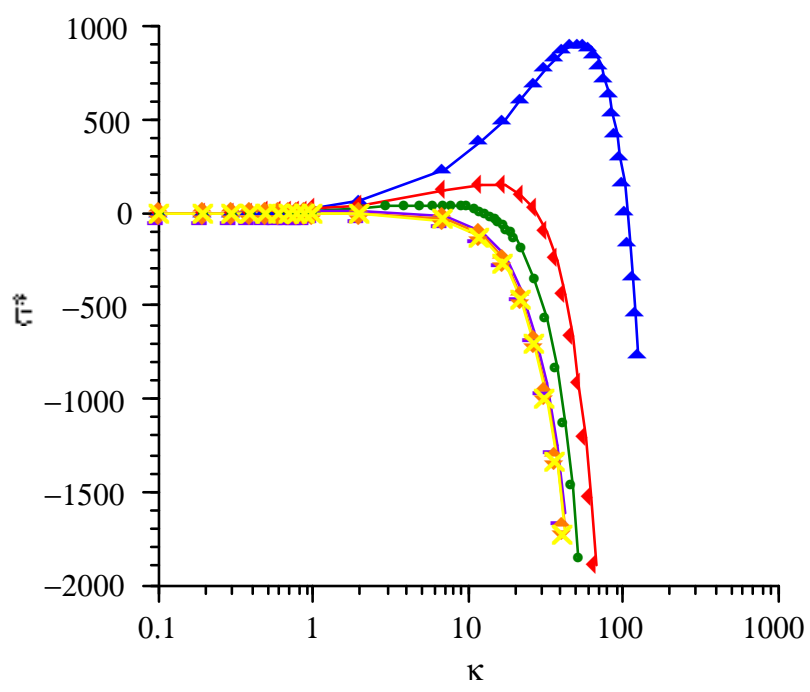

(a) 


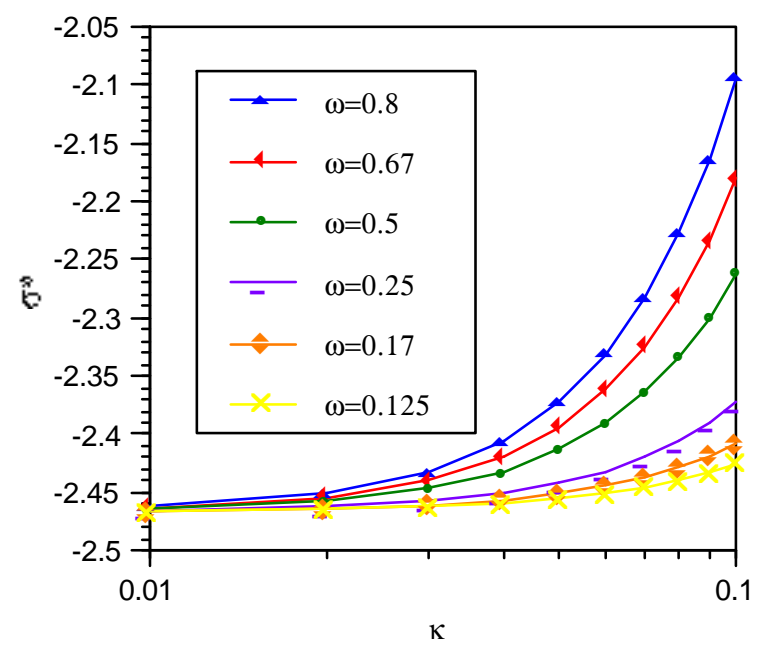

(b)

Figure 4. (a) The $\sigma^{*}-\kappa$ relation for different values of $\omega$ at constant $M=50$. (b) Inset of the plot at small $\kappa$.

Figure 5 shows corresponding result for the case of fixed $\omega$ and variable $M$. The configuration is shown to become more destabilized as the two-phase Rayleigh number $\mathrm{M}$ increases. In all these calculations the other parameters affecting the Rayleigh number definition above, for example the latent heat of vaporization, were held constant. Under this condition, it was found that all the data collapsed on the same curve, if variables $\omega$ and $\mathrm{M}$ were combined so that the Rayleigh number was constant. However, this should not be interpreted to imply that the Rayleigh number is the only relevant parameter of the problem (see below). 


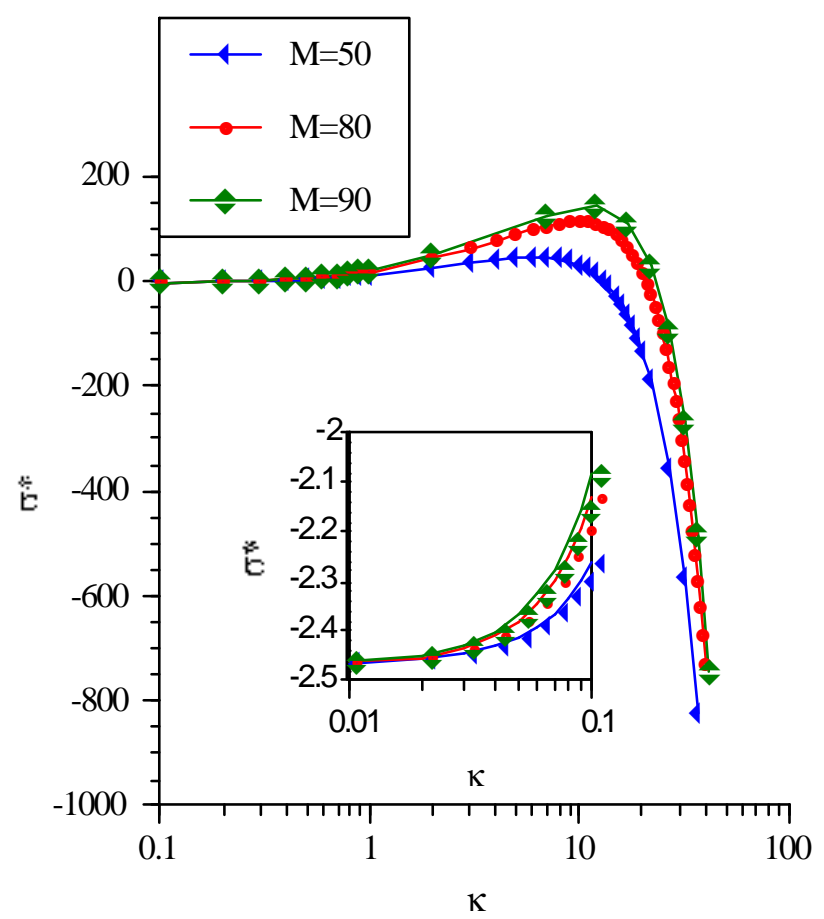

Figure 5. The $\sigma^{*}-\kappa$ relation for different values of $M$ at constant $\omega=0.5$, and inset of the plot at small $\kappa$.

As shown by Ramesh and Torrance (1990), a critical Rayleigh number, $\mathrm{Ra}_{\text {crit }}$, exists which is a function of the parameters of the problem, and in particular $\omega$. Fig. 6 shows a plot of $\mathrm{Ra}_{\text {crit }}$ versus $\omega$ obtained assuming constant latent heat. It is shown that $\mathrm{Ra}_{\text {crit }}$ is considerably smaller than the critical number corresponding to either single-phase natural convection or the liquid dominated problem treated by Ramesh and Torrance (1990). Furthermore, $\mathrm{Ra}_{\text {crit }}$ is found to increase as $\omega$ decreases. Both Figs. 5 and 6 are demonstrating that at higher $\omega$ the system is more unstable and also that the effect of buoyancy at two-phase zone is not as important as the effect of Ra and $\omega$. The effect of $\omega$ on stability is further investigated in the asymptotic limit, leading to almost the same behavior as for the single-phase problem, as explained in the next section.

To test the effect of the phase change process, we considered the sensitivity of the results to the latent heat, by keeping the Rayleigh number constant. Results are shown in Fig. 7. The problem becomes more unstable as the latent heat decreases, indicating the smaller energy requirements to sustain a destabilizing heat pipe above the vapor region as the latent heat is smaller. By changing Lv, but keeping the Rayleigh number constant, $\omega$ 
will vary in inverse proportion, and the same is true for the velocity of vapor and liquid in two-phase zone. However, decreasing Lv at a constant $\omega$ may show a different effect on stability depending on the resulting value of the Rayleigh number. At a constant $\omega$, decreasing Lv leads to smaller values of $\mathrm{Ra}$, which will in turn stabilize the system.

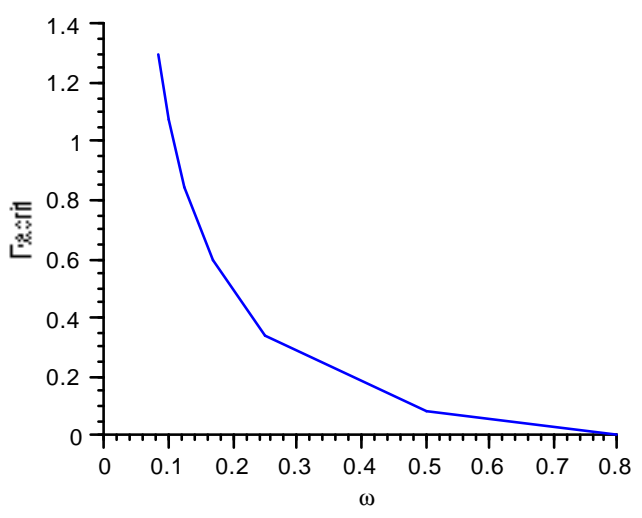

Figure 6. The dependence of $\mathrm{Ra}_{\text {crit }}$ on $\omega$.

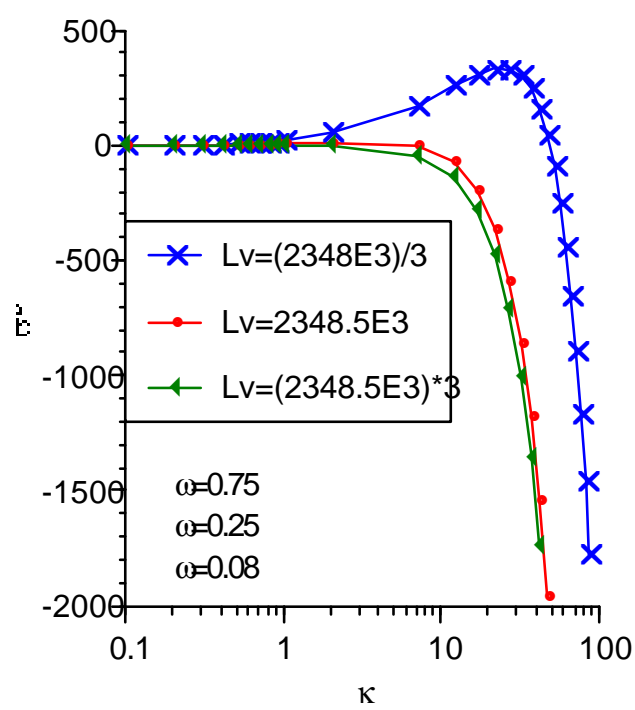

Figure 7. The effect on stability of the variation of the latent heat (in $\mathrm{J} / \mathrm{Kg}$ ).

Figure 8 is a graph of the $\sigma^{*}-\kappa$ relation for different $\mathrm{Ra}$ values at a constant $\omega$. The behavior shown is characteristic of other values of $\omega$, as long as it stays below a maximum value beyond which a heat pipe zone can not exist. As expected and shown in the figure, the system becomes more unstable as the Rayleigh number increases. 


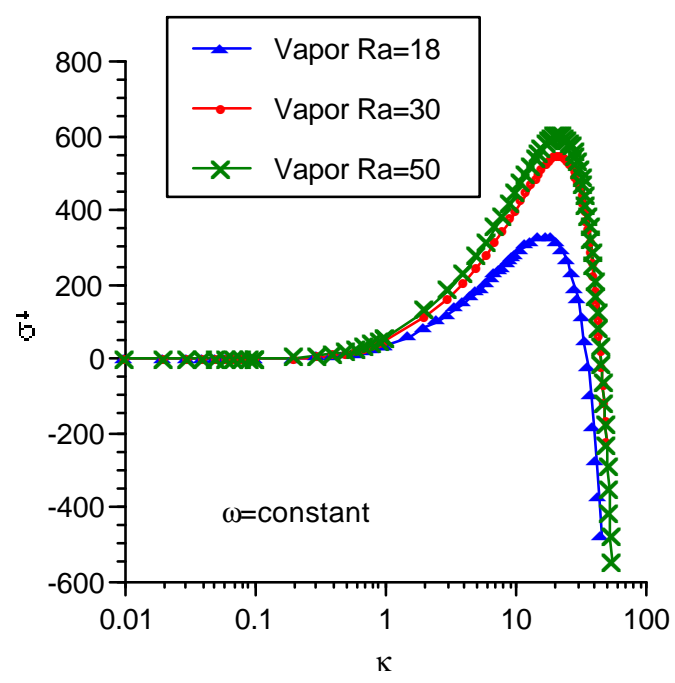

Figure 8. The $\sigma^{*}$ - $\kappa$ relation for different values of $\mathrm{Ra}$ at constant $\omega$.

Finally, Figure 9 demonstrates the effect of $\omega$ on the stability at a fixed typical Rayleigh number. It is shown that as $\omega$ decreases, the configuration is less unstable, reflecting the fact that the density of the heat pipe region diminishes at smaller values of $\omega$. In other words, for smaller values of $\omega$, the saturation of vapor in the two-phase zone increases and therefore the gravitational instability effect decreases (see also Fig. 1.)

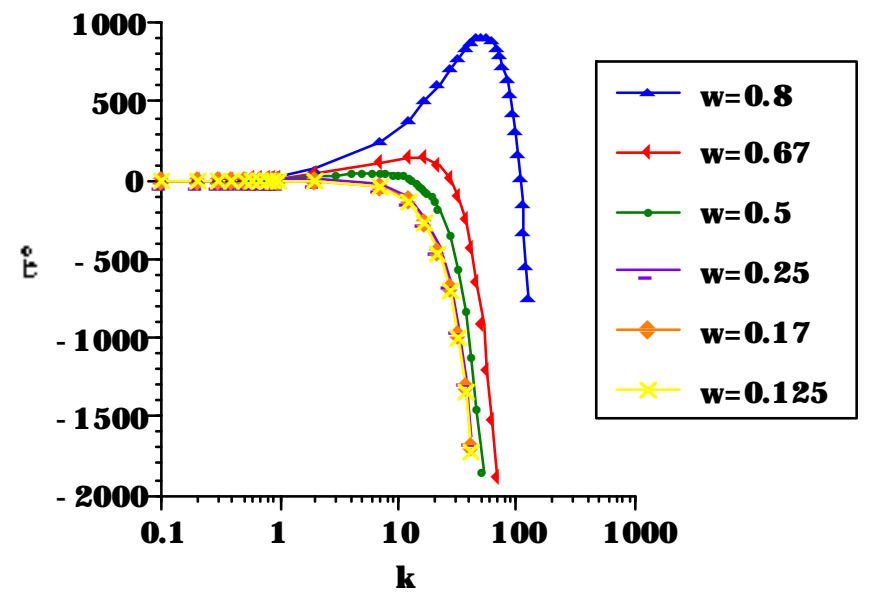

Figure 9. The $\sigma^{*}-\kappa$ relation for different values of $\omega$ at constant $\mathrm{Ra}$. 
The effect of two-phase Rayleigh number, $\mathrm{Ra}_{2 \phi}$, was also studied on the stability of the system. It was found that this parameter had a negligible effect on stability.

\section{Comparison between liquid-dominated and vapor-dominated systems}

A better perspective in our problem can be obtained by comparing it to the case of a liquid-dominated two-phase layer underlying a liquid zone (liquid case.) To obtain a valid comparison between these two cases, we used the same analytical approach, subject to similar boundary conditions as in the vapor-dominated case. Coverning equations, boundary conditions and dimensionless parameters for this problem are given in Appendix A. One interesting conclusion from the analysis is that the small $\kappa$ behavior is identical to that for the vapor-dominated case (namely, equation (59)). Note, however, that the value of $\beta_{1}$ is now different from the vapor case.

The effect of the Rayleigh number and $\omega$ was studied assuming an identical base state for both cases. Fig.10 shows a comparative behavior of the two cases for the same Rayleigh number. The vapor case shows a much more unstable behavior than the liquid case. The reason may be due to less viscose and conduction stabilizing factors in vapor compared to liquid.

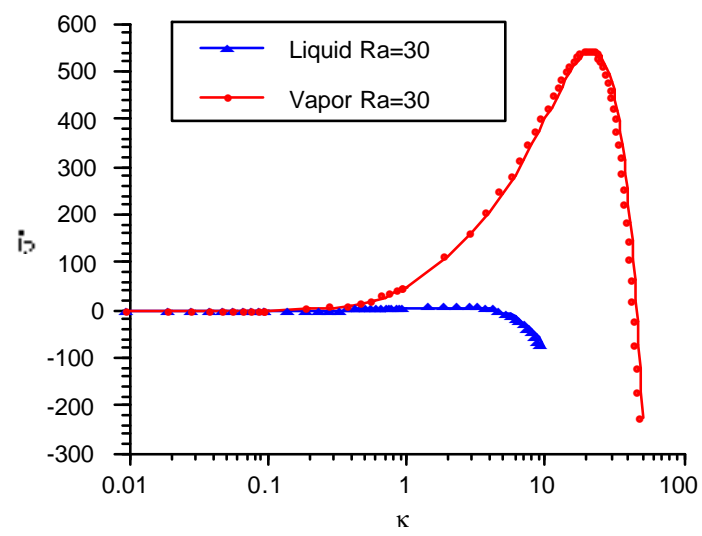

Figure 10. Comparison between vapor- and liquid-dominated cases at constant Ra. 
Figure 11 shows the effect of $\omega$ at a fixed Rayleigh number. As shown in the figure, the liquid-dominated case shows a behavior opposite to that in the vapordominated case. As $\omega$ decreases, the liquid-dominated system becomes more unstable, although it is much less sensitive to the changes compared to the vapor-dominated case. It can be concluded that the gravitational destabilizing factor in liquid case is not as important as the case in vapor.

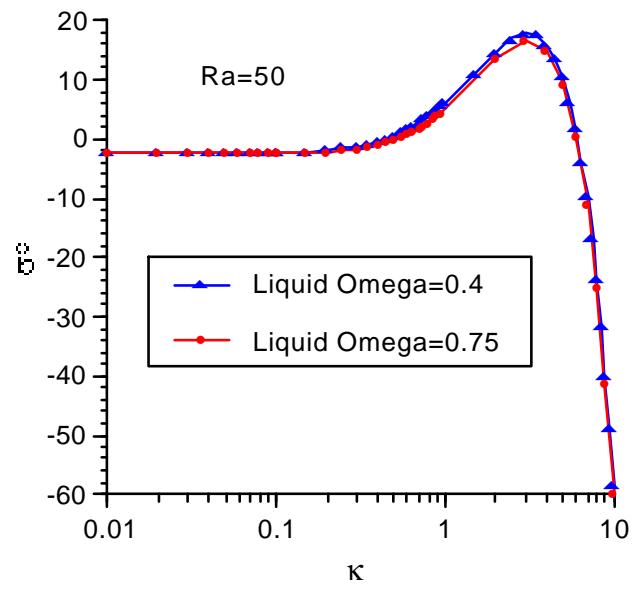

Figure 11. The effect of $\omega$ in the liquid-dominated case for a constant Ra.

The same trends are found on the effect of changes in Lv (Figure 12). The results show an opposite behavior and the changes are not as significant compared to the vapor case.

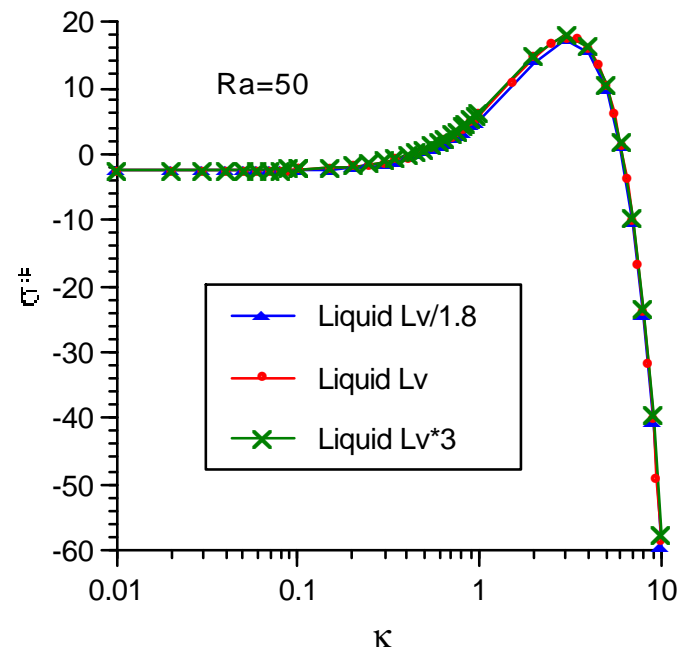

Figure 12. The effect of latent heat on liquid case $(\mathrm{Lv}=2348.5 \mathrm{E} 3 \mathrm{~J} / \mathrm{Kg})$. 
The relation between $\mathrm{Ra}_{\text {crit }}$ and $\omega$ with different values of $\mathrm{Lv}$ is shown in Fig. 13. With decreasing $\omega$ the system becomes more stable for the vapor-dominated case, as shown before, whereas the liquid-dominated becomes less stable. The other observation is that the vapor case shows more variations: Since the two-phase Rayleigh number is decreasing as $\omega$ increases, it can be presumed that the liquid case is more sensitive to two-phase Rayleigh number than the vapor case. Finally, it is also shown that in the limit of small values of $\omega$ the two cases do approach the same $\mathrm{Ra}_{\text {crit }}$.

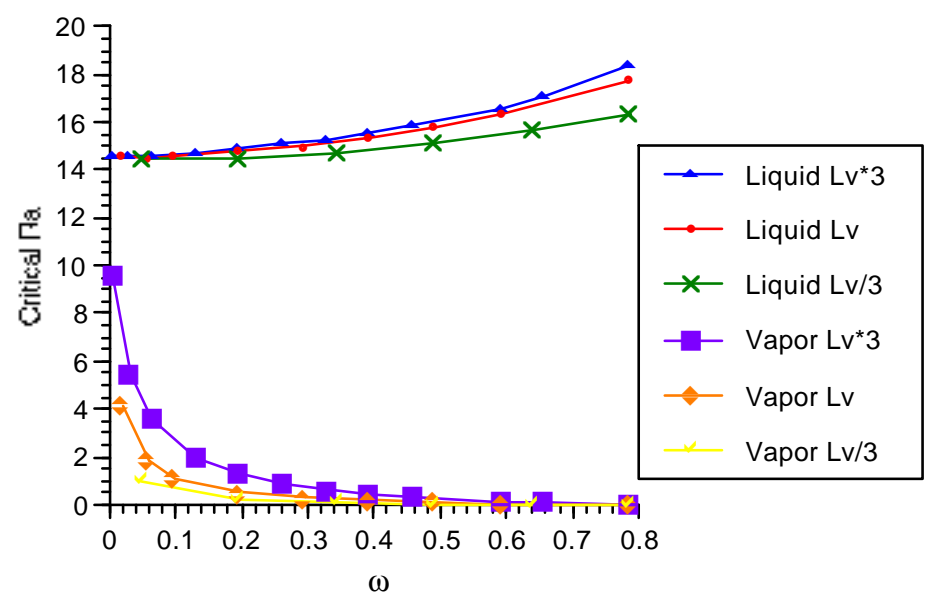

Figure 13. The dependence of $\mathrm{Ra}_{\text {crit }}$ on $\omega$ for liquid and vapor ( $\left.\mathrm{Lv}=2348.5 \mathrm{E} 3 \mathrm{~J} / \mathrm{Kg}\right)$.

As $\omega$ approaches zero, each system reduces to its respective single phase and thus a single phase behavior is to be expected. Lapwood (1948) showed that the critical Rayleigh number in the case of natural convection is $4 \pi^{2}$, under the assumption of constant temperature and impermeable boundary conditions at the top and the bottom. However in our case due to different boundary conditions, the critical Rayleigh number is different than the one reported by Lapwood.

We close by noting that at this point, vapor compressibility was not considered. To determine the effect of compressibility, the problem was reformulated and solved taking into account this parameter. This effort is not being presented here for the sake of brevity. The effect of compressibility is shown in Figure 14. It is clear that the effect is indeed not significant in the stability analysis, and can be readily neglected, justifying our assumptions. 


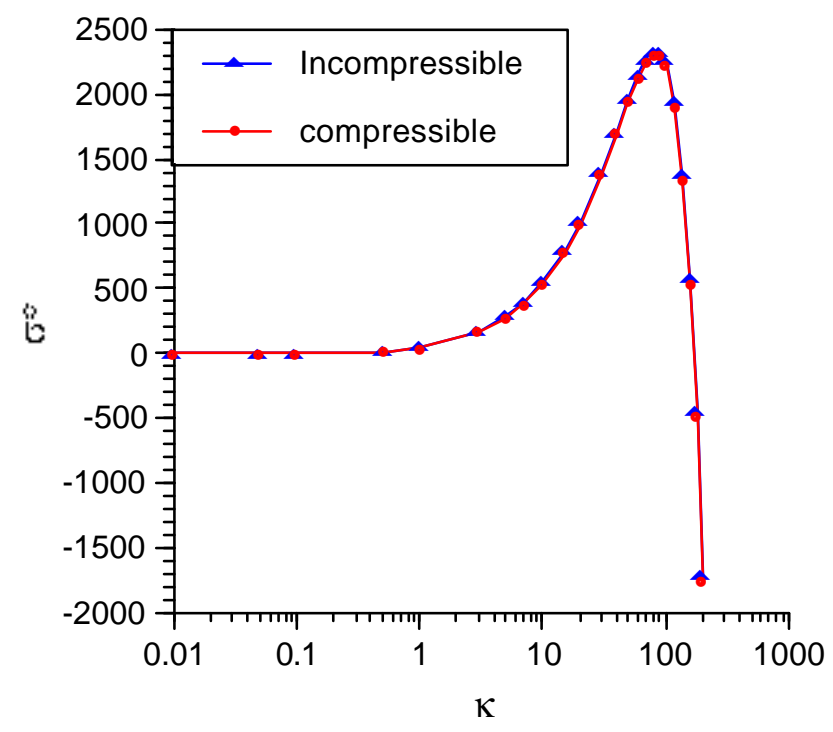

Figure 14. The effect of vapor compressibility.

\section{CONCLUSIONS}

In this paper, we studied the linear stability of a two-phase heat pipe zone (vaporliquid counterflow) in a porous medium, overlying a superheated vapor zone. It was found that the problem has similarities with the liquid-dominated case, in that long and short waves are stable, but intermediate wavelengths can be unstable, depending on the parameter values. A critical Rayleigh number was identified and shown to be different than in natural convection under single-phase conditions in two respects: The critical value is significantly smaller, while the critical value is shown to also depend on the other parameters of the problem. In particular, we found that the latent heat affects the stability of the problem. Comparison with the liquid-dominated case shows that the two problems are different, with the vapor-dominated problem being more unstable and more sensitive to the variations of parameters. The results find applications to steam-water flows as they apply in enhanced oil recovery, geothermal systems, as well as to the conditions of the proposed Yucca Mountain nuclear waste repository. 


\section{NOMENCLATURE}

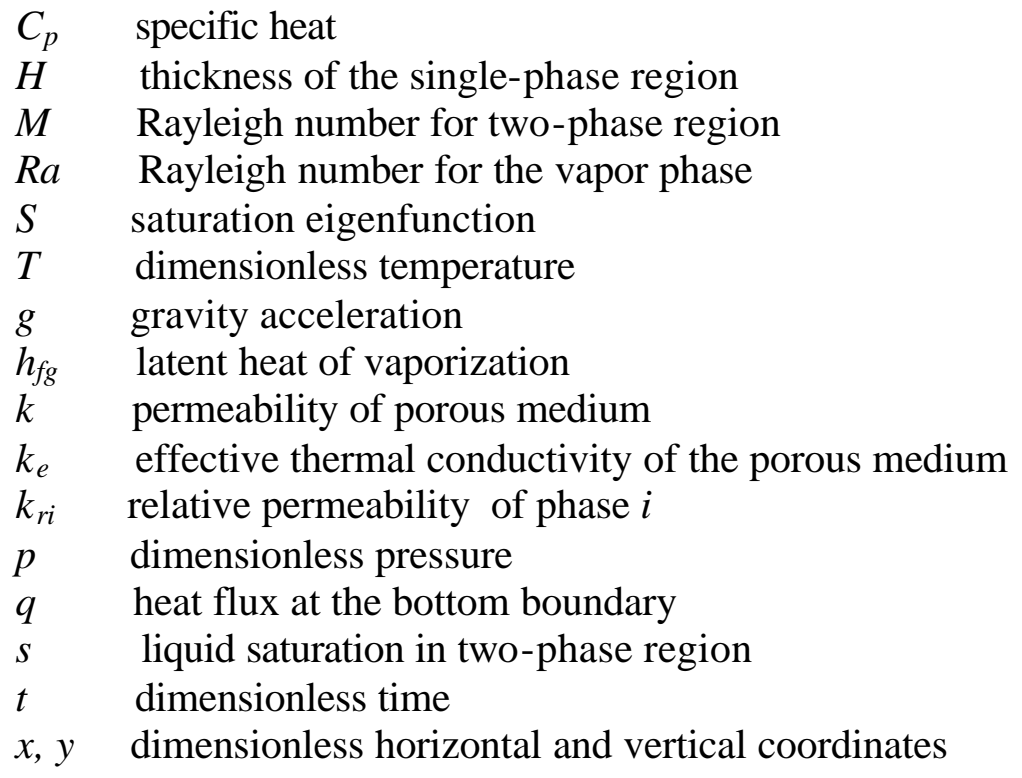

Greek symbols

$\begin{array}{ll}\Pi & \text { pressure eigenfunction } \\ \Theta & \text { temperature eigenfunction } \\ \alpha_{v} & \text { vapor thermal diffusivity } \\ \beta_{v} & \text { vapor thermal expansion coefficient } \\ \beta_{1} & \text { the ratio of heat capacities for rock and vapor } \\ \delta & \text { vapor-heat pipe stationary state interface position } \\ \phi & \text { porosity } \\ \kappa & \text { wave number of the disturbance } \\ \mu & \text { dynamic viscosity } \\ \nu & \text { kinematic viscosity } \\ \rho & \text { density } \\ \sigma & \text { rate of growth of the disturbance } \\ \omega & \text { dimensionless heat flux }\end{array}$

Subscripts and superscripts

$\begin{array}{ll}c r & \text { critical } \\ l & \text { liquid } \\ \text { min } & \text { minimum } \\ v & \text { vapor } \\ 0 & \text { base state } \\ l & \text { perturbed state } \\ * & \text { dimensional }\end{array}$




\section{REFERENCES}

Bau, H. H., and Torrance, K. E., 1982, "Boiling in Low-Permeability Porous Materials," International Journal of Heat Mass Transfer, 25, 45-54.

Drazin, P. G. and Reid, W. H. 1981, Hydrodynamic Stability. Cambridge University Press.

Gebhart, B., Jaluria, Y, Mahajan, R., and Sammakia, B., (1988, Bouyancy-Induced Flows and Transport. Hemisphere Publishing Corporation.

Kneafsey, T. J. and Pruess, K., 1999, "Laboratory Experiments on Heat-Driven TwoPhase Flows in Natural and Artificial Rock Fractures”, Water Res. Res., in press.

McGuinness, M. J., 1996a, "Steady Solution Selection and Existence in Geothermal Heat Pipes -I. The Convective Case", International Journal of Heat Mass Transfer, 39, 259274.

McGuinness, M. J., 1996b, "Steady Solution Selection and Existence in Geothermal Heat Pipes -II. The Conductive Case", International Journal of Heat Mass Transfer, 40, 311321.

McGuinness, M. J., Blakeley, M., Pruess, K. \& O’Sullivan, M. J., 1993, “Geothermal Heat Pipe Stability: Solution Selection by Upstreaming and Boundary Conditions," Transport in Porous Media, 11, 71-100.

Lapwood, E. R., 1948, Proc. Cambridge Philos. Soc. 44, 508.

Pestov, I., 1998, "Stability of Vapor-Liquid Counterflow in Porous Media," Journal of Fluid Mechanics, 364, 273-295. 
Ramesh, P. S. and Torrance, K. E., 1990, "Stability of Boiling Porous Media," International Journal of Heat Mass Transfer 33, 1895-1908.

Ramesh, P. S. and Torrance, K. E., 1993, “ Boiling in Porous Layer Heated from Below: Effects of Natural Convection and a Moving Liquid/Two Phase Interface" Journal of Fluid Mechanics, 257, 289-309.

Satik, C., Parlar, M., and Yortsos, Y. C., 1991, "A Study of Steady-State Steam-Water Counterflow in Porous Media", International Journal of Heat Mass Transfer 34, 17551771.

Stubos, A. K., Satik, C., and Yortsos, Y. C., 1993, "Effects of Capillary Heterogeneity on Vapory-Liquid Counterflow in Porous Media", International Journal of Heat Mass Transfer, 28, 485-495

Udell, K. S., 1985, "Heat Transfer in Porous Media Considering Phase Change and Capillarity - the Heat Pipe Effect", International Journal of Heat Mass Transfer 28, 485495.

White, D. E., Muffler, L. J., and Truesdell, A. H., 1971, "Vapor-Dominated Hydrothermal Systems Compared with Hot-Water Systems", Economic Geol, 66, 75-97. 


\section{Appendix A}

The dimensionless governing equations in a liquid-dominated heat pipe overlaid by a liquid layer are as follows:

\section{Liquid Zone:}

$$
\begin{gathered}
\nabla\left(\mathrm{v}_{l}\right)=0 \\
\mathrm{v}_{l}=-\nabla p+R a T j \\
\beta_{1} \frac{\partial T}{\partial t}+\mathrm{v}_{l} \nabla T=\nabla^{2} T
\end{gathered}
$$

Two-phase Zone:

$$
\begin{gathered}
\beta_{2} \frac{\partial s}{\partial t}+\nabla\left(\overline{\rho_{\mathrm{v}}} \mathrm{v}_{\mathrm{v}}+\mathrm{v}_{\mathrm{l}}\right)=0 \\
\mathrm{v}_{\mathrm{v}}=-k_{\mathrm{rv}} \overline{\mu_{l}}\left(\nabla p+R a_{2 \phi} j\right) \\
\mathrm{v}_{l}=-k_{\mathrm{rl}} \nabla p \\
-\phi \frac{\partial s}{\partial t}+\nabla\left(\mathrm{v}_{\mathrm{v}}\right)=0
\end{gathered}
$$

where

$$
\beta_{2}=\phi\left(1-\overline{\rho_{\mathrm{v}}}\right)
$$

The dimensionless parameters and coefficients are as follows:

Dimensionless velocity: $\quad \mathrm{v}=\frac{\mathrm{v}_{l}^{*} \mathrm{H}}{\alpha_{l}}$ 
Dimensionless length: $\quad y=\frac{y^{*}}{H}$

Dimensionless Pressure: $\quad \mathrm{p}=\frac{\mathrm{k}}{\mathrm{v}_{l} \alpha_{l}} \frac{\mathrm{p}^{*}-\mathrm{p}_{\mathrm{o}}^{*}-\rho_{\mathrm{vr}} g \mathrm{y}^{*}}{\rho_{l \mathrm{r}}}$

Single-phase Rayleigh number: $\quad R a=\frac{k H g \beta_{l}\left(T_{s a t}^{*}-T_{o}^{*}\right)}{\alpha_{l} \mathrm{v}_{l}}$

Vapor thermal diffusivity: $\quad \alpha_{l}=\frac{k_{e}}{\rho_{l} C p_{l}}$

Two-phase Rayleigh number: $\quad R a_{2 \phi}=\frac{k H g\left(1-\overline{\rho_{\mathrm{v}}}\right)}{\alpha_{l} \mathrm{v}_{l}}$

Dimensionless temperature: $\quad T=\frac{T_{s a t}^{*}-T^{*}}{T_{s a t}^{*}-T_{o}^{*}}$

Unity vector in Y direction: $\quad j=\frac{g}{g}$

Dimensionless time: $\quad t=\frac{\alpha_{l}}{H^{2}} t^{*}$

The ratio of heat capacity of vapor and rock: $\quad \beta_{1}=\frac{\phi \rho_{l} C p_{l}+(1-\phi) \rho_{r} C p_{r}}{\rho_{l} C p_{l}}$

Vapor and liquid density: $\quad \overline{\rho_{\mathrm{v}}}=\frac{\rho_{\mathrm{v}}}{\rho_{\mathrm{l}}}$

Liquid and vapor ratio:

$$
\overline{\mu_{l}}=\frac{\mu_{1}}{\mu_{\mathrm{v}}}
$$

Dimensionless latent heat: $\quad \lambda=\frac{L_{\mathrm{v}}}{\mathrm{Cp}_{l}\left(T_{\text {sat }}^{*}-T_{o}^{*}\right)}$ 
where $\mathrm{H}$ is the thickness of the liquid layer at the base state.

\section{Eigenvalue Problem:}

\section{Liquid Zone:}

$$
\begin{gathered}
\kappa^{2} \pi^{-}+R a \frac{d \theta}{d y}-\frac{d^{2} \pi^{-}}{d y^{2}}=0 \\
\left(\beta_{1} \sigma-R a+\kappa^{2}\right) \theta+\frac{d \pi^{-}}{d y}-\frac{d^{2} \theta}{d y^{2}}=0
\end{gathered}
$$

Two-phase Zone:

$$
\begin{gathered}
\kappa^{2} \pi^{+}-\frac{\phi \sigma}{k_{r v 0} \overline{\mu_{l}}} \Sigma+\frac{1}{k_{r v 0}}\left(\frac{d p_{0}^{+}}{d y}+R a_{2 \phi}\right) \frac{d \Sigma}{d y}-\frac{d^{2} \pi^{+}}{d y^{2}}=0 \\
\kappa^{2} \pi^{+}+\frac{\phi \sigma}{k_{r l 0}} \Sigma-\frac{1}{k_{r l 0}} \frac{d p_{0}^{+}}{d y} \frac{d \Sigma}{d y}-\frac{d^{2} \pi^{+}}{d y^{2}}=0
\end{gathered}
$$

\section{Boundary Conditions:}

At $\mathrm{y}=0$ :

$$
\left\{\begin{array}{l}
\theta=0 \\
\frac{d \pi^{-}}{d y}=0
\end{array}\right.
$$

At $\mathrm{y}=\mathrm{y}_{\mathrm{t}}$ : 


$$
\left\{\begin{array}{l}
k_{r l 0} \frac{d \pi^{+}}{d y}+\frac{d p_{0}^{+}}{d y} \Sigma=0 \\
-k_{r v 0} \frac{d \pi^{+}}{d y}+\left(\frac{d p_{0}^{+}}{d y}+R a_{2 \phi}\right) \Sigma=0
\end{array}\right.
$$

At $\mathrm{y}=1$ :

$$
\begin{gathered}
\theta=\Delta \\
\pi^{-}-\pi^{+}+\frac{\Delta}{\lambda k_{r l 0}}=0 \\
R a \theta-\left(\alpha_{1} \sigma\right) \Delta+\left[\frac{d p_{0}^{+}}{d y}-\overline{\rho_{\mathrm{v}}} \overline{\mu_{l}}\left(\frac{d p_{0}^{+}}{d y}+R a_{2 \phi}\right)\right] \Sigma+\left(k_{r l 0}+\overline{\rho_{\mathrm{v}}} \overline{\mu_{l}} k_{r v 0}\right) \frac{d \pi^{+}}{d y}-\frac{d \pi^{-}}{d y}=0 \\
\left(\alpha_{2} \sigma\right) \Delta-\left(\lambda \overline{\rho_{\mathrm{v}} \mu_{l}}\left(\frac{d p_{0}^{+}}{d y}+R a_{2 \phi}\right)\right) \Sigma+\frac{d \theta}{d y}+\left(\lambda k_{r v 0} \overline{\rho_{\mathrm{v}}} \overline{\mu_{l}}\right) \frac{d \pi^{+}}{d y}=0
\end{gathered}
$$

where $\alpha_{1}$ and $\alpha_{2}$ are defined as:

$$
\left\{\begin{array}{l}
\alpha_{1}=\phi\left(1-\overline{\rho_{\mathrm{v}}}\right)\left(1-s_{0}\right) \\
\alpha_{2}=\phi \lambda \overline{\rho_{\mathrm{v}}}\left(1-s_{0}\right)
\end{array}\right.
$$





\title{
A 2-D Pore-Network Model of the Drying of Single-Component Liquids in Porous Media
}

\author{
Andreas G. Yiotis, Athanassion Stubos, Andreas Boudouvis and Yannis C. Yortsos \\ INTRODUCTION
}

Drying of porous solids is a subject of significant scientific and technological interest in a number of industrial applications including coatings, food, paper, textile, wood, ceramics, building materials, granular materials, electronic devices and pharmaceuticals $[1,4,7,19,20,27,28]$. In a different context, drying in porous media is involved in distillation and vaporization processes associated with soil remediation [11], as well as in the recovery of volatile hydrocarbons from oil reservoirs by gas injection [15]. A variation of the latter is the main problem addressed here.

Generally, in drying, a single- or multi-component liquid phase gradually evaporates and is removed from the complex porous structure via combined heat and mass transfer processes. The traditional description relies on phenomenological approaches, in which the porous medium is a continuum, the dependent variables, like moisture content, are volumeaveraged quantities and the relation of fluxes to gradients is through empirical coefficients $[9,17,31,32,33]$. Such approaches essentially ignore the effect of the pore microstructure which is of key importance for a quantitative understanding of the process. In fact, drying involves many pore-scale mechanisms, for example the motion of individual gas-liquid menisci residing in the pore space, diffusion in the gas phase (for a single-component liquid) and the liquid phase (for a multi-component liquid), viscous flow in both phases, capillarity and possibly liquid flow through connected films, all of which need to be accounted for. We should note that although it also involves the receding of liquid-vapor interfaces, drying is not a typical external displacement process, like external drainage, which has been well studied in the past. Rather similarities exist to processes like solution gas drive and/or boiling in porous media, where the displacement of the liquid phase is driven internally by mass or heat transfer and which have been only relatively recently investigated [16,25].

The physical context of the problem considered here is schematically depicted in Fig. 1a, which shows an exaggerated schematic of a fractured network. Liquid is trapped in the matrix and may vaporize as a result of an injected purge gas flowing primarily in the 
fractures. The actual overall problem is quite complex, requiring the consideration of the network of fractures and the matrix continuum, gas flow and mass transfer in the fracture network and the multi-dimensional mass transfer from the matrix continuum to the fracture network. For simplicity, we will consider the much simpler geometry of the pore-network model shown in Fig. 1b, which involves a 2-D rectangular matrix block, all but one boundaries of which are impermeable to flow and mass transfer. We will also neglect gravity. Extension to 3-D geometries is, in principle, straightforward, although computationally costly, while equally feasible is the consideration of gravity effects. We consider isothermal conditions, under the assumption that heat transfer in the solid operates much faster than mass transfer in the gas phase. Finally, a single-component liquid phase is assumed. It is of note that this approach applies also to the vaporization of trapped NAPL's in low-permeability layers or regions [11].

At any time during the process, evaporation of the liquid leads to the receding of the liquid (drying) front, leaving behind disconnected clusters of liquid and liquid films (in corners of capillaries, or as thin films), the size and location of which change continuously with time. In general, four different spatial regions can be identified [30] (Fig. 1c):

(i) a far-field (from the fracture) region consisting of the initial liquid;

(ii) a region where both liquid and gas phases are macroscopically connected (this region may be absent in certain 2-D square pore-networks for topological reasons);

(iii) a region where the liquid phase is disconnected and consists of individual clusters of variable sizes (trapped islands of liquid); and

(iv) a near-field (to the fracture) region consisting primarily of the connected gas, with the liquid phase in the form of pendular rings, corner films or thin films on the solid surface, the thickness of which is progressively reduced towards a "totally dry" regime.

Evidently, the gas phase is macroscopically continuous in the last three regimes. On the basis of his experiments, Shaw [26] has postulated that liquid films may also provide hydraulic conductivity to the liquid phase in these regimes. We must point out that these four regimes only develop after the end of the Constant Rate Period (CRP). Prior to this, regions (iii) and (iv) cannot arise. Our model accounts for the full evolution of all these regimes, including the $\mathrm{CRP}$, as will be shown below. 
The conventional approach to modeling drying is based on a continuum description, the simplest model for which involves only the two limiting regions (the far-field and the near-field), separated by a planar drying front. The latter recedes due to diffusion-controlled mass transfer obeying a square-root time dependence. More sophisticated continuum models have also been developed, as discussed in Luikov [17], and Whitaker [33], among others. Recently, Tsimpanogiannis et al. [30] proposed a more complex 1-D model using transverse-averages. Their model involves various coefficients, which need to be evaluated from a pore-network study, however (see also Stubos and Poulou [29]). Tsimpanogiannis et al. [30] also developed a theory based on pore-scale considerations, for the description of the scaling properties of the drying front. Specifically, they showed that consideration of viscous effects makes drying equivalent to Invasion Percolation in a Stabilizing Gradient (IPSG) (e.g. see Xu et al. [34]). In particular, they emphasized that liquid flow in the porous matrix is driven by capillary pressure gradients (the so-called capillary pumping or capillary wicking effect, Ceaglske and Hougen [2]). The allowance of film flow could be an essential element of that theory. Based on the fact that drying is controlled by both capillarity and diffusion, in contrast to external drainage which is controlled by the injection rate, a power law relation of the evaporating front width with a modified, diffusion-based capillary number was obtained and shown to be compatible with the experimental data of Shaw [26]. However, other important features of the process, including the partition of the liquid phase in various regimes, the mass transfer rates, etc., were not explored.
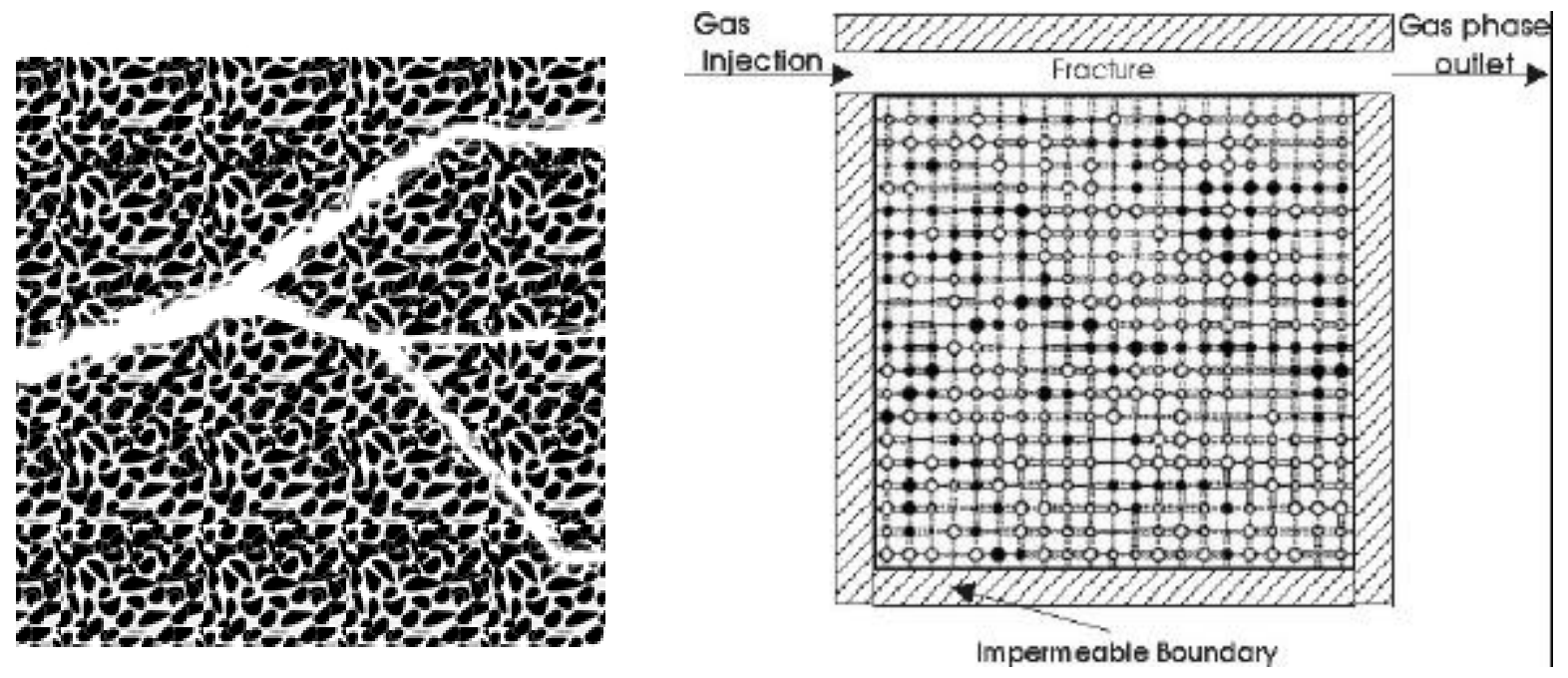


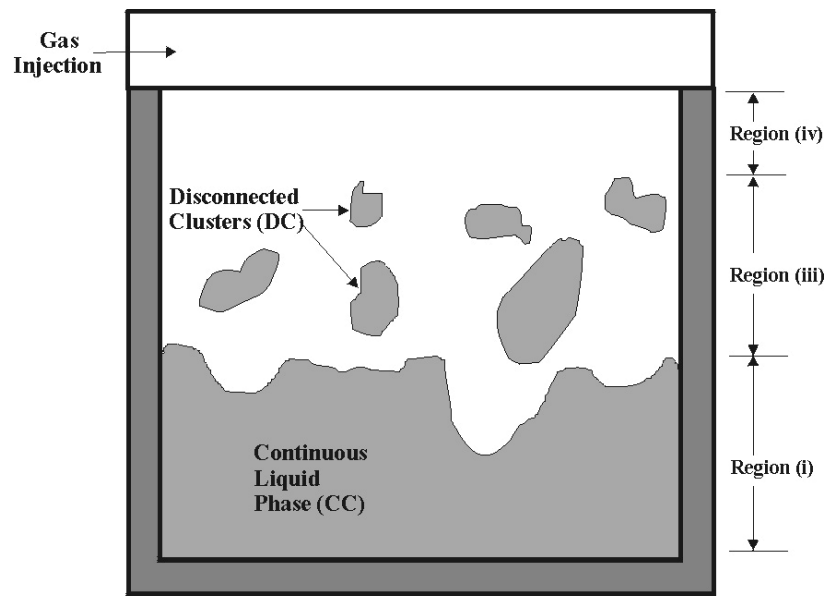

Figure 1a Schematic representation of fractured porous media. Figure 1b Pore-network model representation of a porous medium. Figure 1c Classification of typical saturation patterns.

Following recent trends in describing processes in porous media, several studies in recent years used a pore-network approach to model drying. Key to these approaches is the consideration of mass transfer, elements of which were described by Li and Yortsos [16] and Jia et al. [12], among others. Various pore-network models with specific applications to drying were proposed originally by Nowicki et al. [18], and more recently in a series of papers by Prat and co-workers [13,14,22,23]. In parallel, Pot et al. [21] used lattice-gas automata to simulate evaporation in a 2-D lattice. Nowicki et al. [18] presented a numerical simulation of the process at the pore-network level. However, the authors did not expand on the particular patterns and regimes obtained or on the associated effects on drying rates. Prat's studies represent the first attempt to characterize theoretically drying patterns and their rate of change in porous structures. Prat [22] studied the formation of drying patterns assuming capillary control, neglecting viscous effects and considering mass transfer only by quasi-static diffusion. Laurindo and Prat [14] provided a macroscopic assessment of the importance of liquid films. Based on percolation patterns and isothermal conditions, they computed drying rates by solving a quasi-static diffusion equation in the gas phase. Prat and Bouleux [23] focused on diffusional mass transfer and the effect of gravity on the front 
structure, but also commented on viscous effects. In earlier experiments using horizontal glass-bead packs (Shaw [26]), viscous forces were found to be important for explaining the formation of an evaporating front (separating continuous liquid from gas) of a finite size. More generally, we expect that advection and viscous effects will have an impact on patterns and drying rates. Existing pore-network models address mostly slow drying, controlled by capillarity and/or gravity and by diffusion, ignoring advection and/or viscous effects. We note that consideration of advection in the gas phase requires knowledge of the pressure field, which is coupled to viscous flow in the two phases and capillary effects. Thus, these two processes must be treated simultaneously.

The present paper is motivated by the above lack of completeness and attempts to shed light to mass transfer and/or viscous effects on the development of drying patterns. It is also motivated by the need to provide reliable coefficients in the appropriate continuum models, and in a sense it represents a continuation of the work by Tsimpanogiannis et al. [30], except that film flows are ignored. We consider the isothermal drying (at room temperature) of a porous block, initially saturated with liquid (hexane), subject to flow of air in the fracture and in the absence of gravity. The paper is organized as follows: First, we present details of the pore-network approach. The various mechanisms described previously (diffusion in gas phase, viscous flow in both phases, capillarity and capillary wicking) are addressed. Then, simulation results are obtained for the drying patterns and rates, for various values of the dimensionless parameters governing the process. The latter involve a mass transfer-based capillary number and the Peclet number based on the gas velocity in the fracture. The results are subsequently analyzed using simpler statistical theories, such as Invasion Percolation (IP). Various limiting cases are identified and analyzed. In particular, we pay attention to the trapped islands surrounding the drying front, which due to their screening effect on mass transfer play an important role in the problem. Scale-up issues as well as effects of various parameters not considered in the pore network are discussed in the last section.

\section{FORMULATION}

A discrete pore network model is used to simulate the drying of a porous block. The porous medium is represented by the standard approximation of a 2-D network of spherical 
pore bodies connected through cylindrical pore throats [3,5] (Figure 1b). The pore bodies serve as containers for either of the two phases and it is assumed that they have no capillary or flow resistance. Therefore, when a liquid-gas interface lies within a pore body, the pressures of both phases are taken to be equal and the interface recedes without any capillary forces restraining its movement (Figure 2b). This simplification can be relaxed at the expense of higher computational cost and could be subject to the uncertainty of the precise pore body geometry. The throats serve as conductors of the flow and mass transfer and they act as capillary barriers. When a stationary liquid meniscus (interface) lies at the entrance of a throat adjacent to a liquid pore (Figure 2a), an interfacial pressure difference, roughly equal to $2 ? / r$, where ? is the surface tension and $r$ the radius of the throat, develops between the two neighboring pores. The meniscus remains stationary until the pressure difference between the two pores exceeds that capillary pressure threshold. Then the meniscus will recede instantly (Figures $2 \mathrm{c}$ and $2 \mathrm{~d}$ ), since it is assumed that the throat has no volume and the gas phase penetrates the pore. In our simulations, pore body and throat radii were uniformly distributed in the range $0.37-0.74 \mathrm{~mm}$ and $0.16-0.32 \mathrm{~mm}$, respectively. The lattice length $\ell$ (pore center to pore center) was taken to be $2 \mathrm{~mm}$.
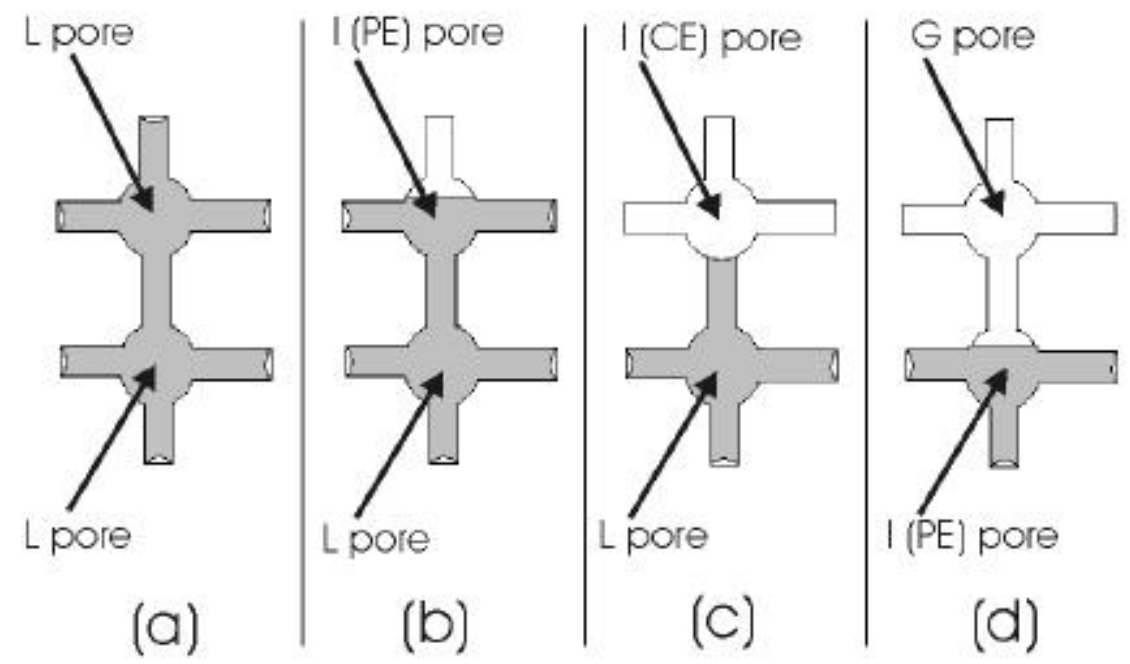

Figure 2 The evolution of drying at an isolated liquid cluster (DC) consisting of two liquid pores. (a) Initially the cluster is surrounded by I pores of type CE. (b) When the pressure difference at the upper throat exceeds its capillary resistance, the meniscus recedes and the pore is penetrated by the gas phase. The same transition takes place between (c) and (d). 
The 2-D network has three sides impermeable to flow and mass transfer. Alternatively, one could impose periodic boundary conditions at the sides. Along the fourth side runs a fracture, which is represented as 1-D chain of pore bodies and throats. The two ends of the fracture are open to flow and mass transfer. Pore body and throat radii are taken equal to $0.77 \mathrm{~mm}$ and $0.275 \mathrm{~mm}$, respectively. We note that the dimensions of the fracture considered in the simulations are comparable to the mean dimensions of the network. This is rather unrealistic, since one would expect that a real fracture would have larger dimensions than the network. However, it is adopted here, in order to emphasize viscous effects in the porous medium. Higher fracture dimensions would require unrealistically high gas flows for the pressure drop along the fracture to be sufficiently high to exceed the capillary pressure threshold at the throats of the network in direct contact with the fracture. The study of the viscous effects is one of the objectives of this work.

Initially the network is saturated by a single component liquid (hexane). The fracture, however, contains only air at the beginning of the drying process. At one end of the fracture a purge gas (air) is injected at a constant volumetric rate. The concentration of the liquid component vapors is assumed to be zero at the entrance and the exit of the fracture at all times. Perhaps, a more appropriate Dankwerts type boundary condition, in which the concentration gradient is zero at the exit, could be used. The present condition exaggerates slightly the drying rate. The pressure is assumed equal to atmospheric at the exit of the fracture and variable at the entrance of the flow, in order $\mathrm{b}$ maintain the constant rate condition. The gas injection results to a pressure gradient along the fracture, which eventually develops inside the porous block as well. As a result of the gas flow, the liquid evaporates initially at the interface pore throats along the fracture. Vapor flows by advection and diffusion.

Throughout the process, isothermal conditions are assumed, heat diffusion in the solid being faster than mass diffusion in the gas. This assumption will certainly not apply in the case of poorly conducting solids, however. The 2-D pore network is horizontal, thus gravity effects, which could be dominant, depending on the magnitude of the gravity Bond number [23], are not included. These can be readily implemented in the pore-network simulation. However, gravity can mask important mass transfer effects, while the 
understanding of the simpler drying process in its absence, is still incomplete, as noted above. Finally, the temperature is sufficiently far from the boiling point of the liquid, so that evaporation rates are diffusion-controlled and the binary mixture in the gas phase can be assumed to be dilute. Thus, the physical properties of the binary gas phase do not depend on the concentration of the liquid component vapors and are assumed to be constant and equal to those of the purge gas.

As a result of the ensuing drying process, the liquid will reside, in general, in two different and evolving regions (Fig. 1c): a "continuous" cluster (CC), which is part of the initial liquid cluster and can be defined as being "sample-spanning" across the two lateral edges of the matrix block; and various "disconnected" clusters (DC), which have become disconnected from the $\mathrm{CC}$ and from one another, and they are not "sample spanning" (see below). Their geometry, size, configuration and location are important to drying patterns and rates. Of course, initially, the porous medium is only occupied by a CC. It is the subsequent rate of change of CC and the formation of DC clusters, which is a main objective of this work. Liquid may also reside in films (thicker corner films or thin films) in gas-occupied pores, that may provide hydraulic continuity between clusters and/or with the fracture. These are not considered here, although in all likelihood, they have non-trivial effects on drying patterns and rates, as will be discussed below. Receding of the liquid-gas interface in the various clusters occurs when the capillary pressure across a meniscus first exceeds the capillary threshold at the pore where the meniscus resides. Accounting for capillarity requires the consideration of pressure fields in the liquid and gas phases. Flow in the latter is assumed to be slow and viscous-controlled. We will discuss this in more detail below.

In drying applications we can distinguish three types of pore bodies (Figure 2): Those fully occupied by gas (belonging to the gas phase and denoted by G), those fully occupied by liquid (belonging to the liquid phase and denoted by L) and those at the gasliquid interface (in which a meniscus resides, denoted by I). The latter may be further subdivided in completely empty (CE) and partly empty (PE) pores [30]. As noted above, this classification does not account for corner films. 
Liquid evaporates at the gas-liquid interface at rates determined by mass transfer in the gas phase, which are governed by advection and diffusion. The evaporation rate at I-type pores is equal to

$$
F_{i j}=D \frac{C_{i}-C_{j}}{\ell}
$$

where $F_{i j}$ is the evaporation flux through a throat connecting neighboring pores i and $\mathrm{j}, D$ is the diffusion coefficient of the liquid component vapors in the gas phase through the porous medium, $C_{i}$ is the concentration at the liquid pore $\mathrm{L}$, which is by default equal to the equilibrium concentration $C_{e}$ of the liquid component vapors and $C_{j}$ is the concentration at the I pore of type CE. Equation (1) also applies to I pores of type PE, where the interface resides within the pore, the concentration in such pores also being equal to $C_{e}$, since they contain liquid. Then $C_{j}$ is the concentration of the adjacent $\mathrm{G}$ pore (See Figure 2d).

Mass transfer of the vapor in the gas phase obeys the convection-diffusion equation

$$
\frac{\partial C}{\partial t}+\boldsymbol{u} \cdot \nabla C=D \nabla^{2} C
$$

where $C$ is the vapor concentration and $\boldsymbol{u}$ is the gas-phase velocity. In a $\mathrm{G}$ pore, this is further discretized as

$$
\mathrm{V}_{\mathrm{i}} \frac{\Delta \mathrm{C}_{\mathrm{i}}}{\Delta \mathrm{t}}=\sum_{j}\left[\mathrm{Dpr}_{\mathrm{ij}}^{2} \frac{\mathrm{C}_{\mathrm{i}}-\mathrm{C}_{\mathrm{j}}}{\ell}\right]+\sum_{j}\left[\frac{\mathrm{pr}_{\mathrm{ij}}^{4}\left(\mathrm{P}_{\mathrm{i}}-\mathrm{P}_{\mathrm{j}}\right)}{8 \mu_{g} \ell} \overline{\mathrm{C}_{\mathrm{ij}}}\right]
$$

where $V_{i}$ is the volume of pore i, $\Delta C_{i}$ is the change in $C_{i}$ during the elapsed time $\Delta t, r_{i j}$ is the radius of the throat connecting pores $\mathrm{i}$ and $\mathrm{j}, \mu_{g}$ is the gas viscosity, $P$ is the pressure and $C$ is the concentration at the pore. Note that the advection term is upstream weighted, namely

$$
\bar{C}_{i j}=C_{i} \quad \text { if } P_{i}>P_{j}, \quad \text { and } \quad \bar{C}_{i j}=C_{j} \quad \text { if } P_{j}>P_{i}
$$


In I pores of type $\mathrm{CE}$ the advection term of equation (2) is not accounted for

$$
\mathrm{V}_{\mathrm{i}} \frac{\Delta \mathrm{C}_{\mathrm{i}}}{\Delta \mathrm{t}}=\sum_{j}\left[\mathrm{Dp} r_{\mathrm{ij}}^{2} \frac{\mathrm{C}_{\mathrm{i}}-\mathrm{C}_{\mathrm{j}}}{\ell}\right]
$$

The pressure fields are obtained from a separate computation to be discussed below. We note the use of a simplified Poiseuille-type approximation for the flow across two adjacent pores, and the assumption that mass transfer between the pores is by diffusion and convection (namely, dispersion in a single pore is not considered). However, mass transfer between two adjacent pores, one of which resides in the fracture, is enhanced by considering a velocity-dependent mass transfer coefficient [24]

$$
\left(\frac{K_{f} \ell}{D}\right)=1+P e^{a}
$$

where the Peclet number, $P e$, is defined as

$$
P e=\left(V_{f} \frac{\ell}{D}\right)
$$

$a$ is an exponent that depends on the geometry of the fracture and $V_{f}$ is the linear gas velocity through the fracture. Equation (5) introduces a quasi-empirical macroscopic diffusion coefficient at the boundary between the matrix block (porous medium) and the free area of the fracture. This diffusion enhancement is an attempt to model the influence of the gas flow rate to the mass transfer enhancement along the convective layer within the fracture. When a neighboring pore contains a meniscus (PE), its concentration is the vapor equilibrium concentration. The boundary conditions for mass transfer involve zero flux at the lateral boundaries, a constant volumetric flux at the entrance of the fracture and zero concentration at the entrance and exit of the fracture. The latter could be modified to a zeroconcentration gradient condition, but this was not considered. 
The single-component liquid in the liquid phase and the non-condensable gas in the gas phase satisfy continuity equations. For their calculation, we solve for the pressure fields in $\mathrm{G}$ and $\mathrm{L}$ pores. Fluxes between adjacent pores of the same type are computed by Poiseuille-law type flow resistances, where the viscosity is taken constant

$$
\begin{gathered}
Q_{i j}=\left(\frac{P_{i}-P_{j}}{\ell}\right) \frac{p r_{i j}^{4}}{8 \mu} \\
\sum_{j} Q_{i j}=0
\end{gathered}
$$

where $\mu$ is the viscosity of either of the two phases. The volumetric flux $Q$ between an I of type $\mathrm{CE}$ pore and a $\mathrm{L}$ pore depends on the pressure difference between the two pores and the capillary pressure threshold of the connecting throat. We need to distinguish two cases: If the pressure difference between the two pores (capillary pressure) is not large enough for the gas phase contained at the I (CE) pore to penetrate the connecting throat, the meniscus remains stationary. However, as long as evaporation continues, there is a net liquid flow (but no non-condensable gas flux) in that direction. Then, in this pore we assign only a gas pressure value and zero mass flux rates of gas towards the L pore. In the next time step, the meniscus may be subject to a sufficiently high capillary pressure that can lead to the subsequent penetration of an adjacent pore and liquid displacement. Then, the meniscus recedes instantly and the L pore becomes of type I, partly empty (PE). In I pores, a mass balance of the evaporating liquid gives the liquid velocity $u_{\ell n}$ at the interface as a function of the rate of emptying of the pore and the diffusive flux in the gas phase, namely [30]

$$
-\mathrm{D} \frac{\partial \mathrm{C}}{\partial \mathrm{n}}=\rho_{\ell}\left(\mathrm{u}_{\ell \mathrm{n}}-\mathrm{u}_{\mathrm{n}}\right)
$$

where $?_{\ell}$ is the mass density of the liquid, $u_{n}$ is the normal velocity of the meniscus and $n$ denotes the normal to the interface. Likewise, conservation of mass of the non-condensable gas leads to [30] 


$$
u_{n}=u_{g n}
$$

where $u_{g n}$ is the gas velocity at the meniscus. These were used as interface conditions coupling the flow fields in the two phases. Therefore at liquid pores L adjacent to I pores of type $\mathrm{CE}$, or at I pores of type PE adjacent to $\mathrm{G}$ pores, equation (7) has the following form which takes into account the above mentioned boundary conditions at the interface:

$$
\mathrm{Q}_{\mathrm{ij}}=\left(\frac{\mathrm{P}_{\mathrm{i}}-\mathrm{P}_{\mathrm{j}}}{\ell}\right) \frac{\mathrm{pr}_{\mathrm{ij}}^{4}}{8 \mu}+D \frac{C_{i}-C_{j}}{\ell \rho_{l}}
$$

The first term on the right accounts for hydraulic flows towards (or from) adjacent L pores and the second term accounts for the evaporating volumetric flux towards the adjacent $G$ pores or I pores of type CE. Taking into account the volumetric evaporation rate at all interface pores from equation (1) and the boundary conditions imposed to the interface by equations (9) and (10) results to the well-known phenomenon of capillary pumping or wicking. This corresponds to a liquid viscous flow from I pores of type PE, where there is no capillary resistance to the receding of the interface, towards L pores adjacent to I pores of type $\mathrm{CE}$, where the menisci remain stationary due capillary effects. The volumetric rate of flow is equal to evaporation flux so that the menisci remain stationary and the mass balance is satisfied. We note that the above treatment of mass transfer applies in the dilute limit. In the case of non-dilute mixtures, counter-diffusion in the gas phase needs to be considered as well. Finally, the gas saturation at I pores of type PE is calculated by the following mass balance

$$
S_{i}^{t+1}=S_{i}^{t}+\frac{\Delta t}{V_{i}} \cdot \sum_{j} Q_{i j}
$$

where $S_{i}^{t+l}$ is the gas saturation inside the pore at the next time step, $S_{i}^{t}$ is the gas saturation at current time and ?t is the time step during which we assume constant flow rates $Q$.

For completeness, we provide a schematic of the way our algorithm accounts for the capillary pumping mechanism. As shown in Figure 3, where two areas A and B along the 
interface are highlighted, if the throats $R$ of the interface at area A have smaller radii than those of area $\mathrm{B}$, they will result in higher capillary pressures $P c$ at the interfaces in area $\mathrm{A}$. If the pressure in the gas phase is taken to be constant then we will have the logical sequence

$$
R_{A}<R_{B} \rightarrow P c_{A}>P c_{B} \rightarrow \text { Pgas }_{A}-P_{\text {Pliq }}>\text { Pgas }_{B}-\text { Pliq }_{B} \rightarrow \text { Pliq }_{B}>\text { Pliq }_{A}
$$

namely, due to the difference in radius, between the two sections of the interface, there results a capillary-induced liquid flow from area B to area A. In fact, during drying, the menisci in area A usually remain stationary and the evaporation flux from the interface of this section is balanced by the liquid flow towards section A from section B.

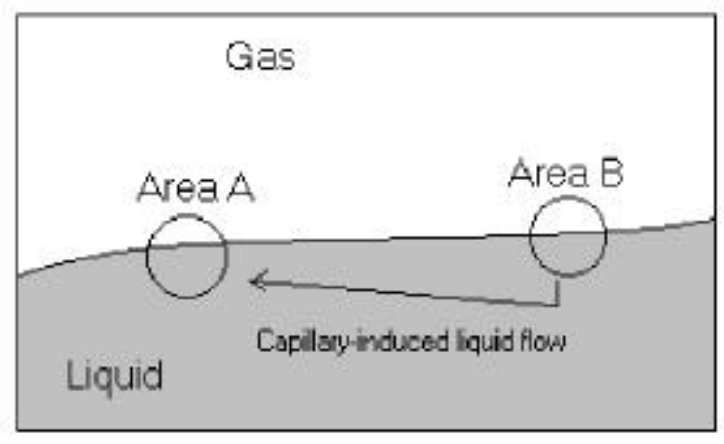

Figure 3 Schematic representation of the capillary pumping phenomenon.

The simulation procedure in the network, the updating of interfaces and the marching in time is described in the Appendix A and can be qualitatively summarized as follows. At any given time, sites have the designation, G, L or I, and pressure and concentration fields are known. The L sites can be part of the original liquid cluster (CC) or they belong to disconnected finite-size clusters (DC), which become trapped through the evaporation process. In the subsequent time step, the overall rate of evaporation from each of the liquid clusters is evaluated. Pressure fields are calculated and PE pores of type I are 
emptied according to the appropriate mass balances. The time step is selected such that it equals the minimum time required to empty completely any of the available PE pores. If at the current time, no PE pores are available to any (or all) of the clusters (namely all I pores are of the CE type) the throat with the smallest capillary threshold in the perimeter of any given cluster is the next throat to be invaded, at which time, the corresponding invaded pore becomes an I pore of the PE type. To determine this throat the liquid pressure is lowered uniformly in space inside the cluster, until the capillary pressure exceeds for the first time the smallest capillary threshold. Equivalently, this can be obtained by invading the throat with the smallest difference between the gas pressure in the pore and the corresponding capillary pressure threshold of the throat. Implicit to the above is the absence of hydraulic continuity between disconnected clusters, namely the absence of liquid films. Nonetheless, invasion must occur, since due to evaporation there is a continuous loss of mass from the liquid clusters. At the conclusion of the time step, concentration fields in the gas phase are computed and the process continues. This algorithm is essentially the same one used by $\mathrm{Li}$ and Yortsos [16] and Satik and Yortsos [25] in the related problems of phase change by solution gas-drive or boiling. All calculations are done explicitly in time. Pressure fields are computed using Successive Over-Relaxation, while concentration fields are obtained from (3) and (4) in a straightforward manner.

From a dimensional analysis of the problem, there are three main dimensionless groups: (i) a diffusion-based capillary number, $\mathrm{Ca}$, defined as

$$
\mathrm{Ca}=\mathrm{D} \mu_{\ell} \mathrm{C}_{\mathrm{e}} / \gamma \ell \rho_{\ell}
$$

where $\mu_{\ell}$ is the viscosity of the liquid phase, expressing the ratio of viscous to capillary forces, based on a diffusion-driven velocity; (ii) a Peclet number, $P e$, defined by (6), expressing the ratio of advection to diffusion in the gas phase, based on the linear velocity assuming only flow in the fracture; and (iii) the viscosity ratio, $M$, between liquid and gas viscosities. The latter is typically large and will not be considered in the sensitivity analysis to follow. We point out that one may also define a capillary number based on the fracture velocity. Clearly, the latter would control high-rate processes at early stages, while the diffusion-based capillary number controls the process at later times. These two capillary 
numbers are linearly related through the Peclet number, thus only two of these parameters are linearly independent. In the presence of gravity, an additional dimensionless number, the Bond number

$$
\mathrm{B}=\frac{\mathrm{g}\left(\rho_{\ell}-\rho_{\mathrm{g}}\right) \mathrm{k}}{\gamma}
$$

expressing the ratio of gravity to viscous forces, must also be considered, where $k$ is the permeability of the porous medium. Geometric parameters include the number of pores in the linear dimension $N=L /$, where $l$ is the typical pore length, the aspect ratio between mean pore size and pore length, as well as between mean pore size and mean throat size, a scaled variance of the size distribution, and the aspect ratio of the matrix block (here taken equal to 1). In this study, all geometric parameters are taken fixed, the aspect ratio of the matrix block is equal to 1, and emphasis is placed on the effect of the capillary and Peclet numbers. The effect of $N$ will be addressed in the discussion on scale-up.

\section{RESULTS}

A number of runs were conducted to simulate drying of liquid hexane in a matrix block of size 50x50. The corresponding physical parameter values are shown in Table 1. To carry out a sensitivity study, we varied the injection rate in the fracture, the interfacial tension and the diffusion coefficient. Table 2 shows the set of runs conducted. The characteristic time $t^{*}$ denotes the time at which the matrix block empties at the maximum drying rate (which occurs at the first time step at which all liquid-gas interfaces are at the fracture-matrix boundary) and it is used to non-dimensionalize time. All simulations correspond to a fixed realization of the pore network, which allows studying the sensitivity of drying patterns and rates to the other parameters. Results on drying patterns, concentration profiles, transversely-averaged saturation profiles and drying rates were obtained. We note that in order to demonstrate strong advection effects, the values of the Peclet number used in certain runs in the above table are high (perhaps unrealistically high). 
Table 1: Values of physical properties used.

\begin{tabular}{ll}
\hline Parameter & Value \\
\hline Volumetric gas flow rates & $0.0-1.0^{*} 10^{-6} \mathrm{~m} / \mathrm{s}$ \\
Surface Tension & $19 * 10^{-3} \mathrm{~N} / \mathrm{m}$ \\
Diffusion Coefficient & $6.38^{*} 10^{-6} \mathrm{~m}^{2} / \mathrm{s}$ \\
Equilibrium concentration & $0.266 \mathrm{~kg} / \mathrm{m}^{3}$ \\
Liquid phase viscosity & $2.85 * 10^{-4} \mathrm{~Pa} * \mathrm{~s}$ \\
Gas phase viscosity & $1.71 * 10^{-5} \mathrm{~Pa} * \mathrm{~s}$ \\
Liquid phase density & $650 \mathrm{~kg} / \mathrm{m}^{3}$ \\
Gas phase density & $4.4 \mathrm{~kg} / \mathrm{m}^{3}$ \\
\hline
\end{tabular}

Table 2: Set of simulation runs.

\begin{tabular}{|c|c|c|c|c|c|c|c|}
\hline$\overline{\text { Run }}$ & $\begin{array}{l}Q \\
\left(\mathrm{~m}^{3} / \mathrm{s}\right) * 10^{-6}\end{array}$ & $\begin{array}{l}\gamma \\
(\mathrm{N} / \mathrm{m}) * 10^{-3}\end{array}$ & $\begin{array}{l}D \\
\left(\mathrm{~m}^{2} / \mathrm{s}\right) * 10^{-6}\end{array}$ & $a$ & $C a$ & $P e$ & $\begin{array}{l}t^{*} \\
(\mathbf{s})\end{array}$ \\
\hline 1 & 1.00 & 19 & 6.38 & 0.33 & $1.68 * 10^{-6}$ & 1325 & 14238 \\
\hline 2 & 0.45 & 19 & 6.38 & 0.33 & $1.68 * 10^{-6}$ & 596 & 17983 \\
\hline 3 & 0.25 & 1.9 & 6.38 & 0.33 & $1.68 * 10^{-5}$ & 331 & 21340 \\
\hline 4 & 0.25 & 19 & 6.38 & 0.33 & $1.68 * 10^{-6}$ & 331 & 21340 \\
\hline 5 & 0.25 & 60 & 6.38 & 0.33 & $5.33 * 10^{-7}$ & 331 & 21340 \\
\hline 6 & 0.25 & 100 & 6.38 & 0.33 & $3.20 * 10^{-7}$ & 331 & 21340 \\
\hline 7 & 0.25 & 19 & 6.38 & 1.00 & $1.68 * 10^{-6}$ & 331 & 500 \\
\hline 8 & 0.25 & 19 & 63.8 & 0.33 & $1.68 * 10^{-5}$ & 33 & 4002 \\
\hline 9 & 0.20 & 19 & 6.38 & 0.33 & $1.68 * 10^{-6}$ & 265 & 22742 \\
\hline 10 & 0.10 & 19 & 6.38 & 0.33 & $1.68 * 10^{-6}$ & 132 & 27645 \\
\hline 11 & 0.05 & 1.9 & 6.38 & 0.33 & $1.68 * 10^{-5}$ & 66 & 33325 \\
\hline 12 & 0.05 & 19 & 6.38 & 0.33 & $1.68 * 10^{-6}$ & 66 & 33325 \\
\hline 13 & 0.015 & 19 & 6.38 & 0.33 & $1.68 * 10^{-6}$ & 19 & 45611 \\
\hline 14 & 0.005 & 19 & 6.38 & 0.33 & $1.68 * 10^{-6}$ & 6.7 & 57819 \\
\hline 15 & 0.0005 & 19 & 6.38 & 0.33 & $1.68 * 10^{-6}$ & 0.66 & 88752 \\
\hline 16 & 0.0 & 19 & 6.38 & 0.33 & $1.68 * 10^{-6}$ & 0.0 & 166132 \\
\hline
\end{tabular}

We will use run 2 and run 15 to illustrate typical features from the simulation. These runs are typical of two limiting regimes, one in which capillary forces are dominant and mass transfer occurs by diffusion, and another in which viscous forces dominate and mass transfer is by advection. In addition, we will discuss a case (run 12) involving capillary control in the phase partition, but advection control in mass transfer. As schematically depicted in Fig. 1c, the results show that the liquid phase consists of a main cluster (CC) and 
a number of discontinuous (DC) clusters at the perimeter of the $\mathrm{CC}$ (see Figs 4 and 5 for runs 2 and 15, respectively). These result from trapping of the liquid as the gas invades the liquid-occupied region. The patterns of the CC and/or the DCs depend on the value of the capillary pressure across the perimeter of each of these clusters as follows.

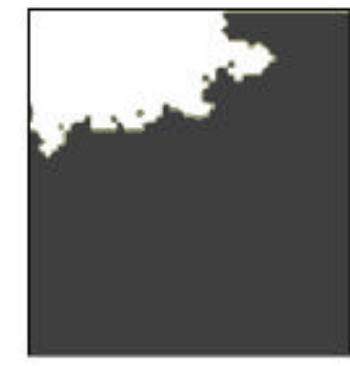

A

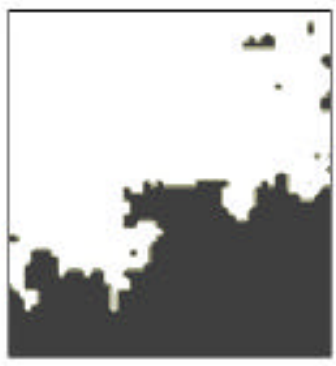

$c$

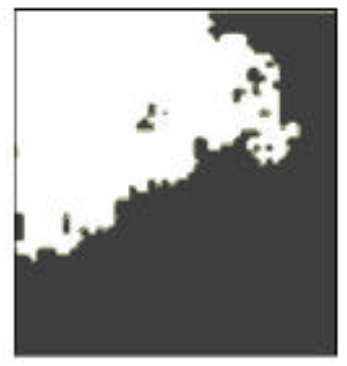

B

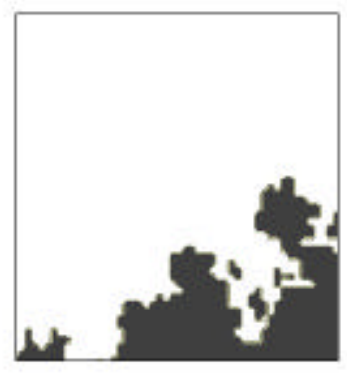

D

Figure 4 Phase distribution patterns for run $2\left(\mathrm{Pe}=596, \mathrm{Q}=0.45^{*} 10^{-6} \mathrm{~m}^{3} / \mathrm{s}\right)$ at four different gas saturation fractions corresponding to $20 \%, 40 \%, 60 \%$ and $80 \%$ of the total pore volume. The liquid phase is black and the gas phase is white.

If viscous forces are not sufficiently strong across a given cluster (for example, as in run 15 , Fig. 5), the capillary pressure variation is negligible, and the cluster takes the pattern of Invasion Percolation (IP), in which the next throat to be invaded by the gas is that with the smallest capillary threshold (here, the one with the largest size) among all perimeter throats of that cluster. This condition depends on the value of the capillary number, the rates of drying (which also set viscous pressure gradients [30]) and the size of the cluster. Under otherwise similar conditions, small DCs are more likely to follow an IP pattern. A detailed check of the sequence of invasion showed that run 15 followed IP rules at any time during the process, while run 2 did not. Patterns corresponding to IP and diffusion-only mass 
transfer were obtained in run 16 , where $P e=0$. The corresponding saturation patterns are shown in Fig. 6. Comparison with Figs. 4 and 5 shows substantial differences in the patterns between runs 2 and 15 (or 16) and some difference (at early times) between runs 15 and 16 . The latter reflects mass transfer effects, due to the different Peclet numbers in the two runs.

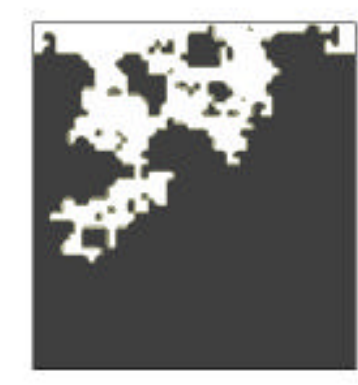

A

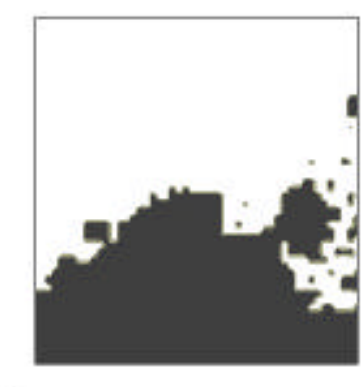

C

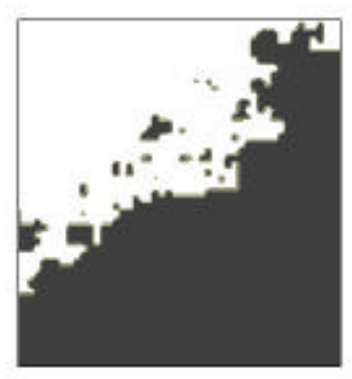

B

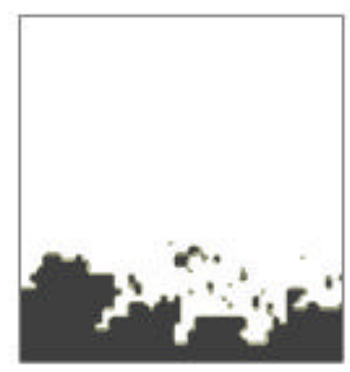

D

Figure 5 Phase distribution patterns for run $15\left(\mathrm{Pe}=0.66, \mathrm{Q}=0.5^{*} 10^{-9} \mathrm{~m}^{3} / \mathrm{s}\right)$ at four different gas saturation fractions corresponding to $20 \%, 40 \%, 60 \%$ and $80 \%$ of the total pore volume. The liquid phase is black and the gas phase is white.

Because of their relevance to real problems and the fact that capillary-dominated patterns eventually appear as late-time regimes, we provide here some additional discussion of their properties. We first recall that all simulations in this paper were conducted assuming the absence of film flows, thus DCs are hydraulically disconnected from each other and the CC. Under these conditions, in a capillary-controlled pattern (such as run 15 and 16), while each cluster obeys locally IP rules, the sequence of penetration is dictated by the mass transfer rates across the perimeter of each cluster. In the related study of Li and Yortsos [16] this process was termed local percolation. Clusters closer to the open boundary are subject to a faster evaporation, compared to those further away, and are emptied faster. The end 
result is the development of gradients in the size of the isolated liquid clusters, with clusters closer to the fracture having smaller size (see also below). These gradients reflect mass transfer, rather than viscous effects. Nonetheless, the pattern of the CC is still dictated by IP rules. Clearly, however, the overall pattern would be a function of the drying rates, namely of the value of the $P e$. We must note that under this regime of local percolation, different clusters may have different-size throats being invaded at the same time. In other words, it is possible that the drying of a given DC occurs by the emptying of a throat $i$, which is smaller than the largest perimeter throat, $j$, of another cluster, which due to negligible mass transfer is not being invaded (of course, as a result of capillary control, throat $i$ is the largest among all perimeter throats of the first cluster).

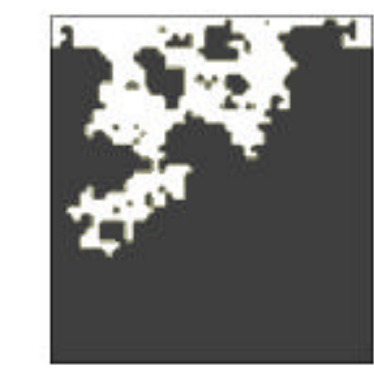

A

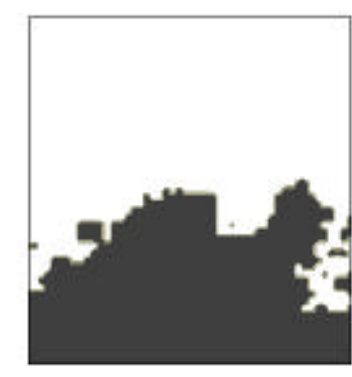

c.

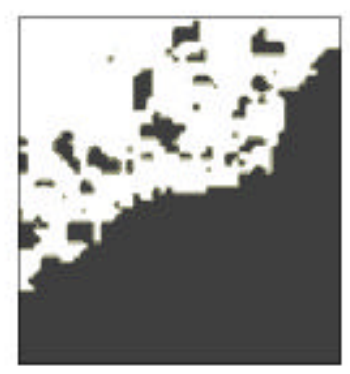

B

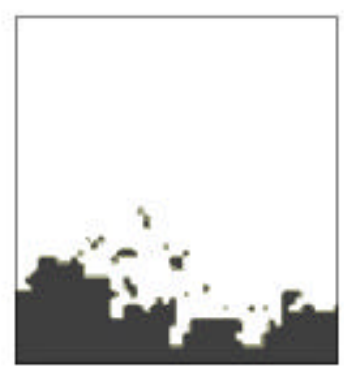

D

Figure 6 Phase distribution patterns for run $16\left(\mathrm{Pe}=0, \mathrm{Q}=0.0 \mathrm{~m}^{3} / \mathrm{s}\right)$ at four different gas saturation fractions corresponding to $20 \%, 40 \%, 60 \%$ and $80 \%$ of the total pore volume. The liquid phase is black and the gas phase is white.

The above analysis of capillary-controlled patterns is based on the assumption of negligible film flow. If, on the other hand, hydraulic continuity exists among all clusters (for example through liquid films) then, in the small $\mathrm{Ca}$ limit, the next throat to be penetrated 
will be the throat with the smallest threshold among all perimeter throats of all clusters. In the liquid-to-gas phase change study of $\mathrm{Li}$ and Yortsos [16], this was termed global percolation. In the absence of viscous or gravity gradients, such a process could lead to a deep penetrating front of a fractal nature, and liquid saturation gradients will not develop, in the sense that the proximity of a particular front site to the open boundary cannot influence the time at which it is invaded. Gradients in saturation will develop in the case of the viscous-capillary-gravity competition. This regime was implied in many previous studies, including the work of Tsimpanogiannis et al. [30]. We also note that deep penetrating fronts of fractal nature were not observed in the experiments of Ho and Udell [10]. Nonetheless, because of possible qualitative differences, it is evident that establishing hydraulic continuity is an important role played by liquid films and needs to be further explored.

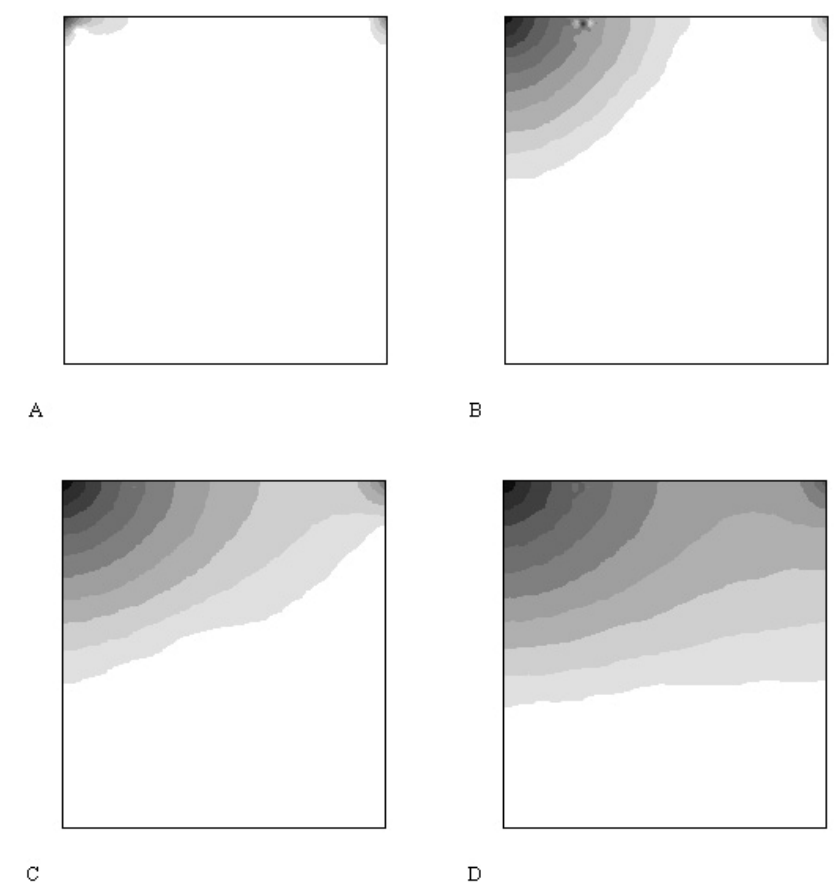

Figure 7 Concentration patterns for run $15\left(\mathrm{Pe}=0.66, \mathrm{Q}=0.5^{*} 10^{-9} \mathrm{~m}^{3} / \mathrm{s}\right)$ at the four different times.

In the limit when capillarity is negligible (as in run 2), the pattern deviates substantially from IP and almost follows a piston-like displacement (PD) (Fig. 4). Under these conditions, the capillary resistance of a throat is negligible, and the pattern is 
exclusively determined by mass transfer considerations, much like in the dissolution of a solid. The rate of generation of DCs and their size are smaller and the liquid phase consists mostly of a CC. Such conditions are not likely to persist for a long time in the typical case, however, where late-time drying patterns are likely to be of the IP type. We need to add that when viscous forces in the gas phase are important in setting the pattern (as is the case in run 2 , for example), the receding of the CC has some of the properties of IPSG in a fracturematrix system (Haghighi et al. [8]). In such cases, the higher capillary pressure upstream leads to a preferential invasion in the matrix in the upstream direction, thus leading to patterns that appear to be slanted (from the upstream to the downstream direction) as shown in run 2 (Fig. 4).

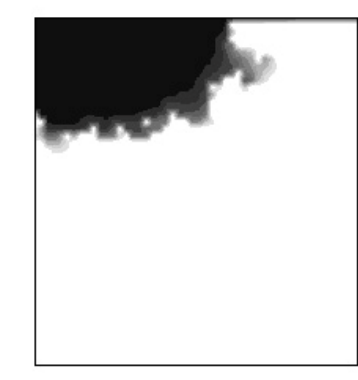

A

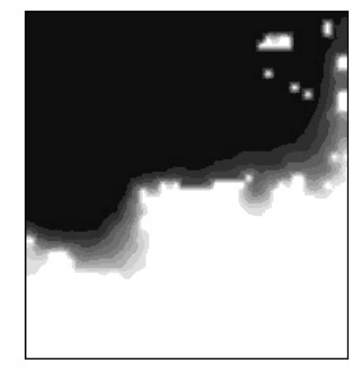

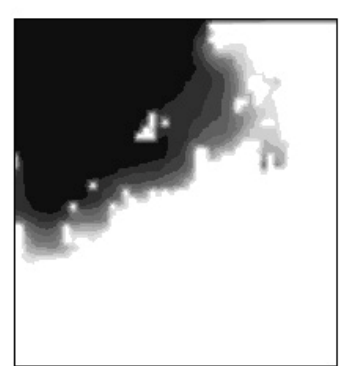

B

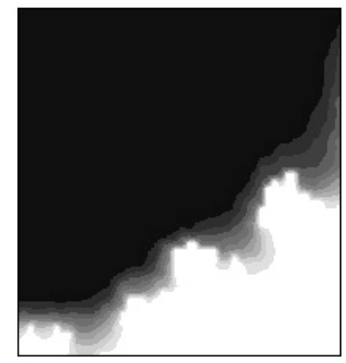

D

Figure 8 Concentration patterns for run $2\left(\mathrm{Pe}=596, \mathrm{Q}=0.45^{*} 10^{-6} \mathrm{~m}^{3} / \mathrm{s}\right)$ at the four different occupation fractions. Darker colors indicate smaller concentrations.

Regardless of the magnitude of the capillary forces, the presence of discontinuous clusters is very significant in the development of the pattern. These clusters result in the 
screening of a part of the $\mathrm{CC}$ from the fracture, hence from the region of high concentration gradients, and lead to a balanced rate of drying between the two types of clusters, through the following stabilizing feedback mechanism. For example, if the mass transfer rates from the $\mathrm{CC}$ are too large, they will result in a faster rate of consumption of the $\mathrm{CC}$, leading to the generation of a larger number of DCs, which in turn screen the $\mathrm{CC}$ from further mass transfer and slow down its rate of drying. Conversely, if the rate of drying of the $\mathrm{CC}$ is too small, most of the reduction in liquid saturation occurs from the DCs, the size and number of which decrease, leading to an increased mass transfer and a subsequent increased rate of consumption of the CC. It follows that for fixed values of the capillary and Peclet numbers, the statistics of the DCs, namely their number density and size distribution, remain approximately constant. In particular, the region where the DCs reside (which we may denote as the front region) is approximately of a constant width (Figs. 46). We should mention that similar findings were observed by Prat and Bouleux [23], although in the different situation where the gradients are provided by gravity rather than mass transfer. The size of the frontal region can be estimated using arguments from Invasion Percolation with Trapping (IPT) as described in the Appendix B.

The screening of the $\mathrm{CC}$ from the region of high mass transfer is evident in the concentration profiles (Figs. 7-8). Depending on the value of the Peclet number and the location of the front, the concentration field in the gas phase may or may not be sensitive to the detailed structure near the front. For small values of the Peclet number (as in run 15, Fig. 7), the process is almost diffusion-controlled and the concentration resembles the much smoother concentration field surrounding an effective continuum (as also found by Li and Yortsos [16]). This despite the rather complex geometry of the front (see Fig. 5), which is rather complex (and in fact, fractal, e.g. see Feder [6]). Almost identical patterns were obtained for run 16 and they are not shown. As advection increases and at early times, however, the region of concentration gradients is a narrow boundary layer surrounding the front. At later times (panel 3 in Fig. 8), this is not uniformly developed, as poorly accessible regions cannot be effectively probed by the flow field. This mass transfer effect is also evident in the simulations of run 12 (Fig. 9). This run has a Peclet number almost two orders of magnitude larger than in run 15, although the sequence of the liquid patterns is almost identical (and is not shown here for simplicity). However, because of the much stronger 
advection effects, concentration profiles are much steeper in most places in run 12, resulting in proportionally higher drying rates (Fig. 9). At the same time, the effect is non- uniform spatially, and there exist regions in which diffusion is controlling. Strong advection effects similar to these were also reported in a related problem of bubble growth driven by heat transfer [25].

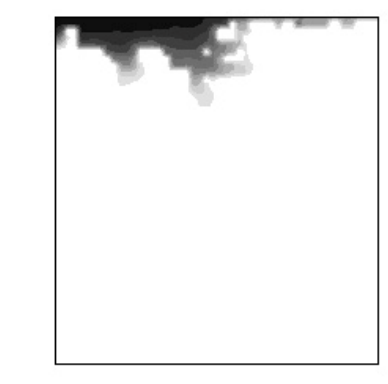

A

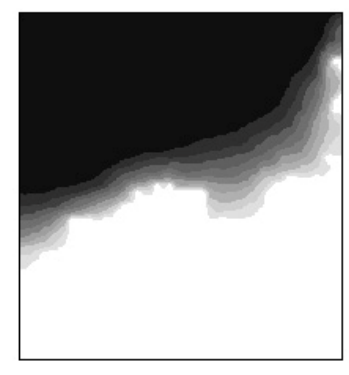

$\mathrm{C}$

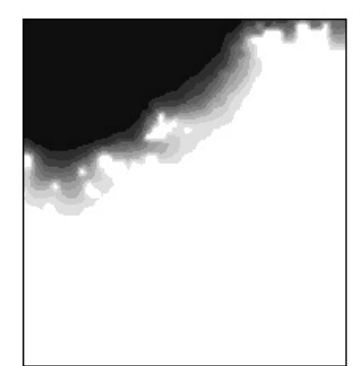

B

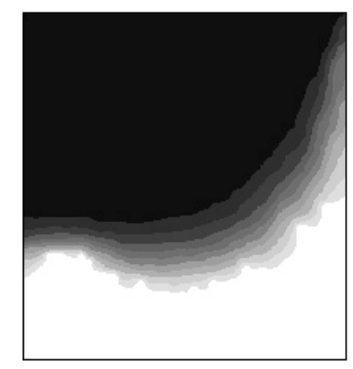

Figure 9 Concentration patterns for run $12\left(\mathrm{Pe}=66, \mathrm{Q}=0.05^{*} 10^{-6} \mathrm{~m}^{3} / \mathrm{s}\right)$ at the four different occupation fractions. Darker colors indicate smaller concentrations.

Runs 2 and 15 are typical of the two limiting regimes and can be analyzed in a relatively straightforward fashion. Intermediate patterns are more difficult to analyze, as they have features from both regimes. When both capillarity and viscous forces compete, the receding of the drying front does not follow strictly IP or PD rules. Depending on the rates of evaporation as well as the size of the individual clusters, there may exist more than one pore of the PE type at the same time, and these pores may not necessarily correspond to the IP or the PD sequence. We must point out that Tsimpanogiannis et al. [30] estimated the spatial extent over which the front follows IP rules using scaling arguments, from which the following relation was developed for 3-D patterns 


$$
s_{f t} \propto\left(\frac{2 S X_{f t}}{C a}\right)^{0.47}
$$

where $\sigma_{f t}, \mathrm{X}_{f t}, \Sigma$ are non-dimensionalised front width, frontal position and variance of the pore size distribution, respectively. Implicit to this relation was the assumption of global percolation (namely of hydraulic continuity via film flow). This result is not expected to hold here. Nonetheless, clusters of small size will erode following local IP rules. In largersize clusters, however, the spatial extent over which IP rules apply would be limited. Thus, the problem acquires some of the characteristics of IPSG [30]. As time increases, drying rates decrease, as the front recedes further away, and the width of the front, where IP is exhibited, increases accordingly. One expects that after sufficiently long time, the process will approach a state where IP applies for all clusters. Some arguments from IPT are furnished in the Appendix B to estimate the size of the front in this problem.
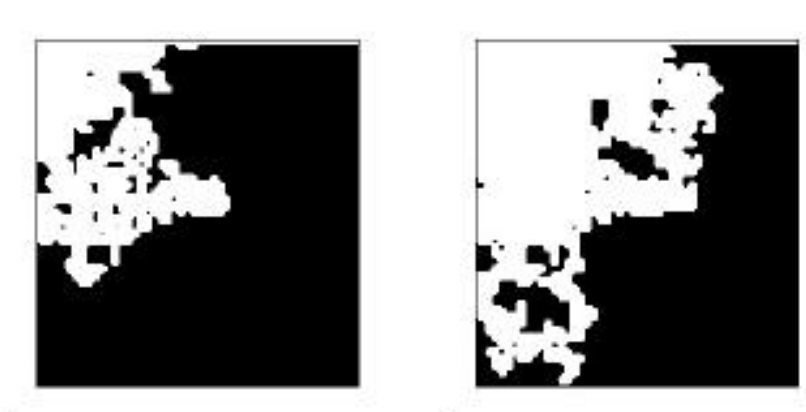

A.

B
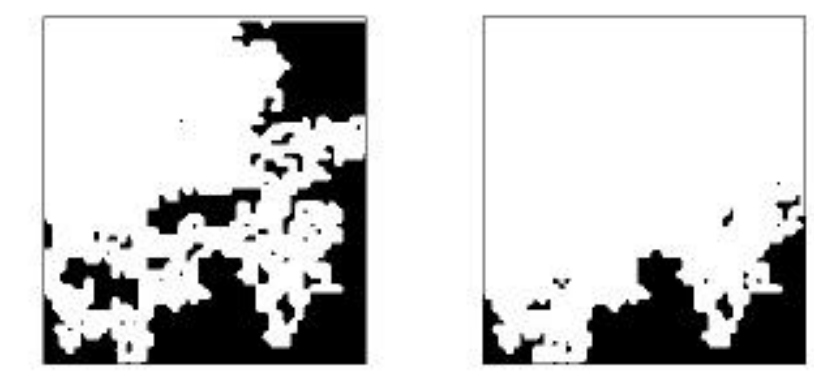

c

D

Figure 10 Phase distribution patterns for run $4\left(\mathrm{Pe}=331, \mathrm{Q}=0.25 * 10^{-6} \mathrm{~m}^{3} / \mathrm{s}\right)$ at four different gas saturation fractions corresponding to $20 \%, 40 \%, 60 \%$ and $80 \%$ of the total pore volume.

The liquid phase is black and the gas phase is white. 
A typical case in which viscous and capillary forces compete is run 4, shown in Figs. 10-11. The invasion pattern belongs to neither of the two limiting regimes. There is evidence of IPSG in the matrix, similar to run 2, but also a multiple number of DCs, similar to run 15. The concentration profiles for this run (Fig. 11) are similar to those of run 12, and suggest substantial concentration gradients in boundary layer regions near the front. At the same time, regions far from the fracture are effectively being screened, despite the large Peclet number in this run, leading to mass transfer similar to the diffusion-controlled case (run 15).

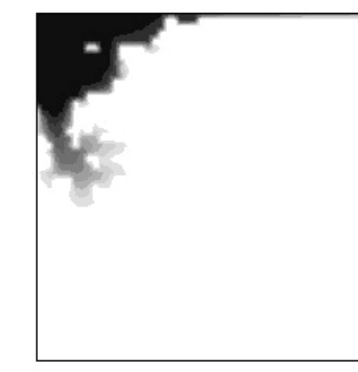

A

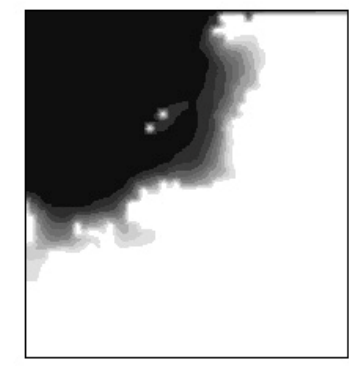

$\mathrm{C}$

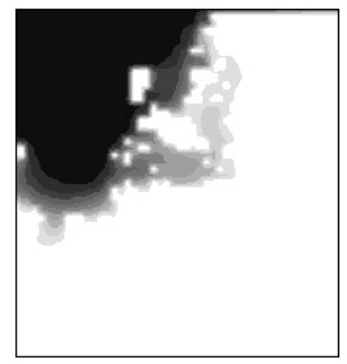

B

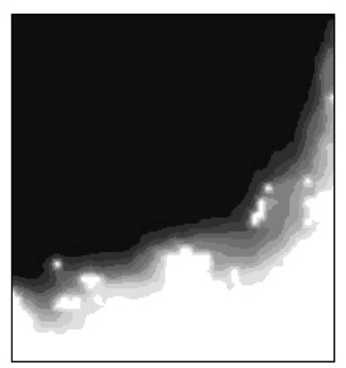

D

Figure 11 Concentration patterns for run $4\left(\mathrm{Pe}=331, \mathrm{Q}=0.25^{*} 10^{-6} \mathrm{~m}^{3} / \mathrm{s}\right)$ at the four different occupation fractions. Darker colors indicate smaller concentrations.

In macroscopic continuum models a quantity of interest is the transverse-averaged saturation profiles. Results for runs 15, 12, 4 and 2 are shown in Figs. 12-15, respectively, for four different values of the fraction of the liquid volume occupying the matrix (and which corresponds to the four panels of the previous figures). As expected, runs 15 and 12 have almost identical profiles (Figs. 12-13), their patterns being dictated by IP rules, even though their mass transfer characteristics are not the same. Differences due to the latter are 
reflected in their drying rates to be discussed below. Gradients in saturation develop, due to the continuous receding of the front. As discussed above and in the Appendix B, these gradients reflect the screening of inaccessible, deeper-lying parts of the liquid front from high concentration gradients, the resulting limited mass transfer, and the development of a frontal region of finite width (which is constant in the absence of viscous effects, but increases with time in their presence). The profiles in runs 4 and 2 (Figs. 14-15) reflect a more uniform penetration, at early times, due to the preferential receding of the liquid in the upstream direction as a result of strong viscous effects. These are not unlike the profiles in the drainage of a matrix block [8]. As time increases, however, all profiles eventually approach a state which progressively becomes capillary- and diffusion-controlled.

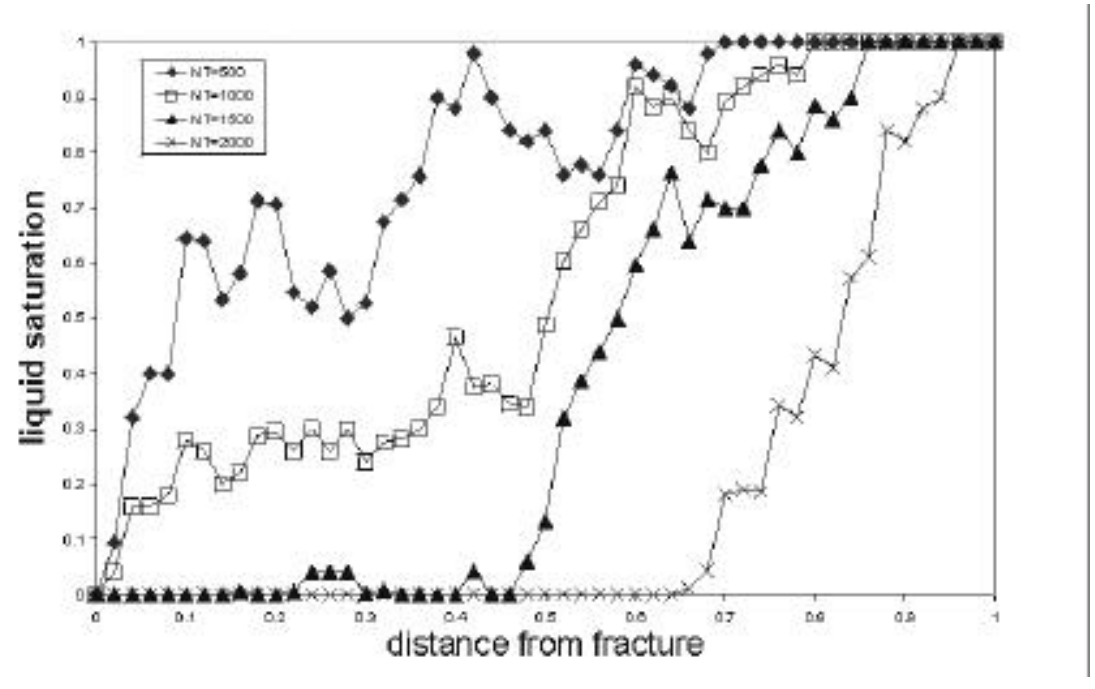

Figure 12 Transversely-averaged saturation profiles for run 15 at the four different occupation fractions. 


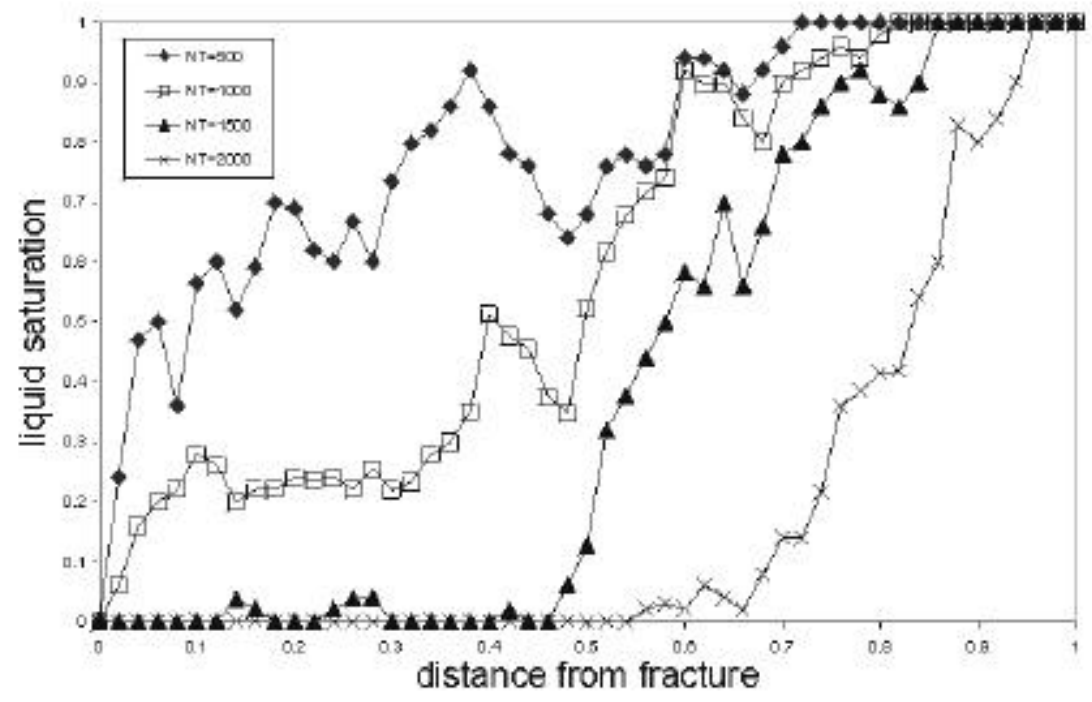

Figure 13 Transversely-averaged saturation profiles for run 12 at the four different occupation fractions.

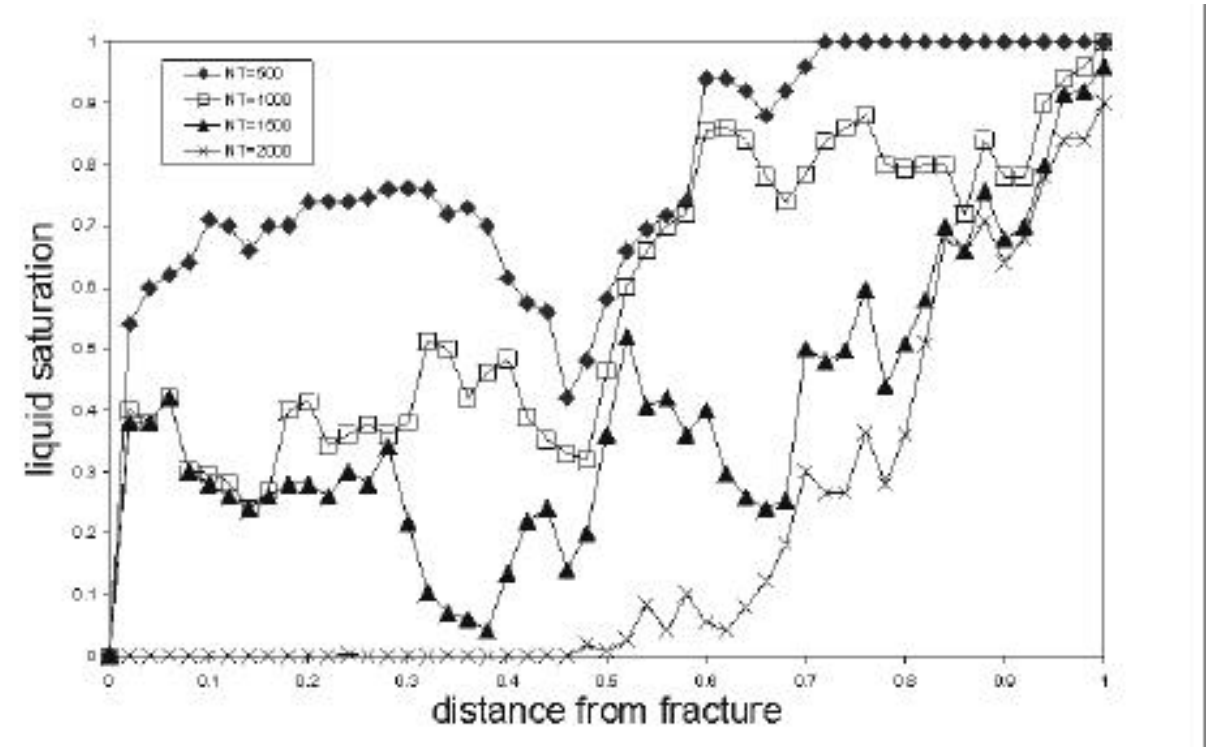

Figure 14 Transversely-averaged saturation profiles for run 4 at the four different occupation fractions. 


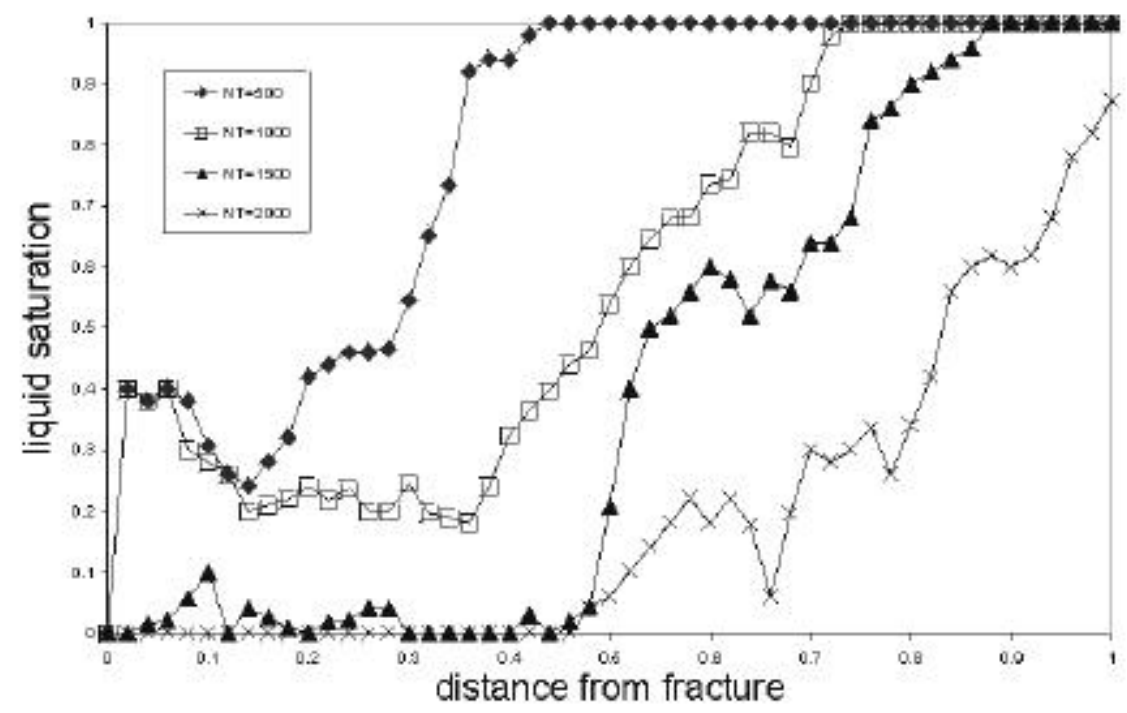

Figure 15 Transversely-averaged saturation profiles for run 2 at the four different occupation fractions.

Fig. 16 shows drying curves for the various runs simulated. As discussed, the time was made dimensionless with the time $t^{*}$ it would take to empty the matrix block under conditions of the maximum rate. It then follows that the slope of the drying curves is the dimensionless rate of drying (relative to the maximum drying rate). Note that because the maximum rate depends on the Peclet number in the fracture (roughly as $P e^{1 / 3}$ ) this plot emphasizes processes under convection control and should be interpreted with care, as far as the upscaling of the process is concerned. The figure shows clearly the existence of the conventional Constant-Rate-Period (CRP) (with the exception that film flow is not involved here) followed by a period of continuously declining rates. From an analysis of the patterns, we have found that the CRP lasts roughly until the time when the $\mathrm{CC}$ has lost continuity with the fracture. This is in qualitative agreement with indirect experimental findings from tests on chalk samples initially containing liquid pentane and dried by methane injection along the fracture [15]. In addition, macroscopic arguments set forth by Stubos and Poulou [29] on the basis of the same experimental data have led to the same conclusion. Indeed, as long as the liquid is continuously connected to the fracture, the combination of enhanced evaporation rate (through the coefficient given by equation (5)) and capillary gradients leads to a constant drying rate. This is evident when considering the low Peclet number run 15 or 
the IP run 16 in Fig. 16 where the CRP is very short due to the fast receding of the liquid phase from the fracture. A close examination of the drying curves shows that the highest rate and the longest CRP are found in run 4. In this "critical" case the gas pressure developing close to the entrance of the fracture is high enough to cause the breaking of menisci in the left side of the matrix block. On the contrary, this cannot happen on the right side sustaining for relatively large times sufficient liquid contact with the fracture (see patterns in Fig. 10). Thus the constant rate during the CRP reflects the detailed mass transfer characteristics during the process and is realization-dependent. By increasing the interfacial tension value in run 4 (runs 5 and 6 of Table 2), we find that the drying rate and the CRP drop to levels similar to the ones determined for the low Peclet number cases (Fig. 17). In fact, the gas pressure is not any more sufficient to overcome the capillary thresholds and the case resembles more and more to IP. When $\gamma$ decreases on the other hand (run 3), all menisci in contact with the fracture break at early times and the CRP is very short. The drying rate also drops accordingly (Fig. 17). For the case of IP-controlled processes, the end of the CRP in terms of the amount of liquid left can be readily estimated from the analysis in the Appendix B.

\section{DISCUSSION}

The above analysis was based on a number of simplifying assumptions, most of which were made to illustrate various effects. We single out the small size of the fracture pores and throats taken, in order to allow for a study of the viscous-capillary competition at the rates considered. At a larger contrast of fracture/matrix capillary and permeability characteristics, the onset of a PD regime will be delayed as far as the flow rate is concerned. We also note that the Peclet number in some of the runs can be considered unrealistically high. Such simulations were purposely made to exaggerate the effect of advection in mass transfer. In general, flow rate-driven effects will dominate the process at early times, and particularly during the CRP, which will be controlled, as a result, by the detailed geometry and process characteristics. It is in this regime and time period, where pore-network simulations, such as these, can have a significant impact. At later stages, the front recedes, viscous effects and advective mass transfer become less important and the process becomes capillary- and diffusion-controlled. In this regime, the analysis discussed in the Appendix B 
applies and various results from the theory of Invasion Percolation with Trapping can be used to describe the process.

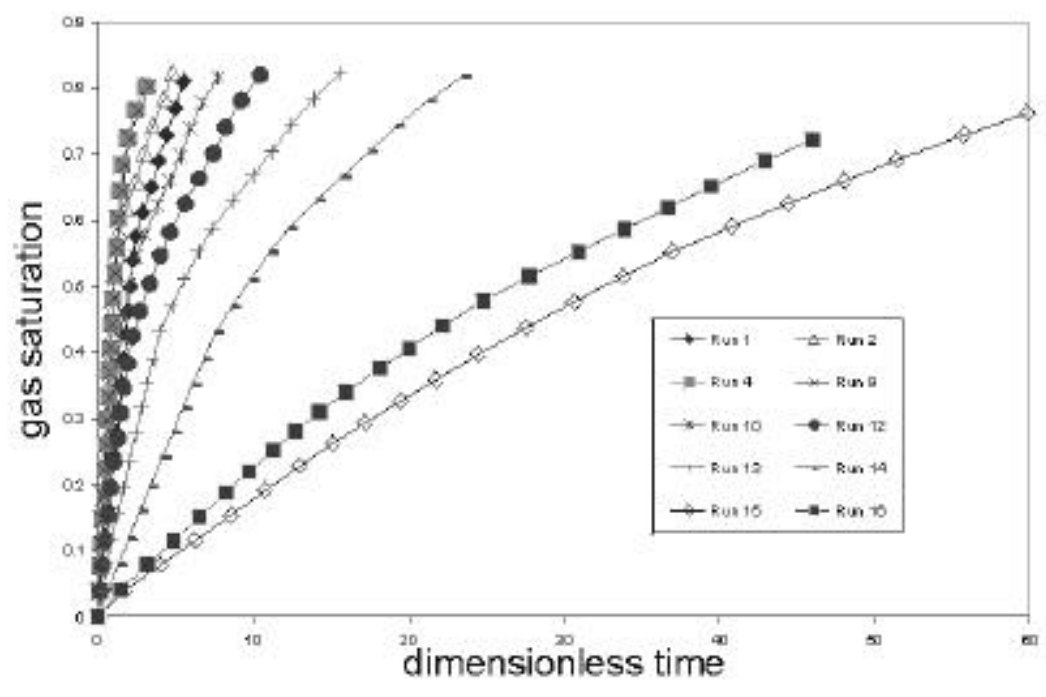

Figure 16 Drying curves (gas volume fraction vs. dimensionless time) for various runs.

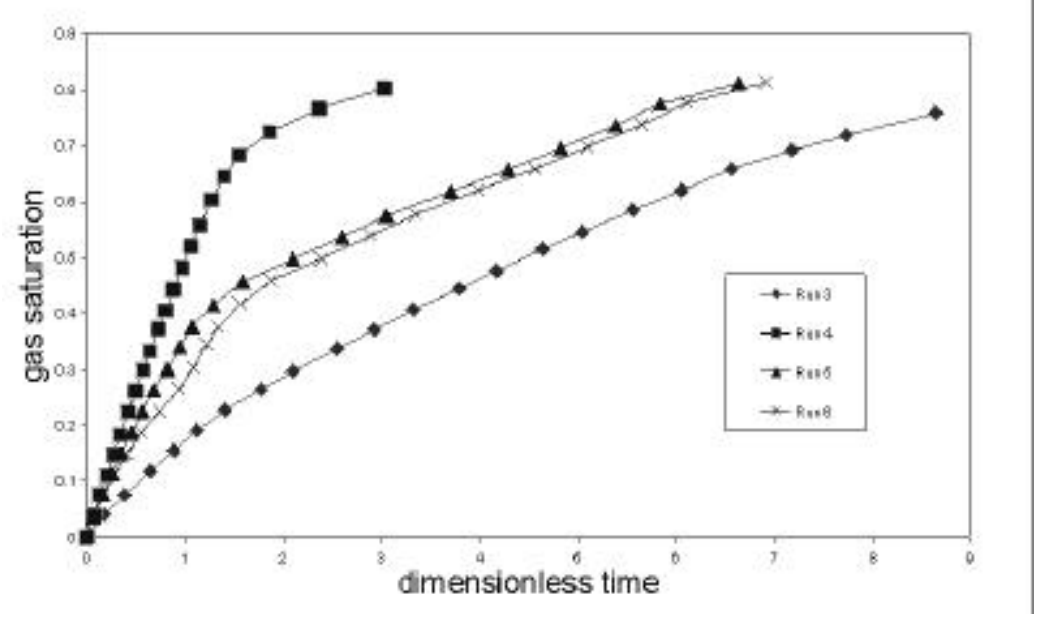

Figure 17 Drying curves (gas volume fraction vs. dimensionless time) for runs 3-6. 
A key simplification made in the paper concerns the absence of liquid films. As discussed, these can be important in providing hydraulic continuity between clusters, which can drastically alter the patterns and the location and size of the DCs and the CC, and can possibly affect macroscopic capillary pressure-saturation relationships. Work in this direction, in particular in the incorporation of film flows in the pore-network simulation, is currently under way. At present, their effect is difficult to assess. We also need to mention the restriction to single-component liquids, which eventually leads to the complete evaporation of the liquid clusters. In the presence of a non-volatile component, the disconnected liquid clusters will not disappear, but will affect adversely the mass transfer rates. Such effects need to be considered as well.

The results can be used for upscaling to macroscopic continuum models. Following the CRP, where the process becomes capillary- and diffusion-controlled, the theory of IPT discussed in the Appendix B can be used to elucidate the distribution of the liquid phase, and the size and extent of the DC region. This information can then be utilized to construct model diffusion problems, the solution of which can be implemented in the calculation of needed coefficients in macroscopic models (for example, as required in Tsimpanogiannis et al. [30]). Such work is also under way. On the other hand, the CRP is not necessarily amenable to such a statistical theory description, given that non-local effects of rate and viscous forces (or finite-size effects in the case of capillary control) are important. The solution of this problem must be sought by conducting extensive pore-network simulations, including the ensemble-averaging of results over many realizations, something that was not done here.

\section{CONCLUSIONS}

In this paper we presented a pore-network model for the drying of a singlecomponent liquid in porous media. The model accounts for various processes at the porescale including mass transfer by advection and diffusion in the gas phase, viscous flow in liquid and gas phases and capillary effects at the gas-liquid menisci in the pore network. A 2-D version of the model under isothermal conditions in a rectilinear horizontal geometry

was formulated. Drying is driven by the flow of a purge gas injected at a constant rate. The 
problem is characterized by two dimensionless parameters, a diffusion-based capillary number, $\mathrm{Ca}$, and a Peclet number, $P e$, in addition to the various geometrical parameters of the pore network. Results on the evolution of the liquid saturation, the trapped liquid islands and the drying rate were obtained as a function of time and the dimensionless parameters. In the absence of liquid film flow, the importance of trapped liquid islands in screening mass transfer to the continuous liquid cluster was emphasized. This results in saturation gradients, and the development of patterns which are different than in the case of flow continuity between the different clusters. For fixed parameter values, the drying front does not in general obey invasion percolation rules. However, as drying progresses, and depending on the relative magnitude of the capillary and Peclet numbers, a transition to a percolationcontrolled problem occurs. In fact, it is likely that such is the case in many realistic situations. Effects of capillarity and mass transfer on saturation profiles and drying rates were discussed. The results provide insight on the process. The pore network simulation can also be used to provide expressions for various coefficients in macroscopic continuum models, currently treated empirically. Work in this direction is in progress.

\section{APPENDIX A}

The essential elements of the algorithm are described below:

1. A random network of pores and throats is created using a uniform size distribution between the specified values.

2. The pressure drop along the fracture is calcula ted using equations (7) and (8).

3. All liquid-gas interfaces of isolated liquid clusters (DC's and the CC) are located and the type (I-CE or I-PE) of their interface pores is identified.

4. Flow conductivities at all network throats are calculated. When a liquid meniscus within a throat is found to be stationary then a zero hydraulic conductivity is assigned to the gas phase adjacent to it.

5. All throats where gas penetration occurs as the local capillary pressure exceeds the capillary pressure threshold are identified. If there is no such throat at a specific cluster, then the gas penetrates the throat that has the lowest capillary threshold along the interface, i.e. the one with the largest radius. 
6. The evaporation rate through every throat across all interfaces is calculated using equation (1).

7. The pressure fields in both liquid and gas phases are solved, using equations (7) and (8) and (11).

8. The I pore of type PE that will be emptied first at the current flow rates is located and the corresponding time required is identified. These pores loose mass due to both evaporation and the viscous flows of the capillary-pumping phenomenon (as shown by equation (11)).

9. The time step is selected taking care not to allow the concentration value at a gas pore to drop bellow zero or exceed the equilibrium concentration.

10. The concentration field is calculated in the gas phase using equations (3) and (4) for the current time step. This calculation is straightforward based on the values of concentration at the end of the previous time step.

11. The gas saturation is calculated through equation (12) at all I pores of type PE for the current flow rates using the current time step.

12. If at the end of the current time step a pore becomes fully filled with gas (I pore of type $\mathrm{CE}$ ) at any of the liquid clusters, the algorithm returns to step 3. If, despite the fact that gas saturation at all penetrated interface pores increases, there is no pore completely filled with gas at the current time step, then the return is to step 6.

The above procedure is repeated until all liquid in the porous media has evaporated.

\section{APPENDIX B}

Discontinuous clusters form as the perimeter of the $\mathrm{CC}$ recedes, due to gas invasion, and the receding phase becomes trapped. In the absence of mass transfer considerations and under capillary control, this is the well-known mechanism of Invasion Percolation with Trapping. In the latter, the statistics of the trapped clusters can be determined from knowledge of the percolation probability, $q$, at which the defending phase (here the liquid) is being invaded. In the particular process under consideration, the invasion of the $\mathrm{CC}$ is a drainage process in which the invading (gas) phase percolation probability is near its percolation threshold

$$
p \sim p_{c}(=1 / 2 \text { for a } 2-\mathrm{D} \text { square lattice })
$$


Then, the defending phase is at percolation probability $q=1-p \sim 1-p_{c}$. Knowledge of $q$ allows one to estimate various statistics of the disconnected, trapped clusters, since the latter can be accurately approximated by the finite-size clusters of a mixed site-bond percolation process at percolation probability $q$. For example, the size of the largest DC follows the percolation scaling

$$
\xi \sim\left|q-\left(1-p_{\mathrm{c}}\right)\right|^{-v}
$$

where $v$ is the correlation length exponent. Statistics are also available (or can be obtained) for other properties of these finite-size clusters. In the above, it is implicitly assumed that $p_{c}$ $<1 / 2$, and that $\xi<<N$. When the latter condition fails, as is the case in the simulations here, where $p_{c}=1 / 2$, the largest (cut-off) size is set by the width of the lattice $N$. The above analysis can be used to estimate the statistics of screened DCs around the front, which are not subject to substantial drying rates, although not of the DCs at the frontier with the gas phase, which are being exposed to strong concentration gradients and a time-varying size.

In the presence of viscous effects, the invasion process is similar to IPSG, in which, in the absence of mass transfer effects, the largest size of the DCs is set by the Bond number of the process, namely

$$
\xi \sim \mathrm{B}^{-\frac{v}{v+1}}
$$

In drying, an equivalent Bond number can be defined as follows [30]

$$
B=\frac{C a}{2 S X_{f t}}
$$

However, the incorporation of an IPSG theory in the trapping problem is yet to be done. In general, we expect that such a theory would lead to the result that the average size of the DCs becomes smaller as viscous forces increase, as is the case with the problem without trapping [30], and also as shown in the simulations. 
We also conjecture that the same length scale, namely the average size of the largest DC, is also the scale for the width of the frontal region, namely the region containing the trapped clusters (DC). Indeed, as we argued in the main text, the mass transfer screening from the DCs sets the balance between them and the CC. We expect that at least for diffusion-controlled problems, this screening will be effectively set by the distance between DCs, which are all sources of constant concentration. Given that the only characteristic length in the problem is the average size of the DCs completes the argument in support of this conjecture.

\section{REFERENCES}

[1] Bruin S, Luyben KCAM. Drying of food materials; A review of recent developments. Advances in Drying 1980;1: 155-215.

[2] Ceaglske NH, Hougen OA. Drying granular solids. Ind Eng Chem 1937;29: 805-813.

[3] Chandler R, Koplik J, Lerman K, Willensen JF. Capillary displacement and percolation in porous media. J Fluid Mech 1982;119: 249-267.

[4] Chen P, Pei DCT. A mathematical model of drying processes. Int J Heat Mass Transfer 1989;32: 297-310.

[5] Fatt I. The network model of porous media. Trans AIME 1956;207: 144-181.

[6] Feder J. Fractals. New York: Plenum, 1988.

[7] Fortes M, Okos MR. Drying theories:Their bases and limitations as applied to food and grains. Advances in Drying 1980;1: 119-154.

[8] Haghigi M, Xu B, Yortsos YC. Visualization and simulation of immiscible displacement in fractured systems using micromodels: I.Drainage. J Coll Interf Sci 1994;166: 168-179.

[9] Hartley JG. Coupled heat and moisture transfer in soil: A review. Advances in Drying 1987;4: 199-248.

[10] Ho CK, Udell KS. An experimental investigation of air venting of volatile liquid hydrocarbon mixtures from homogeneous and heterogeneous porous media. J Contam Hydrol 1992;11: 1-26.

[11] Ho CK, Udell KS. Mass transfer limited drying of porous media containing an immobile binary liquid mixture. Int J Heat Mass Transfer 1995;38: 339-350. 
[12] Jia C, Shing K, Yortsos YC. Visualization and simulation of non-aqueous phase liquids solubilization in pore networks. J Contam Hydrol 1999;35: 363-387.

[13] Laurindo JB, Prat M. Numerical and experimental network study of evaporation in capillary porous media. Phase distributions. Chem Eng Sci 1996;51: 5171-5185.

[14] Laurindo JB, Prat M. Numerical and experimental network study of evaporation in capillary porous media. Drying rates. Chem Eng Sci 1998;53: 2257-2269.

[15] Le Gallo Y, Le Romancer JF, Bourbiaux B, Fernades G. SPE paper 1997;38924.

[16] Li X, Yortsos YC. Theory of multiple bubble growth in porous media by solute diffusion. Chem Eng Sci 1995;50: 1247-1271.

[17] Luikov AV. Heat and mass transfer in Capillary-Porous Bodies. Oxford: Pergamon Press, 1966.

[18] Nowicki SC, Davis HT, Scriven LE. Microscopic determination of transport parameters in drying porous media. Drying Tech 1992;10: 925-946.

[19] Pan SX, Davis HT, Scriven LE. Modeling moisture distribution and binder migration in drying paper coatings. Tappi Journal 1995;78: 127.

[20] Panagiotou NM, Stubos AK, Bamopoulos G, Maroulis ZB. Drying kinetics of a multicomponent mixture of organic solvents. Drying Tech 1999;17: 2107-2122.

[21] Pot V, Appert C, Melayah A, Rothman DH, Zaleski S. Interacting lattice gas automaton study of liquid-gas properties in porous media. J Phys II 1996;6: 1517-1534.

[22] Prat M. Isothermal drying of non-hygroscopic capillary-porous materials as an invasion percolation process. Int J Multiphase Flow 1995;21: 875-892.

[23] Prat M, Bouleux F. Drying of capillary porous media with a stabilized front in two dimensions. Phys Rev E 1999;60: 5647-5656.

[24] Quintard M, Whitaker S. Convection,dispersion, and interfacial transport of contaminants: Homogeneous porous media. Adv Water Res. 1994;17: 221-239.

[25] Satik C, Yortsos YC. A pore network study of bubble growth in porous media driven by heat transfer. J Heat Trans 1995;118: 455-462.

[26] Shaw TM. Drying as an immiscible displacement process with fluid counterflow. Phys Rev Lett 1987;59: 1671-1674.

[27] Simpson WT. Drying wood: A review. Drying Tech 1983;2: 235-264.

[28] Simpson WT. Drying wood: A review. Drying Tech 1984;3: 353-368. 
[29] Stubos AK, Poulou S. Oil recovery potential from fractured reservoirs by mass transfer processes. SPE paper 1999; 56415.

[30] Tsimpanogiannis IN, Yortsos YC, Poulou S, Kanellopoulos N, Stubos AK. Scaling theory of drying porous media. Phys Rev E 1999;59: 4353-4365.

[31] Van Brakel J. Mass transfer in convective drying. Advances in Drying 1980;1: 212267.

[32] Waananen KM, Litchfield JB, Okos MR. Classification of drying models for porous solids. Drying Tech 1993;11: 1-40.

[33] Whitaker S. Heat and mass transfer in granular porous media. Advances in Drying 1980;1: 23-61.

[34] Xu B, Yortsos YC, Salin D. Invasion percolation with viscous forces. Phys Rev E 1998;57: 739-751.

\section{NOMENCLATURE}

\section{Roman Letters}

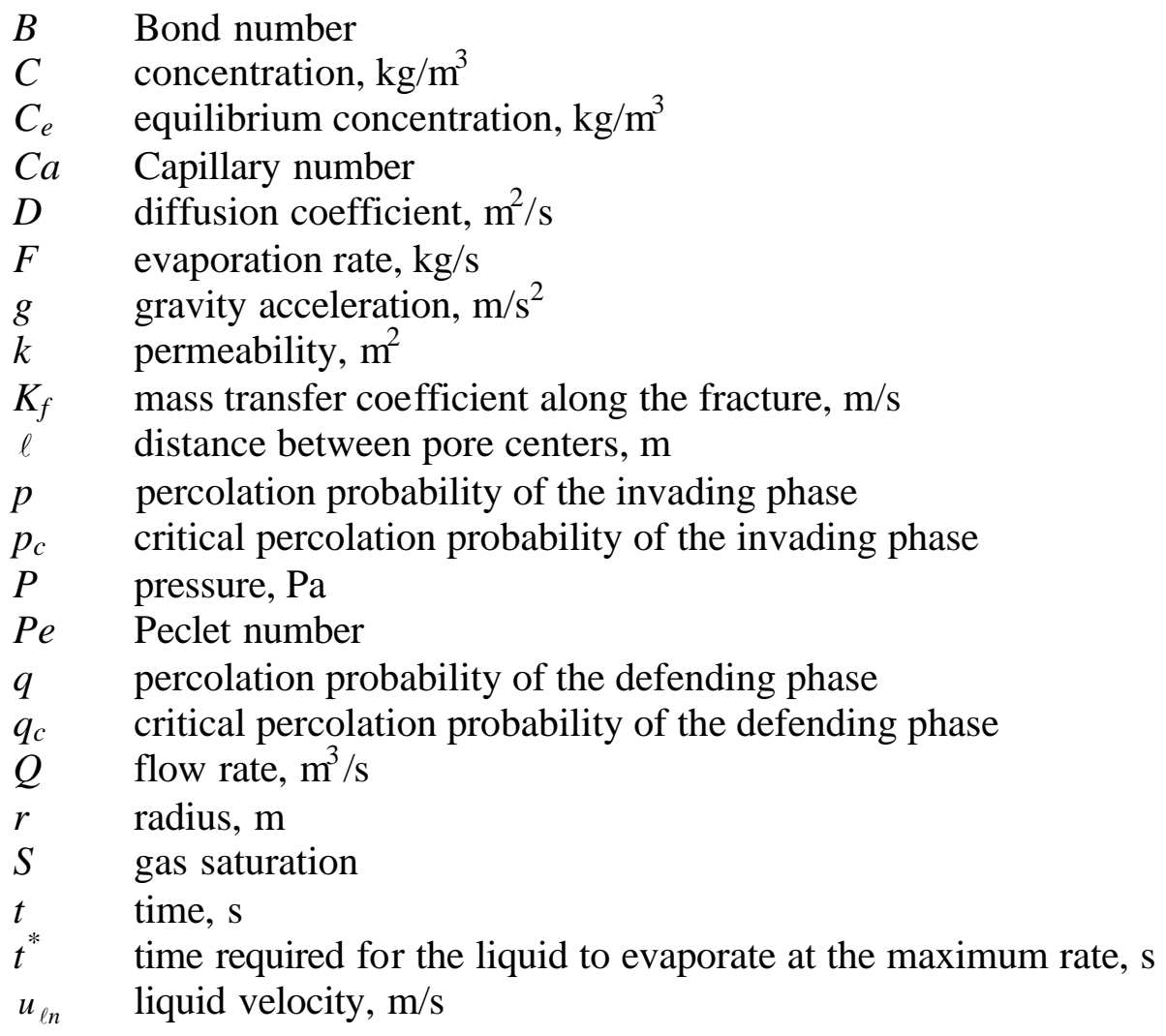


$u_{\mathrm{g} n} \quad$ gas velocity, $\mathrm{m} / \mathrm{s}$

$u_{n} \quad$ velocity of the interface, $\mathrm{m} / \mathrm{s}$

$V_{f} \quad$ gas velocity through the fracture, $\mathrm{m} / \mathrm{s}$

\section{Greek letters}

$a$ exponent of equation (5) that depends on the geometry of the fracture

$\gamma \quad$ interfacial tension, $\mathrm{N} / \mathrm{m}$

$\mu_{g} \quad$ viscosity of the gas phase, $\mathrm{Pa} * \mathrm{~s}$

$\mu_{\ell} \quad$ viscosity of the liquid phase, $\mathrm{Pa}^{*} \mathrm{~s}$

$\rho_{\mathrm{g}} \quad$ density of the gas phase, $\mathrm{kg} / \mathrm{m}^{3}$

$\rho_{\ell} \quad$ density of the liquid phase, $\mathrm{kg} / \mathrm{m}^{3}$

$s_{f t} \quad$ non-dimensionalised front width

$\Sigma \quad$ variance of the pore size distribution

$X_{f t} \quad$ non-dimensionalised frontal position 


\section{DYNAMICS OF IN-SITU COMBUSTION AT VARIOUS SCALES}

A well-established method for the recovery of heavy oils is in-situ combustion. Despite its long history, however, many aspects of the process are not well understood. Two particular aspects were analyzed in this project: The description of the process at the pore-network scale, and its upscaling at the large scale for field applications. A detailed pore-network simulator is described in the second study. It is the first of its kind, as far as we know. The simulator accounts for all relevant phenomena at the microscale, including mass transfer by convection and diffusion, viscous flow, heat transfer in the porespace and the solid matrix and chemical reactions. It predicts small-scale patterns, including particularly the structure of the combustion zone and associated instabilities. The simulator can be used to understand the combustion process from first principles and to delineate ignition, extinction and sustained propagation phenomena.

In the first study, we apply an asymptotic approach to describe the movement of combustion fronts in porous media as gas-dynamic discontinuities. This approach is essential for the upscaling of the process at the field scale and for the assessment of the effect of macrcoscale heterogeneity on issues such as sustained front propagation, extinction, efficiency, etc. The sustained propagation of combustion fronts in porous media is a necessary condition for the success of in situ combustion for oil recovery. In the presence of heat losses, the possibility of ignition and extinction also exists. We address some of these issues by studying the properties of forward combustion fronts propagating at a constant velocity in the presence of heat losses. We use an analytical method to derive expressions for temperature and concentration profiles and the velocity of the combustion font, under both adiabatic and non-adiabatic conditions. Heat losses are assumed to be relatively weak and they are expressed using two modes: (1) a convective type, using an ove rall heat transfer coefficient; and (2) a conductive type, for heat transfer by transverse conduction to infinitely large surrounding formations. In their presence we derive multiple steady-state solutions, with stable low and high temperature branches, and an unstable intermediate branch. Conditions for self-sustaining front propagation are investigated as a function of injection and reservoir properties. The 
extinction threshold is expressed in terms of the system properties. An explicit expression is also obtained for the effective heat transfer coefficient in terms of the reservoir thickness and the front propagation speed. This coefficient is not only dependent on the thermal properties of the porous medium but also on the front dynamics.

Subsequently, we studied the effect of heterogeneity on the sustained propagation of combustion fronts by considering the propagation in a layered system. Much of heavy oil reserves remain in shallow reservoirs, consisting of relatively thin sands separated by nearly impermeable shales. A potential recovery method for heavy oil is in-situ combustion. In the final study in this section analytical models are developed to delineate the combined effects of fluid flow, reaction and heat transfer on the dynamics of combustion fronts in layered porous media, using as parameters the thermal coupling between the layers, the heat transfer to the surroundings and the permeability contrast. We find that in layered systems, the thermal coupling between layers leads to coherent traveling fronts, propagating at the same velocity. This coupling retards greatly fronts in the more permeable layers and accelerates only slightly those in the less permeable ones, until a common front velocity is attained. As in the single-layer case, there exists a unique solution, under adiabatic conditions, and multiple steady-state solutions, under non-adiabatic conditions. The latter lead to ignition and extinction conditions. We show that the layer thickness and the permeability contrast between the layers play a crucial role. Importantly, for a sufficiently large permeability contrast, relatively small layer thickness and under non-adiabatic conditions, steady-state propagation in the two layers cannot be sustained, and the process becomes extinct, even though, under the same conditions, sustained propagation would have been predicted for the equivalent singlelayer problem with the average injection velocity. Simple constraints are derived to delineate this case. The analysis is useful for the understanding of the viability of in situ combustion in heterogeneous porous media.

More generally, related to the general problem of combustion is the area of reactive flow in porous media. We have studied, in this context, a number of problems, including acidization processes, and the upscaling of the information from the pore-scale to the 
scale of the continuum. The $\mathrm{PhD}$ Thesis of $\mathrm{P}$. Kechagia addressed some of these aspects and was partly supported by this contract. For brevity, we refer to the reader to the relevant publications cited.

Publications resulting from this part of the project are listed below:

1. Akkutlu, Y.I, and Yortsos, Y.C., The Effect of Heterogeneity on In-situ Combustion: ThePropagation of Combustion Fronts in Layered Porous Media, SPEJ, submitted; also highlighted in Jour. Pet. Tech., page 56 (June 2002).

2. Kechagia, P., Tsimpanogiannis, I.N., Yortsos, Y.C. and Lichtner, P., On the Upscaling of Reaction-Transport Processes in Porous Media with Fast Kinetics, Chem. Eng. Sci. 57, 2565-2577 (2002).

3. Akkutlu, Y.I, and Yortsos, Y.C., The Effect of Heterogeneity on In-situ Combustion: ThePropagation of Combustion Fronts in Layered Porous Media, paper SPE 75128 , presented at the $13^{\text {th }}$ SPE/DOE Symposium on Improved Oil Recovery, Tulsa, OK (April 13-17, 2002).

4. Akkutlu, I.Y., and Yortsos, Y.C., The Dynamics of Combustion Fronts in Porous Media, Combustion and Flame, submitted (2001).

5. Lu, C., Kechagia, P., Yortsos, Y. C., and Lichtner, P., Non-Local Upscaling of ReactionTransport in Porous Media Using a Hybrid Algorithm, paper presented at the AIChE Fall Meeting, Reno, NV (November 5-8, 2001).

6. Lu, C., and Yortsos, Y.C., A Pore-Network Model of Combustion in Porous Media, paper SPE 69705 presented at the International Thermal Operations and Heavy Oil Symposium (ITOHOS), Margarita Island, Venezuela (March 12-14, 2001).

7. Kechagia, P., Yortsos, Y.C. and Lichtner, P., A Non-Local KPZ Equation to Model Interface Growth, Phys. Rev. E 64, 016315-1-15 (2001).

8. Lu, C., and Yortsos, Y. C., A Pore-Network Model of Smoldering Combustion, paper presented at the AIChE Fall Meeting, Los Angeles, CA (November 12-17, 2000).

9. Kechagia, P., Yortsos, Y. C. and Lichtner, P., On the Constraint of Local Equilibrium in the Upscaling of Reaction and Transport Problems in Heterogeneous Porous Media, paper presented at the AIChE Fall Meeting, Los Angeles, CA (November 12-17, 2000)..

10. Akkutlu, I., and Yortsos, Y.C., The Dynamics of Combustion Fronts in Porous Media, paper SPE 63225 proceedings of the 74th SPE Annual Fall Meeting, Dallas, TX (Oct. 14, 2000).

11. Lu, C. and Yortsos, Y.C., The Dynamics of Combustion in Porous Media at the PoreNetwork Scale, paper presented at the 7th European Conference on the Mathematics of Oil Recovery, Baveno, Lago Maggiore, Italy (Sept. 5-8, 2000). 
The following $\mathrm{PhD}$ Theses were also completed in this specific area:

1. Chuan Lu, Pore-Network Models for Combustion Processes in Porous Media, University of Southern California, May 2003 (expected).

2. Yucel Akkutlu, The Dynamics of Combustion in Porous Media, University of Southern California, April 2002.

3. Persefoni Kechagia, Non-local Upscaling Models for Reaction and Transport Processes in Porous Media, University of Southern California, May 2001. 


\title{
The Dynamics of In-Situ Combustion Fronts in Porous Media
}

\author{
I. Yücel Akkutlu and Yannis C. Yortsos
}

\section{INTRODUCTION}

The propagation of combustion fronts in porous media is a subject of interest to a variety of applications, ranging from in situ combustion for the recovery of oil to catalyst regeneration, coal gasification, waste incineration, calcination and agglomeration of ores, smoldering, and high-temperature synthesis of solid materials. The percolation of the oxidizing fluid plays a crucial role, therefore such processes are often referred to generically as Filtration Combustion (FC). While these problems may differ in application and context, they share a common characteristic that the reaction involves a stationary fuel reactant. The fuel may pre-exist as part of a solid matrix or, as in the case of in situ combustion, may be created in an inert porous medium by processes preceding the combustion region, such as vaporization and low temperature oxidation.

Filtration combustion has been studied extensively in the literature. Among the many articles published we refer to the pioneering works of Aldushin and Seplyarsky [1, 2], where forward and reverse filtration combustion is analyzed in one-dimensional geometries. The structure of the reaction zone was studied by several authors, using matched asymptotic expansions, similar to those in flame analysis (e.g. [3]). Britten and Krantz [4] investigated reverse combustion in the context of coal gasification. In a series of more recent papers, [5], [6], [7], Schult et al. studied smoldering combustion propagation in the context of forced reverse, natural reverse and forced forward combustion.

In Ref. [7] two different traveling waves for forward smoldering combustion were identified: reaction-leading (fuel-deficient) and reaction-trailing (oxygen-deficient). Each structure has two interior layers, separated by regions of constant temperature and propagating with con- 
stant speeds. The reaction-leading structure prevails when the combustion layer travels faster than the heat transfer layer, and conversely for the reaction-trailing case. The authors argued that quenching may exist with the reaction-trailing structure, when the gas mass influx to the reaction front is sufficiently large. Adiabatic extinction limits for forced reverse smoldering waves in the absence of heat losses were studied in Ref. [5]. When the rate of heat transfer to the inert gas exceeds a critical value, extinction occurs.

The above pertained to adiabatic FC. Effects of heat losses were incorporated in other studies, typically using a volumetric sink term to the (one-dimensional) energy balance. Rabinovich and Gurevich [8] considered the oxygen-deficient combustion fronts, where fuel conversion is incomplete. At low injection velocities, they noted the presence of a second solution of unreacted downstream fuel concentration. Britten and Krantz [9] extended their work for reverse coal gasification with an excess of oxygen (the fuel-defficient case) [4] to determine conditions for the extinction of a combustion front. They also identified two steady-state solutions for variables, such as the front temperature. They showed that the front velocity is strongly affected by the heat loss intensity, however the front temperature is only weakly reduced from its adiabatic value. Recently, Aldushin et al. [10] investigated extinction properties of a special kind of forced forward FC, where the combustion and heat transfer layers travel together and thermally support each other.

The objective of this work is to apply an approach similar to the above in the context of in situ combustion for the recovery of oil from subsurface porous media. This problem can also be considered as forced forward $\mathrm{FC}$, where under adiabatic conditions a reaction-leading structure develops. We will assume that the initially available fuel is completely consumed. Many aspects of this problem are common with filtration combustion. However, there exist also certain differences. For example, in FC compared to in-situ combustion: (1) the fuel is a priori available, rather than in-situ generated; (2) phenomena preceding the combustion front are unimportant; (3) the heterogeneity of the flow properties of the porous medium is generally not an issue; (4) the effect of heat losses could be controlled. Addressing these differences is necessary and requires a multi-faceted investigation. In this paper we will focus only on the last of the above aspects, namely the effect of heat losses. Its understanding 
is of importance for sustained in-situ front propagation, and must be addressed before the other issues mentioned. Modeling of this simpler problem is a prerequisite for the analytic description of the more realistic (and more complex) problems, which include the generation of fuel in-situ by preceding reactions and the effect of heterogeneity. The latter two problems can be fruitfully attacked based on the results of the present study. Works in this direction are currently under way [11], [12].

A rigorous description of heat losses to the surroundings requires multi-dimensional modeling, where the heat losses appear as boundary conditions in the reservoir (see Fig. 1a). In the subsurface the surrounding can be considered infinitely large, in comparison to the reservoir thickness, $H$. As an approximation, we will consider the thermally thin limit, in which the problem in an assumed rectilinear homogeneous reservoir can be approximated as onedimensional, with the rate of heat losses represented in the energy balance as a volumetric term. This approximation is valid when the thickness is smaller than the thermal diffusion length, and the Nusselt number is relatively small. For sufficiently low $H$, heat transfer coefficients and front velocities (of the order of meters per day), these constraints are generally satisfied. The heat losses will be modeled by two different modes, convective and conductive. The first is more applicable to laboratory-scale processes (Fig. 1b), and will be expressed as $\tilde{h}\left(\tilde{T}-\tilde{T}_{o}\right) / H$, where $\tilde{h}$ is a heat transfer coefficient. This coefficient will be assumed relatively small, so that the approach of [7] can be directly applied (see also below). The second involves heat conduction in practically semi-infinite strata bounding the porous medium (Fig. 1c). It is more appropriate for subsurface processes and does not require the use of an unknown heat transfer coefficient. Again, for the approach of [7] to be applicable, the heat loss should be relatively weak. We will proceed with an 1-D analysis and neglect in this work the medium heterogeneity and phenomena preceding the combustion front, which are studied separately [11], [12]. The obtained results and analysis extend the work of References [8] and [9]. Therefore, they are new not only to the in situ combustion but to filtration combustion in general.

The paper is organized as follows: First, we briefly describe some general aspects of in situ combustion. The asymptotic framework of [7] is next applied. The results are used to analyze 
1-D moving fronts of constant velocity and to provide a sensitivity study of the effect of the various parameters, with emphasis on ignition, extinction and sustained propagation. The model is a continuum, in which effective values are used for kinetic and transport parameters. A parallel study is also under way addressing the same process, but using a discrete porenetwork model [13].

\section{PRELIMINARIES}

In situ combustion for oil recovery has been studied extensively since the mid $1950 \mathrm{~s}$. The texts by Prats [14] and Boberg [15] summarize the relevant literature on the subject until the late 1980s. A large number of experimental, analytical and numerical studies have been reported. Baily and Larkin [16] provided simple heat transfer models in linear and radial geometries in the presence of a combustion zone of finite thickness. Gottfried [17] treated the combustion front as a discontinuity involving a point heat source, but without exploring the combustion zone structure. Beckers and Harmsen [18] detailed the propagation of various regimes in in situ combustion and its variants, e.g. wet combustion. Burger and Sahuquet [19] analyzed the chemical aspects of the reaction process. Ag̃ca and Yortsos [20] proposed a simplified description, which takes into account the heat losses to the surroundings and discussed sustained propagation and extinction. Their analysis is based on a steadily propagating combustion front, of an a priori known thickness, however. The stability of combustion fronts was analyzed by Armento and Miller [21].

Understanding the dynamics of in situ combustion fronts is important to front stability, the sustained propagation of combustion, the effects of heterogeneity, and the process scale-up. Conventional numerical models do exist for the simulation of in situ combustion. However, because of the thin reaction zone, these models may not be adequate to resolve the front structure, and a more detailed description is necessary. This is particularly the case for process scale-up, which is the averaging of the process over larger scales, for example the subsurface reservoir grid simulation scale. Upscaling is necessary for a realistic description of flow and transport in heterogeneous subsurface formations and it is a subject of great current 
interest [22]. The presence of frontal discontinuities, expected in combustion, adds a novel and important feature to upscaling.

In in situ combustion for oil recovery, one can generally identify the following regions [14]: (1) a burned zone, (2) a combustion zone, (3) a vaporization zone, (4) a steam (condensation) zone, (5) a hot water bank, (6) an oil bank and (7) an initial zone (see schematic of Figure 2). The burned zone contains injected air and possibly a solid residue of burned fuel. Due to heat losses, the temperature in this region increases monotonically downstream, until the location of combustion zone is reached. In this zone, solid fuel and injected oxygen react exothermically to generate combustion gases, such as carbon oxides and superheated steam. In the vaporization zone, lighter components of the hydrocarbons are vaporized and transported by the flowing gas stream.

The combustion reactions of hydrocarbon mixtures are complex: a great number of reaction products are generated by hydrocarbon oxidation over a large temperature range [19]. It is common, however, to classify these groups into two lumped-parameter, single-step reactions: a high-temperature oxidation (HTO) and a low-temperature oxidation (LTO), with HTO occuring in the combustion zone. LTO processes are mostly responsible for fuel generation [11]. Combustion in the HTO region is an oxidation reaction with a large activation energy. Due to the elevated temperatures in this region and upgrading effects of the preceding zones, the fuel consumed in the HTO region is a stationary solid, rather than a gaseous phase, and therefore the reaction is heterogeneous. Previously, Allag [23] and Verma et al. [24] have shown that the fuel vapor consumption reaction is negligible. The combustion stoichiometry is typically represented by a simple exothermic reaction of the form [25]

$C H_{x}+\left(1-\frac{m}{2}+\frac{x}{4}\right) O_{2} \longrightarrow(1-m) C_{2}+m C O+\frac{x}{2} H_{2} O$

where $x$ is the fuel atomic $\mathrm{H}-\mathrm{C}$ ratio and $m$ the fraction of carbon oxidized to $C O$. For the purposes of this paper, however, we will adopt the notation of [7] and use pseudo-components for the fuel and the reaction products, along with their average stoichiometric coefficients, namely 
$[$ Fuel $]+\tilde{\gamma}[$ Oxygen $] \longrightarrow \tilde{\gamma}_{g p}[$ Gaseous Product $]$

Unreacted or bypassed oxygen reacts with the hydrocarbons ahead of the combustion front where the temperature is lower. In this region, LTO tends to increase the density, apparent viscosity and boiling range of the liquid phase oil. Clearly, fuel formation and combustion are inter-dependent. Excessive fuel deposition may retard the rate of advance of the combustion front, while insufficient fuel deposition may not provide enough heat supply to sustain it. In a separate analysis [11] we use the results of the present study to couple LTO and HTO fronts, thus accounting for in-situ fuel generation. Here, however, we will assume a constant amount of available fuel, to first understand the simpler problem of non-adiabatic combustion with a fixed fuel content. For completeness, we note that the vaporization zone is preceded by a steam zone, where in-situ generated steam condenses, and further ahead by a hot water bank, which displaces liquid oil (oil bank). The dynamics of these processes are all coupled to the combustion front. Attempts have recently been made to describe the propagation of an LTO front in the presence of two-phase flow using concepts from wave propagation [26].

\subsection{LARGE-SCALE FEATURES OF THE TEMPERATURE PROFILE}

In this section, we provide a simplified qualitative analysis of the large-scale features of the temperature profile expected in in-situ combustion. Constant rate injection of an oxidizing gas phase and ignition occur at the same point, so that the combustion is forward. Consider a simple 1-D energy balance, with heat conduction negligible compared to convection, and assume that the heat of reaction is a Dirac delta function. For a constant Darcy velocity, $\tilde{v}$, we have

$(1-\phi) c_{s} \rho_{s} \frac{\partial \tilde{T}}{\partial \tilde{t}}+c_{g} \rho_{g} \tilde{v} \frac{\partial \tilde{T}}{\partial \tilde{x}}=\dot{Q} \delta\left(\tilde{x}-\tilde{x}_{f}(\tilde{t})\right)-\frac{\tilde{h}}{H}\left(\tilde{T}-\tilde{T}_{o}\right)$

where the right-hand side involves the heat of combustion, and a convective-type expression for heat losses. Here, $\phi$ represents the effective porosity, $c \rho$ the volumetric heat capacity, with subscript $s$ denoting solid matrix and subscript $g$ the gas phase, and $\dot{Q}$ is the rate of heat 
generation. The coefficient of the transient term includes only the volumetric heat capacity of the solid matrix (which is much larger than the gas). Equation (2) is a hyperbolic equation with a singular source term at the combustion front $\tilde{x}=\tilde{x}_{f}(\tilde{t})$. Initial and injection $(\tilde{x}=0)$ conditions involve a constant temperature $\tilde{T}_{0}$.

Consider the adiabatic case. Outside of the front, the source term is nil, and the solution is obtained with the method of characteristics, which in the present case are straight lines with a constant slope

$\frac{d \tilde{x}}{d \tilde{t}}=\frac{c_{g} \rho_{g} \tilde{v}}{(1-\phi) c_{s} \rho_{s}} \equiv U$

In the adiabatic case, the temperature is constant along the characteristics. In general, there would be two characteristic velocities, one upstream of the front (denoted by subscript III) and one downstream (denoted by subscript I), as the consumption and/or production of gases will affect the mass flux. Let the combustion front move with velocity $V_{F}$. If conditions are such that

$V_{F}>U_{I}$ and $V_{F}>U_{I I I}$

the characteristics from the initial condition (the $\tilde{x}$-axis) will intersect the front trajectory (Figure 3 ), while those from the boundary (the $\tilde{t}$-axis) will not, creating an expanding simple wave region (region II in Fig. 3). In this case, the $(\tilde{t}, \tilde{x})$ plane consists of three regions, one corresponding to the initial (region I, of temperature $\tilde{T}_{o}$ ), another corresponding to the simple wave (region II, of a front temperature to be determined), and a third corresponding to the injection (region III, of temperature $\tilde{T}_{o}$ ). Temperature $\tilde{T}_{f}$ is obtained by integrating (2) across the combustion front,

$\tilde{T}_{f}-\tilde{T}_{o}=\frac{\dot{Q}}{\left[(1-\phi) c_{s} \rho_{s}\right]\left(V_{F}-U_{I}\right)}$

Under conditions (4), the simple wave region II is also spanned by characteristics of slope $U_{I I I}$, except that these now emanate from the combustion front (as shown in Figure 3 ), hence 
they carry temperature $\tilde{T}_{f}$. Thus, the temperature profile at any time consists of two farfield regions with temperature $\tilde{T}_{0}$ and an intermediate expanding region of temperature $\tilde{T}_{f}$. Accounting for conduction will smoothen the discontinuities at the fronts. The conditions in (4) establish a reaction-leading structure. If the inequalities are reversed, the situation is also reversed, the front moves slower, and it is the temperature downstream, which increases to $\tilde{T}_{f}$. Region II precedes, rather than trailing the combustion front, the simple wave expanding ahead of it. This is the reaction-trailing structure.

In the non-adiabatic case, heat losses to the surrounding cannot be neglected. It is straightforward to solve for the temperature profile, when the heat loss is expressed by (2). The solution is

$\tilde{T}=\tilde{T}_{o}+\left(\tilde{T}_{f}-\tilde{T}_{o}\right) \exp \left[\frac{\tilde{h}\left(\tilde{x}-V_{F} \tilde{t}\right)}{H(1-\phi) c_{s} \rho_{s}\left(V_{F}-U_{I}\right)}\right] \quad U_{I} \tilde{t}<\tilde{x}<V_{F} \tilde{t}$

The temperature in region II is not constant any longer, but decreases upstream exponentially (and, discontinuously, at the boundary II-III). Likewise for the case of conductive heat losses. Detailed analysis of these problems is provided in subsequent sections. For both of these cases, the validity of conditions (4) will be probed after the solution of the problem is obtained.

Having obtained a qualitative understanding of the large-scale features of the problem, we will now proceed with a quantitative analysis.

\section{FORMULATION}

We assume throughout a 1-D geometry, of the type shown in Fig. 1. Conservation equations are written for the total energy, the oxygen mass, the total gas mass and the fuel mass. For the latter, we define the fuel density per total volume, $\rho_{f}$, and introduce the extent of conversion depth, $\eta(\tilde{x}, \tilde{t})=1-\rho_{f}(\tilde{x}, \tilde{t}) / \rho_{f}^{o}$, such that $\eta=0$ corresponds to the initial state (denoted by superscript $o$ ) and $\eta=1$ to complete consumption. Dependent variables are the temperature, $\tilde{T}(\tilde{x}, \tilde{t})$, the oxygen mass fraction, $\tilde{Y}(\tilde{x}, \tilde{t})$, the average gas density, $\rho_{g}(\tilde{T}, \tilde{p})$, and the fuel conversion depth. We use Darcy's law for gas flow in the porous medium. 
In formulating the conservation equations we make the following assumptions: pore space and solid are in thermal equilibrium and a one-temperature model is used for the energy balance; heat transfer by radiation, energy source terms due to pressure increase, and work from surface and body forces are all negligible; the ideal gas law is the equation of state for the gas phase; thermodynamic and transport properties, such as conductivity, diffusivity, heat capacity of the solid, heat of reaction, etc., all remain constant. The dimensional form of the equations (superscript tilde) is, then,

$$
\begin{aligned}
& (1-\phi) c_{s} \rho_{s} \frac{\partial \tilde{T}}{\partial \tilde{t}}+c_{g} \rho_{g} \tilde{v} \frac{\partial \tilde{T}}{\partial \tilde{x}}=\lambda \frac{\partial^{2} \tilde{T}}{\partial \tilde{x}^{2}}+\tilde{Q} \rho_{f}^{o} W-\dot{Q}_{h}, \\
& \phi \frac{\partial\left(\tilde{Y} \rho_{g}\right)}{\partial \tilde{t}}+\frac{\partial\left(\tilde{Y} \rho_{g} \tilde{v}\right)}{\partial \tilde{x}}=D_{M} \frac{\partial}{\partial \tilde{x}}\left(\rho_{g} \frac{\partial \tilde{Y}}{\partial \tilde{x}}\right)-\tilde{\mu} \rho_{f}^{o} W \\
& \phi \frac{\partial \rho_{g}}{\partial \tilde{t}}+\frac{\partial}{\partial \tilde{x}}\left(\rho_{g} \tilde{v}\right)=\tilde{\mu}_{g} \rho_{f}^{o} W
\end{aligned}
$$

and

$\frac{\partial \eta}{\partial \tilde{t}}=W$

where, $\tilde{Q}$ is the heat of combustion, $\dot{Q}_{h}$ is the heat loss term and $W$ the rate of reaction. In the above, $c_{i}$ denotes the average specific heat capacity of species $i$ (gas or solid) at constant pressure, $\rho_{i}$ is the volumetric density of species $i, \lambda$ is the effective thermal conductivity, $D_{M}$ is an effective diffusion coefficient in the gas phase, while $\tilde{\mu}=\tilde{\gamma} M_{o} / M_{f}, \tilde{\mu}_{g}=\tilde{\mu}_{g p}-\tilde{\mu}$, and $\tilde{\mu}_{g p}=\tilde{\gamma}_{g p} M_{g p} / M_{f}$ are mass-weighted stoichiometric coefficients for oxygen, net products and gaseous products, respectively. Thus, $\tilde{\mu}_{g}>0$ or $\tilde{\mu}_{g}<0$ corresponds to net gas mass production or consumption, respectively. For the rate of reaction, we use the law of mass action

$$
W=k(\tilde{T}) \tilde{a}_{s}^{m}\left(\frac{\tilde{Y} \tilde{p}}{R \tilde{T}}\right)^{n} \psi(\eta)
$$


where in the applications to follow, exponents $m$ and $n$ will be set equal to one,

$k(\tilde{T})=k_{o} e^{-E / R \tilde{T}}$

$E$ is the activation energy and $k_{0}$ the pre-exponential factor. The dependence on $\eta$ is through the dimensionless function $\psi(\eta)$, the evaluation of which requires a more elaborate pore-level study [13]. Clearly, $\psi(1)=0$. The rate is implicitly dependent on porosity, through the specific surface area per unit volume, $\tilde{a}_{s}$. Finally, we have Darcy's law

$\frac{\partial \tilde{p}}{\partial \tilde{x}}=-\frac{\eta_{g}}{K(\eta)} \tilde{v}$

where $K(\eta)$ is the absolute permeability, $\eta_{g}$ the gas viscosity, and $\tilde{v}$ the Darcy velocity, and the equation of state, assuming ideal gases

$\tilde{p} M_{g}=\rho_{g} R \tilde{T}$

As noted above, the expression for the heat losses takes two different forms. For a convective mode (Fig. 1a), we have

$\dot{Q}_{h}=\frac{\tilde{h}}{H}\left(\tilde{T}-\tilde{T}_{o}\right)$

in terms of the overall heat transfer coefficient, $\tilde{h}$. For a conductive mode (Fig. 1b) the expression is more elaborate, as shown in [27],

$\dot{Q}_{h}=\frac{2 \sqrt{ }\left(\lambda_{h} c_{h} \rho_{h}\right)}{H \sqrt{ } \pi} \int_{0}^{\tilde{t}} \frac{\partial \tilde{T}}{\partial \tau} \frac{d \tau}{\sqrt{ }(\tilde{t}-\tau)}$

This expression is obtained by solving the 1-D heat conduction equation in the semi-infinite domain $0<\tilde{t}, 0 \leq \tilde{z}<\infty$, normal to the flow direction, with a time-dependent temperature at the boundary $\tilde{T}(\tilde{t}, \tilde{x})=\tilde{T}(\tilde{t}, \tilde{x}, \tilde{z}=0)$. It reflects heat loss by transverse conduction in the semi-infinite domain bounding the porous medium. Here, subscript $h$ denotes surrounding properties. 


\section{SCALING AND NON-DIMENSIONALIZATION}

In the reaction zone, reaction and diffusion balance, and the temperature is spatially uniform. The reaction zone is embedded within the heat transfer layer, as shown in Figure 4 . In the combustion zone, convection, conduction and heat losses balance, but reaction is negligible.

Next, we introduce dimensionless space and time variables using the characteristic scales, $l^{*}=\frac{\alpha_{s}}{v_{i}}$ and $t^{*}=\frac{l^{*}}{v_{i}}=\frac{\alpha_{s}}{v_{i}^{2}}$, where $v_{i}$ is the injection velocity and $\alpha_{s}$ the effective thermal diffusivity. This notation is different from [7], although the subsequent analysis is similar. Scaling temperature with $\tilde{T}_{o}$, we obtain the dimensionless conservation equations

$\frac{\partial \theta}{\partial \hat{t}}+a \rho v \frac{\partial \theta}{\partial \hat{x}}=\frac{\partial^{2} \theta}{\partial \hat{x}^{2}}+q \Phi-\dot{Q}_{h D}$

$\phi \frac{\partial(Y \rho)}{\partial \hat{t}}+\frac{\partial(Y \rho v)}{\partial \hat{x}}=\frac{1}{L e} \frac{\partial}{\partial \hat{x}}\left(\rho \frac{\partial Y}{\partial \hat{x}}\right)-\mu \Phi$

$\phi \frac{\partial \rho}{\partial \hat{t}}+\frac{\partial(\rho v)}{\partial \hat{x}}=\mu_{g} \Phi$

and

$\frac{\partial \eta}{\partial \hat{t}}=\Phi$

where

$\Phi=W t^{*}=Z a_{s}\left(\frac{Y(1+\Pi p)}{\theta}\right) \psi(\eta) \exp \left(Z \theta_{f}^{2}\left(\frac{1}{\theta_{f}}-\frac{1}{\theta}\right)\right)$

and in addition

$\frac{\partial p}{\partial \hat{x}}=-\kappa v \quad$ and $\quad \rho \theta=1+\Pi p$. 
In the above, we introduced the following variables and parameters

$$
\begin{aligned}
\hat{x} & =\frac{\tilde{x}}{l^{*}}, \hat{t}=\frac{\tilde{t}}{t^{*}}, \quad \theta=\frac{\tilde{T}}{\tilde{T}_{o}}, \quad \theta_{f}=\frac{\tilde{T}_{f}}{\tilde{T}_{o}}, \quad Y=\frac{\tilde{Y}}{Y_{i}}, \quad p=\frac{\tilde{p}-\tilde{p}_{i}}{\tilde{p}_{i n j}-\tilde{p}_{i}}, \quad \rho=\frac{\rho_{g}}{\rho_{g i}}, \quad v=\frac{\tilde{v}}{v_{i}}, \\
\Pi & =\frac{\tilde{p}_{i n j}-\tilde{p}_{i}}{\tilde{p}_{i}}, \quad \mu=\frac{\tilde{\mu} \rho_{f}^{o}}{\rho_{g i} Y_{i}}, \quad \mu_{g}=\frac{\tilde{\mu}_{g} \rho_{f}^{o}}{\rho_{g i}}, \quad a=\frac{c_{g} \rho_{g i}}{(1-\phi) c_{s} \rho_{s}}, \\
q & =\frac{\tilde{Q} \rho_{f}^{o}}{(1-\phi) c_{s} \rho_{s} \tilde{T}_{o}}, \quad \kappa=\frac{\eta_{g} \alpha_{s}}{K\left(\tilde{p}_{i n j}-\tilde{p}_{i}\right)}, \quad \dot{Q}_{h D}=\frac{\dot{Q}_{h} t^{*}}{(1-\phi) c_{s} \rho_{s} \tilde{T}_{o}}, \\
\alpha_{s} & =\frac{\lambda}{(1-\phi) c_{s} \rho_{s}}, \quad L e=\frac{\alpha_{s}}{D_{M}}, \quad a_{s}=\frac{\tilde{a}_{s}}{a_{s}^{*}}, \quad a_{s}^{*}=\frac{Z R \tilde{T}_{o}}{t^{*} k_{o} Y_{i} \tilde{p}_{i} \mathrm{e}^{-\mathrm{E} / \mathrm{R} \tilde{T}_{f}}}
\end{aligned}
$$

The final step is to convert to coordinates moving with the combustion front, which in the fuel-deficient case can be defined, e.g. as the position at which $\eta=1 / 2$. In the moving coordinates $\xi=\hat{x}-f(\hat{t})$, and $t=\hat{t}$, the non-dimensional equations read

$$
\begin{aligned}
& \frac{\partial \theta}{\partial t}+\left(a \rho v-f_{t}\right) \frac{\partial \theta}{\partial \xi}=\frac{\partial^{2} \theta}{\partial \xi^{2}}+q \Phi-\dot{Q}_{h D} \\
& \phi \frac{\partial(Y \rho)}{\partial t}+\frac{\partial\left(Y \rho\left(v-\phi f_{t}\right)\right)}{\partial \xi}=\frac{1}{L e} \frac{\partial}{\partial \xi}\left(\rho \frac{\partial Y}{\partial \xi}\right)-\mu \Phi \\
& \phi \frac{\partial \rho}{\partial t}+\frac{\partial\left(\rho\left(v-\phi f_{t}\right)\right)}{\partial \xi}=\mu_{g} \Phi
\end{aligned}
$$

$$
\frac{\partial \eta}{\partial t}-f_{t} \frac{\partial \eta}{\partial \xi}=\Phi
$$

$v=-\frac{1}{\kappa} \frac{\partial p}{\partial \xi}$

$$
\rho \theta=1+\Pi p
$$

The boundary conditions depend on the extent of combustion. This paper is concerned with the asymptotics of steady-state combustion fronts, obtained at large times, in the case of complete fuel combustion. Consider, first, the case of heat losses. Then, the temperature in 
the far-field behind the front ultimately reduces to the initial temperature (which is also the injection temperature), and the boundary conditions read

$Y=1, \quad, \theta=1 \quad \eta=1 \quad ; \quad \hat{x} \rightarrow-\infty$

$\frac{\partial Y}{\partial \hat{x}}=0, \quad \theta=1, \quad \eta=0 \quad ; \quad \hat{x} \rightarrow \infty$

For the adiabatic case, we consult the diagram of characteristics of Figure 3. As shown in Figure 3, region II is preceeded upstream by the simple-wave region III, where the temperature, in the absence of conduction, is equal to that at injection. The front separating regions II and III moves slower than the combustion front, however. The front retardation is due to the requirement to heat the rock matrix from the initial (injection) to the front temperature. Because of the increasing separation between the two fronts, the relevant boundary condition for our problem of interest, which is the steady-state in a frame of reference moving with the combustion front, would be

$\frac{\partial \theta}{\partial \hat{x}}=0$

This also implies that $\theta \rightarrow \theta_{f}$ as $\hat{x} \rightarrow-\infty$, for the adiabatic case. Of course, the temperature profile around the front separating regions III and II can also be found, but it is of no interest to this paper.

The above conditions imply that $\theta \rightarrow \theta_{f}$ (adiabatic), $\theta \rightarrow 1$ (non-adiabatic) at $\hat{x} \rightarrow-\infty$ and $Y \rightarrow Y_{b}$ at $\hat{x} \rightarrow \infty$, where $\theta_{f}$ and $Y_{b}$ must be determined.

Having completed the formulation, we proceed next with the analysis of the structure of the reaction zone and then with that of the combustion zone. The analysis is very similar to that presented in [7] and for this reason many details will be omitted. 


\section{REACTION ZONE}

Because the reaction rate is strongly temperature dependent, the combustion reactions are confined to a thin reaction zone at the combustion front, where temperature, pressure and concentrations are approximately constant. This approximation is a result of the condition $Z \equiv \frac{E \tilde{T}_{o}}{R \tilde{T}_{f}^{2}} \gg 1$ (see $[7]$ ). To proceed, we stretch the longitudinal moving coordinate, $X=Z \xi$, and expand the dependent variables in terms of $Z^{-1}$, as follows

$$
\begin{aligned}
\theta & \sim \theta^{\circ}(t)+Z^{-1} \theta^{1}(X, t)+\ldots, \\
Y & \sim Y^{\circ}(t)+Z^{-1} Y^{1}(X, t)+\ldots \\
p & \sim p^{\circ}(t)+Z^{-1} p^{1}(X, t)+\ldots \\
\rho & \sim \rho^{\circ}(t)+Z^{-1} \rho^{1}(X, t)+\ldots \\
\eta & \sim \eta^{\circ}(X, t)+\ldots \\
v & \sim v^{\circ}(X, t)+\ldots \\
f & \sim f^{\circ}(t)+\ldots
\end{aligned}
$$

After substitution in the governing equations, we find the following. To leading-order, the pressure is constant within the reaction zone. Combining the energy equation with the fuel balance shows that to leading-order only conduction in the $X$ direction and reaction participate

$$
\frac{\partial^{2} \theta^{1}}{\partial X^{2}}=q f_{t}^{\circ} \frac{\partial \eta^{\circ}}{\partial X}
$$

To leading order, the heat loss term is $Z^{-1} \dot{Q}_{h D}$ and does not contribute in the reaction zone. Integration yields

$\frac{\partial \theta^{1}}{\partial X}=-q f_{t}^{\circ}\left(1-\eta^{\circ}\right)$ 
where we used the boundary conditions $\partial \theta^{1} / \partial X=0, \eta^{\circ}=1$ as $X \rightarrow-\infty$. The boundary condition on temperature is exact for the adiabatic case, but requires a constraint of relatively weak heat losses, for the non-adiabatic case (see below). For the oxygen mass balance, the leading-order terms are convection, diffusion and reaction,

$\operatorname{Lep}^{\circ} Y^{\circ} \frac{\partial v^{\circ}}{\partial X}-\rho^{\circ} \frac{\partial^{2} Y^{1}}{\partial X^{2}}=\mu \operatorname{Lef} f_{t}^{\circ} \frac{\partial \eta^{\circ}}{\partial X}$

where we have taken into account that $\rho^{\circ}$ is constant in the reaction zone. A second integration gives

$L e \rho^{\circ} Y^{\circ} v^{\circ}-\rho^{\circ} \frac{\partial Y^{1}}{\partial X}=\mu L e f_{t}^{o} \eta^{\circ}+$ const

The total gas mass balance reads

$\rho^{\circ} \frac{\partial v^{\circ}}{\partial X}=-\mu_{g} f_{t}^{\circ} \frac{\partial \eta^{\circ}}{\partial X}$

and after integration

$\rho^{\circ} v^{\circ}=-\mu_{g} f_{t}^{o} \eta^{\circ}+$ const.

From the above, we can determine the jumps in heat, gas mass and oxygen mass fluxes across the front in terms of the jump in the depth of fuel conversion, to be used for the subsequent evaluation of the variables in the combustion zone. We obtain,

$$
\begin{aligned}
& \left.\frac{\partial \theta}{\partial \xi}\right|_{-\infty} ^{\infty}=\left.\frac{\partial \theta^{1}}{\partial X}\right|_{-\infty} ^{\infty}=-q f_{t}^{\circ} \\
& \left.\rho^{\circ} v^{\circ}\right|_{-\infty} ^{\infty}=\mu_{g} f_{t}^{o} \\
& \left.\left.\rho^{\circ} \frac{\partial Y}{\partial \xi}\right|_{-\infty} ^{\infty} \frac{\partial Y^{1}}{\partial X}\right|_{-\infty} ^{\infty}=\left(\mu+Y^{o} \mu_{g}\right) \text { Le } f_{t}^{o}
\end{aligned}
$$


Finally, the leading-order equation for the fuel mass is

$f_{t}^{\circ} \frac{\partial \eta^{\circ}}{\partial X}=-a_{s} \frac{Y^{\circ}\left(1+\Pi p^{\circ}\right) \psi\left(\eta^{\circ}\right) e^{\theta^{1}}}{\theta^{\circ}}$

Multiplying equation (35) by (43) and re-arranging yields

$q\left(f_{t}^{\circ}\right)^{2}\left(\frac{1-\eta^{\circ}}{\psi\left(\eta^{\circ}\right)}\right) \frac{\partial \eta^{\circ}}{\partial X}=a_{s}\left\{\frac{Y^{\circ}\left(1+\Pi p^{\circ}\right)}{\theta^{\circ}}\right\} e^{\theta^{1}} \frac{\partial \theta^{1}}{\partial X}$

which can be further integrated across the reaction zone to give

$f_{t}^{o}=\sqrt{\frac{a_{s}\left\{Y^{\circ}\left(1+\Pi p^{\circ}\right)\right\}}{q \theta^{\circ} I_{\eta}}}, \quad I_{\eta}=\int_{0}^{1} \frac{\left(1-\eta^{\circ}\right)}{\psi\left(\eta^{\circ}\right)} d \eta^{\circ}$.

The above expression specifies the velocity of the front in terms of the mole fraction $Y^{\circ}$ and the temperature $\theta^{\circ}$ of the reaction zone. In deriving the above result we made use of the far-field boundary condition $\partial \theta^{1} / \partial X=0$ as $X \rightarrow-\infty$. In the remaining we will take for simplicity $I_{\eta}=1$.

\section{THE COMBUSTION ZONE}

Downstream of the reaction front, the reaction rates are exponentially small, upstream of the front the reaction ceases due to the assumed complete fuel consumption. Across the two regions, the jump conditions derived previously apply. The analysis is for steady-state propagation, which is the sought asymptotic state. In the moving frame of reference, the total gas, oxygen, and fuel mass balances read respectively,

$$
\begin{aligned}
& \frac{\partial(\rho B)}{\partial \xi}=0 \quad \text { where } \quad B=v-\phi f_{t}^{\circ} \\
& \rho B \frac{\partial Y}{\partial \xi}-\frac{1}{L e} \frac{\partial}{\partial \xi}\left(\rho \frac{\partial Y}{\partial \xi}\right)=0
\end{aligned}
$$


$\frac{\partial \eta}{\partial \xi}=0$

Equation (46) shows that $\rho B$ is a constant. In $\xi>0$, the solution for $Y$ is also a constant, $Y_{b}$, because $B>0$. In $\xi<0$, we integrate (47) using the boundary condition $Y=1$ at $\xi \rightarrow \infty$. Making use of the jump condition across the reaction front, and after some calculations, we find

$Y=\left\{\begin{array}{cl}1-\left(1-Y_{b}\right) \exp \left(L e \int_{0}^{\xi} B^{-} d \xi\right) & : \quad \xi<0 \\ Y_{b} & : 0<\xi\end{array}\right.$

where

$Y_{b}=\frac{1-(\phi+\mu) V_{D}}{1+\left(\mu_{g}-\phi\right) V_{D}} \cong \frac{1-\mu V_{D}}{1+\mu_{g} V_{D}}$.

and we have denoted $V_{D} \equiv f_{t}^{\circ}$. Note that for $Y_{b}>0$, the condition $1>\mu V_{D}$ must apply, namely the total gas mass flux should be sufficiently large. Equation (48) gives

$\eta= \begin{cases}1 & : \quad \xi<0 \\ 0 & : 0<\xi\end{cases}$

assuming, again, complete fuel combustion. Finally, taking $\Pi \ll 1$, and inserting equation (49) into equation (45) gives

$V_{D}^{2} \approx \mathcal{A} \theta_{f} \exp \left(-\frac{\gamma}{\theta_{f}}\right)\left(\frac{1-\mu V_{D}}{1+\mu_{g} V_{D}}\right)$

where we have defined the dimensionless variables

$\mathcal{A}=\frac{\tilde{a}_{s} \alpha_{s} k_{o} Y_{i} \tilde{p}_{i}}{q E v_{i}^{2}}$ and $\gamma=\frac{E}{R \tilde{T}_{o}}$

Equation (50) is one expression relating the unknown leading-order velocity of the front, $V_{D}$, to the front temperature, $\theta_{f}$. A second relation is obtained by solving the heat balance in the combustion zone. For this, we must consider two cases, adiabatic and non-adiabatic combustion. 


\subsection{THE ADIABATIC CASE}

In the adiabatic case, the heat loss term does not contribute and the energy balance reads

$A \frac{\partial \theta}{\partial \xi}-\frac{\partial^{2} \theta}{\partial \xi^{2}}=0$

where

$A=a \rho v-V_{D}<0$

Because $A<0$, the only possible solution for $\xi<0$ is a constant, the front temperature. A flat temperature profile upstream is consistent with the assumption $\partial \theta^{1} / \partial X=0$, as $X \rightarrow-\infty$, made in the reaction zone. For $\xi>0$, integrating (52) and making use the jump condition at the front leads to

$\theta=\left\{\begin{array}{cc}\theta_{f}(t) & : \quad \xi<0 \\ 1-\frac{q V_{D}}{A} \exp (A \xi) & : \quad 0<\xi\end{array}\right.$

which shows that the temperature decays exponentially fast downstream of the combustion front. Continuity of temperature at $\xi=0$, gives the expression for the dimensionless front temperature,

$\theta_{f}=1-\frac{q V_{D}}{a(\rho v)^{+}-V_{D}}$.

When the effects of gas mass influx and the net gas production at the front are negligible, namely when $A \approx-V_{D}$, the front temperature depends only on the available heat content of the fuel, parameter $q$. Figure 5 shows schematic profiles of temperature, mass fraction and conversion across the combustion zone for the adiabatic case.

\subsection{THE NON-ADIABATIC CASE}

Consider, next, the temperature profile for the non-adiabatic case. We will consider separately two different modes, convective and conductive heat losses. 


\subsubsection{Convective Heat Losses}

In the convective case the energy equation reads

$A \frac{\partial \theta}{\partial \xi}-\frac{\partial^{2} \theta}{\partial \xi^{2}}=-h(\theta-1)$

where $h$ is the dimensionless heat transfer coefficient

$h=\frac{\tilde{h} t^{*}}{(1-\phi) c_{s} \rho_{s} H}$

The solution of (55) with boundary conditions $\theta \rightarrow 1$ as $\xi \rightarrow \pm \infty$ is

$\theta= \begin{cases}1+\left(\theta_{f}-1\right) \exp \left\{\frac{1}{2}\left(A^{-}+\sqrt{\left(A^{-}\right)^{2}+4 h}\right) \xi\right\} & : \quad \xi<0 \\ 1+\left(\theta_{f}-1\right) \exp \left\{\frac{1}{2}\left(A^{+}-\sqrt{\left(A^{+}\right)^{2}+4 h}\right) \xi\right\} & : 0<\xi\end{cases}$

We recall that equation (50) was based on the condition $\partial \theta^{1} / \partial X=0$, as $X \rightarrow-\infty$. Con-

sistency with the above expressions requires that $\frac{1}{2}\left(A^{-}+\sqrt{\left(A^{-}\right)^{2}+4 h}\right)=O(1 / Z)$, namely that the heat loss coefficient $h$ is sufficiently small (e.g. $h / V_{D} \approx O(1 / Z)$ ). The validity of this constraint needs to be checked after the solution of the full problem.

Using the jump condition for the heat flux across the reaction front, then, gives

$\theta_{f}=1+\frac{q}{\sqrt{1+\frac{4 h}{V_{D}^{2}}}}$

and where we have assumed that $A^{-} \approx A^{+} \approx-V_{D}$. Corresponding temperature profiles for this case are shown in Figure 6, for different values of the volumetric heat transfer coefficient. 


\subsubsection{Conductive Heat Losses}

When heat losses occur by conduction in a direction perpendicular to the flow direction (Fig. 1b), the heat transfer coefficient is intrinsically time-dependent and the previous analysis does not apply. An expression for the heat losses in this case was developed by Yortsos and Gavalas [27], in terms of the local temperature history. In a dimensional moving coordinate system $\tilde{\xi}=\tilde{x}-V \tilde{t}$ the steady-state dimensional energy balance reads [27]

$\left(c_{g} \rho_{g} \tilde{v}-(1-\phi) c_{s} \rho_{s} V\right) \frac{\partial \tilde{T}}{\partial \tilde{\xi}}=\lambda \frac{\partial^{2} \tilde{T}}{\partial \tilde{\xi}^{2}}+\frac{2 \sqrt{ }\left(\lambda_{h} c_{h} \rho_{h} V\right)}{H \sqrt{ } \pi} \int_{0}^{\infty} \frac{\partial \tilde{T}}{\partial \tilde{\xi}}(\tilde{\sigma}+\tilde{\xi}) \frac{d \tilde{\sigma}}{\sqrt{ } \tilde{\sigma}}$,

We may use the previous notation to express the above equation in terms of the dimensionless moving coordinate $\xi$. For convenience, however, we will introduce a slightly different notation and use the new dimensionless variable

$\xi^{\prime}=\frac{\xi}{x_{*}}$

where

$x_{*}=\frac{\tau}{V_{D}^{1 / 3}}$ and $\tau \equiv\left(\frac{H v_{i}}{2 \alpha_{s}}\right)^{2 / 3}$

In this notation, the dimensionless balance is

$\frac{1}{\bar{\mu}} \frac{\partial \theta}{\partial \xi^{\prime}}=\frac{\partial^{2} \theta}{\partial \xi^{\prime 2}}+\frac{\epsilon}{\sqrt{ } \pi} \int_{0}^{\infty} \frac{\partial \theta}{\partial \xi^{\prime}}\left(\sigma+\xi^{\prime}\right) \frac{d \sigma}{\sqrt{ } \sigma}$

where

$\bar{\mu} \approx \frac{V_{D}^{1 / 3}}{A \tau}$

and

$\epsilon=\sqrt{\frac{\lambda_{h} c_{h} \rho_{h}}{\lambda(1-\phi) c_{s} \rho_{s}}}$ 
Parameter $\epsilon$ controls the heat loss intensity and needs to be sufficiently small for equation (50) to be valid. Defining $\varphi=\partial \theta / \partial \xi^{\prime}$, equation (61) further simplifies to

$\frac{1}{\bar{\mu}} \varphi\left(\xi^{\prime}\right)=\varphi^{\prime}\left(\xi^{\prime}\right)+\frac{\epsilon}{\sqrt{ } \pi} \int_{0}^{\infty} \varphi\left(\sigma+\xi^{\prime}\right) \frac{d \sigma}{\sqrt{ } \sigma}$.

For its solution we need to consider the two different regions, ahead and behind the front.

i - Ahead of the Front, $\xi^{\prime}>0$

In this region (subscript + ), Equation (64) becomes

$\frac{1}{\bar{\mu}} \varphi_{+}\left(\xi^{\prime}\right)=\varphi_{+}^{\prime}\left(\xi^{\prime}\right)+\frac{\epsilon}{\sqrt{ } \pi} \int_{0}^{\infty} \varphi_{+}\left(\sigma+\xi^{\prime}\right) \frac{d \sigma}{\sqrt{ } \sigma}$

which must be solved subject to the boundary conditions

$$
\begin{array}{ll}
\xi^{\prime}=0, & \theta=\theta_{f} \\
\xi^{\prime} \rightarrow \infty, & \theta \rightarrow 1
\end{array}
$$

The solution is an exponential

$\varphi=c_{3} \exp \left(-z_{1} \xi^{\prime}\right), \quad \xi^{\prime}>0$

where $z_{1}>-1 / \bar{\mu}$ is the real positive root of the equation

$\frac{\epsilon^{2} \bar{\mu}^{2}}{z_{1}}-\left(1+\bar{\mu} z_{1}\right)^{2}=0$

Integrating we find the exponential decay

$\theta=1+\left(\theta_{f}-1\right) \exp \left(-z_{1} \xi^{\prime}\right)$

where the front temperature, $\theta_{f}$, is to be determined. 
ii - Behind the Front, $\xi^{\prime}<0$

Behind the front (subscript -) the energy balance reads

$\frac{1}{\bar{\mu}} \varphi_{-}\left(\xi^{\prime}\right)=\varphi_{-}^{\prime}\left(\xi^{\prime}\right)+\frac{\epsilon}{\sqrt{ } \pi} \int_{0}^{\infty} \varphi\left(\sigma+\xi^{\prime}\right) \frac{d \sigma}{\sqrt{ } \sigma} \quad, \quad \xi^{\prime}<0$

This integro-differential equation also includes information ahead of the front, however. When the temperature profile is smooth across the front, the integral reads as follows

$\frac{1}{\bar{\mu}} \varphi_{-}\left(\xi^{\prime}\right)=\varphi_{-}^{\prime}\left(\xi^{\prime}\right)+\frac{\epsilon}{\sqrt{ } \pi}\left(\int_{0}^{-\xi^{\prime}} \frac{\varphi_{-}\left(\sigma+\xi^{\prime}\right) d \sigma}{\sqrt{ } \sigma}+\int_{-\xi^{\prime}}^{\infty} \frac{\varphi_{+}\left(\sigma+\xi^{\prime}\right) d \sigma}{\sqrt{ } \sigma}\right) \quad, \quad \xi^{\prime}<0$

The second term in parantheses can be expressed using the information ahead of the front, (69). We obtain

$\frac{1}{\bar{\mu}} \varphi_{-}\left(\xi^{\prime}\right)=\varphi_{-}^{\prime}\left(\xi^{\prime}\right)+\left(1-\theta_{f}\right) \epsilon \sqrt{ } z_{1} \exp \left(-z_{1} \xi^{\prime}\right) \operatorname{erfc}\left(\sqrt{ }\left(-z_{1} \xi^{\prime}\right)\right)+\frac{\epsilon}{\sqrt{ } \pi} \int_{0}^{-\xi^{\prime}} \frac{\varphi_{-}\left(\sigma+\xi^{\prime}\right) d \sigma}{\sqrt{ } \sigma}$.

Defining $\zeta=-\xi^{\prime}, \varphi_{-}\left(\xi^{\prime}\right) \equiv h(\zeta)$, this further reads

$\frac{1}{\bar{\mu}} h(\zeta)=-h^{\prime}(\zeta)+\left(1-\theta_{f}\right) \epsilon \sqrt{ } z_{1} \exp \left(z_{1} \zeta\right) \operatorname{erfc}\left(\sqrt{ }\left(z_{1} \zeta\right)\right)+\frac{\epsilon}{\sqrt{ } \pi} \int_{0}^{\zeta} \frac{h(\varrho) d \varrho}{\sqrt{ }(\zeta-\varrho)}$

For simplicity, we will solve this equation by neglecting the conduction term along the flow direction (first term on the RHS). In Laplace space we have

$\mathcal{H}(s)=\left(\frac{\left(1-\theta_{f}\right) \epsilon \bar{\mu} \sqrt{ } z_{1}}{\left(\sqrt{ } s+\sqrt{ } z_{1}\right)(\sqrt{ } s-\epsilon \bar{\mu})}\right)$

the inversion of which gives

$h(\zeta)=\frac{\left(1-\theta_{f}\right) \epsilon \bar{\mu} \sqrt{ } z_{1}}{\epsilon \bar{\mu}+\sqrt{ } z_{1}}\left(\sqrt{ } z_{1} \exp \left(z_{1} \zeta\right) \operatorname{erfc}\left(\sqrt{ }\left(z_{1} \zeta\right)\right)+\epsilon \bar{\mu} \exp \left(\epsilon^{2} \bar{\mu}^{2} \zeta\right) \operatorname{erfc}(-\epsilon \bar{\mu} \sqrt{ } \zeta)\right)$ 
To find the temperature, we use the boundary conditions

$$
\begin{array}{ll}
\xi^{\prime}=0, & \theta=\theta_{f} \\
\xi^{\prime} \rightarrow-\infty, & \theta \rightarrow 1 .
\end{array}
$$

After one more integration, the temperature behind the front is found

$$
\begin{aligned}
\theta \approx & \theta_{f}+\frac{\left(1-\theta_{f}\right) \epsilon \bar{\mu} \sqrt{ } z_{1}}{\epsilon \bar{\mu}+\sqrt{ } z_{1}} \\
& \times\left(\frac{1}{\sqrt{ } z_{1}}\left[1-\exp \left(-z_{1} \xi^{\prime}\right) \operatorname{erfc}\left(\sqrt{ }\left(-z_{1} \xi^{\prime}\right)\right)\right]+\frac{1}{\epsilon \bar{\mu}}\left[1-\exp \left(-\epsilon^{2} \bar{\mu}^{2} \xi^{\prime}\right) \operatorname{erfc}\left(-\epsilon \bar{\mu} \sqrt{ }\left(-\xi^{\prime}\right)\right)\right]\right),
\end{aligned}
$$

Equations (69) and (77) along with the jump conditions at the front can be used to evaluate the front temperature. We find

$\theta_{f}=1+\frac{q \tau V_{D}^{2 / 3}}{\left(z_{1}-\epsilon \bar{\mu} \sqrt{ } z_{1}\right)}$.

Typical temperature profiles for the case of conductive losses are shown in Figure 7 . The three profiles correspond to three different steady-state solutions, obtained as will be explained subsequently.

Before proceeding further, we mention that in case the temperature ahead of the front is flat and a discontinuity arises at the front, namely for

$\theta=\mathcal{H}\left(-\xi^{\prime}\right)\left[\theta\left(\xi^{\prime}\right)-1\right]+1$

the energy balance behind the front (70) takes the different expression

$\frac{1}{\bar{\mu}} \varphi_{-}\left(\xi^{\prime}\right)=\varphi_{-}^{\prime}\left(\xi^{\prime}\right)+\frac{\epsilon}{\sqrt{ } \pi} \int_{0}^{-\xi^{\prime}} \frac{\varphi_{-}\left(\sigma+\xi^{\prime}\right) d \sigma}{\sqrt{ } \sigma}-\frac{\epsilon}{\sqrt{ } \pi} \frac{\left(\theta_{f}-1\right)}{\sqrt{ }\left(-\xi^{\prime}\right)}$

This equation can also be solved by methods similar to the previous. 
In summary, by analyzing the heat transfer outside the reaction zone, we have obtained a second relation, in addition to $(50)$, to relate the front temperature to the front velocity. This relation is given by (54), (57) or (78), for the adiabatic case, the convective heat loss case or the case of conductive heat loss, respectively, and involves a combination of various parameters including $q, \gamma, h, \tau$ and $\epsilon$. The solution of the set of the two equations are analyzed for the three different cases using the data set in Table 1.

\section{$7 \quad$ RESULTS}

\subsection{THE ADIABATIC CASE}

In the adiabatic case, the two coupled equations have one unique solution. Thus, in the absence of heat losses, there is no multiplicity and a unique front velocity exists. The variables controlling the solution of this problem are $q, \mu_{g}, \mu, \gamma$ and $\mathcal{A}$. The latter includes the combined effects of $\tilde{a}_{s}$, the kinetics, the pressure and the injection velocity. The effect of the injected oxygen mass fraction also enters through $\mu$, while that of the initial fuel content enters also through both $\mu$ and $\mu_{g}$. For $\mu_{g}>0$, there is always one solution, $V_{D}<1 / \mu$. This is not necessarily the case for $\mu_{g}<0$, where two solutions can exist, of which, however, only one is acceptable, i.e., less than $1 / \mu$.

Figure 8 illustrates the combined effects of mass influx and net gas production due to reaction to the front temperature. The two effects cause the front temperature to increase slightly (for the specific parameter values) with the injection velocity. Under these conditions, the approximation in equation (53), and therefore the preceding analysis of the adiabatic and non-adiabatic models are quite reasonable.

The effect of parameter $\mathcal{A}$ on the front velocity can be assessed by a simple asymptotic analysis

of $(50)$. At large $\mathcal{A}$, for example at small velocities, we have $v \sim \frac{v_{i} \rho_{g i} Y_{i}}{\tilde{\mu} \rho_{f}^{\circ}}$, thus the front velocity increases linearly with the injected oxidant mass flux, and it is inversely proportional to the fuel density. An example of this behavior is shown in Figure 9 where the front velocity is plotted as a function of the injection velocity for different values of the injected oxygen mass 
fraction. The front velocity increases non-linearly with the injection velocity, the rate of increase being constant at low velocities, while decreasing at higher velocities. As expected, increasing the injected mass fraction leads to an increase in the front velocity. Figure 10 shows the non-dimensional unburned oxygen mass fraction at the front, as a function of the injection velocity. The effect is analogous to that of the front velocity.

\subsection{THE NON-ADIABATIC CASE: a. Convective Heat Losses}

Subsequently, we examined the non-adiabatic case. The sensitivity to variations in parameters, in particular the volumetric heat transfer coefficient $\tilde{h} / H$, for the values of Table 1 , was studied.

In contrast to the unique solution found for the adiabatic case, a multiplicity exists for the case of heat losses, provided that the injection velocity exceeds a certain threshold, $E_{c}$. As shown in Figures 11 and 12, the solution now consists of a low-temperature branch in the vicinity of the initial temperature, a high-temperature branch, corresponding to proper combustion and an intermediate branch. The lower branch corresponds to extinction. Above the threshold, three separate solutions exist for any given injection velocity. The existence and stability of these branches were investigated in [11]. Upper and lower branches are stable, while the intermediate branch is unstable. These findings were also confirmed with a direct numerical solution of the problem.

Figure 12 shows that if the solution lies on the high-temperature branch, where rigorous combustion takes place, and subsequently the injection velocity decreases, then due to the increasingly dominating heat losses, an infinitely large slope is reached, at a particular threshold. Since the intermediate branch is unstable, a rapid transition to the lower branch occurs, corresponding to extinction. Threshold $E_{c}$ is the extinction point. Such behavior is extensively reported in the literature for similar systems in reaction engineering.

The upper branch is the solution corresponding to a proper combustion front. In the approximations made in this paper, it runs parallel to the adiabatic temperature solution. The sensitivity of the front temperature to the injection velocity is very large near the threshold, 
but becomes almost negligible above it (again subject to the approximations made). Such behavior is typical of multiple solutions in other areas in reaction engineering. An increase in the heat transfer coefficient or a decrease in the reservoir thickness, both result in lowering the ultimate combustion temperature, and in increasing the ignition temperature and the velocity threshold. This is expected, as higher rates of heat transfer and/or thinner formations lead to larger heat losses. We remark that the lowest value of heat transfer coefficient used in Figure 12 is about double the value used by Gottfried [17] in his investigation.

Figure 13 illustrates the effect of the injected oxygen concentration. As the concentration decreases, the system approaches the extinction point $E_{c}$ at a larger front temperature and injection velocity. This behavior is similar to the effect of varying the heat loss intensity. Figures 14- 15 show plots of the front velocity as a function of the injection velocity for different parameter values. The multiplicity in velocity is also apparent, with the hightemperature branch showing behavior similar to the adiabatic case. From a similar analysis, we can obtain the effect of the heat losses on the the unburned oxygen concentration at the front, Figure 16. The multiplicity is associated with both the front velocity and the unburned mass fraction. There exists a mass fraction branch that practically coincides with the injected mass fraction. The unburned oxygen mass fraction increases with the injection rate and concentration, and decreases with the rate of heat losses.

Finally, Figure 17 illustrates the effect of $q$. This parameter includes the combined effects of parameters such as heat of reaction, initially available fuel, volumetric heat capacity of the solid and initial reservoir temperature. Although the stable temperature branch decreases and the unstable branch increases substantially as $q$ decreases, the front temperature at the extinction point is not very sensitive to changes in $q$. Similar was the effect of $\gamma$.

Subsequently, we investigated the critical extinction threshold (defined using the condition $\left.\left(\partial V_{D} / \partial \mathcal{A}\right) \rightarrow \infty\right)$, and denoted in the following by subscript $c$. Along with equations $(50)$ and (57) this allows us to compute the critical parameters $V_{D c}, \theta_{f c}$ and $\mathcal{A}_{c}$. The parameter space of injection velocity, oxygen mass fraction and volumetric heat transfer coefficient is divided in two regions, one corresponding to sustained propagation and another to extinction (Figure 18). A surface delineates the two regions. Analogous plots can be obtained for 
other parameters. Such plots are useful for practical applications. The values obtained here correspond to the parameters of Table 1.

We close this section by commenting on the effective Damköhler number. The characteristic time for reaction can be defined as $[7]$

$t_{R} \equiv Z\left(\frac{k_{o}\left(Y_{i} \tilde{p}_{i}\right)^{n} e^{-E / R \tilde{T}_{f}}}{l_{R}^{m}\left(R \tilde{T}_{o}\right)^{n}}\right)^{-1}$

A characteristic time for the frontal advance (residence time) can be obtained using

$t_{F}=\frac{l_{T}}{V_{D} v_{i}}$

where $l_{T}$ is the combustion zone thickness also given in [7]. The Damköhler number, Da= $t_{F} / t_{R}$, could be used as an indication of how strongly the reaction kinetics control the combustion front. The calculated ratios for adiabatic and non-adiabatic cases are shown in Figure 19. When the system is adiabatic and the injection rate is small $(O(1) \mathrm{m} /$ day), then $D a$ is much larger than one and the process is controlled by advection. This observation is in agreement with Kumar and Garon's experimental investigation of in situ combustion [29]. However, if the rate is larger or the system is non-adiabatic we observe that it is controlled by the kinetics of the reaction. A multiplicity corresponding to the three branches is also observed.

\subsection{THE NON-ADIABATIC CASE: b. Conductive Heat Losses}

Subsequently, we considered the effect of conductive heat losses. Equations (78) and (50) describe the steady-state solution in the presence of conductive heat losses. The two equations were solved simultaneously. Figures 20 and 21 show the front temperature behavior versus the injection velocity for varying thicknesses of the porous medium. A multiplicity analogous to the case of convective heat losses is apparent. We studied the effect of heat losses by varying the reservoir thickness $H$, all other parameters being fixed. We note that the sensitivity of the 
extinction threshold to the reservoir thickness is significant for thickness values of the order of $1 \mathrm{~m}$ or less. As $H$ decreases, the extinction threshold rapidly increases, thus it requires an increasingly larger velocity for the reaction to be sustained. Figure 22 illustrates the effect of variable oxygen concentration on the front temperature.

By matching the temperatures from the expressions for the two non-adiabatic models, equations (57) and (78), an expression for the effective Nusselt number and the effective heat transfer coefficient can be obtained for the case in which the actual heat losses are by conduction in a semi-infinite medium, but the heat losses are approximated by a convective model. Previously, the transient behavior of such coefficients for thermal fronts in porous medium was analyzed in the absence of heat generating reactions, such as hot water or steam injection. Experiments and analytical works show that its value decreases with respect to time and in the large-time limit, when steady-state condition becomes valid, levels off to a constant value $[30]$.

Defining the Peclet number (based on the front velocity) $\mathrm{Pe}=\frac{H V}{2 \alpha_{s}}$ and re-arranging, the effective Nusselt number, $N u$, is found to be

$N u \equiv \frac{\tilde{h} H}{\lambda}=\operatorname{Pe}^{2 / 3} z_{1}^{2}+2 \epsilon z_{1}^{3 / 2}+\epsilon^{2} \mathrm{Pe}^{-2 / 3} z_{1}-\mathrm{Pe}^{2}$

In the above, we made the approximation that $A=-V_{D}$, as consistently made in the previous as well. In the same notation, $z_{1}$ is the root of the cubic

$\frac{\epsilon^{2}}{\mathrm{Pe}^{4 / 3} z_{1}}=\left(1-\frac{z_{1}}{\mathrm{Pe}^{2 / 3}}\right)^{2}$

The expression (83) is implicitly dependent on the front temperature through the dependence of the non-adiabatic front velocity $V$. Figure 23 shows a plot of the calculated coefficient for varying reservoir thickness and injection velocity. We note that this coefficient first increases and subsequently decreases with the injection velocity, depending on the reservoir thickness. 


\section{CONCLUDING REMARKS}

In this paper, we proposed a method for modeling the propagation of combustion fronts in porous media, by treating the reaction region as a place of discontinuity in the appropriate variables, which include, for example, fluxes of heat and mass. The reaction and combustion fronts have a spatially narrow width within which heat release rates, temperatures and species concentrations vary significantly. The narrow width calls for an approach in which these fronts are treated as surfaces of discontinuity.

Using a perturbation approach, similar to that in smoldering combustion [7], we derived appropriate jump conditions that relate the change in the variables across the front. The conditions account for the kinetics of the reaction between the oxygen and the fuel, the changes in the morphology of the pore space and the heat and mass transfer in the reaction zone. Then, the modeling of the problem reduces to the modeling of the dynamics of a combustion front, on either side of which convective transport of momentum (fluids), heat and mass, but not chemical reactions, must be considered. Properties of the two regions are coupled using the derived jump conditions. This methodology would ultimately allow us to explicitly incorporate permeability heterogeneity effects in the process description, without the undue complexity of the coupled chemical reactions.

The analytical approaches developed reduce the rather complex combustion front propagation problem to a system of two coupled algebraic equations - for the front temperature and its propagation speed. Assuming sufficiently low heat losses, the latter equation (50) consists of a nondimensional quantity $\mathcal{A}$, reflecting the combined effects of inlet conditions as well as physical and chemical properties. The effect of varying this parameter on the system dynamics was studied. Non-adiabatic combustion fronts were investigated by considering the heat losses to the surrounding with two different approaches, a convective type and a conductive type. The expressions obtained are valid for sufficiently small values of the heat loss rate. Analyses for a steadily propagating planar front were presented for typical values of in situ combustion. The presence of heat losses leads to multiplicity and an extinction behavior. The appropriate parameter space was briefly explored for a set of parameter values. 
The observed multiplicity of the forward in situ combustion has similarities to the previously published work of Rabinovich and Gurevich [8] and Britten and Krantz [9]. In addition to their work we found the presence of another stable branch at low temperatures that is connected to the unstable middle branch. The front temperature, velocity and unreacted oxygen concentration solutions for varying injection velocity appear as S-shaped curves. This type of behavior is characteristic of reactive systems in reaction engineering and has been extensively reported in the literature. By comparing the two non-adiabatic models, we were also able to obtain an explicit expression for the overall heat transfer coefficient, equation (83). This coefficient is not only affected by the reservoir thickness, but also by the front dynamics. Equation (83) could be used to extrapolate the non-adiabatic behavior of the combustion front in the laboratory to subsurface conditions.

An interesting behavior of the system is observed when the heat content of the fuel is changed. This could be achieved by varying either the heat of the reaction, $Q$, or the initially available fuel amount, $\rho_{f}^{o}$. The effect of the fuel amount on the front propagation speed has been discussed in in situ combustion literature several times based on adiabatic combustion tube experiments (see for example [31]). Our analytical observation is in agreement with these experiments and brings an insight to this particular behavior of the front.

The planar front analysis has not only given us insight to the nonadiabatic nature of the combustion fronts in porous media but also has revealed and, to a certain extent, quantified the importance of the natural environment where in situ combustion process may have limited applications. We have found that although, due to typical reservoir reservoir thicknesses, the majority of in situ combustion processes may take place under nearly adiabatic conditions, extinction could play an important role on the dynamics of the combustion front.

The models developed do not include effects of the preceding zones, which are quite complex. The vaporization of interstitial hydrocarbons and water downstream, and the thermal cracking of the oil ahead of the unburned region should contribute to the dynamics of combustion front in terms of additional heat losses. On the other hand, LTO reactions between the oxygen left unreacted in the combustion front and the liquid oil phase could be a source of additional heat. Inclusion of these effects to an analytical work, such as presented here, is under consideration. 


\section{References}

[1] Aldushin, A.P. and Seplyarsky, B.S., Sov. Phys. Dokl. 23:483 (1978).

[2] Aldushin, A.P. and Seplyarsky, B.S., Sov. Phys. Dokl. 24:928 (1979).

[3] Matalon, M. and Matkowsky, B.J., J. Fluid Mech. 124:239 (1982).

[4] Britten, J.A. and Krantz, W.B., Combust. Flame 60:125 (1985).

[5] Schult, D.A., Matkowsky, B.J., Volpert, V.A., and Fernandez-Pello, A.C., Combust. Flame 101:471 (1995).

[6] Schult, D.A., Bayliss, A. and Matkowsky, B..J., SIAM J. Appl. Math. 58:806 (1998).

[7] Schult, D.A., Matkowsky, B.J., Volpert, V.A., and Fernandez-Pello, A.C., Combust. Flame 104:1 (1996).

[8] Rabinovich, O.S. and Gurevich, I.G., Combust. Explos. Shock Waves 20:29 (1984)

[9] Britten, J.A. and Krantz, W.B., Combust. Flame 65:151 (1986).

[10] Aldushin, A.P., Rumanov, I.E. and Matkowsky, B.J., Combust. Flame 118:76 (1999).

[11] Akkutlu, I.Y., Dynamics of Combustion Fronts in Porous Media Ph.D. Dissertation, U. of Southern California, Los Angeles (2002).

[12] Akkutlu, I.Y. and Yortsos, Y.C. The Effect of Heterogeneity on In-situ Combustion: The Propagation of Combustion Fronts in Layered Porous Media Presented at the SPE/DOE Thirteenth Symposium on Improved Oil Recovery held in Tulsa, Oklahoma SPE 75128 (2002).

[13] Lu, C. and Yortsos, Y.C. A Pore-Network Model of In Situ Combustion in Porous Media. Presented at the Society of Petroleum Engineers (International) Thermal Operations and Heavy Oil Symposium. Paper SPE 69705 (2001).

[14] Prats, M. Thermal Recovery. SPE Monograph Series SPE of AIME 1982. 
[15] Boberg, T.C. Thermal Methods of Oil Recovery. An Exxon Monograph Series 1988.

[16] Baily, H.R. and Larkin B.K., Petroleum Trans. AIME 219:320 (1960).

[17] Gottfried, B.S., Soc. Pet. Eng. J. 196-210 (1965).

[18] Beckers, H.L. and Harmsen, G.J., Soc. Pet. Eng. J. 231-49 (1970).

[19] Burger, J.G. and Sahuquet, B.C., Soc. Pet. Eng. J. 54-66 (1972).

[20] Ag̃ca, C. and Yortsos, Y.C. Steady-State Analysis of In Situ Combustion. Presented at the Annual California Regional Meeting of the Society of Petroleum Engineers of AIME. Paper SPE 13624 447-362 (1985).

[21] Armento, M.E. and Miller, C.A. Soc. Pet. Eng. J. 423-430 (1977).

[22] Durlofsky, L.J., Jones R.C. and Milliken, W.J., Advances in Water Resources 20:335 (1997).

[23] Allag, O., An Experimental Investigation of the Kinetics of Dry, Underground Combustion Ph.D. Dissertation, U. of Tulsa, Oklahoma (1978).

[24] Verma, V.B., Reynolds, A.C. and Thomas, G.W. A Theoretical Investigation of Forward Combustion in a One-dimensional System. Presented at the 53th. Annual Meeting of the Society of Petroleum Engineers of AIME, held in Houston (1978).

[25] Benham A.L. and Poettmann, F.H., Petroleum Trans. AIME 213:28 (1958).

[26] Da-Mota J.C., Dantas, W.B., Gomes, M.E. and Marchesin, D., Mat. Contemp. 8:129 $(1995)$.

[27] Yortsos, Y.C. and Gavalas, G.R., Int. J. Heat Mass Transfer 305:305 (1982).

[28] Vossoughi, S., Bartlett, G.W., Willhite, G.P., Soc. Pet. Eng. J. 656-663 (1985).

[29] Kumar, M. and Garon, A.M., SPE Reservoir Eng. 55-61 (1991). 


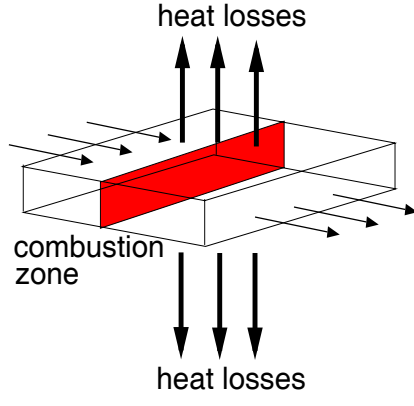

(a)

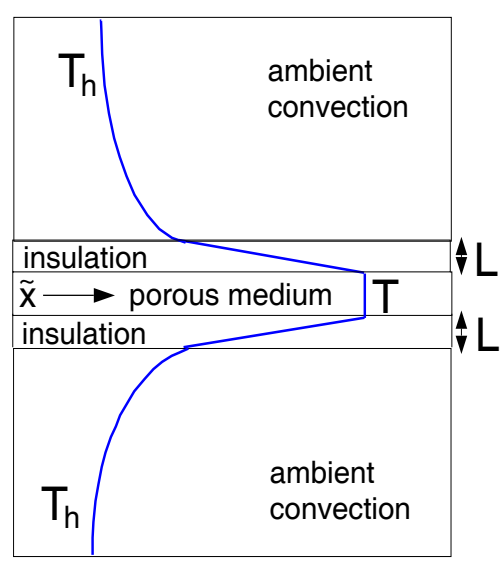

(b)

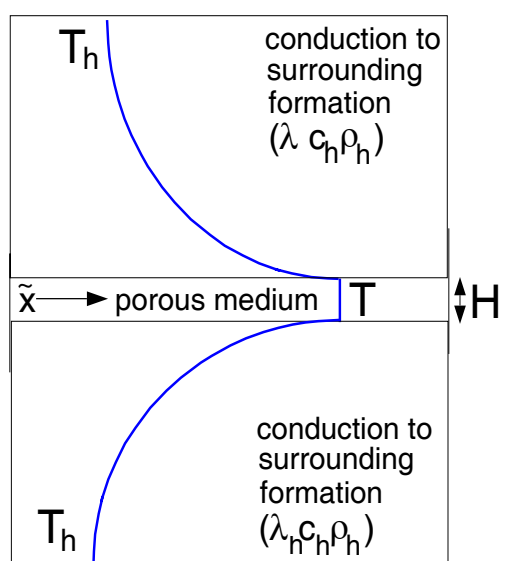

(c)

Figure 1: Heat loss representation: (a) Schematic of the reservoir geometry, with (b) convective and (c) conductive modes.

[30] Zolotukhin, A.B. Analytical Definition of the Overall Heat Transfer Coefficient. Presented at the Annual California Regional Meeting of the Society of Petroleum Engineers of AIME. Paper SPE 7964 (1979).

[31] Martin, W.L., Alexander, J.D. and Dew, J.N., Petroleum Trans. AIME 213:28 (1958).

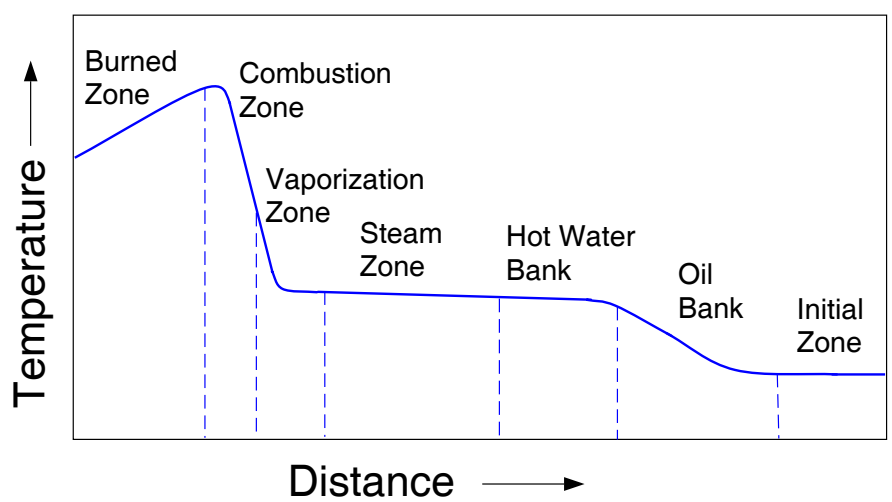

Figure 2: Characteristic regions of in situ combustion process (from [14]). 
Table 1: Typical values of parameters for in-situ combustion

\begin{tabular}{|c|c|}
\hline Parameter & Value \\
\hline$\tilde{Q}$ & $39542 \mathrm{~kJ} / \mathrm{kg}$ fuel \\
\hline $\mathrm{E}$ & $7.35^{*} 10^{4} \mathrm{~kJ} / \mathrm{kmole}$ \\
\hline $\mathrm{R}$ & $8.314 \mathrm{~kJ} / \mathrm{kmole}-\mathrm{K}$ \\
\hline$\tilde{a}_{s}$ & $1.41^{*} 10^{5} \mathrm{~m}^{2} / \mathrm{m}^{3}$ \\
\hline$k_{0}$ & $227 \mathrm{~kW}$-m/atm-kmole \\
\hline$\tilde{T}_{0}$ & $373.15 \mathrm{~K}$ \\
\hline $\mathrm{m}, \mathrm{n}, \alpha$ & 1 \\
\hline$\tilde{p}_{i}$ & $1 \mathrm{~atm}$. \\
\hline$Y_{i}$ & 1.0 \\
\hline$D_{M}$ & $2.014^{*} 10^{-6} \mathrm{~m}^{2} / \mathrm{s}$ \\
\hline$\lambda$ & $8.654^{*} 10^{-4} \mathrm{~kW} / \mathrm{m}-\mathrm{K}$ \\
\hline$\phi$ & 0.3 \\
\hline$c_{g} \rho_{g i}$ & $1.2339 \mathrm{~kJ} / \mathrm{m}^{3}-\mathrm{K}$ \\
\hline$\rho_{f}^{o}$ & $19.2182 \mathrm{~kg} / \mathrm{m}^{3}$ \\
\hline & $1.0936 \mathrm{~kJ} / \mathrm{kg}-\mathrm{K}$ \\
\hline$(1-\phi) c_{s} \rho_{s}$ & $2.012^{*} 10^{3} \mathrm{~kJ} / \mathrm{m}^{3}-\mathrm{K}$ \\
\hline$M_{f}$ & $235 \mathrm{~kg} / \mathrm{kmole}$ \\
\hline$\psi(\eta)$ & $1-\eta$ \\
\hline H/C Ratio & 1.65 \\
\hline$\tilde{\mu}$ & 3.018 \\
\hline$\tilde{\mu}_{g}$ & 1.000 \\
\hline
\end{tabular}

Source: References [14],[15], [20] and [28] 


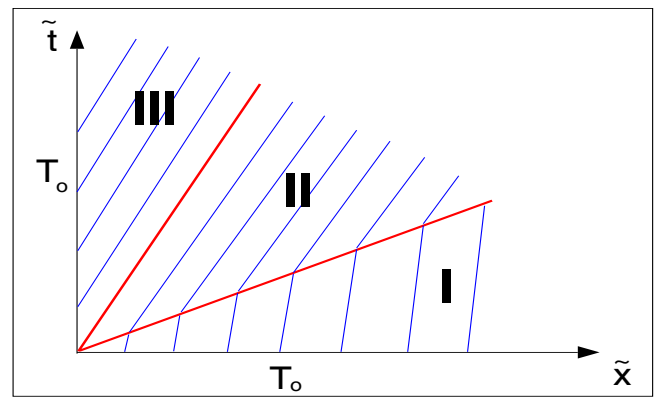

Figure 3: Characteristics diagram for combustion in porous media.

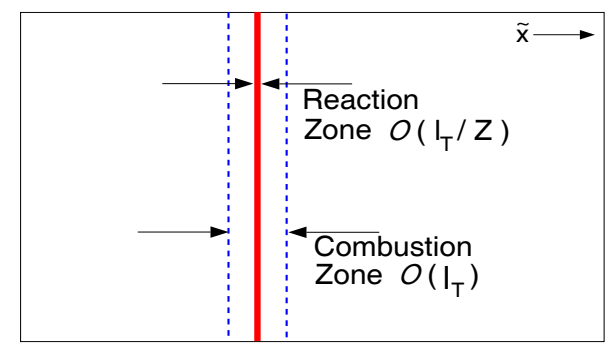

Figure 4: Schematics of reaction and combustion zones. 


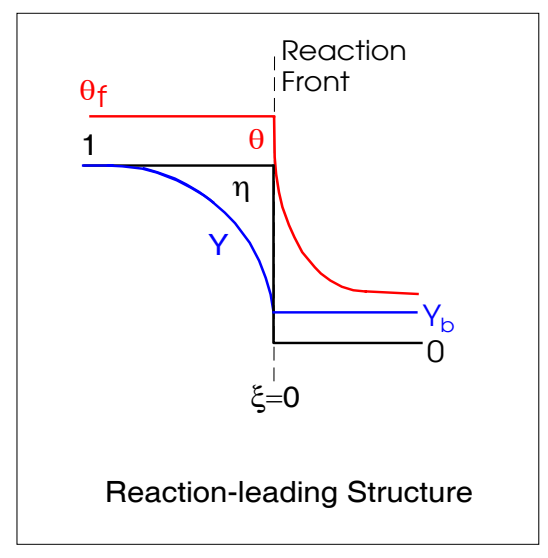

Figure 5: The Adiabatic Case: Schematic profiles of temperature, oxygen mass fraction and fuel conversion in the combustion zone.

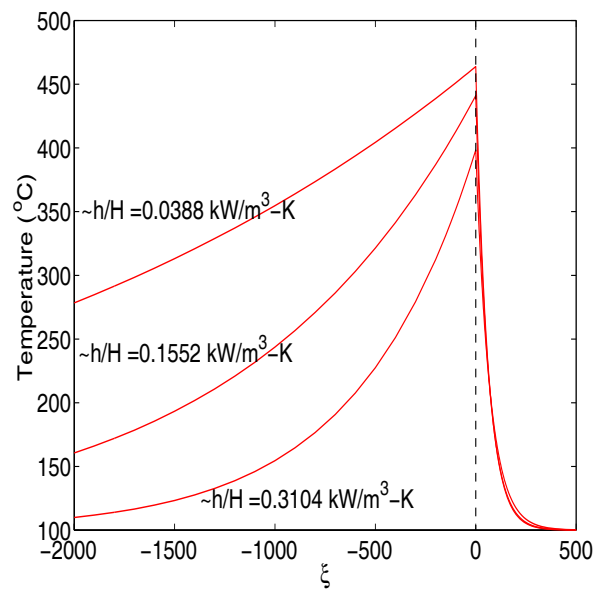

Figure 6: Temperature profiles for nonadiabatic combustion fronts with convective type heat losses, for an injection velocity of $100 \mathrm{~m} /$ day. Only the stable upper branch is shown.

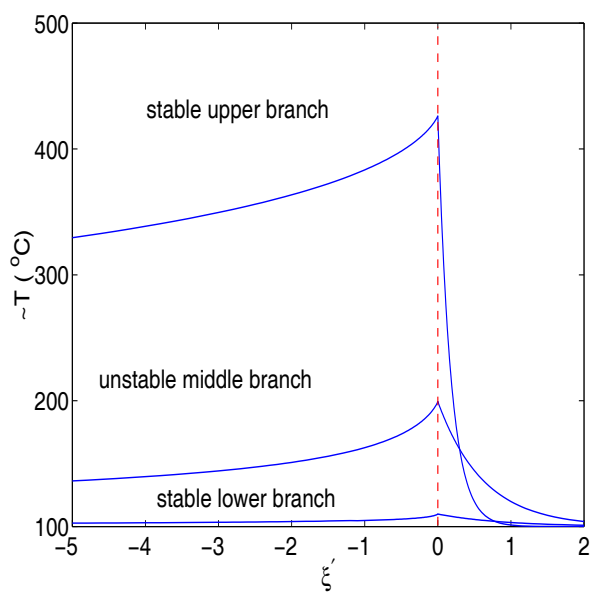

Figure 7: Temperature profiles for nonadiabatic combustion fronts with conductive type heat losses $(\mathrm{H}=0.5 \mathrm{~m})$. The three temperature branches corresponding to the three multiple solutions are shown.

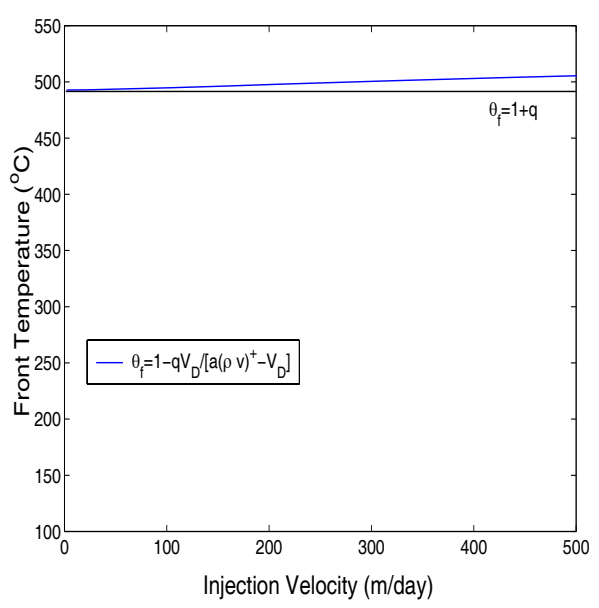

Figure 8: Adiabatic front temperature versus injection velocity, showing the combined effects of mass influx and net gas production. 


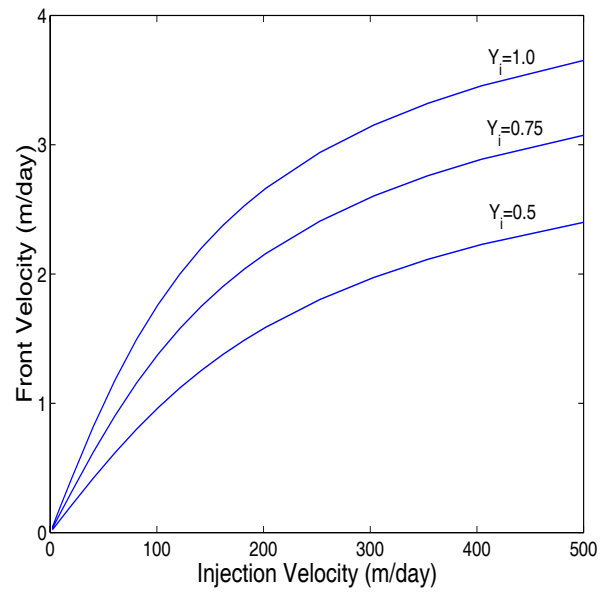

Figure 9: Adiabatic front velocity versus injection velocity for different values of the injected oxygen mass fraction $Y_{i}$.

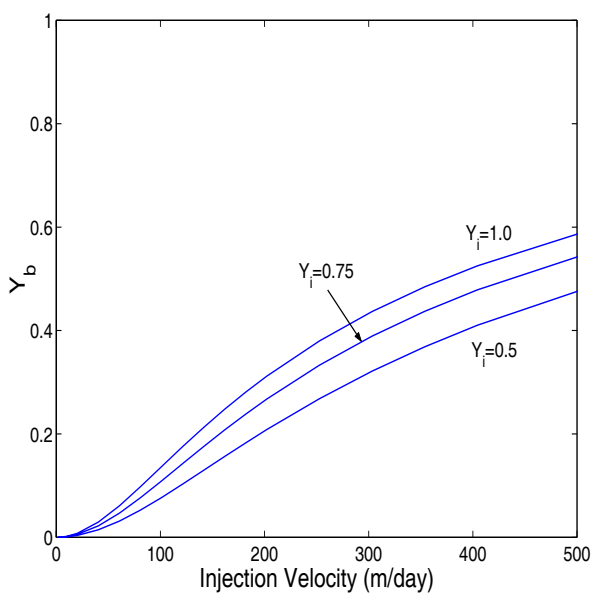

Figure 10: Unburned oxygen concentration for adiabatic front versus injection velocity for different values of the injected oxygen mass fraction $Y_{i}$. 


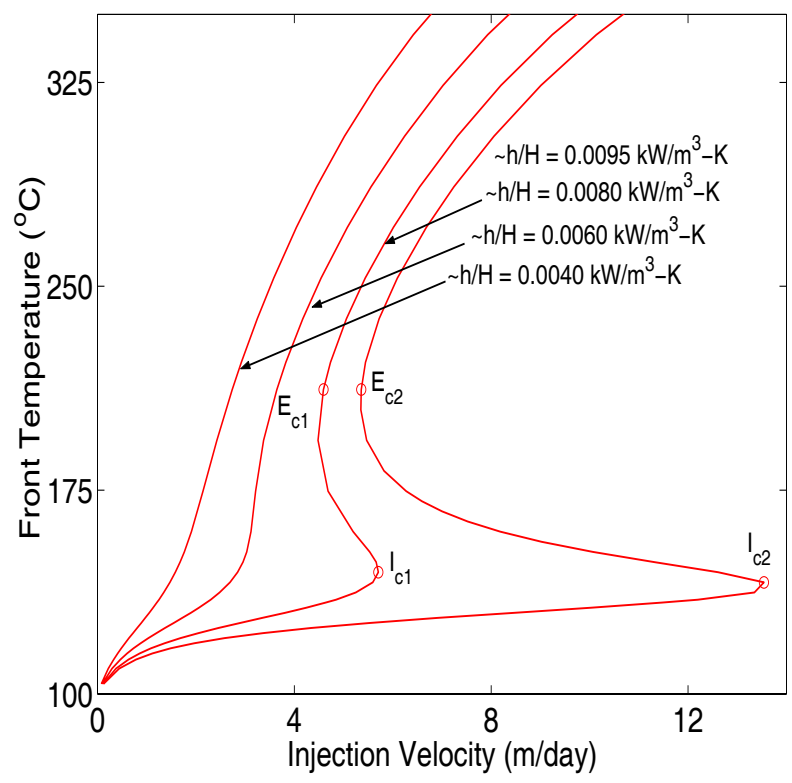

Figure 11: Non-adiabatic front temperature versus injection velocity for different values of the volumetric heat transfer coefficient, $\tilde{h} / H$, for low heat loss rates. The extinction thresholds are $I_{c 1}=(5.7,144.8)$, $E_{c 1}=(4.6,211.4), I_{c 2}=(13.5,141.0), E_{c 2}=(5.4,211.9)$.

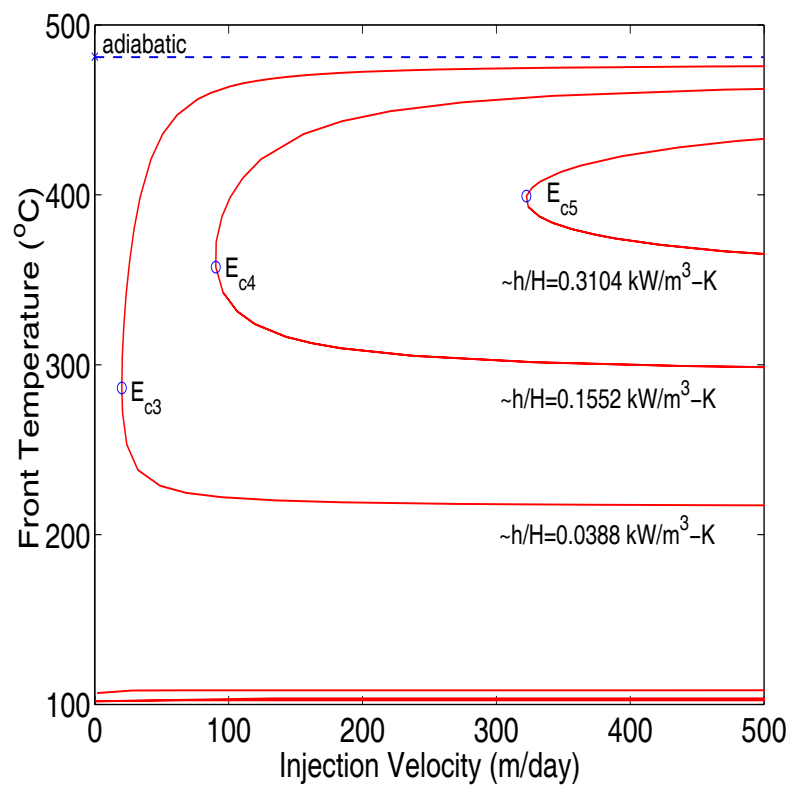

Figure 12: Non-adiabatic front temperature versus injection velocity for different values of the volumetric heat transfer coefficient, $\tilde{h} / H$, for higher heat loss rates. The extinction thresholds are $E_{c 3}=(20.2,286.2)$, $E_{c 4}=(90.4,357.5)$ and $E_{c 5}=(322.5,399.3)$. 


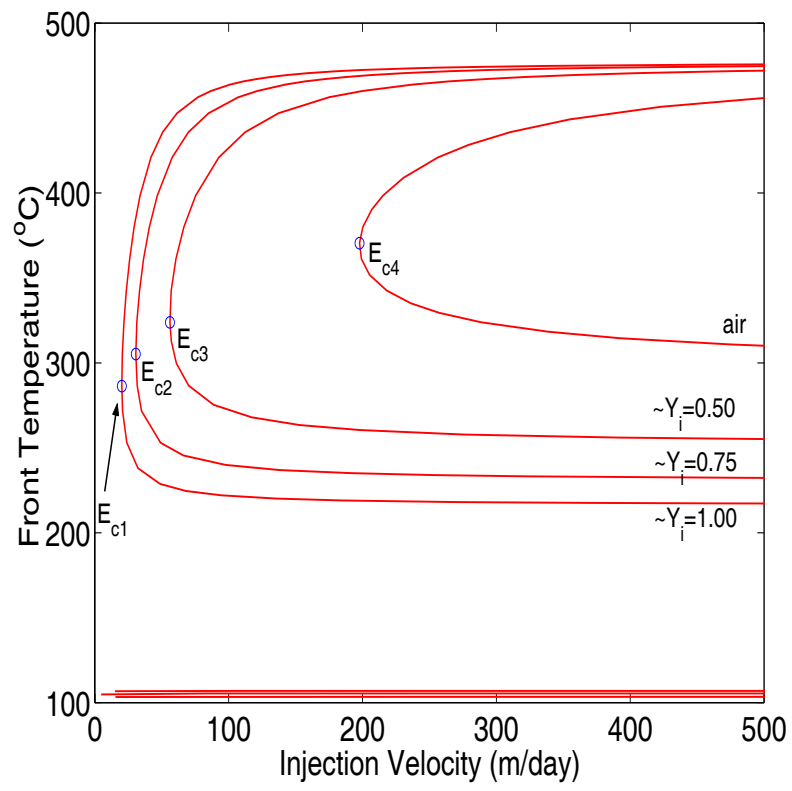

Figure 13: Non-adiabatic front temperature versus injection velocity for different values of the injected oxygen mass fraction $Y_{i} . \quad h / H=0.0388 \mathrm{~kW} / \mathrm{m}^{3}-\mathrm{K}, E_{c 1}=(20.2,286.2), E_{c 2}=(30.6,305.2), E_{c 3}=(56.1,323.9)$, $E_{c 4}=(197.8,370.5)$. 


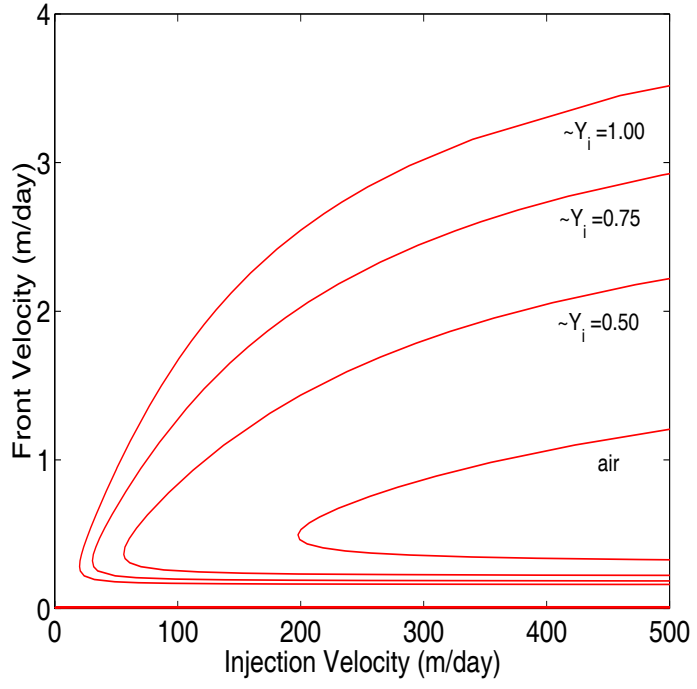

Figure 14: Non-adiabatic front velocity versus injection velocity for different values of the injected oxygen mass fraction $Y_{i}$.

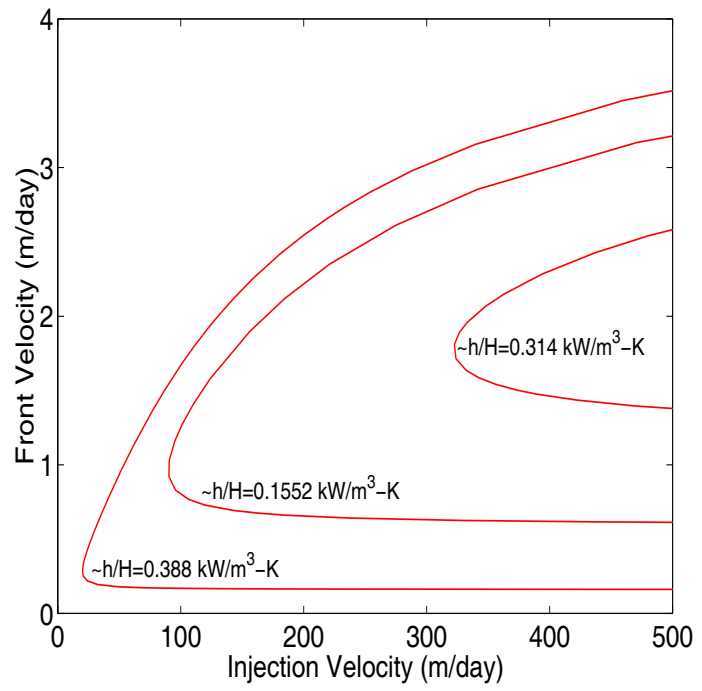

Figure 15: Non-adiabatic front velocity versus injection velocity for different values of the volumetric heat transfer coefficient.

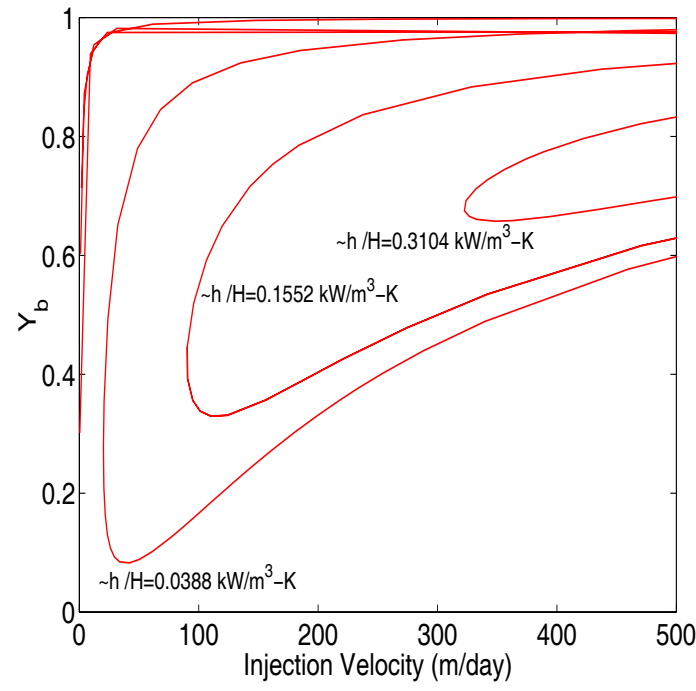

Figure 16: Unburned oxygen mass fraction for the non-adiabatic case versus the injection velocity, for different values of the volumetric heat transfer coefficient. 


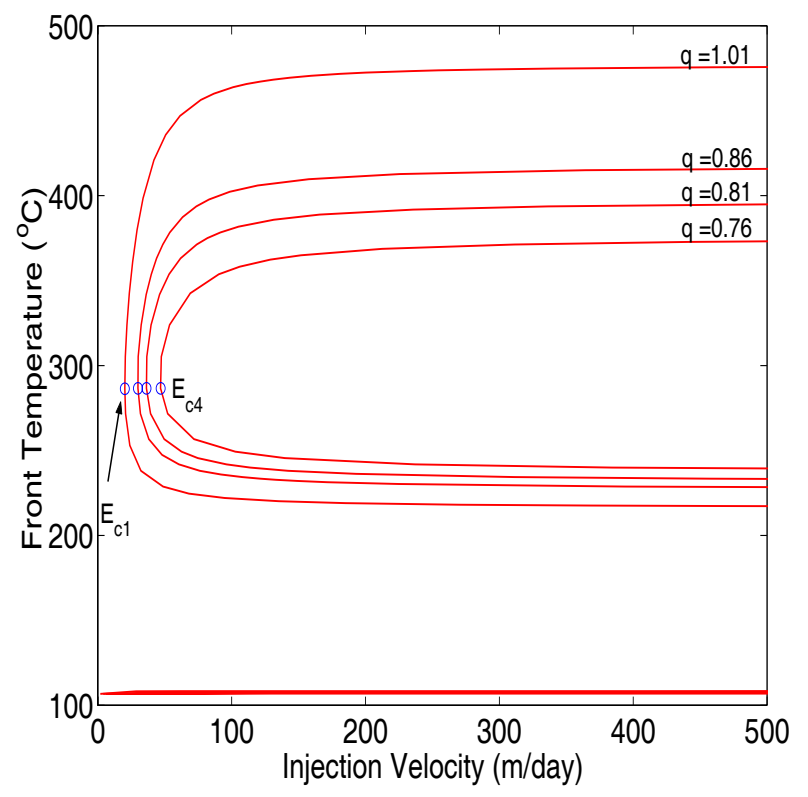

Figure 17: Non-adiabatic front temperature versus injection velocity for different values of $q . \hat{h} / H=0.0388$ $\mathrm{kW} / \mathrm{m}^{3}-\mathrm{K}, E_{c 1}=(20.2,286.2), E_{c 2}=(30.0,286.6), E_{c 3}=(36.3,286.6), E_{c 4}=(46.9,286.6)$.

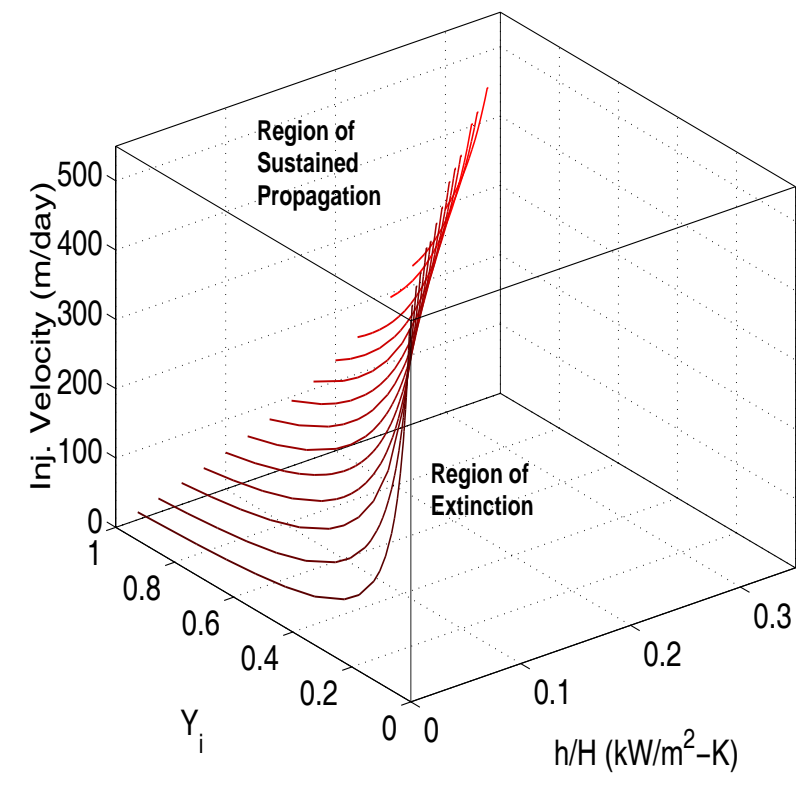

Figure 18: Injection velocity $v_{i}$ versus injected oxygen mass fraction $Y_{i}$ and the volumetric heat transfer coefficient $\tilde{h} / H$. The dividing surface corresponds to the critical injection velocities for the parameters of Table 1. 


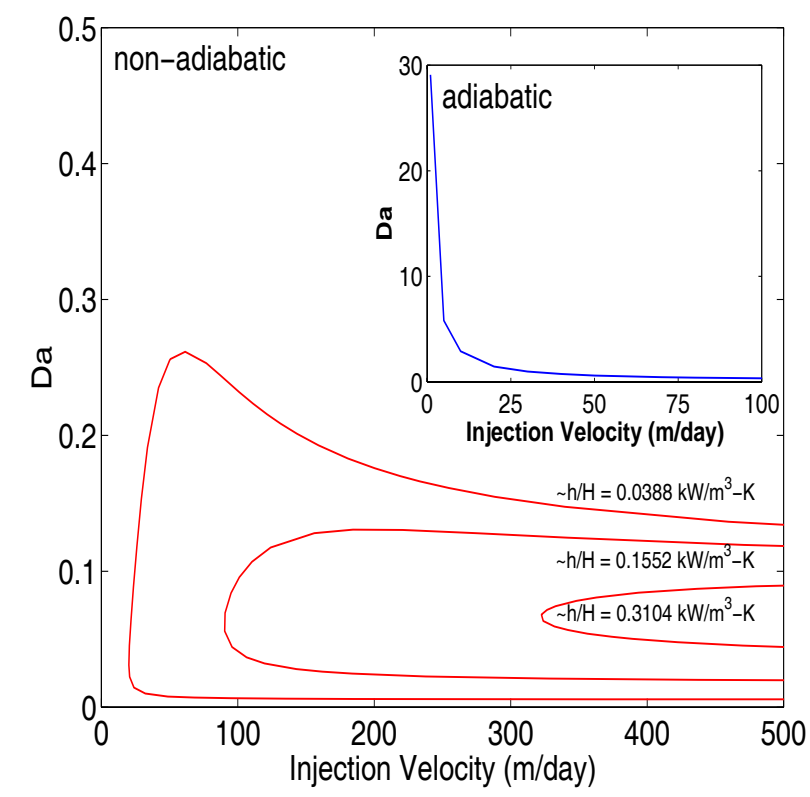

Figure 19: Damköhler number versus injection velocity.

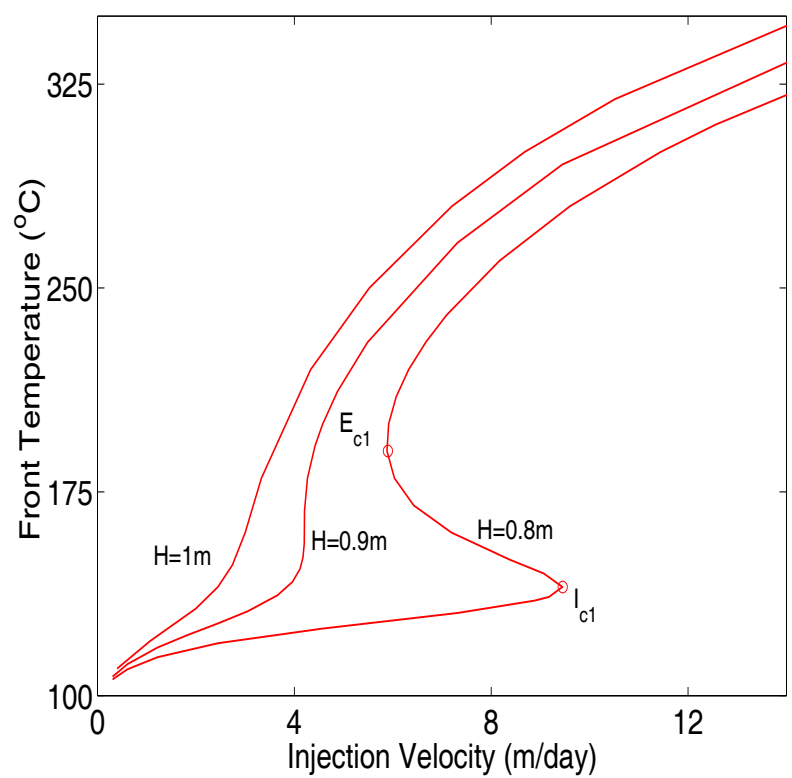

Figure 20: Non-adiabatic front temperature versus injection velocity obtained using the conductive heat loss model (low heat loss rate). $I_{c 1}=(9.45,140.0), E_{c 1}=(5.9,190.0)$. 


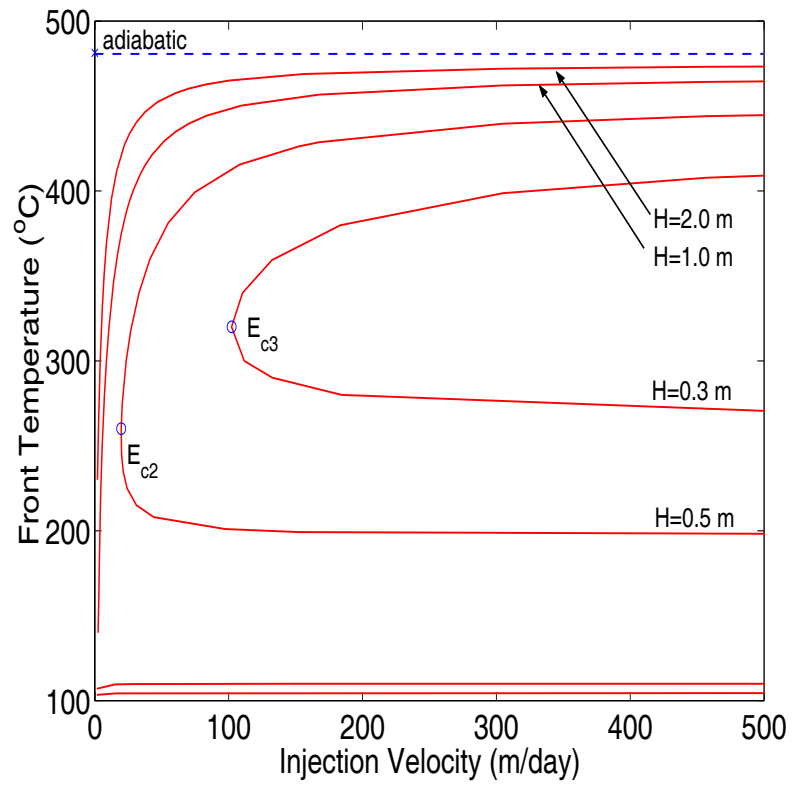

Figure 21: Non-adiabatic front temperature versus injection velocity obtained using the conductive heat loss model (higher heat loss rates). $E_{c 2}=(19.7,260.0), E_{c 3}=(102.1,320.0)$.

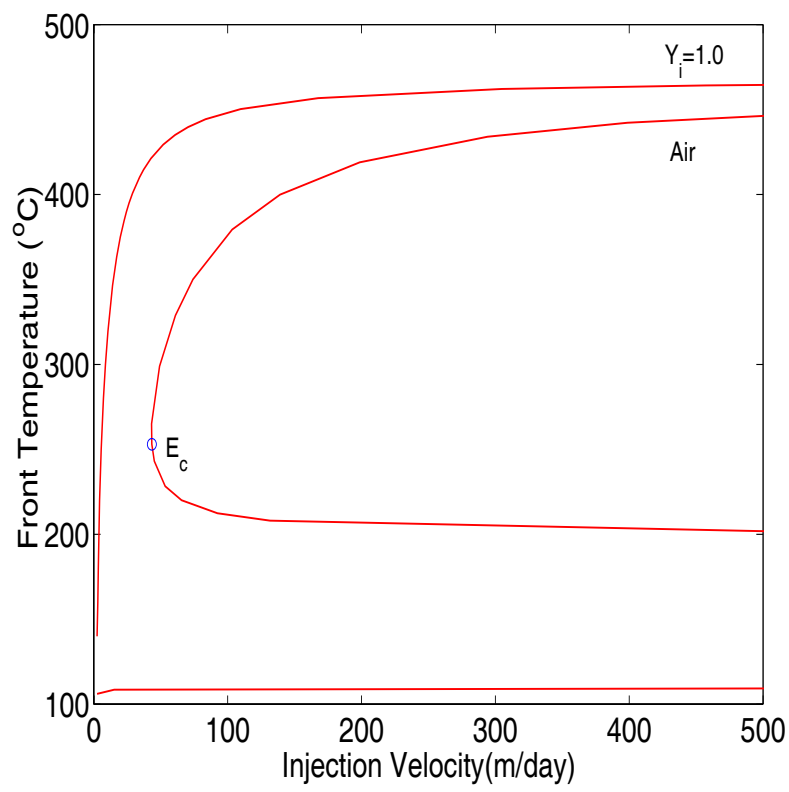

Figure 22: Non-adiabatic front temperature versus injection velocity for the cases of pure oxygen and air injection obtained using the conductive type heat loss model. $H=1 \mathrm{~m}, E_{c}=(43.3,253)$. 


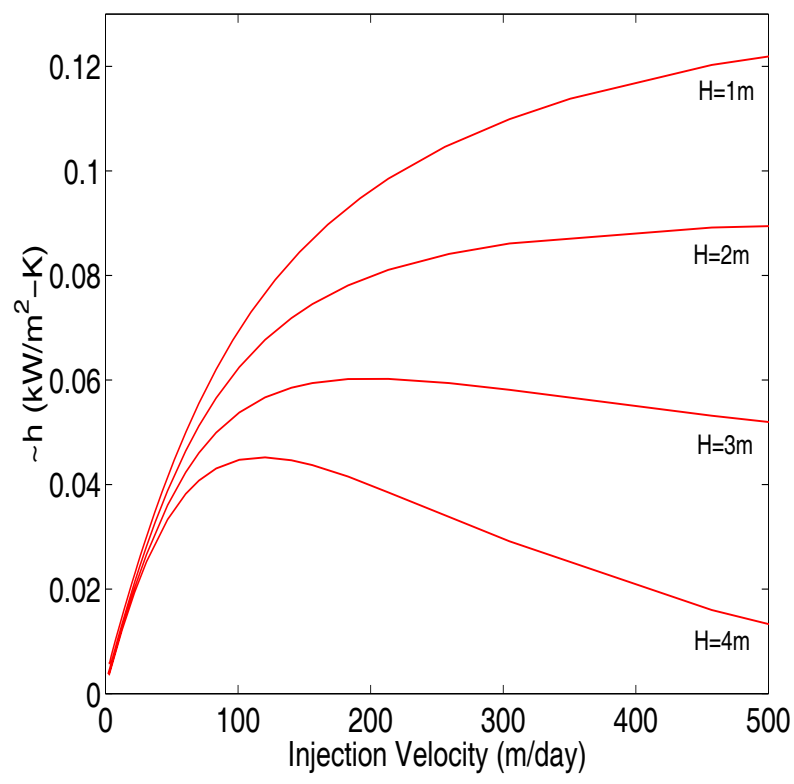

Figure 23: The effective overall heat transfer coefficient $\tilde{h}$ versus injection velocity $v_{i}$ for varying reservoir thickness. 


\title{
A Pore-Network Model of In-Situ Combustion in Porous Media
}

\author{
Chuan Lu and Yannis C. Yortsos
}

\section{INTRODUCTION}

In-situ combustion (ISC) is a well-known process for the recovery of heavy oil from oil reservoirs. Extensive reviews of the method and its field applications have been provided in the literature (Prats, 1982). Even though one of the oldest techniques for heavy oil recovery, however, ISC is also one of the most complex. In addition to the common mechanisms it shares with conventional recovery methods, such as waterflooding and steamflooding, ISC involves the additional complexity of the oxidation reactions. These serve to, first, form the fuel in a regime of Low Temperature Oxidation (LTO) and, subsequently, provide the main combustion reaction under conditions of High Temperature Oxidation (HTO). The main goal of ISC is the sustained propagation of combustion fronts, to supply the necessary heat for viscosity reduction and the self-sustaining of the overall process. As a result, issues of front stability, sustained front propagation and possible extinction are of fundamental importance.

A large number of studies have been published in the literature on ISC, addressing a wide range of issues, from the detailed kinetics of the reaction processes to mathematical models to field applications. Of specific interest to this study is the modeling of the combustion process. Typically, this is done using conventional continuum models, in which reaction rates, concentrations and temperatures are volume-averaged continuum variables. The solution of such models is then sought in the various applications of interest, including laboratory and field scales. This approach has validity as long as the following conditions hold: that the variables of interest, such as concentrations, temperature and reaction rates, do not involve large gradients in space at the underlying microscale, for volume-averaged quantities to be meaningful; and that the effective parameters used in the continuum models reflect fairly accurately the actual processes. Because of the strong non-linearities involved in reaction kinetics, particularly of the Arrhenius dependence of the true reaction rate on temperature,

$$
r \propto C \exp \left(-\frac{E}{R T}\right)
$$

the volume average of the rate will not have the same dependence, namely

$$
<r>\neq<C>\exp \left(-\frac{E}{R<T>}\right)
$$

if the local concentration or temperature vary significantly over the averaging volume. 
Large gradients at the microscale may occur for a number of reasons. The development of sharp propagating fronts is one of them. In displacement processes, fronts arise naturally, due to the different characteristic velocities of the initial and the injected states (a classical example of which is the shock front in a Buckley-Leverett type displacement). The thickness of such fronts is controlled by dissipation, like diffusion, capillarity and conduction. For the case of ISC, however, sharp fronts also result from the fast kinetics of the combustion reactions in the HTO region and the depletion of some of the various chemicals that participate in the reaction. The resulting reaction zones are thin, and give rise to large gradients within the microstructure. The possible development of spatial instabilities is another important cause of large gradients.

The second concern is, in general, the effect on the overall process of small-scale heterogeneities of fluid flow, mass and heat transfer, and chemical reactivity. Local heterogeneities arise due to the randomness of the microstructure, and may result in a distribution of the local variables that can affect the volume-averaged rates as noted above. In addition, of importance to sustained propagation and extinction is the spatial distribution of the fuel in the pores of the microstructure. If the fuel is sparsely distributed, the sustained spreading of combustion across the pore network requires certain conditions on the rates of reaction, heat and mass transfer. Otherwise, the process will become extinct. For problems in which sustained propagation or extinction are critical issues, having an accurate assessment $f$ the phenomena at the microscale becomes then an important objective. This is possible with the development of a pore-network model of ISC, which forms the objective of this paper.

As is well known, there are several distinct regions during an ISC proess (Prats, 1982, Boberg, 1988). From the injection well, they are the swept region, the combustion/cracking zone, the vaporization and LTO zone, the steam zone, the hot water bank, the oil bank and the initial zone (see schematic of Figure 1). For simplicity and by necessity, in this paper we will focus on the HTO regime and ignore the other downstream regimes. Thus, the problem to be modeled is the combustion of a solid fuel, deposited in the porespace, by the injection of a gas-phase oxidant. In a sense, the problem under consideration is similar to what is known as Filtration Combustion (FC) (Aldushin, 1993). The latter finds a variety of applications and has been analyzed in considerable detail in recent years, using continuum models. Some of these res ults will be reviewed below.

Pore-network models have been successfully used to model a variety of problems in porous media in the absence of reaction, from immiscible displacements to boiling (Lenormand 
et al., 1988, Blunt et al., 1991, Li and Yortsos, 1995, Satik and Yortsos, 1996). This has allowed for an accurate representation of effective parameters and for a better incorporation of smallscale mechanisms in continuum models. In processes involving reactions, applications of pore networks have been mainly in the areas of heterogeneous reactions on catalysts, catalysts deactivation, acidization, dissolution of trapped pollutants, etc. [8-11]. Discrete models for FC, and more specifically for ISC processes, have not appeared in the literature to our knowledge (see also below). This paper attempts to fill this gap. The approach to be implemented is analogous to an earlier effort to model thermal processes at the pore-network scale, for example in heat transfer driven bubble growth. The important difference is that here the exothermic combustion reaction at the pore surface must also be included.

Before proceeding, we need to mention that similarities exist between ISC and the process of self-propagating high temperature synthesis (SHS) for the development of new materials (Hwang et al., 1997, 1998). The difference is that in the latter process, the reaction rate depends only on temperature and not on gas-phase concentrations. Experimental investigations have provided much information concerning SHS processes. An understanding of the microscopic processes is crucial to predicting the microstructure and properties of the final product. A numerical model akin to a pore-network model was developed by Hwang et al. (1998), based on which they were able to simula te effects of heterogeneity on the conversion patterns, as a function of various parameters.

The model to be developed in this paper is considerably more complex than that of Hwang et al. (1998). In addition to heat transfer, it accounts for the convection and diffusion of the injected oxidant, its chemical reaction with the fuel and the possible enhancement of permeability as a result of the HTO. Effects of heterogeneity in permeability, fuel distribution, and other variables are also included. The paper is organized as follows: First, we describe the aspects of the pore-network model. Before we provide numerical simulation results, we also review some findings from continuum analyses of FC, in order to verify the validity of the model in special cases. Then, we provide numerical simulation results to examine the sensitivity of the system to various parameters. Finally, we discuss their implication regarding continuum models.

\section{PORE-NETWORK MODEL AND NUMERICAL METHOD}

In this section, we describe the pore-network model and the numerical method used for its solution. Findings from the continuum model of FC are also discussed. 


\section{Model Description}

The pore-network model consists of a dual network of pores and throats, which is embedded in a network of solid sites, representing the solid matrix (Figure 2). The pores (sites) are the places where reaction occurs and contain solid fuel of constant composition. They are interconnected via throats (bonds), which control transport of mass, momentum and heat, and which have distributed sizes. The network of solid sites is needed to account for heat transfer in the solid (e.g. see Satik and Yortsos, 1996). The coupling of solid and pore-space occurs through the heat transfer between pores and solid sites as shown in Figure 2.

In the 2-D simulations to be reported below, both networks are square lattices, of an adjustable length and width. The features on mass and heat transport include the following:

1. In 2-D, each solid site communicates with 4 solid sites (shown in Figure $2 \mathrm{a}$ by the thin solid line between solid sites) and 1 pore site (the dash line between solid and pore sites), through heat conduction. A heat transfer coefficient is used to model the pore-solid exchange.

2. Injection of a mixture of oxidant and inert gas occurs at one end, where pressure, temperature and composition are specified. The outlet end is at a constant pressure to maintain the injection rate. Flow in the pore throats is governed by Poiseuille's law. The oxidant in the gas phase is delivered by gas-phase diffusion and convection.

3. Within a pore site, thermodynamic equilibrium is assumed, such that concentrations, pressure and temperature are uniform. This also implies that fuel and gas are at thermal equilibrium. However, heat transfer does take place between adjacent pore sites, between pore and solid sites and between adjacent solid sites. Though this configuration and our code allow for different thermodynamic and transport parameters, they are taken constant to avoid extra complexity. Variables assignedto sites and bonds are listed in Table 1.

4. The following heterogeneous reaction is assumed,

$$
\text { Solid Fuel }+\mu \text { Gas Oxidant } \rightarrow \mu_{1} \text { Solid product }+\mu_{2} \text { Gas Product }
$$

where $\mu, \mu_{1}$, and $\mu_{2}$ are the stoichiometric coefficients. Implicit in the above is the assumption that the reaction is heterogeneous and does not involve the propagation 
of flame in the pore network. The reaction rate is expressed by the one-step kinetic model

$$
R=k_{r} A_{r} P X_{O} H\left(V_{f}\right) \exp \left(-\frac{E_{a}}{R T}\right)
$$

where $H$ is the step function, $V_{f}$ is the volume of fuel, $P$ is the total pressure, $k_{r}$ is the kinetic constant, $A_{r}$ is the interface area in an individual pore site, $X_{o}$ the mass fraction of the oxidantand $E_{a}$ the activation energy. More complex kinetics, including multi-step, parallel or serial schemes, could also be adopted.

\section{Governing Equations}

The governing equations for FC have been developed for some time now. Due to the multi-phase nature of the process, they must be able to account separately for the properties of the different phases and to also describe their interaction mechanisms. Ohlemiller (1986) provided a formalism that includes single particle/bulk condensed phase equations, to de scribe the behavior of the solid phase temperature and mass balance, and bulk gas equations, to determine the temperature, concentration, pressure and velocity profiles in the gas phase. Currently, most models of the FC processes adapt the bulk equations for both gas and condensed phases along with considerable other simplifications $[15,16]$.

Using appropriate mass and energy balances, we can write conservation equations for each site in the pore network, as follows:

Pore Site I, Adjacent to Pore-Site j:

Gas Phase Component k Mass Balance

$$
\begin{aligned}
\frac{\Delta\left(\frac{P_{i} V_{i}}{R T_{i}} y_{i, k} M_{k}\right)}{\Delta t}= & -\sum_{j} u_{i j} r_{i j}^{2} \frac{P_{i} y_{i, k}}{R T_{i}} M_{k}-\frac{D_{e}}{l R} \sum_{j} r_{i j}^{2}\left(\frac{P_{i}+P_{j}}{2}\right)\left(\frac{2}{T_{i}+T_{j}}\right)\left(y_{i, k}-\right. \\
& +\mu_{k}\left(k_{r} A_{i}^{r} \frac{P_{i} y_{i, O_{2}}}{R T_{i}} \exp \left(-\frac{E_{a}}{R T_{i}}\right)\right) H\left(V_{i, f}\right)
\end{aligned}
$$

\section{Solid Fuel Conservation}




$$
\rho_{f} \frac{\Delta V_{i, f}}{\Delta t}=k_{r} A_{i}^{r} \frac{P_{i} y_{i, O_{2}}}{R T_{i}} \exp \left(-\frac{E_{a}}{R T_{i}}\right) H\left(V_{i, f}\right)
$$

Energy Balance

$$
\frac{\Delta E_{i}}{\Delta t}=-\sum_{j} u_{i j} r_{i j}^{2} \frac{E_{g, i}}{V_{i}}-\frac{K_{g s}}{l} \sum_{j} r_{i j}^{2}\left(T_{i}-T_{j}\right)-h_{s} \sum_{S} A_{i s}^{h}\left(T_{i}-T_{s}\right)-h_{L, P} A_{i, l}^{h}\left(T_{i}-T_{0}\right)
$$

where, we defined the energy content of a site by

$$
\begin{aligned}
& E_{i}=E_{f, i}+E_{g, i} \\
& E_{f, i}=m_{f, i}\left(C_{p, f} T_{i}+\Delta_{c} H_{f}\right) ; E_{g, i}=\sum_{k} \frac{P_{i} V_{i}}{R T_{i}} M_{k} y_{i, k}\left(C_{p g, k} T_{i}+\Delta_{c} H_{f}\right)
\end{aligned}
$$

\section{Momentum Balance}

$$
u_{i j}=\frac{r_{i j}^{4}}{8 \mu l}\left(P_{i}-P_{j}\right)
$$

Solid Site Energy Balance

$$
\rho V C_{p s} \frac{\Delta T}{\Delta t}=-\frac{k_{s}^{h}}{l} \sum_{s^{\prime}} A_{s s^{\prime}}^{h}\left(T_{s}-T_{s^{\prime}}\right)+h_{s} \sum_{j} A_{j s}^{h}\left(T_{j}-T_{s}\right)-h_{L, S} A_{s, l}^{h}\left(T_{s}-T_{0}\right)
$$

The sum in the energy balances express the heat exchange between pore and solid sites, while $h_{L, S}$ denotes heat loss to the surroundings. In addition, ideal gas behavior was assumed. Finally, the bond radius was correlated to the depth of conversion.

To make the equations dimensionless we introduce the characteristic values listed in Table 2 . The corresponding dimensionless groups obtained are shown in Table 3.Together with other parameters, such as the initial temperature, the volume ratio of solid site to the pore site, the average initial fuel amount in pore sites and the oxidant concentration at injection, the dimensionless parameters constitute the main set of variables affecting the process. 


\section{Remarks}

- The above model allows for simulating both forward and reverse combustion. These are differentiated by controlling the ignition point, which is the first row in the inlet for the case of forward combustion, and the row before the last in the outlet, for the reverse case (Figure 3). Our emphasis in this paper is on forward combustion, although some brief comments on reverse combustion will also be made, where appropriate.

- Although the relation between flow rate and pressure difference depends on the Reynolds number, we will only assume laminar, Poiseuille flow. At the continuum level, this is equivalent to Darcy's law, an assumption which is very often made in the literature.

- Though radiation may play an important role because of the high temperature at the fronts, most analytical solutions and numerical simulations omit its effect. It was neglected in our model as well.

- As mentioned above, the kinetics of ISC are rather complicated. They can be accompanied by pyrolysis, evaporation and condensation, multi-step reactions and also by some gas-phase reactions $[3,17,18,19]$. However, in contrast to the well-developed flame kinetics, a detailed reaction mechanism for heterogeneous systems is still not widely used. Instead, one-step kinetics, as assumed above, is commonly accepted for the combustion of solid fuels.

- Finally, in our model, the heat released by the reactions is expressed in an implicit form. This differs from most previous works. The advantage of this formalism lies in the fact that one can ignore the detailed kinetics and the associated physical processes. The shortcomings are that it cannot describe processes at ve ry high flow rates, which can break the thermal equilibrium between the gas phase and the fuel sites.

\section{Numerical Method}

The dimensionless equations were solved by standard implicit schemes. Because of the stiffness of the problem, time steps were confined to less than 1s. However, because the spatial scale needs to be large enough, it takes quite a long time to obtain reasonably accurate results. In previous works in homogeneous problems and stability investigations, adaptive pseudo-spectral algorithms have been applied (Bayliss and Matkowsky, 1987). Since the pore-network model has many features in common with a discretization scheme, we can assign transport properties,

either by making the scheme $2^{\text {nd }}$ order accurate or of an upwind character. In our calculations, it was found that numerical dispersion affects the results only when the injection rate is sufficiently 
high $(\mathrm{Pe}>\mathrm{O}(1))$. For such cases, special methods were adopted. An implicit predictor-corrector method was employed for the temporal evolution. This method is $2^{\text {nd }}$ order accurate in most cases.

In order to speed up the computations, further simplifications were necessary. To this effect, we introduced the following assumptions:

- Constant pressure

Since the gas viscosity is small, the pressure perturbation can be ignored in all governing equations, except in Poiseuille's law (Equation (7)). This assumption is based on the condition that the flow rate is significantly smaller than the speed of sound, and is widely used in the simulations of laminar flames in open space. The computational advantages are that the momentum equation becomes decoupled, while the continuity equation is linearized.

- Quasi-steady state in the gas phase

Because of the difference between the densities of gas and solid, the corresponding time scales are greatly different. Using dimensional analysis, Ohlemiller (1985) and Aldushin and Matkowsky (1998) pointed out that the transient gas terms can be neglected; then, the bulk gas as well as the gas within the condensed phase can be treated as quasi-steady.

Numerical simulation results showed that use of these assumptions dramatically decrease the computation time, without significant loss in accuracy in the pressure, temperature and concentration profiles.

In the general case, the equations are nonlinear and were solved by a Newton-Raphson method. A high-performance linear equations solver is needed. We have tried a traditional SOR method, a preprocessed conjugated gradient (PCCG) method and LSOR methods, which are effective for heterogeneous systems (where properties are different in different directions). The results show that at the beginning stage, the PJCG method converges faster. Then, the LSOR or the SOR methods have better performance. All of these three methods were used as appropriate. We must add that once a step diverges or the solid fuel depletes, the time step should be cut and integration must be repeated. This approach is similar to the PICO technique [22]. Instead of setting a constraint on the number of iteration imes, however, we reduce the time step if convergence cannot be reached after 5 iterations in order to maintain accuracy. Although modified several times, and its performance sped up by more than 20 times, the computational scheme is still time consuming. For 2-D cases, its accuracy and speed are acceptable. More effort 
on the optimization of the code is still needed, however, before it can be employed in 3-D simulations.

\section{RESULTS AND DISCUSSION}

In this section we present results from the numerical simulations. First, we test the validity of the model in some simple cases.

\section{1-D Simulations}

To test the validity of the pore-network model we first conducted $1 \mathrm{D}$ simulations of forward combustion and compared the results with the analysis of Aldushin et al. (1999). These author showed that the dimensionless parameter $\delta=\frac{c_{g} \mu \rho_{f 0}}{C_{s} a_{0}}$ determines the condition for the existence of three different structures, a reaction-leading pattern $(\delta>1)$, where the reaction front leads the thermal front, a reaction-trailing pattern $(\delta<1)$, where the reaction front trails the thermal front, and a wave with maximum energy accumulation $(\delta=1)$, in which the two fronts coincide and the temperature at the front increases with time, according to the equation $T_{b}-T_{0}=Q_{A} a_{0} \sqrt{\frac{G_{0} l}{\pi \lambda c_{g}}}$. Our numerical results plotted in the left panel of Figure 4 are in good agreement with the theoretical predictions. In the first two cases, it is apparent that a traveling wave solution is rapidly reached, which propagates at a constant velocity. This velocity and the extent of the separation between fronts depend on a number of parameters, such as injection rate, heat conductivity, capacity, etc. Our model allows also to investigate the sensitivity of the results to heat losses. The right panel of Figure 4 shows the temperature profiles corresponding to the three cases, but with heat losses included. Although parameter $\delta$ appears to still remain the determining factor in the classification of patterns, the heat loss affects both the propagation velocity and the temperature patterns.

\section{2-D Simulations: 1. Base case}

We subsequently report on $2 \mathrm{D}$ results. The porous medium taken has relatively large particles (the distance between pore sites is of the order of $0.5 \mathrm{~mm}$ ), the fuel is uniformly partitioned in the pore sites, heat losses occur from the pore network to the surroundings at a rate which is $5 \%$ of the heat transfer rate between the gas and the solid, while the Peclet number for mass transfer is 0.05 , for the relatively low injection rate used in the base case. The main local heterogeneity in the base state was on the pore throats, the size of which was randomly 
distributed. In addition, for the particular stoichiometry used, no net production or loss of moles in the gas phase can occur, namely the volumetric flux of the gas phase remains constant. The base case does not include a change of the pore throat size due to the fuel depletion. These have implications on the front stability, as will be discussed below.

For the values of the base state $(\delta<1)$, the pattern is reaction-leading. Typically, the basic structure consists of a leading reaction zone, where most of the combustion reaction occurs, the oxidant and the fuel are depleted fast, and the temperature rises sharply. This combustion front rapidly reaches a steady-state and propagates at constant velocity. Figure 5 shows typical simulation results for the fuel conversion depth, the temperature, the pressure (with respect to the pressure at the outlet end, and multiplied by a factor for illustration purposes) and the oxidant concentration. The extent of the reaction zone is estimated to be of the order of $1.5 \mathrm{~mm}$, which in this case is equivalent to 3 pore lengths. This is consistent with estimates obtained from continuum calculations (Akkutlu and Yortsos, 2000). The profiles have some roughness, departing from strictly piston-like displacement, and temperature and concentrations (particularly the former) exhibit fluctuations. Averaging these fluctuations for use in continuum models would be an important consideration, as noted in the Introduction. However, there is no indication of fingering or instability in this base case. The pressure profiles indicate that the pressure varies significantly near the combustion region, as a result of its high temperature. In addition, there is almost complete consumption of the injected oxidant at the front, thus there is no oxidant leakage across the front.

\section{2-D Simulations: 2. Sensitivity Study}

Using the base-case parameters, we next proceeded to a sensitivity study in order to assess the following effects: The possibility of extinction, instead of sustained propagation, the development of instabilities at the pore-network scale, the effect of spatially correlated porespace and the effect of the distribution of the fuel in the pore network.

\section{a. Extinction}

The sustained front propagation was influenced mainly by the following factors: ignition, the oxidant concentration, the amount of heat losses and the fuel density. In all simulations, ignition was found necessary for the onset of the combustion reaction. For this, the temperature of the inlet row was raised to a sufficiently high value, while that of the adjacent row was also 
slightly raised (thus mimicking an initially sharp exponential profile) (see also Figure 3). Ignition was also found necessary for reverse combustion. In its absence or when the ignition temperature was low, the front quickly became extinct.

Extinction was also promoted by increased heat losses and decreased concentrations of the injected oxidant. For otherwise constant values of the base-case parameters, we found that extinction occurred if heat losses were increased to about $50 \%$ or higher of the rate of heat transfer between the porespace and the solid sites. For a more accurate determination of the threshold additional simulations are needed, however the onset of extinction as the rate of heat losses increases is consistent with continuum model predictions (Akkutlu and Yortsos, 2001). These also suggest that for otherwise constant values, extinction can be circumvented by an increase in the injection rate. The effect of the injection rate is currently being studied. In addition, it was found that sustained front propagation is not possible if the mole fraction of the oxidant at the inlet is reduced to $28 \%$. Again, this value is only indicative of a lower bound rather than a true threshold. It does indicate, however, that injection of a sufficiently rich mixture is required to sustain combustion. A detailed analysis is still in progress.

Before proceeding we show simulation results for the dependence of the front velocity on the injection rate for the base-case but in the absence of heat losses. As shown in Figure 6, the front velocity varies linearly with the injection rate and it is about two orders of magnitude smaller. The dependence in this region can be explained by simple mass balance arguments, given that both oxidant and fuel are completely consumed at the front. We note that in these simulations there is front propagation, even at very small injection rates, reflecting the fact that in this region combustion is promoted by diffusion only. However, it is unlikely that such a front will propagate further in an extended porous medium. We must also note that if the injection rate increases beyond the range shown in Figure 6, the front slows down.

\section{b. Instabilities}

From continuum analyses it is known that forward combustion fronts become stabilized by heat conduction, diffusion, and the net reduction in the number of gas moles due to the combustion reaction. They become destabilized by the net increase in the number of gas moles (expansion) and by an increase in the permeability of the burned region. As mentioned, in this paper we only considered the case in which the next expansion is zero, an assumption which 
greatly facilitates computations. As a result, in the base case the fronts are stable (Figure 5), reflecting the stabilizing influence of conduction and diffusion. To investigate the onset of viscous fingering instability we considered cases in which the pore throat radii of the burned region increase by a constant multiplier. We considered two cases in which the multiplier was 2 and 4, respectively. Given the use of Poiseuille's law, this translates into an effective increase of the permeability of the burned region by $2^{4}=16$, and $4^{4}=256$, respectively. Figure 7 shows the corresponding results for the second case. The effect of this rather substantial permeability contrast (which is analogous to a displacement with a mobility ratio of 256) is evident. The conversion depth acquires features of a viscous fingering pattern, the extent of reaction is nonuniform and the fluctuations of temperature and concentrations are substantial, despite the small size of the pore network. A distinct feature is that the corrugation of the front now gives rise to incomplete conversion in certain regions and a corresponding leakage of oxidant. This phe nomenon was also observed when the pore structure was correlated (see below). Similar, but not as strong, effects were found in the case of the milder permeability contrast of 16.

The simulations in Figure 7 indicate that pore-network scale instabilities are possible provided that the permeability enhancement is sufficiently strong. While the level of such enhancement may be unrealistic, such instabilities will also develop when there is gas expansion at the front as a result of the combustion reaction. Our simulations cannot answer at present the question whether or not these instabilities at the pore-network scale will ultimately lead to process extinction. For their suppression requires a decrease of the injection rate, to promote the stabilizing effects of conduction and diffusion. A study of this effect is currently in progress. Such a decrease must not be too strong, however, since it may lead to extinction as a result of heat losses, as discussed above.

In passing, we must note that strong instabilities do develop in the case of reverse combustion. Now, diffusion is destabilizing, and at sufficiently small injection rates the developed pattern is unstable. For the sake of completeness we show in Figure 8 the results from a typical simulation of reverse combustion. Patterns reminiscent of viscous fingering, including shielding, trapping and tip splitting are obtained. These patterns are accentuated as the injection rate decreases. An elaborate account of this problem will be presented elsewhere.

\section{c. Correlated porespace}


Sensitivity studies were subsequently conducted to investigate the effect of the pore-size distribution and its spatial correlation. First, we examined variations in the pore-size distribution. Figure 9 shows results for the base case, but with the (uniform) pore-size distribution stretched so that the ratio of the end points increased from 3 (which is the base case) to 10 . The simulations show that front roughness, reaction zone thickness and temperature and concentration fluctuations all increase. It is interesting to note, however, that there is no leakage of oxidant across the front. The effects shown in Figure 9 reflect the increased contrast in flow rates, hence in the convected flux of the gas oxidant, among the various pores. Subsequently, the effect of spatial correlations was investigated, by taking a pore-size distribution obeying fBm statistics (Du et al., 1996). These statistics reflect long-range correlations and, for the specific problem considered here, correspond to a correlation length equal to the network size. Corresponding results are shown in Figure 10 for a Hurst exponent $\mathrm{H}=0.7$, which represents smooth fields with channellike features. One effect of the correlated porespace is to increase the roughness of the front, as well as the fluctuations in temperature and concentrations. This is expected, due to the features of the correlated noise. What is important, in the correlated case, however, is that the gas oxidant is not completely consumed at the front. Rather, many regions develop at the front where the oxidant leaks through, partly unreacted (Figure 10). The incomplete combustion is a rather striking effect of the spatial correlation and was observed also in other simulations. The fact that the underlying pore structure influences the pattern to be developed is shown in Figure 11, for the case of a "microfissure" in the porespace. In these simulations, one row in the pore-network has a uniform pore-size distribution the end points of which were four times larger than in the rest. The results of Figure 11 correspond to an injection rate 10 times larger than in the base case. With this rate, the process becomes convectioncontrolled and the patterns reflect clearly the underlying features of the pore-space. The process is similar to the displacement of one fluid by another in a fractured medium. Whether or not this pattern will persist for a long time, however, cannot be answered by the pore-scale simulations, and must be addressed by different means. We must note that by decreasing the injection rate, the "imprint" of the microstructure on the combustion pattern waned, as expected.

\section{d. Distribution of fuel}

The final effect studied in the sensitivity analyses was the distribution of fuel in the porespace. The issue that arises is the following. Assume that the fuel is randomly distributed in 
the sites of the pore-network, namely that there is a probability $p$ that a site contains fuel. The question is whether there exists a critical value (a percolation threshold) $p_{c}$, above which sustained propagation is possible, but below which extinction occurs. It is clear that this value will depend on a number of parameters, an important one being the thermal conductivity of the solid. The role of the latter is not trivial: If conductivity is small, then the sustained propagation of combustion requires that fuel sites, which here act as a heat source, are sufficiently close, hence that $p_{c}$ is large. In that region, we expect that as the thermal conductivity increases, then $p_{c}$ will decrease. However, as the thermal conductivity is sufficiently large it is possible that the heat generated spreads out more than needed resulting in the extinction of the process, unless $p_{c}$ increases. Results from simulations are shown in the graph of Figure 12. Consistent with above, the curve of $p_{c}$ vs. thermal conductivity is non-monotonic. At low values of the thermal conductivity the percolation threshold is a decreasing function, then reaches a relatively flat plateau, and subsequently increases with further increase in thermal conductivity. It is interesting to note that in this process values larger than the typical percolation threshold of 0.59 for site percolation in a square lattice can be obtained. The results of such simulations suggest that the volume-averaged fraction of fuel per unit volume must exceed a critical value for the sustained propagation of a combustion front. This critical value reflects not only intrinsic thermodynamic and kinetic properties, but also the spatial arrangement of the fuel in the porespace.

\section{CONCLUDING REMARKS}

In this paper we developed a pore-network model to simulate the High Tempe rature Oxidation region of in-situ combustion in porous media. The pore-network model is based on dual pore networks (pores and solid sites) and has the objective to incorporate the effect of microstructure on combustion processes in porous media. The model accounts for flow and transport of the gas phase in the porespace, where convection predominates, and for heat transfer by conduction in the solid phase. Gas phase flow in the pores and throats is assumed to be

governed by viscous forces. Heterogeneous combustion with one-step finite kinetics was assumed. The validity of the model was tested against existing 1-D solutions. A variety of 2-D simulations were conducted to investigate the effects of parameters. In particular, we focused on the patterns of the extent of conversion, temperature, pressure and oxidant concentration.

In the absence of gas phase expansion at the front, the combustion fronts are found to be 
generally stable and of a finite degree of roughness. The latter increases with a more broad poresize distribution and with spatially correlated pores. In the base case, both fuel and oxidant are fully consumed within a thin reaction zone, the thickness of which does not exceed a few pore lengths. When spatial correlations occur, however, the oxidant leaks through the front, along the correlated pathways. The development of sustained front propagation was studied as a function of various parameters, which included heat losses, instabilities, and the distribution of fuel. Particular attention was paid to the latter, where it was found that the geometric distribution of the fuel in the pore sites is also a parameter that affects the extinction boundaries. The problem can be posed as an extended percolation like process from which percolation-like thresholds can be computed.

The implications of the study to the validity and the application of continuum models are many-fold. Even in the absence of 2-D effects, the pore-network study shows that the HTO zone is very thin and of the order of a few pore lengths, a fact that makes the application of continuum models questionable, given that the latter presume the absence of gradients over the volume of averaging (which is of the order of hundreds of pores). When 2D and 3-D effects are included, fronts additionally show roughness, instabilities or non-uniform properties. Existing continuum models, based on a direct extrapolation of intrinsic kinetics to the continuum scale, do not account for many of these effects. Salvaging the continuum description can be datained by incorporating the findings at the pore-network scale. One such method would be to derive effective kinetic parameters, such as rate constants and activation energies, by matching the steady-state predictions from continuum and pore-network modek. Such a study is currently underway. The results for the effect of the spatial fuel distribution on the sustained propagation of combustion fronts should also be incorporated in continuum models, as the latter are intrinsically incapable for such an account.

\section{REFERENCES}

1. Prats, M., Thermal Recovery, SPE Monograph Series SPE of AIME (1982).

2. Boberg, T.C., Thermal Methods of Oil Recovery, An Exxon Monograph Series (1988).

3. Aldushin, A.P., New results in the theory of filtration combustion, COMBUSTION AND FLAME 94: 308-320 (1993).

4. Lenormand, R., Touboule, C. and Zarcone, C., Numerical Models and Experiments on 
Immiscible Displacements in Porous Media, J. FLUID MECH. 189: 165 (1988).

5. Blunt, M., and King, P., Relative Permeabilities from Two-and Three-Dimensional Pore-Scale Network Modeling, Transp. Porous Media 6, 407-433 (1991).

6. Li, X., and Yortsos, Y.C., Visualization and Simulation of Bubble Growth in Pore Networks, AIChEJ 41, 214 (1995).

7. Satik, C., and Yortsos, Y.C., A Pore-Network Study of Bubble Growth in Porous Media Driven by Heat Transfer, J. HEAT TRANSFER 118, 455-462 (1996).

8. Andrade, J.S., Street, D.A., Stanley, H.E., Diffusion and Reaction in Percolating Pore Networks, PHYS. REV. E 55, 772-777 (1997).

9. Rieckmann, C., and Keil, F.J., Multicomponent diffusion and reaction in three-dimensional networks: general kinetics, IND. ENGNG. CHEM. RES. 36, 3275 (1997).

10. El-Nafaty, U.A., and Mann, R., Support-pore architecture optimization in FCC catalyst particles using designed pore networks CHEM. ENG. SCI. 54, 3475-3484 (1999).

11. Jia, C., Shing, K. and Yortsos, Y.C., Visualization and Simulation of Non-Aqueous Phase Liquids Solubilization in Pore Networks, J. CONTAM. HYDR., 35, 363 (1999).

12. Hwang, S., Mukasyan, A.S., Rogachev, A.V., and Varma, A., Combustion wave microstructure in gas solid reaction systems: experiments and theory, COMBUST. SCI. AND TECH. 123, 165 (1997).

13. Hwang, S., Mukasyan, A.S., and Varma, A., Mechanisms of combustion wave propagation in heterogeneous reaction systems, COMBUST. AND FLAME 115, 354363 (1998).

14. Ohlemiller, T.J., Modeling of smoldering combustion propagation, PROG. ENERGY COMBST. SCI. 11, 277-310 (1985).

15. Henneke, M.R., and Ellzey, J.L., Modeling of filtration combustion in a packed bed, COMBUSTION AND FLAME 117, 832 (1999).

16. Goldfarb, I., Gol'dshtein, V., Kuzmenko, G., and Sazhin, S., Thermal radiation effect on thermal explosion in gas containing fuel droplets, COMBUSTION THEORY AND MODELLING 3, 769-787 (1999).

17. Summerfield, M., Ohlemiller, T.J., Sandusky, H.W., A thermophysical mathematical model of steady-draw smoking and predictions of overall cigatte behavior, COMBUSTION AND FLAME 33, 263-279 (1978).

18. Kisler, J.P., and Shallcross, D.C., An improved model for the oxidation processes of light crude oil, Trans IchemE 75A, 392-400 (1997). 
19. Leach, S.V., Rein, S., Ellzey, J.L., Ezekoye, O.A., and Torero, J.L., Kinetics and fuel property effects on forward smoldering, COMBUSTION AND FLAME 120, 346-358 (2000).

20. Bayliss, A., and Matkowsky, B.J., Fronts, relaxation oscillations, and period doubling in solid fuel combustion, J. COMPUTATIONAL PHSICS 71, 147-168 (1987).

21. Aldushin, A.P., and Matkowsky, B.J., Instabilities, fingering and the Saffman-Taylor problem in filtration combustion, COMBUST. SCI. TECHN. 133, 293-341 (1998).

22. Issa, R.I., Gosman, A.D., and Ahmadi-Befrui, B., Solution of the implicitly discretised reacting flow equations by operator splitting, J.COMPUT. PHYS. 93, 388-410 (1991).

23. Aldushin, A.P., Rumanov, I.E., and Matkowsky, B.J., Maximal energy accumulation in a superadiabatic filtration comnustion wave, COMBUSTION AND FLAME 118, 17-90 (1999).

24. Akkutlu, I.Y., and Yortsos, Y.C., The Dynamics of Combustion Fronts in Porous Media, paper SPE 63225, presented at the 2000 SPE Fall Meeting, Dallas, Tx (Oct. 2000).

25. Akkutlu, I.Y., and Yortsos, Y.C., in preparation (2001).

26. Du, C., Satik, G., and Yortsos, Y.C., Percolation in a Fractional Brownian Motion Lattice, AICHEJ 42, 2392-2395 (1996). 
Table 1. Variables Assigned to Sites and Bonds

\begin{tabular}{|c|c|c|}
\hline $\begin{array}{c}\text { Pore Site } \\
\text { Properties }\end{array}$ & $\begin{array}{l}\text { Solid Site } \\
\text { Properties }\end{array}$ & $\begin{array}{c}\text { Bond } \\
\text { Properti } \\
\text { es }\end{array}$ \\
\hline Void volume & Volume & Length* \\
\hline $\begin{array}{l}\text { Interface area for } \\
\text { reaction }\end{array}$ & $\begin{array}{l}\text { Heat transfer } \\
\text { area between } \\
\text { solid sites }\end{array}$ & Radius \\
\hline $\begin{array}{l}\text { Heat capacity of } \\
\text { gas and fuel }\end{array}$ & $\begin{array}{l}\text { Heat capacity } \\
\text { of } \\
\text { material }\end{array}$ & Pressure \\
\hline $\begin{array}{l}\text { Heat transfer } \\
\text { area with solid } \\
\text { site }\end{array}$ & $\begin{array}{l}\text { Heat transfer } \\
\text { area with pore } \\
\text { site }\end{array}$ & $\begin{array}{l}\text { Tempera } \\
\text { ture }\end{array}$ \\
\hline Pressure & Temperature & $\begin{array}{l}\text { Gas } \\
\text { phase } \\
\text { velocity }\end{array}$ \\
\hline Temperature & & \\
\hline $\begin{array}{l}\text { Fuel amount } \\
\text { Initial fuel } \\
\text { amount }\end{array}$ & & \\
\hline $\begin{array}{l}\text { Component } \\
\text { concentration }\end{array}$ & & \\
\hline
\end{tabular}

* Except for this term, all other variables can be spatially distributed.

Table 2. Characteristic Parameters

\begin{tabular}{|c|c|c|}
\hline Variable & Characteristic & Approxi \\
& Quantity & mate \\
& & Value \\
\hline Temperature $\left(\mathrm{T}^{*}\right)$ & $\Delta_{\mathrm{r}} \mathrm{H} / \mathrm{C}_{\mathrm{p}, \mathrm{f}}$ & $5000 \sim 16000 \mathrm{~K}$ \\
\hline Density & $\rho_{\mathrm{f}}$ & $0.5 \sim 1.5^{*} 10^{3} \mathrm{Kg} / \mathrm{m} 3$ \\
\hline
\end{tabular}




\begin{tabular}{|c|c|c|}
\hline Pressure & $\mathrm{P}_{0}$ & $1.01325^{*} 10^{5} \mathrm{~Pa}$ \\
\hline Length & L(Bond Site Length) & $5^{*} 10^{-4} \mathrm{~m}$ \\
\hline Time & $\mathrm{L}^{2} / \mathrm{D}_{\mathrm{e}}$ & $5^{*} 10^{-3} \mathrm{~s}$ \\
\hline $\mathrm{K}_{0}=\mathrm{K}_{\mathrm{r}} \exp \left(-\mathrm{Ea} / \mathrm{RT}^{*}\right)$ & & $10 \sim 1.6^{*} 10^{5}$ \\
\hline Activation energy & $\mathrm{E}_{\mathrm{a}}$ & $1,2 \sim 1.6^{*} 10^{6} \mathrm{~J} / \mathrm{mol}$ \\
\hline Diffusion coefficient & $\mathrm{D}_{\mathrm{e}}$ & $5^{*} 10^{-5} \mathrm{~m}^{2} / \mathrm{s}$ \\
\hline
\end{tabular}

Table 3. Dimensionless Groups

\begin{tabular}{|c|l|c|}
\hline Dimensionless group & Definition & Approximate Value \\
\hline Th (Thiele modules) & $\left(\mathrm{K}_{0} \mathrm{LD}_{\mathrm{e}}^{-1} \mathrm{M}_{\mathrm{k}}{ }^{*}\right)^{1 / 2}$ & $1 * 10^{4} \sim 1 * 10^{9}$ \\
\hline $\mathrm{Pe}$ & $\mathrm{u}_{0} 1 \mathrm{De}^{-1}$ & $0.001 \sim .0 .1$ \\
\hline $\mathrm{Le}$ & $\mathrm{K}_{\mathrm{f}} \rho_{\mathrm{f}}^{-1} \mathrm{Cp}_{\mathrm{f}}^{-1} \mathrm{De}^{-1}$ & $0 \sim 10$ \\
$\mathrm{Nu}$ & $\mathrm{h}_{\mathrm{s}} 1 \mathrm{k}^{-1}$ & $0 \sim 1$ \\
\hline $\mathrm{Nu}, \mathrm{L}$ & $\mathrm{h}_{\mathrm{L}, \mathrm{S}} 1 \mathrm{k}_{\mathrm{s}}^{-1}$ & $0 \sim 1$ \\
\hline $\mathrm{Nu} \mathrm{P}_{\mathrm{L}}$ & $\mathrm{h}_{\mathrm{L}, \mathrm{p}} 1 \mathrm{k}_{\mathrm{s}}^{-1}$ & $0.01 \sim 1$ \\
\hline $\mathrm{RLSQ}$ & $\mathrm{r}^{2} \mathrm{l}^{-2}$ & .001 \\
\hline $\mathrm{KKGS}$ & $\mathrm{K}_{\mathrm{g}, \mathrm{f}} \mathrm{k}^{-1}$ & \\
\hline
\end{tabular}




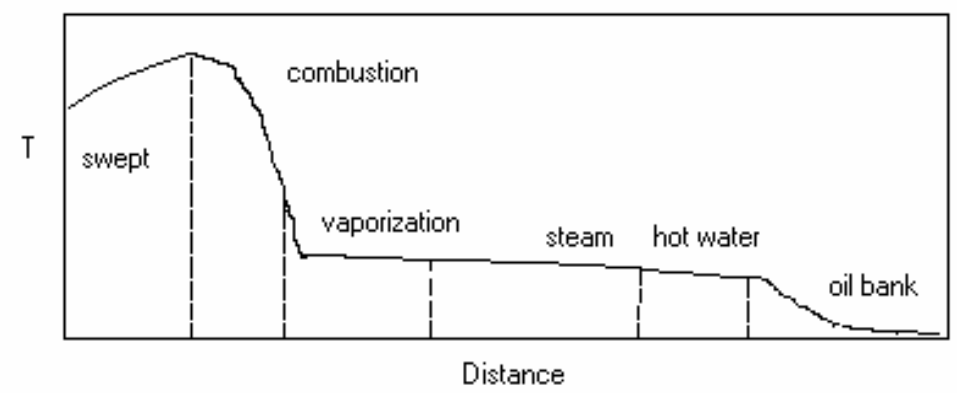

Figure 1. Schematic of Regimes in In-Situ Combustion.
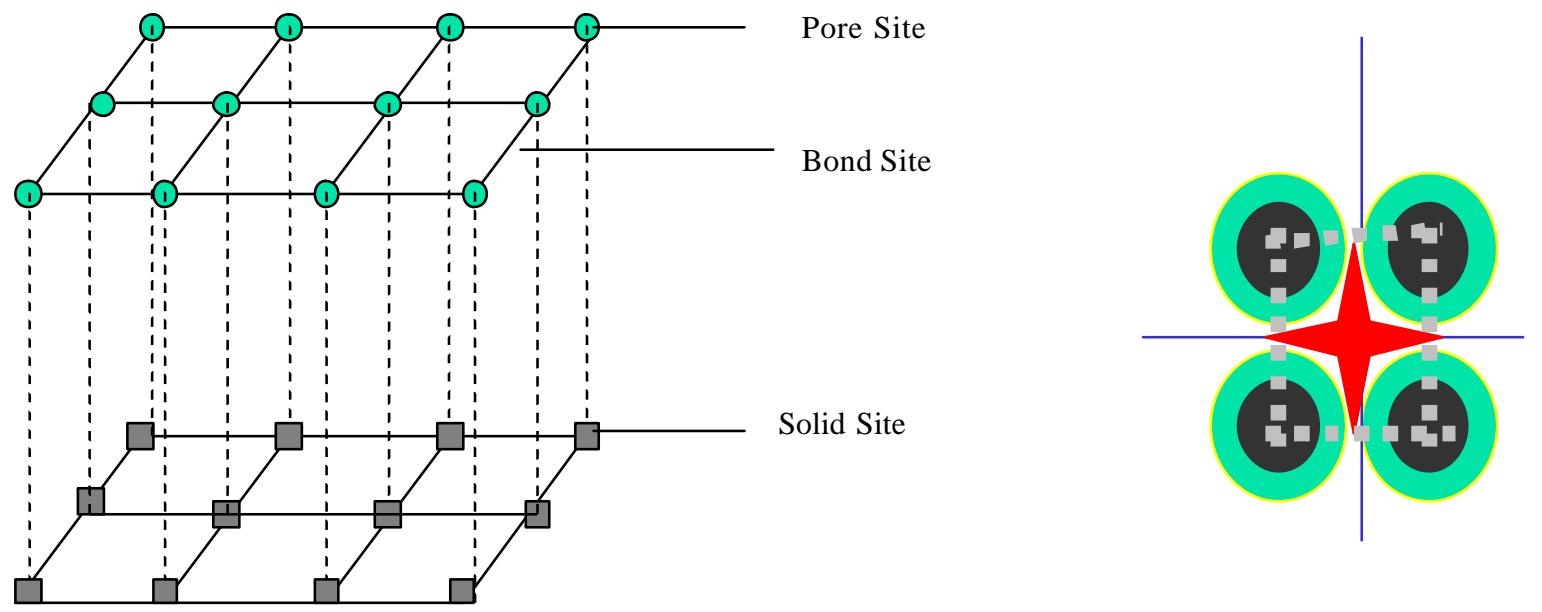

A. The pore-network model

B. Structure of a single pore site containing fuel

Figure 2. Structure of the pore network. 
Cold Injection

Oxidant

Inert Gas

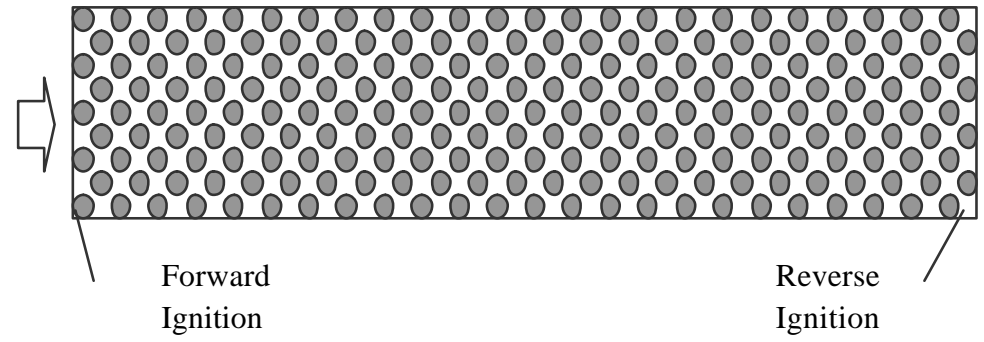

Figure 3. Sketch of the forward and reverse combustion ignition method.

A
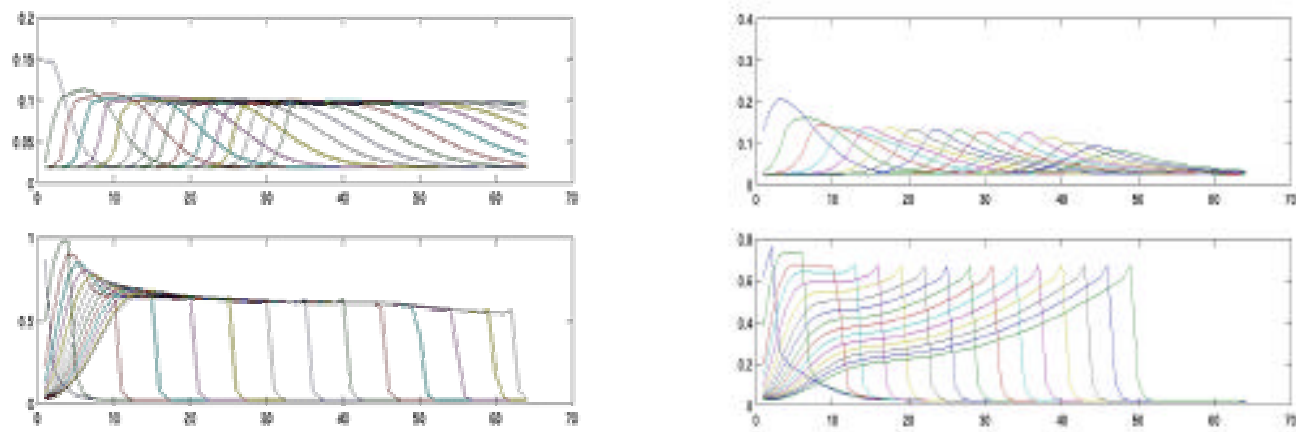

$\mathrm{C}$
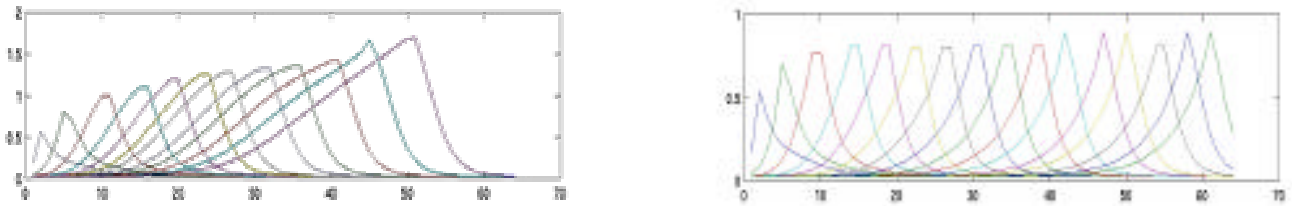

A: Reaction trailing $(\delta>1)$, B: Reaction leading $(\delta<1)$, C: Maximum Accumulation $(\delta=1)$

Figure 4. 1-D simulation results for three different values of $\delta$. The simulations on the right include heat losses. 

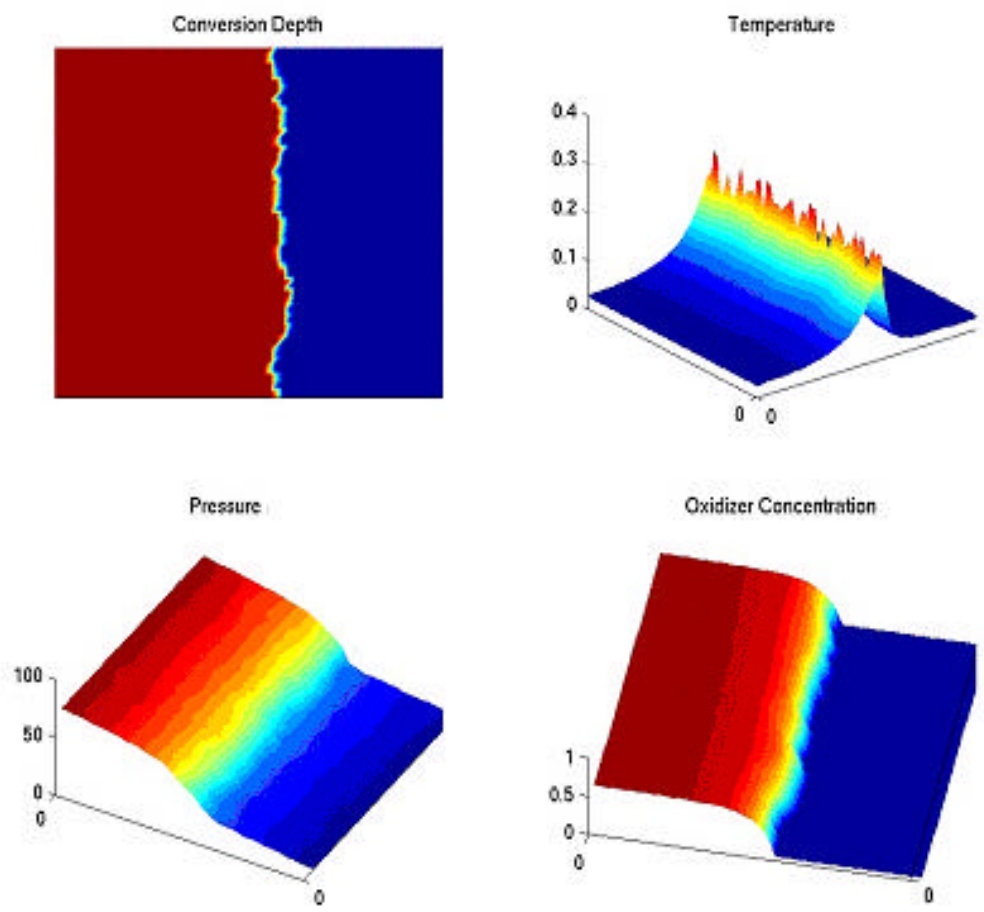

Figure 5. Patterns of forward combustion for the base case.

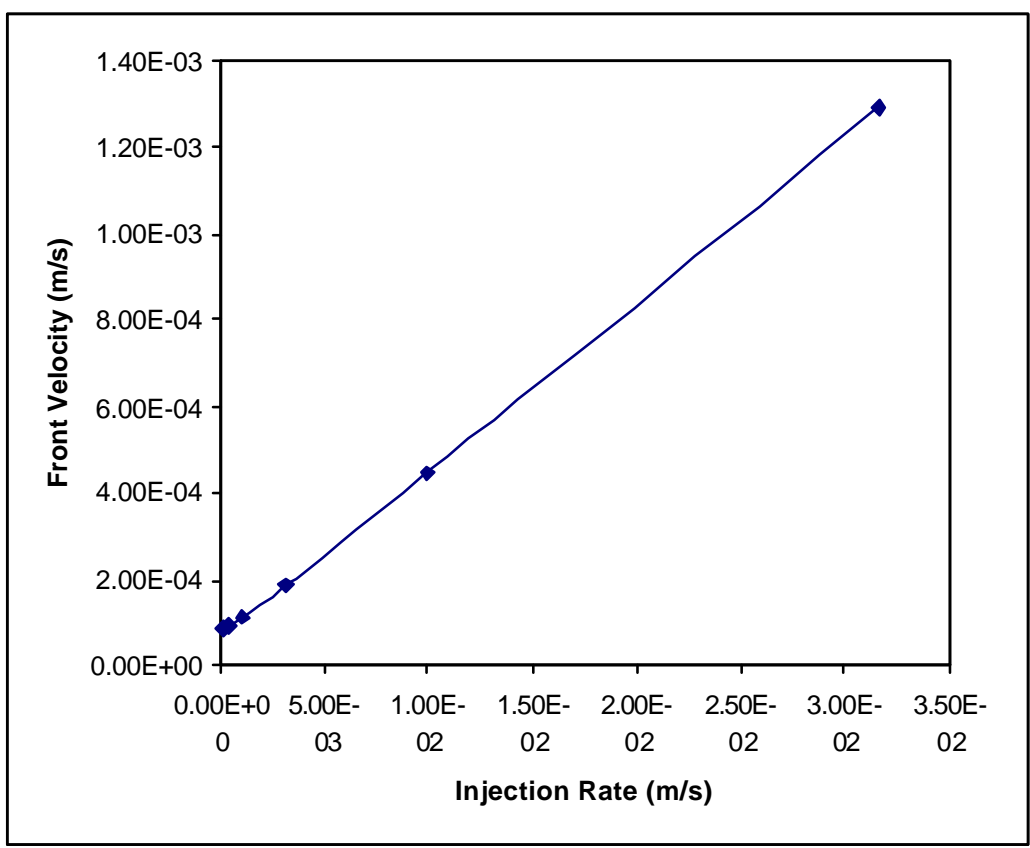

Figure 6. The steady-state front velocity as a function of the injection rate for the base-state. 

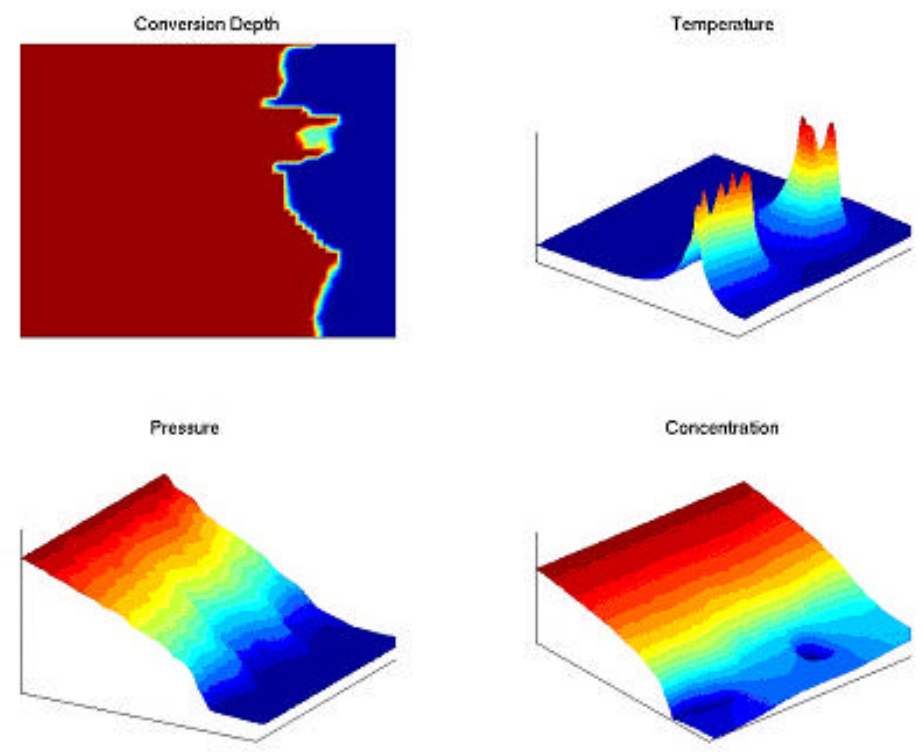

Figure 7. Patterns of forward combustion for the case of enhanced permeability.
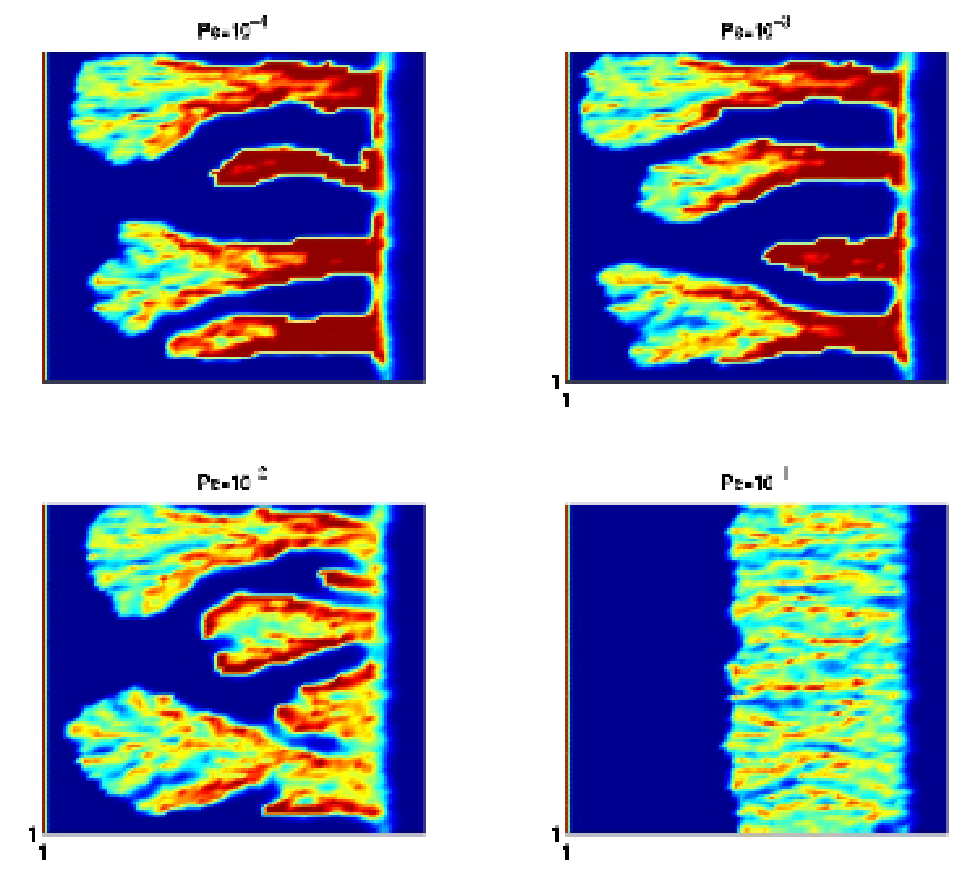

Figure 8. Conversion depth patterns for different values of the Peclet number in reverse combustion: A) $\mathrm{Pe}=10^{-4}$; B) $\mathrm{Pe}=10^{-3}$; C) $\mathrm{Pe}=10^{-2}$; D) $\mathrm{Pe}=10^{-1}$. 

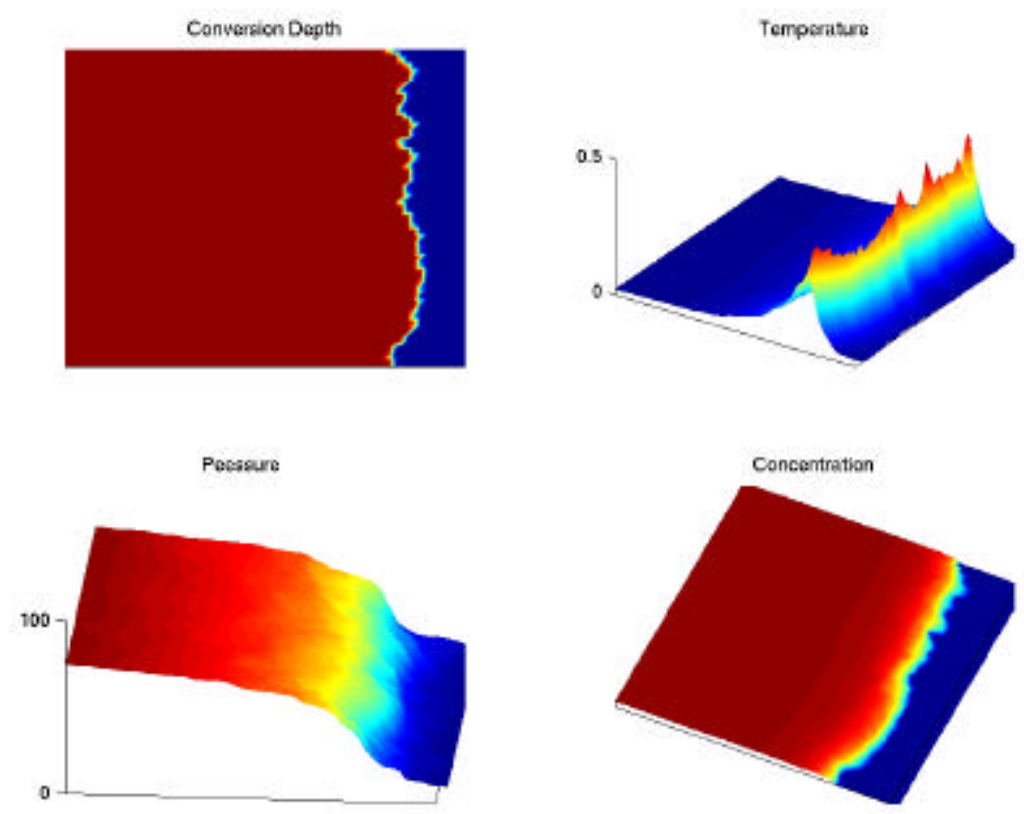

Figure 9. Patterns of forward combustion for the case of a broad pore-size distribution.
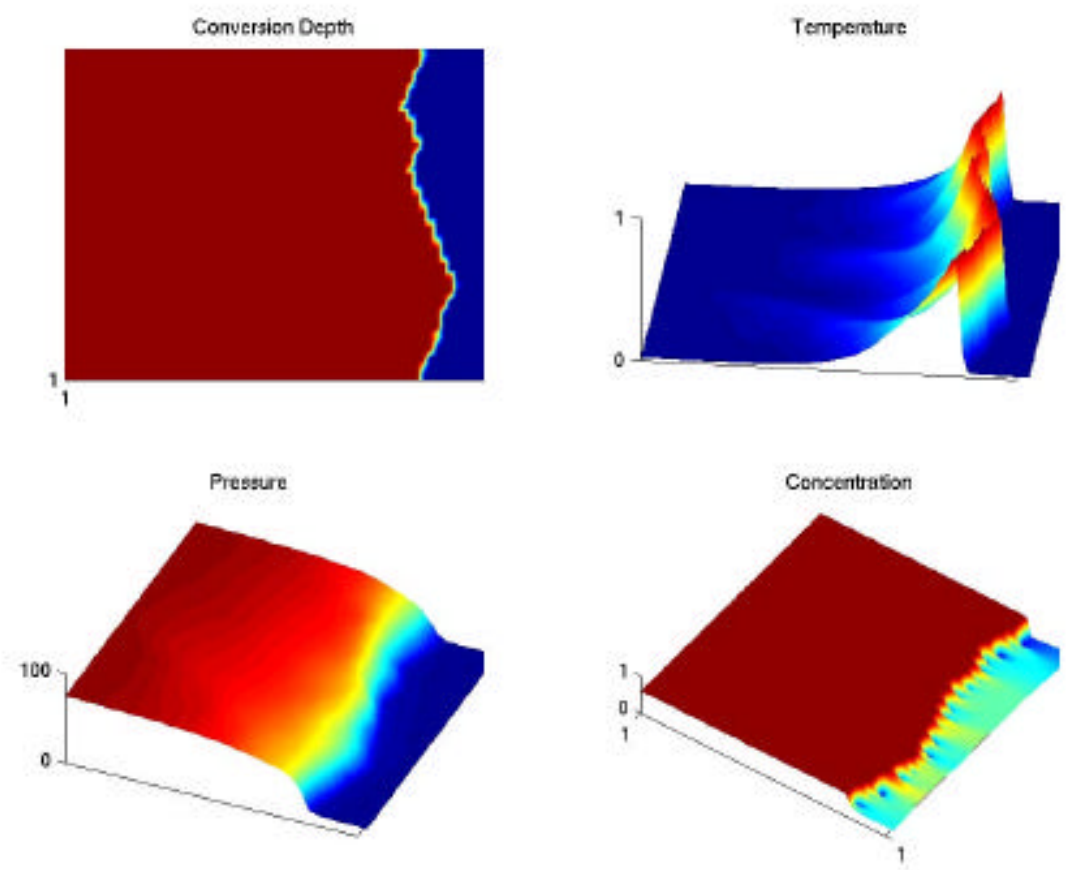

Figure 10. Patterns of forward combustion for the case of $\mathrm{fBm}$ pore-size distribution $(\mathrm{H}=0.7)$. 

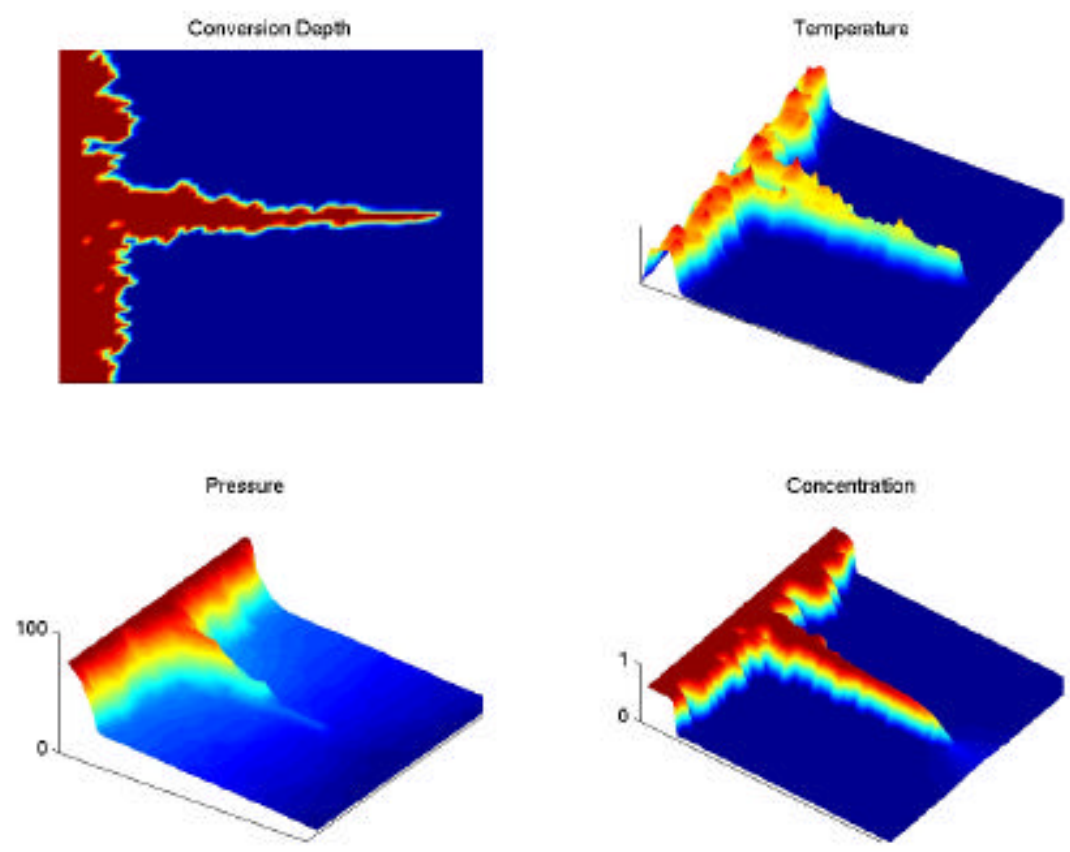

Figure 11. Patterns of forward combustion for the case of a "microfissure" in the middle of the lattice.

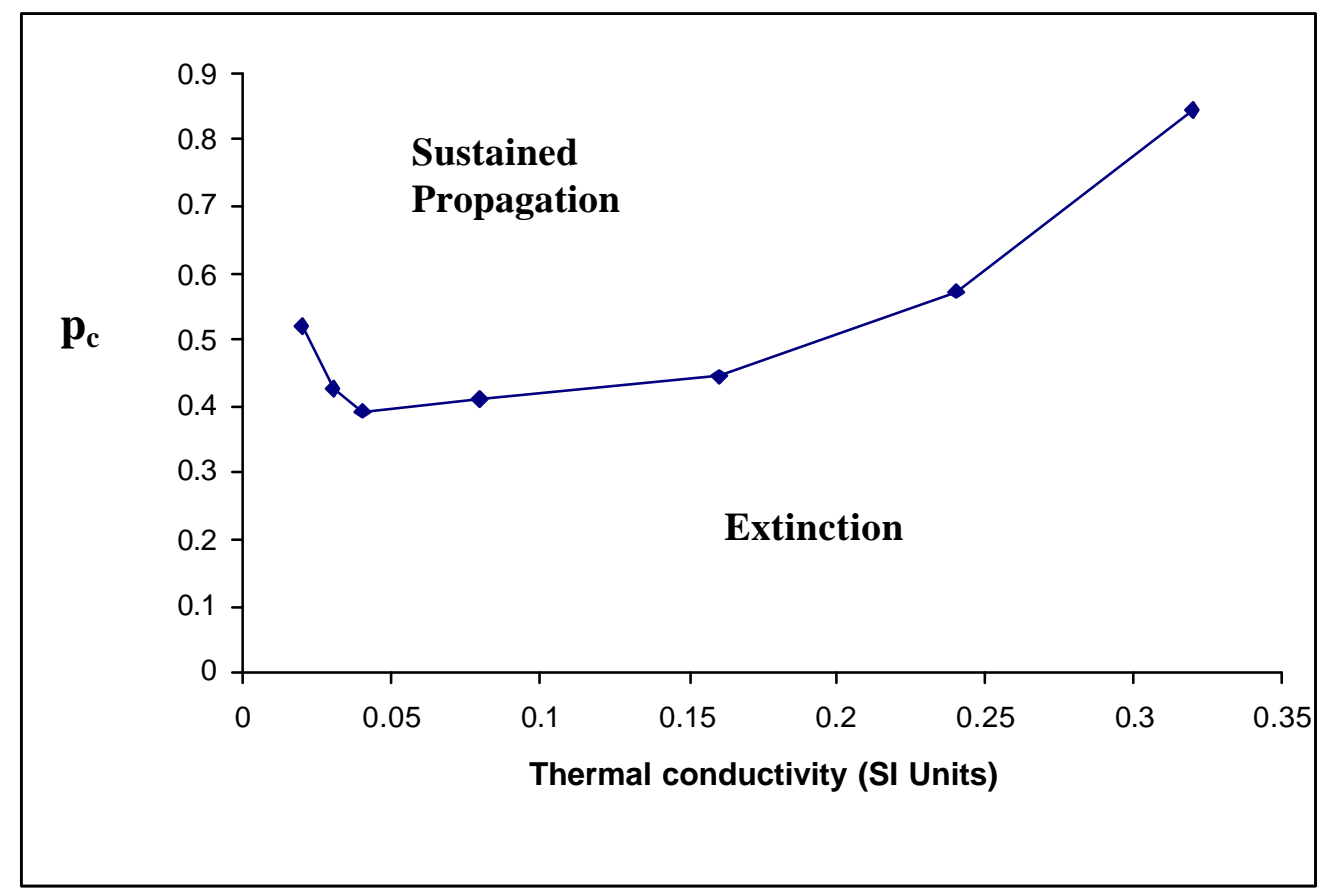

Figure 12. The percolation threshold for the onset of sustained front propagation as a function of the thermal conductivity. 



\title{
The Effect of Heterogeneity on In-situ Combustion: The Propagation of Combustion Fronts in Layered Porous Media
}

\author{
I. Yücel Akkutlu and Yannis C. Yortsos
}

\section{Introduction}

The sustained propagation of a combustion front is necessary for the recovery of oil using in situ combustion. Compared to other methods, in situ combustion involves the complexity of exothermic reactions and temperature-dependent reaction kinetics. The combustion dynamics are influenced by the fluid flow of injected and produced gases, the heat transfer in the porous medium and the surroundings, the rate of combustion reaction(s) and the heterogeneity of the porous medium. In the presence of heat losses, the possibility exists of extinction (quenching) as well as the necessity of ignition for sustained propagation.

Combustion fronts in porous media have been studied extensively in the context of filtration combustion. Analytical treatments of the combustion front dynamics is possible, by using methods similar to the analysis of laminar flames (gaseous phase combustion in the absence of porous medium). Using the property that the activation energy of the overall reaction is large in comparison with the thermal enthalpy [1], Britten and Krantz [2, 3] provided an asymptotic analysis in one-dimensional systems of reverse combustion in coal gasification. In detailed works, Schult et al. [4, 5] investigated the adiabatic combustion of a homogeneous porous medium, in the contexts of fire safety and the synthesis of compacted metal powders (SHS processes). More recently, forward and reverse filtration combustion in a non-reacting porous medium was studied using a pore-network model by Chuan and Yortsos [6]. In parallel, a detailed analysis of the propagation of planar combustion fronts in porous media was undertaken by the present authors [7]. They addressed the issue of steady-state propagation under both adiabatic and non-adiabatic conditions, but emphasized the effect of heat losses to the surroundings. The latter were modeled both by conduction (for subsurface applications) and by convection (for laboratory applications). A number of important results were obtained, which are briefly summarized in the next section. 
In this paper, we consider the use of the same type of approach in an attempt to answer the important question of the effect of the porous medium heterogeneity on the sustained propagation of combustion fronts. As in other contexts, a simple representation of heterogeneity is through the use of layers. For example, layered systems have been employed to investigate heterogeneity effects on processes such as miscible and immiscible displacement [8]. In the latter processes, the effect of heterogeneity typically enters through fluid mobility (where the displacement in a more permeable layer is further accelerated in the case of unfavorable mobility ratio, and conversely retarded in the case of a favorable mobility ratio). In the combustion case of interest here, however, the coupling enters through the heat transfer between the layers, to be expressed by a simple convective-type model. The assumption is rigorously valid if the layers are sealed from one another, or if the fluid mobility remains constant through the process, which is a good assumption, when the net rate of gas generation is small. Then, the injection rate in each layer is constant in time, and proportional to the layer permeability. The analysis will be conducted for two simple geometries, a two-layer system and a symmetric three-layer system, under both adiabatic and non-adiabatic conditions. Our emphasis is on understanding how the heat transfer coupling affects the front propagation in the different layers, on whether or not a state of coherent traveling fronts develops and on whether or not a sufficiently sharp permeability contrast can lead to the extinction of the process. Throughout the paper, we will use methods similar to the single-layer problem of [7]. Because of the relevance of those results to the present problem, they are briefly summarized below.

\section{Preliminaries: Combustion in a Single Layer}

Under adiabatic conditions, it is found that there is always sustained propagation, where the front temperature is given by

$\theta_{f} \equiv T_{f} / T_{o} \approx 1+q$, where $q=\frac{Q \rho_{f}^{o}}{(1-\phi) c_{s} \rho_{s} T_{o}}$

we denoted the heat of reaction by $Q$, the initially available fuel content per total volume by $\rho_{f}^{o}$ and the volumetric heat capacity of the porous medium by $(1-\phi) c_{s} \rho_{s}$. Clearly, equation (1) shows that the front temperature is practically independent of the front velocity. 
Under non-adiabatic conditions, however, front temperature and front velocity are coupled. When the heat losses are modeled by heat conduction in semi-infinite surroundings, the temperature of the front is obtained from the different equation

$\theta_{f}=1+\frac{q}{\omega^{2 / 3} z_{1}-\omega^{4 / 3} \sqrt{z_{1}}} \quad$ where $\quad \omega=\frac{2 \alpha_{s}}{H v_{i}}$

and $z_{1}$ is the positive root of the algebraic equation

$\frac{\bar{\mu}^{2}}{z_{1}}=\left(1+\bar{\mu} z_{1}\right)^{2}, \quad$ with $\quad \bar{\mu}=-\left(\frac{\omega}{V_{D}}\right)^{2 / 3}$.

In (2), $H$ is the thickness of the porous medium, $v_{i}$ the injection velocity, $V_{D}=V / v_{i}$ is the dimensionless front velocity normalized with the injection velocity, and $\alpha_{s}=\lambda /(1-\phi) c_{s}$ is the effective thermal diffusion coefficient. A similar equation applies for the convective heat losses case.

In all cases, the front velocity is related to the front temperature through the following equation

$V_{D}^{2}=\mathcal{A} \theta_{f} \exp \left(-\frac{\gamma}{\theta_{f}}\right)\left(\frac{1-\mu V_{D}}{1+\mu_{g} V_{D}}\right)$

where $\gamma=E / R T_{0}$ is the Arrhenius number, $E$ is the activation energy, $R$ the universal gas constant, $\mu$ and $\mu_{g}=\mu_{g p}-\mu$ are dimensionless stoichiometric coefficients for oxygen and produced gas due to reaction, respectively (see [7] for more details), and

$\mathcal{A}=\frac{a_{s} \alpha_{s} k_{o} Y_{i} p_{i}}{q E I_{\eta} v_{i}^{2}} \quad$ where $\quad I_{\eta}=\int_{0}^{1} \frac{(1-\eta)}{\psi(\eta)} d \eta$

In addition, in Equation (5), $a_{s}$ is the specific surface area per unit volume, $k_{0}$ the preexponential factor, $p_{i}$ the initial gas pressure, $\eta=1-\rho_{f} / \rho_{f}^{o}$ the extent of fuel conversion depth and $\psi(\eta)$ is a dimensionless function representing the dependence of reaction on $\eta$.

In the adiabatic case, there is always a solution for the front velocity as a function of the injection velocity. This relation is plotted in Figure 1 for typical parameter values. It shows that the front velocity is proportional to the injection velocity at sufficiently small injection rates, and increases more slowly as the injection velocity becomes larger. In thermally decoupled layers, under adiabatic conditions, we should expect, therefore, that combustion fronts 
in high permeability layers would travel faster, according to the dynamics portrayed in Figure 1, for example.

On the other hand, in the non-adiabatic case, the coupling between velocity and temperature has significant implications. Figures 2 and 3 show results obtained for the front temperature versus the injection velocity for a varying thickness of the porous medium. The corresponding variation of the front velocity with the injection velocity is shown in Figure 4. For fixed thickness and injection velocity, the system typically shows multiplicity in the solutions, and for sufficiently thin layers, extinction and ignition points, $E_{c}$ and $I_{c}$, exist in temperature (Figures 3, 2). As $H$ decreases, the extinction threshold rapidly increases, namely it requires an increasingly larger injection velocity for the reaction to be sustained, as shown in the Figures. Between these thresholds, there exist three separate solutions for given injection and reservoir parameters, consisting of a stable low temperature (and velocity) branch in the vicinity of the initial conditions, a stable high temperature (and velocity) branch, where rigorous combustion takes place, and an unstable intermediate branch connected to the latter. Such behavior is typical of multiple solutions in other areas in reaction engineering. The upper branch is the solution corresponding to a proper combustion front. It approaches and runs parallel to the adiabatic solution. For a given $H$, the sensitivity of the front variables to the injection velocity is very large near the threshold, but becomes almost negligible above it. Likewise, the sensitivity of the extinction threshold $E_{c}$ to the reservoir thickness is significant for values of $H$ the order of $1 \mathrm{~m}$ or less, for the parameters assumed here. As $H$ decreases, the extinction threshold rapidly increases, namely it requires an increasingly larger injection velocity for the reaction to be sustained, as shown in the Figures. Conversely, at larger $H$, the threshold decreases, and for sufficiently large values, multiplicity disappears altogether. Analogous results are obtained when the heat losses are of the convective type, which would be appropriate for a laboratory application. In fact, in such cases, the system equations are simpler. One can combine the two applications [7], to obtain an expression for the effective heat transfer coefficient $\tilde{h}$ in a system controlled by heat conduction. In terms of the Nusselt number, we have

$$
\mathrm{N}_{\mathrm{u}} \equiv \frac{\tilde{h} H}{\lambda}=\frac{H V}{2 \alpha_{s}}\left(\omega_{i}^{1 / 3} z_{i}^{2}+2 \omega_{i} z_{i}^{3 / 2}+\omega_{i}^{5 / 3} z_{i}-\omega_{i}^{-1}\right)
$$




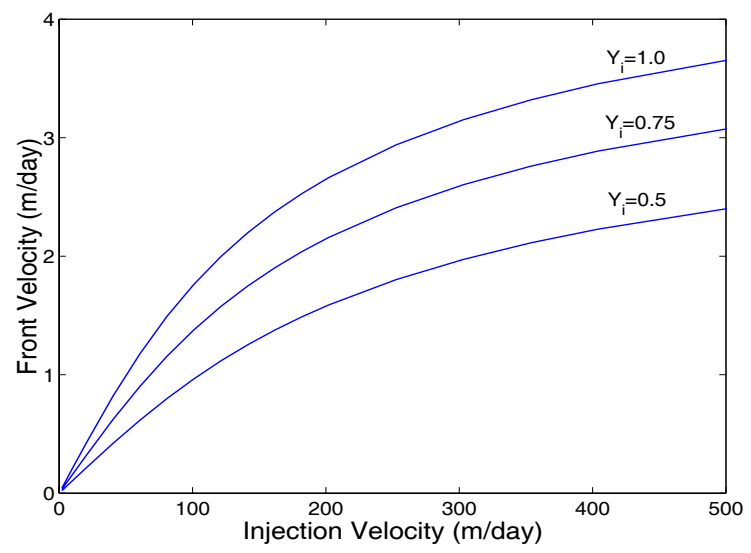

Figure 1: Steady-state front velocity versus injection velocity for different injected oxyen concentration for a single layer porous medium under adiabatic conditions.

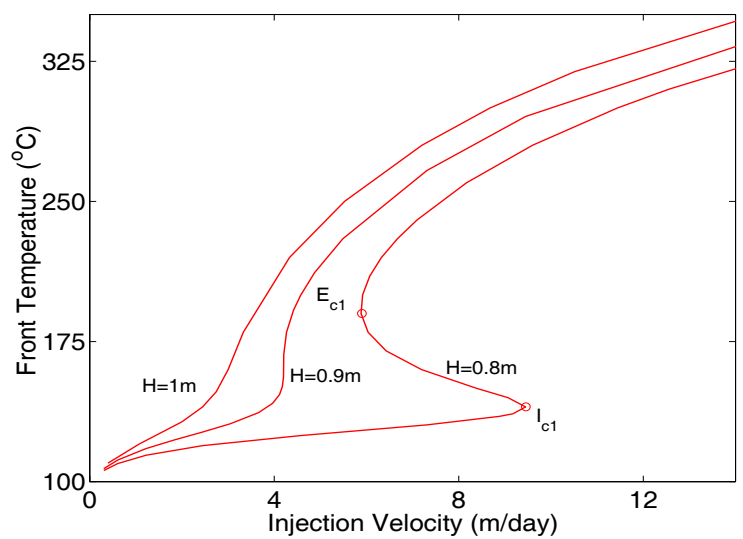

Figure 2: Front temperature versus injection velocity for a single layer under non-adiabatic conditions. $I_{c 1}=(9.45,140.0), E_{c 1}=(5.9,190.0)$. 


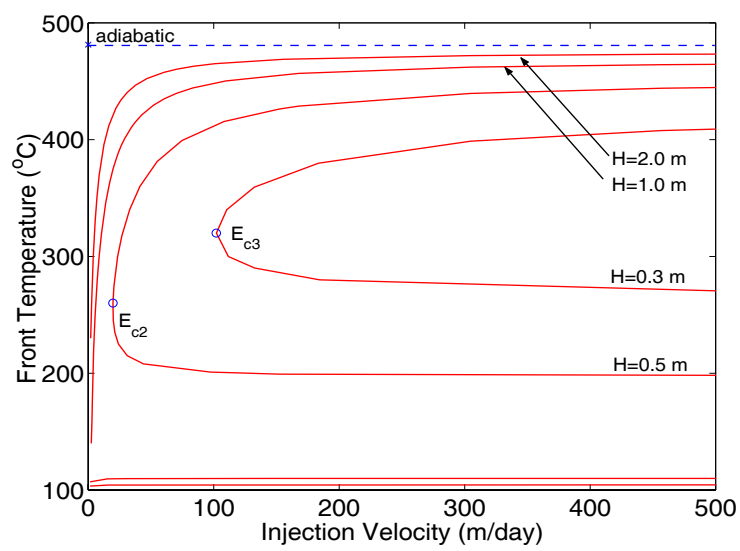

Figure 3: Front temperature versus injection velocity for a single layer under non-adiabatic conditions. $E_{c 2}=(19.7,260.0), E_{c 3}=(102.1,320.0)$.

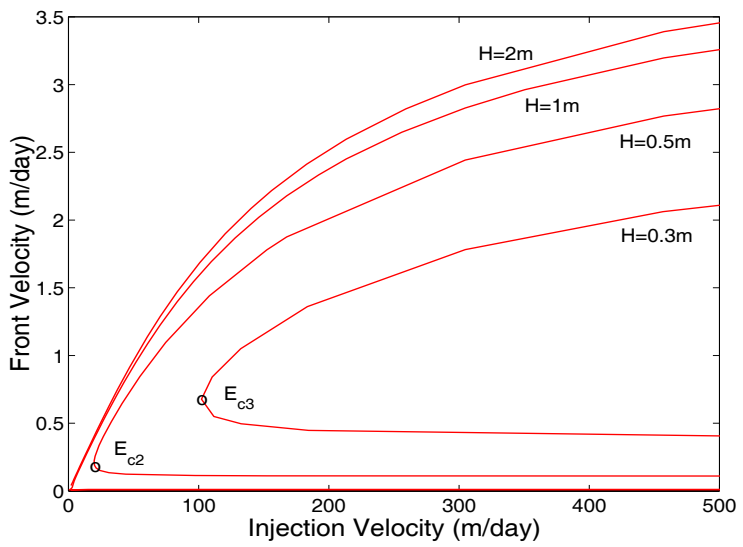

Figure 4: Front velocity versus injection velocity for a single layer under non-adiabatic conditions. $E_{c 2}=(19.7,260.0), E_{c 3}=(102.1,320.0)$. 

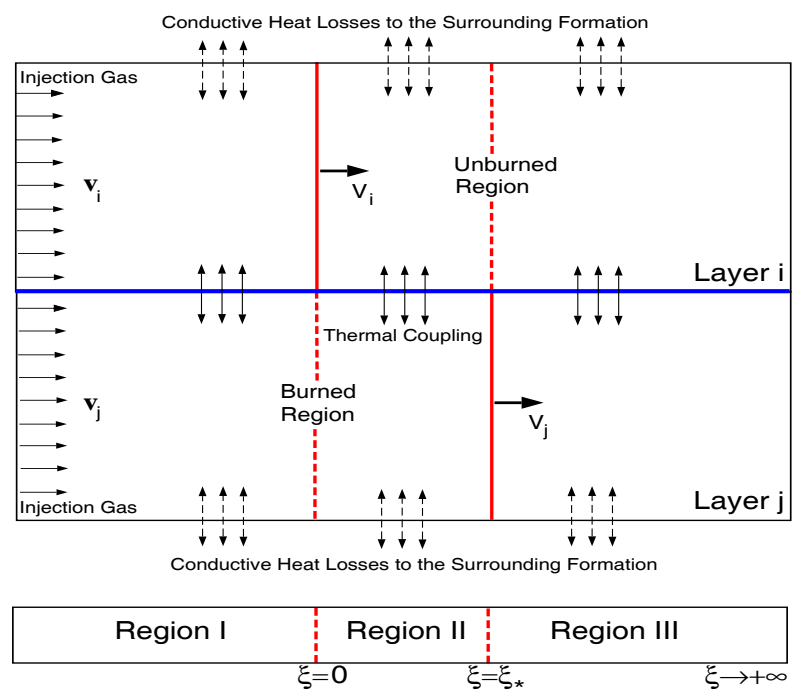

Figure 5: Schematic of the notation used for the propagation of combustion fronts in a two-layered porous medium.

Further details can be found in [7].

\section{Combustion in a Two-Layered Porous Medium}

Consider, now, the application of the same approach to a layered porous medium. The first geometry to be considered is a two-layered system, as shown in the schematic of Figure 5 .

The steady-state propagation of combustion in the two layers $i$ and $j$ is considered. The layers are homogeneous, but with different permeabilities (with layer $j$ being more permeable), and hence different injection velocities. In the absence of mobility variation effects, these are proportional to the layer permeability. We assume only thermal coupling across the layers, which will be expressed in terms of a convective-type heat model. Due to thermal coupling, it is apparent that isolated front propagation in each layer with front velocities dictated, e.g. by Figure 4, cannot take place. Indeed, we expect that the faster traveling front in layer $j$ will slow down due to heat losses to layer $i$ the front of which will accelerate until a coherent state is reached and the front velocities are the same in each layer. In the moving coordinate with respect to the combustion front, $\xi=x-V_{D} t$, where $x=\tilde{x} / l_{*}$ and $t=\tilde{t} / t_{*}$ are the dimensionless space and time variables with $l_{*}=\alpha_{s} / v_{i}$ and $t_{*}=l_{*} / v_{i}$, the dimensionless 
thermal energy balances for the two layers read

$A_{i} \theta_{i}^{\prime}=\theta_{i}^{\prime \prime}+\sigma\left(\theta_{j}-\theta_{i}\right)-h_{i}\left(\theta_{i}-1\right)$

$A_{j} \theta_{j}^{\prime}=\theta_{j}^{\prime \prime}+\sigma\left(\theta_{i}-\theta_{j}\right)-h_{j}\left(\theta_{j}-1\right)$

where prime denotes derivatives, and we have introduced

$A_{i}=a \rho v_{i}-V_{D} \cong-V_{D}, \quad A_{j}=a \rho v_{j}-V_{D} \cong-V_{D}$

The dimensionless parameter $a \ll 1$ represents the ratio of the volumetric heat capacity of the gas to the solid matrix, $\sigma$ is the non-dimensional coefficient for the heat exchange between the two neighboring points in the direction transverse to the propagation, and we have also allowed for heat loss to the surroundings using the heat loss coefficients

$h_{i}=\mathrm{Nu}\left(\frac{\alpha_{s}}{H_{i} v_{i}}\right)^{2}, \quad h_{j}=\mathrm{Nu}\left(\frac{\alpha_{s}}{H_{j} v_{j}}\right)^{2}$

The solution of this problem will be considered in the two different cases of adiabatic and non-adiabatic conditions.

\subsection{Adiabatic conditions}

In the adiabatic case, equation (8) simplifies to

$\theta_{i}=\theta_{j}+\frac{1}{\sigma}\left(A_{j} \theta_{j}^{\prime}-\theta_{j}^{\prime \prime}\right)$

Inserted into equation (7) this gives the following differential equation

$\theta_{j}^{(/ \mathrm{v})}-B \theta_{j}^{\prime \prime \prime}+D \theta_{j}^{\prime \prime}+\sigma B \theta_{j}^{\prime}=0$

where we defined

$B=A_{i}+A_{j}, \quad D=A_{i} A_{j}-2 \sigma$

Its solution is readily obtained,

$\theta_{j}=\tilde{c}_{0}+\tilde{c}_{1} e^{r_{1} \xi}+\tilde{c}_{2} e^{r_{2} \xi}+\tilde{c}_{3} e^{r_{3} \xi}$ 
where $r_{1}>0, r_{2}, r_{3}<0$ are the real roots of

$$
r^{3}-B r^{2}+D r+\sigma B=0
$$

The solution for $\theta_{i}$ follows using equation (11), namely

$$
\begin{aligned}
\theta_{i}=\tilde{c}_{o}+\tilde{c}_{1} e^{r_{1} \xi} & +\tilde{c}_{2} e^{r_{2} \xi}+\tilde{c}_{3} e^{r_{3} \xi} \\
& +\frac{1}{\sigma}\left(A_{j}\left(\tilde{c}_{1} r_{1} e^{r_{1} \xi}+\tilde{c}_{2} r_{2} e^{r_{2} \xi}+\tilde{c}_{3} r_{3} e^{r_{3} \xi}\right)\right. \\
& \left.\quad-\tilde{c}_{1} r_{1}^{2} e^{r_{1} \xi}-\tilde{c}_{2} r_{2}^{2} e^{r_{2} \xi}-\tilde{c}_{3} r_{3}^{2} e^{r_{3} \xi}\right)
\end{aligned}
$$

Because of the jump conditions at the two combustion fronts, it is convenient to consider three different regions, as shown in Figure 5. Using the far-field boundary conditions

$\xi \rightarrow-\infty \quad: \quad \theta_{i}=\theta_{j}=\theta_{I}$,

$\xi \rightarrow \infty \quad: \quad \theta_{i}=\theta_{j}=1$

we then have

\section{Region I}

$\theta_{i}=\theta_{I}+c_{1} e^{r_{1} \xi}+\frac{1}{\sigma}\left(A_{j} c_{1} r_{1} e^{r_{1} \xi}-c_{1} r_{1}^{2} e^{r_{1} \xi}\right)$

$\theta_{j}=\theta_{I}+c_{1} e^{r_{1} \xi}$

\section{Region II}

$$
\begin{gathered}
\theta_{i}=c_{o}+c_{2} e^{r_{1} \xi}+c_{3} e^{r_{2} \xi}+c_{4} e^{r_{3} \xi} \\
+\frac{1}{\sigma}\left(A_{j}\left(c_{2} r_{1} e^{r_{1} \xi}+c_{3} r_{2} e^{r_{2} \xi}+c_{4} r_{3} e^{r_{3} \xi}\right)\right. \\
\left.-c_{2} r_{1}^{2} e^{r_{1} \xi}-c_{3} r_{2}^{2} e^{r_{2} \xi}-c_{4} r_{3}^{2} e^{r_{3} \xi}\right)
\end{gathered}
$$

\section{Region III}

$$
\begin{aligned}
\theta_{i}=1 & +c_{5} e^{r_{2} \dot{\xi}}+c_{6} e^{r_{3} \xi}+\frac{1}{\sigma}\left(A_{j}\left(c_{5} r_{2} e^{r_{2} \dot{\xi}}+c_{6} r_{3} e^{r_{3} \dot{\xi}}\right)\right. \\
& \left.-c_{5} r_{2}^{2} e^{r_{2} \xi}-c_{6} r_{3}^{2} e^{r_{3} \xi}\right)
\end{aligned}
$$


$\theta_{j}=1+c_{5} e^{r_{2} \xi}+c_{6} e^{r_{3} \xi}$

To complete the problem requires formulating jump conditions across the combustion fronts. These read as follows:

At $\xi=0$ :

$\left[\theta_{i}\right]_{\xi=0^{-}}^{\xi=0^{+}}=\left[\theta_{j}\right]_{\xi=0^{-}}^{\xi=0^{+}}=\left[\theta_{j}^{\prime}\right]_{\xi=0^{-}}^{\xi=0^{+}}=0$

$\left[\theta_{i}^{\prime}\right]_{\xi=0^{-}}^{\xi=0^{+}}=-q V_{D i}$

and at $\xi=\xi_{*}$ :

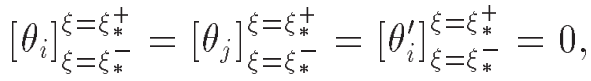

$\left[\theta_{j}^{\prime}\right]_{\xi=\xi_{*}^{-}}^{\xi=\xi_{*}^{+}}=-q V_{D j}$.

Because the fronts travel with the same speed $\left(V_{i}=V_{j}\right)$, both the front and the distance $\xi_{*}$ between them must be determined. In essence, these constants are the eigenvalues of this system of ten equations (seven integration constants, $\xi_{*}, V_{D}$, and $\theta_{I}$ ). In general, the system is non-linear, due to the intricate dependence between front velocity and heat transfer. The ten equations required for its solution consist of the 8 jump conditions, and the application of the expression (4) for the front velocity twice (note that this equation remains valid, regardless of the coupling between the two layers). Details for the solution are given in [9]. Numerical results will be discussed in a later section.

\subsection{Non-adiabatic conditions}

Working likewise, we can formulate the problem in the presence of heat losses. Now, additional terms describing the interaction with the surroundings must be included. Using equation (8) to substitute $\theta_{i}$ in terms of $\theta_{j}$, we have

$\theta_{i}=\left(1+\frac{h_{j}}{\sigma}\right) \theta_{j}+\frac{1}{\sigma}\left(A_{j} \theta_{j}^{\prime}-\theta_{j}^{\prime \prime}-h_{j}\right)$

Inserting into (7) and re-arranging we get

$\theta_{j}^{(\prime \mathrm{v})}-B \theta_{j}^{\prime \prime \prime}+E \theta_{j}^{\prime \prime}+F \theta_{j}^{\prime}+G \theta_{j}-G=0$ 
where

$E=A_{i} A_{j}-2 \sigma-\left(h_{i}+h_{j}\right)$

$F=A_{i}\left(\sigma+h_{j}\right)+A_{j}\left(\sigma+h_{i}\right)$

$G=h_{i} h_{j}+\sigma\left(h_{i}+h_{j}\right)$

the general solution of which is

$\theta_{j}=1+\tilde{c}_{1} e^{r_{1} \xi}+\tilde{c}_{2} e^{r_{2} \xi}+\tilde{c}_{3} e^{r_{3} \xi}+\tilde{c}_{4} e^{r_{4} \xi}$

where we have identified the real roots $r_{1}, r_{2}>0$ and $r_{3}, r_{4}<0$ of

$r^{4}-B r^{3}+E r^{2}+F r+G=0$.

Again, we have to distinguish different expressions in different regimes, which are as follows:

\section{Region I}

$$
\begin{aligned}
\theta_{i}=1 & +\left(1+\frac{h_{j}}{\sigma}\right)\left(c_{1} e^{r_{1} \xi}+c_{2} e^{r_{2} \xi}\right)+\frac{1}{\sigma}\left(A _ { j } \left(c_{1} r_{1} e^{r_{1} \xi}\right.\right. \\
& \left.\left.+c_{2} r_{2} e^{r_{2} \xi}\right)-c_{1} r_{1}^{2} e^{r_{1} \xi}-c_{2} r_{2}^{2} e^{r_{2} \xi}-h_{j}\right) \\
\theta_{j}=1 & +c_{1} e^{r_{1} \xi}+c_{2} e^{r_{2} \xi}
\end{aligned}
$$

\section{Region II}

$$
\begin{aligned}
\theta_{i}= & 1+\left(1+\frac{h_{j}}{\sigma}\right)\left(c_{3} e^{r_{1} \xi}+c_{4} e^{r_{2} \xi}+c_{5} e^{r_{3} \xi}+c_{6} e^{r_{4} \xi}\right) \\
& +\frac{1}{\sigma}\left(A_{j}\left(c_{3} r_{1} e^{r_{1} \xi}+c_{4} r_{2} e^{r_{2} \xi}+c_{5} r_{3} e^{r_{3} \xi}+c_{6} r_{4} e^{r_{4} \xi}\right)\right. \\
& \left.-c_{3} r_{1}^{2} e^{r_{1} \xi}-c_{4} r_{2}^{2} e^{r_{2} \xi}-c_{5} r_{3}^{2} e^{r_{3} \xi}-c_{6} r_{4}^{2} e^{r_{4} \xi}-h_{j}\right) \\
\theta_{j}= & 1+c_{3} e^{r_{1} \xi}+c_{4} e^{r_{2} \xi}+c_{5} e^{r_{3} \xi}+c_{6} e^{r_{4} \xi}
\end{aligned}
$$

\section{Region III:}

$$
\begin{aligned}
\theta_{i}= & 1+\left(1+\frac{h_{j}}{\sigma}\right)\left(c_{7} e^{r_{3} \xi}+c_{8} e^{r_{4} \xi}\right)+\frac{1}{\sigma}\left(A _ { j } \left(c_{7} r_{3} e^{r_{3} \xi}\right.\right. \\
& \left.\left.+c_{8} r_{4} e^{r_{4} \xi}\right)-c_{7} r_{3}^{2} e^{r_{3} \xi}+c_{8} r_{4}^{2} e^{r_{4} \xi}-h_{j}\right)
\end{aligned}
$$



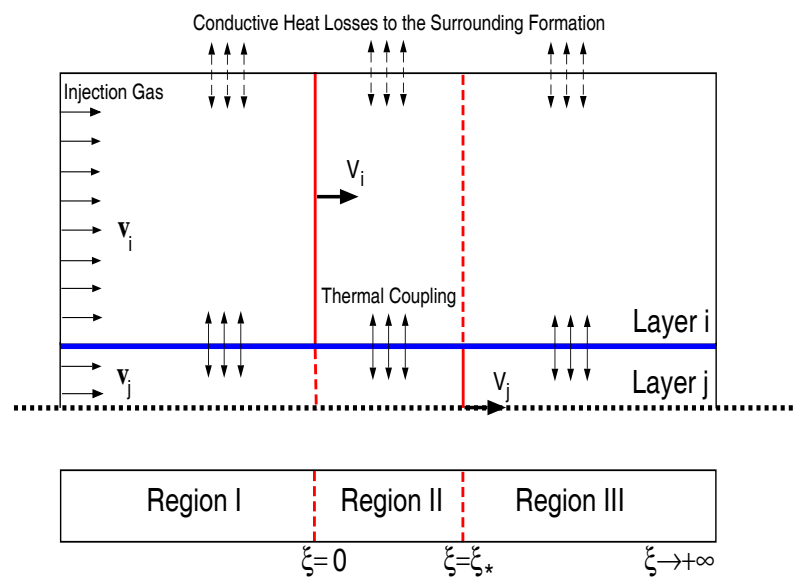

Figure 6: Schematic of the notation used for the propagation of combustion fronts in a two-layered porous medium.

$\theta_{j}=1+c_{7} e^{r_{3} \xi}+c_{8} e^{r_{4} \xi}$

Application of the same jump conditions as before gives rise to a set of ten equations in terms of the ten unknowns (integration constants, the distance between the fronts and the front velocity). Details of the solution are found in [9].

\subsection{Non-adiabatic, symmetric, three-layered porous medium}

The same approach can be applied to the solution of a symmetric three-layered medium, when the two outer layers have the same properties. This type of geometry is useful in the investigation of the effect of a middle layer that plays the role of a permeable thief zone. Because of the symmetry assumed, velocity and temperature of the outer reaction fronts are taken to be identical, as shown in Figure 6 .

Then, the governing energy balances become

$A_{i} \theta_{i}^{\prime}=\theta_{i}^{\prime \prime}+\sigma_{i}\left(\theta_{j}-\theta_{i}\right)-h_{i}\left(\theta_{i}-1\right)$

$A_{j} \theta_{j}^{\prime}=\theta_{j}^{\prime \prime}+\sigma_{j}\left(\theta_{i}-\theta_{j}\right)$

Working as before, equation (41) gives $\theta_{i}$,

$\theta_{i}=\theta_{j}+\frac{1}{\sigma_{j}}\left(A_{j} \theta_{j}^{\prime}-\theta_{j}^{\prime \prime}\right)$ 
Inserted into (40) gives

$\theta_{j}^{(/ \mathrm{v})}-B \theta_{j}^{\prime \prime \prime}+\bar{E} \theta_{j}^{\prime \prime}+\bar{F} \theta_{j}^{\prime}+\sigma h_{i} \theta_{j}-\sigma h_{i}=0$

where

$\bar{E}=A_{i} A_{j}-\sigma_{i}-\sigma_{j}-h_{i}$

$\bar{F}=B \sigma_{j}+A_{j} h_{i}$

the solution of which is obtained as before, in terms of a combination of exponentials, with exponents the real roots $r_{1}, r_{2}>0$ and $r_{3}, r_{4}<0$ of the characteristic equation

$r^{4}-B r^{3}+\bar{E} r^{2}+\bar{F} r+\sigma_{j} h_{i}=0$.

The mathematical procedure is similar to the previous and will not be repeated (see [9] for more details).

\section{Results}

The numerical solution was studied using typical in situ combustion data [7, 9]. Results were obtained for the temperature $T_{f}$ and velocity $V$ of the fronts as well as their distance $\tilde{\xi}_{*}=\xi_{*} \times l_{*}$, in terms of the velocity (hence, permeability) ratio $R=v_{i} / v_{j}$, the thermal coupling coefficient $\sigma$, the velocity of the layers, and, in the non-adiabatic cases, the layer thicknesses $H_{i}$ and $H_{j}$. We considered two velocity cases, one in which the larger velocity is fixed to $v_{j}=100 \mathrm{~m} /$ day (case $\mathrm{j}$ ), and another in which lower velocity $v_{i}$ is fixed to $v_{i}=100 \mathrm{~m} /$ day (case i). In either case $R$ was varied between its limits 0 and 1 .

\subsection{Adiabatic Two-layer Case}

The procedure applied during the calculations is explained in detail in [9]. Figures 7 and 8 show the effect of $R$ on the temperature profiles and the front velocity for constant $\sigma$, and case j. We note the following: The system recovers the single-layer solution (with $V=1.7346$ $\mathrm{m} /$ day) in the single-layer case $R=1$ (Figure 7). Here the two fronts collapse, and their

distance is nil. When $R=0.5$ (Figure 8 ), the separation between the fronts is clear. The 
front in layer $j$ has slowed down, and has a lower temperature than that of layer $i$, which has accelerated to a common velocity (equal to $V=1.0033 \mathrm{~m} /$ day). The temperature profile is more diffuse than in the single-layer case, with heat being transferred from layer $j$ to layer $i$ downstream and from $i$ to $j$ upstream. Interestingly, the temperature profile in the lower-permeability layer has a peak, which is not present in the single-layer problem. Nonetheless, the far-field temperature upstream is equal to the adiabatic temperature. The common front velocity is much closer to the single-layer velocity for the lower-permeability layer (corresponding to an injection velocity of $50 \mathrm{~m} /$ day, rather than the arithmetic average injection velocity of $75 \mathrm{~m} /$ day). This reflects strong non-linear coupling effects. The effect of the thermal coupling parameter $\sigma$ is shown in Figure 9. Interestingly, as $\sigma$ decreases the coupling is not weakened, but rather enhanced. Clearly, the front separation has increases, the temperature peaks increased, while the common front velocity has further increased $(V=1.0161 \mathrm{~m} /$ day $)$.

While these results point out to an important effect of $R$, the latter also depends on the actual velocity values. Figures 10-12 show two sets of the front temperatures, front velocities and front distance, as a function of $R$ for $\sigma=0.01$ and the two cases $\mathrm{j}$ and $\mathrm{i}$, respectively. Recall that case $\mathrm{j}$ corresponds to fixed $v_{j}=100 \mathrm{~m} /$ day, while case $\mathrm{i}$ to fixed $v_{i}=100 \mathrm{~m} /$ day. Of course, ideally one would like to have a three-dimensional plot with $v_{i}$ and $v_{j}$ as the independent variables. However, these computations can be time consuming and in the present we will restrict ourselves to only a few slices of this diagram.

It is clear from Figure 10 that the effect of the actual velocity levels is not great on the front temperatures (except for that of the leading front at small values of $R$ ). In fact, the far-field temperature behind the two fronts is not influenced at all by the variations in $R$ or $\sigma$ as its value is always the adiabatic temperature, as pointed out above. Thus, for the adiabatic case, the temperature is roughly only a function of $R$. However, the effect is significant on the front velocities and the front distance. In case $j$, where it is the larger injection velocity which is kept fixed, the front velocity decreases as the smaller injection velocity decreases, almost proportionally to it, while in case $i$, where the smallest velocity is fixed, the variation is insignificant. Analogous is the effect on the front distance.

These results suggest that essentially the behavior of the system is controlled by the layer with 


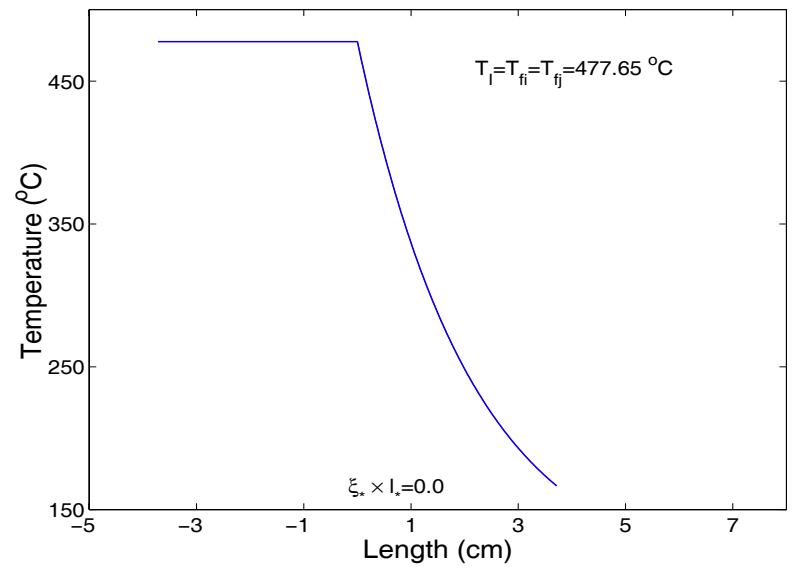

Figure 7: Temperature profiles for the two-layer adiabatic case. $R=1.0, \sigma=0.01$.

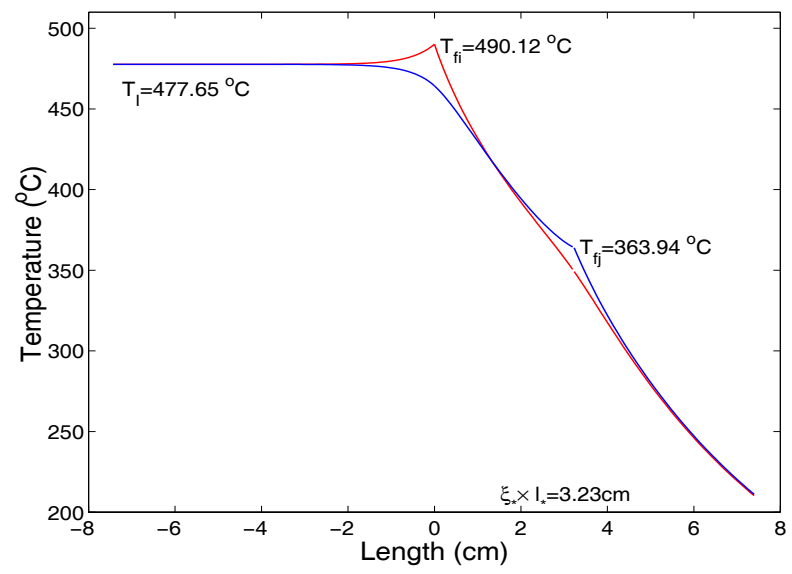

Figure 8: Temperature profiles for the two-layer adiabatic case. $R=0.5, \sigma=0.01$. 


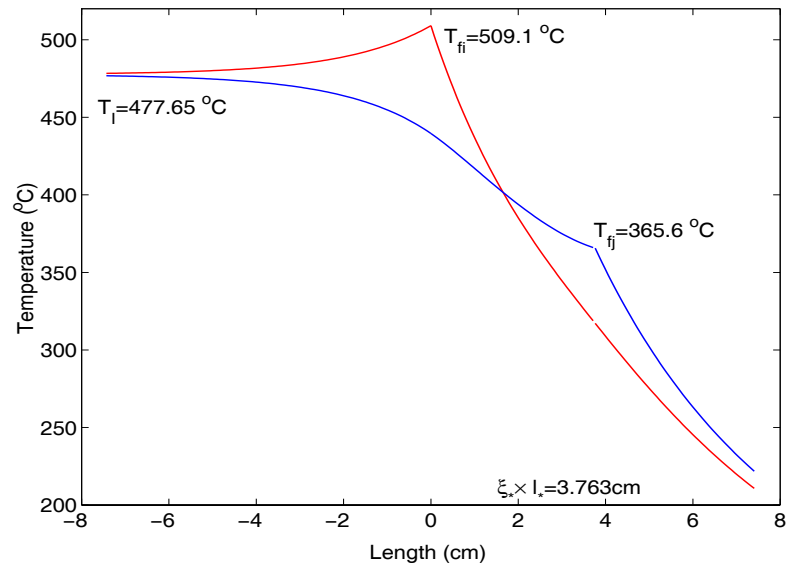

Figure 9: Temperature profiles for the two-layer adiabatic case. $R=0.5, \sigma=0.001$.

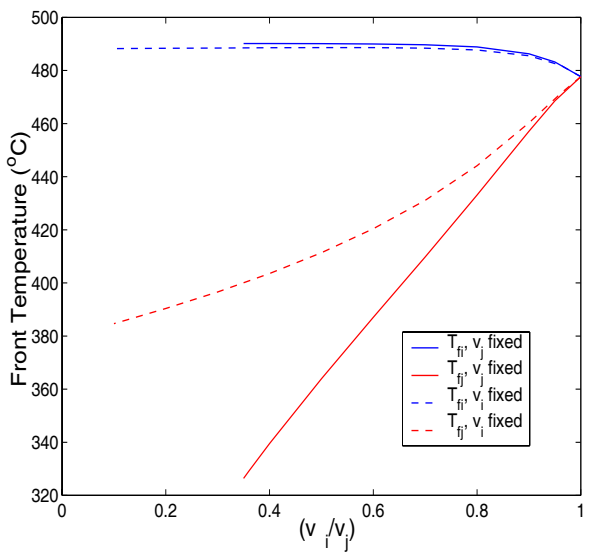

Figure 10: Front temperatures versus $R$ for the two-layer adiabatic case. Solid lines denote case j, dashed lines denote case $\mathrm{i}$. 


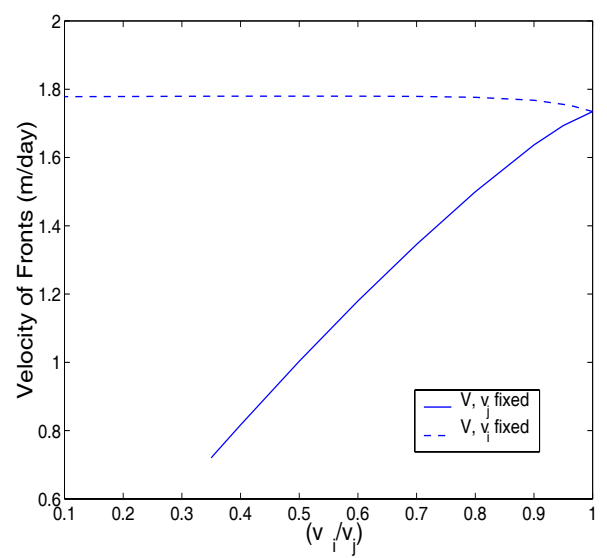

Figure 11: Front velocity versus $R$ for the two-layer adiabatic case. Solid lines denote case j, dashed lines denote case i.

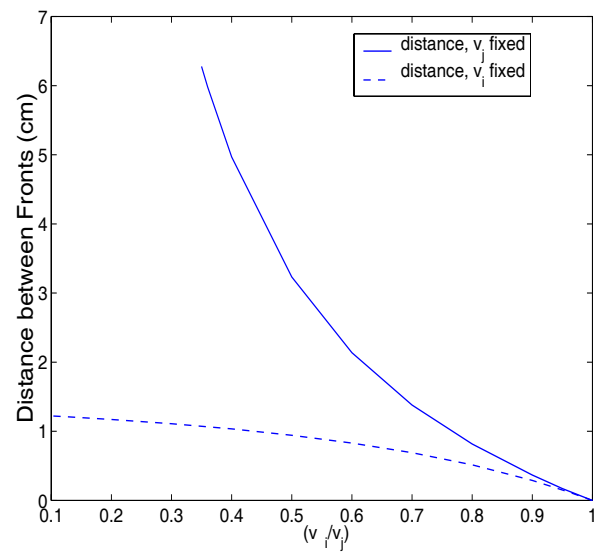

Figure 12: Distance between the fronts versus $R$ for the two-layer adiabatic case. Solid lines denote case $\mathrm{j}$, dashed lines denote case i. 
the smallest injection velocity, with the front velocity in particular almost being a slave of that variable. The implications of this finding are important. For the adiabatic case they simply affect the rate of front propagation. However, for the non-adiabatic case, discussed below, they may have more dramatic consequences, regarding the possibility of process extinction.

\subsection{Non-adiabatic Two-layer Case}

Using the formulation described in the previous sections, numerical results were obtained for the non-adiabatic case in the two-layer system. Now, in addition to the previous, an important additional parameter is the layer thickness, which was taken in all simulations shown as the same for the two layers.

When the layer thickness is sufficiently large (approximately $2 \mathrm{~m}$, for the parameters shown here) the solution of the problem and its sensitivity to $R$ and the velocities is qualitatively the same as in the adiabatic case. Unique solutions exist and the main difference is that the temperature profile is more spread out, has somewhat more structure and, of course, asymptotically tends to the initial value. Characteristic examples are shown in Figures 1315. The observed similarity of the non-adiabatic model results when $H=2 \mathrm{~m}$ to the results of the adiabatic case is consistent with the results of single layer analysis - the combustion fronts propagate as if the system is in the adiabatic mode, given that sufficient gas is injected into the layers.

On the other hand, when the thickness becomes small, the qualitative picture changes. As in the single-layer case, the possibility of multiplicity arises. Figures 16 and 17 show features very similar to the single layer. Thus, for case $\mathrm{j}$, where the lower injection velocity can become sufficiently small in magnitude, extinction and ignition limits arise. The multiplicity arises simultaneously in both fronts, and both fronts ignite and get extinct simultaneously. The corresponding curves for case $\mathrm{j}$ are similar both qualitatively and quantitatively to the single layer case. The results for case i are somewhat different. Here, because the lowest velocity remains fixed (at $100 \mathrm{~m} /$ day), multiplicity does not arise until the layer thickness is sufficiently small (contrast Figures 16 and 17). By comparing with the single-layer results, this effect is somewhat unexpected. If we were to assume that the front basically follows the front velocity corresponding to the lower injection velocity, the curves corresponding to case $\mathrm{i}$ could be 
interpreted from the single-layer results as those corresponding to the upper branch. This would mean that intermediate and lower branches would also exist. These do not appear in Figure 16, although they do in Figure 17, which corresponds to a smaller thickness layer. One infers that when the velocities are sufficiently large, the composite, two-layer system behaves as one with an effectively larger thickness, compared to the case when the layer velocities are relatively small. This interpretation is also supported in the velocity and front distance curves shown in Figures 18 and 19. However, and contrary to the adiabatic case, another effect is also present here, namely, an intrinsic heterogeneity effect through the parameter $R$. For example, the above figures illustrate through case i, that by increasing the heterogeneity of the layers, extinction will eventually set in, even though the lower-permeability layer has a fixed injection velocity. This effect is non-trivial and unexpected. For completeness, we examined the sensitivity of these results to the thermal parameter $\sigma$. Very small differences were found as $\sigma$ was decreased by a factor of 10 .

The implications of these results are important. They point out that increasing the permeability contrast between the layers can have dramatic effects on the propagation of a combustion front. Namely, given an overall injection rate, and for sufficiently small layer thickness, there is a sufficiently large permeability contrast, such that the process becomes extinct. Depending on the parameters, this contrast can be as low as 10. Strongly layered (and by extension, strongly heterogeneous) systems may thus be not good candidates for in-situ combustion.

The above results gave only one indication of the ballpark values for this to occur. A more systematic analysis would require the development of 3 -D diagrams using the two velocities as coordinates and the resulting identification of extinction and ignition limits. Figure 21 illustrates the calculated propagation speed and front temperatures in $v_{i}-v_{j}$ space. The surface corresponds to $H=1 \mathrm{~m}$ and $\tilde{h}=0.008 \mathrm{~kW} / \mathrm{m}^{2}-\mathrm{K}$, same for both layers. At the extinction points, the low injection velocity is nearly constant $(45-65 \mathrm{~m} /$ day) while the higher one varies in between the range. The figure is in agreement with the previous $2-\mathrm{D}$ results, and shows clearly that front extinction is controlled by the lower injection velocity of a layered system. 


\subsection{Non-adiabatic Three-layer Case}

For completeness, we also analyzed the symmetric, three-layer geometry. Now, the middle layer is shielded and does not lose heat directly to the surroundings. The results obtained were qualitatively similar to the previous non-adiabatic problem. In this geometry, we investigated the sensitivity of the results to the ratio of the thickness of the two layer, which here were taken unequal. In the calculations, we also kept the injection velocity of the surrounding layers fixed, and varied $v_{j}$ (case i). Front temperature results are shown in Figure 20 . It is shown that when the shielding layers are thick enough (dashed lines in Figure 20) the behavior approaches the adiabatic case, where there exists a unique solution. If the layer thickness decreases, then multiplicity sets in, with characteristics similar to the ones discussed above.

\section{Concluding Remarks}

In this paper we extended the approach of [7] to heterogeneous systems, by considering the simpler case of in-situ combustion in layered porous media. Two simple geometries were considered, a two-layer model and a symmetric three-layer model. Analytical models were developed to delineate the combined effects of fluid flow, reaction and heat transfer on the dynamics of combustion fronts in the layers, using as parameters the thermal coupling between the layers, the heat transfer to the surroundings and the permeability contrast. We find that in layered systems, the thermal coupling between layers leads to coherent traveling fronts, propagating at the same velocity. This coupling retards greatly fronts in the more permeable layer and accelerates only slightly those in the less permeable one, until a common front velocity is attained. In essence, the problem becomes slave to the injection velocity in the lower permeability layer. As in the single-layer case, there exists a unique solution, under adiabatic conditions, and multiple steady-state solutions, under non-adiabatic conditions. The latter lead to ignition and extinction conditions. Importantly, for a sufficiently large permeability contrast, relatively small layer thickness and under non-adiabatic conditions, steady-state propagation in the two layers cannot be sustained, and the process becomes extinct, even though, under the same conditions, sustained propagation would have been predicted for the equivalent single-layer problem with the average injection velocity. In a sense, the problem 
becomes controlled by the extremes of the permeability distribution. Such behavior can be detrimental to the success of in-situ combustion in highly heterogeneous layered media. In addition, it raises serious questions on the ability of conventional reservoir simulators to capture it. Conventional models average flow and kinetic behavior over substantially large distances, where effects, such as the above, which are dominated by the extremes of the permeability field, cannot be adequately represented. Precise conditions for the delineation of the above behavior need to be further developed. We anticipate that similar conclusions will hold in the case of heterogeneous media. Work in this direction is currently in progress.

\section{References}

[1] Williams, F.A. Combustion Theory, Benjamin and Cummings Publishing Company Inc. (1985).

[2] Britten, J.A. and Krantz, W.B., Combust. Flame, 60:125 (1985).

[3] Britten, J.A. and Krantz, W.B., Combust. Flame, 65:151 (1986).

[4] Schult, D.A., Matkowsky, B.J., Volpert, V.A., and Fernandez-Pello, A.C., Combust. Flame, 104:1 (1996).

[5] Schult, D.A., Bayliss, A. and Matkowsky, B.J., SIAM J. Appl. Math., 58:806 (1998).

[6] Chuan, L. and Yortsos, Y.C. A Pore-Network Model of In Situ Combustion in Porous Media, paper SPE 69705 presented at the Society of Petroleum Engineers International Thermal Operations and Heavy Oil Symposium, Santa Margarita, Venezuela, March $15-17(2001)$.

[7] Akkutlu, I.Y. and Yortsos, Y.C. Combust. Flame, submitted (2001).

[8] Willhite, G.P. Waterflooding, SPE Textbook Series Vol. 3 (1986)

[9] Akkutlu, I.Y. Dynamics of Combustion Fronts in Porous Media, PhD Dissertation, U. of Southern California (2002, expected). 


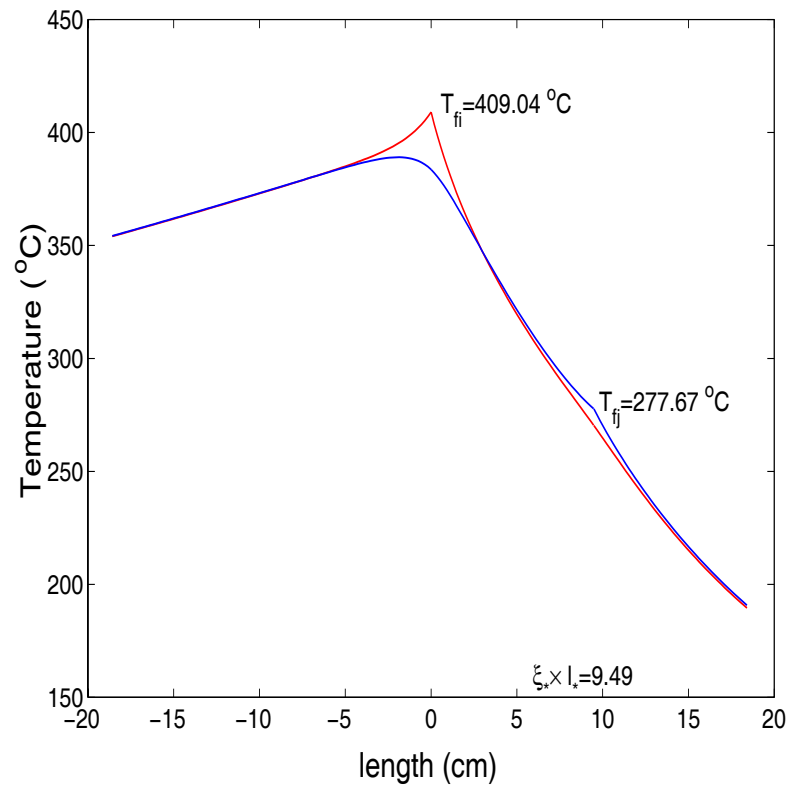

Figure 13: Temperature profiles for the non-adiabatic two-layer model. $H_{i}=H_{j}=2 \mathrm{~m}, R=0.20, \sigma=0.01$, calculated $V=0.4070 \mathrm{~m} /$ day.

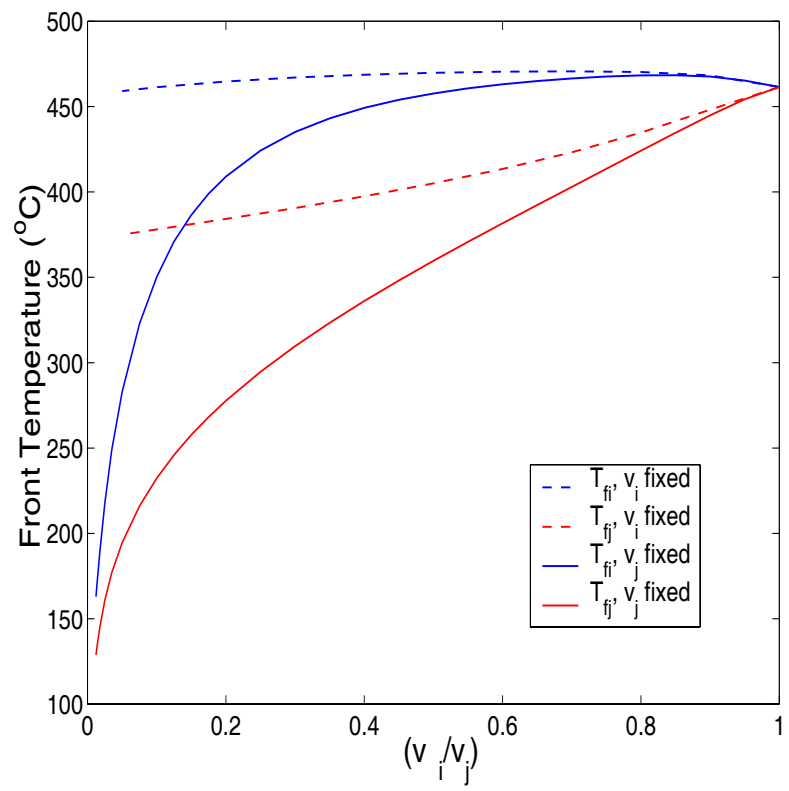

Figure 14: Front temperatures versus $R$ for the non-adiabatic two-layer model. Solid lines denote case $\mathrm{j}$, dashed lines denote case i. $H_{i}=H_{j}=2 \mathrm{~m}$., $\sigma=0.01$. 


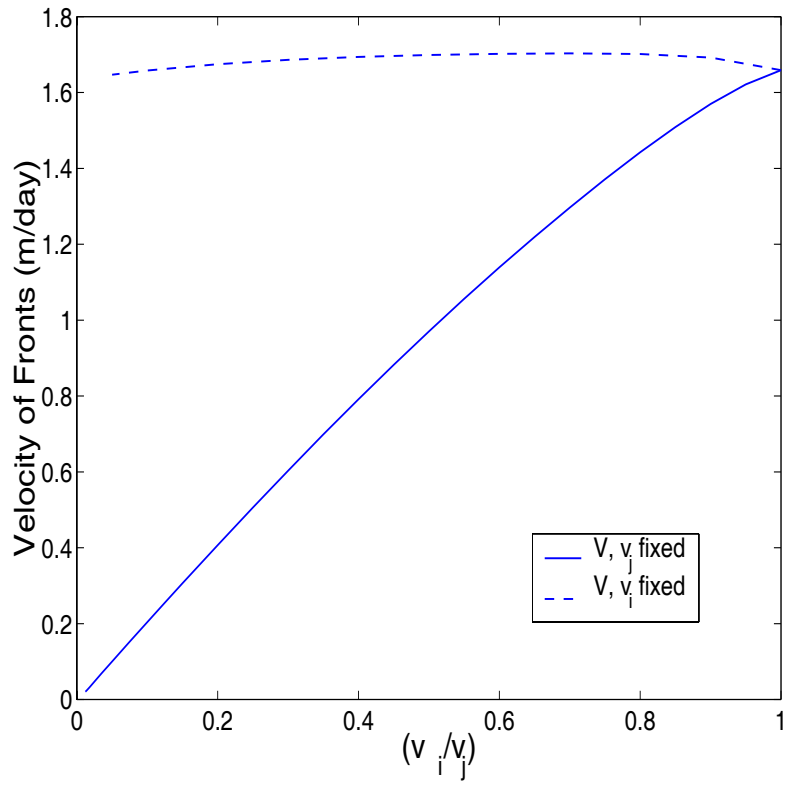

Figure 15: Velocity of the fronts versus $R$ for the non-adiabatic two-layer model. Solid lines denote case j, dashed lines denote case i. $H_{i}=H_{j}=2 \mathrm{~m} ., \sigma=0.01$.

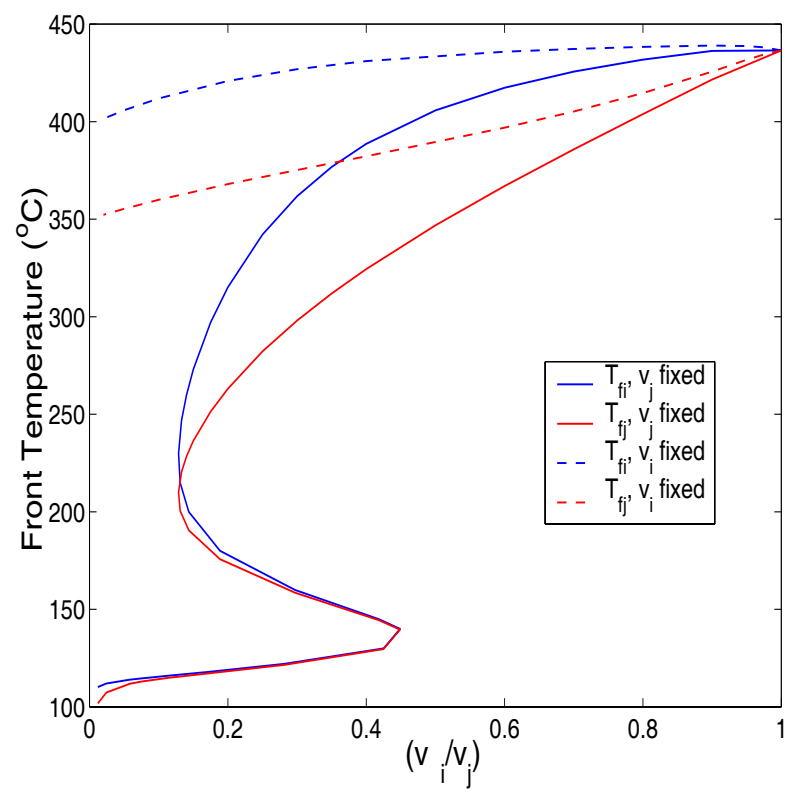

Figure 16: Nonadiabatic front temperatures for layers $i$ and $j$ versus $R$. Solid lines denote case $\mathrm{j}$, dashed lines denote case i. $H_{i}=H_{j}=0.8 \mathrm{~m} ., \sigma=0.1$. 


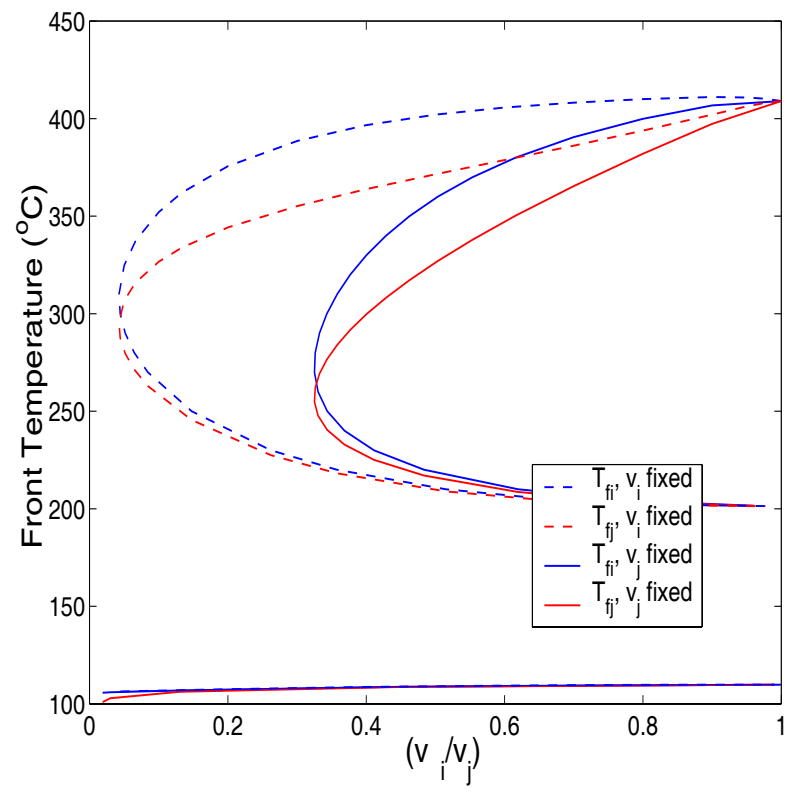

Figure 17: Nonadiabatic front temperatures for layers $i$ and $j$ versus $R$. Solid lines denote case $\mathrm{j}$, dashed lines denote case i. $H_{i}=H_{j}=0.5 \mathrm{~m} ., \sigma=0.1$.

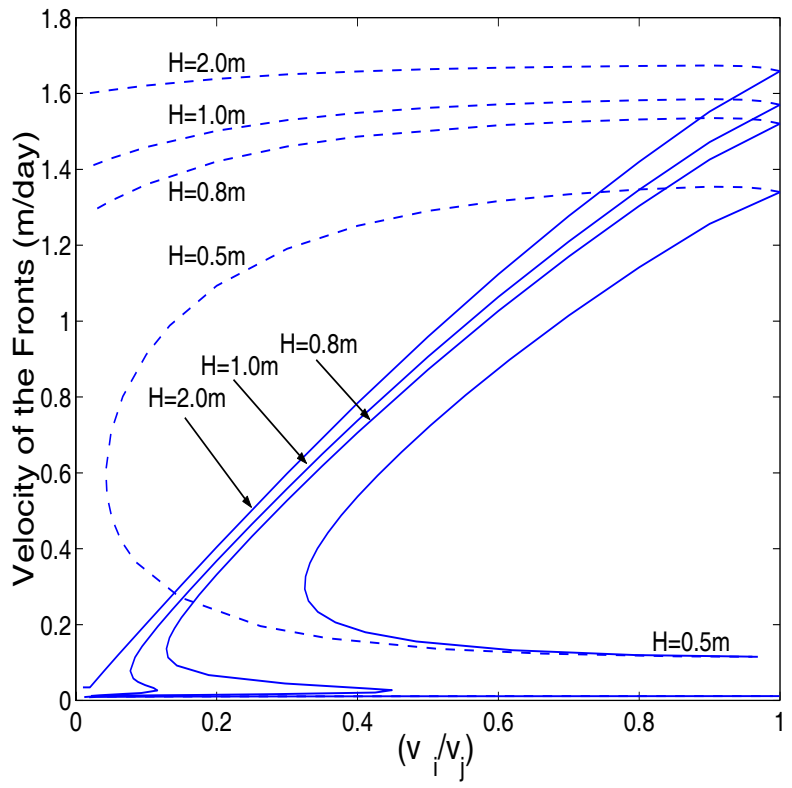

Figure 18: Nonadiabatic front velocity for layers $i$ and $j$ versus $R$ for varying $H$. Solid lines denote case $\mathrm{j}$, dashed lines denote case i. $\sigma=0.1$. 


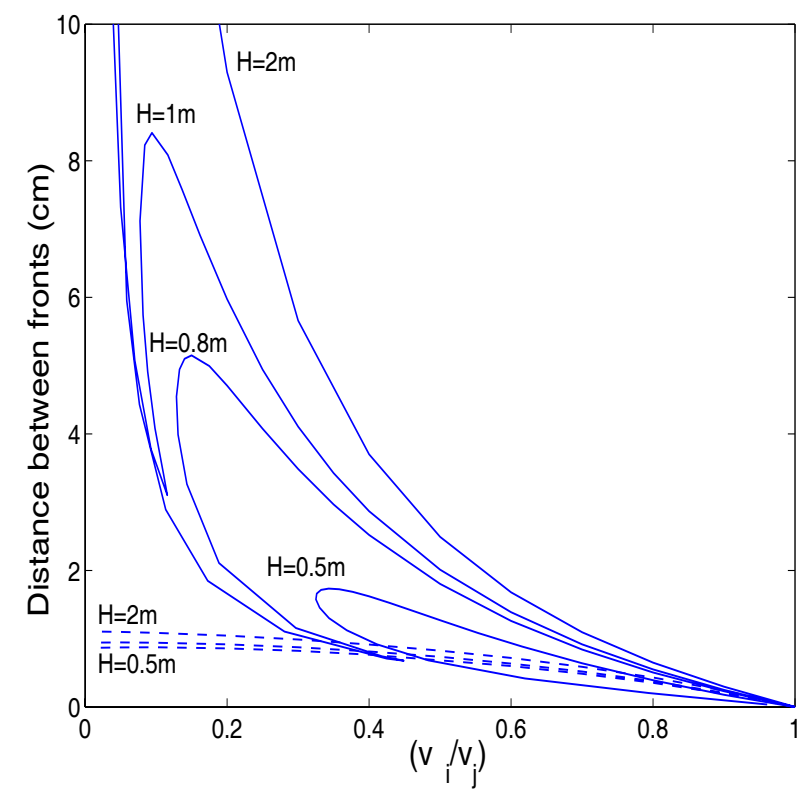

Figure 19: Distance between the fronts in layers $i$ and $j$ versus $R$ for varying $H$ and the non-adiabatic case. Solid lines denote case j, dashed lines denote case i. $\sigma=0.1$.

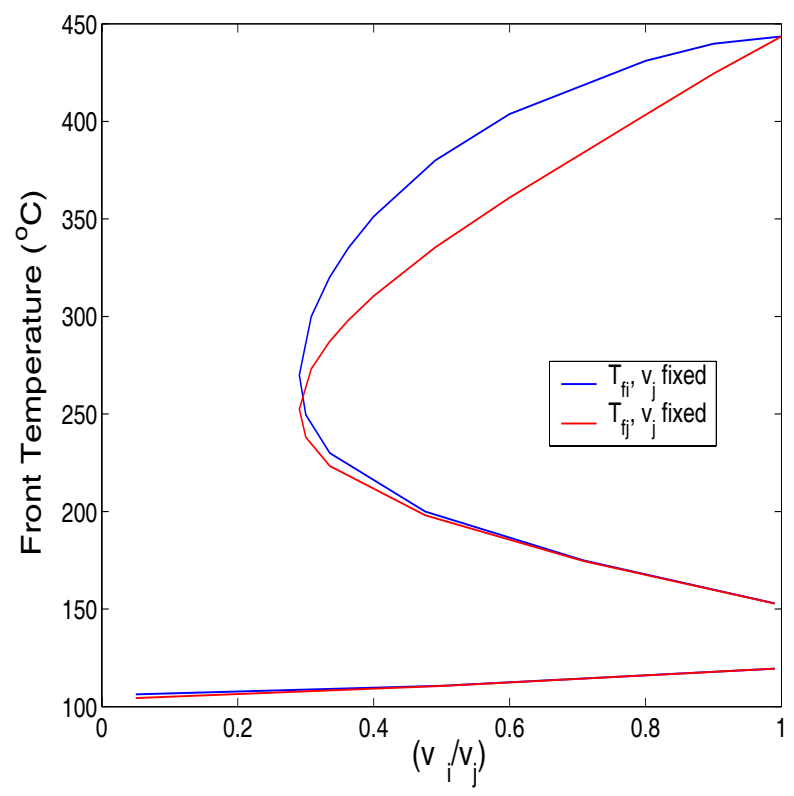

Figure 20: Front temperature versus $R$ for the non-adiabatic symmetric, three-layer case. Solid line denotes thickness of the shielding layers equal to $0.5 \mathrm{~m}$, dashed lines denote thickness equal to $2 \mathrm{~m}$. $\sigma_{i}=0.1$ 


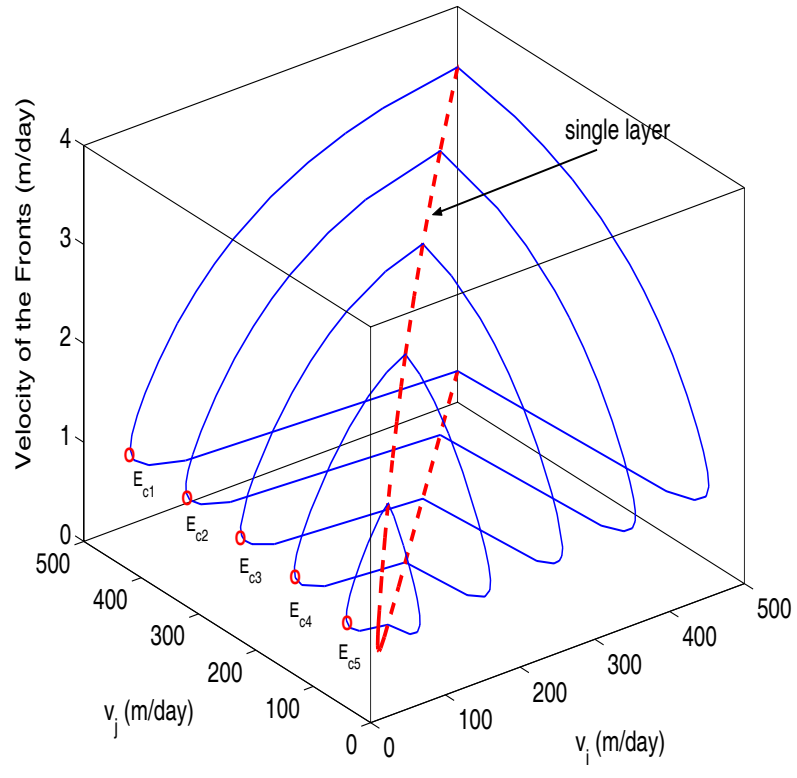

Figure 21: Front velocity versus injection velocities $v_{i}$ and $v_{j} . H_{i}=H_{i}=1 \mathrm{~m} ., \tilde{h}_{i}=\tilde{h}_{j}=0.08 \mathrm{~kW} / \mathrm{m}^{2}-\mathrm{K}$ 


\section{FLOW AND DISPALCEMENT OF FLUIDS WITH YIELD STREES AND UNSTABLE FLOWS IN POROUS MEDIA}

Heavy oils and heavy-oil associated recovery processes involve non-Newtonian rheology. Of specific importance is that of Bingham plastics (fluids with yield stress), which characterize the flow of foams, heavy oils containing asphaltenes, as well as the rheology during the formation of wormholes in sand production. This topic forms the main aspect of the Thesis of M. Chen. In addition, we have conducted a number of studies on flow and displacements in porous media, particularly on the effect of heterogeneity, the role of fractured reservoirs, the identification of heterogeneity and the effect of viscous instabilities on displacement processes. While many of these are ge neric to displacements in porous media, they are also applicable to the displacement of heavy oil. The work in this general area is described in the list of publications and Theses attached. In this final report we present two characteristic examples of the work conducted.

The flow and displacement of fluids with a yield stress is important to many applications in heavy oil recovery. The first report presented in this section describes our efforts to provide specific algorithms to solve this non-linear problem. We develop a non-trivial modification of the algorithm Invasion Percolation with Memory, previously developed in our group, to study the mobilization and viscous flow of such fluids. Based on the new algorithm, we may now include viscous flow effects, which have not been included before in such problems. Applications range from the flow of foams in porous media to the understanding of the mechanisms of the development of wormholes during heavy oil production. This approach is now being used to obtain a number of flow properties for these applications. Then, we applied this approach to describe the displacement of fluids with non-Newtonian rheology of this type, in which viscous effects are also included.

In the second study reported in this section, the general problem of unstable displacements in heterogeneous porous media is addressed. This problem is generic to a number of applications, including instabilities of displacements related to heavy oil. In the report, we study two asymptotic regimes of unstable miscible displacements in porous 
media, in the two limits, where a permeability-modified aspect ratio, $R_{L}=\frac{L}{H} \sqrt{\frac{k_{V}}{k_{H}}}$, becomes large or small, respectively. The first limit is known as Transverse (or Vertical) Equilibrium, the second leads to the problem of non-communicating layers (the DykstraParsons problem). In either case, the problem reduces to the solution of a single integrodifferential equation. Although at opposite limits of the parameter $R_{L}$, the two regimes coincide in the case of equal viscosities. By comparison with High-Resolution Simulation we investigate the validity of these two approximations. The evolution of transverse averages, particularly under viscous fingering conditions, depends on $R_{L}$. We investigate the development of a model to describe viscous fingering in weakly heterogeneous porous media under Transverse Equilibrium conditions, and compare with the various existing empirical models (such as the Koval, Todd-Longstaff and Fayers models).

The following publications resulted from this part of the project:

1. Talon, L., Martin, J., Rakotomalala, N., Salin, D. and Yortsos, Y.C., Lattice BGK Simulations of Macrodispersion in Heterogeneous Porous Media, Water Res. Res., to appear (2003).

2. Yang, Z., Yortsos, Y.C., and Salin, D., Asymptotic Regimes of Unstable Miscible Displacements in Random Porous Media, Adv. Water Res., special anniversary issue, 25, 885-898 (2002).

3. Zhan, L. and Yortsos, Y.C., The Shape of a Gravity Finger in a Rectangular Channel in a Homogeneous Porous Medium, Transport in Porous Media 49, 77-97 (2002)..

4. Laroche, C., Chen, M., Yortsos, Y.C., and Kamath, J., Time Scaling of the Rates of Produced Fluids in Laboratory Displacements, SPEJ, submitted (2001).

5. Shariati, M. and Yortsos, Y.C., Stability of Miscible Displacements Across Stratified Porous Media, Phys. Fluids 13, 2245-2257 (2001).

6. Yortsos, Y.C., Xu, B., and Salin, D., Delineation of Microscale Regimes in Fully Developed Drainage and Implications for Continuum Models, Comp. Geosc. 5, 257-278 (2001). 
7. Zhan, L. and Yortsos, Y.C., A Direct Method for the Identification of the Permeability Field of an Anisotropic Porous Medium, Water Res. Res. 37, 19291938 (2001).

8. Lajeunesse, E., Martin J., Rakotomalala, N., Salin, D., and Yortsos, Y.C., The Threshold of Instability in Miscible Displacements in a Hele-Shaw Cell at High Rates, Phys. Fluids 13, 799 (2001).

9. Zeng, J., Yortsos, Y. C., and Salin, D., On the Brinkman Correction in Hele-Shaw Flows, paper presented at the APS-DFD meeting, San Diego, CA (Nov. 18-20, 2001).

10. Yortsos, Y.C., The Permeability Variogram from Pressure Transients of Multiple Wells, in "Theory, Modeling, and Field Investigation in Hydrogeology: A Special Volume in Honor of Shlomo P. Neuman's 60th Birthday" (D. Zhang, and C.L. Winter, eds.), Geological Society of America Special Paper 348, 19-23 (2000).

11. Yortsos, Y.C., Physical Considerations in the Upscaling of Immiscible Displacements in a Fractured Medium, in "Dynamics of Fluids in Fractured Rock" (B. Faybisenko, P.A. Witherspoon and S.M. Benson, eds.), Geophysical Monograph Series 122, American Geophysical Union, 235-251 (2000).

12. Zhan, L., and Yortsos, Y.C., Identification of the Permeability Field of a Porous Media from the Injection of a Passive Tracer, Phys. Rev. E 62, 863-879 (2000).

13. Zhan, L. and Yortsos, Y.C., A Direct Method for the Identification of the Permeability Field of an Anisotropic Porous Medium, paper SPE 62976 proceedings of the 74th SPE Annual Fall Meeting, Dallas, TX (Oct. 1-4, 2000).

14. Zhang, Y., Shariati, M. and Yortsos, Y.C., The Spreading of Immiscible Fluids in Porous Media Under the Influence of Gravity, Transport in Porous Media 38, 117140 (2000).

15. Shariati, M., and Yortsos, Y. C., The Effect of Heterogeneity on the Stability of Miscible Displacements in Porous Media, paper presented at the AGU Fall Meeting, San Francisco, CA (December 16, 1999).

16. Shariati, M., and Yortsos, Y. C., Effect of Heterogeneity on the Stability of Displacement Processes in Porous Media with Non-Monotonic Viscosity, paper presented at the AIChE Fall Meeting, Dallas, TX (November 1-5, 1999).

17. Zhan, L., and Yortsos, Y. C., Identification of the Permeability Heterogeneity of Porous Media from the Displacement of a Passive Tracer, paper presented at the AIChE Fall Meeting, Dallas, TX (November 1-5, 1999). 
The following $\mathrm{PhD}$ Theses were also completed in this specific area:

1. Min Chen, Investigations on the Flow and Displacement of Fluids with Yield Stress, University of Southern California, December 2003 (expected).

2. Lang Zhan, Identification of the Permeability Heterogeneity of Porous Media by the Injection of a Passive Tracer, University of Southern California, June 2000.

3. Maryam Shariati, Effect of Non-Monotonic Mobility at Various Scales in Porous Media, University of Southern California , May 2000. 


\title{
Mobilization and Displacement of Fluids with Yield Stress in Porous Media
}

\author{
Min Chen and Yannis C. Yortsos
}

\section{INTRODUCTION}

The flow or displacement of fluids with yield stress is an important research area of non-Newtonian fluid studies, which is also encountered in a variety of industry applications. Examples include the flow of heavy oils, which can be often represented as Bingham plastics, the production of sand during cold heavy oil production, and the flow and mobilization of foams in porous media.

Foams are a typical fluid of engineering interest appear to exhibit yield behaviors, which are widely used in the oil industry in various applications, to improve reservoir sweep efficiency, block swept channels, and in gas storage and acidizing operations. These applications rely on the substantial reduction of the gas mobility in rocks obtained in the presence of foams. Some issues in this regard include whether there exists a minimum pressure gradient or a critical gas velocity, above which the porous medium can be mobilized and the relation between the pressure gradient and the flow velocity.

In the context of the flow of Bingham fluid, similar problems arise. Typical Bingham fluids include paint, slurries, pastes and food substances like margarine, mayonnaise and ketchup. Bingham fluids possess nonzero yield stresses, so that they flow when an applied stress exceeds some levels of stress, and they show little or no deformation up to the stresses. Because of the complex property of this kind of fluids, much effort has been made to study the flow and mobilization processes of these fluids. In addition, due to the complexity brought out by the combination of the non-Newtonian fluid rheology with the porous media geometry, the state of the art of Bingham fluid flow is not complete.

Many authors in the Russian literature have approximated the rheology of heavy oils as that of a Bingham fluid [1,2]. Some other studies were also carried out, including flow of foams [3], ground water flow in certain clayey solids, and drilling and hydraulicfracturing fluids [4]. Rossen and Mamun [5] proposed a percolation approach, consisting 
of occupying elements with thresholds below a certain value. In the study of the foam flow, Rossen and Gauglitz [3] developed a model for foam mobilization in porous media, for both continuous and discontinuous gas regimes. In the process, they employed percolation theory to find the cluster size, and verified the fact that a minimum pressure gradient is needed to displace lamellae out of a pore throat. Because of lack of data and also the complexity of the system, their conclusions are not complete.

Wu et al. [4] investigated the transient flow of Bingham fluids in porous media, including single- and multiphase flow, using an ad-hoc extrapolation of the singlecapillary expressions for the Bingham flow. In the context of reservoir engineering, most existing studies are phenomenological and consist of solving effective continuum equations.

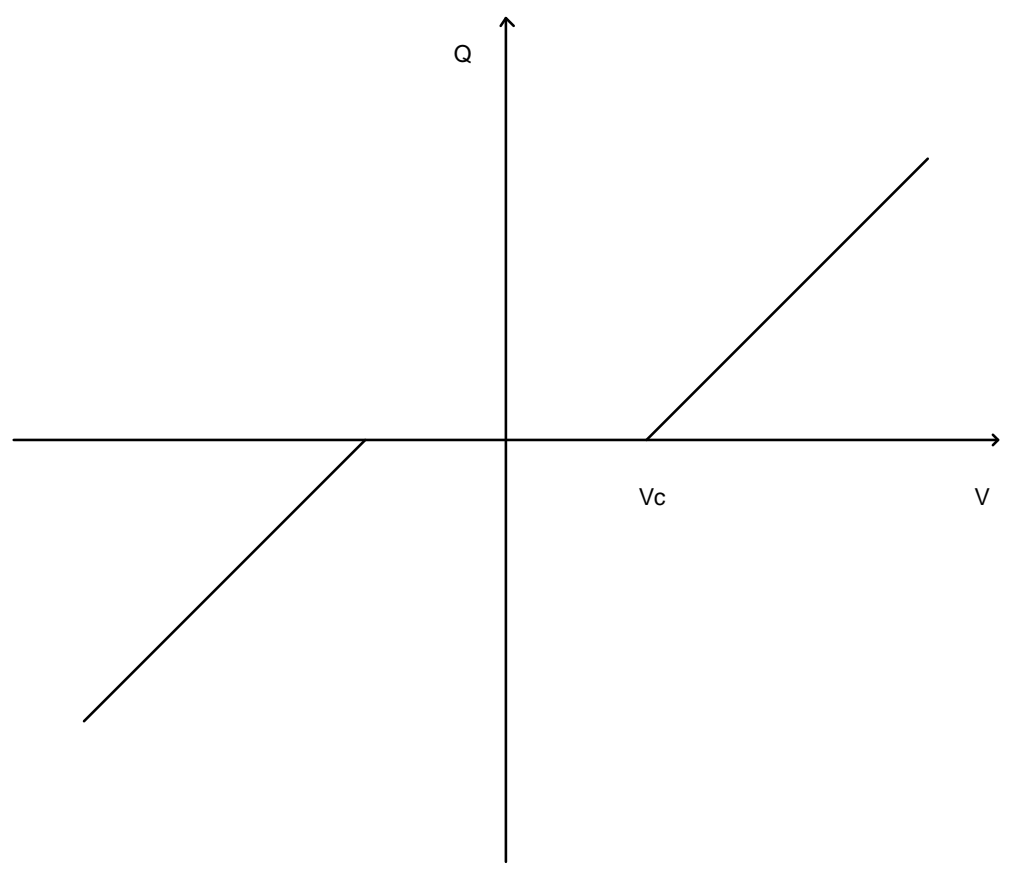

Fig.1 Voltage - current relation of an individual resistor.

A fundamental and now widely accepted work was done by Roux and Herrmann [6]. They used a 2-D network, which is composed of resistors. Each of the resistors has a threshold below which it becomes an insulator. The resistors are distributed randomly in a network. Their results show that the relation between current and the voltage is nonlinear. The macroscopic current $Q$ has the following relation with $V$ and $V_{c}$, 
$Q \sim\left(V-V_{c}\right)^{\delta}$

where $\delta=2$ or $\delta \sim 0.5$ for intermediate part (if for each of the resistor, $Q$ is linearly determined by $V$ when $|V|$ is larger than threshold, as shown in Fig.1, and $V_{c}$ is the minimum voltage to have current in the network. In their studies, the thresholds of the resistors are distributed between 0 and 1 .

Sahimi [7] used EMA to predict that in a certain range, $Q$ depends quadratically on $V$. His Monte Carlo simulation results agree with the prediction quite well. Zhou and Stenby [8] studied the displacement of oil trapped in water-wet reservoirs using percolation theory. They obtained the CDC curve based on pore structure of the medium, which is in good agreement with the measured data. Kharabaf et al. $[9,10,11]$ developed a different algorithm for the construction of the minimum threshold path (MTP), based on which its properties can be studied, such as the connection of the threshold-lattice problem to percolation and also the relation of the MTP with the minimum path of percolation. The algorithm has a similar simulation process as that of an invasion process; the difference is that the advance of the front depends on the front history. It is referred as Invasion Percolation with Memory (IPM). They studied the mobilization of Bingham fluid, immiscible displacement of Bingham fluid by Newtonian fluid and also displacement of Bingham fluid by another kind of Bingham fluid. The same algorithm is used in the study of foam formation and propagation in porous media.

Even though some work has been done, the understanding of single- and multiphase flow of fluids with yield stress in porous media is still limited. In considering and simulating mobilization of fluids with yield stress, Kharabaf used a static model, which will be modified further here. In his work, the potential of a pore is only determined by thresholds, and the pressure distribution effect of the flowing fluid was not considered. In this paper, a new dynamic algorithm for mobilizing fluids with yield stress based on IPM is developed, in which the contribution of flowing fluid is taken into account. Simulation results for different yield stress distributions are compared and discussed. The difference between the new algorithm and Kharabaf's static model is shown. 


\section{Algorithm}

1) Invasion Percolation with Memory (IPM)

Because the IPM process is the basis of the new algorithm, the algorithm of IPM is described with some details [9]. A network composed of bonds and pores are used, in which bonds have thresholds, $\tau_{i}$, randomly assigned from a distribution in $(0,1)$. The invading front invades one site at a time. An arbitrary site currently on the front is denoted by $F$, a site which is a neighbor of $F$ by $F^{\prime}$, the site from which the front will advance by $F_{G}$, the site which will actually be invaded by $F_{G}^{\prime}$. Each site that has been invaded has a value of $V_{1}(F)$. For a site $F$ on the front and one of its neighbors $F^{\prime}$, a sum $S=V_{1}(F)+\tau_{F F^{\prime}}$ can be formed. The minimum one of all possible $S$ can easily be found, the corresponding $F$ and $F^{\prime}$ become $F_{G}$ and $F_{G}^{\prime}$, respectively, and $V_{1}\left(F_{G}^{\prime}\right)=V_{1}\left(F_{G}\right)+\tau_{F_{G} F_{G}^{\prime}}$. If the boundary condition $V_{1}(R)=0$ for all sites $\mathrm{R}$ on the right boundary is applied, the first site invaded on the left boundary has the MTP value, which is $\sum_{i} \tau_{i}$. By this method, the MTP of the lattice can be found, and $V_{1}$ of each site is its smallest resistance to the starting side. The patterns before and after a growth step are shown in Fig. 2.

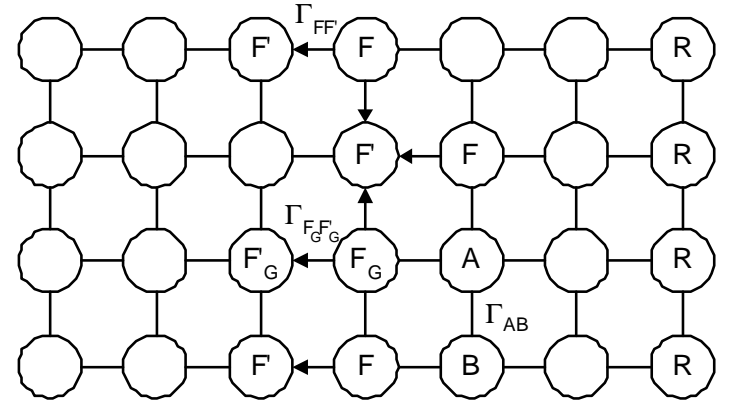

(a)

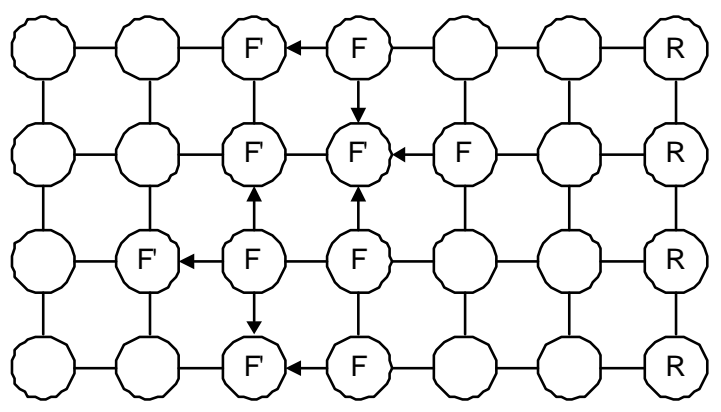

(b)

Fig. 2 Description of the invasion rule, before (a) and after (b) a growth step. 


\section{2) New algorithm on mobilization of fluids with yield stress}

Fluid with yield stress is a kind of special one, belonging to non-Newtonian fluids. A typical example is Bingham fluids, which exhibit a finite yield stress at zero shear rate and they correspond to the extreme case of pseudoplasticity. Bingham model is the most often used model for a Bingham material. The behavior of this kind of fluid described by Bingham model is shown in Fig. 3. For low stress values, the fluid does not deform, and above the critical value $\tau_{0}$, it flows like an inelastic non-Newtonian fluid.

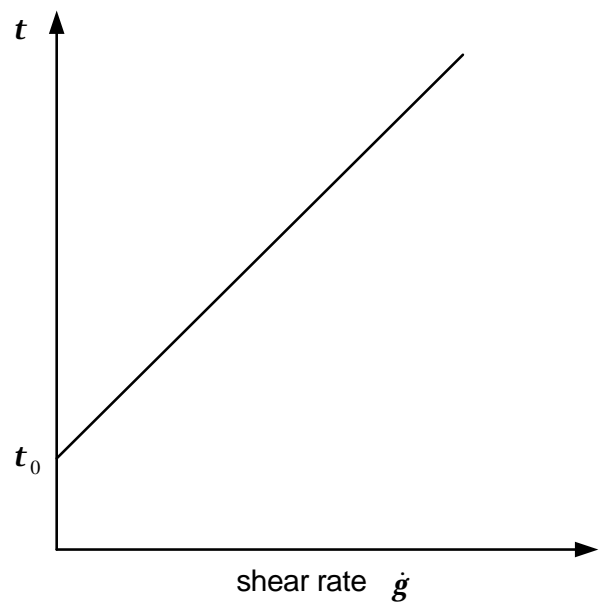

Fig.3 Rheological behavior of a Bingham plastic.

$$
\begin{gathered}
\tau=\tau_{0}-\mu_{p} \dot{\gamma}, \text { when } \tau>\tau_{0} \\
\text { and } \\
\gamma=0 \text {, when } \tau<\tau_{0}
\end{gathered}
$$

If the viscous forces are neglected for single-phase flow of a Bingham fluid, yield stress is the only force affecting its flow. While for Newtonian fluids, when the pressure drop applied is greater than zero, there is flow in the system, for Bingham fluids, the situation is completely different.

When simulation in network-like porous media is considered, the flow of the fluid in a single throat (bond) can be expressed by the following equation [12]

$q_{i}=\frac{\pi r_{i}^{4}}{8 \mu_{p} l}\left(1-\frac{4}{3}\left(\frac{\tau_{0}}{\tau_{i}}\right)+\frac{1}{3}\left(\frac{\tau_{0}}{\tau_{i}}\right)^{4}\right) \Delta p_{i}$ when $\tau_{i}>\tau_{0}$

and 
$q_{i}=0$ when $\tau_{i} \leq \tau_{0}$

where $q_{i}$ is the volumetric flow rate of the fluid in the throat, $\Delta p_{i}$ is the pressure drop applied to the throat, and $\tau_{i}$ is the wall shear stress

$\tau_{i}=\frac{\Delta p_{i} r_{i}}{2 l}$

Just for simplicity, we use the equation below in the algorithm, for throat $i$,

$q_{i}=\Delta p_{i}-\frac{1}{r_{i}}$ when $\Delta p_{i}>\frac{1}{r_{i}}$

and

$q_{i}=0$ when $\Delta p_{i} \leq \frac{1}{r_{i}}$

where $r_{i}$, is distributed with a size distribution, $\alpha(r)$.

This condition (3) is similar to the one used by Roux and Herrmann [6] for a single resistor. So it can be described by Fig.1, too. In equation (3), the variables are all in dimensionless form. It is clear from the simplified equation that the onset of the flow of the Bingham fluid in the network is controlled by the flow situation of each of the throats in the path. For the first path in the system, there is a minimum pressure difference between the two boundaries.

$\Delta P_{\min }=\sum_{i} \frac{1}{r_{i}}$

where the summation is over all of the throats in the first single path.

It is obvious that the first path in our algorithm is completely the same as the static one obtained by Kharabaf et al. But after that, the pressure drop of the system, which is needed to open a new path is not only determined by the throat radius (or $\frac{1}{r_{i}}$ ), but also by the flow, and more exactly the pressure distribution of the mobilized path, which is the factor that is not considered in Kharabaf's mobilization algorithm. In our new algorithm, the two factors, radius and pressure distribution are combined together to be $\tau_{i}$, which is updated every time when a new path is open. In the following description, 
$\tau_{0 i}$ is used, instead of $\frac{1}{r_{i}}$, and $\tau_{0 i}$ is corresponding to the size distribution function $\beta\left(\tau_{0}\right)$

The mobilization of the fluid is the same as finding the paths which connect one side of the network with the other. Each of the paths is corresponding to a specific pressure drop across the network. Here, the pressure at the left side of the network is fixed and equal to zero. As described in previous work, IPM is an effective method to find the minimum path from the right side of the network to the left side. After we find the first path and the critical pressure $\Delta P_{\min }=P_{\min }$, we need a little higher pressure to open a new path. The new path may be a branch or a loop connected to the old or a separate one. We should notice that when the new path is just opened, the flow rates in the newly mobilized throats in the paths are zero and they have no contribution to the total flow rate in the network. If the new path is just opened and connected to the old (AB) one at point $\mathrm{C}$ (Fig.4), for any bond $\mathrm{j}$ in the path $\mathrm{CD}$, the flow rate is

$q_{j}=\Delta p_{j}-\tau_{0 j}=0$

therefore, $\Delta p_{j}=\tau_{0 j}$

For any bond in the path $\mathrm{AC}$, the flow rate is

$q_{k}=\Delta p_{k}-\tau_{0 k}$

If we let

$\Delta p_{k}=q_{k}+\tau_{0 k}=\tau_{k}^{\prime}$

the pressure drop between the two sides of the network for the new path ACD determined using IPM is

$P_{A C D}=\sum_{A C} \tau_{i}^{\prime}+\sum_{C D} \tau_{0 i}=\sum_{A C D} \tau_{i}^{*}$

which is the MTP with updated thresholds $\tau_{i}^{*}$, in the new part CD, $\tau_{i}^{*}=\tau_{0 i}$, and in the shared part AC, $\tau_{i}^{*}=\tau_{i}^{\prime}$

For throats in the old path $\tau_{i}^{*}=\tau_{i}^{\prime}$, so for the old path ACB

$$
P_{A C B}=\sum_{A C B} \tau_{i}^{\prime}=\sum_{A C B} \tau_{i}^{*}
$$


From all above, we can see that the apparent way to find the next path to be opened is to determine the MTP in IPM with updated thresholds. So, in our algorithm, we first use IPM to find MTP with all initial $\tau_{0}$. Then we increase the pressure drop $P_{A C B}$, and at the

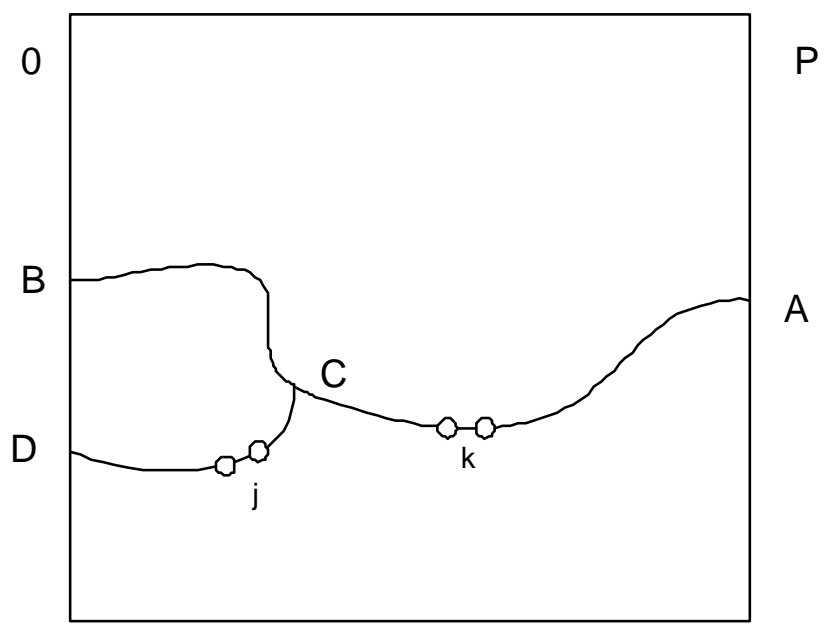

Fig.4 The flow pattern when a new path (ACD) is open.

same time update the thresholds of the bonds in opened paths to be $\tau$ ' according to Eq. (4). After that, we use IPM again to determine the MTP at this pressure drop to find the potential $\left(P_{A C D}\right)$ of site $\mathrm{D}$ at the left boundary. If the pressure drop between the two boundaries is high enough, we can always find a new path ACD. Then decrease the pressure drop. These steps are repeated until the new path with smallest $P_{A C B}=P_{A C D}$ is found. Just as mentioned before, the new path is not necessarily connected to the old ones like the one shown, it may be a loop or even a new path, but the algorithm is the same for all of the situations.

In determining the pressure distribution of the mobilized fluid, the fluid is assumed to be incompressible, and for any site $i$ in the continuous path, the below equation is obeyed,

$$
\sum_{j}^{Z} q_{i j}=0
$$

where $q_{i j}$ is the volumetric flow rate from site $i$ to its neighbor site $j$, and $\mathrm{Z}$ is the coordination number, $Z=4$ for 2-D system and $Z=6$ for 3-D system. At a site, which is the intersection of the four bonds, there may no flowing fluid in several bonds, but 
because of viscous forces, the static fluid in the throat(s) left may make contribution to the flow of the fluid in the other throats. But if the Bingham number of the fluid is high enough, this contribution is negligible. Consequently, in our study, this effect is not considered. Flow rate in a bond is computed according to equation (3).

In 2-D and 3-D systems, fluids flow from right to left, and periodic boundary conditions are used for the other boundaries. The size distribution of $\tau_{0}$ is $\beta^{n}$, where $\beta$ is uniform in the region $(0,1)$. Then for $\tau_{0}$, the probability density function (PDF), the arithmetic mean and standard deviation are

$$
f\left(\tau_{0}\right)=\frac{1}{|n|} \tau_{0}^{(1 / n)-1},\left\langle\tau_{0}\right\rangle=\frac{1}{1+n}, \sigma_{\tau_{0}}=\frac{n}{(1+n) \sqrt{1+2 n}}
$$

respectively. From Eq. (9), it is apparent that for the existence of the arithmetic mean we must have $n>-1$, while for that of the variance, $n>-1 / 2$. Therefore, for finite first and second moments of general threshold distributions, we must restrict Eq. (9) to $n>-1 / 2$. However, the problem with smaller $\mathrm{n}$ will also be considered.

\section{RESULTS AND DISCUSSION}

\section{The case $n=1$}

The relation between the flow rate of the network and the pressure exerted across the network is shown in Figs. 5(a) and 5(b). At a certain pressure drop $P_{\min }$, the first path is open; this is the onset of the mobilization process. Then with the increase of the pressure, more and more bonds are mobilized and the flow rate increases as well. In Fig. 5 (b), we found that

$$
4 u \mu / r * \tau_{0} \sim\left(\left(\Delta P-\Delta P_{\min }\right) / 2 \tau_{0} N\right)^{\alpha}
$$

$\alpha$ is about 1.9 , which is close to 2 . In Kharabaf's work, he showed the mobilized paths of network without flow. His results are much different from what we have here. Fig.6 and Fig.7 are the mobilized paths at different stages. Under the two situations, the first paths opened are the same. After that, the static ones have more compact patterns, while with the new algorithm, we need higher pressure to mobilize the same amount of bonds or to have the same amount of sites in continuous paths (Fig. 8). 
The quantity $8 \mu Q / N \pi r^{*} \Delta P$, which is an indication of the permeability of the fluid, is increasing with the pressure across the network (Fig. 9a) and so is the fraction of pores in the continuous paths (Fig. 9b).
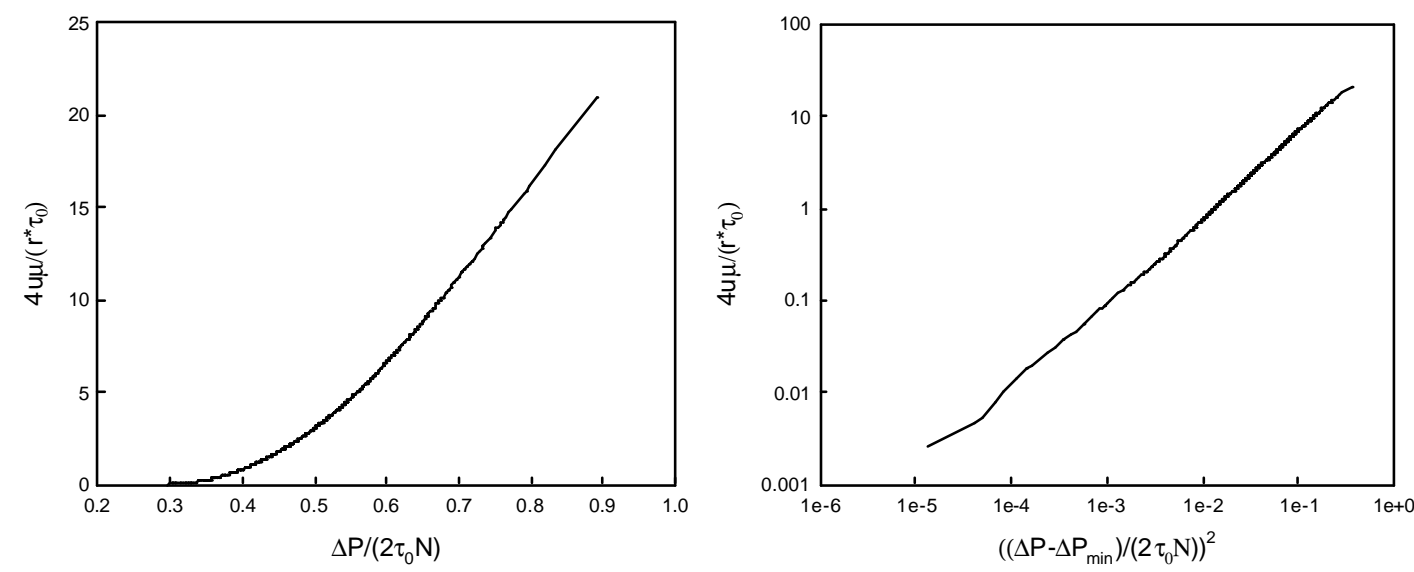

Fig. 5 a) the relation between $4 u \mu / r * \tau_{0}$ and $\Delta P / 2 \tau_{0} N$; b) the relation between $4 u \mu / r * \tau_{0}$ and $\left(\left(\Delta P-\Delta P_{\min }\right) / 2 \tau_{0} N\right)^{2}$

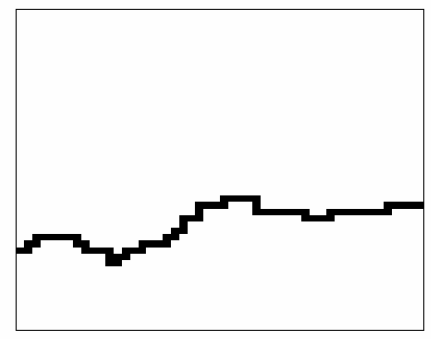

a

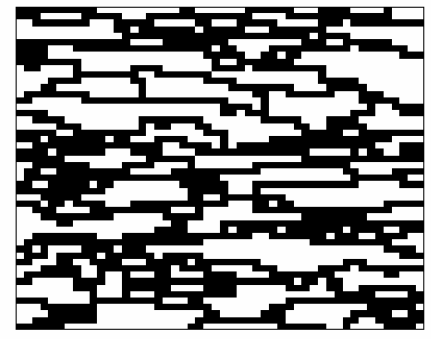

c

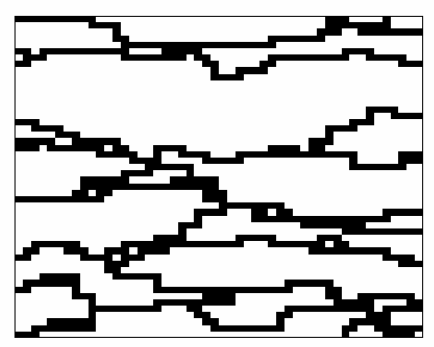

$\mathrm{b}$

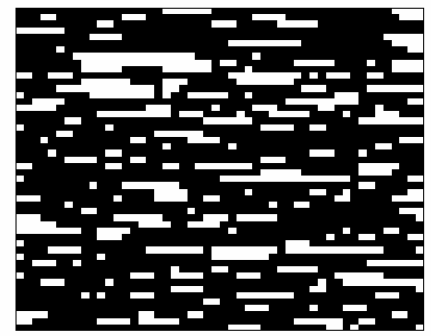

d

Fig.6 The flow patterns at different stages: (a) First path (MTP), (b) One fourth of the sites in continuous paths, (c) half, (d) three fourths. 


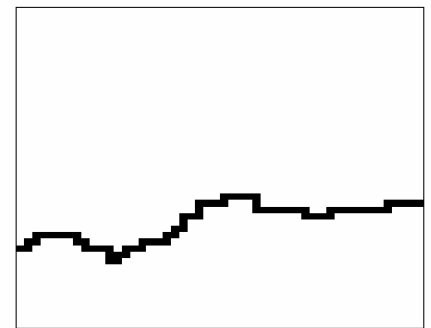

a

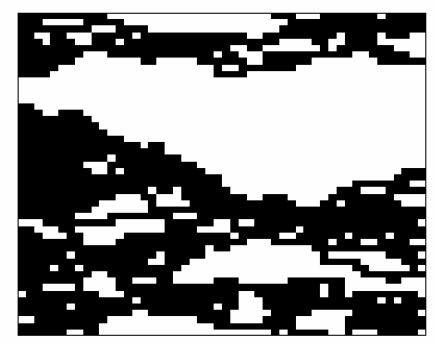

$\mathrm{c}$

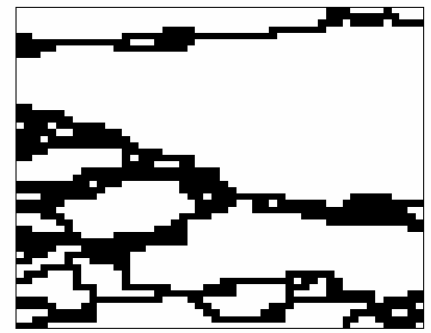

b

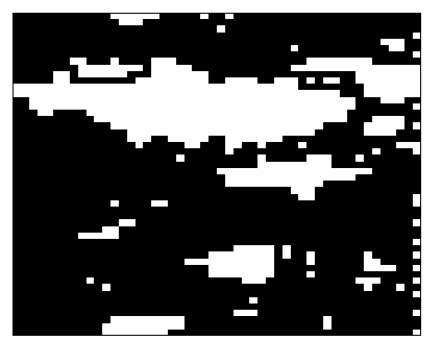

d

Fig.7 The static situation:

(a) The first path (MTP) (b) One fourth of the sites are in continuous paths (c) Half (d) Three fourths.

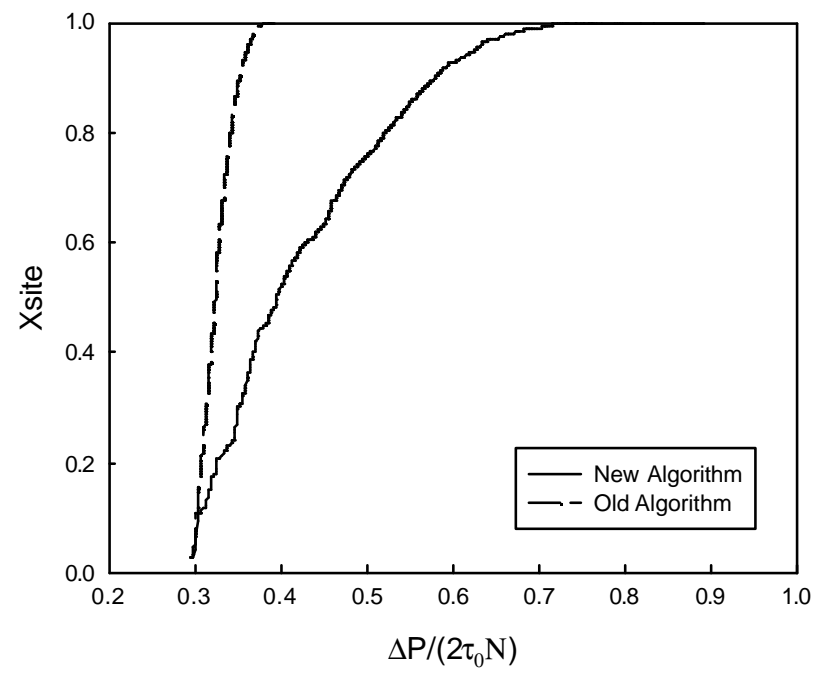

Fig.8 Fraction of pores on the continuous path and the pressure drop exerted. 

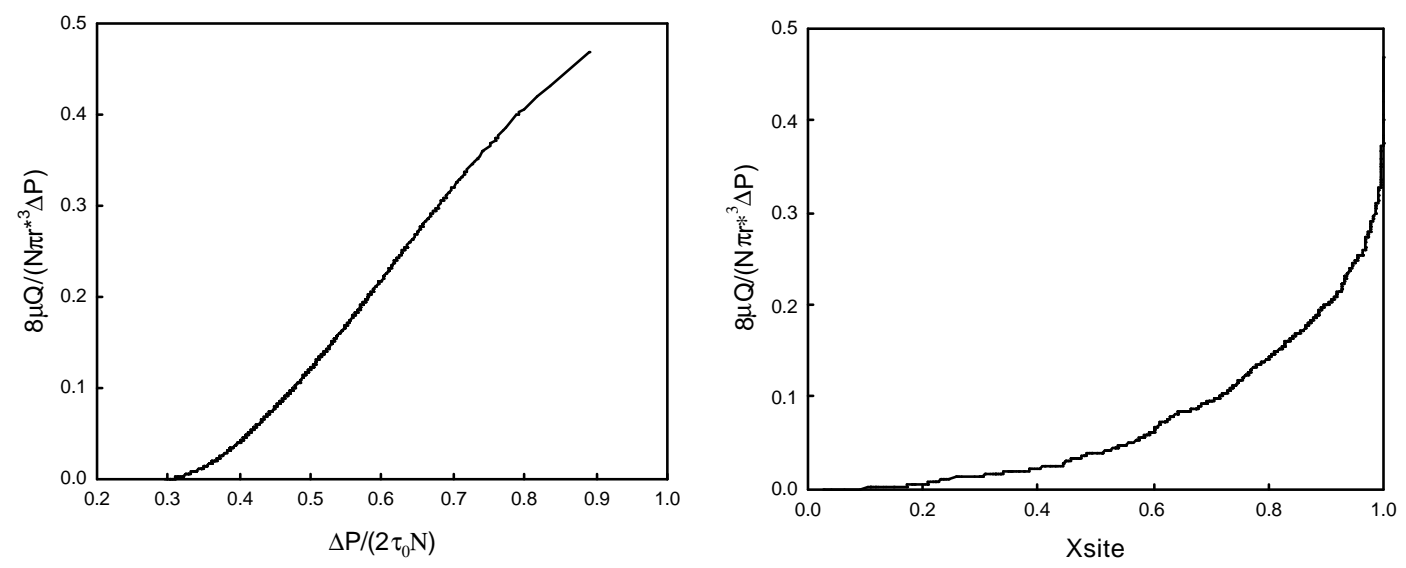

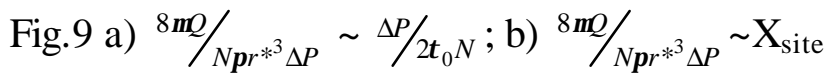

Because the first path in the system is the MTP, the critical pressure to open the first path is increasing when the lattice gets larger and is totally determined by the threshold distribution. For different sizes of lattices, similar $4 u \mu / r_{*} \tau_{0} \sim \Delta P / 2 \tau_{0} N$ relation to those in Fig. 5 can be observed.

\section{The case for different $n$}

For different values of $\mathrm{n}$ in $\beta^{n}$, the relations between the flow rate $\mathrm{Q}$ and pressure drop $\mathrm{P}$ are similar to that of $n=1$. The main difference is the flow pattern of the mobilized fluid. As shown in Figs. 10-Fig.14, with increase of the absolute value of n, the flow paths are more tortuous. In Fig. 10 and Fig. 1.11, the paths are almost straight lines. This is because the values of $\tau_{0}$ are almost the same, close to 1 , thus we do not need much higher pressures to open new paths. 

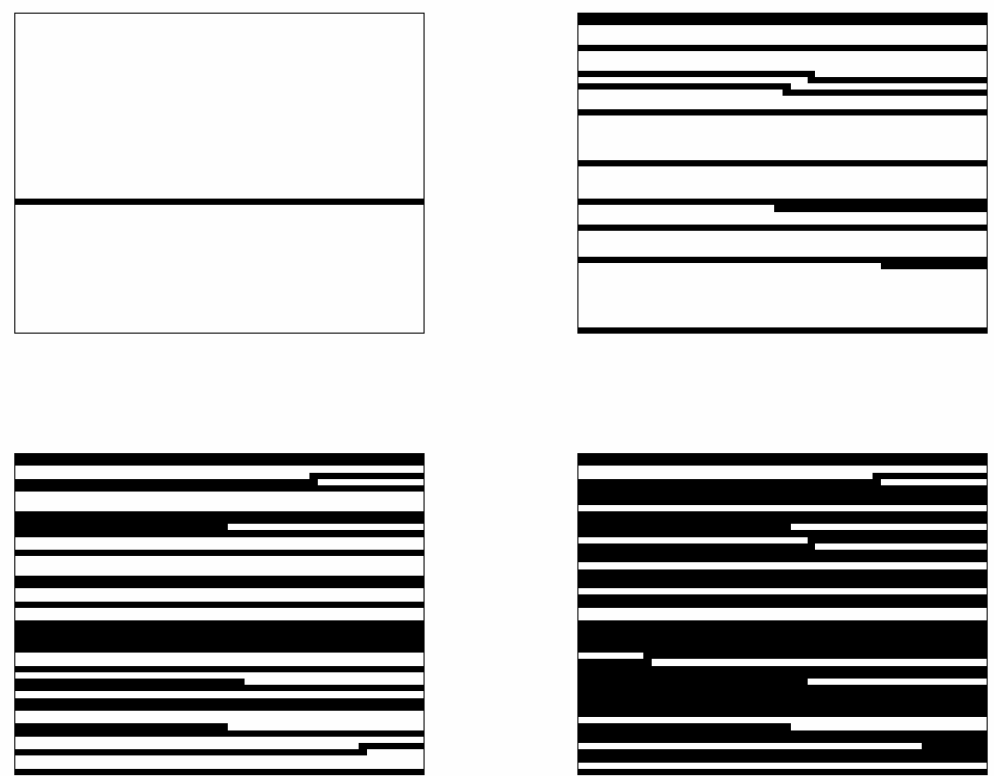

Fig.10 Flow patterns at different stages, $n=-0.1$.
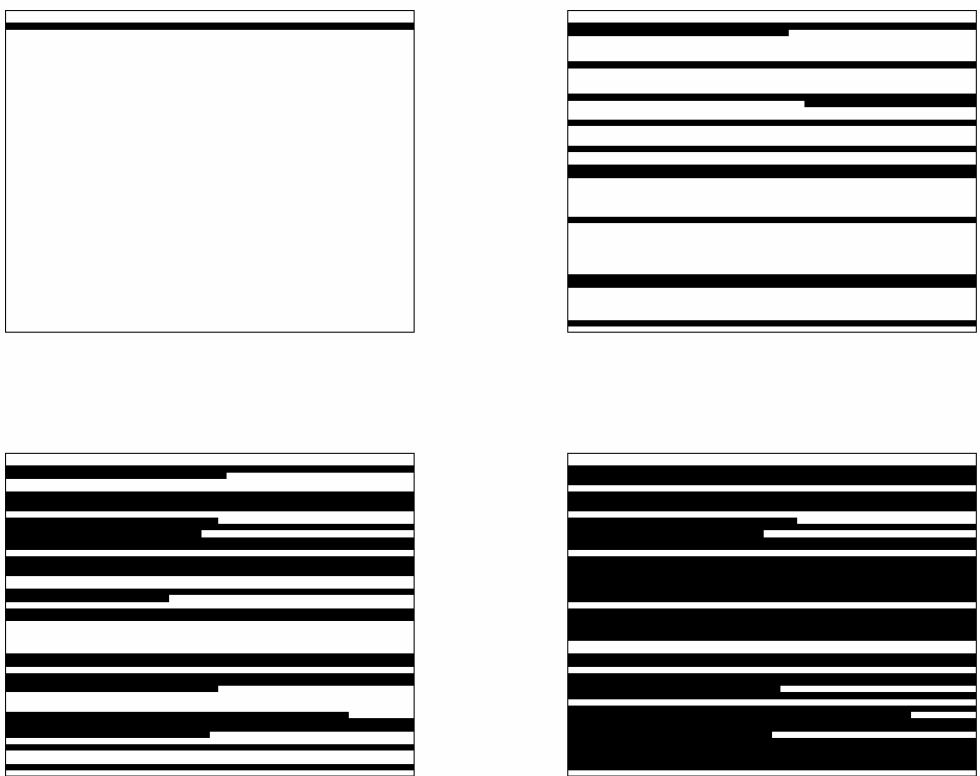

Fig.11 Flow patterns at different stages, $n=0.1$. 

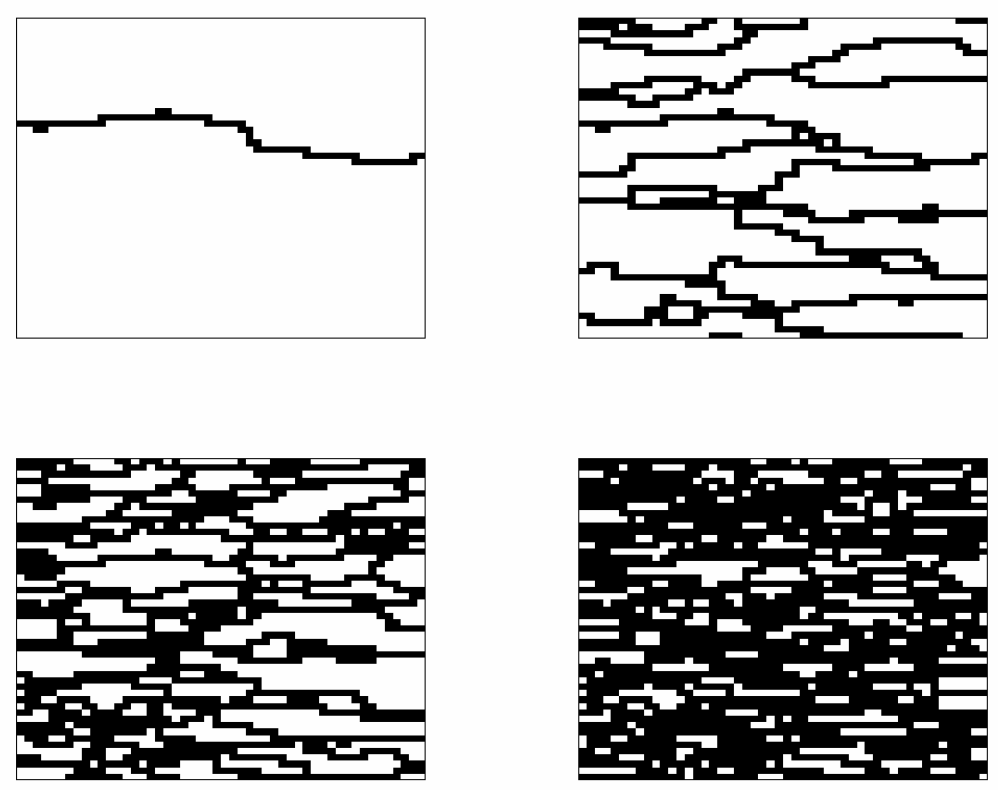

Fig.12 Flow patterns at different stages, $n=-1$.
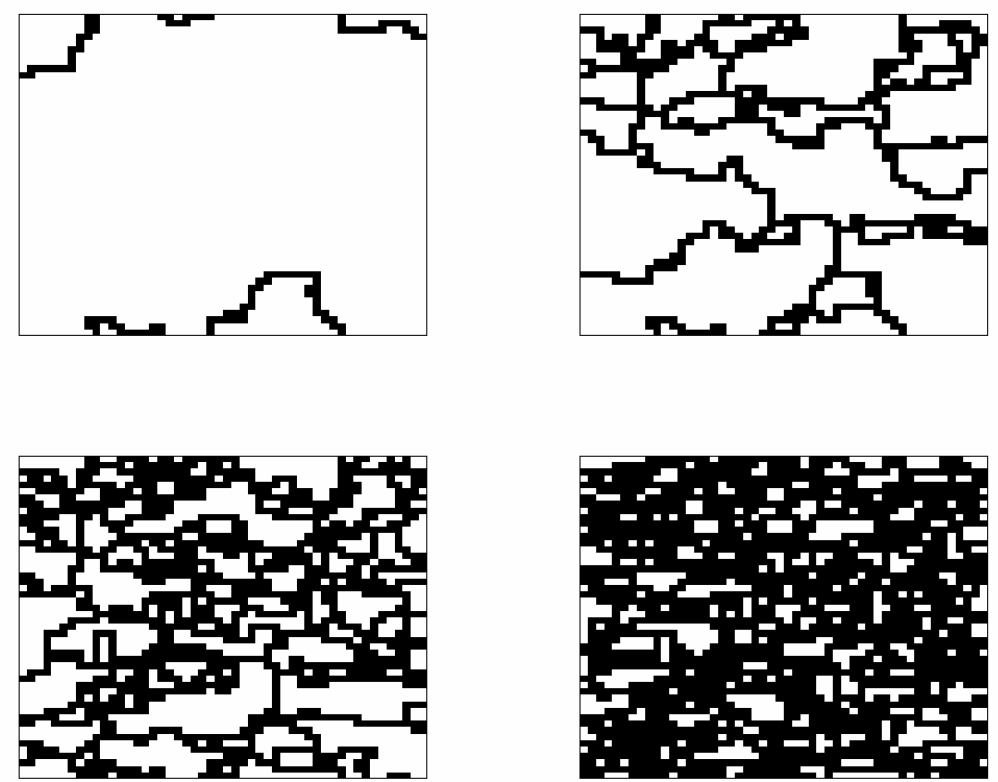

Fig.13 Flow patterns at different stages, $n=5$. 

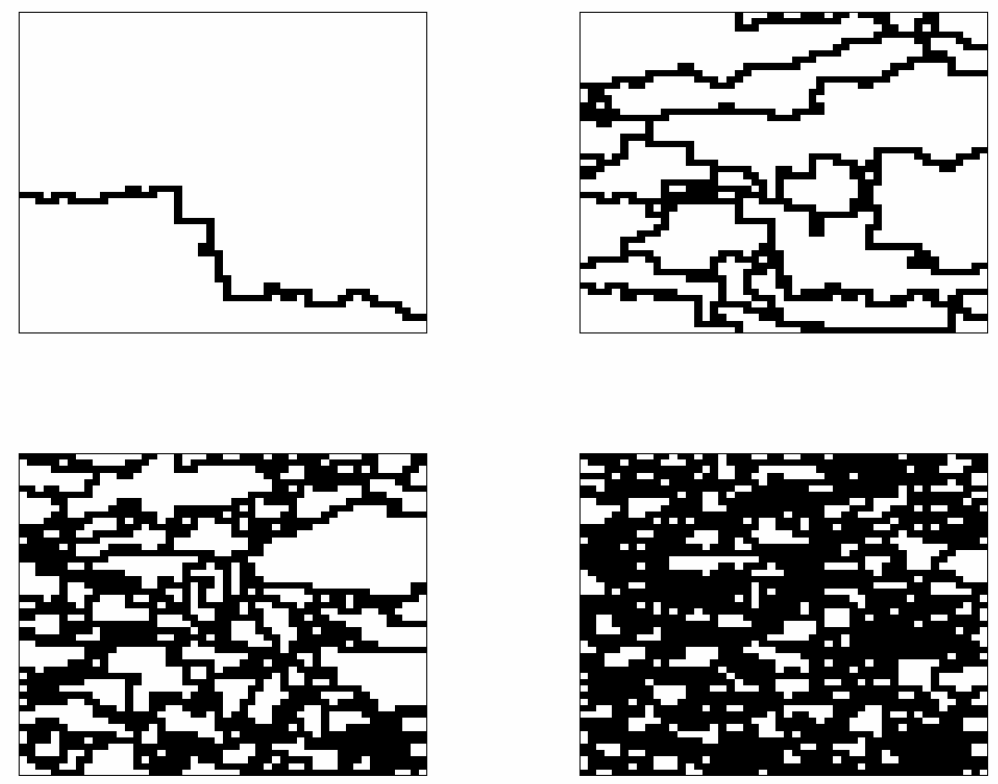

Fig.14 Flow patterns at different stages, $n=-5$.

In previous simulations, the volumetric flow rate in a bond is calculated based on equation (3). If we modify the equation to that shown below,

$q_{i}=r_{i}^{3}\left(\Delta p_{i}-\frac{1}{r_{i}}\right)$

the algorithm works well too. Here, in the simulation process, $\tau_{i}$ is updated in the way shown below.

$\tau_{i}^{*}=\Delta p_{i}=\frac{q_{i}}{r_{i}^{3}}+\frac{1}{r_{i}}=\frac{q_{i}}{r_{i}^{3}}+\tau_{0 i}$

Fig.15 and Fig.18 show simulation results and flow patterns. In Fig. 15, the $4 u \mu / r * \tau_{0}$ and $\Delta P / 2 \tau_{0} N$ relation is not the same as the one we get for $n=1$, while the qualitative change of the curve is in agreement with Roux's result. At the most part, the curve is more like a straight line, and there is an intermediate part, where the slope is different. In the previous discussion for $n=1$, the pressure needed to mobilize the bonds after all of the pores are in continuous paths is very high. In Fig. 17 we see that with the new equation, all of the 
bonds in the system can be mobilized with relatively smaller pressures. The patterns in Fig.18 are similar to the ones we present before.

In Fig.19, the interesting patterns of mobilizing the fluid from the center are shown. As expected, the paths towards the corners are open later. We can see that even though the distance from the center is not the determining fictor, it still has obvious effect on the mobilization process.
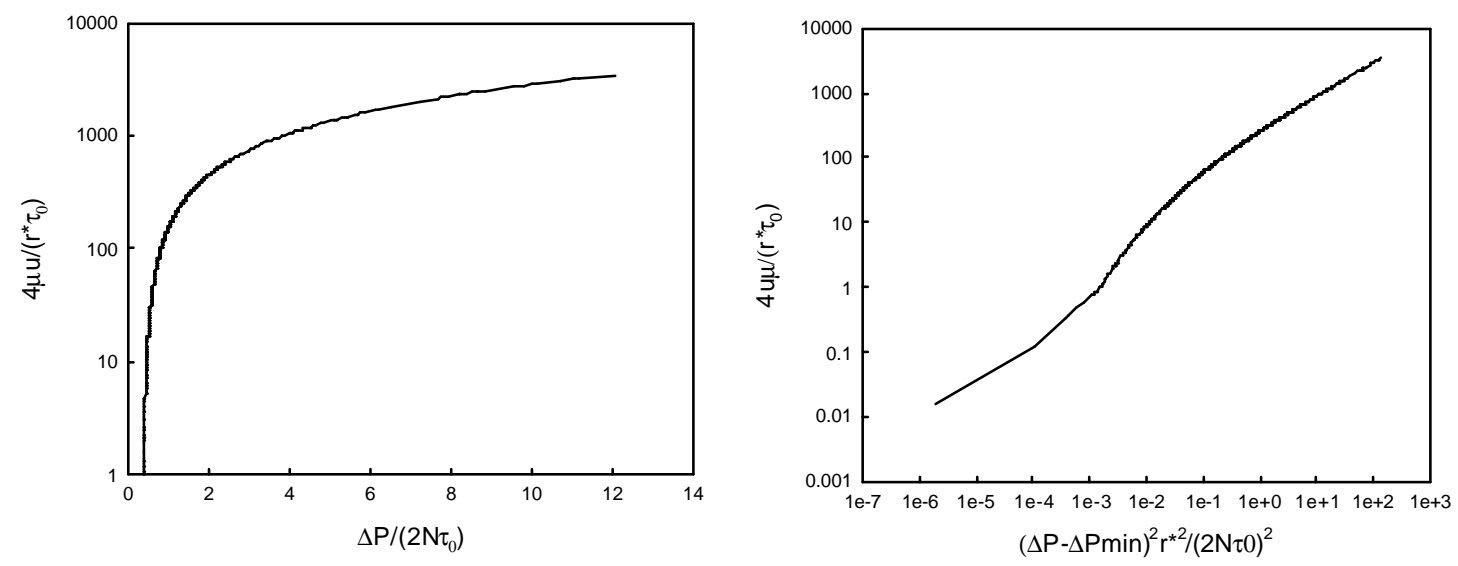

Fig.15 Relation between $4 u \mu / r^{*} \tau_{0}$ and $\Delta P / 2 \tau_{0} N$;

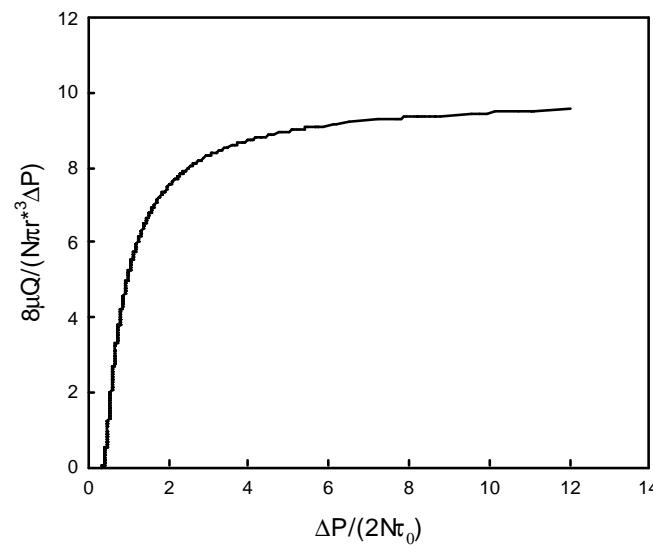

Fig.16 $8 \mu Q / N \pi *^{*} \Delta P=\Delta P / 2 \tau_{0} N$

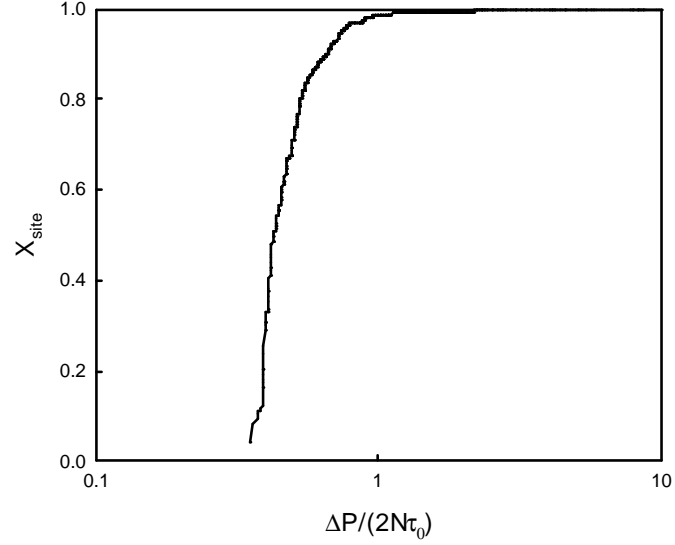

Fig. $17 \mathrm{X}_{\text {site }} \sim \Delta P / 2 \tau_{0} N$ 

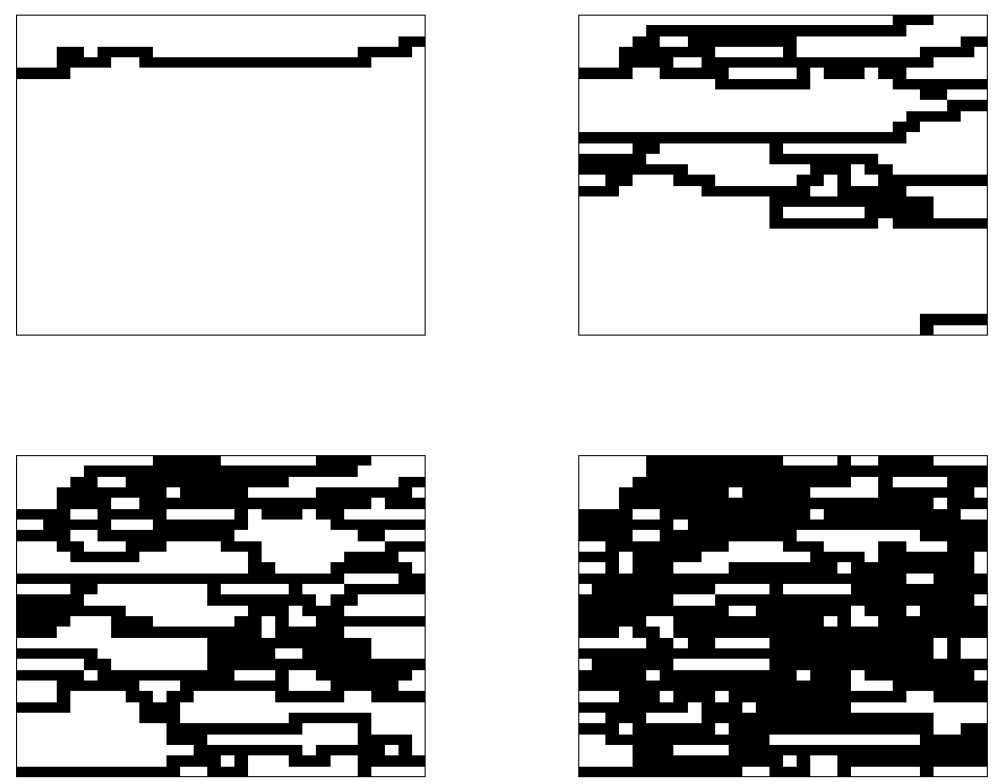

Fig.18 Flow patterns of the fluid at different stages.
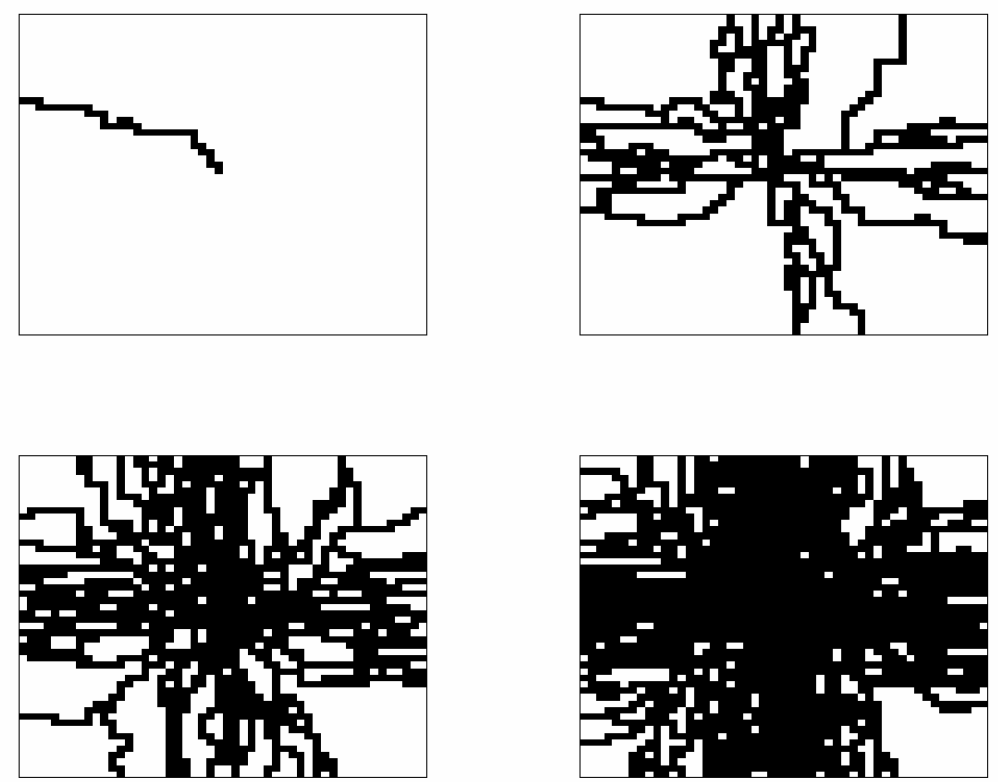

Fig.19 Flow patterns of the fluid mobilized from the center of the network. 


\section{Examples on mobilization of Bingham fluid in complex system}

Subsequently, the mobilization of Bingham fluid in more complex system was also considered. The patterns of confined flows of Bingham fluids in converging and diverging networks at different stages are shown in Fig 20 and Fig 21. To compare the result to the flow process in complex geometries, the bond size in this part is evenly distributed in a very narrow interval, from 1.0 to 1.0001. From Fig. 20 and Fig.21, we can find that the mobilization process is very similar to the ones shown in Fig. 10 and Fig. 11, where the bond sizes are very close to 1 . The difference between the ones presented here and the characteristics claimed by Lipscomb [13] is that in our results yielding is not happening everywhere. This is understandable, because even though the bond size is very close to one, there are still differences between bond sizes and, consequently, the initial yields stresses which lead to the necessity that different paths need different minimum pressure drop to open. If the bond sizes were completely the same, then yielding would have occurred over the entire flow field when the pressure difference applied is high
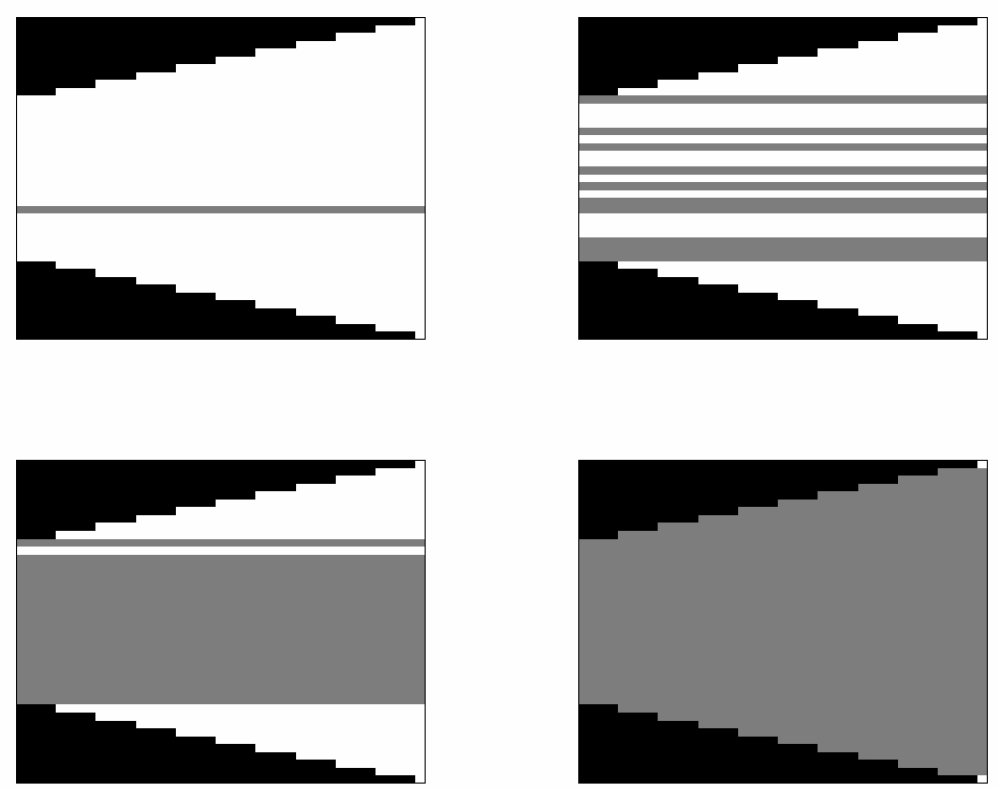

Fig.20 Flow in converging geometry: first path, one quarter, half of all sites and almost all available sites are in continuous paths. 

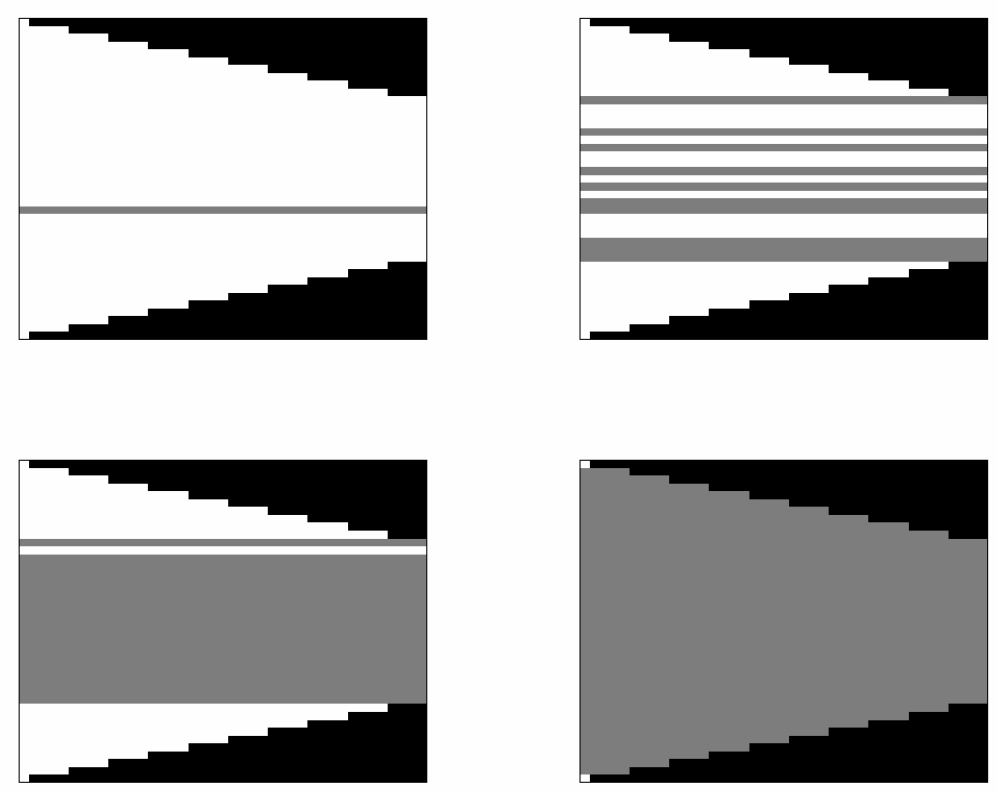

Fig.21 Flow in diverging geometry: first path, one quarter, half of all sites and almost all available sites are in continuous paths.
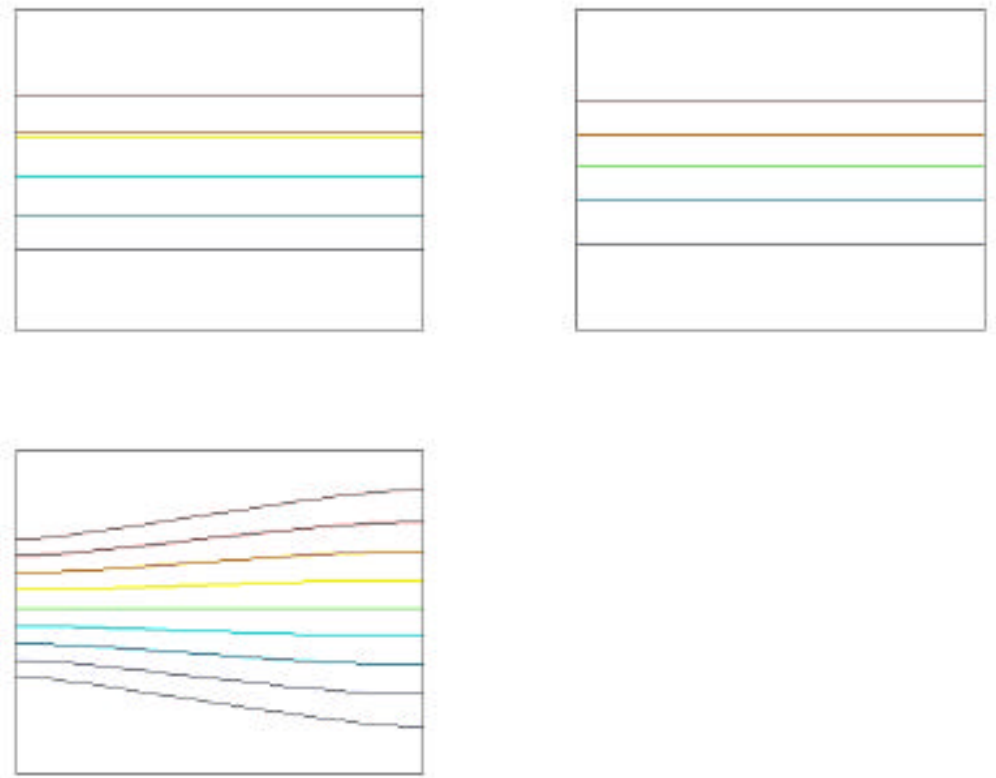

Fig.22 Streamlines of the patterns in a converging geometry. 
enough to open a path. In these figures, black part is the walls, the gray part is the mobilized fluid and white part is static fluid. Fig.22 shows the streamlines corresponding to the last three patterns in Fig.20.

Next, flow of Bingham fluids in a geometry with an obstacle at the center was studied. The mobilization process is presented in Fig.23. The black part at the center is an obstacle that cannot be mobilized, gay denotes the sites with flowing fluid and white denotes the static fluid, except for the last figure, where the white part is the mobilized fluid. Because of the size difference between bonds, the paths become open one by one and each at a certain pressure drop. In Fig.24, streamlines at various mobilization stages are plotted. Because of the Darcian property of the flow, finally all of the available sites in the system are occupied by mobilized fluid.
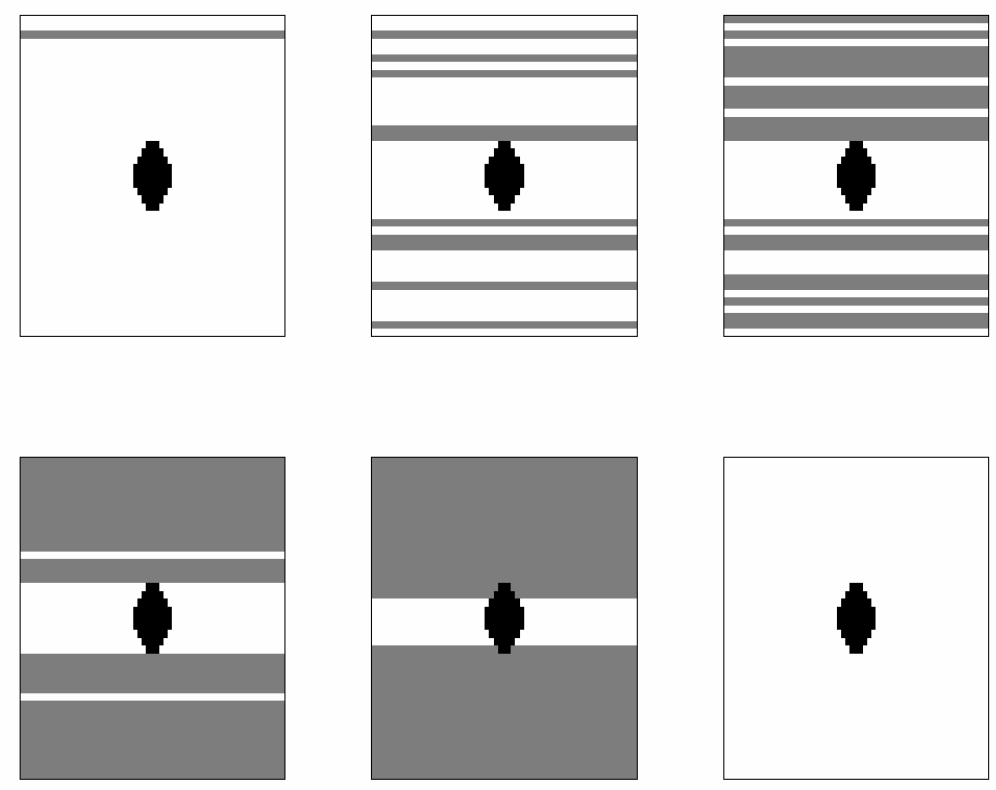

Fig.23 Flow in a geometry with an obstacle at the center: first path, about one quarter, half, three quarters, $85 \%$ of all sites and all available sites are in continuous paths. 

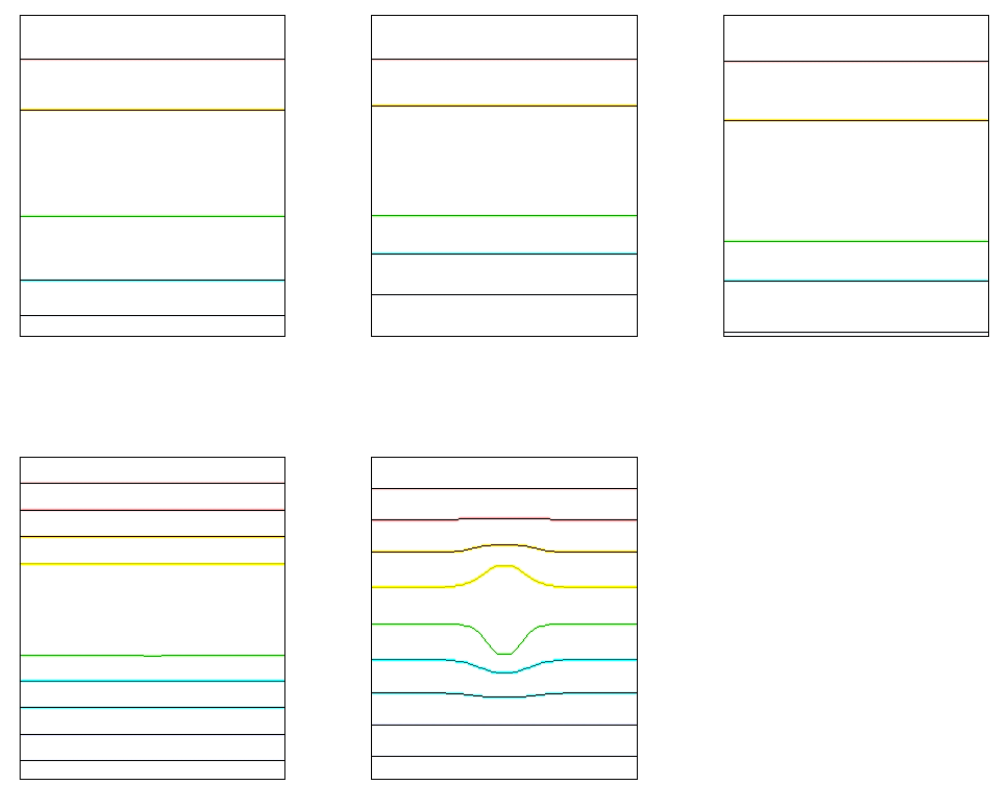

Fig.24 Streamlines in a geometry with an obstacle at the center.

\section{Displacement of a Bingham fluid by a Newtonian fluid}

The displacement of a Bingham fluid by a Newtonian fluid is encountered widely in oil recovery processes. The efficiency of the displacement is affected by the properties of the two fluids. This problem can also be tackled with our new algorithm. In this part, capillary forces are neglected.

First, let's consider a case in which the Newtonian fluid has almost zero viscosity. This means that the pressure drop is almost zero in the part occupied by the Newtonian fluid. The interface advances by selecting the site which fills out the fastest. Every other interface moves a distance determined by its velocity in the same time interval. If we use the same equation as (3) for the flow of the non-Newtonian fluid, and calculate the pressure distribution with the constraints of mass conservation at each pore in the mobilized path, we get the patterns shown in Fig.25. The algorithm is similar to the one discussed before. In this case, only the $\tau_{i}$ s of the non-Newtonian fluid are updated. In Fig. 25 and all the figures in this section, the white part is the non-Newtonian fluid, the gray part is the mobilized 

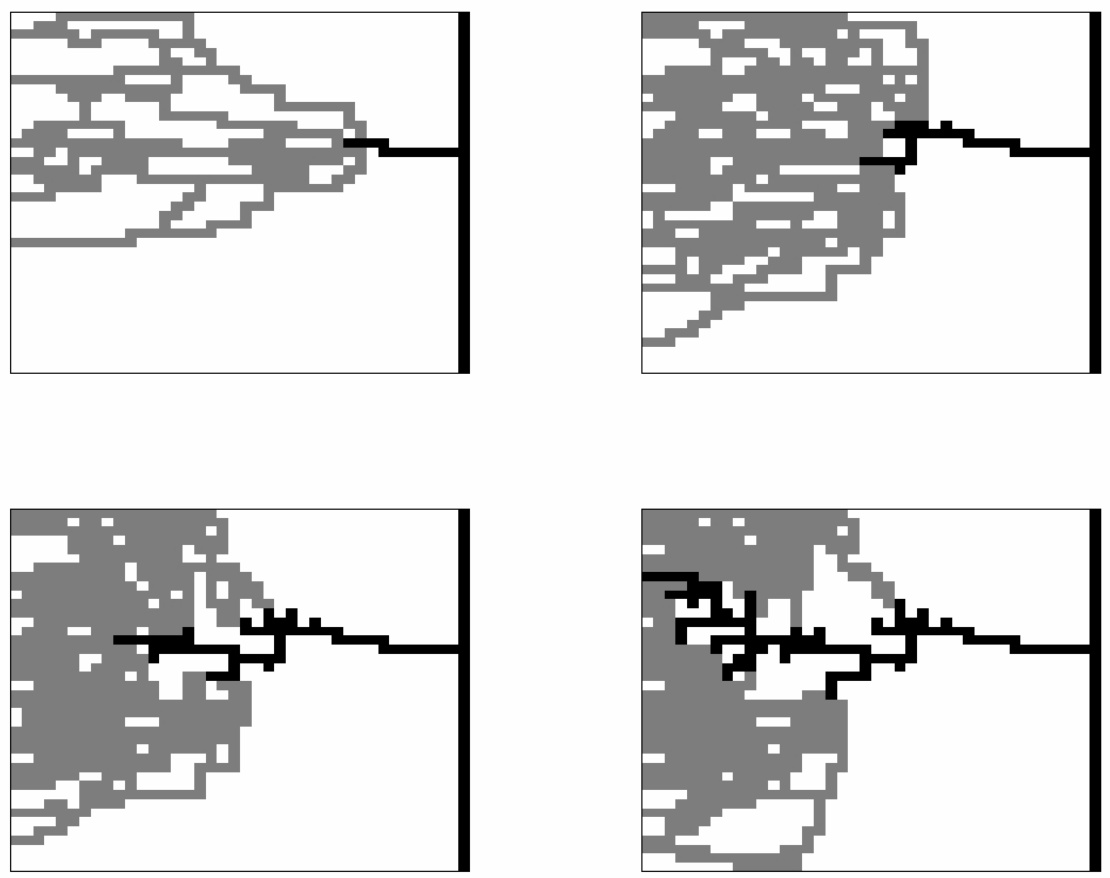

Fig.25 Patterns of displacement process at different stages, viscosity ratio=0.

non-Newtonian fluid and the black part denotes Newtonian fluid. Before the breakthrough of the Newtonian fluid, the pressure is kept constant at the value corresponding to the initial pressure to open the first path. With time increasing, more and more paths are open and the front of the Newtonian fluid moves deeply into the lattice gradually. If we consider situations with larger viscosity ratios (1 in Fig.26 and 20 in Fig.27), the number of mobilized paths in the system does not increase considerably. The patterns at larger viscosity are much different from the one with zero viscosity, while the latter one can have more mobilized paths. One reason for this difference is the larger pressure drop in the former case. The pressures are not large enough to overcome the thresholds.

\section{Mobilization of a Bingham fluid trapped in a Newtonian fluid}

In oil recovery processes, waterfloods of Bingham rheology heavy oils are of much importance. During this kind of displacement process, the Bingham fluid may be trapped 

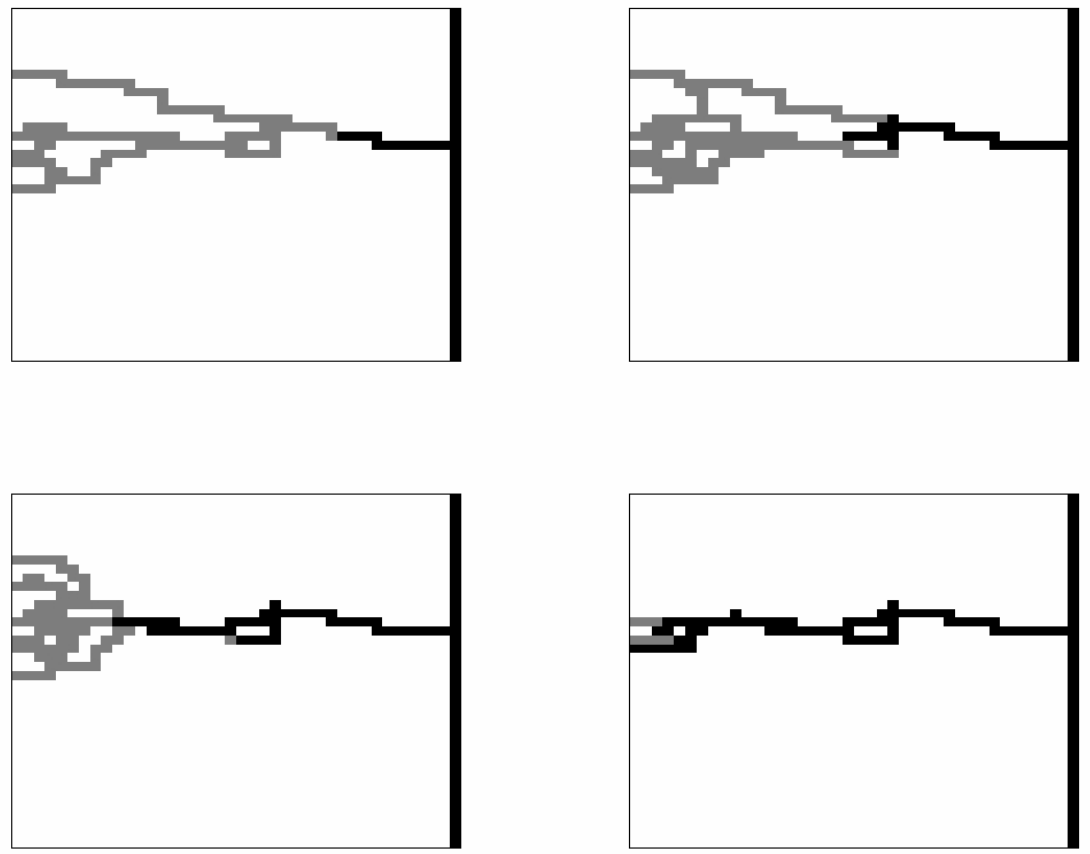

Fig.26 Displacement patterns at different stages, viscosity ratio=1.
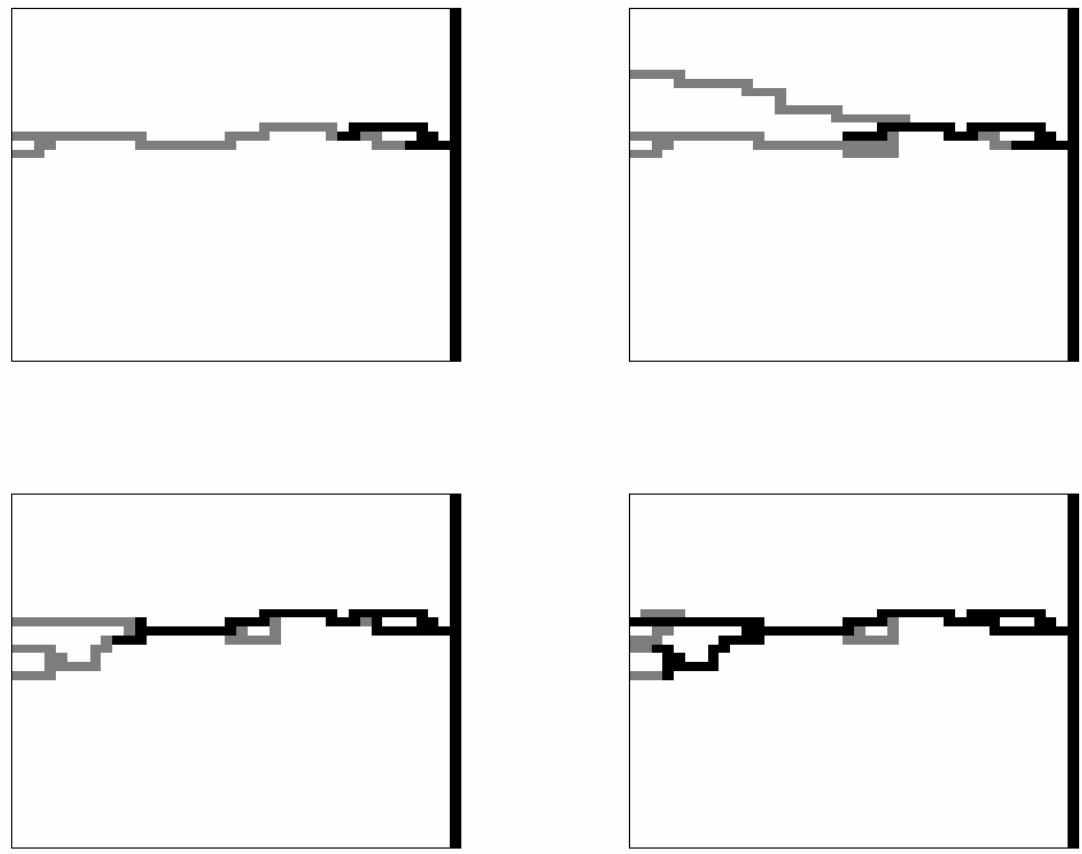

Fig.27 Displacement patterns at different stages, viscosity ratio=20. 
until the pressure becomes sufficiently high. Immiscible displacement now involves not only the yield stress, but also the capillary forces. Viscous forces in the Bingham fluid are still neglected, assuming the displacement takes place very slowly.

To use the new algorithm developed to mobilize the trapped Bingham fluid, $\tau_{i}$ should be correspondingly updated every time. For Newtonian fluid, the initial value is $\tau_{0 i}=0$, and

$\tau_{i}^{*}=\Delta p_{i}$

For Bingham fluid, $\tau_{0 i}$ keeps constant, $\tau_{0 i}=\frac{1}{r_{i}}$

At the interfaces of the two fluids, the capillary condition should be considered, $P_{c}=\frac{2 \gamma}{r}$

In each bond occupied by Newtonian fluid, we have $q_{i}=\frac{\pi r_{i}^{4}}{8 l \mu} \Delta p_{i}$

First, consider the mobilizing process of a single point of Bingham point trapped. Fig.28 shows the trace of the point. It starts from the right boundary of the network. This is a quasi-steady state process. If the pressure of the Newtonian fluid around the Bingham fluid is high enough to overcome both the thresholds and the capillary effect, the Bingham fluid moves in the direction of decreasing pressure, otherwise, it remains trapped until the pressure applied to the system increases. In every step, the pressure distribution of the Newtonian fluid is computed. When a block of Bingham fluid trapped in a Newtonian fluid is mobilized, the Bingham fluid would undergo a fragmentation process, and result into a set of disconnected ganglia, which are mobilized almost one by one. Fig.29 shows some patterns of the mobilization process. It is possible that some trapped points can never be moved out the system. 


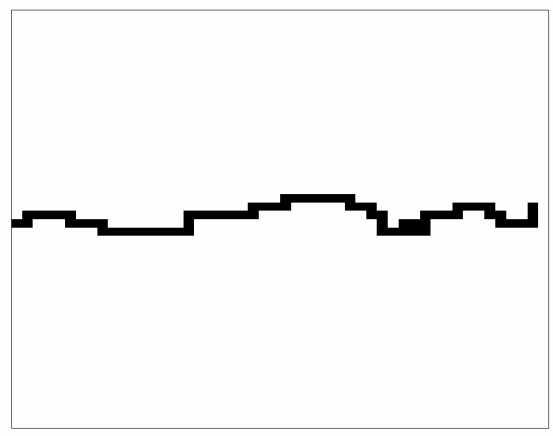

Fig.28 Mobilization trace of a single point.
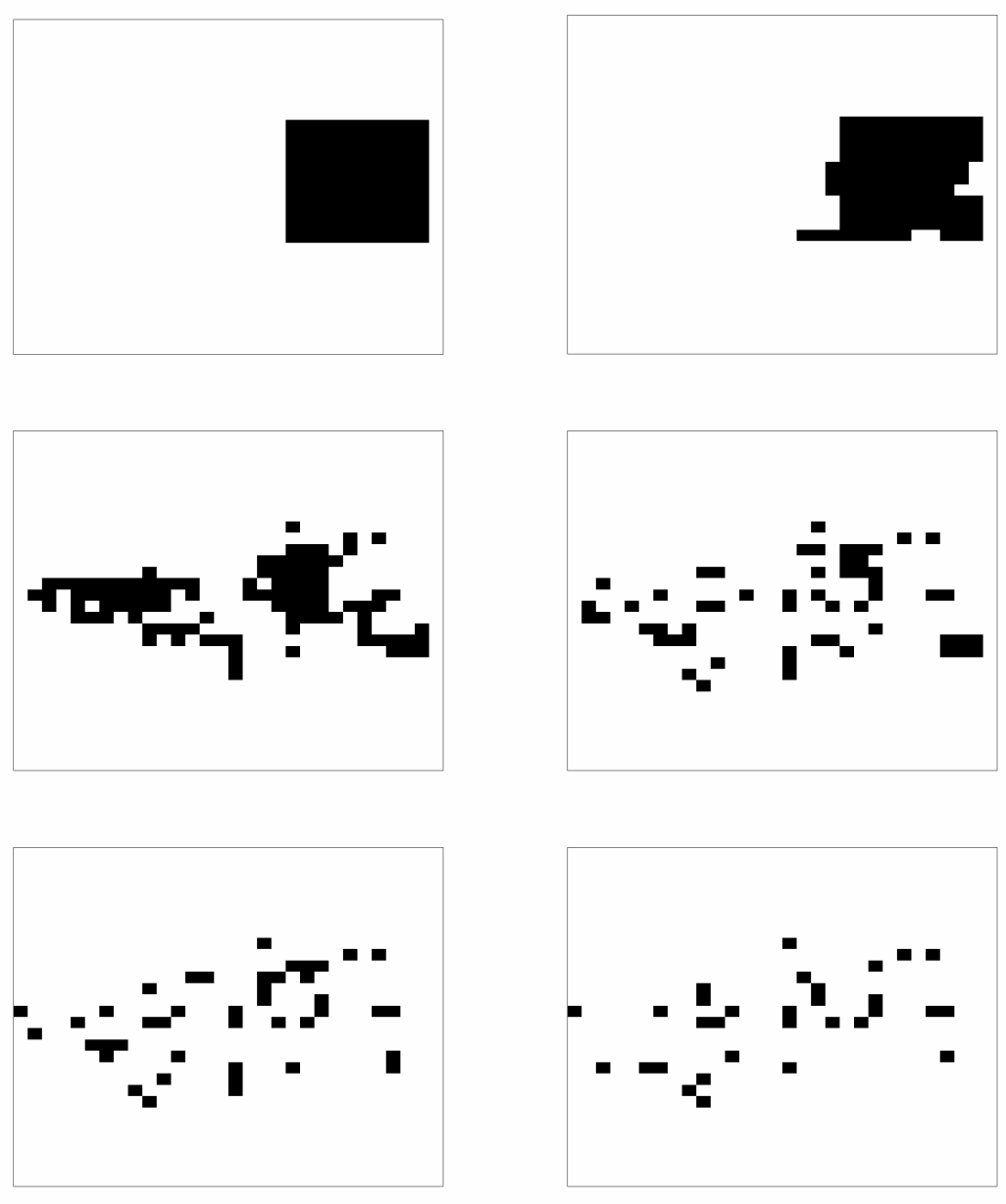

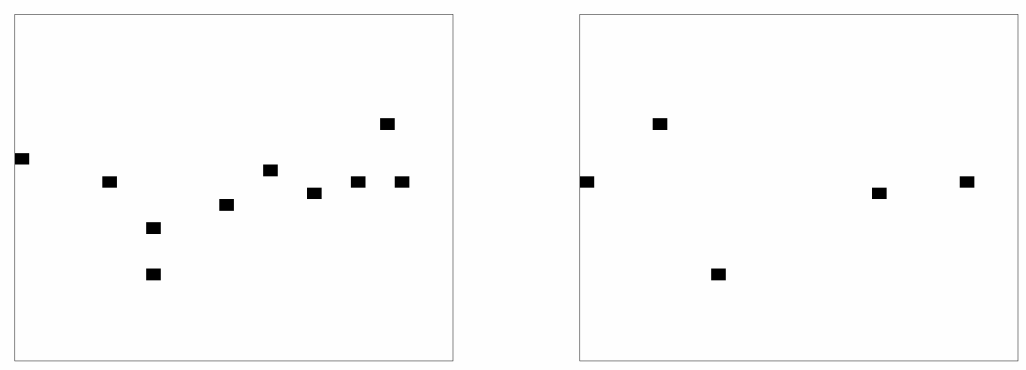

Fig.29 Snapshots of the mobilization process.

\section{CONCLUSIONS}

A new algorithm is developed, which can effectively solve the problem of the mobilization of a single fluid with yield stress in a network. The simulation results are in good agreement with the work done by Roux et al. The model was successfully used to model various situations. The distribution of the yield stress has obvious effects on the mobilization process. With a larger disorder (increase of the power $n$ ), the paths are more tortuous. A variety of geometries was studied. The algorithms were also used to model the displacement of a non-Newtonian fluid by a Newtonian fluid. We find that the patterns are affected by the viscosity ratio of the Newtonian fluid to the non-Newtonian fluid, in addition to the effect of the rheological parameters. Finally, the model is employed to describe the mobilization of a Bingham fluid trapped in a Newtonian fluid. As found in experiments, the Bingham fluid block is mobilized in the form of disconnected smaller ganglia, which move one by one.

\section{REFERENCES}

1 Barrenblatt, G.I., Entov, V.M., and Ryzhik, V.M.: Theory of fluid flows through natural rocks, Kluwer Academic Publishers, Netherlands, 1990.

2 Bedrikovetsky, P.: Principles of oil and gas production, Kluwer Academic Publishers, Netherlands, 1995.

3 Rossen, W. R. and Gauglitz, P.A.: "Percolation theory of creation and mobiliztion of foam in porous media”, AIChE J., 36(8) Aug. 1990, pp. 1176-1188.

4 Wu, Y.S., Pruess, K., and Witherspoon, P.A., SPERE, (1992) 369. 
5 Rossen, W.R. and Mamun,C.K.: "Minimal path for transport in networks", Phys. Rev. B 47(18) 1993-II, pp. 11815-11825.

6 Roux, S., and Herrmann, H.J.: "Disorder-induced nonlinear conductivity", Europhys. Lett., 4(11), 1987, pp. 1227-1231.

7 Sahimi, M.: "Non-linear transport processes in disordered media", AIChE J. 39(3) 1993, pp. 369-385.

8 Zhou, D. and Stenby, E.: "Displacement of trapped oil from water-wet reservoir rock", Transport in Porous Media, vol. 11, 1993, pp. 17-31.

9 Kharabaf, H. and Yortsos, Y.C.: "Invasion Percolation with memory," Phys. Rev. E (1997) 55, 7177.

10 Kharabaf, H. and Yortsos, Y.C.: "Pore Network Model for Foam Formation and Propagation in Porous Media", SPE J. March 1998 pp. 42-53.

11 Kharabaf, H.: PhD Dissertation, U. of Southern California, Los Angeles, California (1996).

12 Bird, R.B., Stewart, W.E., and Lightfoot, E.N.: Transport Phenomena, Wiley, New York (1960).

13 Lipscomb, G.G., and Denn, M.M.: "Flow of Bingham fluids in complex geometries," Journal of Non-Newtonian Fluid Mechanics 14(1984) 337-346. 



\section{Asymptotic Regimes in Unstable Miscible Displacements in Random Porous Media \\ Zhengmin M. Yang, Yannis C. Yortsos and Dominique Salin}

\section{INTRODUCTION}

In the past literature, two different regimes, the Transverse Equilibrium (TE) and the Dykstra-Parsons $(D P)$ regimes, have been used to model displacements in porous media. Transverse equilibrium is a commonly made assumption to describe fluid displacements $[12,18,27]$. In this regime, transverse (vertical) flow is extensive. The parameter $R_{L}=$ $L / H\left(k_{v} / k_{h}\right)^{1 / 2}$ was suggested [12] as the controlling factor for a system to approach $T E$ and was analytically shown to be so by Yortsos [27]. Here, $L$ and $H$ denote the length and width of the porous medium, $k_{h}$ and $k_{v}$ are mean values of the longitudinal and transverse permeabilities, respectively. By contrast, the Dykstra-Parsons (DP) approach [4] is used to describe processes when there is no transverse flow (for example, in the case of non-communicating layers). Using either TE or DP leads to considerable simplifications, due to the reduction in the number of primary variables, and facilitates the development of coarse-scale models and process scale-up (see also [16], [21]). Such reduction is of interest to coarse-gridding in large-scale simulations.

Although used extensively, the range of validity of these regimes remains unclear, and even confusing. This is particularly the case for unstable displacements and viscous fingering problems. It is one of the objectives of this paper to delineate the region of their validity in such cases. For this, we will proceed with an asymptotic analysis, from which we show that the $T E$ and $D P$ regimes are obtained in the limits $R_{L} \gg 1$ and $R_{L} \ll 1$, respectively. In either case, the problem is reduced to the solution of a single (and novel) integro-differential equation. The TE limit is akin to the lubrication approximation in viscous flows [9]. The validity of the two asymptotic approximations is investigated using the solution of the full problem with $R_{L}$ as a parameter. We use standard methods of High-Resolution Simulation (HRS) to study displacement patterns and the transverse averages in concentration and fluid mobility. The analysis is for 2-D geometries and for the miscible problem only.

The simulations show that the development of patterns and transverse averages depends 
on the parameter $R_{L}$, in addition to the mobility ratio, $M$. This is of importance, particularly as far as viscous fingering is concerned. As is well known, viscous fingering is a hydrodynamic instability that develops when a more viscous fluid is being displaced by a less viscous one in porous media, or in porous-media-like geometries (such as Hele-Shaw cells) [8, 14, 26]. Even though extensively studied, the evolution of viscous fingers in random porous media is still not completely understood, however. At a coarse scale (for example in terms of the transverse average of the injected fluid concentration), unstable miscible displacement is typically described using semi-empirical models, such as the Koval [11], the Todd and Longstaff [20], and the Fayers model [6] (see also [1]). None of these contain $R_{L}$ as a parameter, yet their validation is typically done using $H R S$ or experimental results at a particular $R_{L}$ value, usually $R_{L}=3$. At the same time, a basic hypothesis in their justification is the assumption of "parallel flow", which is implied in both the TE and the DP limits discussed above. With the exception of Waggoner [22], who made an attempt in the TE limit, a connection to these regimes has not yet been established. At present, none of the viscous fingering models can be derived in a self-consistent manner and must be considered as empirical. It is the second objective of this paper, therefore, to analyze the equations at the $T E$ limit, and to search for an alternative model. This model must be based on the distinction between fingered and non-fingered regions and account for transverse mixing and for the variance of the mobility (and concentration) in the fingered zones.

The paper is organized as follows: First, we proceed with a mathematical description from which we derive the $T E$ and $D P$ approximations, in the two limits $R_{L} \gg 1$ and $R_{L} \ll 1$, respectively. Then, we use $H R S$ to study the dependence of the patterns on $R_{L}$ and show that the two asymptotic limits are consistent with the full problem in the respective limits. The last section deals with the development of a viscous fingering model assuming $T E$ conditions.

\section{MATHEMATICAL FORMULATION}

We consider miscible flow in a heterogeneous, but spatially uncorrelated, porous medium of a two-dimensional, rectangular geometry. We proceed by assuming that the fluids are incompressible and first-contact miscible, and that mixing of fluids does not cause volume change. Based on these assumptions, the mathematical model is described by the following 
standard equations

$$
\begin{gathered}
\phi \frac{\partial C}{\partial T}+\frac{\partial(U C)}{\partial X}+\frac{\partial(V C)}{\partial Y}=\nabla \cdot(\mathbf{D} \nabla C) \\
U=-\frac{K_{h}}{\mu(C)} \frac{\partial P}{\partial X} \\
V=-\frac{K_{v}}{\mu(C)} \frac{\partial P}{\partial Y} \\
\frac{\partial U}{\partial X}+\frac{\partial V}{\partial Y}=0
\end{gathered}
$$

where capital letters stand for the real physical variables, $\phi$ is porosity, $C$ is concentration of the injected fluid (solvent), $U$ and $V$ are longitudinal and transverse velocities, $\mathbf{D}$ is the dispersion coefficient tensor, generally a function of velocity, $K_{h}$ and $K_{v}$ are longitudinal and transverse permeabilities, respectively, and $\mu(C)$ is viscosity, a function of $C$, in general. In this paper, the variation of $\mathbf{D}$ with velocity will not be considered (but see also [29]).

The above can be non-dimensionalized using the dimensionless parameters: $\kappa(x, y)=$ $\frac{K_{h}}{k_{h}}=\frac{K_{v}}{k_{v}} ; \delta=\frac{k_{v}}{k_{h}} ; \epsilon=\frac{H}{L} ; R_{L}=\frac{\sqrt{\delta}}{\epsilon} ; x=\frac{X}{L} ; y=\frac{Y}{H} ; c=\frac{C}{C_{o}} ; t=\frac{T}{\phi L / q} ; u=\frac{U}{q} ; v=\frac{V}{q} ;$ $w=\frac{v}{\epsilon} ; \lambda=\frac{\mu_{i}}{\mu}$; and $p=\frac{P k_{h}}{L q \mu_{i}}$, where subscript $o$ denotes injection, subscript $i$ denotes initial, $q$ is the injection velocity, and $\lambda(c)$ is a dimensionless mobility, generally a function of the concentration (and in the present notation varying in the interval $(M, 1)$ ). In this dimensionless notation, we then obtain the following system

$$
\begin{aligned}
\frac{\partial c}{\partial t}+\frac{\partial(u c)}{\partial x}+\frac{\partial(w c)}{\partial y}=N_{\|} \frac{\partial^{2} c}{\partial x^{2}} & +N_{\perp} \frac{\partial^{2} c}{\partial y^{2}} \\
u & =-\kappa \lambda \frac{\partial p}{\partial x} \\
\frac{1}{R_{L}^{2}} w & =-\kappa \lambda \frac{\partial p}{\partial y} \\
\frac{\partial u}{\partial x} & +\frac{\partial w}{\partial y}=0
\end{aligned}
$$

where we introduced the inverse Peclet numbers, $N_{\|} \equiv \frac{D_{\|}}{q L}$ and $N_{\perp} \equiv \frac{D_{\perp} L}{q H^{2}}$, to express longitudinal and transverse dispersion, respectively. If only molecular diffusion is considered, $N_{\|}=\epsilon N_{\perp}$. For the solution of (5)-(8) we will use conventional initial and boundary conditions: $c=0$ at $t=0 ; w=0$ at $y=0$ and $y=1 ; \int_{0}^{1} u d y=1, w=0$ (namely, $p=$ const 
$(t))$ and $c-N_{\|} \frac{\partial c}{\partial x}=1$ at $x=0$; and $\frac{\partial c}{\partial x}=0$ and $p=0$ at $x=1$. We will also consider the solution of the problem subject to periodic boundary conditions at $y=0$ and $y=1$.

The above formulation contains the dimensionless parameters $N_{\|}, N_{\perp}, R_{L}^{2}$, the functional relation between mobility and concentration, and the two scale ratios $\frac{\Lambda_{h}}{L}$ and $\frac{\Lambda_{v}}{H}$ (the latter arising implicitly in the case of spatially varying permeabilities, where $\Lambda_{i}$ denotes characteristic correlation lengths in the respective directions). Here, however, our focus is only on

displacements in uncorrelated fields of weak heterogeneity, where $\frac{\Lambda_{h}}{L}, \frac{\Lambda_{v}}{H} \ll 1$, hence such dependence will be neglected. As discussed above, the emphasis is placed on the effect of the modified aspect ratio, $R_{L}$. Note that in an isotropic medium, $R_{L}$ becomes the geometric aspect ratio, $R_{L}=\frac{L}{H}$, in which case large or small $R_{L}$ effectively implies large or small aspect ratio, respectively. In the following, we will first consider the asymptotic behavior of the system in the limits of large and small $R_{L}$, respectively.

\section{Large $R_{L}$ Limit}

Consider the asymptotic behavior of (5)-(8) in the limit $R_{L} \gg 1$. By taking a regular asymptotic expansion in terms of $R_{L}^{-2}$, namely

$$
\begin{aligned}
u & =u_{0}+\frac{1}{R_{L}^{2}} u_{1}+\ldots \\
w & =w_{0}+\frac{1}{R_{L}^{2}} w_{1}+\ldots \\
p & =p_{0}+\frac{1}{R_{L}^{2}} p_{1}+\ldots \\
c & =c_{0}+\frac{1}{R_{L}^{2}} c_{1}+\ldots
\end{aligned}
$$

one can show [27] that the pressure variation to leading-order is only a function of $x$, namely

$$
\frac{\partial p_{0}}{\partial x}=-\frac{1}{\int_{0}^{1} \kappa \lambda d y} \quad ; \quad \frac{\partial p_{0}}{\partial y}=0
$$

Then, the longitudinal velocity, $u$, can be explicitly related to the mobility (hence, the concentration) through 


$$
u_{0}=\frac{\kappa \lambda\left(c_{0}\right)}{\int_{0}^{1} \kappa \lambda\left(c_{0}\right) d y}
$$

In this limit, therefore, the concentration evolves according to

$$
\begin{array}{r}
\frac{\partial c_{0}}{\partial t}+\frac{\partial\left(u_{0} c_{0}\right)}{\partial x}+\frac{\partial\left(w_{0} c_{0}\right)}{\partial y}=N_{\|} \frac{\partial^{2} c_{0}}{\partial x^{2}}+N_{T D} \frac{\partial^{2} c_{0}}{\partial y^{2}} \\
u_{0}=\frac{\kappa \lambda\left(c_{0}\right)}{\int_{0}^{1} \kappa \lambda\left(c_{0}\right) d y} \\
w_{0}=-\frac{\partial}{\partial x}\left(\frac{\int_{0}^{y} \kappa \lambda\left(c_{0}\right) d y}{\int_{0}^{1} \kappa \lambda\left(c_{0}\right) d y}\right)
\end{array}
$$

It should be noted that although the pressure is to leading-order independent of the transverse coordinate $y$ (hence the term Transverse or Vertical Equilibrium), there exists substantial transverse mixing, as the transverse velocity $w$ is non-zero to first-order, in general. The transverse velocity could vanish in many cases, for example in tracer diffusion in a layered system, where $\lambda=1$ and $\kappa$ depends on $y$ only. In such cases, the vanishing of the velocity has often led to confusing the $T E$ with the $D P$ regime, where the leading-order transverse velocity is by definition nil (see also below).

Substitution of (16) and (17) into (15) leads to a single integro-differential equation for the evolution of the concentration

$$
\frac{\partial c}{\partial t}+\frac{\kappa \lambda}{\int_{0}^{1} \kappa \lambda d y} \frac{\partial c}{\partial x}-\frac{\partial}{\partial x}\left(\frac{\int_{0}^{y} \kappa \lambda d y}{\int_{0}^{1} \kappa \lambda d y}\right) \frac{\partial c}{\partial y}=N_{\|} \frac{\partial^{2} c}{\partial x^{2}}+N_{\perp} \frac{\partial^{2} c}{\partial y^{2}}
$$

where we have omitted subscript 0 for simplicity. The solution of (18) requires, in addition, a relation for the mobility dependence on concentration. Various conventional mixing rules can be used, including exponential and quarter-power laws [24]. As long as the viscosityconcentration dependence is monotonic, however, the results are generally insensitive to the particular form used, and only depend on the end-point mobility ratio, $M \equiv \frac{\mu_{i}}{\mu_{o}}$. On the other hand, non-monotonic variations can lead to qualitatively different results (e.g. see $[15])$.

Implied in the above is the use of no-flow boundary conditions at the lateral boundaries. As discussed in Yang and Yortsos [24], however, the problem at the TE limit suffers from a boundary effect. This can be avoided if we apply periodic boundary conditions. In this case, 
the expression for $u$ remains as in (16), however, the expression for the transverse velocity is modified to read [24]

$$
w=-\int_{0}^{y} \frac{\partial u}{\partial x} d y+\frac{\int_{0}^{1} \frac{1}{\kappa \lambda} \int_{0}^{y} \frac{\partial u}{\partial x} d y^{\prime} d y}{\int_{0}^{1} \frac{1}{\kappa \lambda} d y}
$$

The numerical solutions to be shown below are based on the use of such periodic boundary conditions.

Before proceeding, we must also point out the tacit assumption in (15)-(17) that the asymptotic expansion (9)-(12) is uniformly valid. Clearly, this assumption will break down when $w$ is large (namely when $v$ is of order one) and/or when $\kappa$ vanishes. The first may occur in places where $\kappa \lambda$ is discontinuous, for example, in places of permeability discontinuity in the case of a passive tracer, or at the tip of sharp fingers (for example, in Saffman-Taylor type problems), in the case of a homogeneous medium. The second will occur in places of permeability barriers.

\section{Small $R_{L}$ Limit}

An other distinct limit is obtained at small $R_{L}$. We now use the asymptotic expansion

$$
\begin{aligned}
u & =u_{0}+R_{L}^{2} u_{1}+\ldots \\
w & =w_{0}+R_{L}^{2} w_{1}+\ldots \\
c & =c_{0}+R_{L}^{2} c_{1}+\ldots
\end{aligned}
$$

and take the limit $R_{L} \rightarrow 0$, to obtain to leading order

$$
w_{0}=0
$$

Inserting in (8) shows that $u_{0}$ is $x$-independent. Using the appropriate boundary conditions, it is then not difficult to derive the following expression for the streamwise velocity

$$
u_{0}=\frac{1}{\int_{0}^{1} \frac{d x}{\kappa \lambda\left(c_{0}\right)} \int_{0}^{1} \frac{d y}{\int_{0}^{1} \frac{d x}{\kappa \lambda\left(c_{0}\right)}}}
$$


The concentration field is now obtained as the solution of the following integrodifferential equation

$$
\frac{\partial c}{\partial t}+\left[\frac{1}{\int_{0}^{1} \frac{d x}{\kappa \lambda(c)} \int_{0}^{1} \frac{d y}{\int_{0}^{1} \frac{d x}{\kappa \lambda(c)}}}\right] \frac{\partial c}{\partial x}=N_{\|} \frac{\partial^{2} c}{\partial x^{2}}+N_{\perp} \frac{\partial^{2} c}{\partial y^{2}}
$$

where subscript 0 was suppressed.

Equations (24) and (25) describe the so-called Dykstra-Parsons limit. Comparison between (18) and (25) shows the difference in transverse flow in the two regimes: Under TE conditions, transverse flow is significant and is driven by mobility or permeability variations. In the $D P$ regime, however, transverse flow is nil. In both cases, the streamwise velocity is explicitly related to concentration and permeability. In the $D P$ problem, it is only dependent on the transverse coordinate $y$ (namely, it is layer-dependent). In the TE regime, $u$ depends on both $x$ and $y$. However, when $c$ and $\kappa$ are only $y$-dependent, then $w$ will also vanish in the TE limit. This may lead to confusing $T E$ with $D P$ (where $w=0$ at all times) (see Lake [12]). Even so, the two expressions for $u$ are different. It is also worth noting that for a passive tracer in a layered system, the $T E$ limit coincides exactly with the $D P$ limit. This has also been another source of confusion between the two regimes in the literature.

\section{NUMERICAL RESULTS}

To study the validity of the above limits, the numerical solution of the full model was considered. Numerical simulations of unstable displacements have used various approaches, including finite-difference schemes [2], spectral methods [29] and methods based on simulation along characteristic directions or streamtubes $[10,19]$. Our simulations were conducted using finite differences based on the Total Variation Diminishing ( TVD) method with flux limiter [17], which is a dynamic weighting between lower- and higher-order difference schemes, depending on the local flow condition. For the simulation of unstable displacements, the instability was triggered by fluctuations either in permeability or in the inlet concentration, either of which resulted into similar patterns. The results to be shown below were obtained using fluctuations in the permeability field. The latter was taken to be random, with a small variance, corresponding to a Dykstra-Parsons coefficient (which is related to the log-normal variance [12]) of $V_{D P}=0.05$. 
The effect of $R_{L}$ on the fingering pattern in an unstable displacement is shown in Figure 1, for $M=10$, at $t=0.3$, and for different values of $R_{L}$. The results for finite $R_{L}$ were obtained using the full model, equations (5)-(8), which accounts for both pressure and concentration. The results for the $T E\left(R_{L} \gg 1\right)$ and $D P\left(R_{L} \ll 1\right)$ regimes were obtained from the respective $T E$ and $D P$ models, equations (18) and (25), which contain only concentration as the independent variable. In either, we made use of periodic boundary conditions at the lateral boundaries. The same computational grid (equal to $100 \times 100$ in this work, although a number of other grids were also used in [24]) was used in all simulations. Although physical dispersion was not included, the numerical scheme did generate some numerical dispersion. Note that variations in $R_{L}$ can be accomplished, for example, by varying the aspect ratio $L / H$, while keeping $k_{v} / k_{h}$ constant. Therefore, the patterns in Figure 1 and the subsequent figures with $R_{L}<1$ must be compressed, while those with $R_{L}>1$ must be stretched, if they were to reflect the actual pattern in physical space.

Figure 1 shows that as $R_{L}$ increases, the viscous fingering pattern shows a constantly decreasing number of fingers, which acquire a progressively larger width and a decreasing concentration. This can be attributed to the increased transverse mixing as $R_{L}$ increases. The emergence of a few dominant fingers at larger $R_{L}$ is consistent with previous works (e.g. Zimmerman and Homsy [29]) which show the coarsening of viscous fingering patterns as the process evolves. Viewed differently, this pattern reflects the fact that because of the large $R_{L}$ value (larger aspect ratio), there is sufficient time for the injected fluid to sample the transverse direction, resulting into larger transverse mixing. The mixing zone appears to decrease as $T E$ conditions are approached. Comparison between the various patterns shows that the TE limit, as described by the single equation (18), describes reasonably well the main features of the full problem at large $R_{L}$. A firm evidence that equation (18) indeed contains the long-wave fingering instability is provided in the Appendix, using a linear stability analysis.

Conversely, as $R_{L}$ decreases, the number of fingers increases, their width decreases and their concentration increases. These characteristics reflect the decrease of transverse flow and of communication between the fingers. Because of the small disorder in permeability (small $\left.V_{D P}\right)$, the $D P$ pattern practically shows no fingering characteristics, resembling a piston- 
like displacement, in contrast to its TE counterpart, which displays a persistent fingering behavior. Viewed differently, the $D P$ limit corresponds to the early part of a displacement process, in which very little time is given for the fluid to sample the transverse direction, thus resulting into negligible mixing. Comparison with the full simulations shows that the $D P$ limit, equation (25), is the correct limit of the full problem as $R_{L}$ tends to zero.

Figures 2-4 show concentration and pressure profiles at $t=0.3$, for $M=10$, and $R_{L}=10$, 1 and 0.01 , respectively. The concentration contours have the features described previously: as $R_{L}$ increases, the number of fingers decreases. The pressure profiles are quite interesting. At large $R_{L}$ (Figure 2), the pressure is practically independent of the transverse direction in accordance with the TE limit, equation (18). The pressure field rapidly approaches $T E$ conditions at a small distance downstream and upstream of the mixing zone. At $R_{L}=1$ (Figure 3 ) the pressure profile is more variable and clearly departs from the $T E$ prediction. Even more pronounced is the pressure fluctuation for small $R_{L}$ (Figure 4). Here, the profile has the characteristics corresponding to displacement in a structure of non-communicating layers. Compared to Figure 2, where the pressure displays characteristics of displacement in a homogeneous system, this difference is quite instructive.

These results indicate that when $R_{L}$ is greater than about 10, the viscous fingering pattern practically converges to that of the TE model, where the pressure contours are approximately independent of the transverse direction. At small $R_{L}$ (less than 0.1 ), on the other hand, the pattern is well described by the $D P$ model. Now, the pressure profile depends only on the mobility variation in the transverse direction as in a layered formation. These results appear to indicate that overall, the $T E$ and $D P$ approximations are good descriptions of the displacement in the respective limits. In reaching this conclusion, one should not neglect, however, the role of dispersion, which is present in all figures through numerical dispersion.

\section{VISCOUS FINGERING MODELS}

In describing viscous fingering in porous media, various empirical models have been devel-

oped. Popular among them are the models of Fayers [6], Koval [11] and Todd and Longstaff [20]. They are all based on a 1-D description in the absence of dispersion, using a transversely averaged concentration, as described in the following 


$$
\frac{\partial \bar{c}}{\partial t}+\frac{\partial F(\bar{c})}{\partial x}=0
$$

where $\bar{c}=\int_{0}^{1} c d y$. The fractional flow function $F=F(\bar{c})$, has a different functional form for the different models. In the Koval and the Todd and Longstaff models, it is defined as

$$
F(\bar{c})=\frac{\bar{c}}{\bar{c}+(1-\bar{c}) / M_{e}}
$$

where the effective mobility ratio $M_{e}<M$ is smaller than the nominal and is calculated as follows: In the Koval model, by making the assumption that viscous fingers have the fixed concentration $c_{f}=0.22$, from which $M_{e}=\frac{\mu_{i}}{\mu\left(c_{f}\right)}$, and in the Todd and Longstaff model by using the expression $M_{e}=\left(\frac{\mu_{i}}{\mu_{o}}\right)^{1-\omega}$, where parameter $\omega$ is determined empirically and it is typically taken to be about $2 / 3$ [20]. Either of these assumptions leads to the lowering of the effective viscosity ratio between injected and displaced fluids, and can be attributed to diffusion and transverse mixing. Equations (26) and (27) lead to the spreading profile

$$
\bar{c}=\frac{\sqrt{\frac{M_{e}}{x / t}}-1}{M_{e}-1}
$$

The model of Fayers incorporates additional physics, it consists of two equations, and has a fractional flow defined as

$$
F(\bar{c})=\frac{\Lambda c_{f}}{\Lambda+(1-\Lambda) \mu\left(c_{f}\right) / \mu_{i}}
$$

where $\Lambda$ is a measure of the "finger width". The latter was related to the "concentration of the finger", $c_{f}$, by ad-hoc expressions [5]-[6]. For example, the following different forms have been proposed

$$
\Lambda_{1}=a+(1-a) c_{f}^{\alpha}
$$

and

$$
\Lambda_{3}=c_{f}^{\beta /(1-\beta)}
$$

where the various unspecified parameters must be determined by fitting with experimental or numerical results.

We used HRS to compare the numerical solution with the various empirical models and to study their dependence on parameters. Figures 5-7 show the comparisons of the Koval, Todd 
and Longstaff, and Fayers (based on expression (31)) models to 2-D HRS results for different $R_{L}$ values. The $H R S$ results show the following features: As $R_{L}$ increases, the curves becomes steeper and the mixing zone length decreases. There is significant fluctuation in the results, which increases with increasing $R_{L}$. The results do not actually collapse on a single curve, but they fluctuate around a mean curve, which must be viewed as relating the expected value of $\bar{C}$ to the convective variable $x / t$. This stochastic aspect reflects the fingering instability. Both the full and the empirical models show that the velocities of the leading and trailing edges of the fingering zone correspond to a reduced viscosity ratio, compared to the actual, as indeed assumed in the empirical models. For example, the leading and trailing velocities of Figure 5 are approximately 1.8 and 0.55 , respectively, which are significantly different than the velocities which would have corresponded to the nominal ratio $M=10$, and which according to (28) (with $M_{e}=10$ ) should be 10 and 0.1 , respectively.

The goodness of the fit between empirical and numerical models depends on the value of $R_{L}$. When $R_{L}$ is of order 1 , the empirical models fit the numerical results very well. As $R_{L}$ increases (Figures 6-7), the agreement is less satisfactory, but the fit remains good. In addition, the fluctuation in the results appears to increase. It is evident that $R_{L}$ has an effect on the average fingering behavior. (As an extreme example to prove this point, consider the small $R_{L}(D P)$ limit, where the displacement is almost piston-like.) Such an effect, however, is actually not represented in the existing models, which do not contain $R_{L}$. At the same time, however, the form of the fractional flow function used in the empirical models reflects parallel flow (indeed the ratio of the flow conductances in $(27)$ is $\frac{\bar{c} M_{e}}{(1-\bar{c})}$, like what would be obtained in the TE limit (see also below)). One could surmise that these models actually pertain to TE. In fact, it can be shown that in theory, a convective model, namely one where variables depend only on the similarity variable $x / t$, as is the case with all these models, is only possible in the limit $R_{L} \rightarrow \infty$. However, a rigorous connection to $T E$ has not yet been established.

In the next section, we will attempt to shed some light to the situation, by deriving an effective model based on the TE limit. Before proceeding, however, it is worth remarking on the general structure of the fractional flow function $F(\bar{c}$; other parameters). 


\section{DISCUSSION}

Consider the transverse average (denoted by overbar) of equation (15), which is valid for any $R_{L}$. We can derive equation (26), by taking the decomposition $c=\bar{c}+c^{\prime}$ and $u=1+u^{\prime}$, which, after some calculations and transverse averaging, leads to the following expression

$$
F=\bar{c}+\overline{u^{\prime} c^{\prime}}
$$

This is a general result, valid for any displacement. Using a small-fluctuation theory $\left(\left|c^{\prime}\right| \ll\right.$ $\bar{c})$, Welty and Gelhar [23] showed that the second term on the RHS of (32) is a dispersive flux. However, in fully-developed viscous fingers, a small-fluctuation assumption is clearly invalid (e.g. see Figure 8), as fingered and non-fingered regions have substantially different properties. In fact, the empirical fingering models suggest that $\overline{u^{\prime} c^{\prime}}$ corresponds instead to a convective, rather than dispersive, term, possibly related to the mean value $\bar{c}$. It is apparent, therefore, that a different expansion than that in [23] is needed.

In addition, by comparing (32) with (27), the above models further suggest that under viscous fingering conditions, velocity and concentration fluctuations are positively correlated,

$$
\overline{u^{\prime} c^{\prime}}>0
$$

For example, for the Todd-Longstaff model we have

$$
\overline{u^{\prime} c^{\prime}}=\frac{\left(M_{e}-1\right) \bar{c}(1-\bar{c})}{M_{e} \bar{c}+1-\bar{c}}
$$

namely, in unstable displacements $\left(M_{e}>1\right)$, the cross-correlation between velocity and concentration is positive, reaches a maximum at the mid-point concentration and vanishes at the end-points.

These properties follow directly in the TE case, where the velocity is explicitly related to concentration and permeability (equation (16)). Indeed, under these conditions, and if we neglect permeability fluctuations (see also below), the cross-correlation reads as

$$
\overline{u^{\prime} c^{\prime}}=\frac{\overline{c^{\prime} \lambda^{\prime}}}{\bar{\lambda}}
$$


Truncating to second-order the expansion for $\lambda^{\prime}$ we have $\overline{c^{\prime} \lambda^{\prime}} \approx \dot{\lambda}(\bar{c}) \bar{c}^{\prime 2}$, hence

$$
\overline{u^{\prime} c^{\prime}} \approx \frac{\dot{\lambda}(\bar{c}) \bar{c}^{\prime 2}}{\bar{\lambda}}
$$

where $\dot{\lambda}(\bar{c})$ is the derivative of $\lambda$. Thus, under TE conditions, the cross-correlation is positive, for the unstable case $(\dot{\lambda}(\bar{c})>0)$, negative for the stable case $(\dot{\lambda}(\bar{c})<0)$, and vanishes at the end points $\left(c^{\prime}=0\right)$. Inserted in the fractional flow function, the latter results would lead to a spreading wave in the first case and a sharp front (piston-like displacement) in the second, as indeed expected.

Towards the development of a viscous fingering model under TE conditions

Consider, next, the derivation of a purely convective fingering model, which as mentioned above, is theoretically possible at conditions of TE. We start with the basic set of TE equations, summarized below for convenience

$$
\begin{array}{r}
\frac{\partial c}{\partial t}+u \frac{\partial c}{\partial x}+w \frac{\partial c}{\partial y}=N_{\|} \frac{\partial^{2} c}{\partial x^{2}}+N_{\perp} \frac{\partial^{2} c}{\partial y^{2}} \\
u=\frac{\kappa \lambda}{\int_{0}^{1} \kappa \lambda d y} \\
\frac{\partial u}{\partial x}+\frac{\partial w}{\partial y}=0
\end{array}
$$

Equivalently, we can use a mobility formulation, instead of (38), by multiplying by $\dot{\lambda}=d \lambda / d c$ (assumed non-zero) to obtain, in place of (37)

$$
\frac{\partial \lambda}{\partial t}+u \frac{\partial \lambda}{\partial x}+w \frac{\partial \lambda}{\partial y}=N_{\perp} \dot{\lambda} \frac{\partial^{2} c}{\partial y^{2}}
$$

where in the latter we omitted longitudinal dispersion.

As in other contexts of unstable flow [13], a sound framework for the development of models for the average (up-scaled) behavior is the method of moments. These can be constructed, for example, for the concentration formulation, by multiplying $(38)$ by $n c^{n-1}(n=1,2,3 \ldots)$ and integrating over $y$ in the interval $[0,1]$ to obtain 


$$
\frac{\partial}{\partial t}\left(\int_{0}^{1} c^{n} d y\right)+\frac{\partial}{\partial x}\left(\frac{\int_{0}^{1} \kappa \lambda c^{n} d y}{\int_{0}^{1} \kappa \lambda d y}\right)=N_{\|} \epsilon^{2} n \int_{0}^{1} c^{n-1} \frac{\partial^{2} c}{\partial x^{2}} d y-N_{\perp} n(n-1) \int_{0}^{1}\left(\frac{\partial c}{\partial y}\right)^{2} c^{n-2} d y
$$

$(n=1,2,3 \ldots)$, where we made use of the lateral boundary conditions. As noted before, the first moment $(n=1)$ provides an expression for the fractional flow function, i.e.

$$
F \equiv \frac{\overline{\kappa \lambda c}}{\overline{\kappa \lambda}}=\frac{\bar{\lambda} \bar{c}+\overline{\bar{c}} \overline{\kappa^{\prime} \lambda^{\prime}}+\bar{\lambda} \overline{\kappa^{\prime} c^{\prime}}+\overline{c^{\prime} \lambda^{\prime}}+\overline{\kappa^{\prime} \lambda^{\prime} c^{\prime}}}{\bar{\lambda}+\kappa^{\prime} \lambda^{\prime}}
$$

This shows that the fractional flow function is the sum of contributions of various crosscorrelation terms. Equivalently, using the mobility formulation, we can construct moments of $\lambda$ by multiplying (40) by $n \lambda^{n-1}$ and integrating over $y$ in the interval $(0,1)$, as before, to get

$$
\frac{\partial}{\partial t}\left(\int_{0}^{1} \lambda^{n} d y\right)+\frac{\partial}{\partial x}\left(\frac{\int_{0}^{1} \kappa \lambda^{n+1} d y}{\int_{0}^{1} \kappa \lambda d y}\right)=-N_{\perp} \int_{0}^{1}\left(\lambda^{n}\right)^{\cdot}\left(\frac{\partial c}{\partial y}\right)^{2} d y
$$

From now on, we will proceed with the simpler mobility formalism.

It was pointed out before that in order to apply a small-fluctuation approach, we need to distinguish between fingered and non-fingered regions (see Figure 8), and define transverse averages over the finger region only. This is necessary, in view of the sharp contrast expected between the variables in the two regions. We can delineate viscous finger regions by requesting that concentrations there exceed a small threshold $c_{\epsilon}(\ll 1)$. This is akin to turbulent flow modeling (e.g. of free shear flows), where the turbulent region is separated from the initial by a contorted surface (the so-called viscous superlayer) [13]. This surface is analogous in our problem, to the viscous finger boundaries. A cut across a constant value of $x$ (as shown in Figure 8) gives rise to a discontinuous curve, separating fingered from non-fingered regions, and characterized by intermittency. Formally, the latter can be defined by the intermittency function,

$$
I=H\left(c-c_{\epsilon}\right)
$$

where $H(z)$ is the Heaviside function. For completeness, we note that the transverse average of $I$ is, in fact, the fraction occupied by fingers and which equals the finger width $\Lambda$, introduced in Fayers' model, 


$$
\bar{I}=\Lambda(x, t)
$$

In this view, therefore, the finger width $\Lambda(x, t)$ is the probability that the point $(x, y, t)$ is occupied by a viscous finger.

Further progress requires an equation for the evolution of $\Lambda$. By taking mass balances across different fingers it can be shown that $\Lambda$ satisfies the following equation, valid for arbitrary $R_{L}$

$$
\frac{\partial \Lambda}{\partial t}+\frac{\partial(\Lambda<u>)}{\partial x}=S
$$

where brackets denote transverse averages over the fingered region. Term $S$ on the RHS denotes transport between fingered and non-fingered regions and contains fluctuations and diffusion contributions. Obviously, the specification of this term is crucial (see below).

Having derived an equation for the evolution of $\Lambda$, we can define moments over the fingered region only. Consider, for example, the first moment of the mobility, $n=1$ in (43). For convenience, we introduce the normalized mobility

$$
\lambda_{f}=\frac{\lambda-1}{M-1}
$$

which varies in the interval $[0,1]$. In this notation, therefore, $\lambda_{f}$ vanishes outside the finger. After various manipulations and by neglecting the fluctuations of permeability, the equation for the first moment can be shown to be

$$
\frac{\partial}{\partial t}\left[\Lambda<\lambda_{f}>\right]+\frac{\partial}{\partial x}\left[\frac{\Lambda\left[<\lambda_{f}>+(M-1)<\lambda_{f}>^{2}\left(1+\frac{\sigma_{\lambda}^{2}}{\left.<\lambda_{f}\right\rangle^{2}}\right)\right]}{1+(M-1) \Lambda<\lambda_{f}>}\right]=-N_{\perp} \Lambda<\ddot{\lambda}_{f}\left(\frac{\partial c}{\partial y}\right)^{2}>
$$

where we defined the variance of the mobility inside the fingered region

$$
\sigma_{\lambda}^{2}=\left(\lambda_{f}-<\lambda_{f}>\right)^{2}
$$

Equation (48) contains the unknown term, $\sigma_{\lambda}^{2}$. For its calculation, an additional equation for the second moment can be constructed, which, however, will involve higher-order moments, 
etc. Instead, we elected to close the system by postulating an equation for the variance, using the HRS results. Figure 9 shows numerical results of the variance plotted vs. the mean value $\left\langle\lambda_{f}\right\rangle$. As expected, the variance vanishes at the end points and reaches a maximum value somewhere in-between. This qualitative behavior is expected, since the endpoints correspond to the tip and base of the mixing zone, where all variances must vanish. Yang [24] found that to a good approximation, this maximum is nearly independent of $M$ for the values tried, and can be approximately fitted with the best-fit expression

$$
\sigma_{\lambda}^{2}=\lambda_{f}^{1.8}\left(1-\lambda_{f}\right)^{2.5}
$$

We will use this approximation to express the unknown variance in (48). Its importance can be seen by noting that although the ratio $\frac{\sigma_{\lambda}^{2}}{\left\langle\lambda_{f}\right\rangle^{2}}$ becomes small at values of $\left\langle\lambda_{f}\right\rangle$ near unity, it cannot be neglected at smaller values of $\left\langle\lambda_{f}\right\rangle$ (which in fact dominate the fingering behavior).

In summary, a viscous fingering model can be constructed by using the system of equations (46),(48) and (50), and solved subject to the boundary conditions $\Lambda(x, 0)=0,<$ $\lambda_{f}(x, 0)>=0, \Lambda(0, t)=1,<\lambda_{f}(0, t)>=0$. Such a model would consist of two differential equations, like Fayers', rather than a single equation, as in Koval, and Todd and Longstaff. This approach is rigorous, assuming TE conditions. However, the solution of the problem depends crucially on the specification of the term $S$. Waggoner [22] attempted a similar VF model at TE. In our notation, his model is equivalent to taking $S=0$ and $\sigma_{\lambda}^{2}=0$. This choice leads, however, to a spreading wave with a leading edge velocity equal to the nominal viscosity ratio $M$, which is much faster than what is found in the simulations. This solution underpredicts the propagation speed of the base and overpredicts the propagation speed of the tip. Yang [24] considered the different case

$$
S=\Lambda \frac{\partial<u>}{\partial x}
$$

and solved the system numerically. Results are shown in Figure 10. The match between the simpler model and the numerical results is quite good. Important features are well captured, especially the steep mobility shock in the finger base region, and the front retardation. However, it is possible that some of the features actually reflect numerical dispersion effects. 
Interpolating between these two case, we have also considered the ansatz $S=(1-$ $\beta) \Lambda \frac{\partial\langle u\rangle}{\partial x}$, where $0<\beta<1$ is an adjustable parameter. Then, an analysis of the resulting hyperbolic system shows that (in the case of negligible variance) the solution paths satisfy the equation

$$
<\lambda_{f}>=(\text { const }) \Lambda^{\frac{1-\beta}{\beta}}
$$

Interestingly, this equation is very similar to Fayers' model (31). Under the latter assumption, the solution of the system gives features similar to Fayers' model.

While several of these approximations appear promising, non-trivial progress will require considerably more insight on this exchange parameter $S$. At this point, the problem can still be considered to be open.

\section{CONCLUSIONS}

In this paper, we studied the asymptotic behavior of miscible displacements in porous media in the two limits, where a permeability-modified aspect ratio, $R_{L}=L / H\left(k_{v} / k_{H}\right)^{2}$, becomes large or small, respectively. The first limit is known as Transverse (or Vertical) Equilibrium (TE), while the second leads to the problem of displacement in a non-communicating layered system (the so-called Dykstra-Parsons (DP) problem). In either case the problem reduces to the solution of a single integro-differential equation. By comparison with HRS results, we showed the important effect of $R_{L}$, particularly for viscous fingering problems. For the latter case, we described the development of a viscous fingering model under TE conditions. The model is based on a small-fluctuation approach for quantities inside the finger regions, and as in Fayers' model, it consists of a set of two equations. The model was shown to depend crucially on the specific expression that describes the interaction between fingered and non-fingered regions. Although good results were found for a specific approximation, the origin and justification of such approximation are lacking and further work is needed.

Before closing, some additional remarks are appropriate. First, it is important to comment on the validity of the TE approximation. While the single integro-differential equation does capture viscous fingering instabilities (see Appendix), and the solution of the problem 
has many common features with the $H R S$ results, this approximation cannot predict the properties of developed fingers in homogeneous porous media (for example, the SaffmanTaylor finger in a homogeneous Hele-Shaw cell [14]). The perturbation expansion becomes singular precisely at the tip of the finger (where the transverse velocity $v$ is $O(1)$ ) and the full solution of the problem is needed in order to predict the properties of the fully-developed finger. This inadequacy for homogeneous problems is in fact more severe if one were to explain, based on such an assumption, the selection principle of the homogeneous SaffmanTaylor problem [3]. The apparent success of the TE approach in the present case must be due to two factors: (1) the existence of the random perturbations to which the displacement front is continuously subject, which lead to continuous tip splitting, a random motion of the fingers and self-cancellations of the TE errors, and (2) the effect of diffusion, which although small, it is sufficient to dilute the sharpness of the fingers. The TE model has the obvious advantage that it contains only one independent variable. Although the final model is still subject to an unknown variable, namely the exchange term $S$, it can be the basis of further study, which can hopefully lead to a more rigorous result. 


\section{References}

[1] Blunt, M.J. and Christie, M.A., "Exact Solutions for Viscous Fingering in Two-Phase, Three-Component Flow", paper SPE 22613 (1991).

[2] Christie, M.A., "High-Resolution Simulation of Unstable Flows in Porous Media", SPE Reservoir Engineering, 297 (1989).

[3] Combescot, R., Dombre, T., Hakim, V., and Pomeau, Y., "Shape Selection of SaffmanTaylor Fingers", Phys. Rev. Lett. 56, 2036 (1986).

[4] Dykstra, H. and Parsons, R.L., "The Prediction of Oil Recovery by Waterflooding, Secondary Recovery of Oil in the United States", 2nd ed., API, 160-174 (1950).

[5] Fayers, F.J., "An Approximate Model with Physically Interpretable Parameters for Representing Viscous Fingering", SPE Reservoir Engineering, 551-558 (1988).

[6] Fayers, F..J., Jouaux, F. and Tchelepi, H.A., "An Improved Macroscopic Model for Viscous Fingering and Its Validation for 2D and 3D Flows - I. Non-Gravity Flows", In Situ 18, 43-78 (1994).

[7] Hickernell, F.J., and Yortsos, Y.C., "Linear Stability of Miscible Displacement in Porous Media in the Absence of Dispersion", Studies in Appl. Math. 24, 93-115 (1986).

[8] Homsy, G.M., "Viscous Fingering in Porous Media", Ann. Rev. Fluid Mech. 19, 271-311 (1987).

[9] Joseph, D.D. and Renardy, Y.Y., Fundamentals of Two-Fluid Dynamics, SpringerVerlag (1992).

[10] King K.J., Blunt M.J., Mansfield M. and Christie M.A., "Rapid Evaluation of the Impact of Heterogeneity on Miscible Gas Injection", paper SPE 26079 (1994).

[11] Koval, E.J., “A Method for Predicting the Performance of Unstable Miscible Displacement in Heterogeneous Media", SPEJ 3, 145-155 (1963).

[12] Lake, L.W., Enhanced Oil Recovery, Prentice Hall, New York (1989). 
[13] Pope, S.B., Turbulent Flows, Cambridge University Press (2000).

[14] Saffman, P.G. and Taylor, G.I., "The Penetration of a Fluid into a Porous Medium or Hele-Shaw Cell Containing a More Viscous Fluid", Proc. Roy. Soc. London Ser. A 245, $312(1958)$.

[15] Shariati, M. and Yortsos, Y.C., "Stability of Miscible Displacements Across Stratified Porous Media", Phys. Fluids 13, 2245-2257 (2001).

[16] Sorbie, K.S., Pickup, G.E., Ringrose, P.S. and Jensen, J.L., "Flow Regimes in Miscible Displacements in Heterogeneous Correlated Random Fields", paper SPE/DOE 24140 (1992).

[17] Sweby P.K.,"High-Resolution Schemes Using Flux Limiters for Hyperbolic Conservation Laws", SIAM J. Numerical Analysis 21, 995-1011 (1984).

[18] Tchelepi, H.A., and Orr Jr., F.M.: "Interaction of Viscous Fingering, Permeability Heterogeneity and Gravity Segregation in Three Dimensions", SPE Reservoir Engineering, 266 (1994).

[19] Thiele, M.R., Batycky, R.P., Blunt, M.J. and Orr, Jr., F.M., "Simulating Flow in Heterogeneous Media Using Streamtubes and Streamlines", SPE Reservoir Engineering, $5(1996)$.

[20] Todd, M.R. and Longstaff, W.J., "The Development, Testing, and Application of a Numerical Simulator for Predicting Miscible Flood Performance", J. Pet. Tech., 874$882(1972)$.

[21] Waggoner, J.R., Castillo, J.L. and Lake, L.W., "Simulation of EOR Processes in Stochastically Generated Permeable Media", SPE Formation Evaluation, 173 (1992).

[22] Waggoner, J.R., “The Growth of Viscous Fingers", Ph.D. Dissertation, University of Texas, Austin (1990). 
[23] Welty, C. and Gelhar, L.W., "Simulation of Large Scale Transport of Variable Density and Viscosity Fluids Using a Stochastic Mean Model", Water Res. Res. 28, 815-827 (1992).

[24] Yang, Z.M., “Application of Transverse Flow Equilibrium in Miscible Displacements", Ph.D. Dissertation, University of Southern California (1995).

[25] Yang, Z.M. and Yortsos, Y.C., "Effect of No-Flow Boundaries on Viscous Fingering in Porous Media of Large Aspect Ratio", SPEJ, 285-292 (1998).

[26] Yortsos, Y.C., "Instabilities in Displacement Processes in Porous Media", J. Phys.: Condens. Matter 2, SA443-SA448 (1990).

[27] Yortsos, Y.C., “A Theoretical Analysis of Vertical Equilibrium”, TiPM 18, 107-129 (1995); also, paper SPE 22612 (1991).

[28] Yortsos, Y.C. and Hickernell, F.J., "Linear Stability of Immiscible Displacement in Porous Media", SIAM J. Appl. Math. 49, 730-48 (1989).

[29] Zimmerman, W.B. and Homsy G.M.,"Viscous Fingering in Miscible Displacements: Unification of Effects of Viscosity Contrast, Anisotropic Dispersion, and Velocity Dependence of Dispersion on Nonlinear Finger Propagation", Phys. Fluids A 4, 1099 (1992). 


\section{APPENDIX}

In this Appendix we carry out a linear stability analysis to determine whether the TE equation (18) admits the conventional viscous instability (e.g., as described in [8] or [26] and references therein). We consider a homogeneous porous medium and take a base state independent of $y$. In the moving coordinate $\xi=x-t$, and neglecting longitudinal dispersion, the base-state is $c=\tilde{c}(\xi)$. We will take the substitution $c=\tilde{c}(\xi)+\zeta$, and look for the evolution

of the disturbance $\zeta$. By taking the linear limit, in which second-order terms are neglected, the following equation describes the evolution of $\zeta$

$$
\zeta_{t}+\left(\zeta-\int_{0}^{1} \zeta d y\right) g(\xi)=N_{\perp} \zeta_{y y}
$$

in which we denoted

$$
g(\xi)=\frac{\mathrm{d} \ln \lambda(\tilde{c})}{\mathrm{d} \xi}
$$

and, without loss, we neglected longitudinal dispersion.

To solve (53) we first note the interesting property

$$
\int_{0}^{1} \zeta d y=\operatorname{const}(\xi) \equiv S_{1}(\xi)
$$

obtained by integrating (53) over $y$ in $[0,1]$ and using the no-flux boundary conditions. Equation (55) implies that the growing disturbances compete and grow at the expense of the others. We next make the substitution, $\zeta=S_{1}+e^{g t} \chi$, to find that $\chi$ solves the heat equation

$$
\chi_{t}=N_{\perp} \chi_{y y}
$$

Its solution is expressed in terms of a Fourier series

$$
\chi=\sum_{0}^{\infty} A_{n}(\xi) \exp \left[-n^{2} \pi^{2} N_{\perp} t\right] \cos (n \pi z)
$$

Thus, in the linear limit the disturbance $\zeta$ evolves according to 


$$
\zeta=S_{1}+\sum_{1}^{\infty} A_{n}(\xi) \exp \left[-\left(n^{2} \pi^{2} N_{\perp}+g\right) t\right] \cos (n \pi y)
$$

It follows that the rate of growth of the disturbance, $\omega$, is related to the wavenumber $n$ through

$$
\omega=-g-n^{2} \pi^{2} N_{\perp} \quad ; \quad n=1,2, \cdots
$$

Parabolic relations between the rate of growth and the wavenumber, similar to (59), have been derived in previous stability analyses of the full problem (e.g. [7], [28]). The importance of the logarithmic derivative of the mobility in controlling the instability was also pointed out before [7].

Equation (59) can be used to infer the stability of the displacement:

(i) If $g>0$ everywhere, all disturbances are stable and decay to a uniform profile, $\zeta \rightarrow S_{1}(\xi)$. Stability, therefore, requires that the logarithmic derivative of the mobility profile is positive everywhere, namely that the mobility increases in the direction of displacement, or, equivalently, that a more viscous fluid displaces a less viscous fluid, as expected.

(ii) If, on the other hand, $g<0$ somewhere, large wavelength modes may become unstable. Instability requires the condition

$$
-g_{m} \geq \pi^{2} N_{\perp} \quad ; \quad-g_{m} \equiv \max -g
$$

Here, the fastest growing mode corresponds to $n=1$. However, wavenumbers larger than a cutoff value, $n^{*}$, where

$$
1<n^{*}=\frac{1}{\pi} \sqrt{\frac{-g_{m}}{N_{\perp}}}
$$

are stable due to transverse dispersion. Finally, if

$$
-g_{m} \leq \pi^{2} N_{\perp}
$$

namely if the mobility profile is not too steep or dispersion is sufficiently strong, stability is unconditional. These results are consistent with conventional viscous instability notions, 
namely that the instability is driven by unfavorable mobility and that dispersion is stabilizing short-wavelength disturbances.

The relevance of the previous stability analyses to the present work is not surprising. TE applies to long and narrow systems, hence all waves in such geometries would correspond to short wavelengths in the unbounded geometries of the previous works. This explains why the rate of growth $\omega$ in the TE problem is not proportional to the wavenumber at small wavenumbers (e.g., as in the Saffman-Taylor problem), but it is instead constant. In the absence of dispersion, all such wavelengths would grow at the same rate [7]. Nonetheless, in both formulations viscous instability is a result of non-local effects and arises from the unfavorable mobility contrast. 


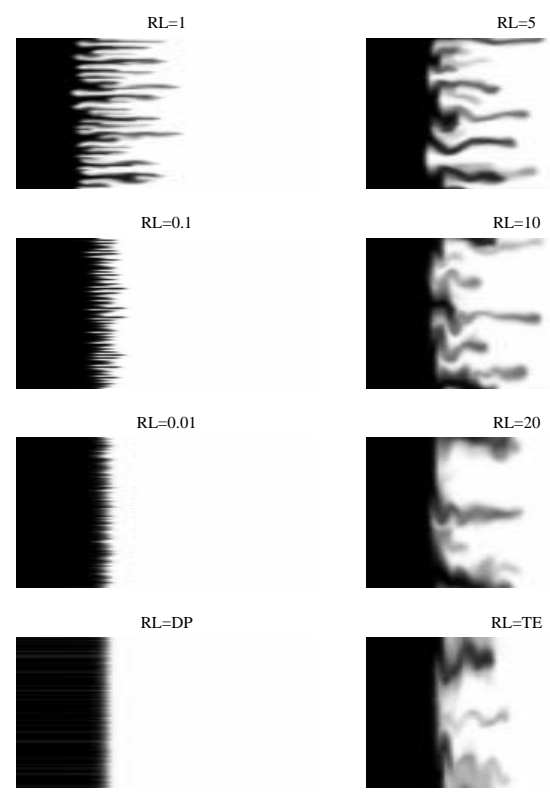

Figure 1: Unstable Displacement Patterns in a Porous Medium of a Rectangular Geometry (where the $x$ and $y$ axes aligned along the horizontal and vertical direction, respectively) for $M=10, t=0.3$ and for Different Values of $R_{L}$. Displacement is From-Left-To-Right.
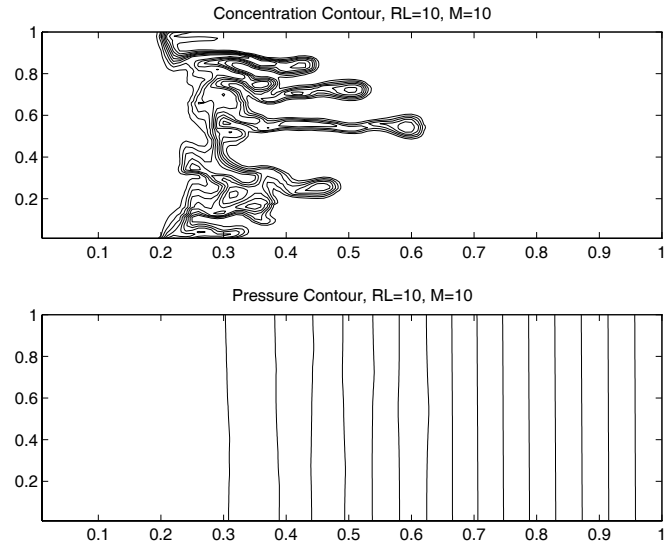

Figure 2: Concentration and Pressure Contours for an Unstable Displacement in the Same Porous Medium as in Figure 1, for $M=10, t=0.3$ and $R_{L}=10$. 

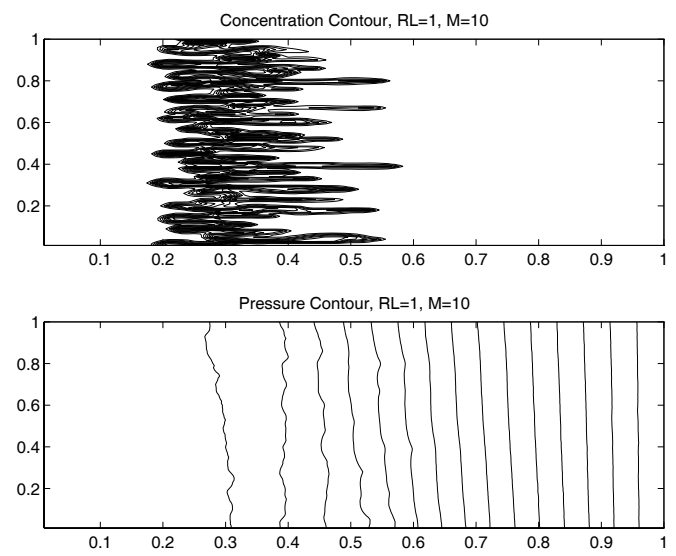

Figure 3: Concentration and Pressure Contours for an Unstable Displacement in the Same Porous Medium as in Figure 1 , for $M=10, t=0.3$ and $R_{L}=1$.
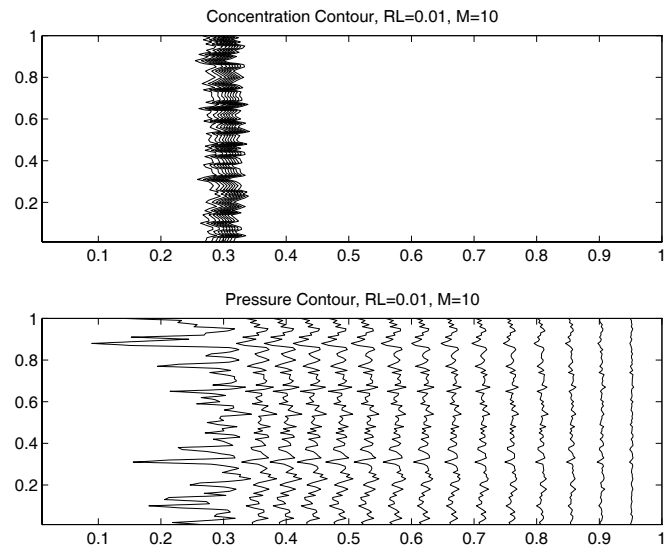

Figure 4: Concentration and Pressure Contours for an Unstable Displacement in the Same Porous Medium as in Figure 1 , for $M=10, t=0.3$ and $R_{L}=0.01$. 


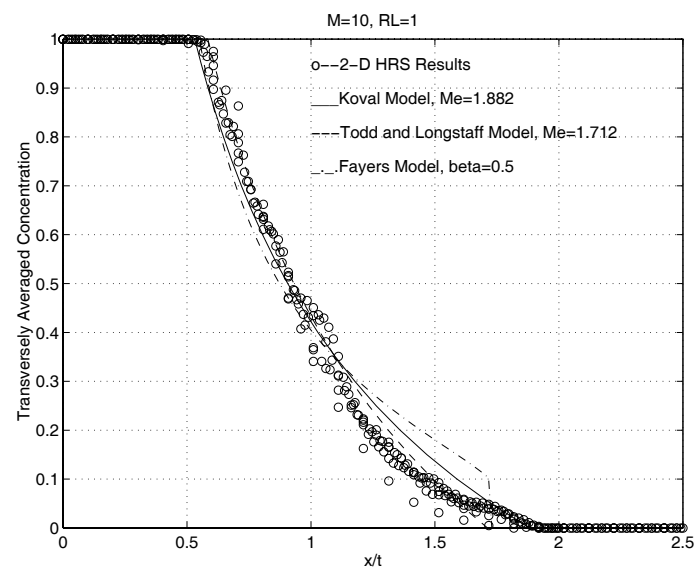

Figure 5: Comparison Between the Koval, Todd-Longstaff and Fayers Models and 2-D HRS Results at $R_{L}=1$.

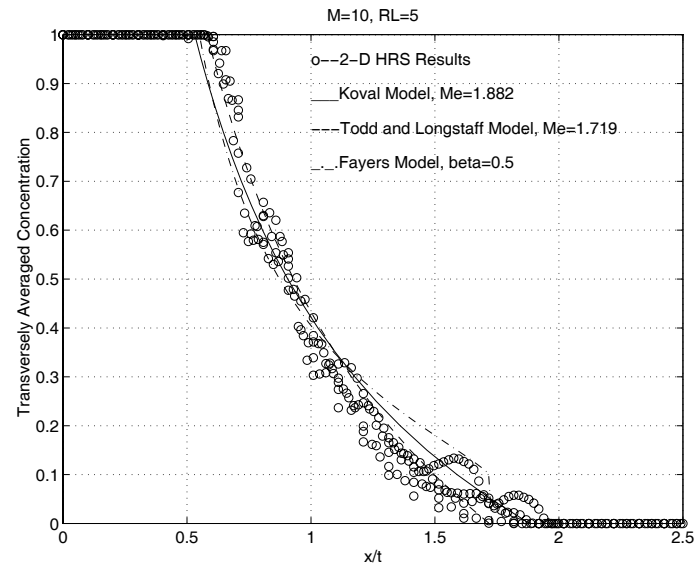

Figure 6: Comparison Between the Koval, Todd-Longstaff, Fayers Models and 2-D HRS Results at $R_{L}=5$. 


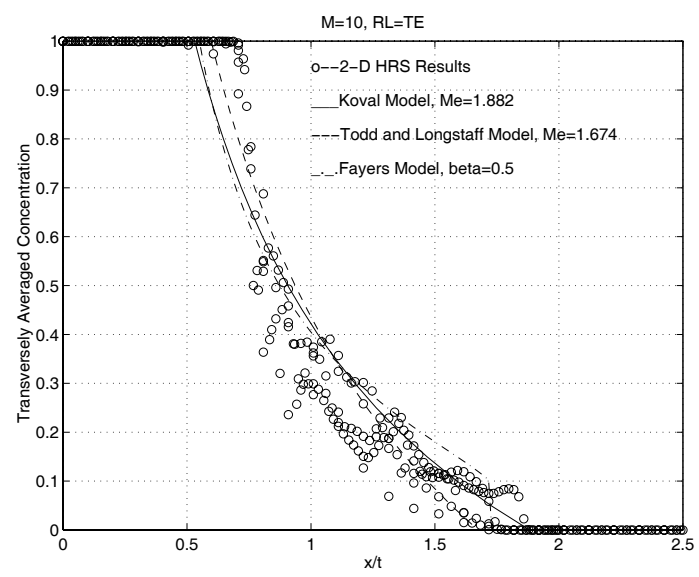

Figure 7: Comparison Between the Koval, Todd-Longstaff, Fayers Models and 2-D HRS Results Under TE Conditions.
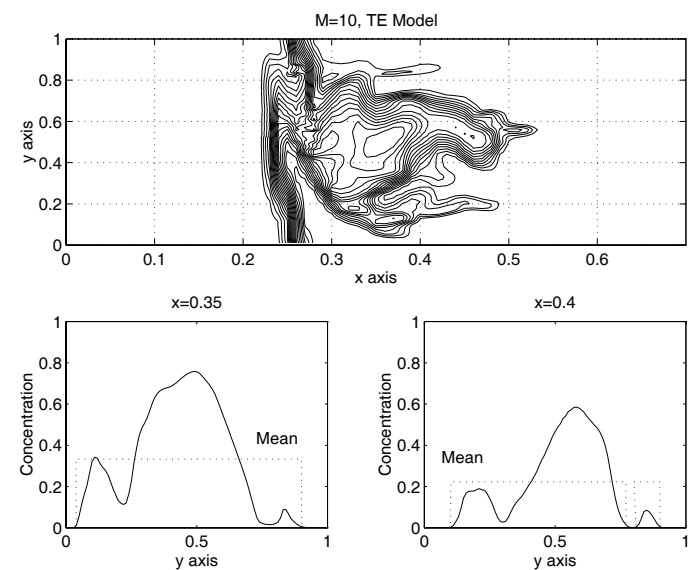

Figure 8: Definition of the Fingered and Non-Fingered Regions. 


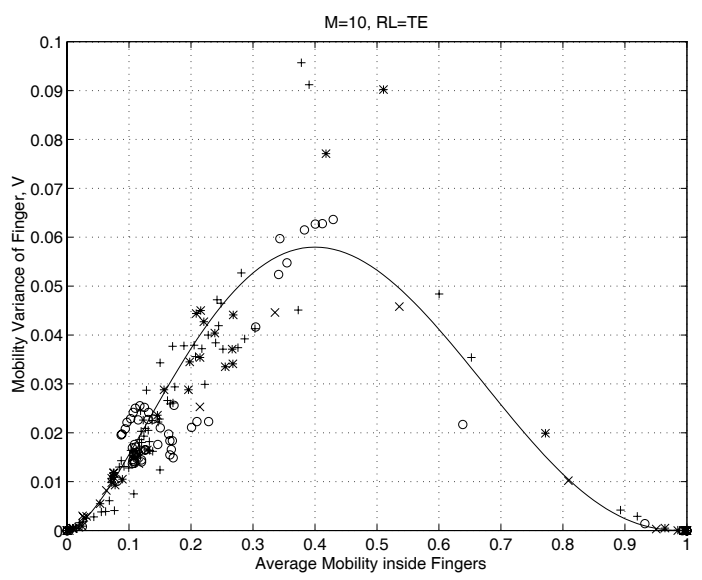

Figure 9: The Variation of the Scaled Finger Mobility Variance $\sigma_{\lambda}^{2}$ vs. the Scaled Average Finger Mobility, $\left\langle\lambda_{f}\right\rangle$. The Solid Line Denotes the Fit with Expression (50) ( $M=10$, $T E)$.
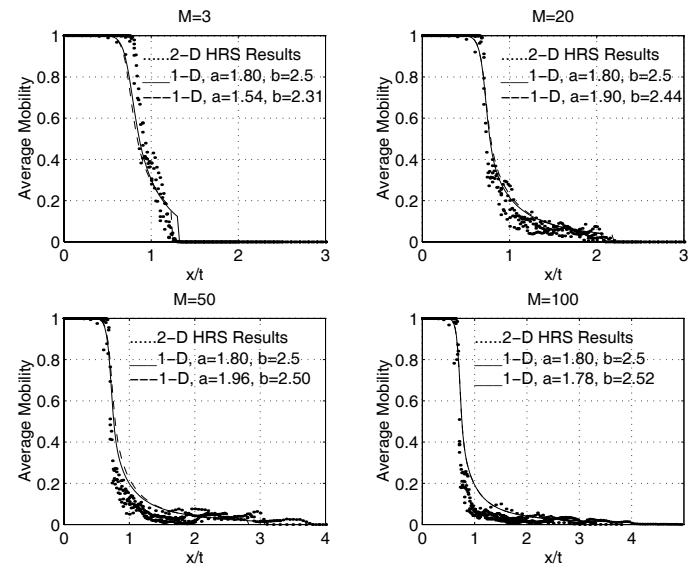

Figure 10: Comparison Between 2-D HRS Results and the Viscous Fingering Model for Different Values of $M$. 